\title{
Actions of lignocellulolytic enzymes on Abies grandis (grand fir) wood for application in biofuel production
}

\author{
Dissertation \\ In Partial Fulfillment of the Requirements for the Degree \\ Doctor of Philosophy $(\mathrm{PhD})$ \\ of the Faculty of Forest Sciences and Forest Ecology \\ Georg-August-University Göttingen
}

Submitted by

Banyat Cherdchim

born in Songkhla, Thailand

Göttingen, 2010 





\title{
Actions of lignocellulolytic enzymes on Abies grandis (grand fir) wood for application in biofuel production
}

\author{
Dissertation \\ In Partial Fulfillment of the Requirements for the Degree \\ Doctor of Philosophy $(\mathrm{PhD})$ \\ of the Faculty of Forest Sciences and Forest Ecology \\ Georg-August-University Göttingen
}

Submitted by

Banyat Cherdchim

born in Songkhla, Thailand

Göttingen, 2010 
D7

$1^{\text {st }}$ Referee: Prof. Dr. Ursula Kües

$2^{\text {nd }}$ Referee Prof. Dr. Stefan Schütz

$3^{\text {rd }}$ Referee Prof. Dr. Edmone Roffael

Date of Oral Examination: 27 ${ }^{\text {th }}$ October 2010 


\section{Zusammenfassung}

Abies grandis ist ein schnell wachsender Nadelbaum, der großes Potential in der nachhaltigen Holzproduktion und deren verarbeitetenden Industrie besitzt. Daher ist es für mögliche Anwendungen notwendig, mehr über das Verhalten von A. grandis Holz gegenüber holzabbauenden Pilzen zu erfahren. In Abbauversuchen nach Norm EN 113 (1996) konnten sowohl Holz als auch Holzprodukte von A. grandis durch Braunfäulepilze abgebaut werden, aber gegenüber Weißfäulepilzen zeigten $A$. grandis Holz eine vergleichsweise gute Resistenz. Früh- und Spätholz von A. grandis wurde in unterschiedlicher Stärke über die Inkubationszeit abgebaut. Nach 19 Wochen zeigten die vom Braunfäulepilz Corniophora puteana besiedelten Früh- und Spätholzproben einen höheren Gewichtsverlust als die Frühholzproben. Im Gegensatz dazu verursachte der Weißfäulepilz Trametes versicolor einen 2mal höheren Gewichtsverlust bei Frühholzproben im Vergleich zu Früh- und Spätholzproben. Behandlung mit Harnstoffformaldehyd oder Ammoniumsulfat steigerte die Beständigkeit des Holzes gegenüber Weißfäulepilzen. Wasser- und Aceton-extrahiertes Holz verlor dagegen an ResistenzRissiges Holz war darüber hinaus auch anfälliger; wahrscheinlich erlauben Risse Pilzen einen leichten Eintritt ins Holz.

Es wurde angenommen, dass Weißfäulepilze oxidative Enzyme (Laccasen, Peroxidasen) sekretieren, um das in den Holzzellwänden vorkommende Lignin zu modifizieren. Diese Modifikation des Lignins resultiert in Chinonstrukturen, die mit MBTH (3-Methyl-2-benzothiazolinon-hydrazon Hydrochlorid Monohydrat) gefärbt werden können, worauf eine rötliche Farbe entsteht. Die Effekte der oxidativen Enzyme auf Holzzellwände wurde untersucht, in dem Holzschnitte mit MBTH inkubiert wurden. Rötliche Färbung der Holzzellwand deutete dabei auf modifiziertes Lignin in der Holzzellwand hin. Die Dicke der Zellwand und deren Färbung verringerten sich durch längere Inkubation mit T. versicolor, wahrscheinlich durch starken Ligninabbau. Innerhalb von Jahrringen nahm der Anteil des gefärbten Zellwandbereichs $\left(\mathrm{A}_{\mathrm{S}}\right)$ und der gesamte Zellwandbereich $\left(\mathrm{A}_{\mathrm{T}}\right)$ in einer s-förmigen Kurvenform vom Früh- zum Spätholz zu. Um den Angriff der Pilze auf das Lignin zu simulieren, wurde das RedOx-Enzym Laccase mit Mediator (HBT: 1-Hydroxybenzotriazol, HBA: 4-Hydroxybenzoesäure; beide Substanzen reagieren nach Oxidation durch Laccase mit Lignin) oder $\mathrm{Mn}^{3+}$-Acetat als ein mit Lignin reagierendes Produkt der pilzlichen Manganperoxidase in A. gran- 
dis Vollholz injiziert. Die rötliche Färbung mit MBTH war etwas besser mit Laccase mit HBT als mit HBA. Auch die Inkubation mit $\mathrm{Mn}^{3+}$-Acetat ergab eine rötliche Färbung der Holzzellwände, wobei die stärkste Färbung bei der höheren $\mathrm{Mn}^{3+}$-Acetat Konzentration von $10 \mathrm{mM}$ auftrat.

Die Weißfäulepilze Pleurotus ostreatus und T. versicolor zählen zu den besten Laccaseproduzenten bei Zugabe von A. grandis Holz als Laccaseinduktor zu Pilzkulturen. Holz und Holzextrakstoffe induzierten in Abhängigkeit von Konzentration die Produktion von extrazellulären Laccasen, aber nicht von anderen lignozellulolytischen Pilzenzymen, wie Manganperoxidase (MnP), Ligninperoxidase (LiP) oder Zellulasen als Enzyme, die ebenfalls mit Lignocellulose reagieren. Aus Kultivierungsversuchen der Pilze in Medium mit Holz und unterschiedlichen $\mathrm{C}$ und $\mathrm{N}$ Gehalten läßt sich folgern, dass das $\mathrm{C} / \mathrm{N}$ Verhältnis des Holzes ein weiterer genereller Faktor in der Laccaseinduktion ist.

Holzextraktstoffe von $A$. grandis zeigten inhibierende Wirkung auf das Wachstum der Weißfäulepilze Coprinopsis cinerea, P. ostreatus und T. versicolor. Die extrahierbaren Holzstoffe wurden mittels Gaschromatographie und Massenspektrometrie (GC-MS) identifiziert. In den Wasser- und den Acetonextrakten wurden neun verschiedene phenolische Stoffe gefunden: 2-Methoxyphenol (Guajacol), 4-Hydroxybenzaldehyd, 1,4-Dihydroxybenzen (Hydrochinon), 3,5-Di-tertbut-4-hydroxytoluol (Butylhydroxytoluol), 3-Methoxy-4-hydroxybenzaldehyd (Vanillin), 4-Hydroxybenzoesäure, 3-Methoxy-4-hydroxybenzoesäure (Vanillinsäure), 4-Hydroxy-zimtsäure ( $p$-Cumarinsäure) und 4-Hydroxy-3-methoxyzimtsäure (Ferulasäure). Einige der natürlichen phenolischen Stoffe, wie 4-Hydroxyzimtsäure, 4-Hydroxybenzaldehyd oder 3,5-Di-tertbut4-hydroxytoluol können als natürliche Induktoren in der Laccaseproduktion mit $P$. ostreatus und T. versicolor eingesetzt werden. Allerdings hemmte die Zugabe von 1,4-Dihydroxybenzen und $p$-Benzochinon zu den Flüssigkulturen in der Konzentration von $1 \mathrm{mM}$ das Wachstum von P. ostreatus und T. versicolor. Durch Holyextrakstoffe in der Produktion induzierte Lasseisoenyzme der beiden Pilze wurden mittels nativer Gelelektrophorese analysiert und mittels MS-Analyse der proteolytisch verdauten Proteine indentifiziert. Im Fall von P. ostreatus wurde das Isoenzym Laccase II und im Fall von $T$. versicolor die Isoenzyme Laccase I und Laccase III gefunden.

Weiterhin wurde $A$. grandis Holz für den technischen Einsatz in der enzymatischen Biokraftstoffherstellung getestet. Dabei konzentrierten sich die Experimente auf chemische, physikalisch-chemische und biologische Vorbehandlungen des Holzes und 
auf die Hydrolyse von Zellulose durch Zellulasen, die entweder allein oder in Kombination mit anderen Enzymen (Xylanase, Laccase oder $\beta$-Glukosidase) eingesetzt wurden, um eine hohe Ausbeute an Glukose aus dem lignozellulosehaltigen Substrat für die Biokraftstoffherstellung $\mathrm{zu}$ erhalten. In biologischen Vorbehandlungen wurden kleine A. grandis Holzblöcke für 19 Wochen nach der Norm EN 113 (1996) mit Pilzen inkubiert. Die biologische Vorbehandlung mit P. ostreatus (im Vergleich zu T. versicolor und C. puteana) ergab exzellente Raten von $42,8 \%$ für die Umsetzung der gesamten Holzmasse in Glukose, was $92,4 \%$ der im A. grandis Holz verfügbaren Zellulose entspricht.

Eine energieaufwendigere Vorbehandlung unter Einsatz von $8 \mathrm{ml}$ einer 85\%igen Phosphorsäure für $1 \mathrm{~h}$ bei $50^{\circ} \mathrm{C}$ resultierte ebenfalls in guten Glukoseausbeuten bei Anwendung von jeweils $4 \mathrm{U} \mathrm{ml}^{-1}$ Zellulase, Xylanase und Laccase. Längere Inkubationszeiten und höhere Konzentrationen und Mengen an Phosphorsäure bei einer Inkubationstemperatur von $50^{\circ} \mathrm{C}$ führten zu Gewichtsverlusten von bis zu $50 \%$ bei gleichzeitiger Steigerung des Zelluloseanteils auf bis zu 100\%. Höchste Glukoseausbeuten (bis zu 100\%) konnten durch eine physikalisch-chemische Vorbehandlung erzielt werden, bei der $1 \mathrm{~g}$ Holzpartikel in $8 \mathrm{ml}$ einer 85\%igen bzw. 80\%igen Phosphorsäure für $20 \mathrm{sec}$ in einer Mikrowelle erhitzt wurde, und eine anschließende Hydrolyse mit einer Mischung aus $4 \mathrm{U} \mathrm{ml}^{-1}$ Zellulase, $4 \mathrm{U} \mathrm{ml}^{-1}$ Xylanase und $4 \mathrm{U} \mathrm{ml}^{-1}$ Laccase oder, alternativ zur Laccase, $4 \mathrm{U} \mathrm{ml}^{-1} \beta$-Glukosidase für $24 \mathrm{~h}$ in $5 \mathrm{ml} 50 \mathrm{mM}$ Natriumacetat-Puffer, pH 5.0. Die Mikrowellenbehandlung erlaubte eine Reduzierung der Phosphoräurekonzentration unter $80 \%$ bis hin zu $40 \%$ und $20 \%$ mit noch annehmbaren Zuckerausbeuten in nachfolgender enyzmatischer Hydrolose der Zellulose (60\% Umwandlung von Zellulose in Glukose), aber nur, wenn Zellulase und Xylanase zusammen mit Laccase eingesetzt wurden. Wasserextrakte aus Holz, bzw. spezifische phenolische Substanzen ( $p$-Cumarinsäure, Vanillinsäure und Ferulasäure) in den Extrakten, zeigten inhibierende Wirkung bei der Hydrolyse von Zellulose mit Zellulase. Phenolische Stoffe in den Holzextrakstoffen können durch Laccaseaktivität abgebaut werden, was einen höheren Umsatz der Zellulasen in der Glukoseproduktion gewährleistet und was den positiven Effekt der Laccase auf die Glukoseproduktion aus Zellulose von A. grandis Holz erklärt. 


\section{Summary}

Abies grandis is a fast growing coniferous tree with a high potential for sustainable wood production and applications in the wood product industry. However, for the possible applications knowledge on behaviour of the wood with wood-decaying fungi was required. In wood block decay tests following norm EN 113 (1996), A. grandis wood as well as wood composites from $A$. grandis wood were easily degraded by brown-rot fungi but the wood showed a comparably good resistance against white-rot species. Earlywood and latewood in A. grandis wood was shown to different degrees to be affected by decay of different species over the incubation time. The brown-rot fungus Corniophora puteana caused higher mass loss for samples of earlywood+latewood than for samples of earlywood alone, as analyzed after 19 weeks of incubation. In contrast, the white-rot fungus Trametes versicolor caused a $2 \mathrm{x}$ higher mass loss of earlywood samples than of earlywood+latewood samples. Teatment of the wood with urea formaldehyde or ammonium sulphate however enhanced the resistance against white rot fungi. In contrast, wood extracted with water and with water+acetone lost resistance. Moreover, broken wood was easily degraded by fungi; likely, broken cells allow fungi an easy entry into wood cells.

White-rot wood decay fungi were expected to secrete oxidative enzymes (laccase, peroxidases) in order to modify lignin within the wood cell walls. This modification of lignin results in quinones structures that can be stained with MBTH (3methyl-2-benzothiazolinone-hydrazone hydrochloride monohydrate) resulting in a reddish color. The effects of oxidative enzymes on modification of wood cell walls were evaluated by staining wood sections with MBTH. Reddish staining of wood cell walls indicated modification of lignin within the wood cell walls. Staining of cell walls and cell wall thicknesses were reduced by longer incubation time of wood with T. versicolor, likely by massive degradation of lignin. Within year rings, the area fraction of stained cell walls $\left(\mathrm{A}_{\mathrm{S}}\right)$ and the total cell wall area $\left(\mathrm{A}_{\mathrm{T}}\right)$ was found to increase in a slightly sigmoid form from earlywood to latewood. The fungal actions on lignin were simulated through injections into solid A. grandis wood blocks using the redoxenzyme laccase with a mediator (HBT: 1-hydroxybenzotriazole, HBA: 4-hydroxybenzoic acid; both substances that interact with lignin upon oxidation by laccase) or individually with $\mathrm{Mn}^{3+}$ acetate, presenting a product of fungal 
Mn-peroxidase known to act on lignocellulose. Reddish MBTH staining was slightly better with laccase with HBT than with HBA. Also, treatment with $\mathrm{Mn}^{3+}$ acetate resulted in a reddish color in the wood cell walls which was strongest with application of higher $\mathrm{Mn}^{3+}$ acetate concentrations (10 mM).

The white-rot fungi Pleurotus ostreatus and T. versicolor are amongst the best laccase producers on $A$. grandis wood material when added to fungal cultures. Wood or wood extractives in concentration-dependent manner induced production of extracellular laccases but no manganese-dependent peroxidases (MnP), lignin peroxidases (LiP) or cellulases as fungal enzymes also acting on lignocellulose. Deduced from sub-culturing the fungi on medium with wood and different $\mathrm{C}$ and $\mathrm{N}$ contents, another general factor in laccase induction was likely the $\mathrm{C}$ to $\mathrm{N}$ ratio of the wood.

Wood extractives from $A$. grandis were shown to inhibit growth of the whiterots Coprinopsis cinerea, $P$. ostreatus and $T$. versicolor, respectively. By gas chromatography-mass spectrometry (GC-MS), extractable wood compounds were identified. Within the water extractives and the acetone extractives, nine distinct phenolic compounds were found: 2-methoxyphenol (guaiacol), 4-hydroxybenzaldehyde, 1,4-dihydroxybenzene (hydroquinone), 3,5-di-tertbut-4hydroxy-toluene (butylhydroxytoluol), 3-methoxy-4-hydroxybenzaldehyde (vanillin), 4-hydroxybenzoic acid, 3-methoxy-4-hydroxybenzoic acid (vanillic acid), 4-hydroxy cinnamic acid ( $p$-coumaric acid), and 4-hydroxy-3-methoxy cinnamic acid (ferulic acid). Some of the natural phenolic compounds, such as 4-hydroxy cinnamic acid, 4-hydroxybenzaldehyde or 3,5-di-tertbut-4-hydroxy-toluene have a potential to act as natural inducers in laccase production of $P$. ostreatus and T. versicolor. Application of 1,4-dihydroxybenzene and $p$-benzochinone at the concentration of $1 \mathrm{mM}$ to liquid cultures however inhibited growth of $P$. ostreatus and $T$. versicolor. Laccase isoenzymes of these two fungi induced in production by extractives were analyzed by native gel electrophoresis and identified by MS-analysis of peptides from specific proteolytic digests. Isoenzyme analysis detected laccase II in case of P. ostreatus and laccase I and laccase III in case of T. versicolor.

A. grandis wood was further tested for potential technical use in enzymatic biofuel production. Experiments focused on effects of chemical, physico-chemical and biological pretreatments of the wood and, subsequently, onto cellulose hydrolysis by cellulase alone and in combination with other enzymes (xylanase, laccase and 
$\beta$-glucosidase) in order to gain the highest glucose yields of transforming lignocellulosic substrate into fermentable sugars for biofuel production. In biological pretreatments, A. grandis wood mini blocks were incubated for 19 weeks with fungi according to EN 113 (1996). The biological pretreatment with P. ostreatus (compared to T. versicolor and C. puteana) gave excellent with conversion of $42.8 \%$ of the total original wood mass into glucose which corresponds to $92.4 \%$ of the cellulose that was originally present in the $A$. grandis wood.

Application of $8 \mathrm{ml} 85 \%$ phosphoric acid in a more energy consuming pretreatment for $1 \mathrm{hr}$ at $50^{\circ} \mathrm{C}$ in a hot water bath resulted also in best glucose yields when applying each $4 \mathrm{U} \mathrm{ml}^{-1}$ of cellulase, xylanase and laccase. Longer incubation times and higher concentrations and amounts of phosphoric acid at a temperature pretreatment at $50^{\circ} \mathrm{C}$ lead to mass losses up to $50 \%$ and simultaneous relative increase of the cellulose content up to $100 \%$. Highest glucose yields (up to $100 \%$ ) were reached in a physico-chemical pretreatment by microwaving $1 \mathrm{~g}$ of wood particles in $8 \mathrm{ml}$ of 85 or $80 \%$ phosphoric acid for $20 \mathrm{sec}$ and applying subsequently a combination of $4 \mathrm{U} \mathrm{ml}^{-1}$ cellulase, $4 \mathrm{U} \mathrm{ml}^{-1}$ xylanase and $4 \mathrm{U} \mathrm{ml}^{-1}$ laccase or, alternatively to laccase, $4 \mathrm{U} \mathrm{ml}^{-1}$ $\beta$-glucosidase in $5 \mathrm{ml}$ of $50 \mathrm{mM}$ sodium acetate buffer, $\mathrm{pH} 5.0$ for hydrolysis for $24 \mathrm{hrs}$. Microwaving allowed reducing a phosphoric acid concentration below $80 \%$ to $40 \%$ and $20 \%$ in order to still obtain reasonable yields of sugars $(60 \%$ of conversion of cellulose into glucose) in subsequent enzymatic hydrolysis of cellulose but only if cellulase and xylanase were applied together with laccase. Water extractives from $A$. grandis wood, respectively specific phenolic compounds ( $p$-coumaric acid, vanillic acid and ferulic acid) within the water extractives were shown to act as inhibitors in hydrolysis of cellulose by cellulase. Phenolic compounds in wood extractives can be degraded by laccase activity, allowing better performance of cellulases in the production of glucose and explaining the observed positive effect of laccase in glucose production from cellulose from A. grandis wood. 


\section{Acknowledgements}

I am indebted to lots of people who have helped me in many cases for a huge variety of reasons.

First of all, I like to express my sincere thanks to my supervisor, Prof. Dr. Ursula Kües. Without the supporting, advising, excellent guiding and editing from her deep knowledge of the subject and professional intellectual input I would never reached up to this stage in this PhD study. I like to thank Prof. Dr. Stefan Schütz and Prof. Dr. Edmone Roffael for agreeing to undertake the examination of this work. I especially express my deep gratitude to Dr. Andrzej Majcherczyk, who enlightened my knowledge in bio-chemistry and on proteins, for his advice, suggestions and supporting me in the way how to plan for doing experiments.

I sincerely thank Assoc. Prof. Dr. Boonsom Siribumrungsukha, president of the Prince of Songkla University (PSU), Assist. Prof. Dr. Sieng Kritsaneepaiboon, vicepresident of PSU, Surat Thani Campus, Assoc. Prof. Kriengsak Pattamarakha, dean of the Faculty of Technology and Management, Dr. Nitas Proukaew, dean of the Faculty of Sciences and Industrial Technology of PSU, for giving me the opportunity and providing the financial support throughout my PhD studies. Moreover, I like to thank for the financial support during the last year of my $\mathrm{PhD}$ study on the one hand the International Student Service, University of Göttingen, and especially Olaf Blümel, head of International Student Service, and Anne Muller, and for the scholarship with care achievement STIBET I (DAAD), University of Göttingen and especially also Prof. Dr. Michael Hörner, coordinator of the program and on the other hand the Katholischer Akademischer Ausländer-Dienst (KAAD), especially Corina Martinas, Dr. Cecilia Leon Ramirez, Peter-Paul König, Dr. Heinrich Geiger, Daniela Ramb, Pater Benedikt Lautenbacher SJ, Karin Bialas and Hania Rose.

I like to thank my master's supervisors, Assist. Prof. Dr. Buhnnum Kyokong and Assist. Prof. Dr. Nirundorn Matan from the Walailak University, Thailand, for guiding me to work as a scientist and advising an interesting topic for a $\mathrm{PhD}$.

I thank Dr. Patrik Hoegger to enlighten me a principal knowledge for DNA and laboratory experimental work. I thank Prof. Dr. Alireza Kharazipour for discussions on wood composites and wood components. I like to give a great thank to Mojtaba Zomorrodi for his technical input on GC-MS analysis of compounds in the liquid supernatant from Abies grandis wood particles treated with laccase and Karin Lange for 
her input on laccase production of white-rot fungi upon addition of wood particles to liquid cultures, and not only for their kind technical help but also for the friendly atmosphere in the lab and for helping me to improve my German language skills. I like to thank Alexandra Dolynska, Gisbert Langer-Kettner, Bernd Kopka, Merle Fastenrath, Thomas Klein, Christine Kettner and Marianne Smiatacz for supporting me to use the scientific and technical equipments of the institute and giving technical services, and I like to thank Christian Hof, Bodo Caspar Kielmann and Bernd Bringemeier from the Division of Wood Biology and Wood Products for support and preparing Abies grandis wood samples, Dr. Markus Euring and Dr. Cora Müller for providing laccase and extraction instruments for this study, Dr. Hubert Vos, Dr. Redelf Kraft, Dr. Pannipa Malanit (University of Hamburg) and Stefan Schütze for providing wood particles and wood milling, Dr. Christa Lang, Dr. Andrea Olbrich, and Dr. Rosemarie Heyser for their support in preparing wood microtome sections and discussing wood anatomy, and Dr. Annette Naumann and Ihtzaz Malik for giving me advices on wood mass loss testing. I thank Dr. Norman Loftfield for the analysis of the component content in Abies grandis wood by using Inductively Coupled Plasma-Atomic Emission Spectrometer (ICP-AES), and Prof. Dr. Edzo Veldkamp for the information on using of ICP-AES.

In addition to those already mentioned above, I like to thank my colleagues at the Division of Molecular Wood Biotechnology and Technical Mycology, all present and past included, the doctors Martin Rühl, Monica Navarro-González, Prayook Srivilai, Wassana Chaisaena, Sudhakar Peddireddi, Jhansi Kalyani Pemmasani, Katerina Svobodova, and Claus Behn, the student colleagues Dorothea Fragner, DongSheng Wei, Olivia Sánchez-Hernández, Samantha Sarai Navarro González, Teresita Spezzia Mazzocco with son Samuel, Carmen de Sena Tomas, Yulei Zhao, Kiran Kumar Reddy, Manuel Alfaro, Martin Siewert, Stephan Nix, Sebastian Dantz, Nina Ritter, Hossein Hosseinkhani, and Neda Nikvash Kolesara, the technician Michael Reichel and to my student colleagues from other Divisions of the Institute including Guan Wu Zhou, Hua Bai, Atef Abo-Ogiala, Widi Sunaryo, Chunxia Zhang, Rodica Pena, and Dejuan Ning for their friendly support and for generating nice atmospheres being great for student life. I like to thank to Dr. Ernst Kürsten (previous) and Dr. Gerhard Büttner (present) for coordinating the international $\mathrm{PhD}$ program that gave me a chance to attend several conferences, meeting, seminars, and courses. Thanks to Prof. Dr. Barry Goodell for giving and discussing the special topic in wood biodeterioration. I thank 
Maritza Ulrich-Totz for teaching and improving my German language and Tony Gallucci for teaching me English.

I like to give a special thanks to all my Thai friends in Göttingen who on the long term participated in several events when I was a Thai-student president for a year and during my time of study as a PhD student at the Georg-August-University. Special thanks to Chakrapong Chaikong, Rungsan Jarernsook and Pitipong Thobunluepop for their discussions on the statistical data analysis and to Chor Hoon Poh and Nattipong Mekkhala for reading my written thesis work. Thanks to Dr. Christoph Köhler for being nice, friendly and helpful. Thanks to all Thai people to support and provide some stuff for my daily life, especially the Asia Markt in Weende, Göttingen, to my relatives in Kassel including Mae-Siriporn Steinbeck, Kurt Steinbeck, Uraiwan Senkaew, Pornpaween Senkaew, Jens Limprecht, Prangthip Wenzel, Mayura Jampasi, Lamphai Schwerdtle, Dieter Schwerdtle, Somkiat Chuerdsuwan, Arissara Nakawisut (Girbig) and Areeya Tansee for providing and taking care of me during the whole four years in Germany, to the Family Geier for their warm kindness and helpfulness, to my relatives in Pegnitz including: Thanom, Eric, Lisa and Kevin Wittmann, to Uraiporn Kaeomanee, Parisut Phonkaset and Kurt Blaser in Interlaken, Switzerland for their kindly friendship and providing some living expense to me and my family when they visited me in Germany. I give a pretty deep thank to Wat Dhammavihara BuddhistenVerein Hannover e.V., particularly to Phramaha Thongnark Duangpon, for supporting great events and providing Thai musical instruments for graduate cerebrations. I like to forward my thanks to colleagues and friends in the Walailak University and the Prince of Songkla University, Thailand, especially to Boonrueng Khaonuan, Prateep Chompoonutch, Nipaporn Kaenpetch, Arwut Kaenpetch, Kanchuree Wongwairatanakul, Tuangporn Puerghom, Somsak Porpan, and Manatsanan Klaikaew who still were keeping contact and friendly relationship with me, to the Thippaka Family, particularly Somchai Thippaka, Poh-Kard Thippaka, Mae-Lek Thippaka and Adjima Thippaka for their kindly encouragement during my study in Germany.

Last but not least, I like to give a specific special thanks to my parents, my father Rerm Cherdchim and my mother Klein Cherdchim, my brothers Paiboon Cherdchim, Suriya Cherdchim and Pramot Cherdchim, my sister Ubonrat Cherdchim and my nephew Methanon Cherdchim for their encouragement, support and for providing me in such way to benefit for my entire life from education and for leading me to an intellectual and emotional development. 



\section{Table of Contents}

Page

Zusammenfassung

Summary

iv

Acknowledgements

vii

Table of contents

xi

\section{CHAPTER I}

Introduction

I.1 Biofuel production from wood 3

I.2 Enzymatic wood hydrolysis $\quad 4$

$\begin{array}{ll}\text { I.3 Methods of pretreatment } & 16\end{array}$

$\begin{array}{ll}\text { I.3.1 Chemical pretreatments } & 17\end{array}$

I.3.2 Physical and physico-chemical pretreatments 19

$\begin{array}{ll}\text { I.3.3 Biological pretreatments } & 20\end{array}$

I.4 Effects of pretreatment on enzymatic glucose production from wood 21

I.5 Economical and environmental aspects of biofuel production

(bioethanol) 22

I.6 Aims of the thesis $\quad 24$

$\begin{array}{ll}\text { I.7 References } & 26\end{array}$

\section{CHAPTER II}

II. 1 Abstract

II. 2 Introduction

II.3 Materials and methods

II.3.1 Fungal strains

II.3.2 Wood block decay test 
II.3.4 Statistical analysis

II.4 Results

II.4.1 Biodegradation of $A$. grandis wood blocks inoculated with different wood decay fungi

II.4.2 Influence of different types of pretreatment of $A$. grandis wood on decay by $T$. versicolor

II.5 Discussion 58

II.6 References 62

II.7 Supplementary material

II.7.1 Further analysis of wood mass values in relation to the moisture content of wood blocks

II.7.2 Plastic staining by volatiles emitted from Heterobasidion sp. cultures

II.7.3 References 70

\section{CHAPTER III}

\section{Effects of fungi and fungal enzymes on Abies grandis wood}

III.1 Abstract 75

III. 2 Introduction 77

III.3 Materials and methods 80

III.3.1 Fungal strains and laccase 80

III.3.2 Wood block decay test 81

III.3.3 Lignin determination

III.3.4 Chemical treatment of $A$. grandis wood

III.3.5 Microscopic analysis of wood sections and image analysis 85

III.3.6 Determining the distance of earlywood and of latewood in a year ring

III.3.7 Statistical analysis 90

III.4 Results 90

III.4.1 Mass loss of $A$. grandis wood by incubation with different decay fungi 
III.4.2 Modification of wood cell walls and wood lignin by incubation with T. versicolor Strain 6 and its oxidative enzymes

III.4.3 Image analysis of MBTH-stained wood after 6 weeks incubation with $T$. versicolor Strain 6

III.4.4 Effects of fungal oxidative enzymes on wood lignin modification

III.5 Discussion

III.5.1 Mass loss and cell wall modification of $A$. grandis wood after inoculation with T. versicolor Strain 6

III.5.2 Further biological observations on the fungus T. versicolor

Strain 6 in growth on $A$. grandis wood

III.5.3 Mimicking the effect of fungal decay by incubation of wood with either isolated laccase or $\mathrm{Mn}^{3+}$, respectively

III.5.4 Comparison of methods for analyzing lignin modification within wood fiber cell walls

III.6 References

III.7 Supplementary material

III.7.1 Density measurements of $A$. grandis wood blocks with earlywood+latewood and of $A$. grandis wood blocks with only earlywood

III.7.2 Mass loss of wood by decay fungi

III.7.3 Staining of microtome sections of A. grandis wood after incubation with $T$. versicolor Strain 6 with MBTH and image analysis

III.7.4 Comparison of parameters of $A$. grandis wood blocks after incubation of 6,12 and 19 weeks with or without $T$. versicolor

\section{CHAPTER IV}

\section{Contrasting functions of Abies grandis wood extractives on}

protection against white-rot fungi and introduction of laccase activity 
IV.1 Abstract

IV.2 Introduction

IV.3 Materials and methods

IV.3.3 Fungal cultivations

IV 3.3.1 Microorganisms and general culture conditions

IV.3.3.2 Fungal cultures with wood, wood extractives or specific phenolic compounds

IV 3.3.3 Influence of nutrients on laccase production

IV.3.4 Wood decay tests following EN 113 and fungal growth test of wood blocks immersed in wood extractives

IV.3.5 Effect of wood extractives on growth of fungi

IV.3.6 Enzymes

IV.3.6.1 Enzyme tests

IV 3.6.2 Laccase treatment of $A$. grandis wood particles

IV.3.6.3 Gel electrophoresis and staining

IV.3.6.4 Protein identification

IV.3.7 Statistical analysis

IV.4.1 Enzyme production in fungal cultures in presence of

A. grandis wood particles

IV.4.2 Effects of wood extractives on laccase production

IV.4.3 Laccase induction by different fractions of $A$. grandis wood extractives

IV .4.4 Identification of $A$. grandis wood extractive compounds by GC-MS

IV.4.5 Laccase induction and inhibition by phenolic compounds

IV.4.6 Enzyme identification

IV.4.7 Spectrophotometric determination of laccase activity on wood particles

IV.4.8 Wood extractives in protection of $A$. grandis wood against fungal decay 
IV.5.1 Phenolic compounds in wood extractives play a role in laccase production 205

IV.5.2 Wood extractives in protection of wood against fungi 212

IV.5.3 General conclusions on functions of wood extractives on fungi 213

$\begin{array}{ll}\text { IV.6 References } & 214\end{array}$

IV.7 Supplementary material 225

IV.7.1 Laccase induction by different fractions of $A$. grandis wood extractives and by DMSO

IV.7.2 Identification of specific compound in A. grandis wood extractive by GC-MS 227

IV.7.3 Inhibition of fungal growth by phenolic compounds 232

IV.7.4 Component composition of $A$. grandis wood 232

IV.7.5 Influence of nutrients on laccase production 235

IV.7.6 References 239

\section{CHAPTER V}

\section{Application of Abies grandis wood for technical use and in} enzymatic biofuel production

V.1 Abstract 243

V.2 Introduction 245

$\begin{array}{ll}\text { V.3 Materials and methods } & 248\end{array}$

$\begin{array}{ll}\text { V.3.1 Wood and straw } & 248\end{array}$

V.3.2 Chemical and physico-chemical pretreatment 249

V.3.3 Microorganism and culture conditions for biological pretreatment 250

V.3.4 Chemicals and enzymes $\quad 250$

V.3.5 Enzymatic cellulose hydrolysis 251

V.3.6 Sugar assay 252

V.3.7 Wood component characterization $\quad 252$

V.3.8 Statistical analysis 253

V.4 Results 253 
V.4.1 Chemical composition of $A$. grandis wood in comparison to Norway spruce wood and wheat straw

V.4.2 Effect of enzymatic hydrolysis on glucose production of lignocellulosic materials

V.4.3 Effects of $A$. grandis wood pretreatments on production of glucose

V.4.3.1 Chemical pretreatment of $A$. grandis wood particles

V.4.3.2 Microwave-chemical pretreatment of $A$. grandis wood particles

V.4.4 Effects of laccase in combination with other enzymes on hydrolysis of cellulose to glucose

V.4.4.1 Chemical pretreatment and effects of laccase and other enzymes on glucose production

V.4.4.2 Microwave-chemical pretreatment and effects of laccase on glucose production

V.4.4.3 Biological pretreatment of A. grandis wood for efficient glucose production

V.4.5 Influence of wood extractives and specific phenolic compounds on cellulase hydrolysis

V.5.1 A. grandis softwood as a resource in environmentally friendly production of glucose for biofuels

V.5.2 Chemical and physico-chemical pretreatment of $A$. grandis wood for glucose production

V.5.3 Effects of laccase and $\beta$-glucosidase in enzymatic hydrolysis by cellulase and xylanase of $A$. grandis cellulosic pellets for glucose production

V.5.4 Biological pretreatment of $A$. grandis wood for enzymatic hydrolysis for glucose production from cellulose 


\section{CHAPTER VI}

\section{Final discussion and prospects}

VI.1 Final discussion

VI.1.1 General overview on the results of this work and their implications for technical applications

VI.1.2 Laccase and wood extractives, colleagues or enemies?

VI.1.3 The possibility to use modification of wood cell wall to calculate stages of decay by fungi

VI.1.4 Enzymatic hydrolysis of wood and pretreated wood material for biofuel production and its technical improvement 320

VI.2 General conclusions and prospects for future own studies 

CHAPTER I

Introduction 



\section{I.1 Biofuel production from wood}

Problems of the world energy consumption come from the rapid growing demand for energy from the limited fossil resources for petroleum and gas and from the global warming by use of fossil fuels. The decrease in crude-oil resources enhances the urgent demand for alternative resources for fuels worldwide. Also by the increased climate concerns about the use of fossil-base energy carriers, and by political commitment, the trend has recently turned towards improved utilization of renewable energy resources. Fossil-based energy resources, such as petroleum, coal and natural gas satisfy so far parts of the energy world's consumption. Alternatives to fossil-based energy resources are nuclear power, hydropower, wind power, and biomass, representing together currently about one of three quarters of the world's primary energy consumption. Biomass is an abundant and carbon-neutral renewable energy resource for the production of biofuels and also of valuable chemicals (Stöcker et al. 2008).

Nowadays, ethanol is the most important renewable fuel in terms of volume and market value. Presently, as the first generation of biofuels, it is produced from sugar and starch-based materials serving also food and fodder purposes. The disadvantages of such productions are evident and lie in competition with food and fodder of animals' and humans' consumption. Therefore, in the second generation of biofuels it is interesting to use non-food and non-fodder material for the production. Lignocellulosic biomasses from trees and other plants is easily renewable and thus have the potential to serve as a sustainable feedstock for future biofuels and value-added bio-based products, compensating for the diminishing crude oil and coal resources ( $\mathrm{Li}$ et al. 2008; Taherzadeh and Karimi 2008).

Conversion of renewable lignocellulosic materials to the end products of ethanol or other value-added chemicals employs three major steps: i. pretreatment to make lignin- and hemicellulose-linked cellulose accessible and to decrease the degree of polymerization of crystalline cellulose counteracting enzymatic digestibility, ii. the conversion of cellulose to glucose by enzymatic hydrolysis, and iii. fermentation of glucose to end products of ethanol or other value-added chemicals. However, as a bottleneck of biofuel production from lignocellulose, the currently available procedures are expensive processes. Pretreatment of the material in order to free cellulose for enzymatic degradation makes up about one third of total production costs. In addition, there is the huge amount of enzymes required to access cellulose and hemicellulose for 
transformation into glucose and other sugars. Therefore, cheap methods of pretreatment with a high degree of performance combined with synergistic enzymatic hydrolysis came into focus as presenting potentially good alternatives for energy (biofuel) production in terms of economical and environmental aspects (Mtui 2009; Taniguchi et al. 2010).

\section{I.2 Enzymatic wood hydrolysis}

Enzymatic hydrolysis of cellulose to glucose is carried out by enzymes called cellulases that are specialized in breaking up the $\beta-1-4$-glycosidic bonds of glucan. The cellulases are divided into three sub-categories, representing three types of activity: endoglucanases, exoglucanases (cellobiohydrolases) and $\beta$-glucosidases. The endoglucanases significantly reduce the degree of polymerization of a cellulosic substrate by randomly attacking the interior parts of cellulose, mainly in the amorphous regions of cellulose. The exoglucanases incrementally shorten the glucan molecules by binding to the glucan ends and releasing mainly cellobiose units. Finally, $\beta$-glucosidases split the disaccharide cellobiose into two units of glucose. The enzymatic hydrolysis is usually performed under mild conditions in contrast to chemical hydrolysis. Therefore, one may expect low corrosion problems of reactors, low consumption and loss of material for unwanted reactions other than for the wanted product (fermentable sugars), and low toxicity of the hydrolyzates when using this type of technological process (Dadi et al. 2007; Jørgensen et al. 2008; Karimi 2007; Olofsson et al. 2008; Taherzadeh and Mtui 2009).

A successful pretreatment of wood and other lignocellulosic plant material has to remove the hemicelluloses and lignin to a large extent, in order to make the celluloses in the cell walls accessible for enzymatic hydrolysis. Historically, industrial cellulose digestion has made use of acid hydrolysis and optimizations of acid hydrolysis of various lignocellulosic materials have been carried out for ethanol producing purpose (Table 1). Acid hydrolysis however is little suitable for subsequent microbial conversion of released glucose into ethanol because of production of toxics in the hydrolyzates affecting the fermenting microorganisms. Moreover, the maximum glucose yield from acidic pretreatments is limited to approximately $60 \%$ in a batch process for kinetic reasons. Enzymatic degradation of the accessible cellulose from less 


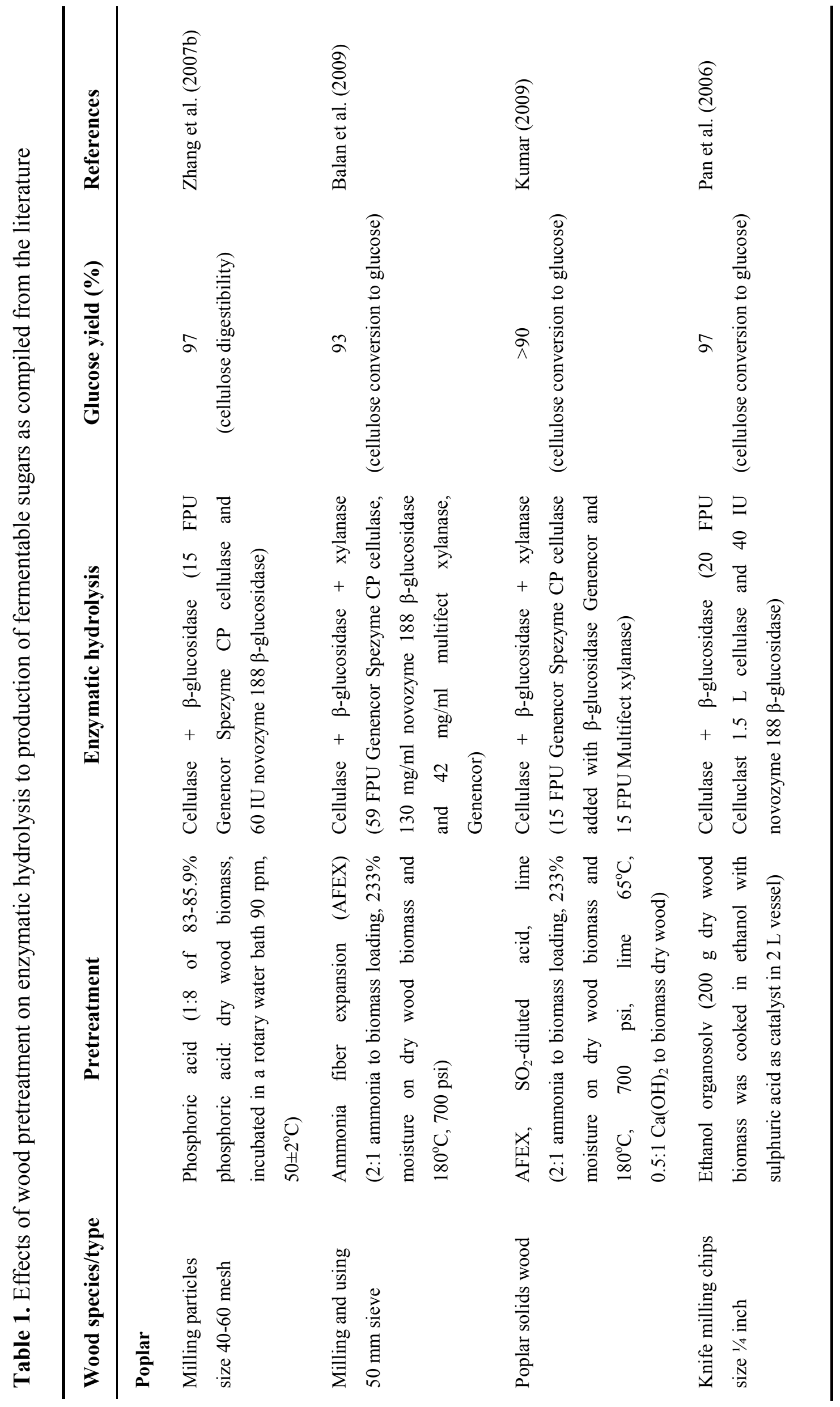




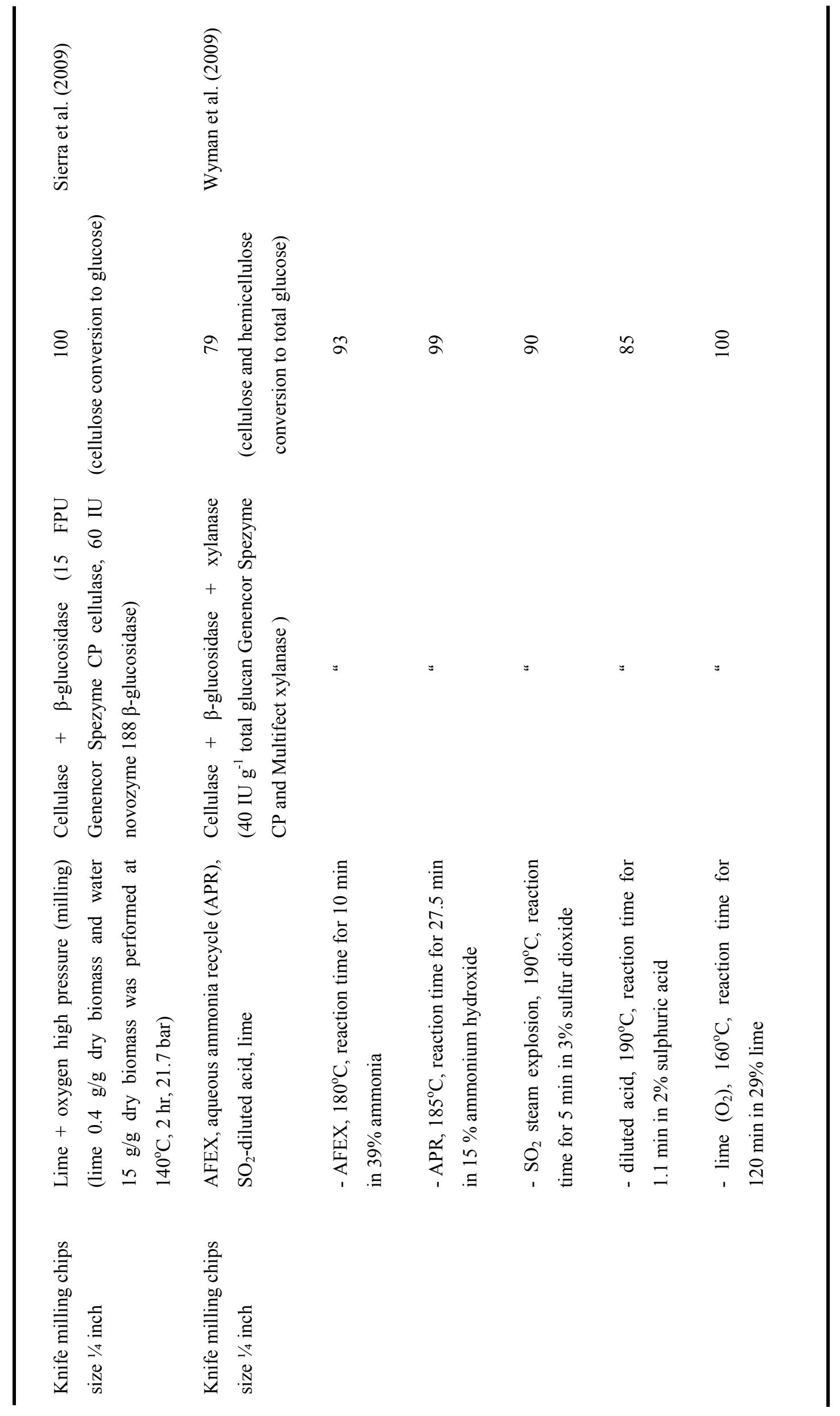




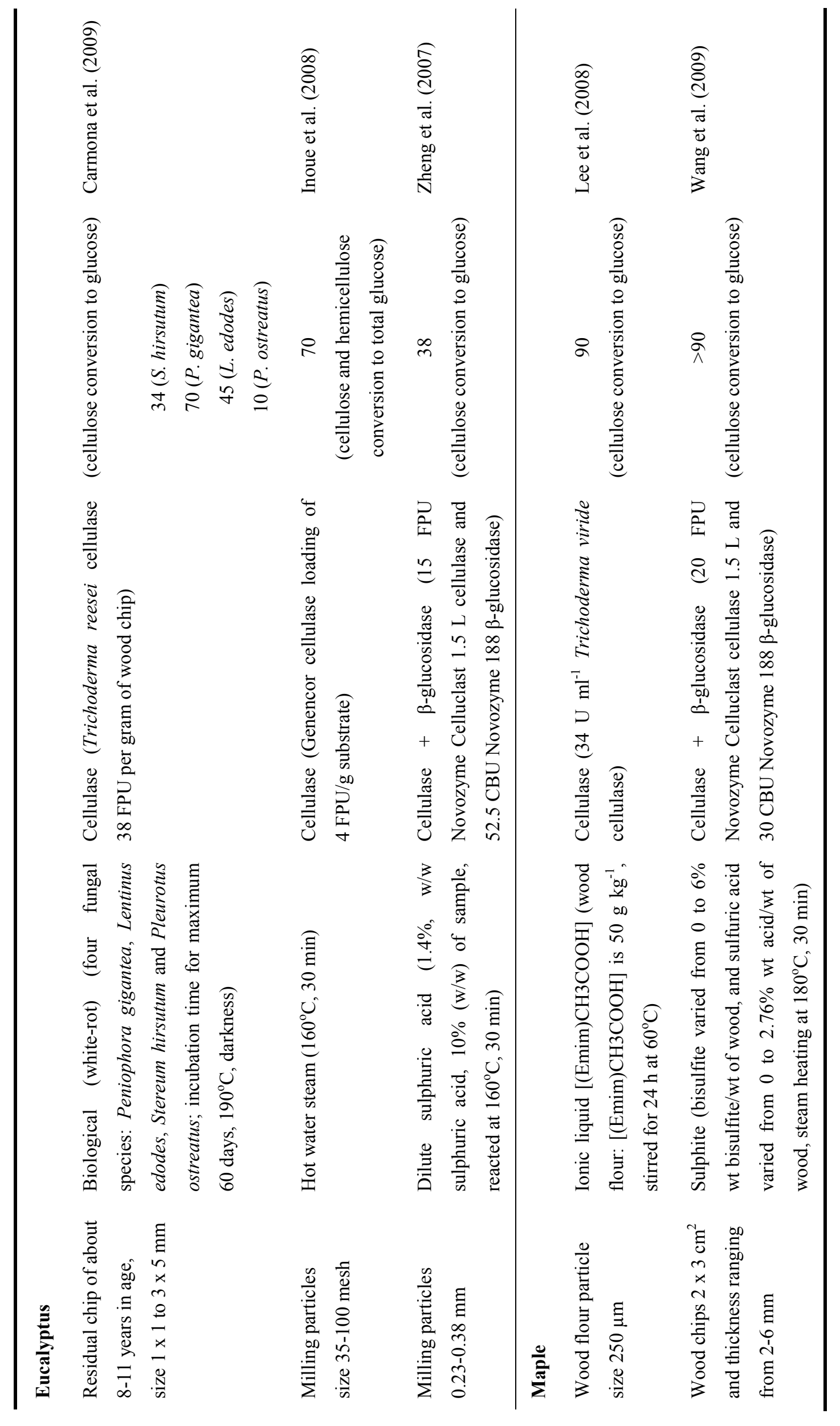




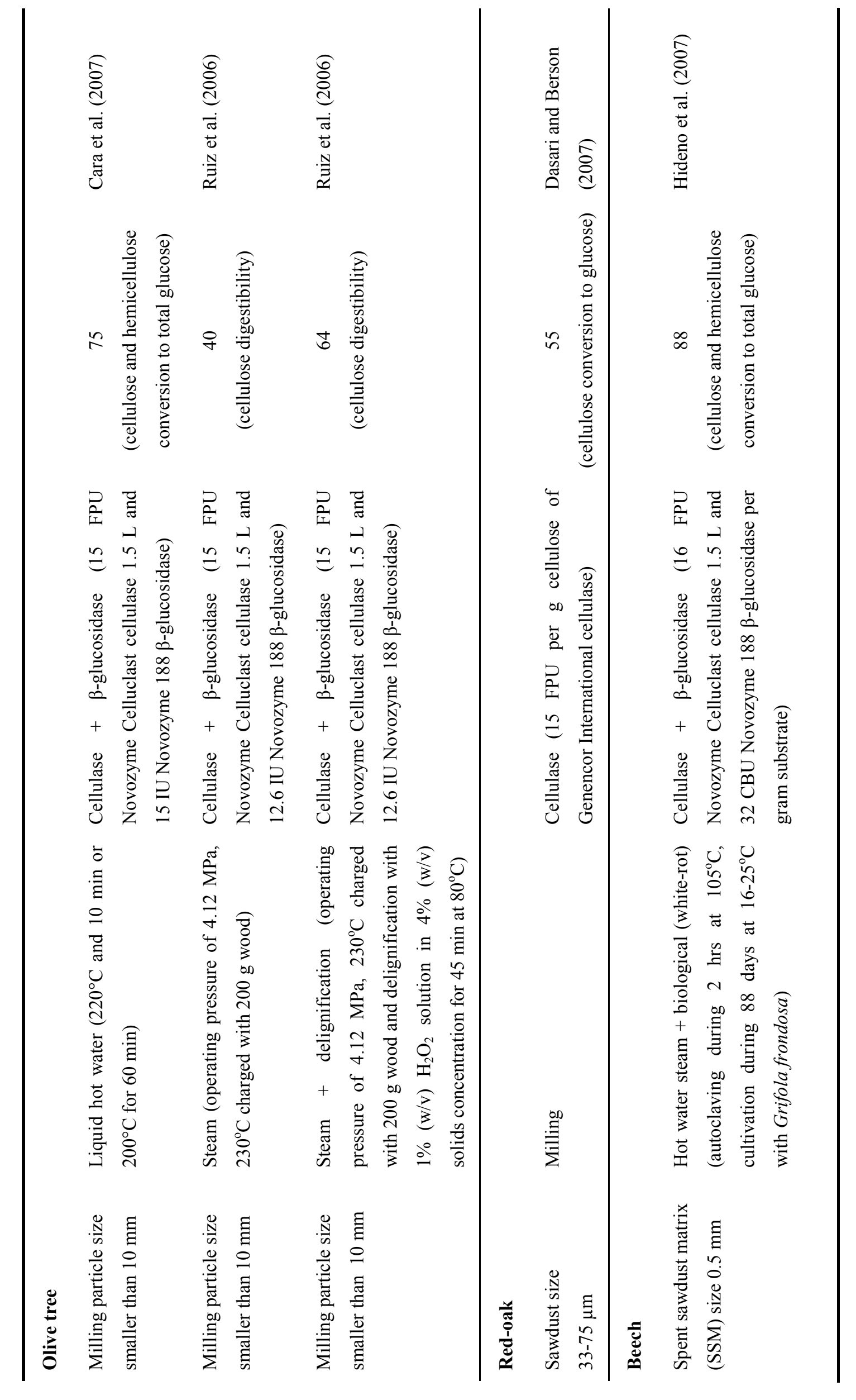




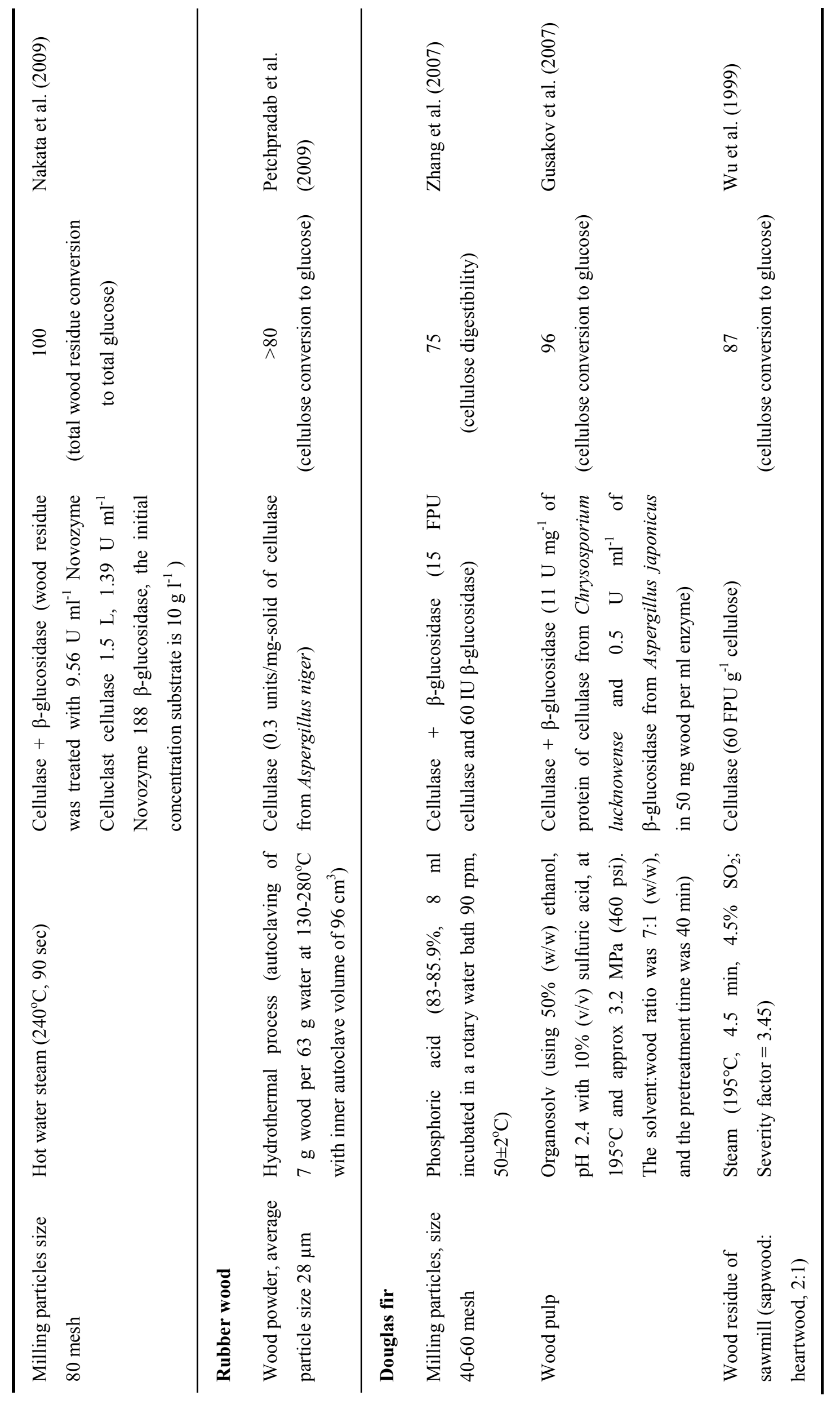




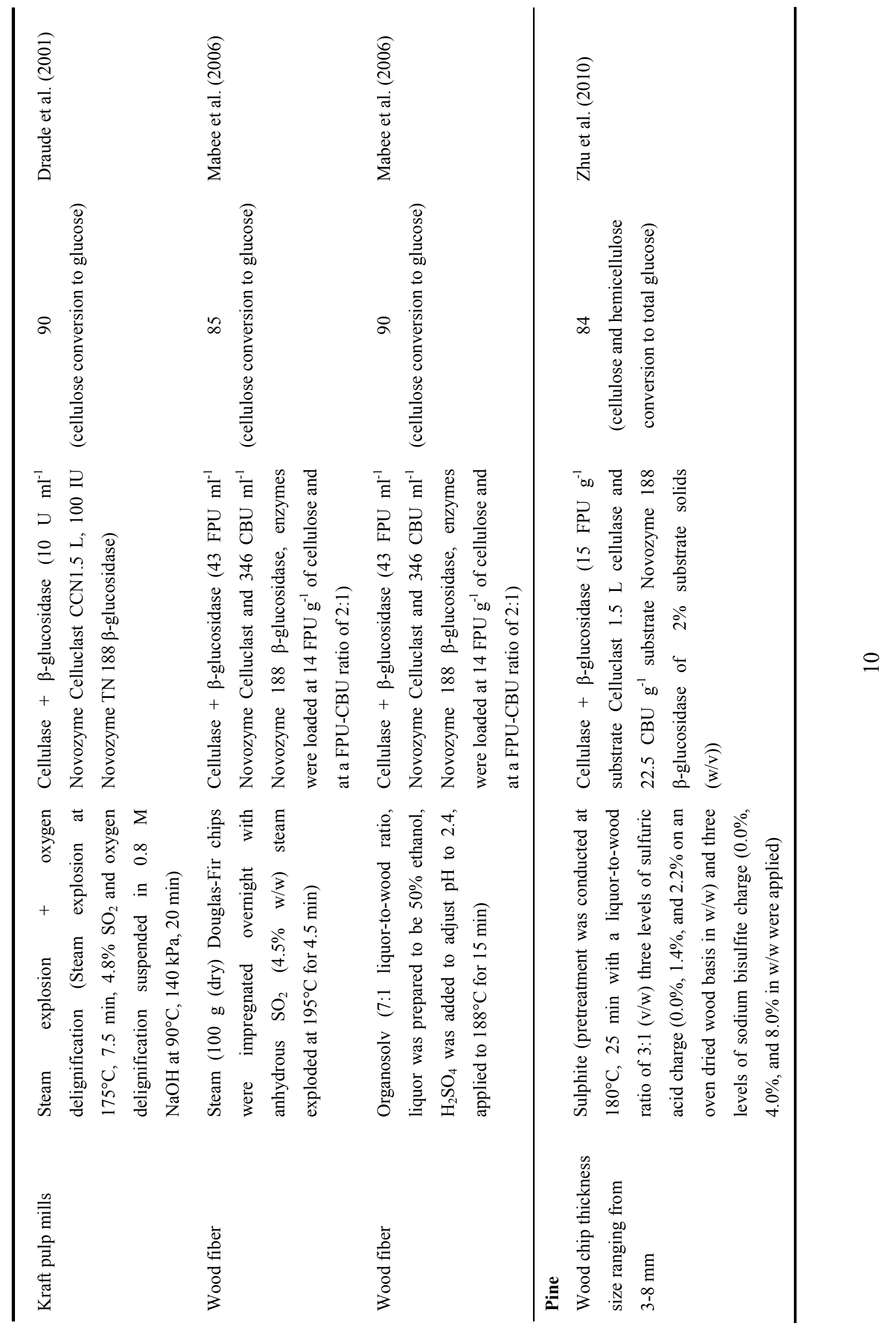




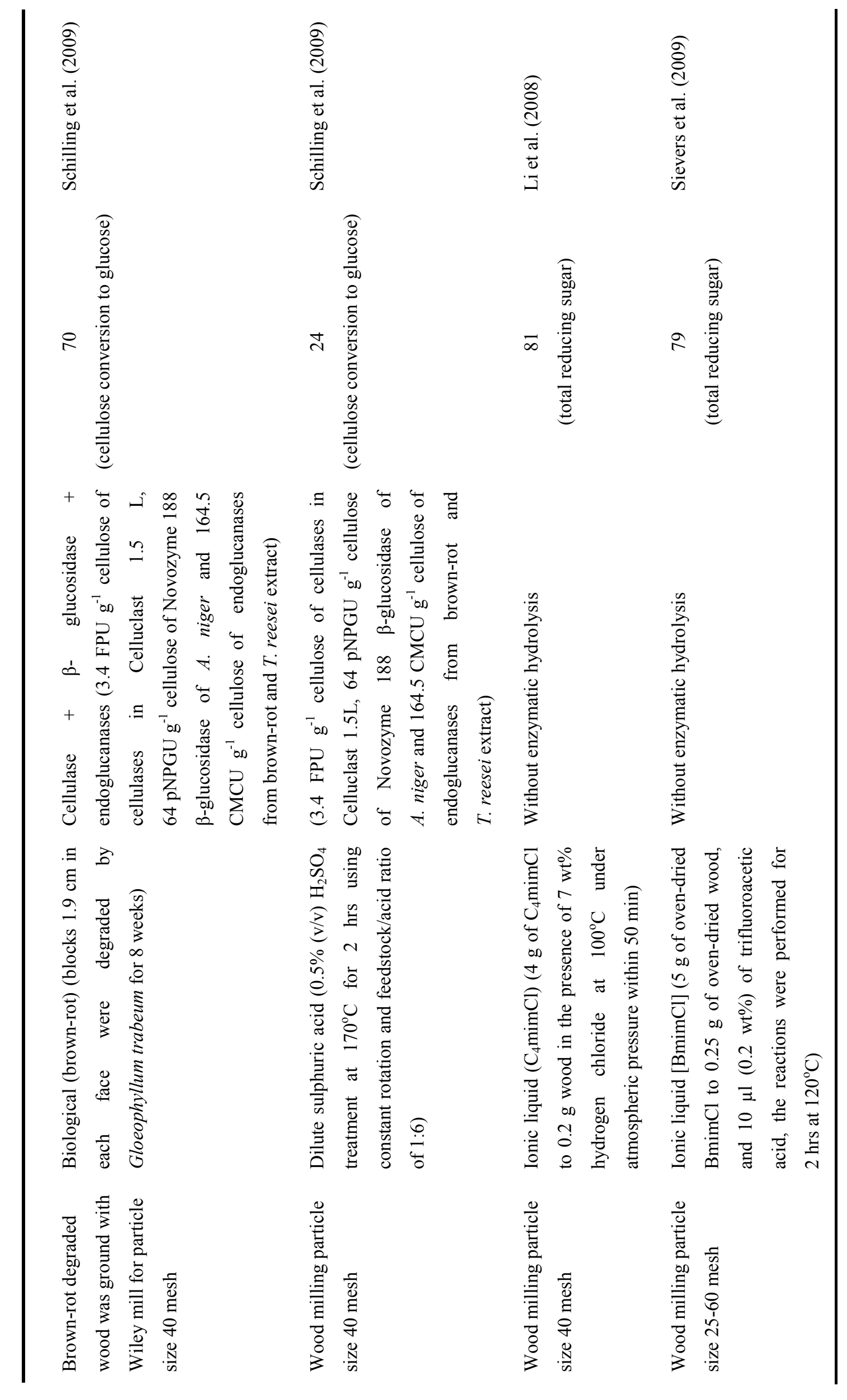




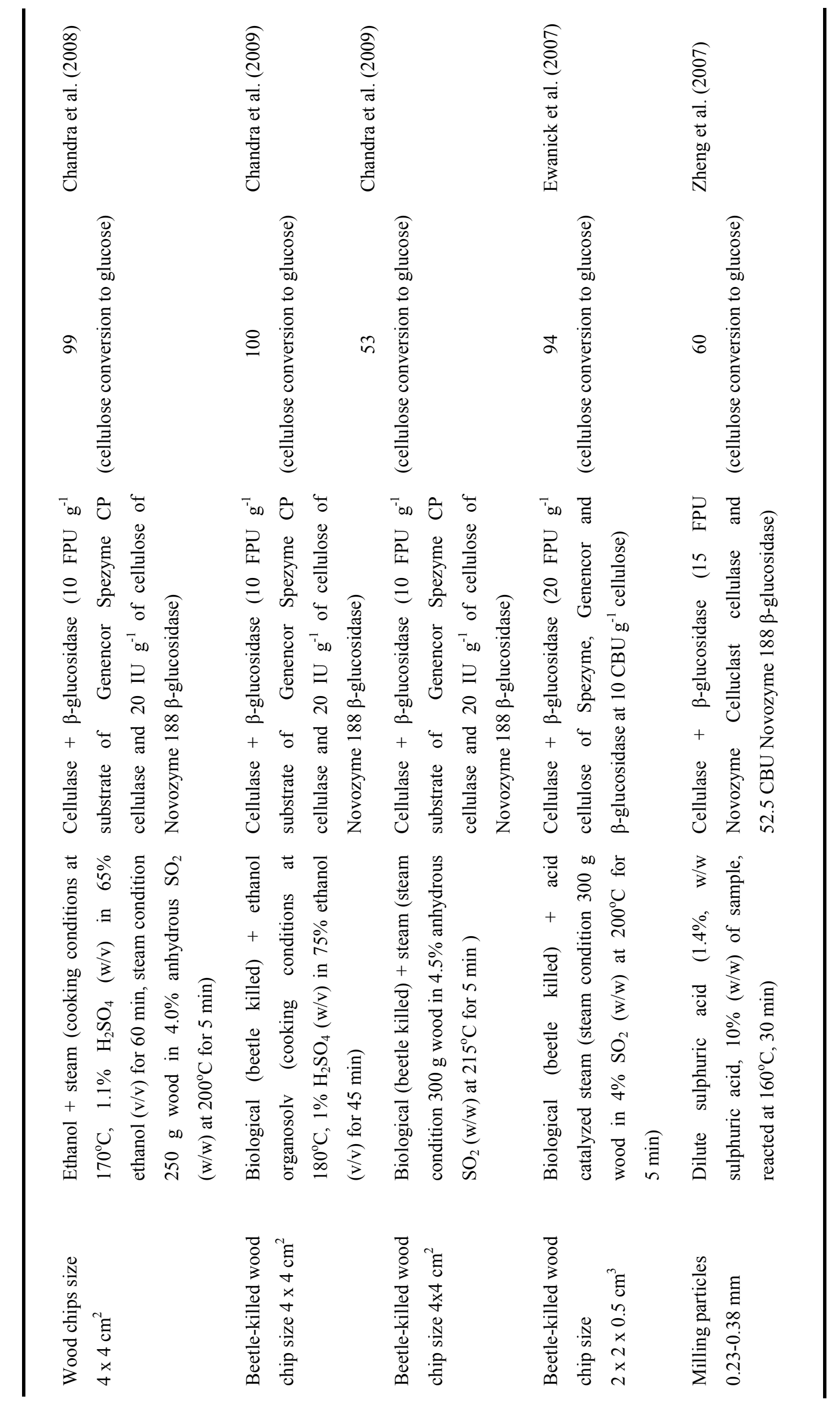




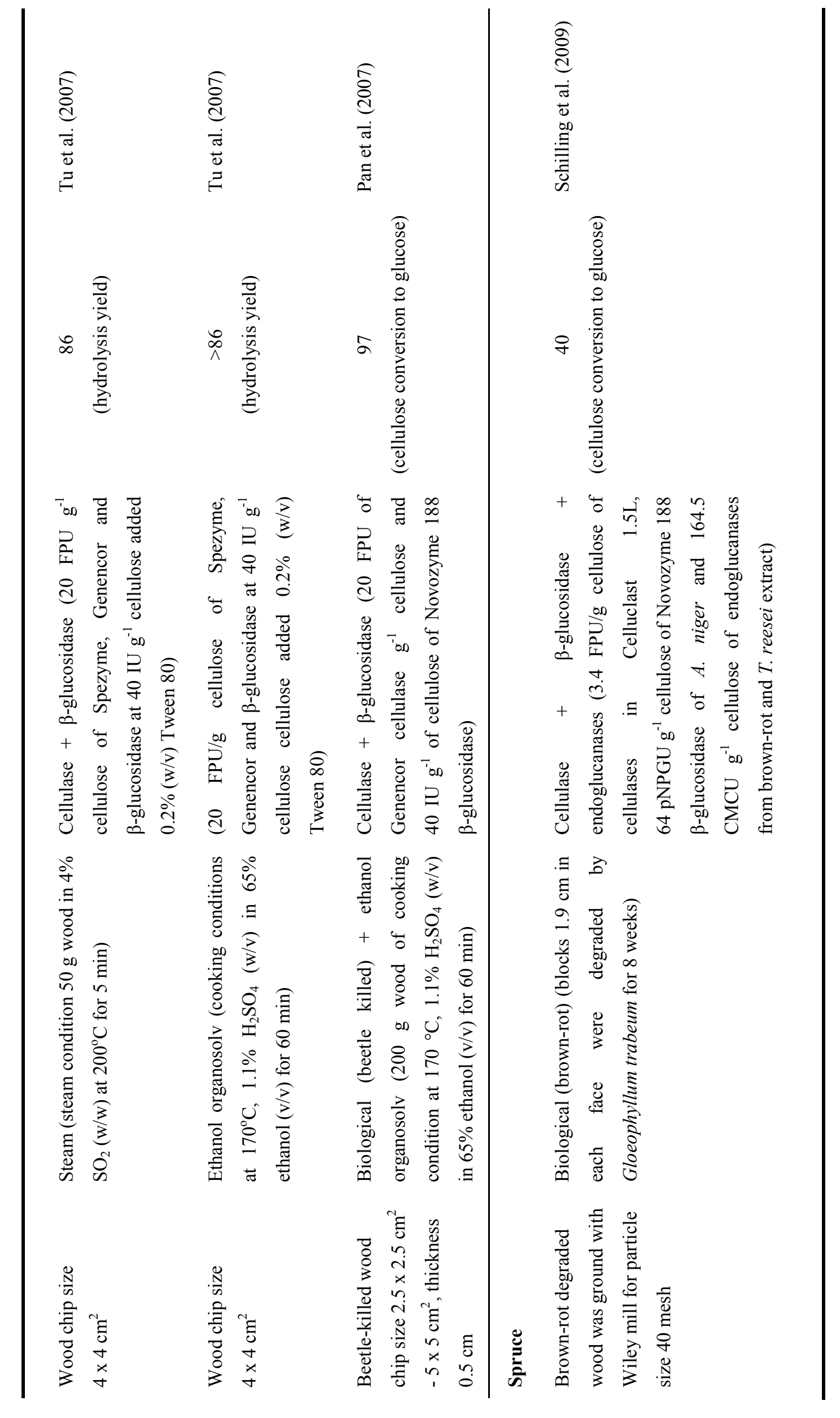




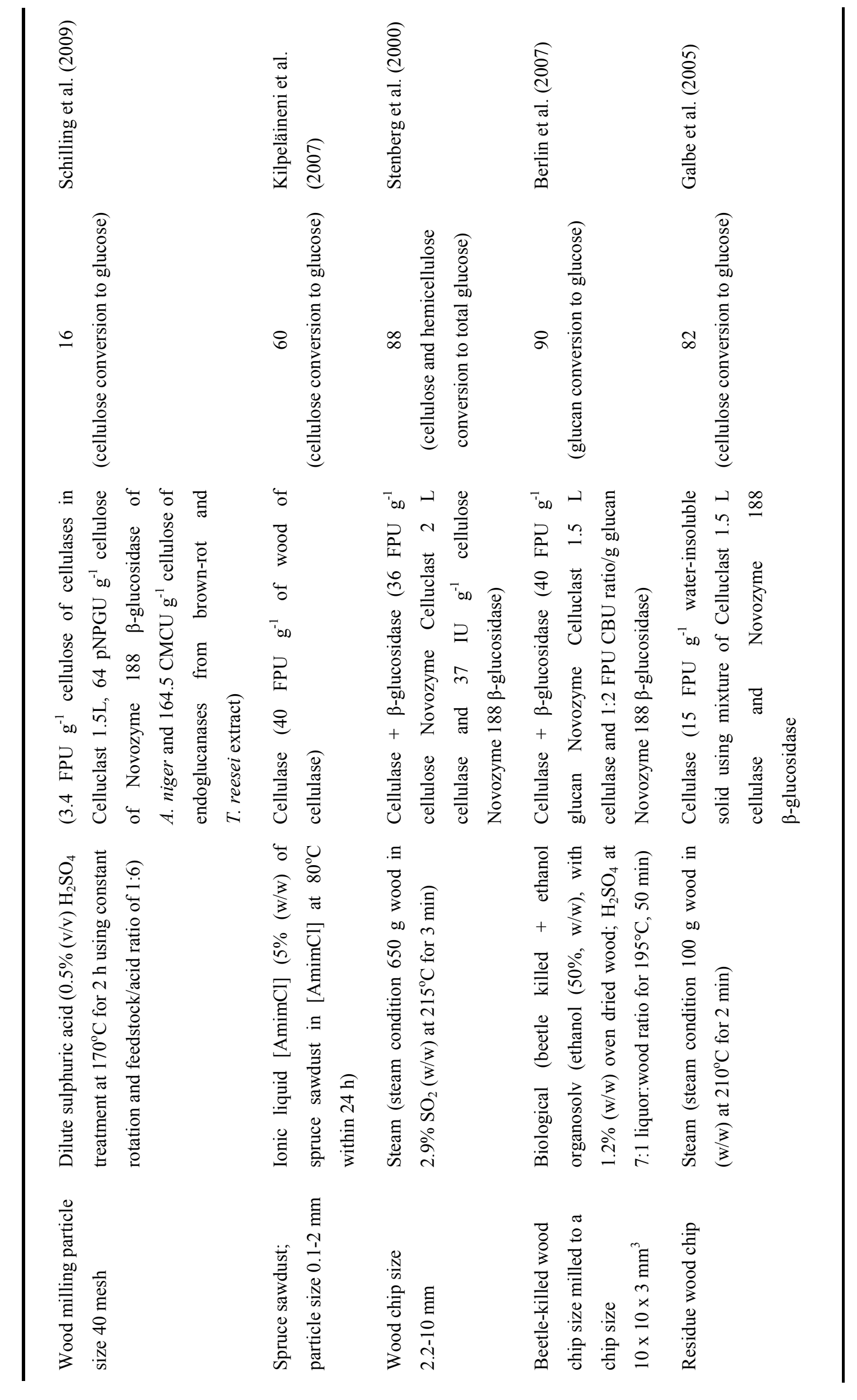




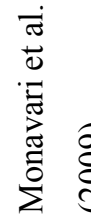

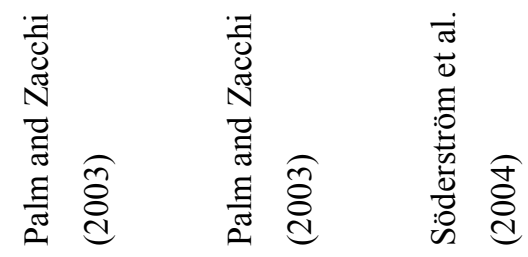

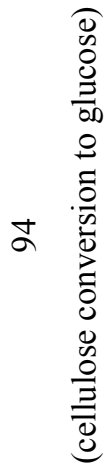

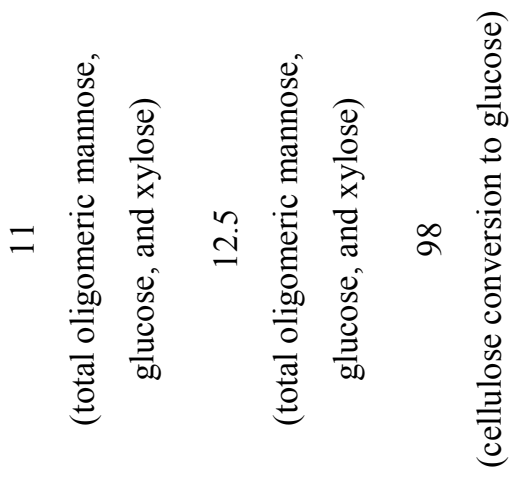

To 苂 $\infty$

Tos 寻 $\infty$

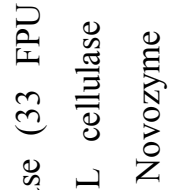

焉

荡 荡

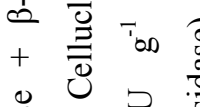

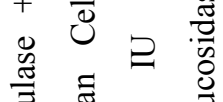

节苛

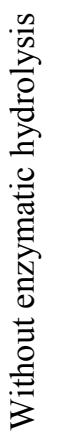

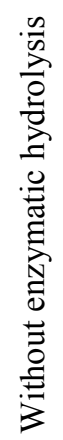

焉表

군

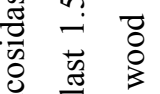

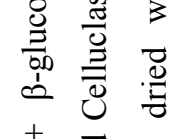

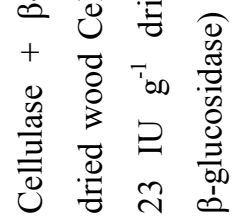

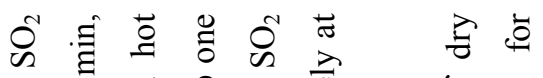

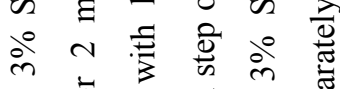

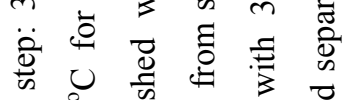

$\begin{array}{lll}4 & 0 & 0 \\ 0 & 0 & 0 \\ 0 & 0 & 0 \\ 0 & 0 & 0\end{array}$

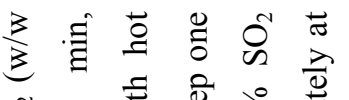

क 0 है ते की

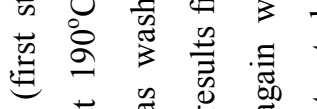

立

ヘิ

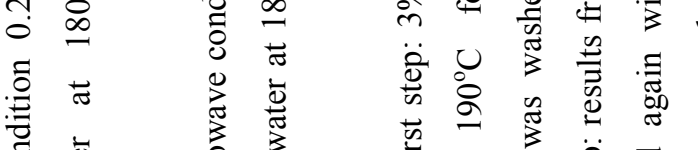



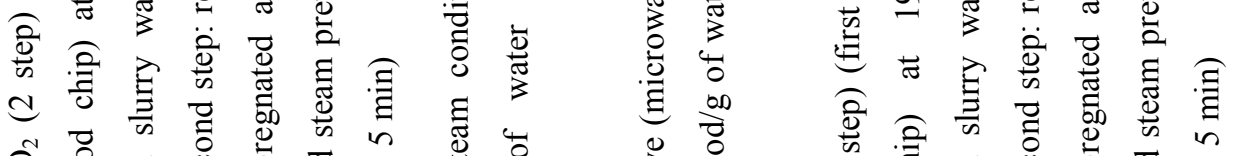

家

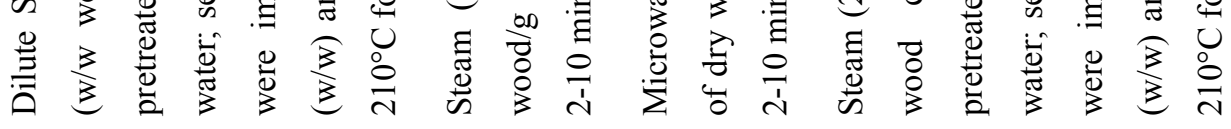

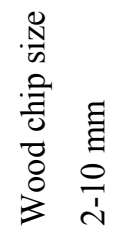

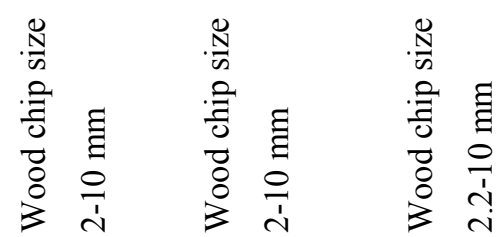

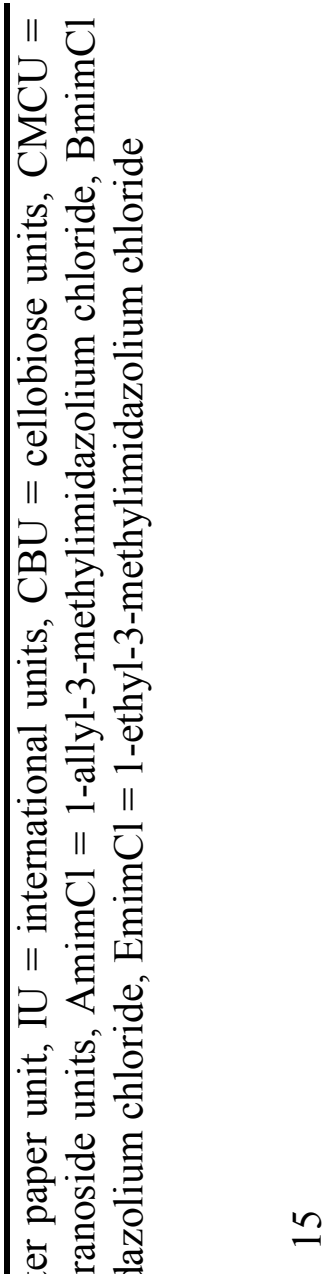


harsh pretreatments has instead the potential of yielding relatively non-toxic hydrolyzates with higher sugar yields (Olofsson et al. 2008).

\section{I.3 Methods of pretreatment}

Pretreatment is used in biofuel production to make cellulose accessible to enzymatic hydrolysis. All of these methods should create the possibility for enzymes to attack cellulose by reducing the crystallinity of the cellulose (Sannigrahi et al. 2010), by enhancing the accessible surface area of the cellulose (Taherzadeh and Karimi 2008) and by removing the protection by lignin and hemicellulose as the main determinants of hindering an efficient hydrolysis (Fig. 1, Mtui 2009). The best suitable pretreatments are developed in respect of various criteria of benefit, according to the effectiveness of reaction and according to economical and environmental aspects. Principally, wood

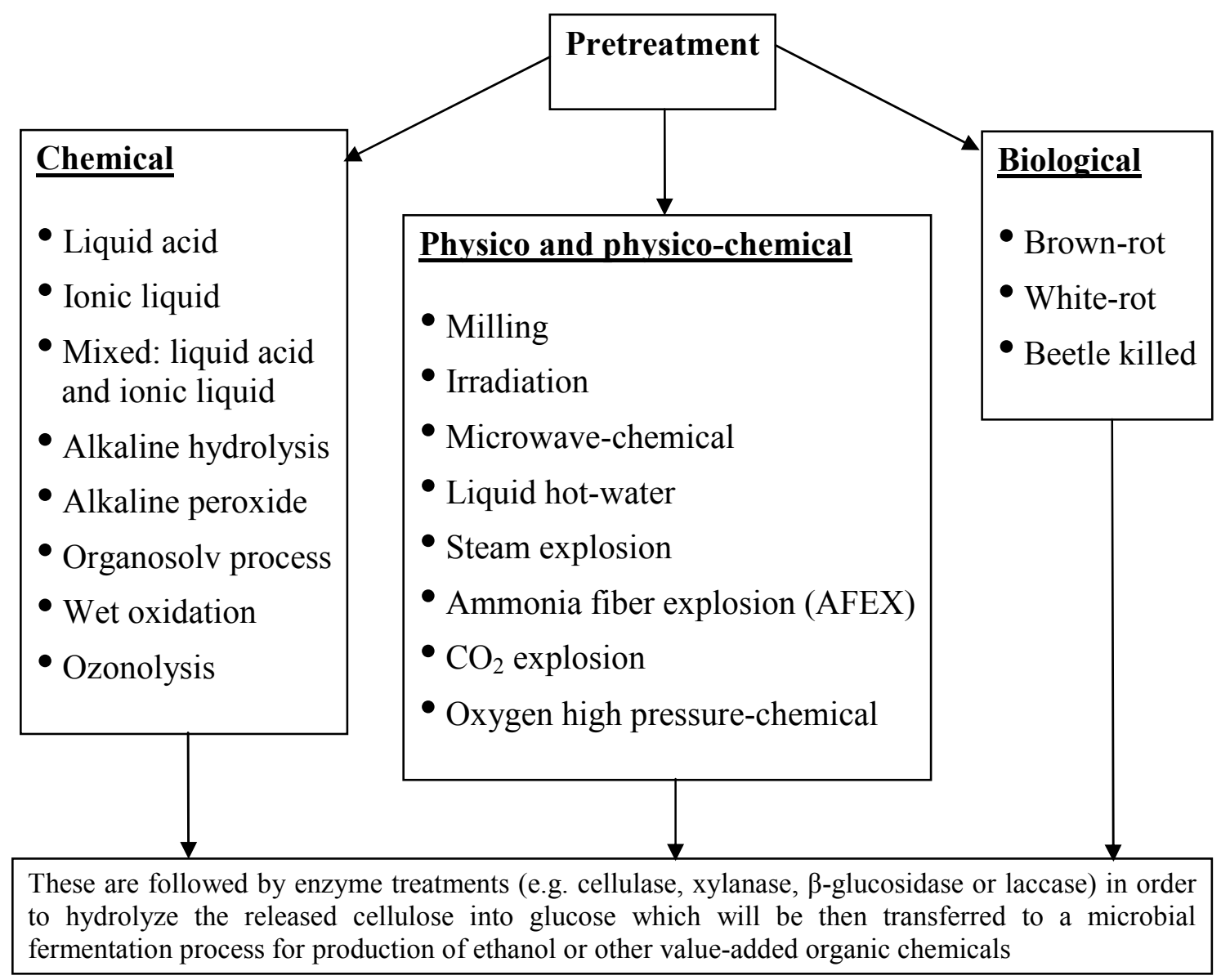

Fig. 1 Overview of types of pretreatments of lignocellulosic material for subsequent enzymatic hydrolysis for producing fermentable sugars 
pretreatment might be chemically, physically or biologically and combinations thereof (Fig. 1, Mtui 2009; Taherzadeh and Karimi 2008). In general, it is however difficult to place the methods under one category only. Depending on the measures applied, methods are classified under "Chemical pretreatment", "Physical and Physico-chemical pretreatment" and "Biological pretreatment" (Galbe and Zacchi 2007; Olofsson et al. 2008).

\section{I.3.1 Chemical pretreatments}

The cellulose microfibrils in the plant cell walls have both crystalline and amorphous regions, but the major part of cellulose is in the crystalline form. It has principally been shown that hydrolytic enzyme (cellulase) can hydrolyze the more accessible amorphous portion of cellulose, while the hydrolytic enzymes are not much effective in degrading the less accessible crystalline portion (Paul et al. 2003; Sannigrahi et al. 2010). Swelling of the pores within cellulose microfibrils can be achieved through alkaline pretreatment by soaking the sample in an alkaline solution (e.g. sodium hydroxide) and incubating the material for a certain time. Swelling enhances the internal surface area of the cellulose, and a decrease in the degree of polymerization and crystallinity follows. It is therefore expected that a decrease in the crystallinity of cellulose will increase the digestibility of the lignocellulose material. However, lignin and hemicellulose have to be solubilized and usually this happens in the pretreatments together. Alkaline pretreatment disrupts the lignin structure and breaks the bonds between lignin and carbohydrates, which makes the carbohydrates more accessible for enzymes to digest. Generally, this delignification is more effective for agricultural residues and herbaceous crops of less lignin content than for wood material. Typically, hardwood contains less lignin than, in general, softwood. Using lime instead of sodium hydroxide in an alkaline pretreatment is especially suited for agricultural residues and hardwood, such as poplar wood (Galbe and Zacchi 2007; Kumar 2009; Sierra et al. 2009; Taherzadeh and Karimi 2008). By the generally high content of lignin, alkaline pretreatment is less suitable for softwood (Taherzadeh and Karimi 2008).

Ionic liquid (ILs) pretreatments use chemicals, such as 1-allyl-3methylimidazolium chloride (AmimCl), 1-butyl-3-methylimidazolium chloride $(\mathrm{BmimCl})$, and 1-ethyl-3-methylimidazolium acetate ([Emim] $\left.\left[\mathrm{CH}_{3} \mathrm{COO}\right]\right)$ which organic salts that usually melt below $100^{\circ} \mathrm{C}$. By their environmentally friendly 
character, these solvents are also called "green solvents". Their chemical properties are excellent to dissolve polar and non-polar organic, inorganic and polymeric compounds. ILs has been shown to decrease the degree of polymerization and crystallinity of cellulose. After dissolution in ILs, wood therefore will be degenerated into an amorphous mixture of its original components. Methods applying ILs are especially used for softwoods, such as pine and spruce, and sometimes also for hardwood, such as maple (Kilpeläineni et al. 2007; Lee et al. 2008; Li et al. 2008; Sievers et al. 2009). In the usual instance, upon ILs pretreatment, the modified material will be afterwards be subjected to enzymatic digestion of the released cellulose. However, Li et al. (2008) and Sievers et al. (2009) reported that they used only ILs to dissolve and hydrolyze cellulose and hemicellulose, and the resulting sugars were obtained without any enzymatic hydrolysis by addition of a catalyst such as $\mathrm{HCl}$ or $\mathrm{H}_{2} \mathrm{SO}_{4}$. The presence of such a catalyst is up to now of high advantage, because enzymatic hydrolysis often suffers a low total rate of conversion of cellulose to glucose and, also, enzymes on the market are usually still (too) expensive for such purpose (Mtui 2009; Taniguchi et al. 2010).

Diluted acid pretreatment is probably the most commonly applied method among all types of chemical pretreatments. This type of method is performed by soaking the material in diluted acid solution and incubating between $140^{\circ} \mathrm{C}$ and $200^{\circ} \mathrm{C}$ for a certain time. Suitable acidic compounds for pretreatment of plant biomass include phosphoric acid, sulphuric acid, sulphur dioxide and sulphite. Under such acidic treatments, the hemicellulose in the plant biomass is hydrolyzed to monomer sugars. Development of lignocellulose pretreatments featuring modest reaction conditions (low temperature and atmospheric pressure) is highly desired because of the decreased energy consumption as a capital investment into the process. In addition, also application of mild conditions reduces the unwanted chemical sugar degradation and the formation of inhibitors acting negatively on microorganism and/or enzymes in ethanol production from the fermentable sugars. To emphasize the importance of the latter, the following recent development is presented at this place: Phosphoric acid was used for a new type of lignocellulose pretreatment in combination with acetone and water as solvent. The organic acetone helps to solve the lignin in the organic phase and to precipitate released cellulose and hemicellulose. The very high sugar yields after enzymatic hydrolysis were attributed to no sugar degradation with the highest enzymatic digestibility ( $\sim 97 \%$ in $24 \mathrm{hrs}$ ), avoided due to the fractionation of cellulose 
from hindering lignin (Monavari et al. 2009; Wang et al. 2009; Zhang et al. 2007; Zheng et al. 2007; Zhu et al. 2010).

Another approach is to use organic acids such as oxalic acid, acetylsalicylic acid and salicylic acid, all of which can be used as catalysts in so called organosolv processes whereby an organic and aqueous organic solvent mixture with inorganic acids $\left(\mathrm{H}_{2} \mathrm{SO}_{4}\right.$ or $\left.\mathrm{HCl}\right)$ are used to break the internal lignin and hemicellulose bonds in plant biomass. The soluble lignin must be washed away with a suitable solvent, such as methanol, ethanol, acetone, ethylene glycol and phenol, prior to enzymatic hydrolysis and fermentation, in order to prevent solvents, lignin and other compounds from degradation, to act as inhibitors of subsequent enzymatic steps (Galbe and Zacchi 2007; Mtui 2009).

\section{I.3.2 Physical and physico-chemical pretreatments}

This category of measures includes physical pretreatment and techniques that combine both chemical and physical pretreatment. Physical pretreatment can increase pores size within cellulose fibrils and accessible surface areas, and it can decrease the degree of polymerization and crystallinity of cellulose (Taherzadeh and Karimi 2007). Different types of physical pretreatment are milling, irradiation by gamma rays, ultrasound, application of electron beams, microwaving, hydrothermal treatment, high pressure steaming, extrusion, expansion and pyrolysis, which may be applied individually or also in certain combinations. In case of physico-chemical pretreatments, the most important processes include explosion techniques (steam explosion, ammonia fiber explosion [AFEX], $\mathrm{CO}_{2}$ explosion and $\mathrm{SO}_{2}$ explosion), liquid hot-water treatments and microwave-chemical treatment (acid and alkaline) (Galbe and Zacchi 2007; Hu et al. 2008; Taherzadeh and Karimi K 2007; Taherzadeh and Karimi 2008).

Steam pretreatment for example can be used together with acid catalysts, such as $\mathrm{H}_{2} \mathrm{SO}_{4}$ or $\mathrm{SO}_{2}$ for hydrolysis of the polymeric carbohydrates and for improvement of enzymatic digestibility in case of softwood that by its high lignin content in general is more difficult to degrade (Taherzadeh and Karimi 2008).

Hydrothermal pretreatment is quite similar in effects to steam pretreatment, but it utilizes lower temperature and lower dry matter content by a generally higher water content compared to steam pretreatment. As a consequence, the resulting sugar yields 
are unfortunately more diluted and, by the heating of a larger water volume, this type of process is more energy demanding (Taherzadeh and Karimi 2008).

Ammonia fiber explosion (AFEX) in presence of water is a method similar to steam pretreatment. In this method, almost no hemicellulose and lignin will be removed. This method can however increase the water holding capacity of plant cell material and the digestibility of the swollen cellulose. AFEX performs best on agriculture residues (Kumar 2009), but some research reported also high sugar yields from poplar wood ( $>90 \%$ ) by using AFEX in combination with $\mathrm{SO}_{2}$ as an acid catalyst and lime or dilute acid (Kumar 2009; Wyman et al. 2009). Also, this method apparently does not produce inhibitors that may inhibit enzymatic hydrolysis (Sun and Cheng 2002).

\section{I.3.3 Biological pretreatments}

Biological pretreatment can be performed by applying lignin and hemicellulose degrading microorganisms, such as white-rot and brown-rot fungi, respectively, to the plant biomass including wood ( $\mathrm{Hu}$ et al. 2008; Taherzadeh and Karimi 2008). On the other hand, also insect-infested wood has a certain potential to affect lignin, carbohydrate and wood extractive contents by the insects grazing on the material (Chandra et al. 2009; Ewanick et al. 2007; Pan et al. 2007). Lignocellulosic-feedinginsects have cellulolytic activities in gut and head-derived fluids that support wood degradation (Oppert et al. 2010). These types of natural pretreatments by microorganisms and insects are considered to be environmentally friendly and energy saving as there is no use of chemicals involved. However, some biomass will be lost with such natural methods and incubation with microorganisms is far too timeconsuming to be done on an industrial scale, whilst targeted incubation with insects might likely not at all be possible. As an optimization of other pretreatments however, such natural treatment of shorter time (in case of microorganisms) or as events that happened in nature (mass infestations of trees by insects) could be used as an initial step in combination with other types of wood pretreatment (Carmona et al. 2009; Pan et al. 2007; Schilling et al. 2009). 


\section{I.4 Effects of pretreatment on enzymatic glucose production from wood}

Effects of wood pretreatment on enzymatic hydrolysis to fermentable sugar production are summarized in Table 1. Briefly, in this table different wood species are divided into the two categories of softwood (Douglas fir, pine and spruce) and hardwood (poplar, eucalyptus, maple, olive tree, red oak, beech, and rubber wood). Effects on different techniques of type of wood substrates (wood chip, wood particles or wood powder), the various methods of wood pretreatment used, and fermentable sugars' yields are summarized. The information in Table 1 also describes in more details: i. Enzymatic hydrolysis yield which is determined from glucose obtained in the enzymatic hydrolysis divided by the potential glucose in the pretreatment materials (Ruiz et al. 2006). ii. Cellulose digestibility which is calculated based on the definition of conversion that is the ratio of the amount of soluble sugars (glucose equivalent) in the liquid phase after hydrolysis to the initial amount of glucose equivalent in the solid cellulose sample before hydrolysis (Zhang et al. 2007a).

Numerous pretreatment investigations for the target of the biofuel production have been carried out using agricultural residues and hardwood and softwood, respectively (Table 1), but with any type of lignocellulosic starting material it is very difficult to compare the yields of fermentable sugar obtained from the different methods and studies. The pretreatment methods are usually evaluated using subsequent enzymatic hydrolysis of a measure of success but different enzyme concentrations, singly or in combination are applied, mainly cellulase and $\beta$-glucosidase, making result also different to compare. A systematic analysis of influencing of pretreatments for any given lignocellulosic material comparing different methods is still missing.

Widely researched softwoods are so far pine and spruce (Table 1). Pine for example was especially investigated in ionic liquid pretreatment. The production of sugar obtained was around $80 \%$ of the theoretically obtainable yield (without enzymatic hydrolysis). In case of steam pretreatment, both of softwood pine and spruce obtained the highest of the theoretically possible yields of sugars of up to $99 \%$, using cellulase + $\beta$-glucosidase for cellulose conversion (Galbe and Zacchi 2007, Table 1). 


\section{I.5 Economical and environmental aspects of biofuel production (bioethanol)}

Ethanol is increasingly used as a transportation fuel. It can also be used in blends with gasoline, which is used commonly in internal combustion engines. Ethanol promotes complete combustion and reduces the emission of pollutants, such as carbon monoxide. It is also effective in increasing the octane number in fuels which defines the quality of fuels in combustion. Because ethanol alone has only a low energy content compared to gasoline, especially when it is used pure, ethanol needs to be supplemented with e.g. gasoline and other additives such as mercury (Briens et al. 2008).

Ethanol is a high priority solution of energy fuels because of its low toxicity and its easy biodegradability. In the past few years, ethanol in the USA was produced in large amounts with 20 billion litters per year and in Brazil with 17 billion litters per year, usually from corn and sugarcane. However, bioethanol production from renewable wood as a non-food and non-fodder resource needs much more advanced processing and technology in order to be applicable for the demands and the supplies of the world fuels consumption (Briens et al. 2008; Olofsson et al. 2008; Sainz 2009).

The first generation of biofuels from food and fodder type of biomass ${ }^{\wedge \wedge}$ has a high potential in the ease of production and in costs of production, but can not be considered to provide a good renewable energy source. As other disadvantages, the first generation of biofuel production from food and fodder type of biomass raised the price of raw materials. Large amounts of wastewater are employed in production ( 11 ethanol production produces 131 of wastewater) and the production of the material such as growth of corn uses more herbicides as well as more nitrogen fertilizers than any other crops produced for example in the USA, giving rise to groundwater and river pollution. Thus, biofuel productions nowadays are moving into the more environmental friendly and low-cost direction helping in addition the $\mathrm{CO}_{2}$, and global climate change problems (Pimentel and Patzek 2005).

The biochemical production of cellulosic ethanol (ethanol obtained from hydrolysis of cellulose into fermentable sugars and converting them into ethanol by microbial fermentation) from wood and other lignocellulosic biomass through a step of enzymatic hydrolysis of cellulose is very promising for the future for applying environmentally friendly biotechnological processes for the production of renewable bioenergy, and in particular bioethanol. 
Pretreatment processes of lignocellulosic biomass are necessary to achieve a good enzymatic degration of polymeric carbohydrates into sugars for production of ethanol by enzymatic hydrolysis in microbial fermentation. An economical and effective pretreatment should address: i. production of reactive cellulosic fibers for enzymatic attack, ii. destruction of hemicelluloses and cellulose as less as possible, ii. avoidance of formation of possible inhibitors of any kind for hydrolytic enzymes and fermenting microorganisms, iv. reduction of the energy demand, $\mathbf{v}$. minimizing costs of size reduction for feedstocks, vi. reduction in cost of materials for construction of pretreatment reactors, vii. production of less residues, viii. consumption of little amounts or of no chemicals and, if required, using cheap chemicals (Taherzadeh and Karimi 2008). The examples in Table 1 show that in this field many activities for improvements are currently ongoing.

The high costs of enzymes present another significant barrier to commercialization of bio-based fuel products. Principally, the costs are determined by the large amounts of enzymes required to access cellulose and hemicellulose to be transformed first into sugars and next the sugars into ethanol. As of the present situation, significant efforts have been done to reduce the costs of hydrolytic enzyme processes by i. optimization of pretreatment methods to be best compatible with enzymes ii. identification of new or more active enzymes, iii. optimization of the combination of hydrolytic enzymes on selected pretreatment methods and iv. using additional enzymes which function as reducers of inhibitor compounds in fullperformance enzymatic hydrolysis (Mtui 2009).

The end product of enzymatic hydrolysis of lignocellulose is mostly a glucose monomer that will be transformed as a raw material in a microbial fermentation process, usually with yeast (Galbe and Zacchi 2007; Kumar et al. 2009). After distillation, pure ethanol is obtained as an end product of bioethanol production from the sugars. To improve this last step in the process from lignocellulose to ethanol even better, there is a worldwide interest in the characterization of C5/C6 sugar-fermenting organisms. Analysis of tolerance to inhibitors in fermentation organisms with rapid growth, improved tolerance to inhibitors of cellulose-to-glucose conversion and the ability of fermentation of multiple sugars under industrial conditions are given priority to (Briens et al. 2008; Galbe and Zacchi 2007; Karimi 2007; Kumar et al. 2009; Hu et al. 2008). Other developments in the field of microorganisms target at increasing natural characters by adding new properties to the organisms through recombinant DNA 
technologies, e.g. that the natural range of fermentable sugars will be increased by additional ones (see the reviews by Abbott et al. 2009; Alper and Stephanopoulos 2009; Dmytruk et al. 2008; Hahn-Hägerdahl et al. 2007; la Grange et al. 2010 and Weber et al. 2010).

To produce only ethanol from cellulosic sugars is not necessarily the best or the only economic possibility to make use of energy and material as stored in wood and other lignocellulosic material. The large market of ethanol makes it likely possible to achieve economies of scale of reduced sugar costs from the lignocellulose, but butanol and other value added bio-based chemicals are further good alternatives for the chemical markets (Mtui 2009; Sun and Cheng 2002).

\section{I.6 Aim of the thesis}

Nowadays, much research has been and is still being performed on transformation of lignocellulose from plant cell walls into fermentable sugars by suitable pretreatments of the material and by enzymatic hydrolysis of released cellulose for bioethanol production from fermentable sugars (Table 1). Of the conversion of plant materials tested by other studies, less work has been investigated on using diverse species of softwood for establishing efficient methods for biofuel production (Galbe and Zacchi 2007). This thesis study therefore focuses especially on wood of Abies grandis which is a fast growing coniferous tree with a high potential for sustainable wood production and applications in the wood products industry (Polle et al. 2008; Spellmann et al. 2010). Research of this thesis targets at wood biomass from $A$. grandis as a potential source for enzymatic treatment technologies for use to unlock the energy stored in the lignocellulose as renewable biomass by converting especially the cellulose of the material into fermentable sugars that can then be applied in biofuel production.

At the beginning of this work, nothing was known on reactions of enzymes with A. grandis wood. Enzymes for enzymatic treatments of lignocellulosic material are usually of microbial origin, often of fungal origin (Eriksson et al. 1990). Also, little was known at the beginning of this study on how decay fungi react with living or dead A. grandis wood. Few reports are available from observations in nature, suggesting a good resistance of the wood against decay fungi (Hawighorst et al. 2010; Kües et al. 2009; Navarro-González et al. 2008). Parallel studies to this work by Dr. Monica Navarro-González on solid wood and on wood composites made from A. grandis 
revealed a certain degree of resistance of against white-rot fungi and sensitivity against brown-rot (M. Navarro-González, personal communication). In this study, it was therefore first a task to further analyze the behavior of $A$. grandis wood with decay fungi and to establish fungal reactions in wood decay. Laccase as a redox-enzyme acting on phenolic structures of lignin (Gómez-Toribio et al. 2009; Hammel et al. 1993; Leonowicz et al. 2001) was found to be active on the A. grandis wood and studied in more detail. Resulting knowledge on laccase was applied later in the development of techniques to convert the $A$. grandis wood into fermentable sugars.

The experimental work of this thesis is presented in four chapters (Chapter II to V) followed by another chapter (Chapter VI) discussing more generally the results of this thesis. The presentation of experiments were subdivided according to research that targeted at elucidation of decay of $A$. grandis wood by fungi (Chapter II), of effects of enzymes on the $A$. grandis cell wall structure (Chapter III), to the biological function of laccase in connection to A. grandis wood and wood extractives (Chapter IV) and application of $A$. grandis wood and enzymes in particularly also laccases into different enzyme mixtures based on cellulases and xylanases for the production of fermentable sugars for the future target of biofuel production (Chapter V). In short detail, the different chapters address:

Chapter II: The fungal attack of $A$. grandis wood was studied using the brownrot fungus Coniophora puteana and various species of white-rot fungi. In fungal degraded $A$. grandis wood, modifications of wood cell walls by extracellular ligninolytic enzymes and cell wall staining methods (with MBTH: 3-methyl-2-benzothiazolinonehydrazone hydrochloride monohydrate) were investigated.

Chapter III: The effects of enzyme actions on modification of wood cell walls (earlywood and latewood) were evaluated by following up changes in cell walls' coloration after staining wood blocks treated with the white-rot Trametes versicolor. Fungal decay of A. grandis wood cell walls was simulated by injecting commercial laccase and $\mathrm{Mn}^{3+}$ acetate as a product of fungal Mn peroxidase (another enzyme known to act in decay of lignocellulose (Mester and Field 1998). Effects were followed up by image analysis and computational analyses of degrees of decay and staining by MBTH. 
Chapter IV: Wood and wood extractives of $A$. grandis wood were used in laccase induction experiments with the white-rot fungi Pleurotus ostreatus and T. versicolor. GC-MS analysis was used to analyze the extractable compounds in wood extractives in order to identify compounds that act as inducers of laccase production. The influence of wood extractives on growth of the wood decay fungi $P$. ostreatus and $T$. versicolor were tested and on growth of the dung fungus Coprinopsis cinerea.

Chapter V: This chapter focuses on the application of lignocellulosic material of A. grandis in producing glucose from its cellulose for potential biofuel production. Different kinds of wood pretreatment were addressed to efficiently release the cellulose from $A$. grandis wood and different kinds of enzymes (cellulase, xylanase, laccase, and $\beta$-glucosidase) and different mixtures of the enzymes were used to convert the modified cellulose in subsequent enzymatic hydrolysis into fermentable sugars. Functions of laccase as well as $\beta$-glucosidase as reducers of inhibitory effects on enzymatic hydrolysis of cellulose was demonstrated.

\section{I.7 References}

Abbot DA, Zelle RM, Pronk JT, van Maris AJA (2009) Metabolic engineering of Saccharomyces cerevisiae for production of carboxylic acids: current status and challenges. FEMS Yeast Research 9(8):1123-1136

Alper H, Stephanopoulos G (2009) Engineering for biofuels: exploiting innate microbial capacity or importing biosynthetic potential?. Nature Reviews Microbiology 7(10):715-723

Balan V, da Costa Sousa L, Chundawat SPS, Marshall D, Sharma LN, Chambliss CK, Dale BE (2009) Enzymatic digestibility and pretreatment degradation products of AFEX-treated hardwoods (Populus nigra). Biotechnology Progress 25(2):365-375

Berlin A, Muňz C, Gilkes N, Alamouti SM, Chung P, Kang KY, Maximenko V, Baeza 
J, Freer J, Mendonça R, Saddler J (2007) An evaluation of British Columbian beetle-killed hybrid spruce for bioethanol production. Applied Biochemistry and Biotechnology 136-140:267-280

Briens C, Piskorz J, Berruti F (2008) Biomass valorization for fuel and chemicals Production - a review. International Journal of Chemical Reactor Engineering $6: 1-49$

Carmona R, Lienqueo ME, Salazar O, García A (2009) Bioenergy II: biological pretreatment with fungi as a tool for improvement of the enzymatic saccharification of Eucalyptus globulus Labill to obtain bioethanol. International Journal of Chemical Reactor Engineering 7:1-13

Cara C, Romero I, Oliva JM, Sáez F, Castro E (2007) Liquid hot water pretreatment of olive tree pruning residues. Applied Biochemistry and Biotechnology 136$140: 379-394$

Chandra R, Ewanick S, Hsieh C, Saddler JN (2008) The characterization of pretreated lignocellulosic substrates prior to enzymatic hydrolysis, part 1: a modified Simons' staining technique. Biotechnology Progress 24:1178-1185

Chandra RP, Ewanick SM, Chung PA, Au-Yeung K, Rio LD, Mabee W, Saddler JN (2009) Comparison of methods to assess the enzyme accessibility and hydrolysis of pretreated lignocellulosic substrates. Biotechnology Letters $31: 1217-1222$

Dadi AP, Schall CA, Varanasis S (2007) Mitigation of cellulose recalcitrance to enzymatic hydrolysis by ionic liquid pretreatment. Applied Biochemistry and Biotechnology 136-140/407-422

Dasari RK, Berson RE (2007) The effect of particle size on hydrolysis reaction rates and rheological properties in cellulosic slurries. Applied Biochemistry and Biotechnology 136-140:289-300

Dmytruk OV, Dmytruk KV, Voronovsky AY, Sibirny AA (2008) Metabolic engineering of the initial stages of xylose catabolism in yeast for the purpose of constructing efficient producers of ethanol from lignocellulosics. Cytology and Genetics 42(2):127-138

Draude KM, Kurniawan CB, Duff SJB (2001) Effect of oxygen delignification on the rate and extent of enzymatic hydrolysis of lignocellulosic material. Bioresources Biotechnology 79:113-120

Eriksson KEL, Blanchette RA, Ander P (1990) Microbial and enzymatic degradation of 
wood and wood components. Springer-Verlag, Berlin

Ewanick SM, Bura R, Saddler JN (2007) Acid-catalyzed steam pretreatment of lodgepole pine and subsequent enzymatic hydrolysis and fermentation to ethanol. Biotechnology and Bioengineering 98(4):737-746

Galbe M, Lidén G, Zacchi G (2005) Production of ethanol from biomass-research in Sweden. Journal of Scientific and Industrial Research 64:905-919

Galbe M, Zacchi G (2007) Pretreatment of lignocellulosic materials for efficient bioethanol production. Advances in Biochemical Engineering Biotechnology 108:41-65

Gómez-Toribio V, García-Martín AB, Martínez MJ, Martínez ÁT, Guillén F (2009) Induction of extracellular hydroxyl radical production by white-rot fungi through quinone redox cycling. Applied and Environmental Microbiology 75(12):3944-3953

Gusakov AV, Salanovich TN, Antonov AI, Ustinov BB, Okunev ON, Burlingame R, Emalfarb M, Baez M, Sinitsyn AP (2007) Design of highly efficient cellulase mixtures for enzymatic hydrolysis of cellulose. Biotechnology and Bioengineering 97(5):1028-1038

Hahn-Hägerdal B, Karhumaa K, Fonseca C, Spencer-Martins I, Gorwa-Grauslund MF (2007) Towards industrial pentose-fermenting yeast strains. Applied Microbiology and Biotechnology 74(5):937-953

Hammel KE, Jensen KA, Mozuch MD, Landucci LL, Tien M, Pease EA (1993) Ligninolysis by a purified lignin peroxidase. Journal of Biological Chemistry 268(17):12274-12281

Hawighorst P, Müller GS, Navarro-González M, Malik I, Kües U, Polle A (2010) Knowledge-based production and durability of innovation beech and grand fir wood-base panels. Forst und Holz 65(1):20-25

Hideno A, Aoyagi H, Isobe S, Tanaka H (2007) Utilization of spent sawdust matrix after cultivation of Grifola frondosa as substrate for ethanol production by simultaneous saccharification and fermentation. Food Science and Technology Research 13(2):111-117

Hu G, Heitmann JA, Rojas OJ (2008) Feedstock pretreatment strategies for producing ethanol from wood, bark and forest residues. BioResources 3(1):270-294

Inoue H, Yano S, Endo T, Sakaki T, Sawayama S (2008) Combining hot-compressed 
water and ball milling pretreatments to improve the efficiency of the enzymatic hydrolysis of eucalyptus. Biotechnology for Biofuels doi: 10.1186/1754$6834 / 1 / 2$

Jørgensen H, Kristensen JB, Felby C (2007) Enzymatic conversion of lignocellulose into fermentable sugars: challenges and opportunities. Biofuels, Bioproducts and Biorefining1:119-134

Kilpeläineni I, Xie H, King A, Granstrom M, Heikkien S, Argyropoulos DS (2007)

Dissolution of wood in ionic liquids. Journal of Agricultural and Food Chemistry 55: 9142-9148

Kumar P, Barrett DM, Delwiche MJ, Stroeve P (2009) Methods for pretreatment of lignocellulosic biomass for efficient hydrolysis and biofuel production. Industrial and Engineering Chemistry Research 48:3713-3729

Kües U, Navarro-González M, Cherdchim B, Majcherczyk A (2009) Anfälligkeit von lebendem und totem Holz der Küstentanne gegenüber Pilzen. Mykologisches Kolloquium, Molekularbiologische Pilzdiagnostik, 7./8.Mai 2009 in Dresden, Institut für Holztechnologie, Dresden:1-14

Kumar R (2009) Effects of cellulase and xylanase enzymes on the deconstruction of solids from pretreatment of poplar by leading technologies. Biotechnology Progress 25(2):302-314

la Grange DC, den Haan R, van Zyl WH (2010) Engineering cellulolytic ability into bioprocessing organisms. Applied Microbiology and Biotechnology 87(4):11951208

Leonowicz A, Cho NS, Luterek J, Wilkolazka A, Wojtas-Wasilewska M, Matuszewska A, Hofrichter M, Wesenberg D, Rogalski J (2001) Fungal laccase: properties and activity on lignin. Journal of Basic Microbiology 41(3-4):185-227

Lee SH, Doherty TV, Linhardt RJ, Dordick JS (2008) Ionic liquid-mediated selective extraction of lignin from wood leading to enhanced enzymatic cellulose hydrolysis. Biotechnology and Bioengineering 102(5):1368-1376

Li C, Wang Q, Zhao YK (2008) Acid in ionic liquid: An efficient system for hydrolysis of lignocellulose. Green Chemistry 10:177-182

Mabee WE, Gregg DJ, Arto C, Berlin A, Bura R, Ilkes N, Mirochnik O, Pan X, E. Pye K, Saddler JN (2006) Updates on softwood-to-ethanol process development. Applied Biochemistry and Biotechnology 129-132:55-70

Mester T, Field JA (1998) Characterization of a novel manganese peroxidase-lignin 
peroxidase hybrid isozyme produced by Bjerkandera species strain BOS55 in the absence of manganese. Journal of Biological Chemistry 273:15412-15417

Monavari S, Galbe Mats, Zacchi G (2009) The influence of solid/liquid separation techniques on the sugar yield in two-step dilute acid hydrolysis of softwood followed by enzymatic hydrolysis. Biotechnology and Biofuels 2(6):1-9

Mtui GYS (2009) Recent advances in pretreatment of lignocellulosic wastes and production of value added products. African Journal of Biotechnology 8(8):1398-1415

Nakata T, Miyafuji H, Saka S (2009) Process integration of ethanol production from Japanese beech as treated with hot-compressed water followed by enzymatic treatment. Journal of Wood Science 55:295-301

Navarro-González M, Wei DS, Kües U (2008) Pilze of Holz der Küstentanne (Abies grandis). Holzbiologie 49(6): 23-25

Olofsson K, Bertilsson M, Lidén G (2008) A short review on SSF - an interesting process option for ethanol production from lignocellulosic feedstocks. Biotechnology and Biofuels 1(7):1-14

Oppert C, Klingeman WE, Willis JD, Opert B, Jurat-Fuentes JL (2010) Prospecting for cellulolytic activity in insect digestive fluids. Comparative Biochemistry and Physiology B-Biochemistry \& Molecular Biology 155(2):145-154

Palm M, Zacchi G (2003) Extraction of hemicellulosic oligosaccharides from spruce using microwave oven or steam treatment. Biomacromolecules 4:617-623

Pan X, Gilkes N, Kadla J, Pye K, Saka S, Gregg D, Ehara K, Xie D, Lam D, Saddler J (2006) Bioconversion of hybrid poplar to ethanol and co-products using an organosolv fractionation process: optimization of process Yields. Biotechnology and Bioengineering 94(5):852-861

Pan X, Xie D, Yu RW, Lam D, Saddler JN (2007) Pretreatment of lodgepole pine killed by mountain pine beetle using the ethanol organosolv process: fractionation and process optimization. Industrial Engineering Chemistry Research 46:2609-2617

Petchpradab P, Yoshida T, Charinpanitkul T, Matsumura Y (2009) Hydrothermal pretreatment of rubber wood for the saccharification process. Industrial Engineering Chemistry Research 48:4587-4591

Pimentel D, Patzek TW (2005) Ethanol production using corn, switchgrass, and wood; biodiesel production using soybean and sunflower. Natural Resources Research 14(1):65-76 
Ruiz E, Cara C, Ballesteros M, Manzanares P, Ballesteros I, Castro E (2006) Ethanol production from pretreated olive tree wood and sunflower stalks by an SSF process. Applied Biochemistry and Biotechnology 129-132:631-643

Sainz MB (2009) Commercial cellulosic ethanol: the role of plant-expressed enzymes. In Vitro Cellular and Developmental Biology-Plant 45:314-329

Sannigrahi P, Miller SJ, Ragauskas AJ (2010) Effects of organosolv pretreatment and enzymatic hydrolysis on cellulose structure and crystallinity in Loblolly pine. Carbohydrate Research 345(7):965-970

Schilling JS, Tewalt JP, Duncan SM (2009) Synergy between pretreatment lignocellulose modifications and saccharification efficiency in two brown rot fungal systems. Applied Microbiology Biotechnology 84:465-475

Sierra R, Granda C, Holtzapple MT (2009) Short-term lime pretreatment of poplar wood. Biotechnology Progress 25(2):323-332

Sievers C, Valenzuela-Olarte MB, Marzialetti T, Musin I, Agrawal PK, Jones CW (2009) Ionic-liquid-phase hydrolysis of pine wood. Industial Engineering Chemistry Research 48:1277-1286

Söderström J, Galbe M, Zacchi G (2004) Effect of washing on yield in one- and twostep steam pretreatment of softwood for production of ethanol. Biotechnology Progress 20:744-749

Stenberg K, Galbe M, Zacchi G (2000) The influence of lactic acid formation on the simultaneous saccharification and fermentation (SSF) of softwood to ethanol. Enzyme and Microbial Technology 26:71-79

Stöcker M (2008) Biofuels and biomass-to-liquid fuels in the biorefinery: catalytic conversion of lignocellulosic biomass using porous materials. Angewandte Chemie International Ed 47:9200-9211

Sun Y, Cheng J (2002) Hydrolysis of lignocellulosic materials for ethanol production: a review. Bioresource Technology 83:1-11

Taherzadeh MJ, Karimi K (2007) Enzyme-based hydrolysis processes for ethanol from lignocellulosic materials: a review. BioResources 2(4):707-738

Taherzadeh MJ, Karimi K (2008) Pretreatment of lignocellulosic wastes to improve ethanol and biogas production: a review. International Journal Molecular Sciences 9:1621-1651

Taniguchi M, Takahashi D, Watanabe D, Sakai K, Hoshino K, Kouya T, Tanaka T 
(2010) Evaluation of fungal pretreatments for enzymatic saccharification of rice straw. Journal of Chemical Engineering of Japan 43(4):401-405

Tu M, Chandra RP, Saddler JN (2007) Recycling cellulases during the hydrolysis of steam exploded and ethanol pretreated lodgepole pine. Biotechnology Progress 23:1130-1137

Wang GS, Pan XJ, Zhu JY, Gleisner R, Rockwood D (2009) Sulfite pretreatment to overcome recalcitrance of lignocellulose (SPORL) for robust enzymatic saccharification of hardwoods. Biotechnology Progress 25(4):1086-1093

Weber C, Farwick A, Benish F, Brat D, Dietz H, Subtil T, Boles E (2010) Trends and challenges in the microbial production of lignocellulosic bioalcohol fuels. Applied Microbiology and Biotechnology 87(4):1303-1315

Wu MM, Chang K, Gregg DJ, Boussaid A, Beatson RP, Saddler JN (1999) Optimization of steam explosion to enhance hemicellulose recovery and enzymatic hydrolysis of cellulose in softwoods. Applied Biochemistry and Biotechnology 77-79:47-54

Wyman CE, Dale BE, Elander RT, Holtzapple M, Ladisch MR, Lee YY, Mitchinson C, Saddler JN (2009) Comparative sugar recovery and fermentation data following pretreatment of poplar wood by leading technologies. Biotechnology Progress 25(2):333-339

Zhang YHP, Schell DJ, McMillan JD (2007a) Methodological analysis for determination of enzymatic digestibility of cellulosic materials. Biotechnology and Bioengineering 96(1):188-194

Zhang YHP, Ding SY, Mielenz JR, Cui JB, Elander RT, Laser M, Himmel ME, McMillan JR, Lynd LR (2007b) Fractionating recalcitrant lignocellulose at modest reaction conditions. Biotechnology and Bioengineering 97(2):214-223

Zheng Y, Pan Z, Zhang R, Labavitch J, Wang D, Teter SA, Jenkins BM (2007) Evaluation of different biomass materials as feedstock for fermentable sugar production. Applied Biochemistry and Biotechnology 136-140:423-436 Zhu JY, Zhu W, OBryan P, Dien BS, Tian S, Gleisner R, Pan XJ (2010) Ethanol production from SPORL-pretreated lodgepole pine: preliminary evaluation of mass balance and process energy efficiency. Applied Microbiology and Biotechnology doi: 10.1007/s000253-009-2408-7 


\section{CHAPTER II}

\section{Degradation of solid Abies grandis wood by fungi}

Contributions of others to this chapter: I am very much thankful to Dr. Mónica NavarroGonzález for kindly providing me the data and her input on fungal degradation of MDF (medium density fiberboards) made of $100 \%$ Abies grandis fibers. 



\section{II.1 Abstract}

Wood mass loss tests were carried out by following norm EN 113 (1996) with 20 weeks of incubation of $A$. grandis wood blocks [30 (longitudinal) x 10 (tangential) x 5 (radial) $\mathrm{mm}$ ] using the brown-rot fungus Coniophora puteana 57 and the white-rot fungi Trametes versicolor Strain 6, Pleurotus ostreatus 8F6, Heterobasidion annosum TC_122-12, P. ostreatus PC9, Heterobasidion sp. strain 121, and Armillaria mellea 901010.3, respectively. C. puteana caused $50 \pm 3.44 \%$ of wood mass loss, $T$. versicolor $36 \pm 6.73 \%$, P. ostreatus $8 \mathrm{~F} 634 \pm 3.51 \%$, and H. annosum TC_122-12 20 $\pm 5.54 \%$. In contrast, no mass loss was obtained by P. ostreatus PC9, Heterobasidion sp. 121 and A. mellea. After fungal incubation, microtome sections of the different wood blocks were prepared and stained with MBTH (3-methyl-2-benzothiazolinone-hydrazone hydrochloride monohydrate). Reddish staining of the wood cell walls indicated chemical cell wall modifications. Reduction of cell wall thickness and mass loss of wood caused by decay by white-rot fungi decreased the amount of staining in later stages of decay. Remaining cell walls were also little stained in later stages of decay, likely due to degradation of chemical compounds that are reactive with MBTH. Degradation by different treatments of wood blocks was further demonstrated in investigations with $T$. versicolor. Wood treated with urea formaldehyde (impregnation under vacuum with $68 \%$ urea formaldehyde in water) or ammonium sulphate (impregnation under vacuum with 33\% ammonium sulphate in water) enhanced the resistance against the fungus, as shown by the reduced low mass loss that was obtained within 12 weeks of incubation with the fungus in comparison to untreated wood. Wood extracted with water, however, caused a loss of resistance. Decay by $T$. versicolor was faster and even higher compared to degradation of the untreated wood by the fungus. Broken wood was also easily degraded by fungi, likely because of the easy entry for the fungus into the wood.

Keywords: Abies grandis wood, Armillaria mellea, Heterobasidion annosum, Heterobasidion parviporum, Pleurotus ostreatus, Trametes versicolor, Coniophora puteana, biodegradation, cell wall modification, urea formaldehyde, ammonium sulphate, mass loss 


\section{II.2 Introduction}

Lignin is a highly branched polyphenolic amorphous polymer in the plant cell wall and is after cellulose the second most abundant chemical on earth (Fengel and Wegener 1983). This polyphenol has a wide range of functional groups provided by the phenyl-propanoid monomers of coniferyl, sinapyl, and $p$-coumaryl alcohols being the basic chemical units that upon polymerization form the lignin. Lignin is thermoplastic in nature; physically, it gives rigidity and stiffness to plant cell walls by forming firm linkages with cellulose and hemicellulose. In many kinds of wood industries, for example in the paper and pulp industry, in textile dye bleaching and in cellulase hydrolysis of wood for potential biofuel production, the presence of lignin is a hindrance for the wood applications since it is responsible for a brown color not wanted in the products. However, lignin can be removed by chemical or by biological bleaching (Chandra et al. 2009; Ewanick et al. 2007; Pan et al. 2007). Laccase (benzenediol: oxygen oxidoreductase, E.C. 1.10.3.2) for example appears to work as an effective enzymatic delignification system on lignocellulosic materials, especially since phenolic substrates are degraded by laccase. Laccase therefore might be applied in bleaching processes (Olofsson et al. 2008; Vivekanand et al. 2008).

White-rot fungi produce various isoforms of extracellular oxidases and peroxidases, including laccase, manganese-dependent $(\mathrm{MnP})$ and -independent peroxidase (MnIP), and lignin peroxidase (LiP), all of which can be involved in the degradation of lignin and/or other wood components (e.g. extractives) in their natural environments (Eriksson et al. 1990) and of which MnP and LiP are mostly distributed in fungi for lignocellulose degradation (Hofrichter 2002; Hofrichter et al. 2010). Laccases belong to the family of blue copper oxidases (Hoegger et al. 2006) and are part of the complex enzymatic system for lignin degradation of many white-rot basidiomycetes. They act specifically towards phenols and poly-phenols (and also towards aromatic amines) and in such way they take part in wood component degradation (Elissetche et al. 2006; Mayer and Staples 2002; Terron et al. 2004). Manganese-dependent MnP is a heme-containing enzyme that oxidizes $\mathrm{Mn}^{2+}$ to $\mathrm{Mn}^{3+}$ that chelates with organic acids. MBTH is thus the principal oxidative agent towards wood lignin (Leonowicz et al. 2001; Mester and Field 1998). Heme-containing LiP, also called ligninase, is similar in structure and mechanism to $\mathrm{MnP}$ and, as $\mathrm{MnP}$, it utilizes $\mathrm{H}_{2} \mathrm{O}_{2}$ and organic peroxides to oxidize a variety of substrates. LiP can catalyze oxidation of lignin monomers, dimers, 
and trimers as well as non-phenolic aromatic compounds (Kersten et al 1985; Shah and Nerud 2002). Catechol oxidase is included in the multi-copper oxidase family and referred to also as diphenol oxidase, $o$-diphenolase, phenolase, polyphenol oxidase or tyrosinase. In case of fungal infection, secreted multi-copper oxidases, such as catechol oxidase, act on monophenols and insert a hydroxyl moiety at the ortho position with respect to the primary $\mathrm{OH}$ group in the monophenols, thereby forming a diphenol structure possibly resulting in a yellow staining upon further oxidation (Dwivedi 2006; Harkin et al. 1973). Furthermore, oxidation of monophenol and diphenol structures by laccases can result in colored $o$ - and $p$-quinones (Dwivedi 2006; Eriksson et al. 1990). In addition, extractable phenolic compounds from the wood (wood extractives $=\mathrm{a}$ complex mixture of terpenoids and steroids, fat and wax, phenolic compounds, and inorganic compounds that are extractable by water; Sjostrom 1993) can undergo similar chemical and enzymatic reactions as lignin, forming insoluble and possibly high molecular quinones (Eriksson et al. 1990). There is widely research done on all these types of enzymes which is supported, on the one hand, by the biological importance of these enzymes in wood decay by fungi and, on the other hand, by the great potential of the enzymes for industrial purposes (Baldrian and Gabriel 2002; Domingues et al. 2007; Kilaru 2006; Kües et al. 2007).

Compared to white-rots, brown-rot fungi follow another strategy of wood decay. They rapidly depolymerize cellulose during early stages of wood decay. The attack on the hemicelluloses is presented in varying degrees; however, degradation of lignin is limited to demethoxylation and other modification reactions. The oxidation reactions performed by brown-rots base on a unique formation of highly reactive hydroxyl radicals by a reaction of hydrogen peroxide with the wood endogenous iron ion $\mathrm{Fe}^{2+}$ (Fenton-reaction):

$$
\mathrm{Fe}^{2+}+\mathrm{H}_{2} \mathrm{O}_{2} \rightarrow \mathrm{Fe}^{3+}+\mathrm{OH}^{\cdot}+\mathrm{OH}^{-}
$$

The resulting hydroxyl radical initiates the break down of glycans (cellulose and hemicellulose) that can be further depolymerized by hydrolytic enzymes (Kremer 1999).

This chapter addresses the behavior of various white- and brown-rot fungi with wood blocks of Abies grandis (grand fir) in order to enhance the basic knowledge for use of this wood. A. grandis is a fast growing coniferous tree with a high potential of 
wood production and, subsequently, for usages of the wood in the wood application technologies (Spellmann et al. 2010; Vos et al. 2010). Its drought tolerance and ecological compatibility (Röhrig 1981) makes A. grandis further well suited for sustainable wood production. So far, the A. grandis wood is, however, little used as building timber or as industrial wood in either compact, chopped or disintegrated form or in the pulp and paper industries (Müller et al. 2009; Vos et al. 2010). For defining possible indoor and outdoor usages of $A$. grandis and for an application in wood products industries, basic knowledge on the wood's behavior with fungal degraders is required. However, little is known in the scientific technical literature on fungal attack of $A$. grandis wood. What is present in the literature often contradicts a special susceptibility of $A$. grandis and its wood against fungi (Kües et al. 2009; NavarroGonzález et al. 2008). Hawighorst et al. (2010) and Kües et al. (2009) published first experiments on behavior of MDF (medium density fiberboards) made from $100 \%$ of A. grandis fibers with the white-rot fungus Trametes versicolor (CTB $863 \mathrm{~A}$ ), the soft-rot fungus Chaetomium globosum (ATCC 6205), and the brown-rot fungus Coniophora puteana BAM Ebw.15 (Fig. 1).
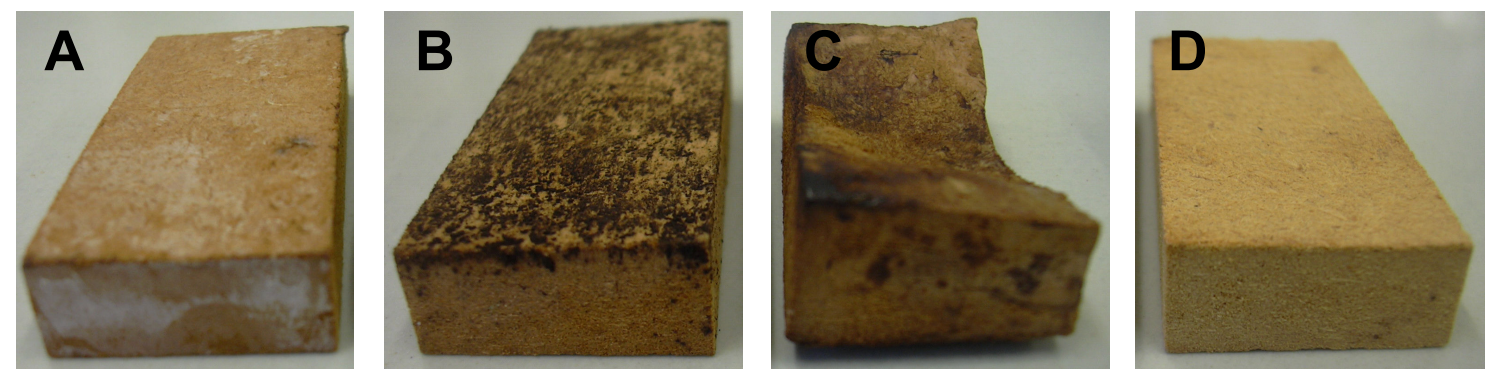

Fig. 1 Mass loss test of MDF made from 100\% A. grandis wood fibers (MDF production processes used either $68 \%$ urea formaldehyde in water or $33 \%$ ammonium sulphate in water). MDF mini blocks $(5 \mathrm{~cm} \times 2.5 \mathrm{~cm} \times 0.8 \mathrm{~cm})$ were incubated for 15 weeks following a modified form of standard EN 113 (1996): A) with the white-rot fungus $T$. versicolor (6.5 $\pm 0.7 \%$ mass loss after 15 weeks of incubation), B) with the soft-rot fungus $C$. globosum (5.1 $\pm 0.5 \%$ mass loss after 15 weeks of incubation), C) with the brown-rot fungus $C$. puteana (33.3 $\pm 8.3 \%$ mass loss after 15 weeks of incubation) and D) for comparison without any fungus $(1.9 \pm 0.7 \%$ mass loss after 15 weeks of incubation). Mass losses were determined from the difference of the dry weights before and after incubation. Photos and data were kindly supplied by Dr. M. Navarro-González (Kües et al. 2009) 
In modified block tests according to standard EN 113 (1996) for durability evaluation, MDF of $A$. grandis fibers revealed a strong susceptibility for the tested brown-rot fungus. Mass loss with $C$. puteana after 15 weeks of incubation were as high as $33.3 \pm 8.3 \%$ and the MDF blocks dramatically changed their size, shape and color (Fig.1). The mass loss after inoculation and incubation with the white-rot fungus T. versicolor was however small, similarly as upon inoculation and incubation with the soft-rot fungus $C$. globosum. These fungi however colonized the surfaces of the MDF blocks and gave them an unpleasant look whilst the shape of the MDF blocks remained unchanged. The results suggested that the A. grandis wood is strongly susceptible to brown-rot but has a certain resistance to white-rots (Hawighorst et al. 2010; Kües et al. 2009).

In the following, an investigation of fungal attack of massive wood of A. grandis is presented using the brown-rot C. puteana and a larger selection of white-rot species. For the first time, modification of wood cell walls of $A$. grandis by extracellular ligninolytic enzymes of fungi was experimentally addressed and investigated by cell wall staining methods.

\section{II.3 Materials and methods}

\section{II.3.1 Fungal strains}

All fungal strains used in this study were from the strain collection of the Division of Molecular Wood Biotechnology and Technical Mycology of the Büsgen-Institute, Göttingen. Solid culture media [malt extract-agar ( $5 \% \mathrm{malt} / 2 \%$ agar)] were inoculated in the middle of the Petri dishes with one mycelium-overgrown agar plug per plate of either one of the white-rot fungi Armillaria mellea 901010.3, Heterobasidion sp. 121, Pleurotus ostreatus PC9, H. annosum TC_122-12, P. ostreatus 8F6 and T. versicolor Strain 6 or of the brown-rot fungus $C$. puteana 57. Inoculated plates were sealed with Parafilm (Laboratory Film, Chicago, USA) and grown for seven days at $25^{\circ} \mathrm{C}$ for use in the EN 113 (1996) wood duration test as described in II.3.2. At this time of incubation in most instances, the fungal mycelia reached the border of the plates (with exception of the cases of A. mellea and P. ostreatus PC9 would respectively grow only as a small of about $2 \mathrm{~cm}$ in diameter on the plate or solid filled the surface of the agar only by three quarter). 


\section{II.3.2 Wood block decay test}

A mini block test of $A$. grandis solid wood according to the European standard EN 113 (1996) was carried out in order to access fungal decay caused by basidiomycetes (Bravery 1978). A. grandis wood was obtained from the Stadtwald Meschede in Sauerland, Germany. The wood was cut into the smaller blocks of 30 (longitudinal) x 10 (tangential) x 5 (radial) mm. Those mini blocks with uneven grain, reaction wood, resin or in which the wood appeared unduly fast or slowly grown were rejected. After oven drying at $103 \pm 2^{\circ} \mathrm{C}$ until a constant mass was achieved (after 24 hrs of incubation), the final dry masses of the mini blocks were determined by weighting and subsequently blocks were sterilized in an autoclave using a dry process, at $121^{\circ} \mathrm{C}$ for $20 \mathrm{~min}$ (ZiRBUS Technology, Bad Grund (Harz), Germany). Sterile disposable Petri dishes measuring $90 \mathrm{~mm}$ in diameter and being $16 \mathrm{~mm}$ deep, each containing approximately $20 \mathrm{ml}$ of solid malt extract-agar medium (5\% malt/2\% agar) covered with fungal mycelia, were used as culture vessels. The depth of the agar was adjusted to this volume in order to ensure that condensed moisture on the inside of the lid did not touch the wood blocks and thereby did not induce water-logging. To further minimize the risk of water logging, the samples were placed onto pre-sterilized metal holders $(1.5 \mathrm{~mm}$ in height) laid onto the mycelial surface after fungal growth occurred on the malt extractagar plates (for fungal growth see II.3.1). Only in case of $A$. mellea and $P$. ostreatus PC9 due to slow fungal growth, the metal holders were (partially) placed onto uncovered agar. Six A. grandis mini blocks per plate were aseptically added on separate metal holders laid on the plates' surfaces, respectively on the surface of the grown mycelia per fungus and per incubation time. Each one plate with six wood blocks was prepared. Control plates without any fungus were similarly amended with six mini blocks. Plates were again sealed with Parafilm and incubated stationary at $22^{\circ} \mathrm{C}$ and $65 \%$ relative humidity $(\mathrm{RH})$ until harvesting. For harvest (after 6, 14 and 20 weeks of incubation, respectively) plates were photographed and, subsequently, the individual wood blocks were collected and freed from surface mycelium. Five of the six wood blocks per treatment were first weighed and then dried at $103 \pm 2{ }^{\circ} \mathrm{C}$ until a constant mass was achieved ( $24 \mathrm{hrs}$ of incubation), weighed again for determining of the dry mass and photographed with a digital CCD camera (Color View II, Soft Imaging System GmbH, Münster, Germany) linked to computer with an installed software tool for images analySIS $^{\circledR}$ (Soft Imaging System GmbH, Münster, Germany). Total losses of wood 
mass were calculated gravimetrically from the difference of the initial dry mass of a wood sample and the dry mass of the treated wood sample. The obtained value of absolute mass loss was set in relation to the original dry mass in order to calculate the relative mass loss in \%. Also for a quality control, dry mass was calculated indirectly by determining moisture content $\left(103 \pm 2^{\circ} \mathrm{C}\right.$ until constant mass) in a wood sub-sample through subtracting the defined dry weight from the wet weight obtained after scraping off the fungal mycelia from the wood blocks (for the detailed results, see supplementary Fig. S1).

In another set of experiments targeted at studying effects of different pretreatments on wood decay, only $T$. versicolor Strain 6 were used in duration tests after EN 113 (1996). A. grandis mini blocks of 30 (longitudinal) x 10 (tangential) x 5 (radial) mm size were aseptically transferred onto metal holders laid onto mycelia of T. versicolor inoculated and grown on malt extract-agar (5\% malt $/ 2 \%$ agar) medium in $90 \mathrm{~mm}$ in diameter Petri dishes as described under II.3.2. A. grandis wood blocks of various physical and chemical pretreatments were tested: i. untreated wood (solid wood), ii. wood extracted by immersing in $60^{\circ} \mathrm{C}$ water for overnight using a metal net as weight to keep the wood underneath the water surface, iii. wood treated with urea formaldehyde (68\% in water) in an exicator for overnight with a metal net on top of the wood to keep it in the liquid over the time, $i v$. wood treated with ammonium sulphate $\left[\left(\mathrm{NH}_{4}\right)_{2} \mathrm{SO}_{4} ; 33 \%\right.$ in water] in an exicator for overnight using also a metal net as a weight on the wood, and $v$. wood that was hammered in order to break the wood down (introducing cracks) for allowing fungal mycelia penetration. Per wood treatment type, two Petri dishes whit three wood samples each were prepared. Wood samples were analyzed after 6,12 and 18 weeks of incubation at $22^{\circ} \mathrm{C}$ and $65 \% \mathrm{RH}$ after EN 113 (1996).

\section{II.3.3 Microscopic analysis}

At each harvest date of the first set of experiments (after 6, 14 and 20 weeks, respectively), from each treatment one out of six wood blocks in total was selected for cellular analysis. After scraping off the mycelia, the wood blocks were cut with a saw about $0.5 \mathrm{~cm}$ from both the edges (cross section) and once in the middle of the block. From the obtained the surfaces of the cutting, each 10 sections of $20 \mu \mathrm{m}$ thickness were excised with a sledge microtome (Jung, No. 25116, 220 volt, 6 Amp). All sections were 
stained with lactophenol blue (Cat. No, 1.13741, Merck, Darmstadt, Germany) for one minute, sections were washed three times with water and stained with $100 \mathrm{mM}$ (in water) of 3-methyl-2-benzothiazolinone-hydrazone hydrochloride monohydrate (MBTH) for a minute in the final staining, followed up by steps of washing with water (3 times). Six randomly chosen sections from total ten sections were each time mounted in $50 \%(\mathrm{v} / \mathrm{v})$ glycerol on slides and examined by light microscopy. Cell walls of individual tissues were inspected under normal white light, to estimate the amount of modified cell walls after staining with lactophenol blue (to observe the fungal mycelia penetrating into wood cells) and MBTH reagent (to stain any modification of wood cell walls). Photographs were taken with a digital CCD camera (Color View II, Soft Imaging System GmbH, Münster, Germany) mounted on a Zeiss Axioplan microscope and linked with computer. Pictures were examined using a software tool for images analySIS $^{\circledR}$ (Soft Imaging System GmbH, Münster, Germany).

\section{II.3.4 Statistical analysis}

Group values for all parameters in the mass loss test were compared by analysis of variance (ANOVA) tests using the Fisher's least significant difference (LSD) and Duncan's test procedure for multiple comparison (SPSS 8.0 for Windows; USA). Relations among the mass losses of the samples were compared between the values obtained for each fungal strain tested at the same time of incubation and also that of the uninfected control in the same week of incubation.

\section{II.4 Results}

\section{II.4.1 Biodegradation of $A$. grandis wood blocks inoculated with different wood decay fungi}

Fig. 2 outlines the general flow chart of a set of experiments performed to evaluate the decay abilities of different white-rot and brown-rot fungi of $A$. grandis wood. Mass loss of $A$. grandis wood by fungi was observed after inoculation periods of 6 weeks, 14 weeks and 20 weeks of incubation, respectively. Mass loss values determined after fungal incubation indicate that the $A$. grandis wood has a good resistance against decay by the white-rots P. ostreatus PC9, Heterobasidion sp. 121 and A. mellea. Even with 
20 weeks of incubation, no mass loss was found in wood samples treated with these fungi, comparable as in the untreated control (Fig. 3).

Wood blocks of $A$. grandis wood 30 (longitudinal) x 10 (tangential) x 5 (radial) mm

Wood blocks individually inoculated with C. puteana 57, T. versicolor Strain 6, P. ostreatus 8F6, H. annosum TC_122-12, P. ostreatus PC9, Heterobasidion sp. 121 and $A$. mellea on 5\% malt 2\% agar media and incubated for 6, 14 and 20 weeks, respectively, at $22^{\circ} \mathrm{C}$ and $65 \%$ relative humidity

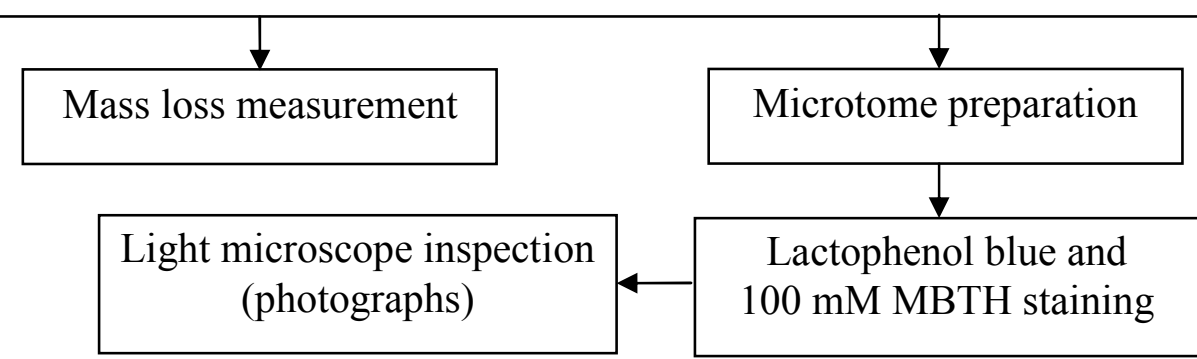

Fig. 2 General flow chart of mass loss measurement and light microscope inspection of solid $A$. grandis wood infected and in some instances also degraded by fungi

Other white-rot species, $H$. annosum TC_122-12, P. ostreatus $8 \mathrm{~F} 6$ and T. versicolor Strain 6, in contrast attacked the A. grandis wood. The mass loss of wood caused by the fungi was higher with longer incubation time. In 20 weeks of incubation, $20 \pm 5.54 \%$ mass loss was obtained by $H$. annosum TC_122-12, $34 \pm 3.51 \%$ mass loss by P. ostreatus $8 \mathrm{~F} 6$ and $36 \pm 6.73 \%$ mass loss by T. versicolor (Fig. 3). Whilst the total mass loss caused by $P$. ostreatus 8F6 and T. versicolor Strain 6 at the end of the incubation time of 20 weeks was not significantly different, $T$. versicolor however was faster in decay of the wood (Fig. 3). However, compared to the brown-rot species C. puteana, A. grandis wood was generally more resistant against white-rot decay fungi. The highest decay of all was obtained with the brown-rot species C. puteana in 20 weeks of incubation with an actual mass loss value of $50 \pm 3.44 \%$. Already after 6 weeks of incubation with C. puteana, the mass loss was as high as $30 \pm 8.5 \%$, and after 14 weeks of incubation, the mass loss was $38 \pm 20.9 \%$. Comparable values for the white-rot fungus $P$. ostreatus 8F6 were only achieved at the end of the 
incubation period of 20 weeks and for T. versicolor only after 14 and 20 weeks of incubation, respectively, whilst incubation with H. annosum TC_122-12 never reached values of such high levels (compare Fig. 3). In conclusion, decay by C. puteana does not only affect stronger the total mass loss but also, it occurs much earlier than with the white-rots.

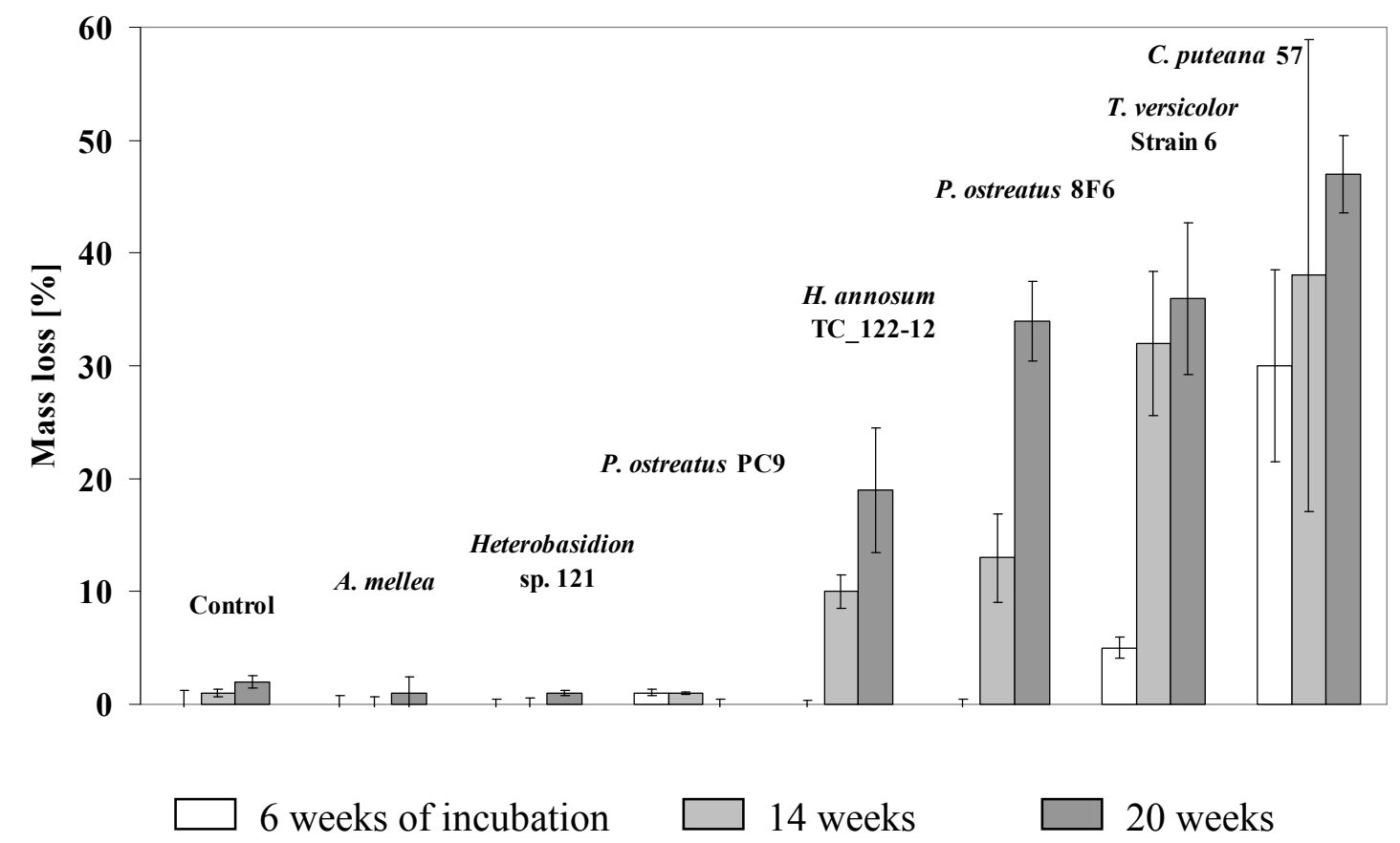

Fig. 3 Mass loss over the time caused by fungal decay of $A$. grandis wood. Average values of each 5 wood blocks per fungus and per time point were calculated and standard deviations are shown. (In the case of $C$. puteana Strain 57, 14 weeks incubation only three mini blocks were considered in calculation). Statistical analysis of the data was performed with SPSS 8.0 for Windows, the different superscripts on the chart (letters in lower case compare the values after 6 weeks of incubation, capital letters compare the values after 14 weeks of incubation, and numbers compare the values after 20 weeks of incubation) indicate values that differ significantly $(p<0.05)$ between treatments as based on analysis of variance (ANOVA)

At each harvest date and for each tested fungus, pictures of $A$. grandis wood blocks colonized with fungi were taken by a digital CCD camera in order to observe growth of fungal mycelia on the wood block's surface. The mycelial growth of all fungi tested is presented in Fig. 4 in comparison to an uninfected wood control of the same age of incubation. Among the seven fungal species, T. versicolor (Fig. 4G) and A. mellea (Fig. 4B) showed the most growth of mycelia on the surface of the wood 
blocks. Already after 6 weeks of incubation, plates were fully filled with white aerial mycelia, even in the case of $A$. mellea that originally without wood showed a very delayed mycelial growth on the agar medium (compare the plate at the first week of incubation in Fig. 4B). By speed of growth on the wood surfaces, amount and density of aerial mycelium, the two species were followed by Heterobasidion sp. 121 (Fig. 4C), P. ostreatus 8F6 (Fig. 4F) and P. ostreatus PC9 (Fig. 4D). The total aerial mycelium of H. annosum TC_122-12 (Fig. 4E) was much lower than that of the other white-rots, as also was the case for C. puteana (Fig. 4H). Special to C. puteana (Fig. 4H), rhizomorphs (compact mycelial strands) appeared in the plates at 14 weeks of incubation. The fungal mycelia from this brown-rot fungus fastest changed color and size of wood blocks that, by the low mycelial density, was visible by brown spots on the wood blocks' surfaces and, also, by the recognizable shrinkages to the wood blocks (Fig. 4H, sample of 14 and 20 weeks of incubation). C. puteana is known to degrade cellulose and in consequence leaves behind the lignin component of wood which then turns brown upon oxidation (Webster and Weber 2007). Accordingly, the brown spots at the surface of the wood samples (see in Fig. 4H in the samples of 14 and 20 weeks of incubation) are expected to be wood decayed by brown-rot. Apparently in these sets of experiments, the overall degree of formation of aerial mycelium by the fungi (Fig. 4) did not correlate with the degree of mass loss of the wood blocks they caused (Fig. 3). For example, A. mellea had highest fungal mycelia growth on wood blocks surfaces (Fig. 4B), but no mass loss was detected with this species (Fig.3), similarly as P. ostreatus PC9 and Heterobasidion sp. 121 that had a medium aerial mycelium production on the wood blocks (Fig. 4D, 4C) and showed no mass loss (Fig. 3). In conclusion, the amount of aerial mycelium produced in a Petri dish by a fungus is no indication for the overall wood degradation ability of a species.

Fig. 5 shows the harvested wood blocks after treatment with fungi, scrapping off the fungal mycelium from the surface and being hot air oven dried at $103 \pm 2^{\circ} \mathrm{C}$ until a constant mass was achieved ( $24 \mathrm{hrs}$ of incubation). In comparison to wood incubated without fungi (control), the sight of all fungal treated wood was of more yellowishorange color (Fig. 5A), especially after longer periods of cultivating with fungi. In case of the white-rot fungi, there were no obvious differences in the red colorization with regards to the severness of decay caused by fungus (Fig. 5B to G). In case of C. puteana-treated wood (Fig. $5 \mathrm{H}$ ), not only the color changed, but the reddish color 

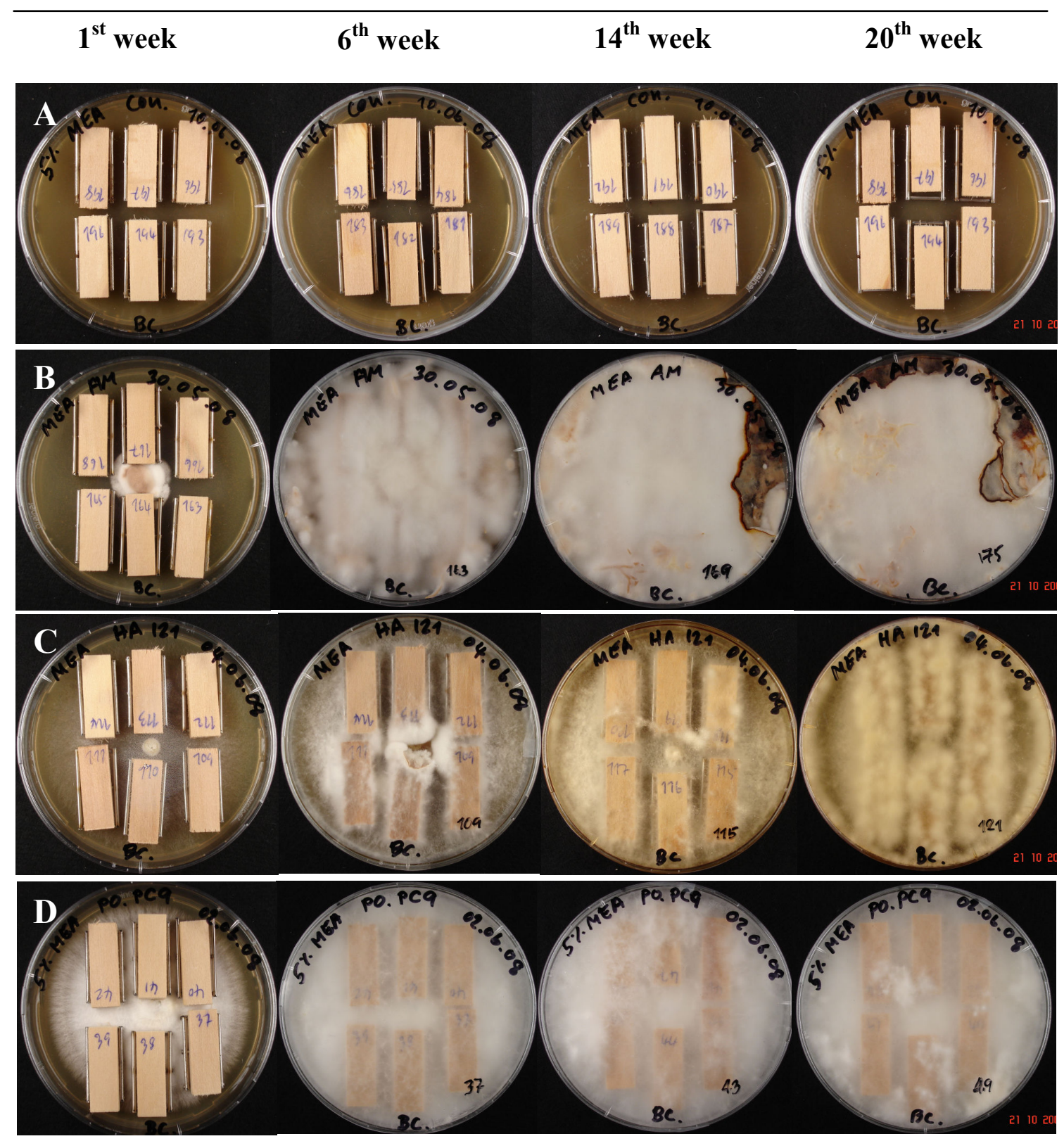

Fig. 4 Petri dishes with $A$. grandis wood blocks incubated on malt extract-agar medium (5\% malt $/ 2 \%$ agar) A) without fungi, or B) with A. mellea, C) Heterobasidion sp. 121, D) $P$. ostreatus PC9, E) $H$. annosum $\mathrm{TC} 122-12$, F) $P$. ostreatus $8 \mathrm{~F} 6$, G)

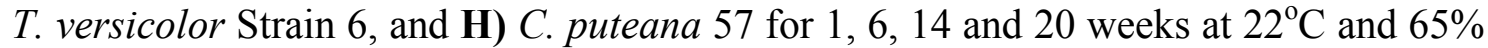
$\mathrm{RH}$, respectively. Note that the yellow color in case of plates of Heterobasidion sp. 121 and $H$. annosum TC_122-12 after 6, 14 and 20 weeks is due to staining of the plastic of the Petri dish by some unknown volatile(s) [further information is given in the supplementary material to this chapter (Fig. S2)]. When opening the Heterobasidion sp. 121 and $H$. annosum TC_122-12 plates, the appearance of the aerial mycelium indeed was white as for all other fungi (Fig. S2B). The time of incubation is indicated above each row of plates 


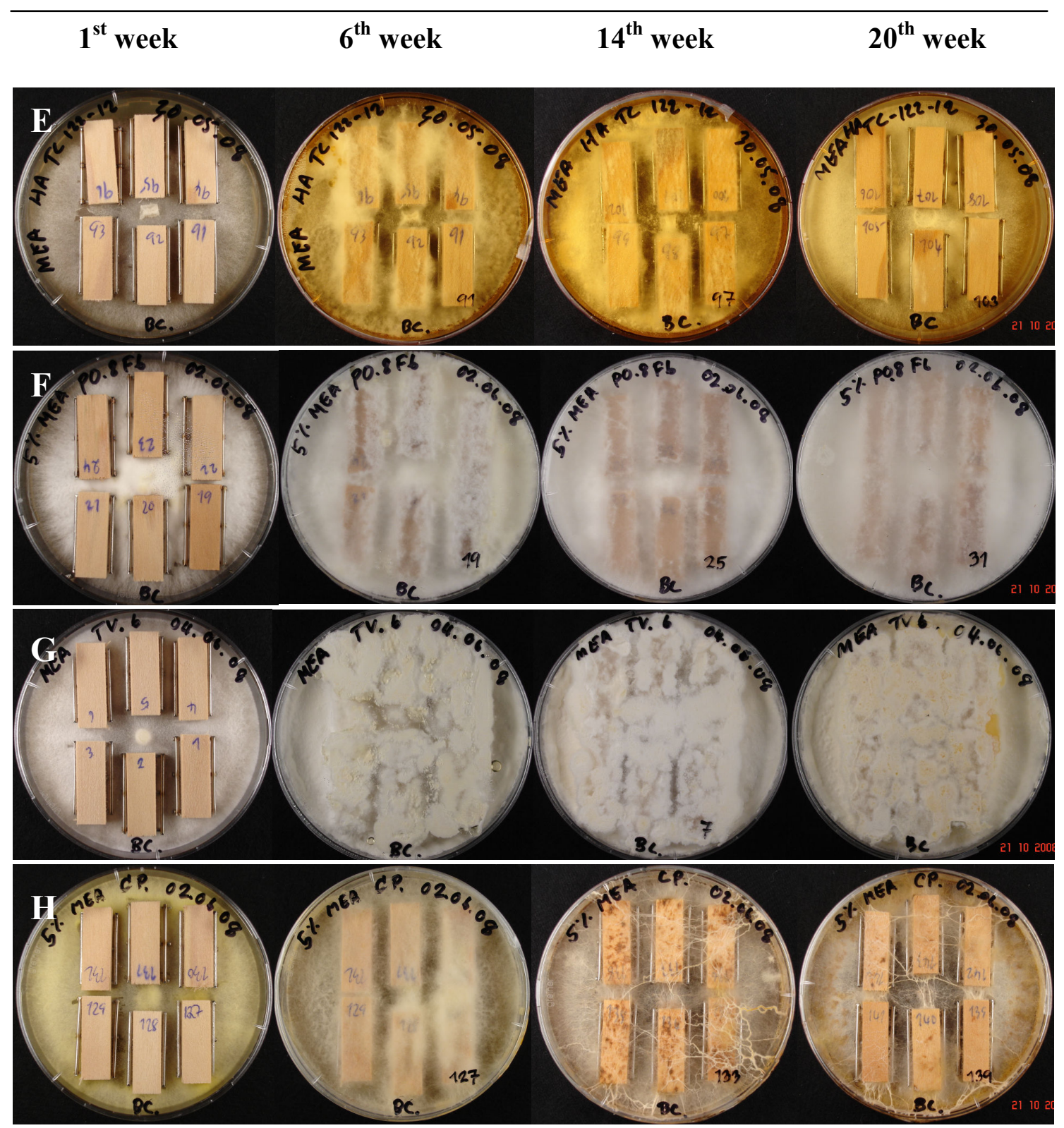

Fig. 4 (continued)

was much more intense than with the white-rot species and many lines of cracking appeared in the wood.

Generally, the color change of $A$. grandis wood blocks inoculated with fungi could indicate a modification of lignin within the wood cell walls. Through the different enzymatic actions expected to happen in case of the white-rot fungi, oxidation of lignin in the wood cell walls will eventually result in formation of quinone structures (Eriksson et al. 1990). Quinones present one of the various chromophoric groups (e.g. phenolic $\mathrm{OH}$-groups, $\alpha$-carbonyl groups, conjugated double bonds, biphenyls and radicals) present in lignin molecules (Fengel and Wegener 1983). In the fungal treated 


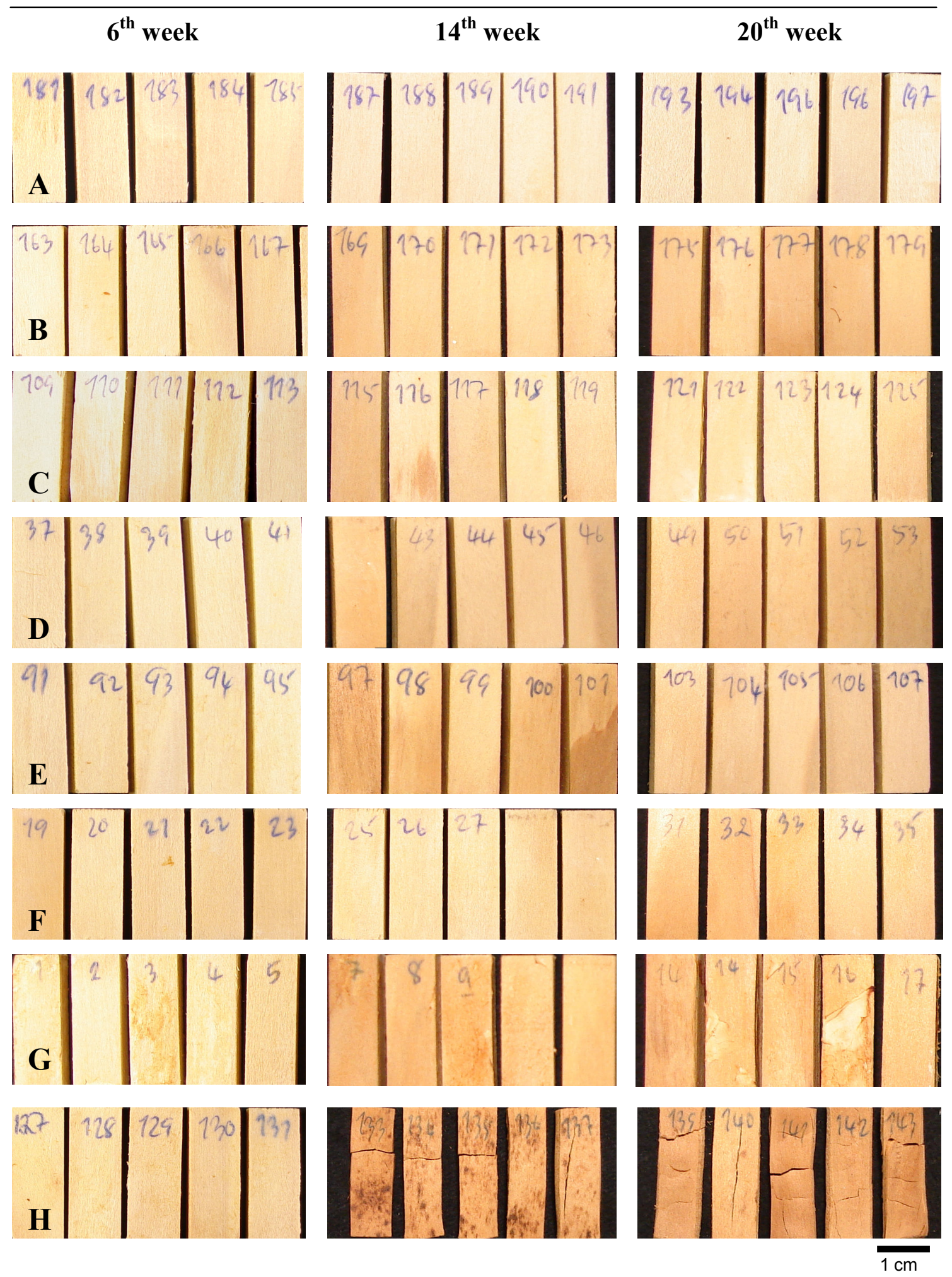

Fig. 5 Appearance of A) A. grandis wood blocks inoculated without fungi, and of wood incubated with either B) $A$. mellea, C) Heterobasidion sp.121, D) P. ostreatus PC9, E) $H$. annosum TC_122-12, F) $P$. ostreatus 8F6, G) T. versicolor Strain 6 and $\mathbf{H})$ C. puteana 57 for 6,14 and 20 weeks at $22^{\circ} \mathrm{C}$ and $65 \% \mathrm{RH}$ after scrapping off the mycelium and oven drying at $103 \pm 2^{\circ} \mathrm{C}$ for $24 \mathrm{hrs}$. The time of incubation is indicated above each row of photos 
samples prior to chemical staining, the light yellow color seen in the microtome sections may come from quinones freed from the lignin with the help of enzymes. The yellow color can possibly also be attributed to structures, such as quinone, quinone methides and stilbenes, created in aging wood by non-enzymatic oxidative processes on lignin and lignin derivative (Eriksson et al. 1990; Jolivalt et al. 2006), but such non-enzymatic processes are expected to take much more time. These possible chemical and enzymatic modifications (oxidations) of lignin and also of wood extractives leading to quinone-structures can become more emphasized by staining with MBTH. Generally, MBTH is used in sensitive spectrophotometric methods as substrate for oxidative enzymes in order to trap enzyme-generated $o$-quinones that are formed by the oxidation of various monophenols as catalyzed by enzymes such as laccase (Dicko et al. 2002; Dwivedi 2006; Espin et al. 1997; Rodríguez-López et al. 1993; Winder and Harris 1991). For example, MBTH traps $o$-quinone products originating from the enzymatic oxidation of phenolic compounds from the wood such as DHPPA (3,4-dihydroxyhydrocinnamic acid) and 4HA (4-hydroxyanisole) and thereby forms a red color adduct (Dicko et al. 2002). However, MBTH is not oxidized itself by oxidative enzymes to strong colored products and, therefore, it can be used as a coupling agent to detect other oxidized compounds with $o$-quinone structures (Espin et al. 1997).

To observe such effects by MBTH in the wood, microtome sections of wood samples have been prepared, stained with lactophenol blue followed by staining with MBTH and these were then inspected under a light microscope (Fig. 6).

Wood incubated for 6 weeks at $22^{\circ} \mathrm{C}$ and $65 \% \mathrm{RH}$ without fungi had a grayish, slightly yellowish color (Fig. 6A). Upon lactophenol blue and MBTH staining, the rays in the wood samples stained brown while no change was observed for the cell walls of the wood fibers (Fig. 6B). In contrast, a section from wood treated 6 weeks with T. versicolor Strain 6 showed a bright red staining of all cell walls, obviously due to the MBTH treatment. Fungal mycelia stained with lactophenol blue appeared in blue color (arrow points in Fig. 6C, D) within the lumina some of the wood fibers, which in the enlargement shown in Fig. 6D was best revealed. Since the technique of lactophenol blue and MBTH staining apparently worked very well, MBTH staining together with lactophenol blue staining to detect fungal mycelium in the wood was done to all microtome sections prepared from fungal treated and non-treated $A$. grandis wood blocks as described in II.3.3. 

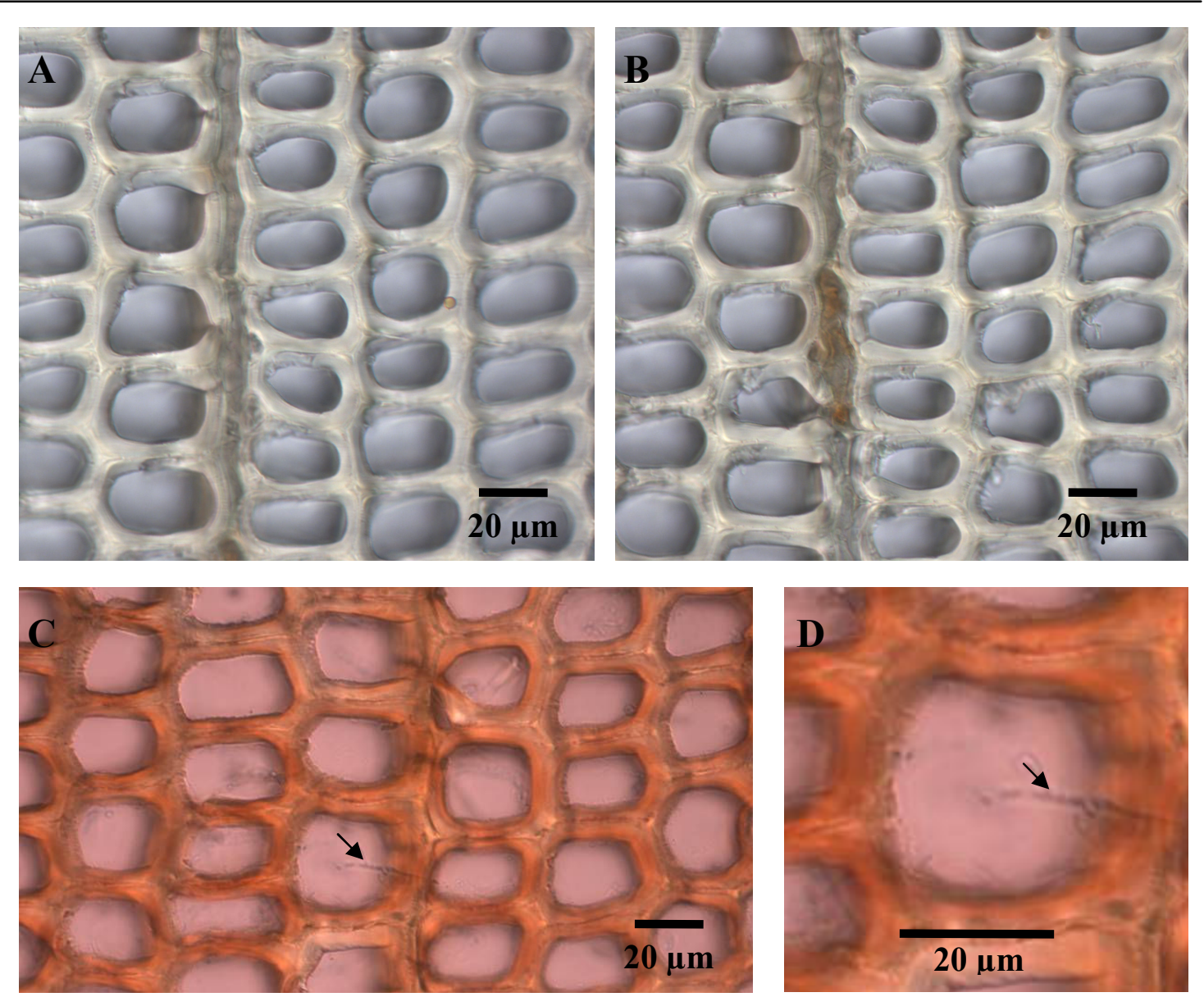

Fig. 6 Microtome sections of $A$. grandis wood blocks A) incubated for 6 weeks without fungi and being not stained with lactophenol blue and MBTH, B) incubated for 6 weeks without fungi, after lactophenol blue and MBTH staining, and C) and D) incubated with T. versicolor Strain 6 for 6 weeks at $22^{\circ} \mathrm{C}$ and $65 \% \mathrm{RH}$, and being successively stained with lactophenol blue and MBTH. The view in D) is enlarged compared to $\mathbf{C}$ ) in order to better observe fungal mycelium (the arrows point to blue-stained fungal mycelium)

For each fungus, as well as for the controls incubated without fungus and for each time point of harvest, all the each six stained microtome sections obtained from the two outer regions of a wood block and all the six stained microtome sections obtained from the center of a wood block were visually inspected under a light microscope. No differences were observed between microtome sections taken from the different positions of a wood block. Therefore, in the following, observations were concentrated on the microtome sections from the centers of wood blocks. Fig. 7 shows a sub-collection of photos made from microtome sections of $A$. grandis wood, after staining with lactophenol blue and MBTH reagent, respectively. For each fungal 
treatment and for the control for each time point of harvest, one selected photograph is presented.

In the microtome sections of the wood incubated without fungi (control) Fig. 7A, as mentioned before, the combined staining caused a brownish decolorisation within the rays. This brown staining occurred regardless of the time of harvest of the wood blocks. In no case, any staining of wood cell walls were observed (Fig. 7A). Thus, even the incubation time of 20 weeks was not long enough to release sufficient amounts of compounds for MBTH reactions from the aging wood.

From the incubations with the white-rot fungi, MBTH staining of cell walls was most seriously effected when the wood was incubated for 6 weeks with $T$. versicolor (Fig. 7G). In the T. versicolor samples, cell wall staining by MBTH as well as staining of the rays were less after 14 and 20 weeks of incubation, respectively, according to the progress in fungal decay of the wood (compare Fig. 3). The cell wall thickness was dramatically reduced, likely by degradation of lignin in the wood cell walls due to enzymatic actions of the fungus. MBTH therefore had less cell wall material to stain and, very likely, many of the apparently formerly present reactive molecules in the cell walls have been decayed by the fungus. Noticeable in the T. versicolor wood samples is that the wood tissues were partially destructed already after 14 weeks of incubation so that some of the wood fibers collapsed during microtome sample preparation (Fig. 7G). Simultaneously with the ongoing decay, the amount of fungal mycelium increased in the samples, being most in the lumina of decayed fibers at 20 weeks of incubation (Fig. 7G).

In microtome samples of wood incubated with $H$. annosum TC_122-12 (Fig. 7E) and P. ostreatus 8F6 (Fig. 7F) that decayed the wood over the time to some degree, respectively, but somewhat slower than T. versicolor Strain 6 (Fig. 3), MBTH cell walls staining was achieved already in the 6 weeks samples and the staining became most intense after 14 weeks of incubation in case of $H$. annosum TC_122-12 (Fig. 7E) and after 20 weeks of incubation in case of P. ostreatus 8F6 (Fig. 7F). At 20 weeks of incubation, MBTH staining of cell walls was nearly abolished in the case of H. annosum TC_122-12 incubation. Cell wall thicknesses in the 20 weeks samples were reduced for both fungi and there was a partial collapse of wood fibers. Fungal mycelium increased for both fungi in the wood fiber lumina with progressing decay. Also with both fungi, staining in the rays of the samples decreased over the time, suggesting that, possibly for nutrition, the fungi were using off the material stained in the rays 


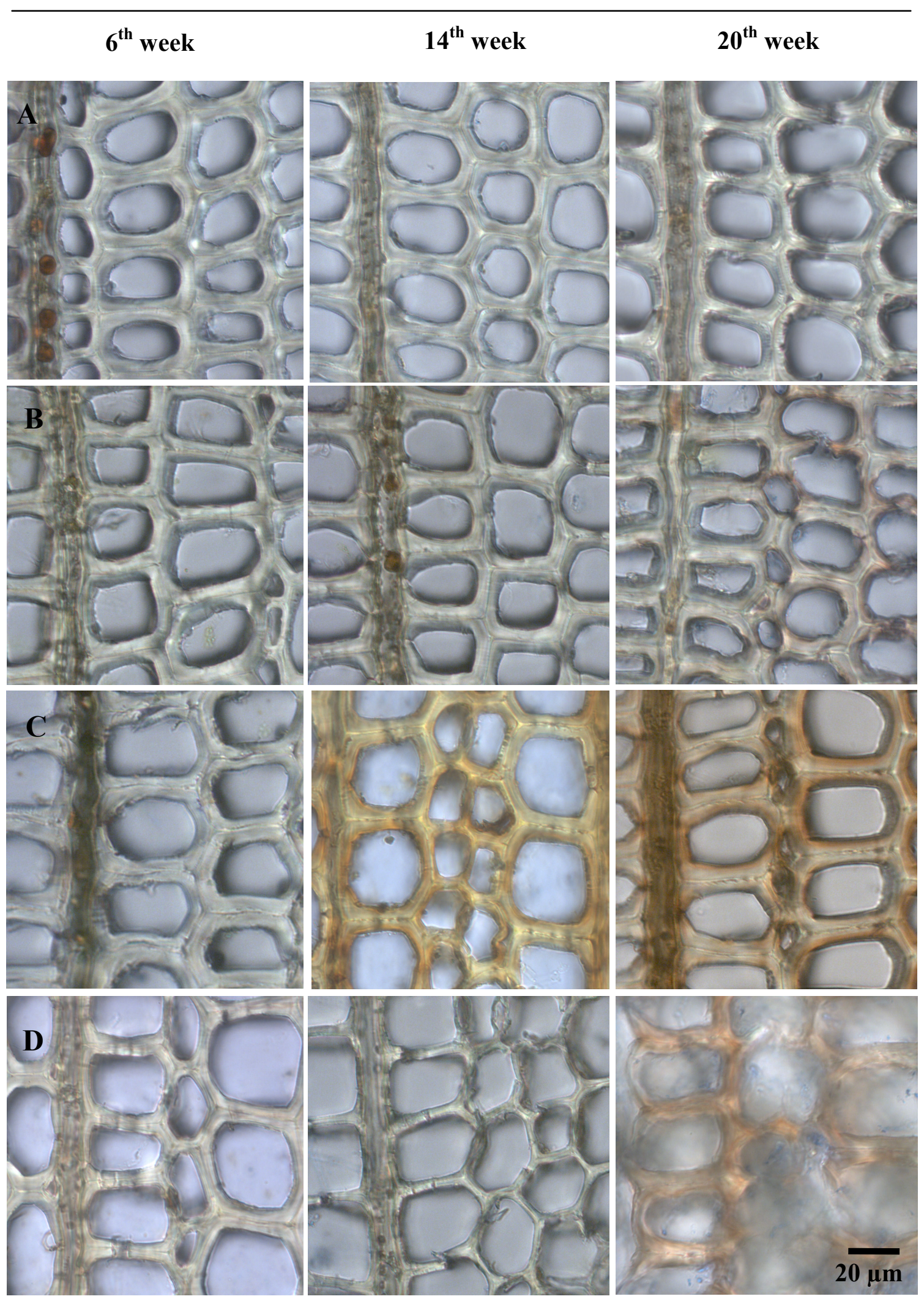

Fig. 7 Microtome sections of $A$. grandis wood blocks incubated A) without fungi, or B) with A. mellea, C) Heterobasidion sp. 121, D) P. ostreatus PC9, E) H. annosum TC 122-12, F) P. ostreatus 8F6, and G) T. versicolor Strain 6, and H) C. puteana 57 for 6,14 and 20 weeks, and stained by lactophenol blue and $100 \mathrm{mM} \mathrm{MBTH}$, respectively. The time of incubation is indicated above each row of photos 


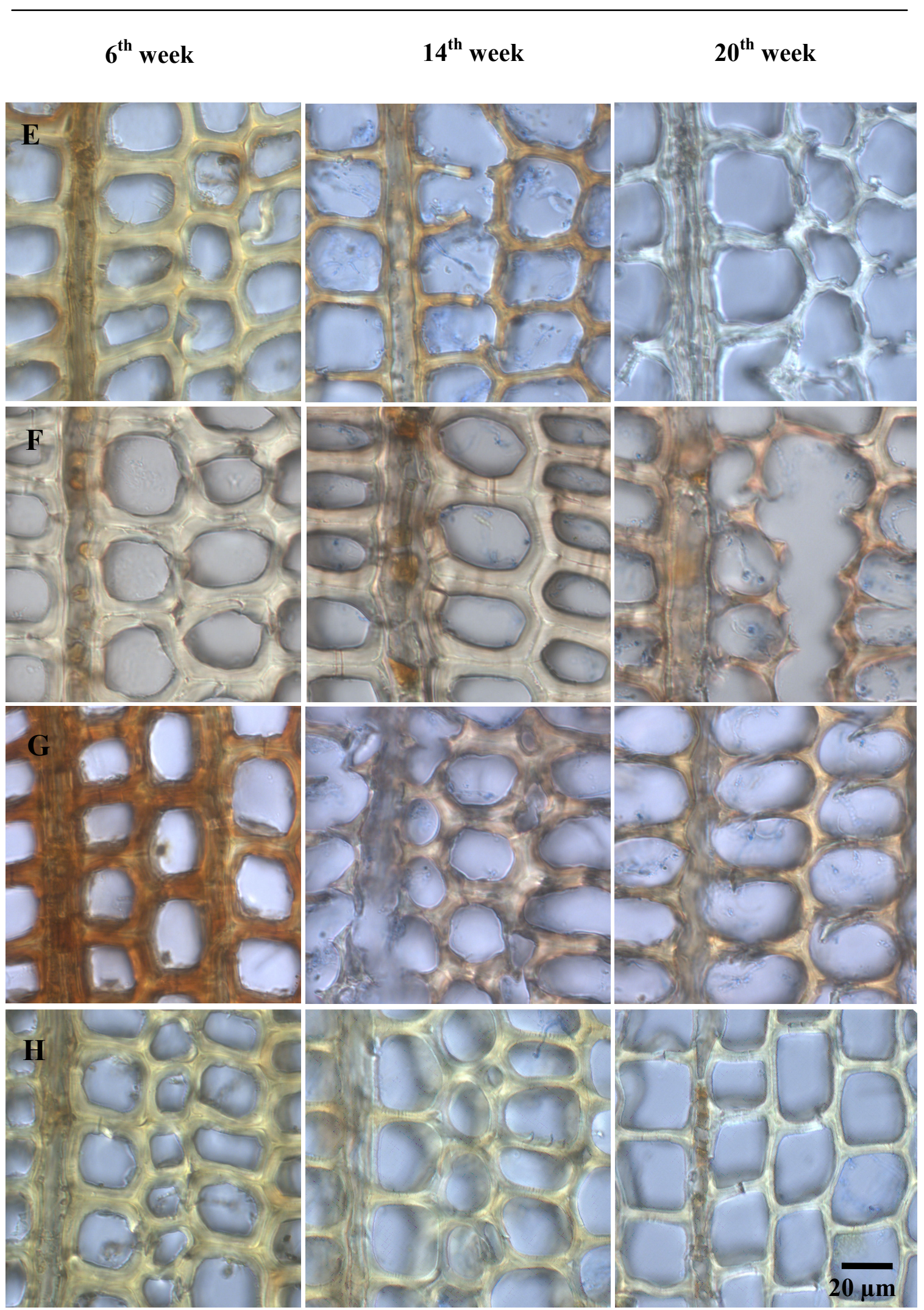

Fig. 7 (continued) 
For the three fungal species that did not give rise of mass loss in this set of experiments (Fig. 3), nevertheless staining of cell walls was encountered. Staining increased over the time with being best in 20 weeks samples. However, the staining intensity after 20 weeks of incubation was not as strong as compared in samples of wood treated with any of the other white-rot fungi (compare Fig. 7B-D to Fig. 7E-H). Fungal mycelium within the samples were in all case less for non-aggressive species (Fig. 7B, 7D and 7E) compared to the white-rot species that caused mass loss. Regarding staining of the rays, there was no obvious reduction in staining with longer time of incubation. Special of the three fungi was P. ostreatus PC9 (Fig. 7D). Although no mass loss was detected upon 20 weeks of incubation (Fig. 3), the fungus affected the structure of the cell walls. Cell walls were softened; making microtome sectioning difficult and resulting wood sections did not keep plain so that focusing with the microscope on a larger sample surface became impossible (Fig. 7D after 20 weeks of incubation).

Wood samples treated with $C$. puteana were different from all other samples (Fig. 7G). Compared to the untreated wood samples, after 6 weeks of incubation with C. puteana, there was only a mild MBTH staining of fiber cell walls. With lower incubation periods, the cell wall material was not anymore stained and, simultaneously, the thickness of the cell walls dramatically decreased due to the decay caused by the fungus (Fig. 7G). There was some fungal mycelium in the 6 weeks samples that increased in amount somewhat over the incubation time. Staining of rays decreased over the time and in the end at 20 weeks of incubation, rays tended to collapse.

\section{II.4.2 Influence of different types of pretreatment of $\boldsymbol{A}$. grandis wood on decay by T. versicolor}

The results presented in Fig. 3 and Fig. $7 \mathrm{G}$ clearly indicate that the white-rot fungus T. versicolor has the ability to decay $A$. grandis wood to a certain extend. This result appears to be in contrast to the report by Kües et al. (2009) and Hawighorst et al. (2010) on a study in which no decay was encountered by $T$. versicolor when testing MDF made $100 \%$ of $A$. grandis fibers. However, for production of MDF, A. grandis fibers were treated with urea formaldehyde (68\% in water) and ammonium sulphate $(33 \%$ in water). In the further MDF production process, preformed fiber mats were pressed by heat $\left(200^{\circ} \mathrm{C}\right)$ into compact sheets (Müller et al. 2009). The chemical treatment, the heat, 
the pressing, or combinations thereof could be reasons for the better resistance of MDF against fungi compared to solid $A$. grandis wood. In the following, results of some experiments are documented that were performed to see effects of different wood pretreatments on $T$. versicolor wood decay.

As a mechanical pretreatment, $A$. grandis mini blocks were hammered to induce cracks in the wood. Other wood mini blocks were soaked $24 \mathrm{hrs}$ in water at $60^{\circ} \mathrm{C}$ to eliminate water soluble extractives. Also to prove chemical effectiveness against decay of fungi on $A$. grandis wood, solid wood mini blocks were impregnated under vacuum with industrial chemicals (either $68 \%$ urea formaldehyde in water or $33 \%$ ammonium sulphate in water) used in MDF production processes. T. versicolor was selected in this set of experiments as the white-rot fungus for testing wood decay after the pretreatments in comparison to untreated wood because of it fastly degrades solid wood (Fig. 3) and it causes serious modifications of the wood cell walls (Fig. 7G).

When comparing broken to compact untreated wood, statistically significant differences in mass loss were observed. The results in Fig. 8 from this set of experiments show that mass loss of broken wood was always in average above that of untreated wood and this was statistically different between the samples of 12 and 18 weeks of incubation in comparison to samples of untreated wood of the same age. The highest mass loss obtained within 18 weeks of incubation of broken wood had in average a value of $49 \pm 11.99 \%$.

The wood extracted with water obtained the highest mass loss within 18 weeks of incubation with an average value of $44 \pm 9.82 \%$. This final average value of mass loss was significantly higher than that of the untreated wood and somewhat lower than the value achieved for broken wood. At the earlier time of 12 weeks of incubation, the extracted wood had the highest mass loss $(36 \pm 1.94 \%)$ of all kinds of wood pretreatments. Likely, the wood lost some inhibitory substrates against degradation by microorganism.

Treatment with urea formaldehyde and ammonium sulphate showed the strongest effect on wood decay by $T$. versicolor. Both chemicals at all times of incubation inhibited the decay by the fungus. Most effective was urea formaldehyde. Mass loss was extremely low compared to untreated wood incubated with the fungus at all three times of harvest (maximum mass loss of untreated wood $35 \pm 17.97 \%$ at 18 weeks of incubation). Urea formaldehyde apparently inhibited the fungal activities. Similarly, the fungus was inhibited by ammonium sulphate but not to the same strength. 


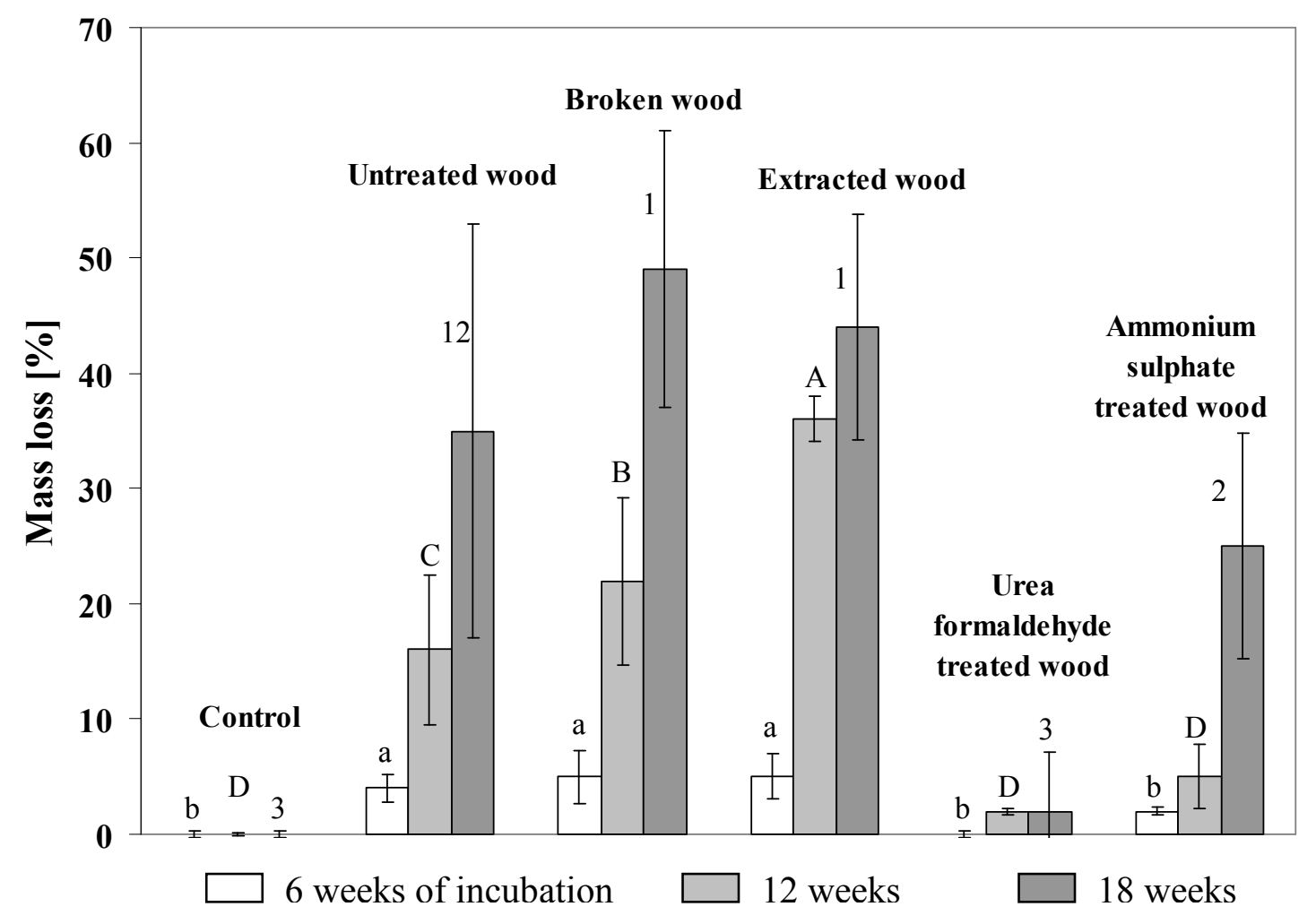

Fig. 8 Mass loss over the time caused by decay of $A$. grandis wood by $T$. versicolor Strain 6. Average values of each 5 wood blocks per treatment and per time point were calculated and standard deviations are shown. Statistical analysis of the data was performed with SPSS 8.0 for Windows, the different superscripts on the chart (letters in lower case compare the values after 6 weeks of incubation, capital letters compare the values after 12 weeks of incubation, and numbers compare the values after 18 weeks of incubation) indicate values that differ significantly $(p<0.05)$ between treatments as based on analysis of variance (ANOVA)

Whilst in the first 12 weeks of incubation, the inhibitor effect by ammonium sulphate on fungal decay was still quiet strong $(5 \pm 2.78 \%$ mass loss after 12 weeks of incubation), at 18 weeks of incubation, there was a reasonable amount of wood decay with $25 \pm 9.77 \%$ of mass loss.

At 6, 12 and 18 weeks of harvest, pictures of $A$. grandis wood blocks colonized with $T$. versicolor were photographed with a digital CCD camera in order to observe growth of fungal mycelia on the wood blocks' surfaces. The mycelia grown on the wood surfaces after different wood pretreatments are presented (Fig. 9C-9F) in comparison to untreated wood controls incubated with (Fig. 9B) and without the fungus (Fig. 9A). After all five different wood pretreatments, all wood surfaces were colonized 

$6^{\text {th }}$ week
$12^{\text {th }}$ week
$18^{\text {th }}$ week

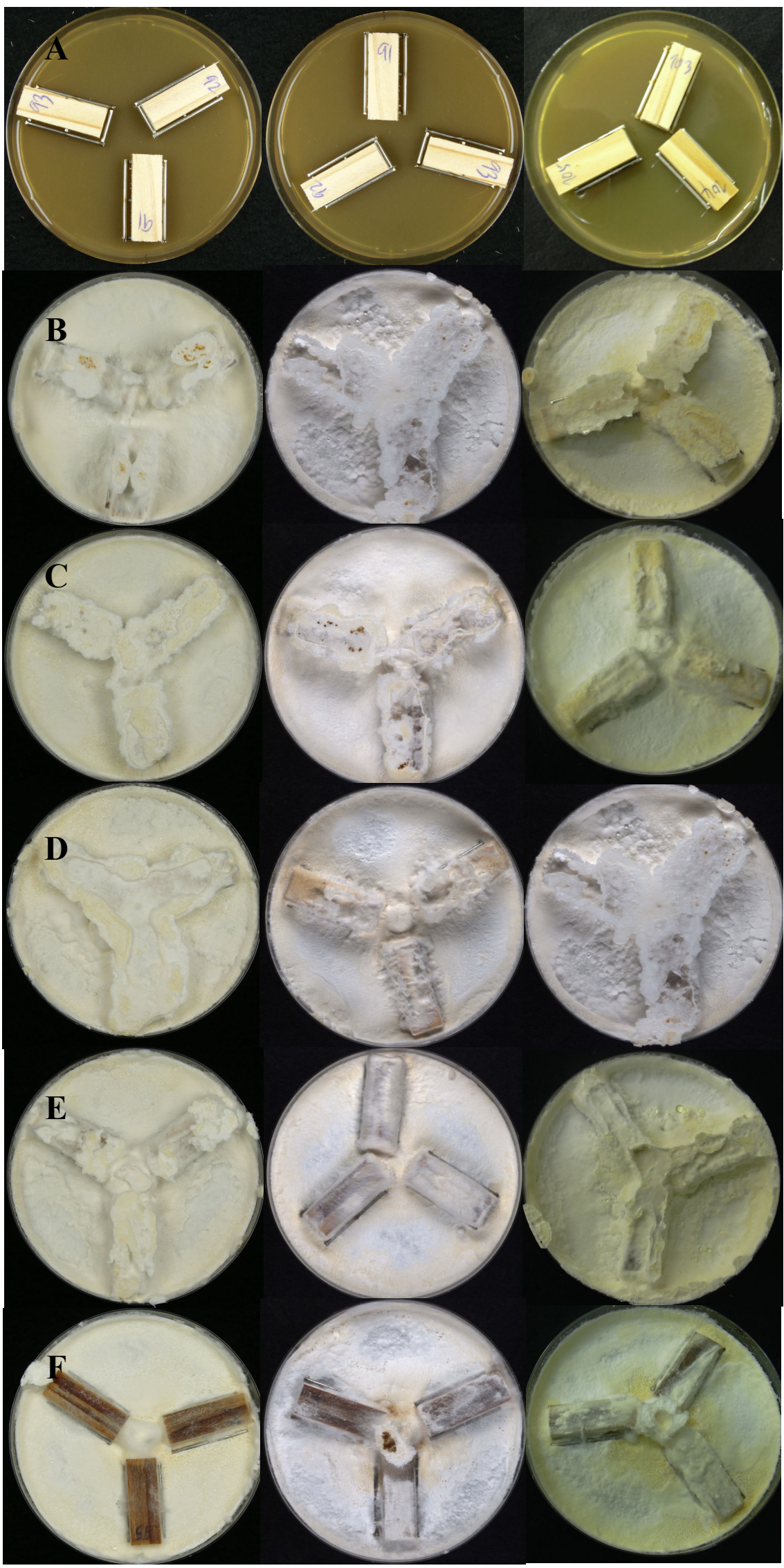

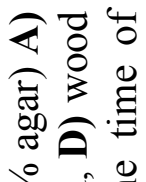

\ें ठํํ

당

일.

in 융

䒠记

छे छ̊

क 3

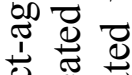

还

区

흥 흔

䓅

б。

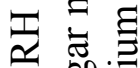

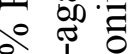

ช

를

记

글

๘

ฮ్

敢

氙 己

䏓

웡

음 혼 음

궁

승

离

ミ

के

षं

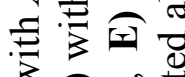

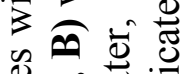

兽

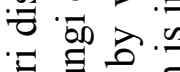

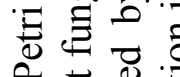

a

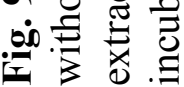


by fungal mycelia. However, differences in the speed of surface colonization were seen. In case of ammonium-sulphate-treated wood, $T$. versicolor first showed few growth of mycelia on the wood surface after the first six weeks of incubation (Fig. 9F). Also after 12 weeks and even after 20 weeks of incubation, the surface growth by $T$. versicolor was still behind that occurring on the surfaces of wood of other pretreatments. The best surface growth of all in contrast was detected with wood of which the extractives were removed through $24 \mathrm{hrs}$ incubation in $60^{\circ} \mathrm{C}$ water (Fig. 9D). Whilst these two cases may correlate with the total in average lower and the total in average higher mass loss observed for the samples in comparison to untreated wood incubated with the fungus (compare Fig. 8), fungal mycelia growth was also very strong on the surface of wood treated by urea formaldehyde, although with this treatment the lowest (nearly no) mass loss was observed (Fig. 8). Thus, the mycelial growth of the fungus on the wood block surfaces can not clarify by sample viewing the effectiveness of mass loss that occurred to the respective wood blocks.

\section{II.5 Discussion}

Degradation of $A$. grandis wood blocks by different fungi and effects by physical and chemical pretreatment of wood on fungal decay have been tested in this work. Of all tested fungi, highest mass loss of $A$. grandis wood was obtained with the brown-rot C. puteana, followed by the white-rots T. versicolor Strain 6, P. ostreatus 8F6 and H. annosum TC_122-12 with average mass loss values of $47 \pm 3.44 \%, 36 \pm 6.73 \%$, $34 \pm 3.51 \%$ and $19 \pm 5.54 \%$, respectively (Fig. 3). There was no mass loss detected during incubation with P. ostreatus PC9, Heterobasidion sp. 121, and A. mellea in comparison to the uninfected control. However, there were some color changes upon staining with MBTH observed in the wood cell walls, also in samples treated with fungi that did not cause wood mass loss (Fig. 6). Color changes were more severe with the white-rots that (partially) degraded $A$. grandis wood, although with enhanced progress of the white-rot such MBTH staining was not anymore possible, likely due to decay of the compounds that can be chemically interactive with MBTH. The various color changes of $A$. grandis wood blocks in early samples incubated with fungi successfully decaying the wood were indicative to later mass loss of wood. The observations of MBTH staining of different strength could be related to the degree of modifications of lignin in the wood cell walls, resulting in stainable quinone structures. It is assumed that enzymes secreted 
by the white-rot fungi can modify lignin within the wood cell walls (Barsberg et al. 2003; Hatakka 1994) and many of the resulting chemical modifications will concern the production of quinones (Eriksson et al. 1990) that are able to react-with MBTH (Dicko et al. 2002; Espin et al. 1997). Already with the naked eye, a color change of the wood treated with fungi was obvious in all cases right from the time of the first harvest. Furthermore, on the micro-scale of inoculated wood blocks with fungi in sectioned microtome samples stained with lactophenol blue and MBTH and inspected with a light microscope, cell wall modifications in particular became better obvious by the MBTH staining.

As a general result from the wood block test, not unexpectedly, C. puteana turned out to be a strong decayer of $A$. grandis wood whilst a number of white-rots were unsuccessful in decaying larger amounts of the A. grandis wood, despite of performing obviously some modifications on the wood cell walls. C. puteana is a brown-rot (Webster and Weber 2007) and does therefore not produce enzymes for the degradation of lignin. Not surprisingly therefore, in staining attempts with MBTH no obvious change in cell wall coloration was detected, not even in the early phase of the experiment (Fig. $7 \mathrm{H})$.

Different from the expectations from published work on MDF made of A. grandis fibers (Hawighorst et al. 2010; Kües et al. 2009), the solid A. grandis wood was of all white-rots best decayed by $T$. versicolor. In the studies present in the literature, MDF incubated was highest sensitive to C. puteana, resulting in high mass loss (33 $\pm 8.3 \%$ mass loss in 15 weeks of incubation) and in block size and color change, whereas decay by $T$. versicolor was neglectable $(6.5 \pm 0.7 \%$ mass loss after 15 weeks of incubation). In contrast to the findings with MDF, in this study in one set of experiments mass loss of the solid wood by decay through $T$. versicolor was $36 \pm 6.73 \%$ after 20 weeks of incubation and in the other set of experiments mass loss by decay through T. versicolor was $35 \pm 17.97 \%$ after 18 weeks of incubation. These values are comparable to each other and T. versicolor Strain 6 apparently is able to decay A. grandis wood.

What were the major differences in the decay experiments of MDF and of solid wood? One possibility is that the T. versicolor strain CTB 863 A used in the test for decay of MDF (Hawighorst et al. 2010; Kües et al. 2009) is generally less aggressive in decay of A. grandis wood. However, EN 113 (1996) tests with solid A. grandis wood blocks and T. versicolor strain CTB 863 A showed that also this strain can decay solid 
A. grandis wood (about 20-25\% mass loss occurred in 16 weeks of incubation at $22^{\circ} \mathrm{C}$ and 65\% RH; Kües et al. (2009) and M. Navarro-González, pers. communication). In conclusion from this, it is likely rather the treatment that the wood fibers experienced in MDF production that will be responsible for resulting in resistance against decay by T. versicolor.

During production, MDF is compact pressed so that possibly little entry openings for the fungi are offered. However, MDF should be less compact compared to solid wood so that fungi might find even better ways into entering the MDF. In studying wood decay fungi on $A$. grandis wood material, humidity conditions play a large role for a success of a fungal attack. Since following up in the MDF as well as in the solid wood decay test the same EN 113 standard, there was not as likely a big difference in humidity. The most obvious difference between MDF and solid wood was due to the addition of urea formaldehyde and ammonium sulphate to the $A$. grandis fibers in MDF production. With this, the total nitrogen amount increases dramatically in the material. Nitrogen is known to negatively effect production of ligninolytic enzymes by T. versicolor (Asgher et al. 2008; Rühl et al. 2007). Therefore to test a possible effect of the nitrogen-containing chemicals, mini wood blocks of $100 \%$ of $\mathrm{A}$. grandis were individually vacuum treated with urea formaldehyde and with ammonium sulphate prior to subjecting the wood blocks to the EN 113 (1996) test with T. versicolor Strain 6.

Urea formaldehyde and ammonium sulphate were proven in this study to prevent decay by white-rot fungus $T$. versicolor, with urea formaldehyde being more effective than ammonium sulphate. However, when considering the chemical concentrations in the solutions used to treat the wood with (either 68\% urea formaldehyde in water or $33 \%$ ammonium sulphate in water) the absolute nitrogen content of the two solutions differed by $22 \%(27 \%$ total nitrogen in the urea formaldehyde solution versus $5 \%$ total nitrogen in the ammonium sulphate solution). In conclusion, considering that the wood blocks will in average have taken up alike volumes of solution, in case of the urea formaldehyde treatment the wood has possibly taken up more than five times the amount of nitrogen than the wood in the case of the ammonium sulphate treatment. However, in this context one should not forget that the chemical type of offered nitrogen differs also between urea formaldehyde and ammonium sulphate on the one hand and in MDF by the polymerized urea formaldehyde resin (Kloeser et al. 2007) on the other hand. Both, urea and volatile ammonium have been reported in the literature at elevated concentrations to have toxic 
effects on Heterobasidion strains (Chang and Chang 1999; Pratt and Redfern 2001; Vasiliauskas et al. 2004). Whether this is also the case for T. versicolor is unclear. There is one report in literature that suggests ammonia to have a negative influence on growth of T. versicolor but not urea (Ejechi 1998). However, the concentration of urea ( $5 \%$ in solution) tested in growth of $T$. versicolor on urea treated wood blocks (Nigerian timber obeche Triplochiton scleroxylon) was by a factor of more than five lower than in the experiments described here.

To my best knowledge, it is also not well known whether T. versicolor can interact with the polymerized urea formaldehyde resin as present in MDF. However, results from decay tests performed with $T$. versicolor on other types of wood composites (particle boards made from sugar cane, straw, and oriented strand boards made from rubberwood) suggest that increasing concentration of polymerized urea formaldehyde hinders or blocks T. versicolor from decay of the wood products (Okino et al. 2009; Teixeira et al. 2009). According to these and the own results, urea formaldehyde and ammonium sulphate both can function as such or in form of a polymerized resin as an inhibitor for fungal growth and for degradation of wood by fungi.

In frame of the same experiments wood when testing effects of nitrogencontaining chemicals on wood decay, also the effects of mechanical damage and the effects of eliminating wood extractives were tested in incubation with $T$. versicolor. Broken wood was easier degraded by the fungus than undamaged wood. Likely, the fungal mycelia penetrated into and passed through the broken wood tissue easier than through the cells of compact wood. Also wood eliminated of its wood extractives was more decayed than the original wood. Whilst in the hammered samples the physical barriers mediated by the densely fitted cell walls in the wood blocks were damaged, in the water incubated samples, the chemical barrier to fungal entry was removed. Water extracted wood after 12 weeks of incubation obtained the highest values of mass loss of all kind of tested treatments (Fig. 8). Likely, wood extracted by water lost resistance with the loss of toxic wood extractives. Toxic effects by wood extractives were analyzed in further experiments as documented in chapter IV of this thesis. 


\section{II.6 References}

Asgher M, Batool S, Bhatti HN, Noreen R, Rahman SU, Asad MJ (2008) Laccase mediated decolorisation of vat dyes by Coriolus versicolor IBL-04. International Biodeterioration \& Biodegradation 62(4):465-470

Baldrian P, Gabriel J (2002) Copper and cadmium increase laccase activity in Pleurotus ostreatus. FEMS Microbiology Letters 206(1):69-74

Barsberg S, Elder T, Felby C (2003) Lignin-quinone interactions: Implications for optical properties of lignin. Chemistry of Materials 15(3):649-655

Bravery AF (1978) A minimized wood-block test for rapid evaluation of wood preservative fungicides. International Research Group on Wood Preservation. Document No. IRG/WP 2113, Stockholm

Chandra RP, Ewanick SM, Chung PA, Au-Yeung K, Rio LD, Mabee W, Saddler JN (2009) Comparison of methods to assess the enzyme accessibility and hydrolysis of pretreated lignocellulosic substrates. Biotechnology Letters $31: 1217-1222$

Chang TT, Chang RJ (1999) Generation of volatile ammonia from urea fungicidal to Phellinus noxius in infested wood in soil under controlled conditions. Plant Pathology 48(3):337-344

Childs RE, Bardsley WG (1975) Steady-state kinetics of peroxidase with 2,2'-azinodi-(3-ethylbenzthiazoline-6-sulphonic acid) as chromogen. Biochemical Journal 145(1):93-103

Dicko MH, Hilhorst R, Gruppen H, Laane C, van Berkel WJH, Voragen AGJ (2002) Zymography of monophenolase and o-diphenolase activities of polyphenol oxidase. Analytical Biochemistry 306(2):336-339

Dominguez A, Gomez J, Lorenzo M, Sanroman A (2007) Enhanced production of laccase activity by Trametes versicolor immobilized into alginate beads by the addition of different inducers. World Journal of Microbiology \& Biotechnology 23(3):367-373

Dorado J, van Beek TA, Claassen FW, Sierra-Alvarez R (2001) Degradation of lipophilic wood extractive constituents in Pinus sylvestris by the white-rot fungi Bjerkandera sp. and Trametes versicolor. Wood Science and Technology 35(12):117-125

Dwivedi RC (2006) Extracellular proteins from lignocellulose degrading 
Basidiomycetes: Redox enzymes from Trametes versicolor and Coprinopsis cinerea. Dissertation, Georg-August University, Göttingen

Ejechi BO (1998) Effect of combined bacterial and urea treatment on biodegradation of wood by basidiomycete fungi. Journal of Phytopathology-Phytopathologische Zeitschrift 146(11-12):533-538

Elissetche JP, Ferraz A, Freer J, Mendonca R, Rodriguez J (2006) Thiobarbituric acid reactive substances, $\mathrm{Fe}^{3+}$ reduction and enzymatic activities in cultures of Ganoderma australe growing on Drimys winteri wood. FEMS Microbiology Letters 260(1):112-118

EN 113 (1996) Wood preservatives - test method for determining the protective effectiveness against wood destroying basidiomycetes - Determination of the toxic values. European Committee for Standardization, Brüssel, Belgium: $35 \mathrm{p}$

Eriksson KEL, Blanchette RA, Ander P (1990) Microbial and enzymatic degradation of wood and wood components. Springer-Verlag, Berlin

Espin JC, Tudela J, Garcia-Canovas F (1998) 4-hydroxyanisole: The most suitable monophenolic substrate for determining spectrophotometrically the monophenolase activity of polyphenol oxidase from fruits and vegetables. Analytical Biochemistry 259(1):118-126

Ewanick SM, Bura R, Saddler JN (2007) Acid-catalyzed steam pretreatment of lodgepole pine and subsequent enzymatic hydrolysis and fermentation to ethanol. Biotechnology and Bioengineering 98(4):737-746

Falck R (1926) Über korrosive and destruktive Holzzersetzung und ihre biologische Bedeutung. Berichte der Deutschen Botanischen Gesellschaft 44:652-664

Fengel D, Wegener G (1983) Wood. Chemistry, ultrastructure, reactions. Walter de Gruyter, Berlin

Gutierrez A, del Rio JC, Gonzalez-Vila FJ, Martin F (1998) Analysis of lipophilic extractives from wood and pitch deposits by solid-phase extraction and gas chromatography. Journal of Chromatography A 823(1-2):449-455

Jolivalt C, Neuville L, Boyer FD, Kerhoas L, Mougin C (2006) Identification and formation pathway of laccase-mediated oxidation products formed from hydroxyphenylureas. Journal of Agricultural and Food Chemistry 54(14):50465054

Harkin JM, Obst JR (1973) Lignification in trees - indication of exclusive peroxidase participation. Science 180(4083):296-298 
Hatakka A (1994) Lignin-modifying enzymes from selected white-rot fungiproduction and role in lignin degradation. FEMS Microbiology Reviews 13(23):125-135

Hawighorst P, Müller GS, Navarro-González M, Malik I, Kües U, Polle A (2010) Knowledge-based production and durability of innovation beech and grand fir wood-base panels. Forst und Holz 65(1):20-25

Hoegger PJ, Kilaru S, James TY, Thacker JR, Kües U (2006) Phylogenetic comparison and classification of laccase and related multicopper oxidase protein sequences. FEBS Journal 273:2308-2326

Hofrichter M (2002) Review: lignin conversion by manganese peroxidase (MnP). Enzyme and Microbial Technology 30(4):454-466

Hofrichter M, Ullrich R, Pecyna MJ, Liers C, Lundell T (2010) New and classic families of secreted fungal heme peroxidases. Appl Microbiol Biotechnol 87(3):871-897

Kersten PJ, Tien M, Kalyanaraman B, Kirk TK (1985) The ligninase of Phanerochaete chrysosporium generates cation radicals from methoxybenzenes. Journal of Biological Chemistry 260:2609-2612

Kilaru S (2006) Identification of fungal multi-copper oxidase gene families:

Overexpression and characterization of Coprinopsis cinerea laccases for applications in biotechnology. Dissertation, Cuvillier Verlag, Göttingen

Kloeser L, Kües U, Schöpper C, Hosseinkhani H, Schütze S, Dantz S, Malik I, Vos H, Bartholme M, Müller C, Polle A, Kharazipour A (2007) Panel boards and conventional adhesives. In Kües (Ed.) Wood Production, Wood Technology, and Biotechnological Impacts. Universitätsverlag Göttingen, Göttingen, pp 297346

Kremer ML (1999) Mechanism of the Fenton reaction. Evidence for a new intermediate. Physical Chemistry Chemical Physics 1(15):3595-3605

Kües U (2007) Molecular wood biotechnology - defining a new field of research. In Kües (Ed.) Wood Production, Wood Technology, and Biotechnological Impacts. Universitätsverlag Göttingen, Göttingen, pp 15-40

Kües U, Navarro-González M, Cherdchim B, Majcherczyk A (2009) Anfälligkeit von lebendem und totem Holz der Küstentanne gegenüber Pilzen. Mykologisches Kolloquium, Molekularbiologische Pilzdiagnostik, 7./8.Mai 2009 in Dresden, Institut für Holztechnologie, Dresden:1-14 
Leonowicz A, Cho NS, Luterek J, Wilkolazka A, Wojtas-Wasilewska M, Matuszewska A, Hofrichter M, Wesenberg D, Rogalski J (2001) Fungal laccase: properties and activity on lignin. Journal of Basic Microbiology 41(3-4):185-227

Majcherczyk A, Johannes C, Hüttermann A (1999) Oxidation of aromatic alcohols by laccase from Trametes versicolor mediated by the 2,2 '-azino-bis-(3ethylbenzothiazoline-6-sulphonic acid) cation radical and dication. Applied Microbiology and Biotechnology 51(2):267-276

Mayer AM, Staples RC (2002) Laccase: new functions for an old enzyme. Phytochemistry 60(6):551-565

Mester T, Field JA (1998) Characterization of a novel manganese peroxidase-lignin peroxidase hybrid isozyme produced by Bjerkandera species strain BOS55 in the absence of manganese. Journal of Biological Chemistry 273:15412-15417

Müller G, Schöpper C, Vos H, Kharazipour A, Polle A (2009) FTIR-ATR spectroscopic analyses of changes in wood properties during particle- and fibreboard production of hard- and softwood trees. Bioresources 4(1):49-71

Navarro-González M, Wei DS, Kües U (2008) Pilze of Holz der Küstentanne (Abies grandis). Holzbiologie 49(6): 23-25

Okino EYA, Alves MVD, Teixeira DE, de Souza MR, Santana MAE, Del Menezzi CHS (2009) Accelerated laboratory test of rubberwood oriented strandboard exposed to wood decay fungi. Ciencia Florestal 19(3-4):333-341

Olofsson K, Bertilsson M, Lidén G (2008) A short review on SSF - an interesting process option for ethanol production from lignocellulosic feedstocks.

Biotechnol Biofuels 1(7):1-14

Pan X, Xie D, Yu RW, Lam D, Saddler JN (2007) Pretreatment of lodgepole pine killed by mountain pine beetle using the ethanol organosolv process: fractionation and process optimization. Industrial and Engineering Chemistry Research 46: 2609261

Pratt JE, Redfern DB (2001) Infection of Sitka spruce stumps by spores of Heterobasidion annosum: control by means of urea. Forestry 74(1):73-78

Rodríguez-López JN, Escribano J, García-Cánovas F (1994) A continuous spectrophotometric method for the determination of monophenolase activity of tyrosinase using 3-methyl-2-benzothiazolinone hydrazone. Analytical Biochemistry 216(1):205-212

Röhrig E (1981) Neuere Grundlagen für den Anbau von Abies grandis. Schriften aus 
der Forstlichen Fakultät der Universität Göttingen und der Niedersächsischen Forstlichen Versuchsanstalt, Sauerländer Verlag, Frankfurt am Main

Rühl M, Kilaru S, Navarro-González M, Hoegger PJ, Kharazipour A, Kües U (2007) Production of laccase and other enzymes for the wood industry. In Kües (Ed.) Wood Production, Wood Technology, and Biotechnological Impacts. Universitätsverlag Göttingen, Göttingen, pp 469-507

Shah V, Nerud F (2002) Lignin degrading system of white-rot fungi and its exploitation for dye decolorization. Canadian Journal of Microbiology 48(10):857-870

Sjostrom E (1993) Wood chemistry fundamentals and applications. Academic Press, San Diego

Spellmann H, Geb M, Nagel J, Nagel R, Schmidt M (2010) Timber product oriented silvicultural strategies for mixed strands of beech ( Fagus silvatica) and grand fir (Abies grandis). Forst und Holz 65(1):12-19

Teixeira DE, Garlet A, Sanches KL (2009) Resistance of particleboard panels made of agricultural residues and bonded with synthetic resins or PVC plastic to woodrotting fungi. CERNE 15(4):413-420

Terron MC, Gonzalez T, Carbajo JM, Yague S, Rana-Cuenca A, Tellez A, Dobson ADW, Gonzalez AE (2004) Structural close-related aromatic compounds have different effects on laccase activity and on lcc gene expression in the ligninolytic fungus Trametes sp I-62. Fungal Genetics and Biology 41(10):954962

Vasiliauskas R, Lygis V, Thor M, Stenlid J (2004) Impact of biological (Rotstop) and chemical (urea) treatments on fungal community structure in freshly cut Picea abies stumps. 31(3):405-413

Vivekanand V, Dwivedi P, Sharma A, Sabharwal N, Singh RP (2008) Enhanced delignification of mixed wood pulp by Aspergillus fumigatus laccase mediator system. World Journal of Microbiology \& Biotechnology 24(12):2799-2804

Vos H, Kharazipour A (2010) Properties of light particle boards manufactured using Abies grandis (grand fir). Forst und Holz 65(1):26-30

Webster J, Weber RWS (2007) Introduction to fungi. University Press, Cambridge

Winder AJ, Harris H (1991) New assays for the tyrosine-hydroxylase and dopa oxidase activities of tyrosinase. European Journal of Biochemistry 198(2):317326 


\section{II.7 Supplementary material}

\section{II.7.1 Further analysis of wood mass values in relation to the moisture content of wood blocks}

Fig. S1 links to the experiment documented in Fig. 3 of this chapter and shows the measured average amount of humidity taken up in the different wood blocks over the time of incubation. Generally, the uninfected control was found to be the lowest in moisture uptake of the different wood blocks tested over the time of incubation (Fig. S1). However, simple ANOVA analysis was not able to proof this to be statistically different (not shown) but a more complex Duncan's test analysis had to be performed in order to document recognizable a degree of difference (Fig. S1).

As a result of this type of analysis at six weeks of incubation, the moisture uptake of wood blocks infested with fungi compared with the wood without a fungus was not significantly different (Heterobasidion sp. 121 and H. annosum TC_122-12), quite similar (P. ostreatus 8F6) or only little more (A. mellea and P. ostreatus PC9) but for the fungal species T. versicolor Strain 6 and C. puteana Strain 57 that start early to degrade the wood (compare mass loss values in Fig. 3). Where an increase in uptake of water was seen compared to the wood sample untreated with a fungus, this might be due to growth of fungal hyphae into the wood and transport of water by them from the agar into the wood.

At 14 weeks of incubation, there was a dramatic increase in uptake of humidity in case of the T. versicolor Strain 6 samples whilst there was no significant or only little clear-cut differences when considering all other values obtained from wood samples infected with fungi compared to each other and compared to the untreated wood (Fig. S1). At this time of incubation, C. puteana Strain 57 already reached a maximum of wood decay (Fig. 3), whereas T. versicolor Strain 6 was in the process of still decaying wood components (Fig. 3). At this time of incubation in the brown-rot samples, already part of the hydrophobic lignin was revealed for the eye in the samples due to brown coloration (see brown spots in Fig. 4H), giving an explanation on the lower uptake of water. In comparison, the samples treated with T. versicolor Strain 6 usually have taken up more water, likely due to decay of hydrophobic lignin but likely also due to the increase of fungal mycelium that grows in the wood and may transport extra water from the agar into the wood. 
At 20 weeks of incubation, there was an even more dramatic increase in uptake of humidity in case of $T$. versicolor Strain 6 samples whilst there was only little further uptake of humidity in wood samples treated with other fungi as compared to the untreated wood. There were no significant or any dramatic differences when considering the average values of moisture uptake obtained from wood blocks infected with any of the other fungi, i.e. A. mellea, Heterobasidion sp. 121, H. annosum TC_122-12, P. ostreatus PC9, P. ostreatus 8F6 and C. puteana Strain 57 in comparison to each other (Fig. S1).

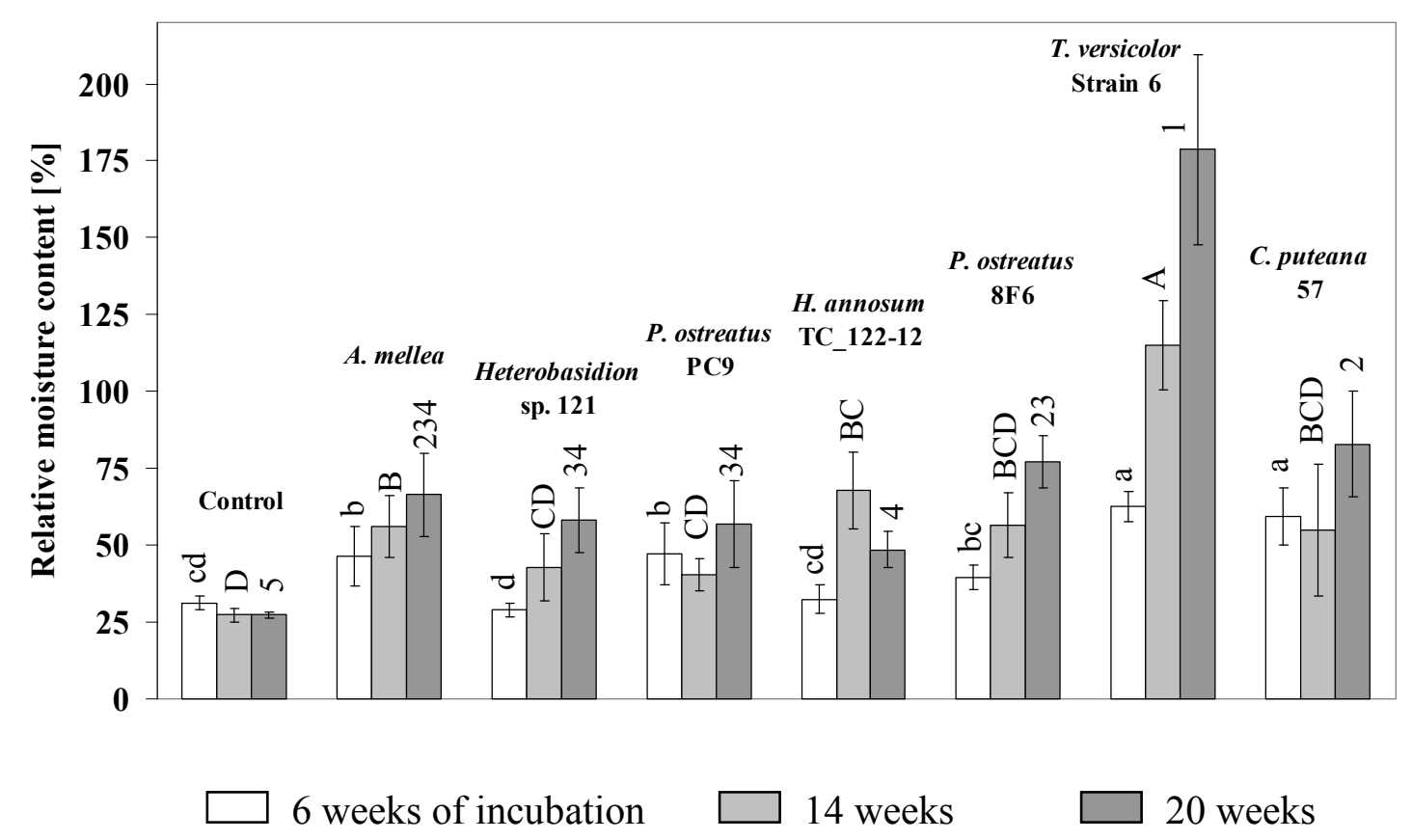

Fig. S1 Relative moisture content of $A$. grandis wood blocks incubated after EN 113 (1996) at $22^{\circ} \mathrm{C}$ and $65 \% \mathrm{RH}$ with or without a fungus. Average values of uptake of humidity of each 5 wood blocks per fungus and per time point were calculated and standard deviations are shown. Statistical analysis of the data was performed with SPSS 8.0 for Windows, the different superscripts on the chart (letters in lower case compare the values after 6 weeks of incubation, capital letters compare the values after 14 weeks of incubation, and numbers compare the values after 20 weeks of incubation) indicate values that differ significantly $(p<0.05)$ between treatments as based on analysis of variance (ANOVA) and then Duncan's test. Results from the Duncan's test are shown in the figure 
In conclusion, there was a dramatic increase in uptake of humidity in case of the T. versicolor Strain 6 at all point of analysis, i.e. after 6, 14 and 20 weeks of incubation, respectively, but with the other fungal species at (nearly) each time point of incubation moisture uptake was more or less comparable as the humidity taken up in the different of wood blocks of the uninfected control. The results generally show that the analysis of uptake of humidity together with knowledge on the amount of total wood mass loss and knowledge on the chemical types of decay a fungus causes can possibly give some indication on the progress of decay but it can not be taken as an absolute measurement of decay and mass loss.

\section{II.7.2 Plastic staining by volatiles emitted from Heterobasidion sp. cultures}

Fig. 4C and 4E in this chapter show Petri dishes with cultures of Heterobasidion strains that obtained a yellow staining of its plastic due to actions of the fungi. This phenomenon has been observed also from other Heterobasidion strains, although staining differed in the intensity of colorization of the plastic staining they caused (Fig. S2A). The yellow staining occurred with plates in presence (Fig. 3) and in absence of wood (Fig. S2A) indicating that the effect is not directly related to wood decay. The yellow staining did not occur in the fungal mycelium (Fig. S2B) and neither in the agar beneath the mycelium (not shown). In order to learn more about the yellow color, the yellow-stained plastic lids were wiped with dry paper tissue, with paper tissue with water, with paper tissue with acetone, and with paper tissue with ethanol. However, in no case the yellow color could be wiped off. Apparently, the fungi produce a volatile that reacts with the polystyrene of the Petri dishes. Suggested by Prof. Dr. Ralph Berger from the Leibniz University of Hanover (LUH), one possibility as the cause for this would be that the fungal strains emit nitrous oxide $\left(\mathrm{N}_{2} \mathrm{O}\right)$ and that this reacts with the polystyrene material of the plastic into a yellow colored azo-dye. On the other hand, Heterobasidion strains are known to produce many different metabolites (e.g. Donnelly et al. 1980; Peippe and Sonnenbichler 1992; Sonnenbichler 1994; Sonnenbichler et al. 1989; Sonnenbichler et al. 1993), amongst volatiles (Glen et al. 1996) that are shown to undergo chemical reactions leading to yellow, orange and red colors (Low et al. 2004). Studying the effect of the colorization of the plastic in more detail is beyond the scope of this thesis work. Accordingly, the answer to the question to the chemical nature of the effect has currently to be left open. 


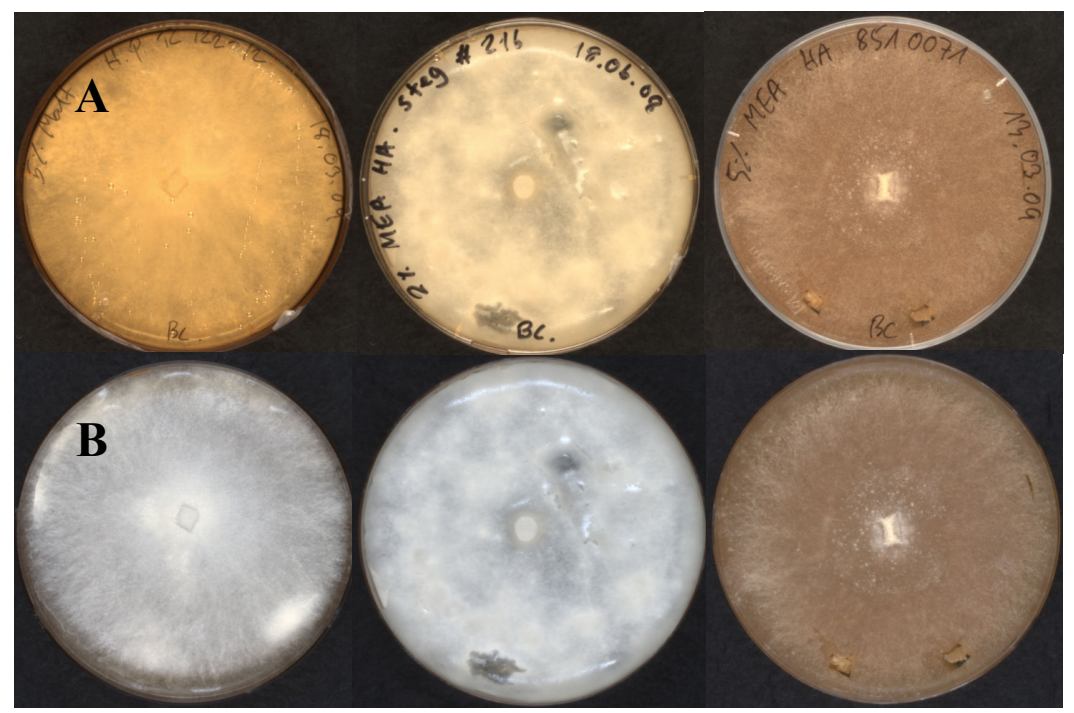

Fig. S2 Petri dishes from (left to right) A) H. annosum TC_122-12, H. parviporum Steg\#216 and $H$. annosum 851007.1 and B) the same order of the plates shown without the Petri dish lids. The fungi were inoculated onto malt extract-agar (5\% malt $/ 2 \%$ agar) and grown at $25^{\circ} \mathrm{C}$ till the mycelium covered the plates. Note the yellow staining of the plastic of the Petri dishes and the white mycelium of the fungi. In case of H. annosum 851007.1, the yellowish-brownish color seen comes from the agar medium shining through the comparably thinly grown mycelium of the strain

\section{II.7.3 References}

Donnelly DMX, Oreilly J, Chiaroni A, Polonsky J (1980) Crystal-structure and absolute-configuration of a new sesquiterpenoid metabolite of Fomes annosus, $7 \alpha, 8 \beta, 11$-trihydroxydrimane. Journal of the Chemical Society-Perkin Transactions I 10:2196-2199

Glen AT, Hutchins SA, Mccorkin NJ (1996) Hexa-1,3,5-triyne-a metabolite of Fomes annosus. Tetrahedron Letters 7(35):4223-4225

Low PJ, Udachin KA, Carty AJ (2004) Reaction of hexa-1,3,5-triyne $\mathrm{Me}_{3} \mathrm{SiC} \equiv \mathrm{CC} \equiv \mathrm{CC} \equiv \mathrm{CSiMe}_{3}$ with $\mathrm{Ru}_{4}(\mathrm{CO})_{13}\left(\mu_{3}-\mathrm{PPh}\right)$ : parallels with the chemistry of alkynes and diynes. Journal of Cluster Science 15(3):277-289

Peipp H, Sonnenbichler (1992) Occurrence of antibiotic compounds in cultures of Amillaria ostoyae growing in the presence of an antagonistic fungus or host plant cells. Biological Chemistry Hoppe-Seyler 373:675-683

Sonnenbichler J, Bliestle IM, Peipp H, Holdenrieder O (1989) Secondary fungal 
metabolite and their biological activities. I. Isolation of antibiotic compounds from cultures of Heterobasidion annosum synthesized in the presence of antagonistic fungi or host plant cells. Biological Chemistry Hoppe-Seyler 370:1295-1303

Sonnenbichler J, Peipp H, Dietrich J (1993) Further metabolites from dual cultures of the antagonistic basidiomycetes Heterobasidion annosum and Gloeophyllum abietinum. Biological Chemistry Hoppe-Seyler 374:467-473

Sonnenbichler J, Dietrich J, Peipp H (1994) Secondary fungal metabolites and their biological activities. V. Investigations concerning the induction of the biosynthesis of toxic secondary metabolites in basidiomycetes. Biological Chemistry Hoppe-Seyler 375:71-79 



\section{CHAPTER III}

\section{Effects of fungi and fungal enzymes on}

Abies grandis wood 



\section{III.1 Abstract}

Wood mass loss tests were carried out by following norm EN 113 (1996) with up to 19 weeks of incubation of $A$. grandis earlywood blocks $[30$ (longitudinal) x 5 (tangential) x 10 (radial)] and earlywood+latewood blocks [30 (longitudinal) x 10 (tangential) x 5 (radial)] of a density of $0.25-0.40 \mathrm{~g} \mathrm{~cm}^{-3}$, using the brown-rot fungus Coniophora puteana Strain 57 and the white-rot fungi Trametes versicolor Strain 6 and Pleurotus ostreatus 8F6, respectively. C. puteana Strain 57 caused $62 \pm 1.02 \%$ of mass loss for samples with only earlywood after 19 weeks of incubation, and $66 \pm 1.02 \%$ of mass loss for samples with earlywood+latewood. After 19 weeks of incubation, samples of earlywood alone ( $43 \pm 6.29 \%$ mass loss) was higher degraded by T. versicolor Strain 6 compared to the earlywood+latewood samples $(26 \pm 1.02 \%$ mass loss). After 19 weeks of incubation in this series of experiments, P. ostreatus 8F6 caused only $4 \pm 2.17 \%$ and $4 \pm 1.47 \%$ mass loss of earlywood and of earlywood+latewood samples, respectively.

The effects of enzyme actions on modification of wood cell walls were evaluated by changes in the cell wall color after staining wood blocks treated with T. versicolor Strain 6 with MBTH (3-methyl-2-benzothiazolinone-hydrazone hydrochloride monohydrate). Reddish staining of wood cell walls indicated chemical cell wall modifications, likely of the lignin. Reduction of cell wall thickness and mass loss of wood caused by decay by T. versicolor Strain 6 decreased the amount of staining in later stages of decay, likely due to degradation of chemical compounds that are reactive with MBTH. Staining with MBTH was best at samples incubated for only 6 weeks with $T$. versicolor Strain 6 . The area fraction of stained cell wall $\left(A_{S}\right)$ was found to increase in a slightly sigmoid form from earlywood to latewood, similar as the total cell wall area $\left(\mathrm{A}_{\mathrm{T}}\right)$. The quotients stained cell wall area by total cell wall area appeared to increase linearly from earlywood to latewood. The observation on A. grandis wood samples in a stage of early decay suggest that the decay type is a selective white-rot acting first on lignin and then with increasing degration of lignin on other components of the cell wall.

In order to decay the wood by attacking the lignin, $T$. versicolor is known from the literature to secrete both, laccases and manganese-dependent peroxidases. The modification of lignin by fungal treatment is expected to result in quinone structures that can be stained by MBTH. The fungal actions on lignin can be simulated by treating 
III. Effects of fungi and fungal enzymes on Abies grandis wood degradation

the wood with either a laccase/mediator system or, alternatively, with $\mathrm{Mn}^{3+}$ acetate mimicking the outcome of Mn-dependent peroxidase oxidation reactions on available $\mathrm{Mn}^{2+}$. Two laccase/mediator systems were used: reddish MBTH staining was slightly better with HBT (1-hydroxybenzotriazole) than with HBA (4-hydroxybenzoic acid). Also treatment with $\mathrm{Mn}^{3+}$ leads to reddish a colorization in the wood cell walls which was strongest with a higher applied $\mathrm{Mn}^{3+}$ concentration $(10 \mathrm{mM})$. In the $\mathrm{Mn}^{3+}$ treatments, the reddish color distributes more over the whole widths of the cell walls as compared to the wood cells treated with laccase/mediators due to the large size of the enzyme molecules. With the living fungus, the staining patterns resembled more the picture as obtained with the isolated laccase plus mediators than the $\mathrm{Mn}^{3+}$-effects. Wood is generally low in Mn-content and this limitation is probably responsible for the lack of MBTH-staining over the whole all cell wall width.

Keywords: mass loss, modification cell wall, area fraction, stained cell wall, cell wall area, latewood, earlywood, C. puteana, T. versicolor, P. ostreatus, oxidative enzyme, MBTH, laccase-mediator, manganese peroxidase 


\section{III.2 Introduction}

Abies grandis (grand fir) is a fast growing coniferous tree with a high potential for sustainable wood production and application in the wood products industry. A. grandis has been introduced from North America into Germany and has recently in a larger governmentally funded project been analyzed in its potential to fastly increase wood production in domestic forests (Polle et al. 2008). This tree species seem to be particularly well suited for planting in Germany because of its drought tolerance and ecological compatibility with climate conditions, soil conditions and native species (Röhrig 1981; Spellmann et al. 2010). Furthermore tested was the possibility to use the A. grandis wood for applications e.g. in solid form (Hof et al. 2008) or in wood composite production (Müller et al. 2009; Vos and Kharazipour 2010). For defining possible indoor and outdoor usages of $A$. grandis, knowledge on its behavior with fungal degraders is required (Kües et al. 2009; Navarro-González et al. 2008). In decay of $A$. grandis wood by white-rot fungi, extracellular oxidative enzymes secreted by the fungi are expected to be involved in degradation of cell wall components. Two classes of oxidative enzymes (called ligninolytic or lignin-modifying enzymes) - peroxidase and laccase - are involved in ligninolysis in order to catalyze the cleavage of carbon-carbon or carbon-oxygen bonds in complex lignin polymers.

Laccases are produced by many white-rot basidiomycetes for lignin and wood component degradation, and these enzymes act specifically towards phenols and poly-phenols. Laccases oxidize preferentially phenolic lignin structures to phenoxy radicals which subsequently oxidize to quinones (Gómez-Toribio et al. 2009; Hammel et al. 1993; Leonowicz et al. 2001). Substrate ranges of laccases and oxidation rates can be increased by adding low molecular weight compounds of high redox potentials to the laccases, resulting in so called laccase-mediator-systems (LMS). Example mediators in LMS are ABTS [2, 2'-azino-bis (3-ethylbenzthiazoline-6-Sulfonate)], HBT (1-hydroxybenzotriazole), HBA (4-hydroxybenzoic acid) and TEMPO (2,2,6,6-Tetramethylpiperidine-1-oxyl) (Call and Mücke 1997; Johannes and Majcherczyk 2000).

Of the different peroxidases [manganese-dependent peroxidases (MnP), manganese-independent peroxidases (MnIP), and lignin peroxidase (LiP)], MnP is an oxidative enzyme that is known to typically oxidize $\mathrm{Mn}^{2+}$ to $\mathrm{Mn}^{3+}$ that in turn chelates with organic acids, such as oxalate, glyoxylate and lactate (Elissetche et al. 2006; 
Leonowicz et al. 2001). MnP itself will not directly act on lignin. However, $\mathrm{Mn}^{3+}$-chelates diffusing into the wood cell walls act as low molecular mass redox mediators subtracting one electron from the lignin structure for these to form radicals; thereby, $\mathrm{Mn}^{3+}$ will be reduced to $\mathrm{Mn}^{2+}$. In follow up reactions, the resulting lignin radicals may be further oxidized and form quinones. In contrast, different to $\mathrm{MnP}$, manganese independent peroxidase attacks directly aromatic groups in the lignin (Mester and Field 1998). In wood degradation, LiP utilizes $\mathrm{H}_{2} \mathrm{O}_{2}$ and organic peroxides to oxidize a variety of organic substrates. LiP can catalyze oxidation of lignin monomers, dimers, and trimers as well as non-phenolic aromatic compounds (Kersten et al. 1985; Shah and Nerud 2002).

Swamy and Ramsay (1999a, b) and Tomšovský and Homolka (2003) for example reported that the white-rot Trametes versicolor strain ATCC 20869 secretes $\mathrm{MnP}$ and laccase during dye decoloration in batch cultures, using modified Kirk's medium. Work by Champagne et al. (2005) supported that MnP and laccase are the major enzymes most likely to be involved in dye decoloration by this strain. Moreover, there are several research groups such as Erden et al. (2009), Even et al. (2009) and Moredo et al. (2003) that observed production of $\mathrm{MnP}$ and laccase by T. versicolor when grown in submerged cultures in presence of different agricultural residues containing lignocellulose. Peroxidase activities other than $\mathrm{MnP}$ is usually not detected in cultivation with T. versicolor (Hiscox et al. 2010; Moredo et al. 2003; Swamy and Ramsay 1999a, b; Tomšovský and Homolka 2003 and others). Both type of enzymes, laccase and MnP has been detected when growing the fungus on different woods (Blanchette 1991; Yu et al. 2008).

In Chapter II of this thesis, I analyzed the effect of incubation of $A$. grandis wood with different fungi by determination of the mass loss of wood measured using norm EN 113 (1996). White-rot decay was generally not as severe than brown-rot. Of six tested white-rot strains, three did not decay the wood over the time of incubation whilst mass loss effects were encountered in increasing order with Heterobasidion annosum strain TC_122-12, Pleurotus ostreatus strain 8F6 and T. versicolor Strain 6, respectively. Staining with MBTH (3-methyl-2benzothiazolinone-hydrazone hydrochloride monohydrate) suggested that the fungi cause chemical changes to the wood fibers. In early stages of decay with $T$. versicolor Strain 6, the fibers had a strong reddish color in the wood upon treatment with MBTH, but with progress of decay the wood cell walls lost the ability to react by a color change 
with MBTH. The results were interpreted in such way, that T. versicolor Strain 6 produces oxidative enzymes such as laccase and $\mathrm{MnP}$ which attack the lignin in the cell walls, thereby causing chemical modifications on the fibers that can be made visible by MBTH reactions. The enzymes possibly also act with wood extractives which also might lead to color reactions upon MBTH treatment. In later changes of decay, when the reactive chemical compounds in the wood have been degraded by enzymatic actions, MBTH would show no effect anymore.

White-rot basidiomycetes, as the most frequent wood rotting organisms, are extensively lignin degrading and they are further characterized by their specific abilities to degrade hemicellulose and cellulose. Selective and simultaneous white-rots are distinguished that respectively either first decay the lignin to get better access to the cellulose or also hemicellulose or that decay lignin and the sugar polymers at the same time (Eriksson et al. 1990). The precise analysis of degradation type (selective or simultaneous white-rot) requires chemical analysis of cellulose and lignin contents in the wood prior and after decay. Depending on the wood type, $T$. versicolor for example exerts selective or simultaneously white-rot (Anagnost 1998; Archibald et al. 1997; Fackler et al. 2006; Mohebby 2004). To better follow this up, it requires the determination of modifications that occurred in the decayed wood by best available techniques, such as the MBTH-staining technique of modified lignin as developed first by Lang (2004) and then further in Chapter II of this thesis, following an original idea of Dr. A. Majcherczyk.

In this chapter, a more deep study on cell wall modifications of $A$. grandis wood as particularly performed by $T$. versicolor Strain 6 is presented together with experiments in which wood was treated with an individual isolated $T$. versicolor enzyme (laccase) or with $\mathrm{Mn}^{3+}$-chelates as the principle primary product of the ligninolytic enzymes MnP.

In Chapter II, the A. grandis wood samples used in mass loss tests with fungal degraders were randomly selected from a wood log, and this might have resulted in a high standard deviation of calculated average values of mass loss reflecting the degree of wood decay (see Fig. 3 and Fig. S1 in Chapter III). In this chapter, wood blocks were specifically obtained from the border region of sapwood and heartwood; wood blocks were carefully selected according to a certain density range $\left(0.25-0.4 \mathrm{~g} \mathrm{~cm}^{-3}\right)$. One group of wood blocks had a complete year ring plus some earlywood plus latewood from an older year ring and some earlywood from a younger year ring (Fig. 1). Wood 
samples of the same density range consisting of only earlywood (Fig. 1) were also considered in mass loss tests by fungal degradation of wood blocks, for better observing the effects of types of wood cells (wood cells with larger lumina in earlywood and wood cells with smaller lumina in latewood) on wood degradation. Next to the whiterot $T$. versicolor Strain 6, for comparison and as controls, wood decay tests were also performed with the white-rot $P$. ostreatus $8 \mathrm{~F} 6$ as a less aggressive species and with the brown-rot Coniophora puteana Strain 57, which follows up a chemical way of wood decay: Brown-rot degradation differs from the white-rot in that lignin is not or nearly not attacked, but the cellulose and hemicellulose in the cell walls by the chemical

Fenton-reaction: $\mathrm{Fe}^{2+}+\mathrm{H}_{2} \mathrm{O}_{2} \rightarrow \mathrm{Fe}^{3+}+\mathrm{OH}^{\bullet}+\mathrm{OH}^{-}$(Eriksson et al. 1990; Kremer 1999). Upon fungal incubation, the remaining wood material was analyzed by MBTH staining (only $T$. versicolor samples) and by chemical analysis of prevalent wood compounds (especially lignin).

Moreover in this chapter, to demonstrate effects of ligninolytic enzymes in white-rot decay, modification of cell walls was analyzed by injecting either a T. versicolor laccase plus mediator (HBT or HBA) solution or a solution of manganese (III) acetate in $100 \mathrm{mM}$ sodium lactate buffer into the wood, then sectioning it with a microtome, staining it with $\mathrm{MBTH}$, and, after photographing it with a digital CCD camera, images were evaluated by analySIS ${ }^{\circledR}$ imaging software for effects that occurred on the wood cell walls. Results were used for comparison to the observations obtained by an extensive image analysis done on MBTH-stained sections of wood blocks incubated for 6 weeks with T. versicolor Strain 6 .

\section{III.3 Materials and methods}

\section{III.3.1 Fungal strains and laccase}

All fungal strains used in this study were from the strain collection of the Division of Molecular Wood Biotechnology and Technical Mycology of the Büsgen-Institute, Göttingen. Solid culture media [malt extract-agar (5\% malt 2\% agar)] were inoculated in the middle of the Petri dishes (90 $\mathrm{mm}$ in diameter) with one mycelium-overgrown agar plug per plate of either one of the white-rot fungi $P$. ostreatus $8 \mathrm{~F} 6$ and T. versicolor Strain 6 or of the brown-rot strain C. puteana Strain 57. Inoculated plates were sealed with Parafilm (Laboratory Film, Chicago, USA) and grown for seven days 
at $25^{\circ} \mathrm{C}$ until plates were filled with mycelium for use in the EN 113 (1996) wood duration test as described in II.3.2.

Laccase III (NCBI accession no. AAL93622.1) used in this study was obtained from earlier work of R. Dwivedi (2006) of T. versicolor Strain 6 grown for 14 days at $25^{\circ} \mathrm{C}$ in $100 \mathrm{ml}$ 2,5-xylidine induced BSM medium [basidiomycetes medium; per liter: 5 g glucose, 0.65 g L-asparagine, $1 \mathrm{~g} \mathrm{KH}_{2} \mathrm{PO}_{4}, 0.5 \mathrm{~g} \mathrm{MgSO}_{4}$ x 7 $\mathrm{H}_{2} \mathrm{O}, 0.5 \mathrm{~g} \mathrm{KCL}, 0.5 \mathrm{~g}$ yeast extract, $50 \mathrm{ml}$ stock solution I $\left(0.2 \mathrm{~g} / 1 \mathrm{FeSO}_{4} \times \mathrm{XH}_{2} \mathrm{O}\right), 50 \mathrm{ml}$ stock solution II $\left(0.16 \mathrm{~g} / \mathrm{M} \mathrm{Mn}\left(\mathrm{CH}_{3} \mathrm{COO}\right)_{2}\right.$ x $4 \mathrm{H}_{2} \mathrm{O}, 0.04 \mathrm{~g} / \mathrm{Z} \mathrm{Zn}\left(\mathrm{NO}_{3}\right)_{2}$ x $4 \mathrm{H}_{2} \mathrm{O}, 1 \mathrm{~g} / 1 \mathrm{Ca}\left(\mathrm{NO}_{3}\right)_{2}$ x $4 \mathrm{H}_{2} \mathrm{O}$, $\left.0.06 \mathrm{~g} / \mathrm{CuSO}_{4} \times \mathrm{H}_{2} \mathrm{O}\right)$ ]. The culture supernatant of the T. versicolor Strain 6 culture (original activity $3.6 \mathrm{U} \mathrm{ml}^{-1}$ ) was concentrated by $\mathrm{R}$. Dwivedi on a preparative DEAE (diethylaminoethyl-dextran)-sepha-rose column and finally purified to homogeneity (1223 $\mathrm{U} \mathrm{ml}^{-1}$ ) using a Mono-Q column and stored at $-20^{\circ} \mathrm{C}$ in 0 to $30 \%$ of $1 \mathrm{M} \mathrm{NaCl}$ in $20 \mathrm{mM}$ potassium phosphate $\mathrm{pH} 6.4$ in a volume of about $50 \mathrm{ml}$ until further use. Aliquots of $1 \mathrm{ml}$ of thawed laccase solution were filled into $1.5 \mathrm{ml}$ Eppendorf tubes and were stored at $-20^{\circ} \mathrm{C}$ for use in this study. Upon thawing again, the enzymatic activity was tested directly prior to use. Enzymatic activities measured in the individual samples reached from $867 \mathrm{U} \mathrm{ml}^{-1}$ (after 3 weeks of storage) to $744 \mathrm{U} \mathrm{ml}^{-1}$ to $475 \mathrm{U} \mathrm{ml}^{-1}$ to $327 \mathrm{U} \mathrm{ml}^{-1}$ to $272 \mathrm{U} \mathrm{ml}^{-1}$ to $211 \mathrm{U} \mathrm{ml}^{-1}$ and to $196 \mathrm{U} \mathrm{ml}^{-1}$ (after 5 months of storage). Laccase activity was determined with ABTS; oxidation of ABTS was followed by an absorbance increase at $420 \mathrm{~nm}$. The enzyme activity was expressed in units defined as $1 \mathrm{U}=1 \mu \mathrm{mol}$ ABTS oxidized $/ \mathrm{min}\left(\varepsilon_{420}=36,000 \mathrm{M}^{-1} \mathrm{~cm}^{-1}\right)($ Childs and Bardsley 1975; Majcherczyk et al. 1999) using a microplate spectrophotometer (Spectra max340PC; Molecular Devices, Inc., USA)

\section{III.3.2 Wood block decay test}

A mini block duration test of $A$. grandis solid wood obtained from the Stadtwald Meschede in Sauerland, Germany, was carried out according to the European standard EN 113 (1996) (Bravery 1978). Wood blocks were separately obtained from the border in between the sapwood and the heartwood: the wood was cut into smaller blocks of 30 (longitudinal) $\times 10$ (tangential) $\times 5$ (radial) $\mathrm{mm}$ to obtain samples with earlywood+latewood (Fig. 1), as described in II.3.2, or into smaller wood blocks of 30 (longitudinal) $\times 5$ (tangential) $\times 10$ (radial) $\mathrm{mm}$ to obtain samples with only earlywood (Fig. 1). Wood blocks were carefully selected according to the position of 
year ring borders and width of year rings. Wood blocks were measured in weight to calculate the density and selected in the range of $0.25-0.40 \mathrm{~g} \mathrm{~cm}^{-3}$ for this experiment (for the detailed results, see supplementary Fig. S1 and S2). Wood blocks used not directly in the EN 113 test were stored in a sealed plastic bag at RT $\left(25^{\circ} \mathrm{C}\right.$, dark). For the EN 113 duration test, for each type of wood samples and each fungus tested, six wood blocks were laid on metal holders (1.5 $\mathrm{mm}$ in height) on fungal mycelium grown on malt extract medium for further treatment as described in Chapter II.3.2. Controls without fungi on the malt extract agar were treated analogously. Samples were inoculated for 6,12 and 19 weeks until harvest. For mass loss determination, each five randomly chosen wood blocks per treatment were scrapped off the mycelium, dried and weighed and mass loss determination was done as described in Chapter II.3.2.

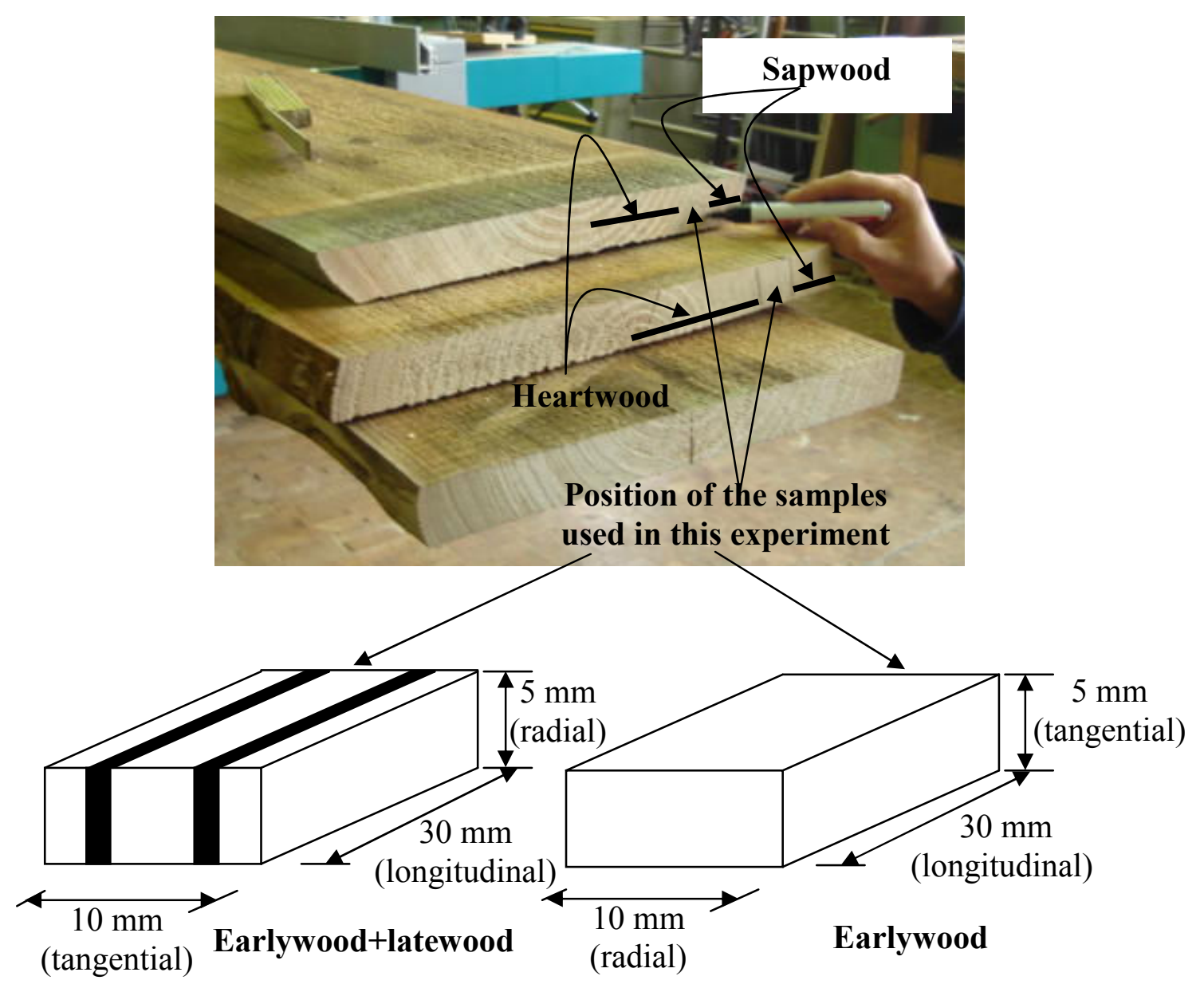

Fig. 1 Preparation of A. grandis wood blocks cut from the border region of sapwood and heartwood (top). Two types of samples were prepared with either both earlywood and latewood (bottom left) or with only earlywood (bottom right). Latewood is marked by black color, earlywood by white color 


\section{III.3.3 Lignin determination}

Lignin contents in wood samples were measured by using Klason lignin following the small scale instructions of TAPPI T 222 om-02 (2002).

\section{III.3.4 Chemical treatment of $\boldsymbol{A}$. grandis wood}

For the chemical treatment tests, a randomly selected wood block sample from the stock of A. grandis wood blocks of a size of 30 (longitudinal) x 10 (tangential) $\mathrm{x} 5$ (radial) $\mathrm{mm}$ and a density of $0.36 \pm 0.06 \mathrm{~g} \mathrm{~cm}^{-3}$ (sample with earlywood+latewood as indicated in Fig.1) and a T. versicolor Strain 6 incubated A. grandis wood block at 6 weeks of incubation (the left sixth wood block from the EN 113 experiment described in Chapter III.3.2) were cross-sectioned at the center of the wood block with a microtome (Jung, No. 25116, 220 volt, 6 Amp) into slides of $20 \mu \mathrm{m}$ thickness. More than 50 microtome sections per wood sample were generated. Each 10 sections per wood block sample were immersed for $30 \mathrm{~min}$ in either one of the following solutions:

- $10 \mathrm{mM} \mathrm{Mn}^{3+}$-acetate, $\mathrm{pH} 7$ in $100 \mathrm{mM}$ sodium lactate buffer $\left(\mathrm{Mn}^{3+}\right.$ sample)

- $10 \mathrm{mM} \mathrm{Mn}^{2+}$-acetate, $\mathrm{pH} 7$ in $100 \mathrm{mM}$ sodium lactate buffer $\left(\mathrm{Mn}^{2+}\right.$ sample)

- $10 \mathrm{U} \mathrm{ml}^{-1}$ T. versicolor Strain 6 laccase in $100 \mathrm{mM}$ sodium lactate buffer

- $10 \mathrm{U} \mathrm{ml}^{-1}$ T. versicolor Strain 6 laccase $+1 \mathrm{mM}$ HBT mediator in $100 \mathrm{mM}$ sodium lactate buffer

- $1 \mathrm{mM}$ HBT mediator in $100 \mathrm{mM}$ sodium lactate buffer

Samples were subsequently washed with running tap water for $10 \mathrm{~min}$. It is expected that quinones are formed upon oxidation of cell wall lignin with $\mathrm{Mn}^{3+}$ that subsequently can be visualized with MBTH staining (Rodríguez-López et al. 1994). Therefore, reduction of quinones by sodium borohydride $\left(\mathrm{NaBH}_{4}\right)$ prior to the staining was used as a control. In case of the $\mathrm{Mn}^{3+}$ treatment, 5 of the total 10 sections were treated with $50 \mathrm{mM} \mathrm{NaBH}_{4}$ in dry methanol solution for $30 \mathrm{~min}$ in order to reduce the quinone compounds within the lignin structure, and then sections were washed with running tap water for $10 \mathrm{~min}$. All wood sections of all treatments were stained with $100 \mathrm{mM}$ MBTH for inspecting and photographing as described under II.3.3.

For chemical injection experiments, wood blocks with earlywood+latewood [30 (longitudinal) x 10 (tangential) x 5 (radial) mm] from the $A$. grandis wood blocks stock were cut with a fret saw into smaller blocks of 8 (longitudinal) x 10 (tangential) 
x 5 (radial) $\mathrm{mm}$. A sledge microtome was used to smooth the cross-section wood surface. Subsequently, these smaller wood blocks were further cut longitudinally with a blade (Apollo; ever-sharp-blade, Solingen, Germany) exactly at the two borders of a given year ring (each considered year ring measured $6 \mathrm{~mm}$ in width) in order to obtain slides having a complete annual wood increment from earlywood to latewood. Wood blocks [8 (longitudinal) x 6 (tangential) x 5 (radial)] were sonicated in distilled water for $5 \mathrm{~min}$ to remove all of stacking (dust from surface smoothing) from the lumina of the wood cells. Wood blocks were mounted into about $1 \mathrm{~cm}$-long rubber tubes $(5 \mathrm{~mm}$ in diameter) in longitudinal direction, the rubber tubes were fitted onto the outlets of $20 \mathrm{ml}$ plastic syringes and these were mounted onto an injector system (Cole Parmer, 74900 series) that provided in a liquid flow rate of $2,106 \mu \mathrm{h} \mathrm{h}^{-1}$ per wood blocks $10 \mathrm{ml}$ of either one of the following solutions:

- $4 \mathrm{mM} \mathrm{Mn}{ }^{3+}$-acetate, $\mathrm{pH} 7$ in $100 \mathrm{mM}$ sodium lactate buffer $\left(\mathrm{Mn}^{3+}\right.$ sample)

- $10 \mathrm{mM} \mathrm{Mn}^{3+}$-acetate, $\mathrm{pH} 7$ in $100 \mathrm{mM}$ sodium lactate buffer $\left(\mathrm{Mn}^{3+}\right.$ sample)

- $10 \mathrm{U} \mathrm{ml}^{-1}$ T. versicolor Strain 6 laccase $+1 \mathrm{mM} \mathrm{HBT}$ in $100 \mathrm{mM}$ sodium lactate buffer

- $10 \mathrm{U} \mathrm{ml}^{-1}$ T. versicolor Strain 6 laccase $+1 \mathrm{mM} \mathrm{HBA}$ in $100 \mathrm{mM}$ sodium lactate buffer.

Per type of solution, 3 different wood blocks were treated. After passing the wood samples, the left-over liquids were collected in a beaker. Wood samples were then washed with running tap water and samples were subsequently cross-sectioned with a sledge microtome into slides of $20 \mu \mathrm{m}$ thickness. Each 10 sections were washed with running tap water. The sections were stained with $\mathrm{MBTH}$ for a minute, then washed again with running tap water, mounted in glycerol on slides, and cell walls of individual tissues were inspected under a light microscope Zeiss Axioplan (Zeiss, Jena, Germany) with white light and photographed with a digital CCD camera (Color View II, Soft Imaging System GmbH, Münster, Germany) that was mounted onto the microscope and linked with a computer with an installed copy of the image software analySIS ${ }^{\circledR}$ (Soft Imaging System GmbH, Münster, Germany). Pictures were examined using the software tool for images analySIS ${ }^{\circledR}$ as described under II.3.3. 


\section{III.3.5 Microscopic analysis of wood sections and image analysis}

For a first type of image analysis, by an own program setting, images of cell walls of A. grandis wood stained with MBTH (Fig. 2A) were transferred from the image analySIS ${ }^{\circledR}$ program (Fig. 2B) to Adobe Photoshop (version 6.0). The gray-shaded lumina of the wood fibers and the complete ray cells of the sections were deleted with the eraser tool leaving the cell lumina and the rays white. Afterwards, images were transferred into the Mathematica program (version 5.1, WOLFRAMRESEARCH, USA) installed on a laptop to evaluate the amounts of stained cell walls. The subsequent steps of the process of image analysis using programming commands of the software Mathematica version 5.1 are explained in the following:

- Import color images in which the grey-shaded lumina ray cells from the wood were already deleted (by using Adobe Photoshop version 6.0)

- Use command colorData and greyData to convert color-images to grey-images, respectively

- Indicate color-code by the following:

○ Black: color-code is 0

- White: color-code is 255

- Set up the color-threshold between 0-255 (in this specific analysis the color-threshold $=140$ was chosen) to indicate the black and white pixels according to the stained wood cell walls (applies for all images; color-threshold must be of the same value)

- Command criteria: If the color-code is of $>140$ threshold, the program will present white pixels, if the color-code is of $\leq 140$ threshold, the program will present black pixels

- Each analyzed image is presented in just the two contrast colors of black and white pixels. The black pixels indicate in this program setting the modification of cell walls as detected by staining with MBTH

The Mathematica program calculates a total numeric amount of all pixels (black and white; value $\mathrm{P}_{\mathrm{BW}}$ ) and of black pixels alone (stained cell walls, see Fig. 2C; value $\left.\mathrm{P}_{\mathrm{B}}\right)$. The area fraction of stained cell walls $\left(\mathrm{A}_{S}\right)$ per image was calculated in percentage from these values as taken from the Mathematica program windows (Fig. 2D) using the following equation (Cherdchim 2004):

$$
A_{S}=\frac{P_{B}}{P_{B W}} \times 100 \%
$$






Fig. 2 Image analysis of an area fraction of stained cell walls $\left(\mathrm{A}_{\mathrm{S}}\right)$ from the $A$. grandis wood section using $\mathbf{A}$ ) a color photo taken by a digital CCD camera and imported into the image analySIS ${ }^{\circledR}$ program, B) a picture in Adobe Photoshop after deleting the grey color from the lumen space of wood cells, and C) a black and white picture after conversion with the Mathematica program D) shows the Mathematica programs' windows of evaluating the black and white pictures

The quality of this half-manual analysis using first Adobe Photoshop and the Mathematica program was controlled by a second type of image analysis using the original photos and the set-up in the specific image analySIS ${ }^{\circledR}$ program (Soft Imaging System GmbH, Münster, Germany) for calculating the stained cell wall areas:

- Open color images

- Click menu: Operate/Intensity/Maximize contrast

- Click menu: Image/Set magnification

- Click menu: Operate/Color separation/intensity (change to grey shading)

- Click menu: Set threshold for Phase I: threshold value was set up between low (0) - high (100); this setting reflects the dark stained cell wall areas: $0=$ black, $100=$ grey shade up to which pixels are counted

- Click menu: Define determination/Execute

- Click menu: File/Save as/save as an Excel-sheet

- Each cell within an image in this analysis is recognized as individual area. Each analyzed image is therefore presented in the values of stained cell wall 
area $\left(\mu \mathrm{m}^{2}\right)$ obtained of the individual cells. The value per one cell as presented with the chosen threshold indicates the complete stained cell wall area the specific cell. The total stained cell wall areas of all cells per image was calculated by importing the values for all individual cells into Microsoft Excel 2002 (Microsoft Corporation, USA) and summarizing these together. From the value obtained, the percentage of the stained cell wall area in an image was calculated in proportion to the total area of the image. [The total area per image was determined in the image analySIS ${ }^{\circledR}$ program by clicking button Frame/ROI Result to mark the total analyzed image area].

Determination of complete cell wall areas per image was also carried out by using the same photos and the image analySIS ${ }^{\circledR}$ program in order to individually calculate the complete cell lumen areas and the complete ray cell areas. These values together were subtracted from the value of the total area of a respective image, thereby yielding the value for the complete area of cell walls within the image. For this analysis, the following parameters in the image analySIS ${ }^{\circledR}$ program were used:

- For calculating cell lumen areas:

- Open color images

- Click menu: Operate/Intensity/Maximize contrast

- Click menu: Image/Set magnification

- Click menu: Operate/Color separation/Hue

- Click menu: Set threshold for Phase II: threshold value was set up between low (101) - high (240); this setting reflects the light cell lumen areas: $101=$ grey shade, $240=$ light grey shade up to which pixels are counted

- Click menu: Define determination/Execute

- Each cell in this analysis is recognized as individual area. Each analyzed image is therefore presented in the values of lumen area $\left(\mu \mathrm{m}^{2}\right)$ of the individual cells. The value per one cell as presented with the chosen threshold indicates the complete cell lumen area of the specific cell. The total lumen area of all cells per image was calculated by importing the values for all individual cells into Microsoft Excel 2002 and summarizing these together. From the value obtained, the percentage of the cell lumen area in an image was calculated in proportion to the total area of the image. 
- For calculating ray cell areas:

- Open color images in which the grey-shaded ray cells from the wood were already deleted by using Adobe Photoshop version 6.0 in order to subtract these values from the value of cell lumen areas

- Click menu: Operate/Intensity/Maximize contrast

- Click menu: Image/Set magnification

- Click menu: Operate/Color separation/Blue

- Click menu: Set threshold for Phase III: threshold value was set up between low (241) - high (55); this setting reflects the whitened areas: $241=$ light grey shade, $240=$ white up to which pixels are counted

- Click menu: Define determination/Execute

- Click menu: File/Save as/save as an Excel-sheet

- Each cell in this analysis is recognized as individual area. Each analyzed image is therefore presented in the values of its different ray areas $\left(\mu \mathrm{m}^{2}\right)$. The total ray cell area per image was calculated by importing the values for all individual rays into the Microsoft Excel 2002 and summarizing these together. From the value obtained, the percentage of the ray cell area in an image was calculated in proportion to the total area of the image.

Both of the total ray and the total cell lumen areas per analyzed image were summarized following importing these values into the Microsoft Excel 2002. The percentage of total cell wall area was calculated in proportion by dividing the total area of rays + cell lumina by the total area of the analyzed image.

In further analysis, done to study of the thicknesses of wood cell walls in photographs of wood sections (photographed with an objective giving a 100x magnification) by using the image analySIS ${ }^{\circledR}$ program, values were calculated from cell walls of $n=30$ different wood fibers from earlywood and from latewood of nondegraded or degraded wood, respectively. The 30 different cells per wood type were selected from 6 to 8 different images of the same wood section. Note that the thickness of the cell walls of decayed wood, particularly of the earlywood in the samples was not fully reliable to measure since by the softened cell wall structure it was not possible to sharply focus on all cut cell walls with a given microtome section. 
The following parameters in the images analysis of the image analySIS ${ }^{\circledR}$ program were used for calculating thicknesses of cell walls:

- Open color images

- Click menu: Image/Set magnification

- Click menu: Measurement/Arbitrary Distance

- Click to mark two points on the outer and inner edge of a cell wall, respectively as a measure of the thickness of the cell wall; per cell, measure in this way the cell wall at 10 different positions

- Click with the right mouse button on the screen window to stop the length marking function

- Click menu: Window/Sheet

- Click menu: File/Save as/save as an Excel-sheet

- Each cell in this analysis is recognized as individual thickness. Each analyzed image is therefore presented in the values of cell wall width $(\mu \mathrm{m})$ of about 5 cells of all the 10 positions measured per cell. The average thickness of cell wall per cell was calculated from the 10 corresponding values by importing the values into Microsoft Excel 2002. Next, the calculated cell wall thicknesses for all 30 different cells of one wood sample were used to calculate in Microsoft Excel 2002 the average cell wall thickness within an analyzed tissue (either earlywood or latewood from non-degraded wood or from degraded wood).

\section{III.3.6 Determining the distance of earlywood and of latewood in a year ring}

To study the staining of cell walls in tissues of different age over a whole year ring (about $6 \mathrm{~mm}$ in width) in relative distance to each other, relative positions within the wood section were calculated from earlywood to latewood by using the following equation:

$$
S=\frac{D-X}{D}
$$

where $S=$ a value proportional to a respective distance from the start of earlywood growth to a region of interest in direction of latewood, $D=$ the width of a year ring $(6 \mathrm{~mm})$ and $X\left(X_{1}, X_{2}, X_{i} \ldots\right)=$ the respective distance in $\mathrm{mm}$ from the start of growth of earlywood (year ring border to last year's wood increment) to the individual place of 
inspection in direction of latewood; $S=0$ at the position of the beginning of the year-ring (earlywood) and $S=1$ at the end of year-ring (latewood) (Fig. 3).

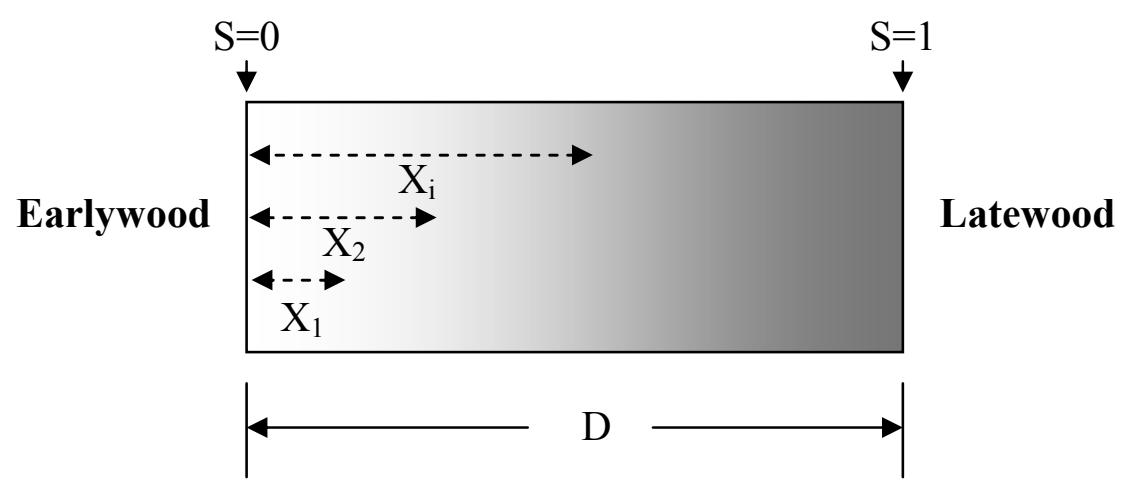

Fig. 3 Diagram of a given year ring from year ring border to year ring border that presents a complete annual wood increment from earlywood to latewood; for definition of $\mathrm{S}, \mathrm{X}_{1}, \mathrm{X}_{2}, \mathrm{X}_{\mathrm{i}}$ and $\mathrm{D}$ see main text

\section{III.3.7 Statistical analysis}

Group values for all parameters in the mass loss test were compared by analysis of variance (ANOVA) tests using the Fisher's least significant difference (LSD) and Duncan's test procedure for multiple comparison (SPSS 8.0 for Windows; USA). Relations among the mass losses of samples were compared for each fungal strain to the uninfected control in the same week of incubation.

\section{III.4 Results}

\section{III.4.1 Mass loss of $\boldsymbol{A}$. grandis wood by incubation with different decay fungi}

Mass loss of $A$. grandis wood blocks (density range of $0.25-0.40 \mathrm{~g} \mathrm{~cm}^{-3}$; density mean value of earlywood+latewood $=0.322 \pm 0.045 \mathrm{~g} \mathrm{~cm}^{-3}$ and density mean values of earlywood alone $=0.346 \pm 0.041 \mathrm{~g} \mathrm{~cm}^{-3}$; for further information see supplementary material of Fig. S1 and S2) by fungi was observed after 6 weeks, 12 weeks and 19 weeks of incubation, respectively. Mass loss values determined after fungal incubation (Fig. 4) indicate that the $A$. grandis wood was comparably resistant against 
decay by the white-rots $P$. ostreatus 8 F6 and T. versicolor Strain 6 unlike against the brown-rot C. puteana Strain 57 (however, compare Fig. S3 in the supplementary material).

Mass loss of samples treated with $P$. ostreatus $8 \mathrm{~F} 6$ was very low as deduced from comparisons with the uninfected control, regardless of whether the samples contained earlywood+latewood or only earlywood. No mass loss was obtained in the first 6 and 12 weeks of incubation with P. ostreatus 8F6, whereas after 19 weeks of incubation average mass loss values of only $4 \pm 1.47 \%$ and $4 \pm 2.17 \%$ were obtained for earlywood+latewood and earlywood samples, respectively (Fig. 4). Samples incubated for 6 weeks with the fungus were analyzed for their relative lignin content (Fig. 5). Consistent with $P$. ostreatus $8 \mathrm{~F} 6$ being a white-rot fungus, there was a slight statistically significant reduction in lignin content in both types of samples tested. Samples of earlywood+latewood had a relative lignin content of $29.76 \pm 0.10 \%$ compared to $30.49 \pm 0.90 \%$ of the control, and samples of only earlywood had a lignin content of $31.94 \pm 0.11 \%$ as compared to $33.77 \pm 1.10 \%$ of the control. It is interesting to note that in the controls the relative lignin content in samples with only earlywood was slightly higher than in the samples of earlywood+latewood (Fig. 5).

C. puteana Strain 57 in contrast degraded A. grandis wood very fast (Fig. 4); already after the six weeks of incubation, the samples of earlywood+latewood showed an average mass loss value of $46 \pm 1.21 \%$ whilst the average mass loss for samples of only earlywood was even higher with $55 \pm 4.57 \%$ (Fig. 4). These considerable values of mass loss coincided well with an increase in the relative amount of lignin in the samples (Fig. 5); at 6 weeks of incubation, the relative lignin content in the samples with earlywood+latewood and in the samples with only earlywood was more than double $(61.51 \pm 1.98 \%$ and $76.24 \pm 0.68 \%$, respectively) as compared to the untreated control $(30.49 \pm 0.90 \%$ and $33.77 \pm 1.10 \%$, respectively). Mass loss further increased over the time, resulting in average mass loss values of $66 \pm 1.02 \%$ for samples with earlywood+latewood after 19 weeks of incubation, and of $62 \pm 1.02 \%$ for samples with only earlywood, respectively (Fig. 4). 


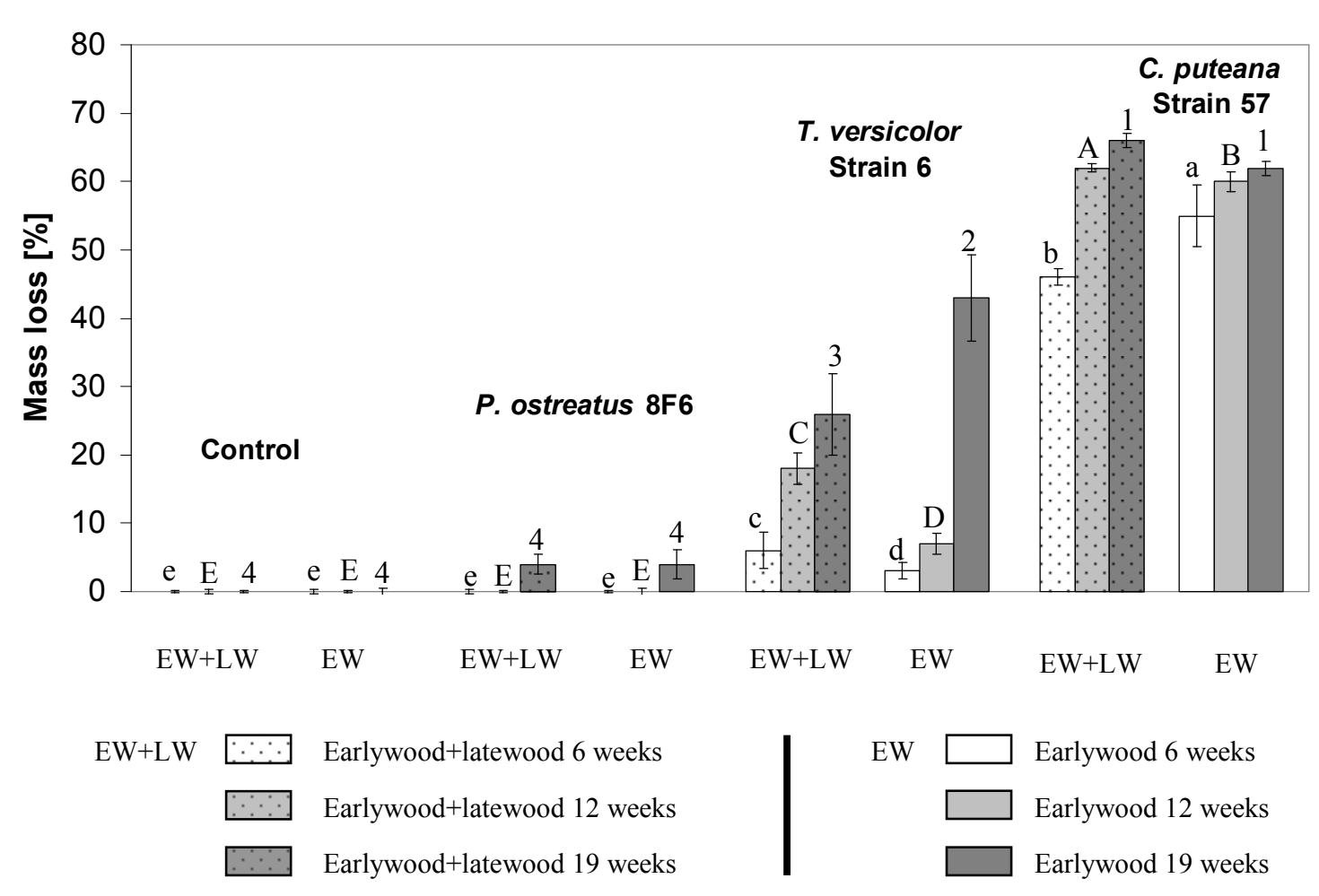

Fig. 4 Mass loss over the time caused by fungal decay of A. grandis wood blocks of earlywood+latewood in comparison to wood blocks of only earlywood. Wood blocks were carefully selected by density and even growth of year rings and incubated for 6 to 19 weeks with or without a fungus on solid malt extract-agar medium $(5 \%$ malt $2 \%$ agar) at $22^{\circ} \mathrm{C}$ and $65 \% \mathrm{RH}$ (relative humidity) in the dark. Per set-up, 6 different wood blocks were incubated with or without a fungus. Upon harvesting, average mass loss values of each 5 dried wood blocks per fungus and per time point were calculated after determining the dry weights. Standard deviations are also shown. Statistical analysis of the data was performed with SPSS 8.0 for Windows, the different superscripts on the chart (letters in lower case compare the values after 6 weeks of incubation, capital letters compare the values after 12 weeks of incubation, and numbers compare the values after 19 weeks of incubation) indicate values that differ significantly $(p<0.05)$ between treatments as based on analysis of variance (ANOVA)

As in the previous experiment documented in Fig. 3 in Chapter II of this thesis, T. versicolor Strain 6 again showed an intermediate effect on decay of $A$. grandis wood. Only low mass loss was detected after 6 weeks of incubation with T. versicolor Strain 6 with an average value of $6 \pm 1.21 \%$ for the samples of earlywood+latewood and with an average value of $3 \pm 1.20 \%$ for the samples of only earlywood (Fig. 4). At this time of incubation, there was a small decrease in the average lignin content of all samples, indicating the beginning of white-rot decay: samples with earlywood+latewood had a relative lignin content of $28.84 \pm 0.50 \%$ compared to the value of $30.49 \pm 0.90 \%$ of the 
control and samples with only earlywood had a relative lignin content of $29.28 \pm 0.32 \%$ compared to $33.77 \pm 1.10 \%$ of the control (Fig. 5). The detected decrease in lignin content by treated with $T$. versicolor Strain 6 suggests that the fungus selectively acts on the lignin of the A. grandis wood. As further shown in Fig. 4, T. versicolor Strain 6 degraded earlywood+latewood faster in the first 12 weeks of incubation $(18 \pm 2.33 \%$ mass loss) than the pure earlywood (mass loss of $7 \pm 1.54 \%$ ), but finally after the longer incubation time of 19 weeks earlywood alone ( $43 \pm 6.29 \%$ mass loss) was higher degraded (see Fig. 4) compared to the earlywood+latewood ( $26 \pm 1.02 \%$ mass loss).

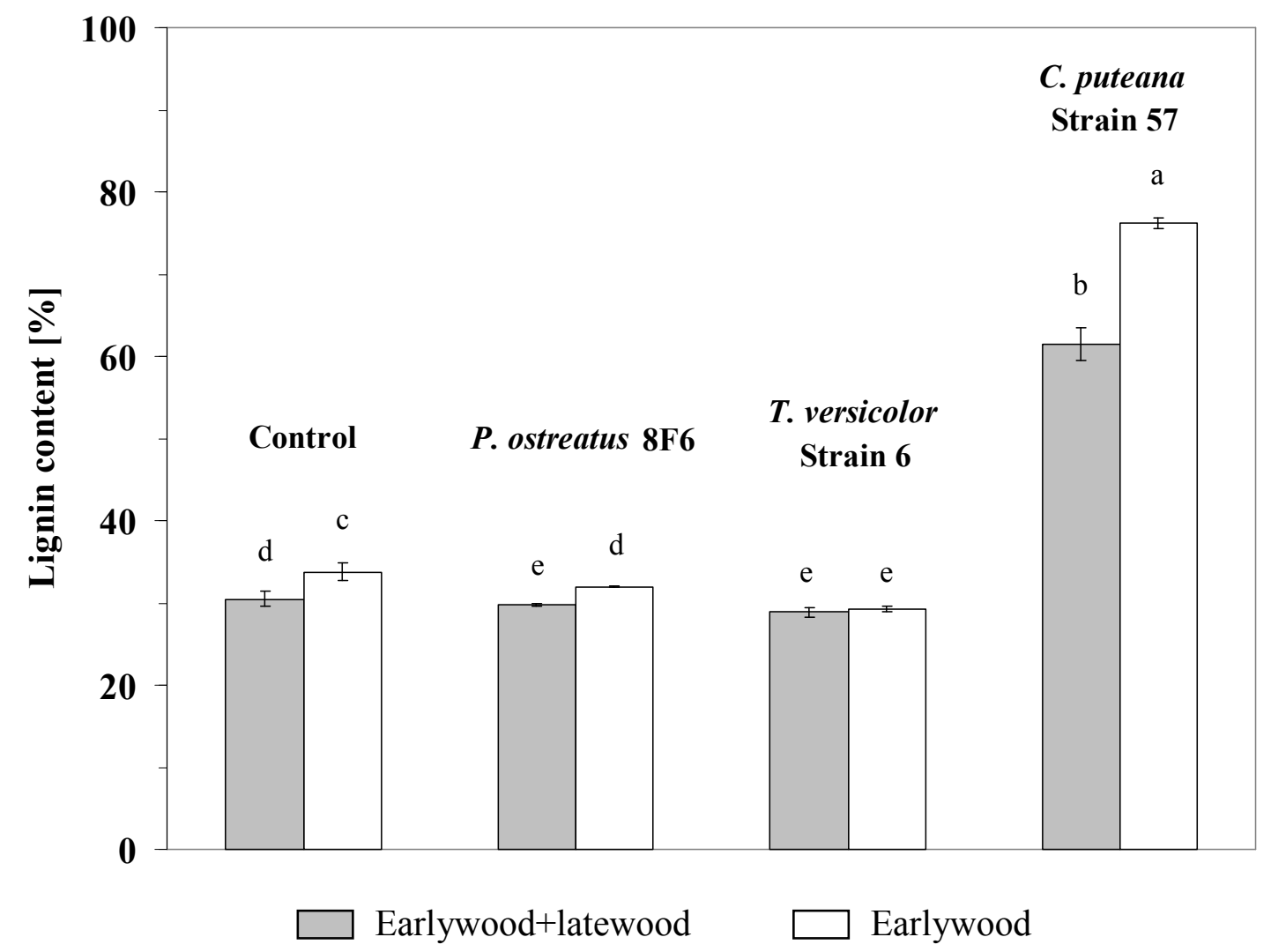

Fig. 5 Relative lignin content of $A$. grandis wood blocks of earlywood+latewood in comparison to wood blocks of only earlywood after 6 weeks of incubation with a fungus or without a fungus (control) on solid malt extract-agar medium (5\% malt $2 \%$ agar) at $22^{\circ} \mathrm{C}$ and $65 \% \mathrm{RH}$ in the dark (for mass loss values of the same samples, compare Fig. 4). Per set-up, 6 different wood blocks were incubated with or without a fungus. Upon harvesting, average lignin content values of 5 dried wood blocks per fungus in 3 technical replications were calculated following TAPPI T 222 om-02 (2002). Standard deviations are also shown. Statistical analysis of the data was performed with SPSS 8.0 for Windows, the different superscripts on the chart indicate values that differ significantly $(p<0.05)$ between treatments as based on analysis of variance (ANOVA) 
$6^{\text {th }}$ week
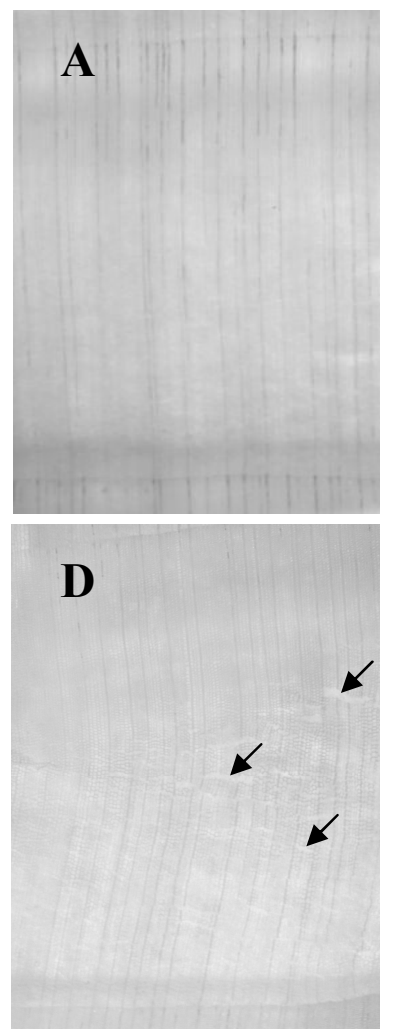

$12^{\text {th }}$ week
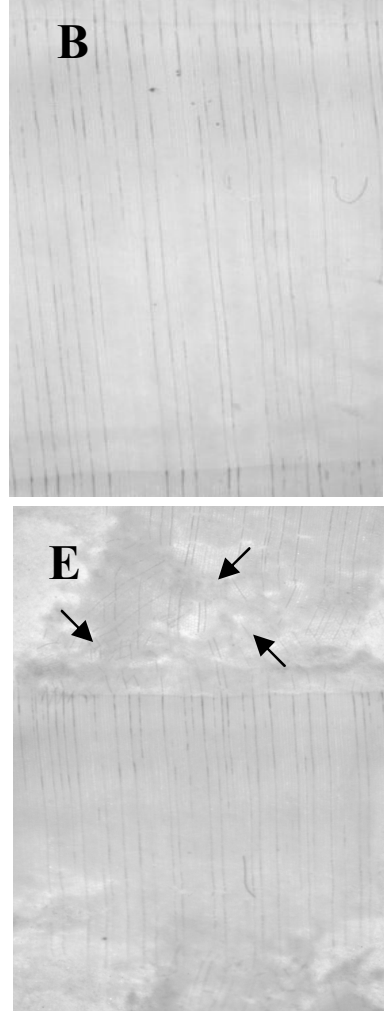

$19^{\text {th }}$ week
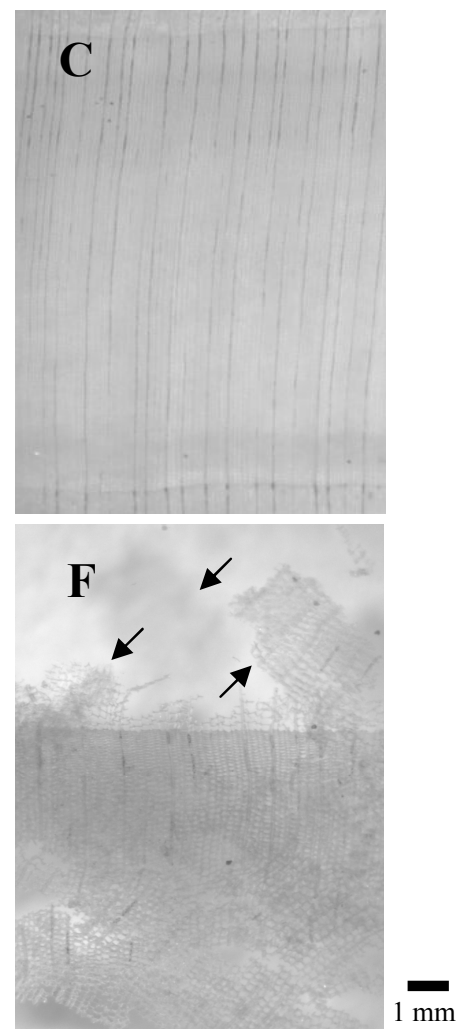

Fig. 6 Appearance of microtome sections of untreated and thus undamaged A. grandis wood blocks A) after 6 weeks, B) after 12 weeks and C) after 19 weeks of incubation at $22^{\circ} \mathrm{C}$ and $65 \% \mathrm{RH}$ on $5 \%$ malt $2 \%$ agar, and of wood blocks treated with $T$. versicolor Strain 6, D) after 6 weeks, E) 12 weeks and F) 19 weeks of incubation, respectively. In case of all untreated controls and in case of the sample of 6 weeks of incubation with T. versicolor Strain 6, overviews on complete year rings are shown. Arrows in Fig. 6D mark small cracks in the wood structure of the sample treated for 6 weeks with T. versicolor Strain 6. In case of 12 weeks and 19 weeks of incubation with T. versicolor Strain 6, overviews on whole year rings were not possible to show because of the strong damage of the wood cells caused by the fungus. The earlywood was so much destroyed that only some broken parts were left (pointed to by arrows). Only in the latewood regions, the tissue structure was still reasonably maintained. The time of incubation is indicated above each row of wood sections

Wood blocks treated with T. versicolor Strain 6 were inspected in more detail (Fig. 6). Per time point, each one wood block of the six incubated with the fungus was chosen for sectioning with a microtome directly after harvesting and scrapping off the surface mycelium. After 6 weeks of incubation, it was still possible to obtain complete microtome sections from the wood. In case of 12 weeks and 19 weeks of incubation, however, it was not possible anymore to section a complete year ring over the whole 
width in one piece from border to border because of wood degradation by the fungus (Fig. 6E and 6F). Compared to the untreated wood (Fig. 6A-6C), in the sections of wood incubated for 6 weeks with $T$. versicolor Strain 6 , a beginning of tissue degeneration became visible by some short cracks that were likely caused by the friction the microtome sledge applied onto the wood surface (Fig. 6D) whilst generating the samples through the process of sectioning.

\section{III.4.2 Modification of wood cell walls and wood lignin by incubation with T. versicolor Strain 6 and its oxidative enzymes}

For white-rot wood decay fungi, we expect that oxidative enzymes secreted by the fungi will modify the lignin within the wood cell walls. This modification of lignin results in quinone structures that can be stained by novel lignin-interacting reagents, such as MBTH. Wei et al. (2010) described that laccase might oxidize hydroquinone to produce semiquinones that act as reductants of $\mathrm{O}_{2}$ and $\mathrm{Fe}^{3+}$. It is to be expected that this enzymatic quinone formation is best to be shown in early steps of decay by white-rot fungi, prior to massive lignin degradation. Accordingly, Fig. 7 shows A. grandis samples with earlywood+latewood treated for 6 weeks with the white-rot $T$. versicolor Strain 6 in comparison to untreated wood and Fig. 8 shows A. grandis samples with only earlywood treated for 6 weeks with the fungus in comparison to the untreated control. From the macro-scale, it was obvious that the treated wood blocks' color was more reddish and intense than that of the untreated wood blocks, indicating chemical alteration on the lignin in the fungal samples (Fig. 7A and Fig. 8A). On the smaller scale showing overviews of the wood tissues (Fig. 7B and Fig. 8B), one can see the modification of cell walls due to the fungal actions even better due to the staining with MBTH done as described in materials and methods of Chapter II.

Whilst there was no staining effect with MBTH in the untreated controls, cell walls of the fungal treated wood had a strong reddish color (Fig. 7B and Fig. 8B). However, in the samples with earlywood+latewood differences in intensity of staining were obvious between earlywood and latewood (Fig. 7B to 7D). Latewood apparently reacted on the whole much stronger with MBTH than earlywood, although the staining was not absolutely even in the latewood (Fig. 7B). This is not just due to a bad staining reaction since a series of consecutive microtome sections from the same T. versicolor Strain 6-incubated wood block showed always at the same areas stronger 
or less strong staining (see Fig. S4 in the supplementary material). Accordingly, it is likely the degree of local fungal enzymatic attack on the wood that has to be made responsible for the observed effects.

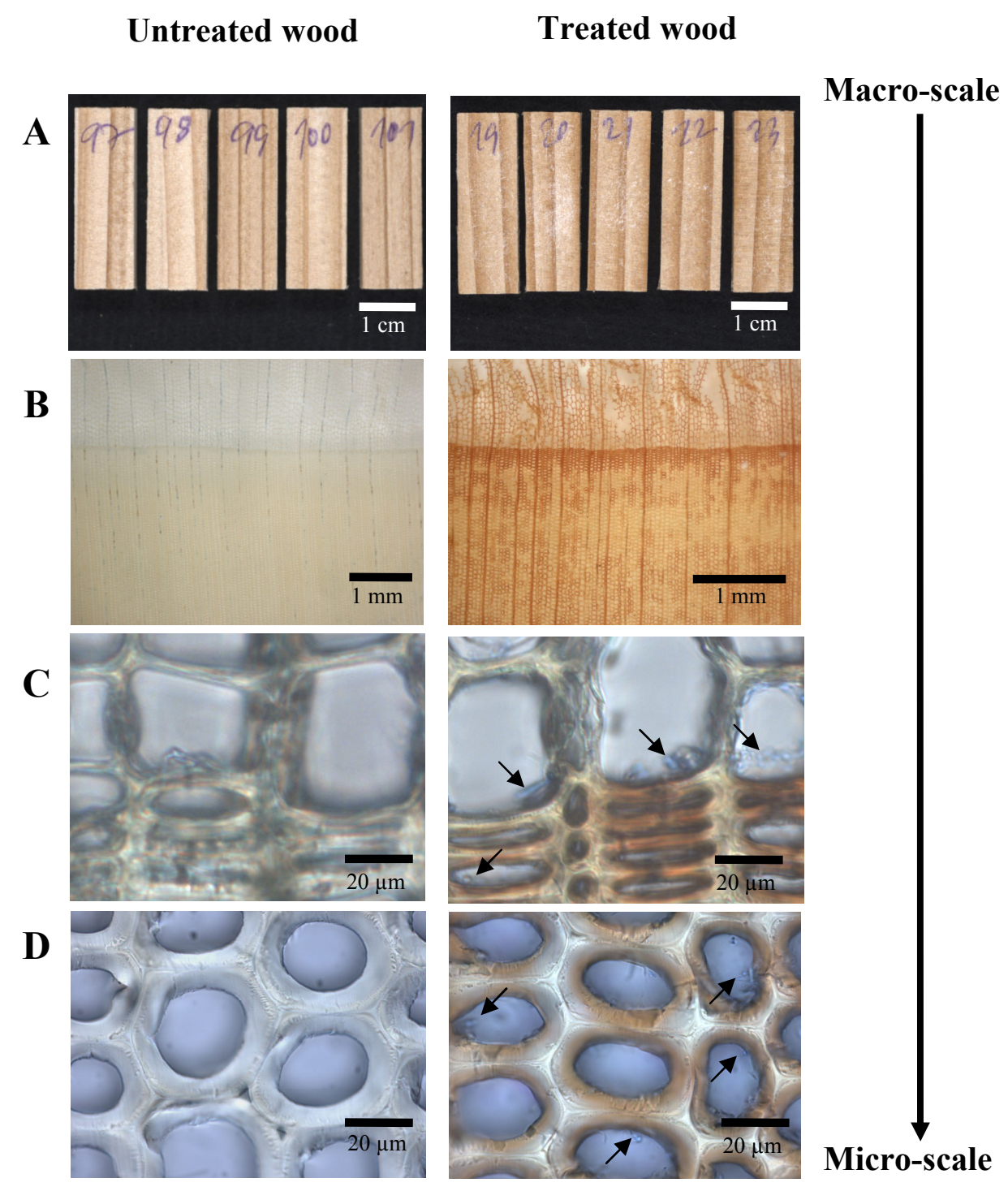

Fig. 7 Appearance of $A$. grandis wood blocks (with earlywood+latewood) after 6 weeks of incubation without fungus (left column) and after 6 weeks of incubation with T. versicolor Strain 6 (right column) and drying the harvested wood blocks in the oven at $103 \pm 2^{\circ} \mathrm{C}$ for $24 \mathrm{hrs}$. A) General overviews of the complete wood blocks after drying, B) overviews of microtome sections made from parallel non-dried wood blocks, showing latewood and earlywood tissues at a year ring border (bottom latewood, top earlywood of next year), C) enlarged views in the microtome sections of cells of all earlywood and D) enlarged views in the microtome sections of cells of the latewood. Microtome sections as shown in $\mathbf{B}$ to $\mathbf{D}$ were stained by lactophenol blue (for fungal staining) and $100 \mathrm{mM} \mathrm{MBTH}$, respectively, as described in Chapter II.3.3. Note the fungal hyphae (see arrows) in the cell lumina of the samples treated with the fungus. The types of wood treatment are indicated above each row of photographs 
Untreated wood

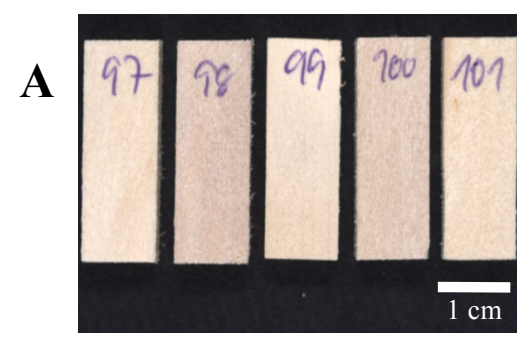

B

C

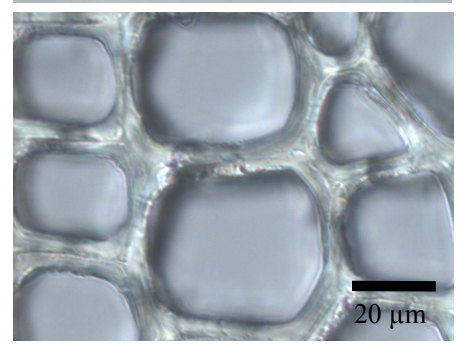

Treated wood

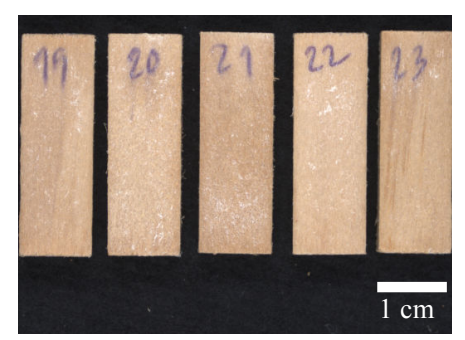

\section{Macro-scale}

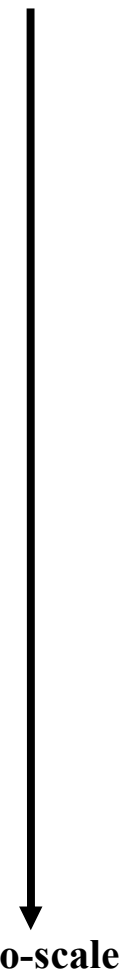

Fig. 8 Appearance of $A$. grandis wood blocks (with earlywood) after 6 weeks of incubation without fungus (left column) and after 6 weeks of incubation with T. versicolor Strain 6 (right column) and drying the harvested wood blocks in the oven at $103 \pm 2^{\circ} \mathrm{C}$ for $24 \mathrm{hrs}$. A) General overviews of the complete wood blocks after drying, B) overviews of microtome sections made from parallel non-dried wood blocks, showing earlywood tissues and $\mathbf{C}$ ) enlarged views in the microtome sections of cells of earlywood. Microtome sections as shown in $\mathbf{B}$ to $\mathbf{C}$ were stained by lactophenol blue (for fungal staining) and $100 \mathrm{mM} \mathrm{MBTH}$, respectively, as described in Chapter II.3.3. Note in $\mathbf{B}$, the different direction of rows of wood cells as shown compared to Fig. 8B is due to the different directions of cutting the wood block with only earlywood (compare Fig. 1). In $\mathbf{C}$, the fungal hyphae in the cell lumina of the samples treated with the fungus are marked by the arrows. The types of wood treatment are indicated above each row of photographs

Whether the effect of a differential degree of staining by MBTH of latewood and earlywood as seen in Fig. 7B is simply due to the broken cell walls and/or due to a higher amount of cell wall modification may be deduced from enlarged views of the wood cells. When comparing earlywood cells from untreated and from $T$. versicolor Strain 6 incubated wood with each other (Fig. 7C, 7D and Fig. 8C), it appears that some cell walls in fungal treated samples might have already lost somewhat in thickness. Staining by MBTH mainly occurred in the layers of the cell walls being most inner to 
the lumina of the cells (Fig. 7C, Fig. 8C, Fig. 9 and Fig. 10). Particularly in the earlywood with the comparably thin cell walls, next to a staining of the inner cell wall layers, there are sometimes places where the staining is continuous over the adjacent cell walls of two neighboring cells (Fig. 8C and Fig. 9). In some instances, in earlywood and in latewood, it is along the pits in between two adjacent cells where staining is strongest and continuous over the whole width of the cell walls (Fig. 11). Some views on cells indicated that the fungal hyphae use the pits to enter from cell to cell (Fig. $11 \mathrm{~B}$ to $11 \mathrm{D}$ and $11 \mathrm{~F}$ to $11 \mathrm{H}$ ). Also within enlarged views of the wood sections, hyphae can be seen growing within the parenchymatic ray cells (Fig. 11A and 11E). Some hyphae appear to grow into the pits connecting the parenchymatic cells to the surrounding wood fiber cells, other hyphae grow along the cell walls of the ray
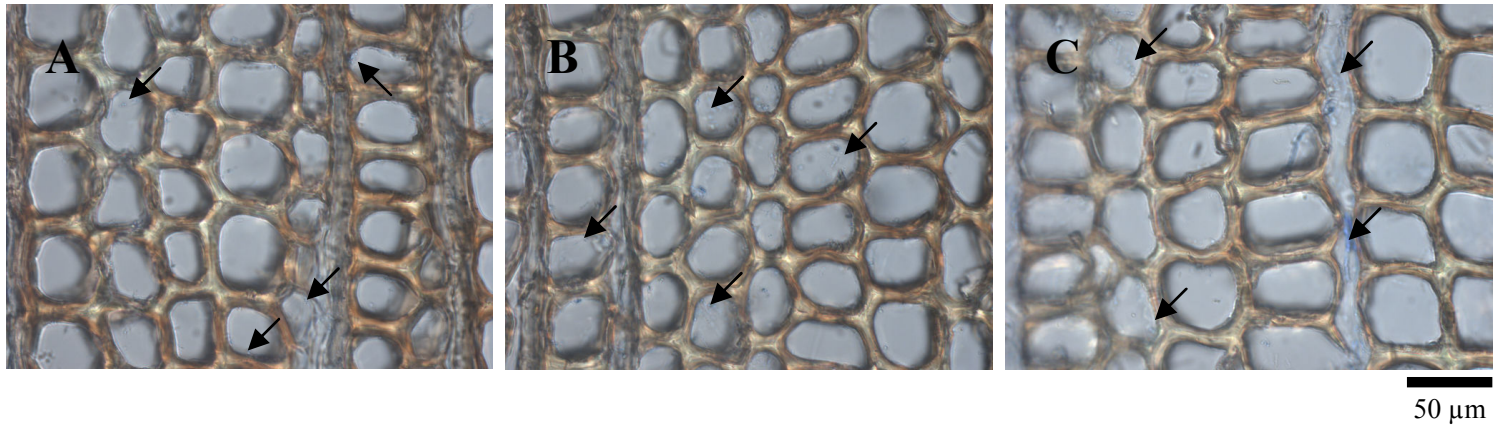

Fig. 9 Microtome sections of an A. grandis wood block (earlywood) incubated for 6 weeks with $T$. versicolor Strain 6, stained by lactophenol blue and $100 \mathrm{mM} \mathrm{MBTH}$, respectively, as described in Chapter II.3.3. A to $\mathbf{C}$ show different areas from the same section. Note the fungal hyphae (see arrows) in the lumina of wood fibers and in broken cells of the rays
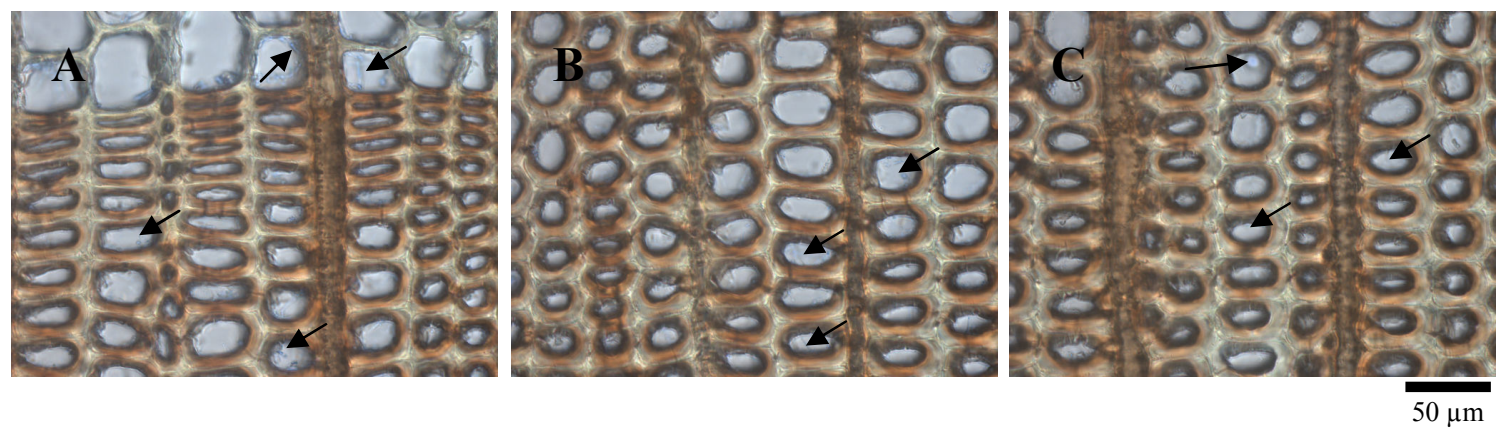

Fig. 10 Microtome sections of an A. grandis wood block (A area at the year ring's border showing mostly latewood and two lines of earlywood; $\mathbf{B}$ and $\mathbf{C}$ areas of only latewood) incubated for 6 weeks with $T$. versicolor Strain 6 , stained by lactophenol blue and $100 \mathrm{mM} \mathrm{MBTH}$, respectively, as described in Chapter II.3.3. Note the fungal hyphae (see arrows) in the cell lumina 

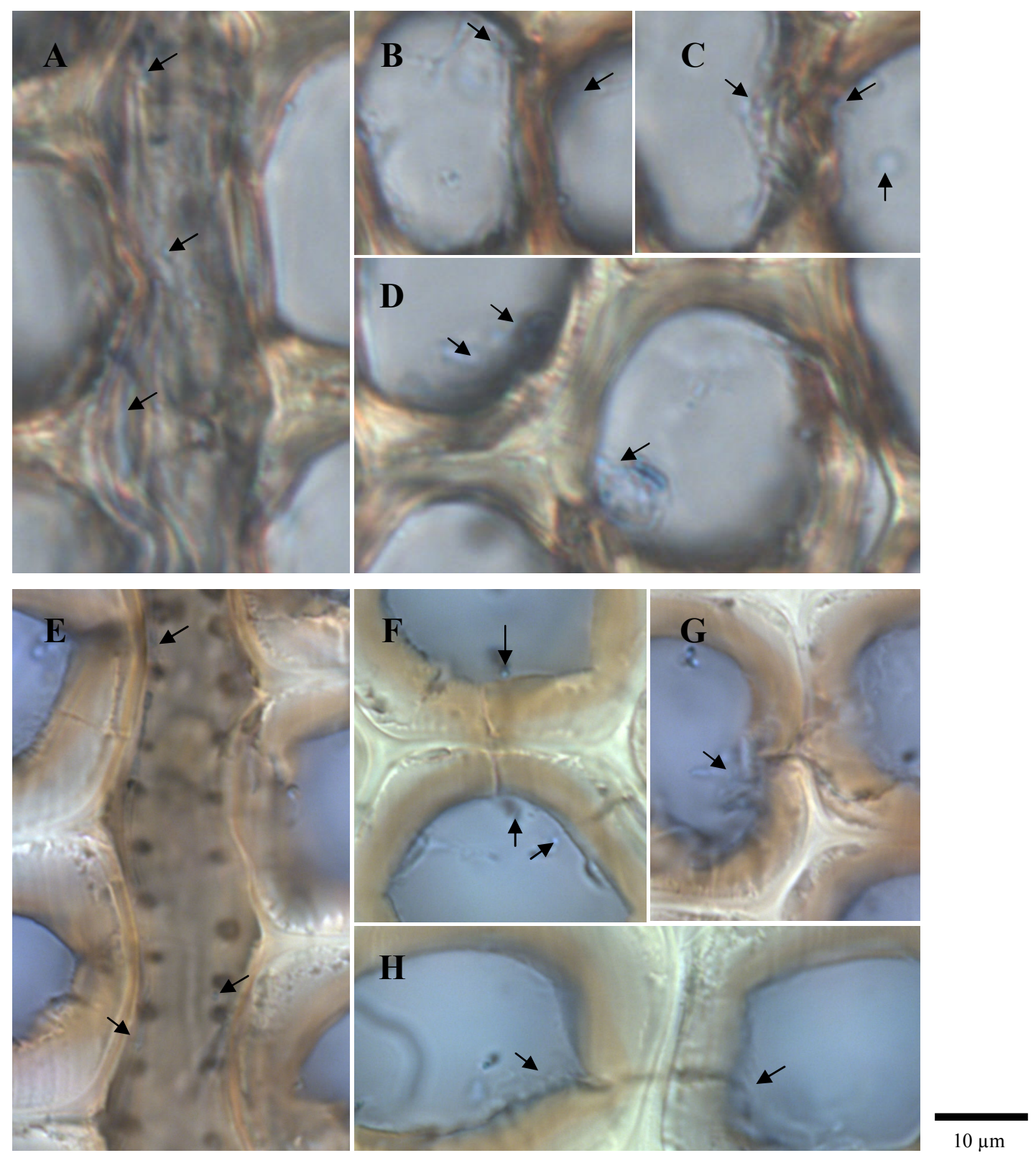

Fig. 11 Enlarged views on microtome sections of $A$. grandis wood blocks incubated for 6 weeks with $T$. versicolor Strain 6 after staining with lactophenol blue and $100 \mathrm{mM} \mathrm{MBTH}$, respectively. A) Ray cells and B) to D) wood fiber cells and pits of earlywood. E) Ray cells and F) to $\mathbf{H}$ ) wood cells and pits of latewood. Arrows indicate growth of fungal hyphae through the rays (A and $\mathbf{B}$ ) or growth of fungal hyphae through the pits ( $\mathbf{B}$ to $\mathbf{D}$ and $\mathbf{F}$ to $\mathbf{H}$ ) in the wood cells of the samples treated with the fungus

cells. At these places, the thin ray cell walls are comparably strongly stained by MBTH, of intensities stronger than those of the inner parts of the neighboring lignified, much thicker cell walls of the wood fibers. 
Sometimes, the decay may already be so advanced that staining by MBTH was possibly not anymore successful, as suggested for example from the left third of the photo of earlywood incubated for 6 weeks with T. versicolor Strain 6 in Fig. 9C, showing cells with weakened cell walls hard to focus on. In many other instances, however, two adjacent cell walls were only stained at the inner layers and not fully throughout the whole cell walls (Fig. 9). In the latewood, in the cell walls of some regions of the tissue, there is only a beginning of staining of the most inner layer of the cell walls of wood fibers, documenting nicely that the fungus attacks the comparably thick cell walls from the inside towards the outside (Fig. 7D and Fig. 10). Although the overall staining of the wood cell walls in total sum was apparently often much stronger in the latewood than in the earlywood at six weeks of incubation with $T$. versicolor Strain 6, a throughout staining of adjacent cell walls of two neighboring cells was still an exception (Fig. 10). Roughly about half of the cell walls of fibers in earlywood and $1 / 2$ to $2 / 3$ of the cell walls of fibers in latewood presented MBTH-stained reddish parts, indicating the ongoing decay over their width. When measuring the width of cell walls of wood treated for 6 weeks with T. versicolor Strain 6 from each 30 independent wood fibers, $2.66 \pm 0.66 \mu \mathrm{m}$ was determined for the average width of cell walls of the earlywood cells and $6.20 \pm 1.33 \mu \mathrm{m}$ for the average width of cell wall of fibers in the latewood, respectively, reflecting a total lower area of staining even if the whole cell walls would have been afflicted. The thickness of wood cell walls was also calculated from $n=30$ different wood fibers from earlywood and $n=30$ different wood fibers from latewood of wood samples not treated with the fungus, respectively. Overall, intact earlywood cell walls measured $2.17 \pm 0.24 \mu \mathrm{m}$ in average width, intact cell walls of latewood from wood not infected with the fungus cover in average about $6.93 \pm 0.66 \mu \mathrm{m}$ in width. Generally, the standard deviations were larger in the measurements for the fungal treated wood as compared to the samples from wood not treated with a fungus. Partially, this might be due to the less sharp contrast of the cell wall borders in the photos of fungal treated wood samples.

ANOVA analysis suggested the values from earlywood and the values from latewood, respectively, to be statistically significant different from each other. However, only two randomly chosen wood samples were compared to each other and the detected differences might rather reflect the differences in individual growth of cells at different regions in a year ring of a tree than effects by the fungus on the cell walls of the infested samples, particularly also since the width measured for the earlywood 
fibers in fungal treated wood was larger than the value obtained for the control sample (for better discussion see supplementary material).

Fig. 12 and 13 show decay of A. grandis wood by T. versicolor Strain 6 in microtome sections stained with lactophenol blue and MBTH after 6 weeks, 12 weeks and 19 weeks of incubation, respectively. Compared to the comparably intact samples of 6 weeks of incubation with the fungus, only stained wood debris of the sections made from wood blocks of 12 and 19 weeks of incubation with the fungus can be shown (Fig. 12). However, it is important to note that MBTH staining in the earlywood of the samples of longer incubation was neglectable compared to that of the earlywood from the sample incubated for six weeks with the fungus (Fig. 12A to 12C). In the less degraded latewood of the older samples, MBTH staining occurred as indicated by a reddish coloration but generally at a lower level as compared to the wood incubated for only 6 weeks with the fungus (Fig. 12D to 12F).

$$
6^{\text {th }} \text { week }
$$


$12^{\text {th }}$ week
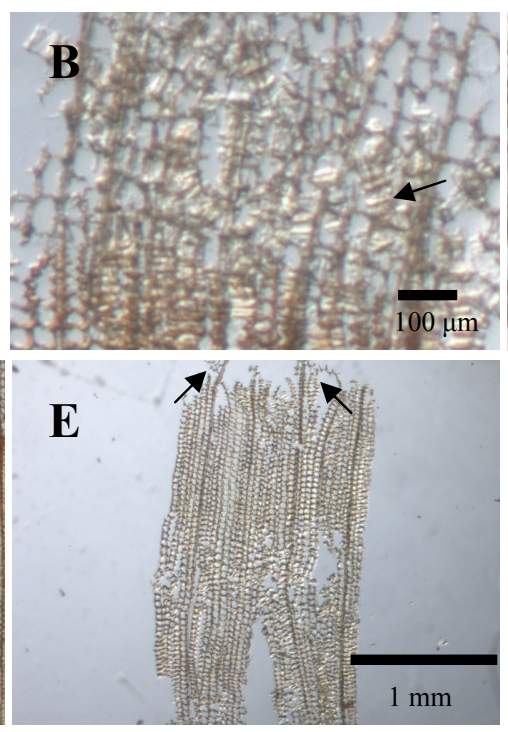

$19^{\text {th }}$ week
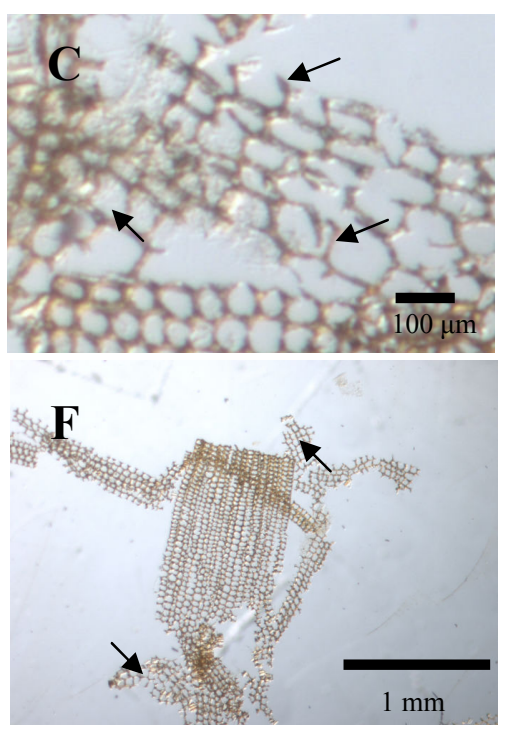

Fig. 12 Appearance of microtome sections of A), B) and C) earlywood and D), E) and F) of latewood of $A$. grandis wood blocks after 6 weeks, 12 weeks and 19 weeks of incubation at $22^{\circ} \mathrm{C}$ and $65 \% \mathrm{RH}$ on $5 \%$ malt $2 \%$ agar with $T$. versicolor Strain 6 and after staining with lactophenol blue and MBTH as described in Chapter II.3.3. In case of 12 weeks and 19 weeks of incubation with $T$. versicolor Strain 6, overviews on whole year rings were not possible to show because of the strong damage of the wood cells as caused by the fungus. The earlywood was so much destroyed that only some broken parts were left (pointed to by arrows). Only in the latewood regions, the tissue structure was still to some extend reasonably maintained. The time of incubation is indicated above each row of wood sections 


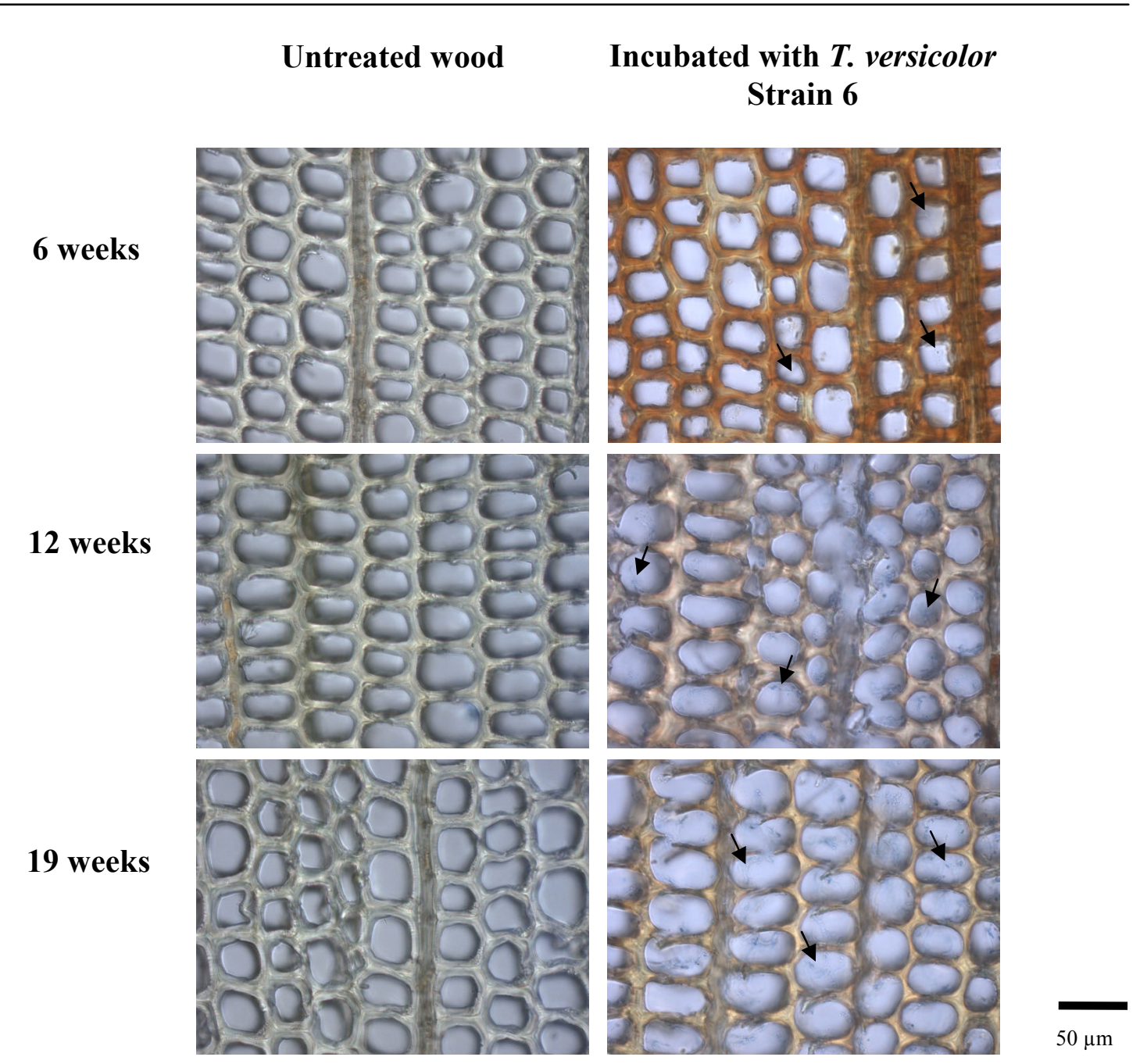

Fig. 13 Enlarged views on latewood in microtome sections of $A$. grandis wood blocks incubated without fungus (left column), or with T. versicolor Strain 6 (right column) after staining with lactophenol blue and $100 \mathrm{mM} \mathrm{MBTH}$, respectively. Arrows indicate fungal hyphae in the cell lumina of the samples treated with the fungus. The time of incubation is indicated at the left of rows of wood sections

\section{III.4.3 Image analysis of MBTH-stained wood after 6 weeks incubation with}

\section{T. versicolor Strain 6}

To follow up the distribution of successful MBTH staining over a wood section (a complete year ring) in more detail, individual digital images of $400 \mu \mathrm{m}$ (vertical) x $550 \mu \mathrm{m}$ (horizontal) were taken from microtome sections of a wood block incubated for 6 weeks with $T$. versicolor Strain 6 that were observed under a light microscope Zeiss Axioplan (Zeiss, Jena, Germany) at a magnification of 20X. From one year ring border to the next, in a series of photos, image for image were taken without any space 
in between (in total 16 photos), thereby following up 15 different series of wood cells being interrupted by two rays of wood parenchyma, respectively (Fig. 14A, 14B and 14D).

Each photo was transferred into the Adobe Photoshop version 6.0, where cell lumina as well as the complete rays of wood parenchyma were marked and eliminated with the erase tool function, resulting in white areas instead. The modified photos were next transferred into the Mathematica program and converted by using the Mathematica commands colorData and greyData into grey-scale images. These images were further converted with the threshold command into black and white diagrams (Fig. 14B and 15B). Thereby, a threshold value of 140 was applied in order to emphasize on the MBTH-stained cell wall areas. In the resulting black and white diagrams, the black staining referred to stained cell wall regions, white to the cell lumina, to ray parenchyma and to unstained cell wall regions. Next in the Mathematica program, the black and white pixels were calculated individually in image for image. For every image, the relations of amounts of black pixels to amounts of white pixels were determined and the results are presented in \% in the column "Stained cell wall area" in Table S1 in the supplementary material. Alike values were calculated when doing the analysis in the image software analySIS ${ }^{\circledR}$ program with the original photos using the parameters as given in the section III.3.4 (see Table S2 in the supplementary material for the individual results).

For better documentation, all images as modified in the Mathematica program were assembled in a jigsaw manner into a continuous diagram (Fig. 14D and 14E). Next, the area fraction of stained cell walls (described in III.3.4) was calculated for the whole analyzed year ring (in total $6.2 \mathrm{~mm}$ in width) in sections of $1 \mathrm{~mm}$ in length and $550 \mu \mathrm{m}$ in width, starting from the border of latewood to earlywood of the next year ring down to the earlywood flanking the previous year ring border (see Table S2 and S3). Generally, the area fraction of stained cell walls was lower at the earlywood region than at the latewood region. The lowest value of stained cell wall fraction per analyzed $\mathrm{mm}$ of wood area was $17.39 \%$ found in the earlywood of the section and the highest value of $44.81 \%$ was found at the latewood bordering the next year's earlywood (Fig. 14E). As shown by the calculated trendline in Fig. 16, the area fraction of stained cell walls increased in a slightly sigmoid fashion from earlywood to latewood. When comparing the position of the inflexion point $\left(A_{S}=28.35 \%\right.$, as shown in Fig. 16 at a relative distance $\mathrm{S}$ of $0.52=3.18 \mathrm{~mm}$ from the year ring border from the start of 


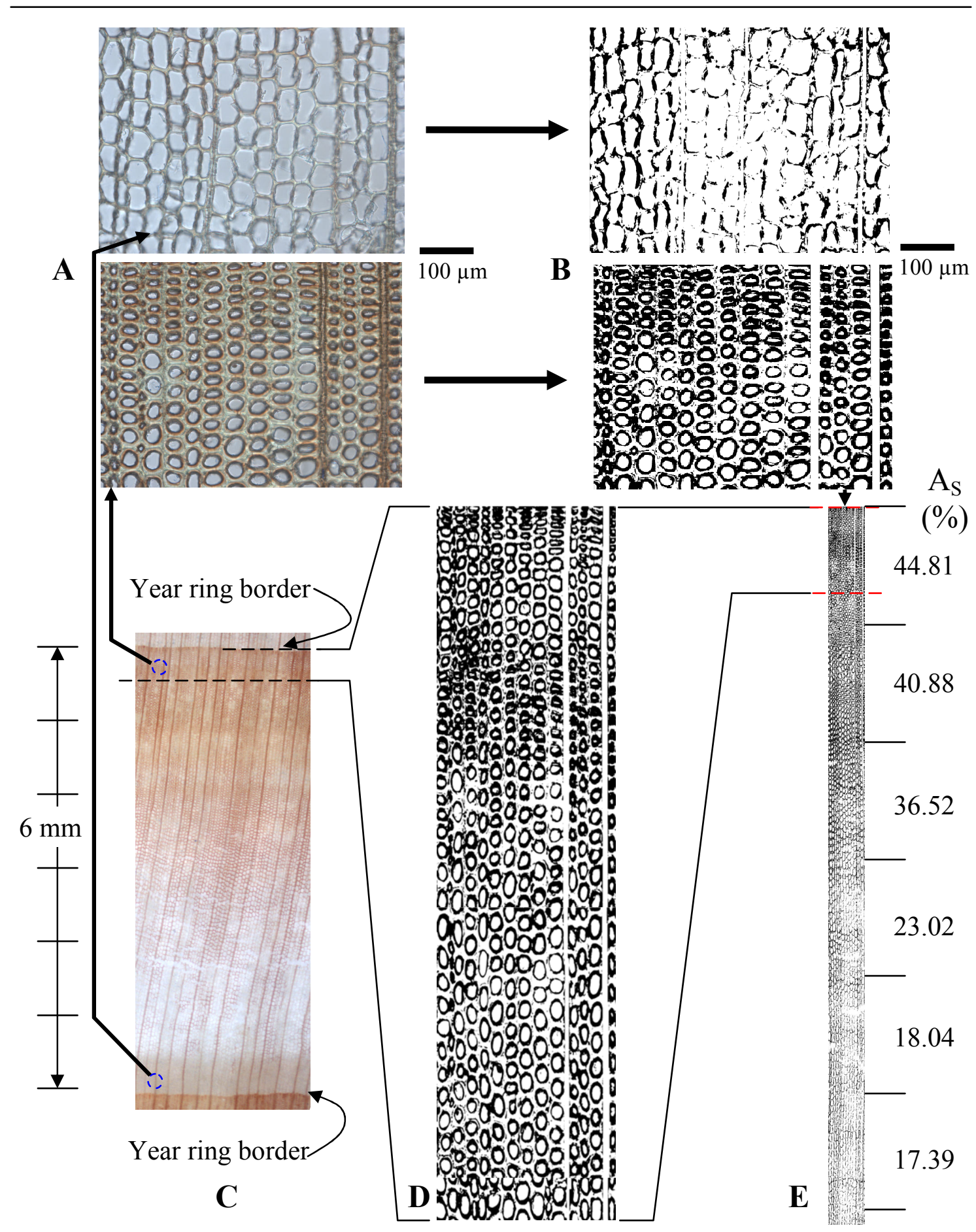

Fig. 14 Microtome sections of an A. grandis wood block incubated for 6 weeks with T. versicolor Strain 6, stained by lactophenol blue and $100 \mathrm{mM} \mathrm{MBTH}$, respectively, as described in Chapter II.3.3. A) Color picture of an area of a microtome section taken by a digital CCD camera, B) black and white picture of the same area after modification in Adobe Photoshop and evaluation with a threshold of 140 with the Mathematica program, C) overview of a microtome section analyzed from year ring border to year ring border, and D) individual diagram of a latewood region as obtained after conversion of individual photos in the Mathematica program from assembling 3 consecutive individual images together and $\mathbf{E}$ ) continuous diagram of in total 16 images as obtained from assembling them into one unit after conversion with Mathematica program. AS in $\mathbf{E}$ refers to the total area of the cell walls stained by MBTH per total section area 
A
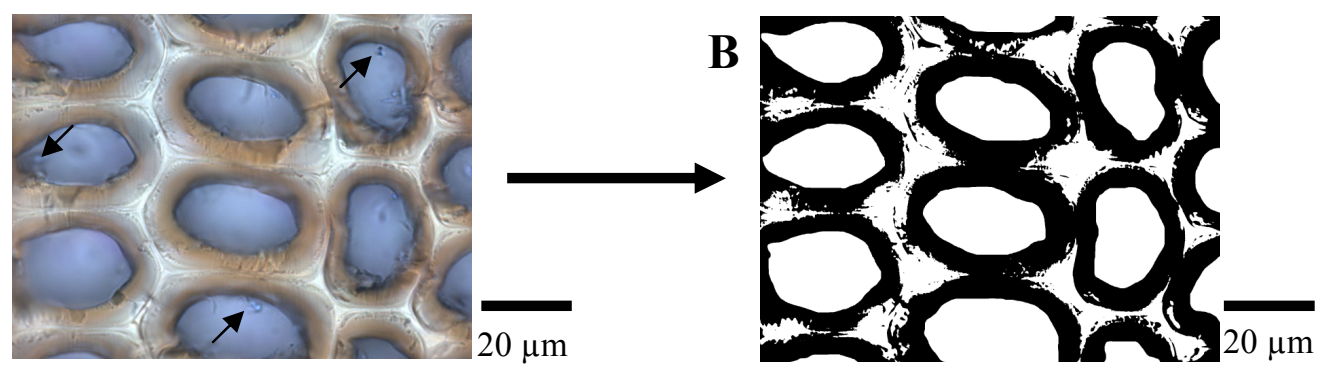

Fig. 15 Enlarged view of a microtome section of an $A$. grandis wood block incubated for 6 weeks with $T$. versicolor Strain 6 , stained by lactophenol blue and $100 \mathrm{mM}$ MBTH, respectively, as described in Chapter II.3.3. A) Enlargement of a color picture of latewood taken by a digital CCD camera, B) black and white picture of $\mathbf{A}$ after conversion with the Mathematica program. Arrows indicate the fungal hyphae in the cell lumina of the samples treated with the fungus

earlywood production) with the distribution of wood tissue in the decayed year ring (Fig. 14A), it corresponded relatively well to the change from earlywood to latewood judged to be at about $3.4 \mathrm{~mm}$ distance (at a relative distance $\mathrm{S}$ of 0.548 as shown in Fig. 16) from the start of earlywood formation (Fig. S5).

As can clearly be seen in Fig. 14A and 14B and in Fig. 15 from the enlarged views of the photos and the converted images of earlywood and latewood of the fungal incubated wood, cell walls were not necessarily completely stained. In most instances, the inner parts of the cell walls were stained but staining usually did not cover the whole width of the cell walls. To study the area fraction of stained cell walls in comparison of the total cell wall areas relative to each other in the earlywood and in the latewood region, respectively, first the complete cell wall areas were calculated with the image analySIS ${ }^{\circledR}$ program as described under III.3.4, using the 16 photos as taken in series from the year ring from the earlywood to the latewood. The obtained individual values $\left(\mathrm{A}_{\mathrm{T}}\right)$ are given in the column "Total cell wall area" in Table S3 in the supplementary material. The area fraction of total cell walls was also found to increase in a slightly sigmoid form from earlywood to latewood, similar as the area fraction of stained cell walls. Also, the position of the inflexion point at $\mathrm{A}_{\mathrm{S}}=28.35 \%$ and the relative distance $\mathrm{S}$ of 0.52 from the start of earlywood production was comparable (Fig. 16). Lowest cell wall areas occurred in the earlywood with $41-43 \%$ of a total section area and highest values in the latewood with $64-65 \%$ of a total section area. These total cell wall areas in principle corresponded to those of a year ring section from 
a wood block incubated for 6 weeks without a fungus (see Table S4 and Fig. S5 and as well Table S5 and S6 and the further discussion in the supplementary material), indicating again that the decay by $T$. versicolor Strain 6 only just started at 6 weeks of incubation. When calculating the quotients stained cell wall areas by total cell wall areas, interestingly for the T. versicolor Strain 6 treated samples, a linear trendline appeared increasing from earlywood to latewood (Fig. 16). As previously estimated in Chapter III.4.3, about 41 to $48 \%$ to cell wall area was stained in the earlywood (roughly a half of the complete cell wall area) and $60-70 \%$ in the latewood area (up to $2 / 3$ of the total cell wall area).

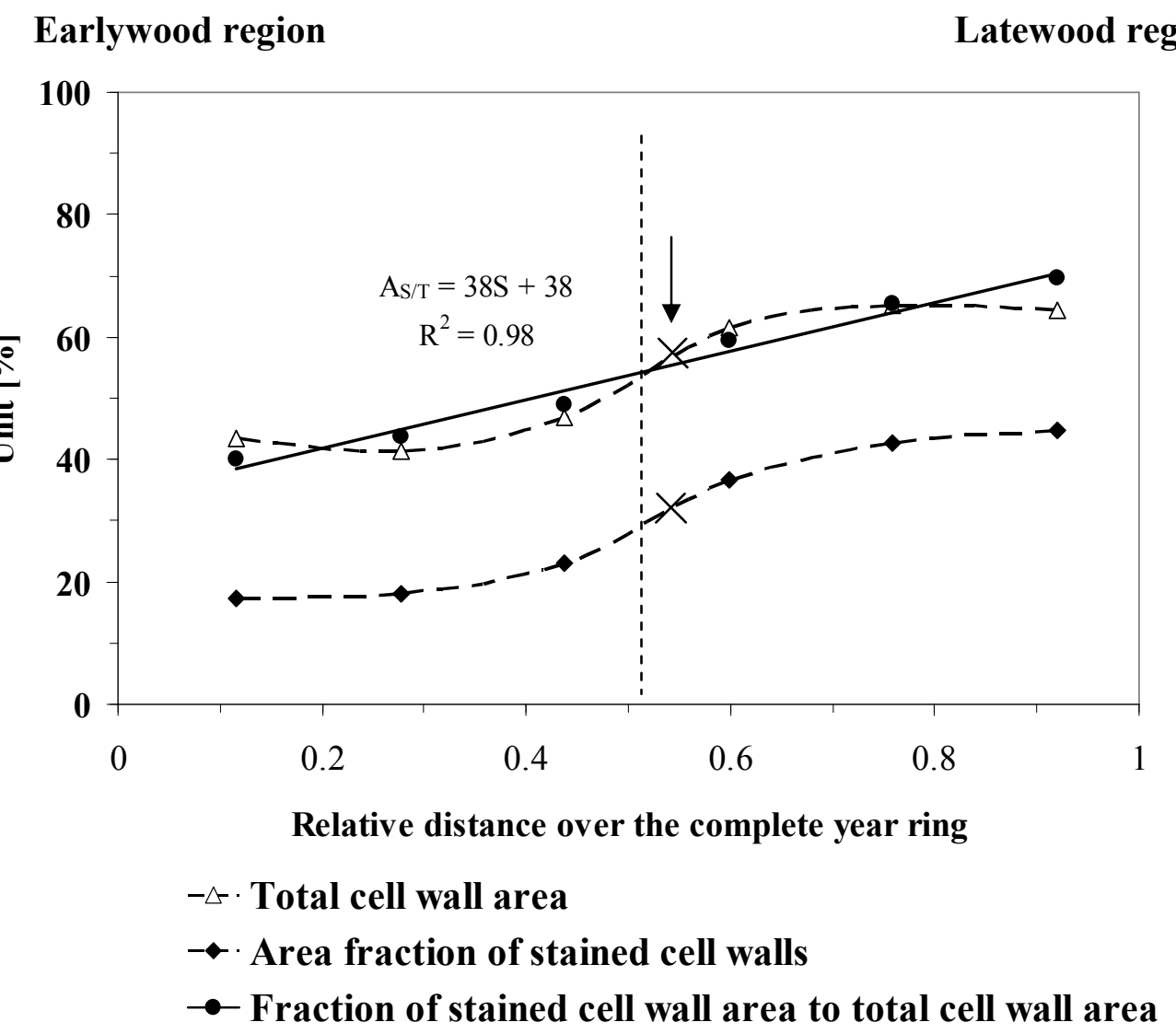

Fig. 16 Total cell wall areas $\left(A_{T}\right)$, the area fractions of stained cell wall area $\left(A_{S}\right)$ and the fractions of stained cell wall area to total cell wall area $\left(\mathrm{A}_{\mathrm{S} / \mathrm{T}}\right)$ as described over a complete year ring from earlywood to latewood within an $A$. grandis wood block after 6 weeks of incubation with T. versicolor Strain 6 at $22^{\circ} \mathrm{C}$ and $65 \% \mathrm{RH}$ on $5 \%$ malt $2 \%$ agar (compare Fig. 13 for image analysis). The inflexion point of the curves is indicated by a vertical dash line and the point of change from growth of earlywood to growth of latewood is presented by crosses $X$ and by a vertical arrow 


\section{III.4.4 Effects of fungal oxidative enzymes on wood lignin modification}

Incubation of $A$. grandis wood with white-rot fungi lead to decay of compounds in the cell walls that caused a reddish color upon staining with MBTH. MBTH is expected to react with quinones in the cell walls and these are expected to be generated by modification of the lignin in the cell walls by actions of enzymes secreted by fungi. In order to proof such postulated enzymatic actions and modifications on cell walls, wood sections were treated with purified laccase with and without a mediator compound (HBT) which should enhance the expected enzymatic reaction on modified lignin. In agreement, laccase without mediator showed low effects, as visualized by the low reddish color obtained upon the MBTH-staining (compare the laccase treated wood to the untreated wood in Fig. 17). In contrast, laccase plus mediator HBT modified wood cell walls with highest effectiveness, whilst the mediator alone was not effective (Fig. 17).

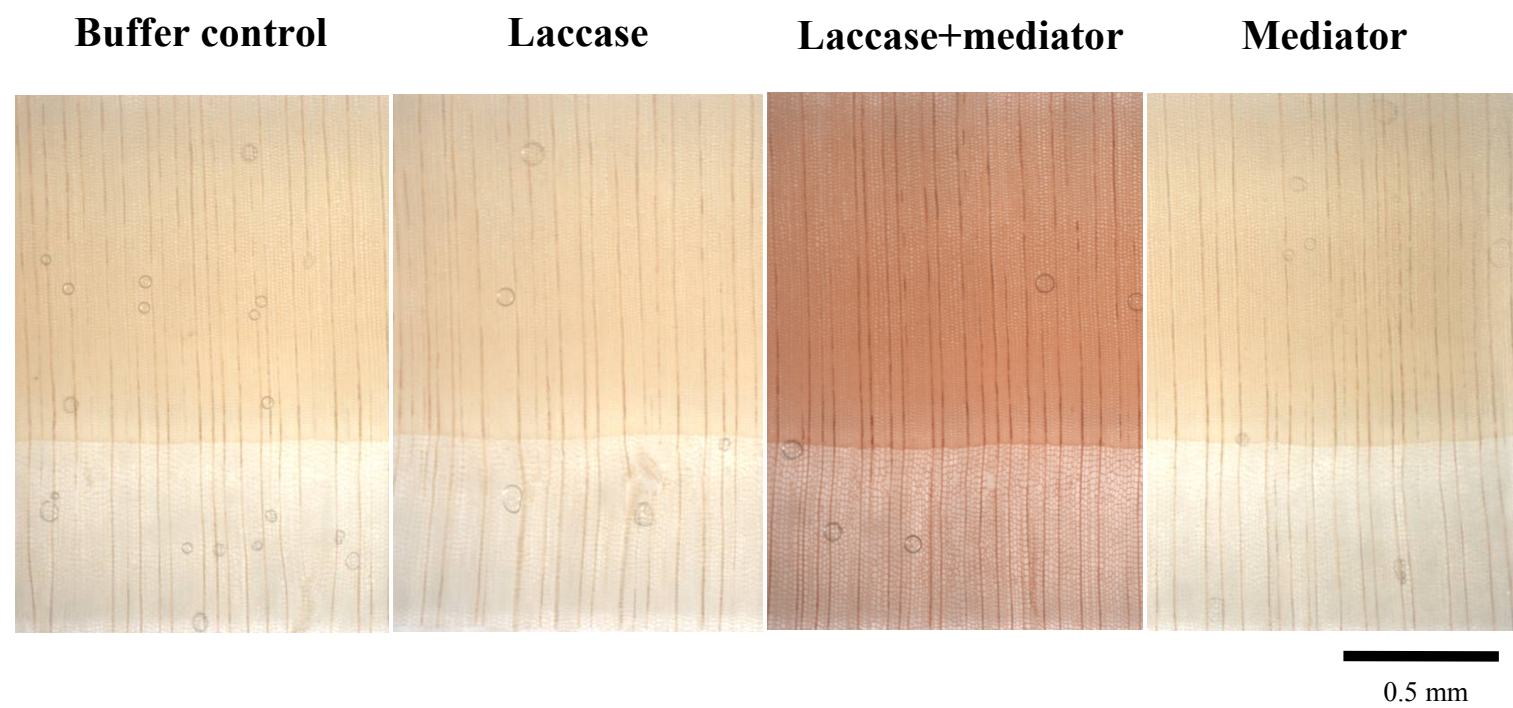

Fig. 17 Microtome sections of an A. grandis wood block incubated (from left to right) for 30 min with $100 \mathrm{mM}$ sodium lactate buffer, with $100 \mathrm{mM}$ sodium lactate buffer with $10 \mathrm{U}^{-1}$ purified $T$. versicolor Strain 6 laccase, with $10 \mathrm{U}^{-1}$ purified $T$. versicolor Strain 6 laccase $+1 \mathrm{mM}$ HBT mediator, and with $1 \mathrm{mM}$ HBT mediator. All samples were subsequently stained by $100 \mathrm{mM} \mathrm{MBTH}$. The types of wood treatment are indicated above the wood sections 


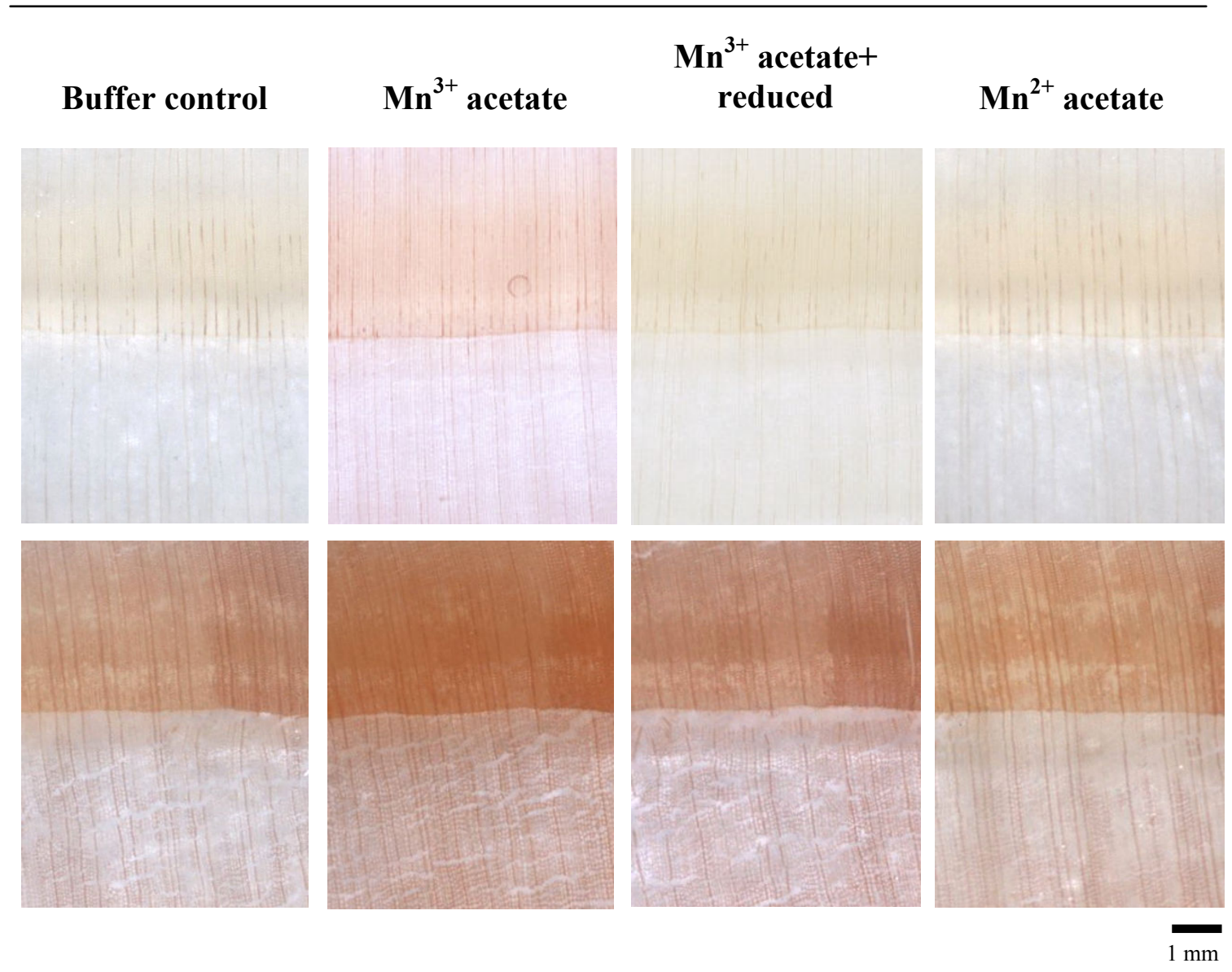

Fig. 18 Microtome sections of $A$. grandis wood blocks incubated without fungus (upper row) and incubated for six weeks with $T$. versicolor Strain 6 (lower row). Wood sections were treated (from left to right) with $100 \mathrm{mM}$ sodium lactate buffer, with $\mathrm{Mn}^{3+}$-acetate, $\mathrm{Mn}^{3+}$-acetate followed by reduction with $50 \mathrm{mM} \mathrm{Na}\left(\mathrm{BH}_{4}\right)$ in dry methanol, and with $\mathrm{Mn}^{2+}$-acetate in $100 \mathrm{mM}$ sodium lactate buffer, $\mathrm{pH}$ 7. All wood sections were stained by $100 \mathrm{mM} \mathrm{MBTH}$. The types of wood treatment are indicated above each row of wood sections

In another set of experiments, sections of wood were treated with active $\mathrm{Mn}^{3+}$ acetate to simulate the actions of manganese-dependent peroxidase or inactive $\mathrm{Mn}^{2+}$ acetate for a control. Parts of $\mathrm{Mn}^{3+}$ acetate-treated samples were reduced after the treatment for further control of $\mathrm{Mn}^{3+}$ action. Both untreated A. grandis wood sections was tested as well as sections of wood incubated for six weeks with T. versicolor. All samples were stained with MBTH to indicate possible lignin oxidation or modification. $\mathrm{Mn}^{3+}$ apparently reacted with wood having not been in touch with the fungus: upon MBTH-staining, there was a reddish color in the wood (Fig. 18). Including the reduction step in between the treatment by $\mathrm{Mn}^{3+}$ and by MBTH gave only a slight reddish color (Fig. 18). In contrast, $\mathrm{Mn}^{2+}$ was not effective on the wood for subsequent 
successful MBTH staining (Fig. 18). MBTH-staining was successful with all samples treated for 6 weeks with the fungus T. versicolor Strain 6. However, there were differences in the degree of the staining. In the buffer control and in the sample treated with $\mathrm{Mn}^{2+}$, there was a medium reddish staining of the wood (Fig. 18) which results from the former incubation with the fungus (compare Fig. 17 and 18). When treating the wood with $\mathrm{Mn}^{3+}$, the wood tissues were very strongly stained. The degree of staining was intermediate when in between $\mathrm{Mn}^{3+}$ and MBTH-treatments a reduction step was included (Fig. 18).

The results shown in Fig. 17 and 18 proof the direct (laccase) or indirect (MnP) action of fungal enzymes on modification of wood cell walls. However, in order to support this even further, in order to simulate enzymes secreted by fungi into the wood cell wall, chemical injections into the cell of wood blocks were performed (Fig. 19). Wood blocks (one per treatment) were injected either with laccase/mediator or $\mathrm{Mn}^{3+}$ solutions in order to investigate their effects on the oxidation or modification of lignin. An A. grandis wood block injected with only buffer served as control. For better comparison, wood treated for 6 weeks with T. versicolor Strain 6 was also included as a control for degrees of MBTH staining.

In no case of wood subjected to injection of a solution, the intensity of the reddish staining by MBTH reached that of the wood treated for 6 weeks with the T. versicolor Strain 6. However, upon MBTH-staining, wood blocks treated with laccase showed light reddish staining in the inner part of the fiber cell walls (Fig. 19). A difference made the type of mediator used: staining was slightly better with HBT than with HBA (Fig. 19). Also treatment with $\mathrm{Mn}^{3+}$ lead to a reddish colorization in the wood cell walls, with the higher applied concentration $(10 \mathrm{mM})$ giving the better results (Fig. 19). Striking is that in the $\mathrm{Mn}^{3+}$ treatments, the reddish color distributed more over the widths of the cell walls as compared to the wood cells treated with laccase and mediators (compare Fig. 19C and 19D with 19E and 19F). By the small molecule size (ion: $1.61 \AA$ in diameter; in the chelated form such as $\mathrm{Mn}^{3+}$-acetate, the size of the molecule is about $3 \AA$, see Force et al. 1997; Pederson and Khanna 1999), $\mathrm{Mn}^{3+}$-ions can easily penetrate the cell walls (pore radius size of 15-30 $\AA$, see Evan and Hedger 2008) whereas the larger enzymes (67 kDa of laccase III from T. versicolor Strain 6 , see Dwivedi 2006; Johansson and Nyman 1996) are blocked from diffusion into the cell walls and they can only attack the inner surface of the cell walls. Altogether, the results strongly support that the oxidative enzymes secreted by the white-rot $T$. versicolor 
Strain 6 or their products can modify lignin within the wood cell walls when the fungus grows in the wood.

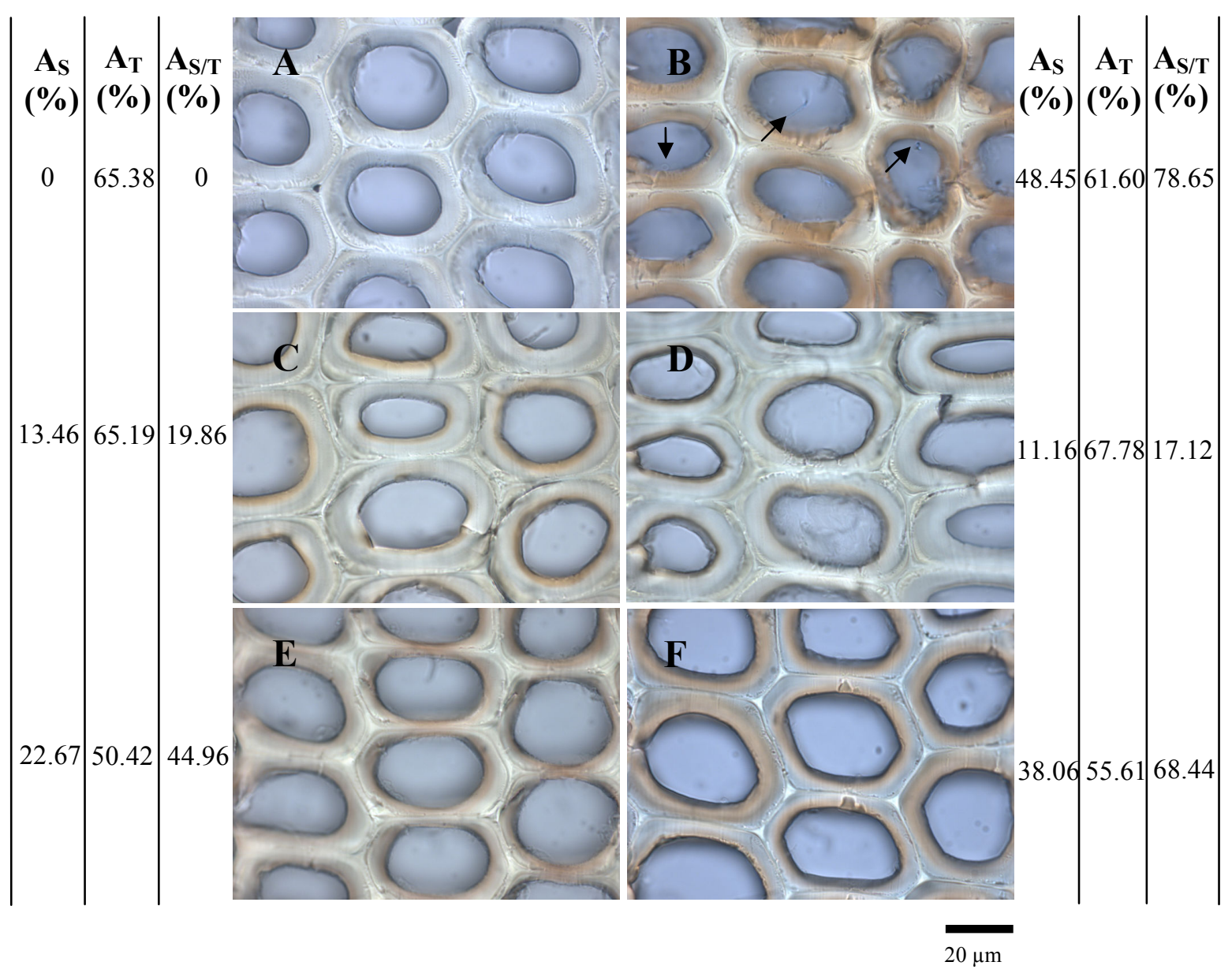

Fig. 19 Enlarged views of microtome sections of $A$. grandis wood blocks A) infiltrated with buffer, or B) incubated for six weeks with $T$. versicolor Strain 6, C, D), wood infiltrated with laccase/mediator: HBT and HBA, respectively and E, F) wood infiltrated with $\mathrm{Mn}^{3+}$-acetate in concentrations of $4 \mathrm{mM}$ and $10 \mathrm{mM}$, respectively. All wood sections were stained by $100 \mathrm{mM} \mathrm{MBTH}$. Total cell wall areas $\left(\mathrm{A}_{\mathrm{T}}\right)$, total stained cell wall area $\left(A_{S}\right)$ and the fractions of stained cell wall area to total cell wall area $\left(A_{S / T}\right)$ in the samples were calculated with the image analySIS ${ }^{\circledR}$ program and put in relation to each other. Note the microtome section of the wood block incubated with $T$. versicolor Strain 6 was also stained by lactophenol blue. Arrows indicate the fungal hyphae in the cell lumina of the samples treated with fungus 


\section{III.5 Discussion}

\section{III.5.1 Mass loss and cell wall modification of $\boldsymbol{A}$. grandis wood after inoculation with $T$. versicolor Strain 6}

Laccase and particularly manganese peroxidase secreted by white-rot fungi such as T. versicolor appear to attack and degrade lignin in the wood (Hiscox et al. 2010; Johansson and Nyman 1996). Several research groups reported on manganese peroxidase obtained from T. versicolor strains able to oxidize $\mathrm{Mn}^{2+}$ to $\mathrm{Mn}^{3+}$, phenols, amine, dyes and more aromatic alcohols. The physiological function of the enzyme in wood decay could be to produce $\mathrm{Mn}^{3+}$-chelates, for the $\mathrm{Mn}^{3+}$-mediated conversion of different phenols and especially also lignin, in addition to direct interaction with the lignin (Johansson et al. 1996; Lundell et al. 2010). Laccases have the ability to catalyze the oxidation of unspecific phenolic compounds and, in the presence of charge-transfer mediators, also the oxidation of non-phenolic compounds. Also fungal laccases including the $T$. versicolor laccases have been repeatedly connected to lignin degradation (Baldrian 2006). To follow these different enzymatic actions on lignin up in decay of $A$. grandis wood by $T$. versicolor, in this study wood degraded by the fungus and wood treated with either isolated T. versicolor laccase or with $\mathrm{Mn}^{3+}$ as the reactive product of $\mathrm{Mn}$-dependent peroxidase was stained with $\mathrm{MBTH}$, a reagent forming pink adducts with oxidized quinone (Rodríguez-López et al. 2004; Fig. 20). Oxidized quinones are amongst the products expected to be obtained from lignin by enzymatic reaction by laccase (Champagne et al. 2005) and by chelated $\mathrm{Mn}^{3+}$ as an oxidative mediator (Mester and Field 1998). Different from the large laccases (laccase III of T. versicolor is about $67 \mathrm{kDa}$; Dwivedi 2006), the small $\mathrm{Mn}^{+3}$-chelates $\left(\mathrm{Mn}^{3+}\right.$ is about $1.61 \AA$ in diameter, $\mathrm{Mn}^{3+}$-acetate is about $3 \AA$ in diameter) easily penetrate cell walls (Blanchette 1984; Evan et al. 1994) and thus it reaches also easily the lignin in the inner cell wall layers.

In this study, first $A$. grandis wood was incubated with T. versicolor Strain 6 for 6, 12 and 19 weeks, respectively. Afterwards, wood blocks were sliced with a microtome and wood sections were stained with MBTH. Longer fungal incubation made the A. grandis wood less responsive for MBTH-staining, likely due to a quite progressed decay of the lignin in the cell walls. At 12 weeks of incubation and even more at 19 weeks of incubation, the microtome sections of the wood blocks were 


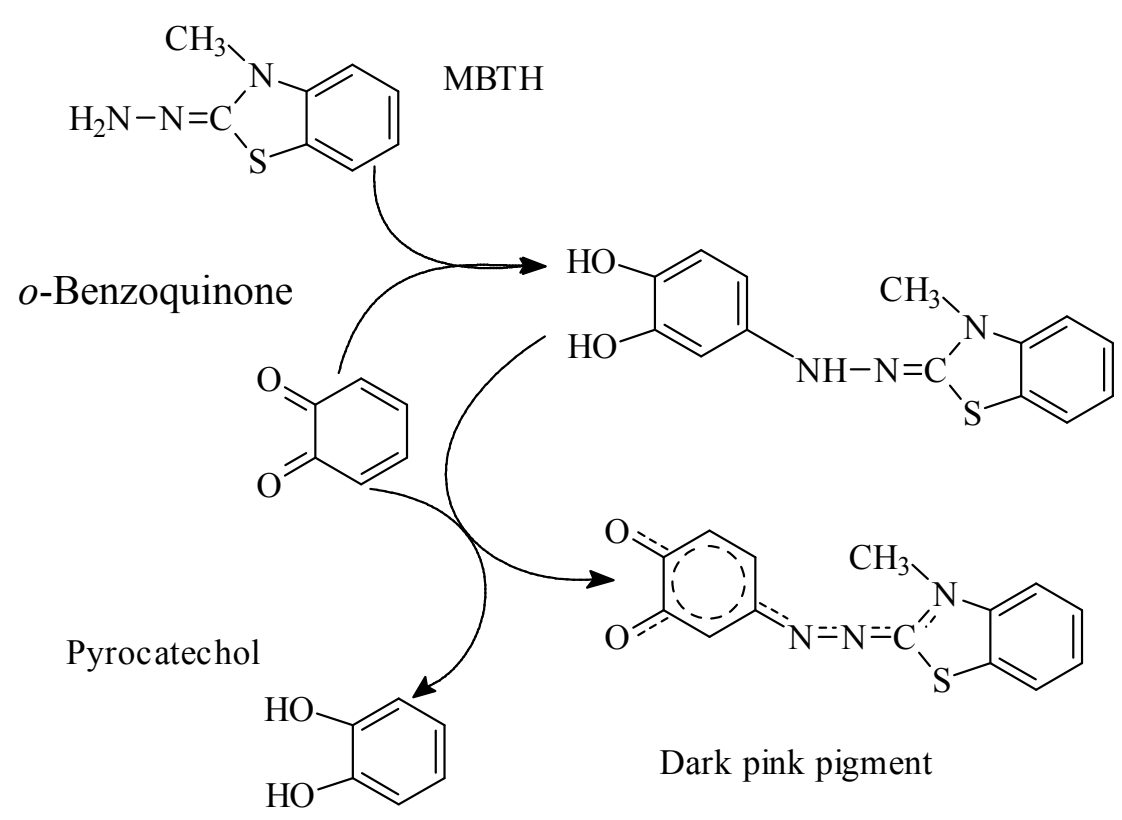

Fig. 20 Scheme of MBTH and reaction scheme using $o$-quinone as substrate to form a dark pink pigment

relatively brittle and the sections broke into smaller pieces. The secondary layers of cell walls were partially or fully consumed in the longer incubated samples (Fig. 12 and 13), leaving not much material that could react with MBTH. Accordingly, some staining was still seen in samples incubated for 12 weeks with the fungus and very little staining in the samples incubated for 19 weeks with the fungus. In contrast, highest intensity was achieved in the samples incubated for six weeks with the fungus and these samples were therefore chosen to study fungal decay by using MBTH staining in more detail (see Chapter III.5.2).

Generally in the fungal decay, this study shows that the degree of MBTH-staining reflects the degree of mass loss. In the beginning, low level mass loss $(6 \%)$ as detected after 6 weeks of incubation with $T$. versicolor Strain 6 was accompanied with strong reddish staining of wood cell walls (Fig. 13). Increasing mass loss $(18 \%)$ reduced the ability of wood cell walls for staining with MBTH such as seen in the samples incubated for 12 weeks with the fungus (Fig. 13). Finally, when mass loss $(26 \%)$ was severe after 19 weeks of incubation and the secondary cell walls to large amounts were decayed, no MBTH-staining was achieved (Fig. 13).

Within a wood sample, one can also distinguish between reactions in the earlywood and in the latewood (Fig. 14). Staining was higher in latewood than in 
earlywood; this related to the generally higher amount of cell wall material in the latewood but also to the higher relative amount of cell wall material that was stained (Fig. 14 and Table S1). This effect of more staining of cell wall material furthermore could be caused by a different percentage of lignin in the earlywood cell walls as compared to the lignin in the latewood cell walls.

Fengel $(1969,1970)$ calculated the distribution of lignin in the cell wall layers of conifer spruce tracheids. The reports described that a higher proportion of the compound middle lamella in earlywood is the reason for the higher percentage of lignin in this part of an annual ring. Species however vary in the lignin distribution over the annual growth rings. In hardwood such as Robinia pseudoacacia and Prunus americana, the lignin content of earlywood is higher than that of latewood but in Gleditsia triacanthos, an opposite situation was found (Orton et al. 2004). Müller (2008) recently studied the lignin content in A grandis wood by FTIR microscopy (Fourier transform infrared microscopy) and STEM-EDX (Scanning transmission electron microscope with energy dispersive X-ray spectroscopy). He found that in the latewood of $A$. grandis the lignin content was highest. Over a cross-section of neighboring walls however, highest lignin content sharply peaks to be middle lamella. In overviews of cross section image analysis of FTIR-pictures highest staining is indicated in the middle lamella in between two cells and in the corner regions where three neighboring cells meet. It might therefore be somewhat surprising that upon treatment with $T$. versicolor Strain 6 not the strongest staining occurred in the middle lamellas and in the corner areas of three adjacent fibers cells. Regularly, instead there was no staining (Fig. 13 and 15), in contrast to the staining of middle lamellas between rays and fiber cells (Fig. 13). From the photos of MBTH-staining, one must therefore conclude that the lignin in the middle lamella between fiber cells was shielded whereas such shield for the middle lamella separating wood fibers and ray cells shield is obviously missing. In consequence of this, middle lamella of ray cells are firstly decayed (Fig. 13) and this is followed by a first breakage of the whole wood tissue along the rays (Fig. 13) since middle lamella functions in gluing cells together (Fengel and Wegener 1984). Furthermore in this context, an high lignin content was detected by the study of Müller (2008) in the cell walls, respectively the middle lamellae of ray cells, whilst the thick S2 layers of neighboring wood fibers had a comparably low lignin content. This may very well explain the on the first view contrasting results of Müller (2008) stating that latewood has higher lignin content than earlywood and of those 
results shown in Fig. 5 presenting that earlywood of $A$. grandis has a relatively higher lignin content than samples of earlywood plus latewood. Müller (2008) in his FTIRmicroscopy study summarizes the relative lignin content as distributed over a defined area but not the relative amount of lignin in the total amount of chemical material present in the area. Thus, throughout the S2 layers of cell walls of latewood fibers the overall lignin content might be lower than throughout the S2 layers of the cell walls of earlywood fibers. Possibly this is responsible for the linear increase in MBTH-staining of amount of stained cell wall area per amount of total cell wall area observed in the wood section treated for 6 weeks with T. versicolor Strain 6 (Fig. 16).

Best seen from the samples of six weeks incubation with T. versicolor Strain 6 (Fig. 15), it is conceivable that cell wall decay in wood fibers start from the inner tertiary (S3) layer over the S2 layer towards the S1 layer to the primary cell wall and the middle lamella. MBTH-staining was strongest in the most inner layers of the cell walls and MBTH-staining of middle lamella were never detected, not even after the longer incubation of 19 weeks. This should indicate that the lignin in the middle lamella was not or only very late attacked after the barriers by the other cell wall layers were destroyed. In spruce, the different layers of the cell wall encompass the following thickness. Primary cell walls + a half of middle lamella are $0.09 \mu \mathrm{m}$ thick. In comparison, the S1 layers take $0.26 \mu \mathrm{m}$ (earlywood) and $0.38 \mu \mathrm{m}$ (latewood), the $\mathrm{S} 2$ layers are $1.66 \mu \mathrm{m}$ (earlywood) and $3.69 \mu \mathrm{m}$ (latewood) and the tertiary layers (also called S3) are $0.09 \mu \mathrm{m}$ in earlywood and $0.14 \mu \mathrm{m}$ in latewood, giving a total cell wall thickness of $2.10 \mu \mathrm{m}$ for earlywood and of $4.3 \mu \mathrm{m}$ for latewood (Fengel and Wegener 1984). In comparison in this study, the total cell walls of decayed A. grandis wood measured $2.17 \pm 0.24 \mu \mathrm{m}$ (earlywood) and 6.93 $\pm 0.66 \mu \mathrm{m}$ (latewood), respectively. Whilst the width of the individual layers was not determined, the majority of the cell wall material is obviously represented by the S2 layers of the cell walls. Interestingly in conifers such as spruce, the lignin content in the S2 layers of earlywood is lower than the lignin content in the latewood (Fengel and Wegener 1984), in contrast to the situations as found in the middle lamella. The lignin content measured in this study for the earlywood cells of $A$. grandis (Fig. 5) possibly also refers to higher lignin content in the middle lamella of earlywood as compared to latewood. It is further interesting to note that the overall weight of cell walls of earlywood is apparently higher than that of cell wall of latewood (see supplementary material Fig. S1, S2 and Fig. 4). All these may 
contribute to the different behavior of earlywood and of latewood in decay noted in this study (Fig. 4).

The decay test with wood samples of earlywood+latewood and with wood samples of only earlywood indicated that in the first 6 to 12 weeks of incubation, decay of samples of earlywood+latewood proceeded faster, but at later time of incubation decay of samples of only earlywood was much more enhanced (Fig. 4). At this late time of incubation, wood samples of only earlywood collapsed in shape (see Fig. S6 and Table S5).

The microscopic analysis of earlywood+latewood samples showed clearly that middle lamellae of wood fibers in earlywood were much destroyed (Fig. 9 and Fig. 12). Although MBTH-staining of middle lamellae were previously not observed in between the earlywood fibers, they should have been destroyed as concluded from the highly broken cell material indicating that neighboring cells parted from each other (Fig. 12 and Fig. 13). Transferring these observations to the samples of only earlywood, it becomes conceivable that the collapses of the wood blocks is based also on decay of middle lamellae in between earlywood fibers.

The results of wood mass loss tests by $T$. versicolor Strain 6 (i.e. that mass loss in the first 6 weeks of incubation was higher in samples of earlywood+latewood than in samples of earlywood alone in contrast to the situation in later steps of decay) and MBTH-staining of cell walls (indicating that the modification of cell walls was higher in samples of latewood than in earlywood) is likely related to the different lignin content in latewood and earlywood, respectively. The white-rot fungus $T$. versicolor Strain 6 clearly started to degrade lignin from the S3 layer towards S2, S1 and then the primary cell wall and at last the middle lamella. This process apparently was first faster in the latewood than in the earlywood. However, finally after 19 weeks of incubation, when the white-rot $T$. versicolor Strain 6 reached the middle lamellae for degradation, a higher mass loss of earlywood (with the apparently higher lignin content in the middle lamellae) was obtained in the end of the investigation. At this point of incubation, the middle lamellae of cells of latewood were apparently more resistant to decay than the middle lamellae of the earlywood. 


\section{III.5.2 Further biological observations on the fungus T. versicolor Strain 6 in growth on $A$. grandis wood}

Various of the figures shown in this chapter document that T. versicolor Strain 6 easily grows within the lumina of wood fiber cells as well as in the parenchymatic ray cells. The fungus probably grows first within the ray cells with easy to degrade organic material (Fig. 11A and 11E) to then move into the neighboring wood fiber cells via the pits (Fig. 11). Also between wood fibers, the fungus appears to make its way via the pits. Often, hyphae are detected at the opening of the pits to the inner cell lumina (Fig. 11). This suggests that hyphae grow through the pits on their way of entering fiber cell per fiber cell. Possibly during growth of fungal hyphae through the pit, secreted enzymes attack the cell wall material, specifically the lignin. Usually, it is also along the pits where continuous staining is seen, regardless of whether in earlywood or latewood (Fig. $11 \mathrm{~B}$ to $11 \mathrm{D}$ and $11 \mathrm{~F}$ to $11 \mathrm{H}$ ). In fungal wood decay, modification/degradation zones occur immediately surrounding the hyphae, and in close vicinity wall components are degraded (Fengel et al. 1983; Eriksson et al. 1990). The staining of all cell wall layers along the pit channel documents that the fungus is enzymatically active on all cell wall layers. This observations confirms previous statements that erosion through hyphae extend deeply into the secondary wall to destroy S3, S2 and S1 layers (Fengel et al. 1983; Eriksson et al. 1990). However, the study here shows that decay by fungal enzymes not only occurs along the pit channels but also starting from the inner S3 layer of the cell walls (Fig. 11).

The fungal hyphae (arrows show in Fig. 14A) penetrate into the cell lumen from cell to cell via the pit structures as reported for white-rot of other woods (Fengel et al. 1983; Eriksson et al. 1990). The observed cell wall modification/degradation documents in this study for A. grandis wood an erosion of cell walls by enzymes of white-rot fungus T. versicolor Strain 6 that occurs from the cell lumen toward the middle lamella.

\section{III.5.3 Mimicking the effect of fungal decay by incubation of wood with either isolated laccase or $\mathrm{Mn}^{3+}$, respectively}

So far, I discussed effects of the living fungus T. versicolor Strain 6 on A. grandis wood. MBTH-staining suggested repeatedly that the fungus is enzymatic active in order to modify cell wall begin with the larger target of cell wall decay. More direct evidence 
for enzymatic actions comes from experiment where isolated laccase III of the fungus or $\mathrm{Mn}^{3+}$-chelates as reaction products of a fungal $\mathrm{MnP}$ reaction were applied to the wood.

The experiments with the soaking wood section in solutions containing either laccase or $\mathrm{Mn}^{3+}$-acetate, respectively with injections of the solutions into wood blocks indicate that both the T. versicolor Strain 6 laccases III and $\mathrm{Mn}^{3+}$-acetate can be active on the lignin in the wood fiber cell walls. When laccase was injected into the cell lumina, only the inner cell wall layers were affected due to the large molecular weight of the protein. In contrast, the small $\mathrm{Mn}^{3+}$-acetate easily migrated through the cell walls as deduced from the broad staining of cell walls over their whole width (Fig. 19). $\mathrm{Mn}^{3+}$-chelates thus are apparently more effective in fast decay of wood fiber walls than the laccase enzymes. However, the $\mathrm{Mn}^{3+}$ concentrations of $4 \mathrm{mM}$ and $10 \mathrm{mM}$ used are much above the $\mathrm{Mn}^{3+}$ expected to be available for the fungus in nature in the wood. $\mathrm{Mn}^{3+}$ concentration values of only $74 \mu \mathrm{g} \mathrm{g}^{-1}$ of $A$. grandis dry wood were determined (see Chapter IV). Wood blocks of the density of $0.25-0.40 \mathrm{~g} \mathrm{~cm}^{-3}$ as used in this study and size of 30 (longitudinal) x 10 (tangential) x 5 (radial) $\mathrm{mm}$ and with an average weight of $0.5 \mathrm{~g}$ should thus contain an Mn content of approximately $37 \mu \mathrm{g}$. Assuming that about $50 \%$ of the wood blocks (of a volume of $1,500 \mathrm{~mm}^{3}$ ) consist of lumina that can be filled in the experiment with liquid, this would correspond to about $0.75 \mathrm{ml}$ total liquid volume and, accordingly, to $160 \mu \mathrm{g} \mathrm{Mn}$ for the $4 \mathrm{mM}$ solution and to $375 \mu \mathrm{g} \mathrm{Mn}$ for $10 \mathrm{mM}$ solution. In conclusion, this is factor of about 4 , respectively 10 times more. Since in nature by the natural low Mn content of the $A$. grandis wood not as much Mn is obviously given to easily diffuse in sufficient amounts through the whole wood fiber cell walls, it is therefore conceivable that the fungus $T$. versicolor for most efficient wood degradation will apply both strategies of enzymatic decay of lignin, i.e. making use of laccases as well as of Mn-peroxidases oxidizing available $\mathrm{Mn}^{2+}$ to $\mathrm{Mn}^{3+}$. All photos taken from wood infected with $T$. versicolor Strain 6 and stained with MBTH, particularly those from 6 weeks of incubation (Fig. 19), agree with such a dual decay strategy. 


\section{III.5.4 Comparison of methods for analyzing lignin modification within wood fiber cell walls}

Decay of wood cell walls by fungi and particularly the decay of lignin are very complex processes that up to now are only poorly understood. Various methods have been described in order to study and to get insights in what is ongoing in the degradation of lignin (see Table 1). Spectroscopic and visual methods such as FTIR (Fourier Transform Infrared Spectroscopy) or electron microscope inspection are widely used in research. Fackler et al. (2007) for example studied beech wood degraded by white and brown-rot fungi by using FTIR. Pandey et al. (2004) reported results of an FTIR analysis of beech and pine wood degraded by brown-rot fungi in comparison to a novel spectrophotometric analysis method by using acetyl bromide for determination of lignin contents in wood powder. Other research groups analyzed wood degraded by fungi by techniques such as high performance liquid chromatography (HPLC), X-ray microdensitometry, pyrolysis gas chromatography mass spectrometry (Py-GC/MS), low temperature scanning electron microscopy (LTSEM), quartz crystal microbalance with dissipation (QCM-D), atomic force microscopy (AFM), nuclear magnetic resonance (NMR), oxygen consumption measurement, size exclusion chromatography (SEC), reverse phase high performance liquid chromatography (RP-HPLC), high performance anion exchange chromatography (HPAEC), matrix-assisted laser desorption ionizationtime of flight mass spectroscopy (MALDI-TOF MS), scanning and transmission electron microscopy (SEM, TEM) etc. (see summaries in Table 1). All these are highly costly methods and need specific equipment, specific sample preparation as well as specific technical skills. For most of these, the wood samples, regardless of whether treated with a fungus or not, need to be powdered for analysis. Only very few allow detection of lignin locally in the wood and detection of local changes in the lignin within cell walls due to fungal decay. Amongst are FTIR-microscopy (Naumann et al. 2005). In this study and in a former master thesis (Lang 2004), a very cheap and easy to handle and quickly to perform MBTH-staining technique was developed and applied to wood from pine trees (Lang 2004) and A. grandis (this work). As a further superior advantage, the MBTH-staining makes easily visible within the wood cell walls where chemical modification of lignin happened. The technique is so easy to do that it can easily be performed in any lab dealing with wood samples without the need to buy any expensive equipment and to specifically train persons. This technique when required 
III. Effects of fungi and fungal enzymes on Abies grandis wood degradation

might add to the traditional wet chemistry analysis, which generally is time-consuming and requires larger samples, but still has an advantage over analysis by high technology methods requiring very specific equipment and skills (Pandey et al. 2004). One of the easiest and cheapest methods for identification of actions of white-rot fungi in wood is

Table 1. Methods for analysis of modified lignin

\begin{tabular}{|c|c|c|c|}
\hline Wood & Fungus & Method* & References \\
\hline \multirow[t]{3}{*}{ Beech } & $\begin{array}{l}\text { Ceriporiopsis } \\
\text { subvermispora, } \\
\text { Trametes versicolor }\end{array}$ & $\begin{array}{l}\text { Fourier transform } \\
\text { infrared (FTIR) }\end{array}$ & Fackler et al. (2007) \\
\hline & $\begin{array}{l}\text { Gloeophyllum } \\
\text { trabeum, Poria } \\
\text { placenta }\end{array}$ & $\begin{array}{l}\text { FTIR and acetyl bromide } \\
\text { method }\end{array}$ & Pandey et al. (2004) \\
\hline & $\begin{array}{l}\text { Coniophora puteana } \\
\text { G. trabeum and } T \text {. } \\
\text { versicolor }\end{array}$ & $\begin{array}{l}\text { X-ray } \\
\text { microdensitometric }\end{array}$ & Bucur et al. (1997) \\
\hline \multirow[t]{3}{*}{ Eucalyptus } & $\begin{array}{l}\text { Trametes villosa and } \\
\text { Panus crinitus }\end{array}$ & $\begin{array}{l}\text { HPLC with UV-VIS } \\
\text { detector }\end{array}$ & Yu et al. (2008) \\
\hline & $\begin{array}{l}9 \text { strains of } \\
\text { basidiomycetes }\end{array}$ & Py-GC/MS & del Rio et al. (2001) \\
\hline & $\begin{array}{l}\text { Dendrophora } \\
\text { albobadia } \\
\text { Lentinus tigrinus } \\
\text { Peniophora cinerea } \\
\text { Peniophora lycii } \\
\text { Phanerochaete } \\
\text { crassa }\end{array}$ & LTSEM & Speranza et al. (2009) \\
\hline
\end{tabular}


III. Effects of fungi and fungal enzymes on Abies grandis wood degradation

\begin{tabular}{|c|c|c|c|}
\hline & $\begin{array}{l}\text { Pycnoporus } \\
\text { cinnabarinus laccase }\end{array}$ & LTSEM & Speranza et al. (2007) \\
\hline & Inocutis jamaicensis & LTSEM and & Martinez et al. (2005) \\
\hline & & $\mathrm{Py}-\mathrm{GC} / \mathrm{MS}$ & \\
\hline & Bjerkandera adusta, & $\mathrm{Py}-\mathrm{GC} / \mathrm{MS}$ & del Rio et al. (2002) \\
\hline & P. lycii, $P$. cinerea & & \\
\hline & Peniophora sp. and & & \\
\hline & P. crassa & & \\
\hline \multirow[t]{5}{*}{ Pine } & C. puteana & SEM & $\begin{array}{l}\text { Karppanen et al. } \\
\text { (2008) }\end{array}$ \\
\hline & C. puteana & $\begin{array}{l}\text { FTIR and acetyl bromide } \\
\text { method }\end{array}$ & Pandey et al. (2004) \\
\hline & G. trabeum and & X-ray & Bucur et al. (1997) \\
\hline & T. versicolor & microdensitometry & \\
\hline & C. subvermispora & $\begin{array}{l}\text { UV absorption } \\
\text { microspectrophotometry }\end{array}$ & $\begin{array}{l}\text { Blanchette et al. } \\
\qquad \text { (1997) }\end{array}$ \\
\hline Poplar & $\begin{array}{l}\text { Phanerochaete } \\
\text { chrysosporium }\end{array}$ & TEM & Ruel et al. (1994) \\
\hline
\end{tabular}

* FTIR $=$ Fourier transform infrared

HPLC $=$ High performance liquid

LTSEM $=$ Low temperature scanning electron microscopy

Py-GC/MS = Pyrolysis gas chromatography mass spectrometry

$\mathrm{SEM}=$ Scanning electron microscopy

$\mathrm{TEM}=$ Transmission electron microscopy

UV-VIS = Ultraviolet-visible 
the here presented MBTH-staining-method. It is based on an oxidation reaction by the formation of a fusion product with a quinone, resulting in a color change in wood cell walls containing lignin that will have been previously by some way (either by laccase action or by $\mathrm{Mn}^{3+}$ action) enzymatically modified by the fungi. As will be shown in the following chapter (Chapter IV), MBTH-staining can also be applied on milled wood fibers decayed by fungi or by enzymes and use can also be made of the MBTH stain by spectrophotometric measurements in detecting organic compounds (likely quinones, see Fig. 20) released from the wood by actions of fungal enzymes.

However, a further special advantage of MBTH-staining is given when wanting to observe effects of white-rot fungi on larger areas of wood tissues. Also this can principally be performed without the need of very special equipment or computer programs. In Chapter III.3.4, an experiment is presented in which with the cheap method of MBTH-staining a whole year ring of $A$. grandis wood infected by T. versicolor Strain 6 was observed by light microscopic inspection after staining wood sections with the MBTH. Individual images were analyzed by some standard programs such as Adobe Photoshop and Mathematica for degree of cell wall staining within wood sections in order to evaluate the degradation of wood by the fungus. The quantitative analysis of stained cell walls for the estimation of the degree of modified/degraded lignin has been carried out in percentage of the area fraction of stained cell walls. This way of computational analysis is somewhat elaborate but releases the researcher from buying relatively extensive and specific image analysis programs such as image analySIS ${ }^{\circledR}$. The comparison of the results from the manual analysis appointing the Mathematica program and of the image analySIS ${ }^{\circledR}$ program indicate that both type of analysis are equally good in the quality of results obtained.

\section{III.6 References}

Anagnost SE (1998) Light microscopic diagnosis of wood decay. IAWA Journal 19(2):141-167

Archibald FS, Bourbonnais R, Jurasek L, Paice MG, Reid ID (1997) Kraft pulp bleaching and delignification by Trametes versicolor. Journal of Biotechnology $53: 215-236$

Baldrian P (2006) Fungal laccases - occurrence and properties. FEMS Microbiology Reviews 30(2):215-242 
III. Effects of fungi and fungal enzymes on Abies grandis wood degradation

Blanchette RA (1991) Delignification by wood-decay fungi. Annual Review of Phytopathology 29:381-398

Blanchette RA (1984) Manganese accumulation in wood decayed by white rot fungi. Phytopathology 74:725-730

Blanchette RA, Krueger EW, Haight JE, Akhtar M, Akin DE (1997) Cell wall alterations in loblolly pine wood decayed by the white-rot fungus, Ceriporiopsis subvermispora. Journal of Biotechnology 53(2-3):203-213

Bravery AF (1978) A minimized wood-block test for rapid evaluation of wood preservative fungicides. International Research Group on Wood Preservation. Document No. IRG/WP 2113, Stockholm, Sweden

Bucur V, Garros S, Navarrete A, deTroya MT, Guyonnet R (1997) Kinetics of wood degradation by fungi with $\mathrm{x}$-ray microdensitometric technique. Wood Science and Technology 31(5):383-389

Call HP, Mücke I (1997) History, overview and applications of mediated lignolytic systems, especially laccase-mediator-systems (Lignozym ${ }^{\circledR}$-process). Journal of Biotechnology 53(2-3):163-202

Champagne PP, Ramsay JA (2005) Contribution of manganese peroxidase and laccase to dye decoloration by Trametes versicolor. Applied Microbiology and Biotechnology 69(3):276-285

Childs RE, Bardsley WG (1975) Steady-state kinetics of peroxidase with 2,2'-azino-di(3-ethylbenzthiazoline-6-sulphonic acid) as chromogen. Biochemical Journal 145(1):93-103

Cherdchim B (2004) Softening behavior of sweet-bamboo culms (Dendrocalamus asper Backer.). Master thesis, Walailak University, Thailand (in Thai)

Dwivedi RC (2006) Extracellular proteins from lignocellulose degrading bacidiomycetes: redox enzymes from Trametes versicolor and Coprinopsis cinerea. Dissertation, Georg-August University, Germany

del Río JC, Gutiérrez A, Martínez MJ, Martínez ÁT (2001) Py-GC/MS study of Eucalyptus asidiom wood treated with different fungi. Journal of Analytical and Applied Pyrolysis 58:441-452

del Río JC, Speranza M, Gutiérrez A, Martínez MJ, Martínez ÁT (2002) Lignin attack during eucalypt wood decay by selected basidiomycetes: a Py-GC/MS study. Journal of Analytical and Applied Pyrolysis 64(2):421-431

Elissetche JP, Ferraz A, Freer J, Mendonca R, Rodriguez J (2006) Thiobarbituric acid 
III. Effects of fungi and fungal enzymes on Abies grandis wood degradation

reactive substances, $\mathrm{Fe}^{3+}$ reduction and enzymatic activities in cultures of Ganoderma australe growing on Drimys winteri wood. FEMS Microbiology Letters 260(1):112-118

EN 113 (1996) Wood preservatives - test method for determining the protective effectiveness against wood destroying basidiomycetes - Determination of the toxic values. European Committee for Standardization, Brüssel, Belgium: $35 \mathrm{p}$

Erden E, Ucar MC, Kaymaz Y, Pazarlioglu NK (2009) New different lignocellulosic materials from Turkey for laccase and manganese peroxidase production by Trametes versicolor. Engineering in Life Science 9(1):60-65

Eriksson KEL, Blanchette RA, Ander P (1990) Microbial and enzymatic degradation of wood and wood components. Springer-Verlag, Berlin, Germany

Evan CS, Dutton MV, Guillen F, Veness RG (1994) Enzymes and small molecular mass agents involved with lignocellulose degradation. FEMS Microbiology Reviews 13:235-240

Evan CS, Hedger JN (2008) Degradation of plant cell wall polymers. In: Gadd GM (Ed.) Fungi in bioremediation. Cambridge University Press, Cambridge, UK, 1 26

Fackler K, Schwanninger M, Gradinger C, Hinterstoisser B, Messner K (2006) Assessing fungal decay of spruce and beech wood using near infrared spectroscopic techniques. Lenzinger Berichte 86:9-13

Fackler K, Schwanninger M, Gradinger C, Hinterstoisser B, Messner K (2007) Qualitative and quantitative changes of beech wood degraded by wood-rotting basidiomycetes monitored by Fourier transform infrared spectroscopic methods and multivariate data analysis. FEMS Microbiology Letters 271(2):162-169

Fengel D (1969) Ultrastructure of cellulose from wood. 1. Wood as basic material for isolation of cellulose. Wood Science and Technology 3(3):203-217

Fengel D (1970) Ultrastructural changes during aging of wood cells. Wood Science and Technology 4(3):176-188

Fengel D, Wegener G (1983) Wood chemistry ultrastructure reactions. Walter de Gruyter, Berlin, Germany

Force DA, Randall DW, Britt RD (1997) Proximity of acetate, manganese, and exchangeable deuterons to tyrosine Y-Z (center dot) in acetate-inhibited photosystem II membranes: Implications for the direct involvement of Y-Z (center dot) in water-splitting. Biochemistry 36(40):1062-1070 
III. Effects of fungi and fungal enzymes on Abies grandis wood degradation

Gómez-Toribio V, García-Martín AB, Martínez MJ, Martínez ÁT, Guillén F (2009)

Induction of extracellular hydroxyl radical production by white-rot fungi through quinone redox cycling. Applied and Environmental Microbiology 75(12):3944-3953

Hammel KE, Jensen KA, Mozuch MD, Landucci LL, Tien M, Pease EA (1993)

Ligninolysis by a purified lignin peroxidase. Journal of Biological Chemistry 268(17):12274-12281

Hiscox J, Baldrian P, Rogers HJ, Boddy L (2010) Changes in oxidative enzyme activity during interspecific mycelial interactions involving the white-rot fungus Trametes versicolor. Fungal Genetics and Biology 47:562-571

Hof C, Kielmann BC, Hapla F (2008) Verwendungsorientierte Untersuchungen am Schnittholz der Abies grandis. Holztechnologie 49:7-10

Johannes C, Majcherczyk A (2000) Natural mediators in the oxidation of polycyclic aromatic hydrocarbons by laccase mediator systems. Applied and Environmental Microbiology 66(2):524-528

Johansson T, Nyman PO (1996) A cluster of genes encoding major isozymes of lignin peroxidase and manganese peroxidase from the white-rot fungus Trametes versicolor. Gene 170(1):31-38

Karppanen O, Venalainen M, Harju AM, Laakso T (2008) The effect of brown-rot decay on water adsorption and chemical composition of Scots pine heartwood. Annals of Forest Science 65(6):610

Kersten PJ, Tien M, Kalyanaraman B, Kirk TK (1985) The ligninase of Phanerochaete chrysosporium generates cation radicals from methoxybenzenes. Journal of Biological Chemistry 260:2609-2612

Kremer ML (1999) Mechanism of the Fenton reaction. Evidence for a new intermediate. Physical Chemistry Chemical Physics 1(15):3595-3605

Kües U, Navarro-González M, Cherdchim B, Majcherczyk A (2009) Anfälligkeit von lebendem und totem Holz der Küstentanne gegenüber Pilzen. Mykologisches Kolloquium, Molekularbiologische Pilzdiagnostik, 7./8.Mai 2009 in Dresden, Institut für Holztechnologie, Dresden: 1-14

Lang C (2004) Redoxenzyme bei Heterobasidion annosum. Master thesis, GeorgAugust University, Göttingen, Germany (in German)

Leonowicz A, Cho NS, Luterek J, Wilkolazka A, Wojtas-Wasilewska M, Matuszewska 
III. Effects of fungi and fungal enzymes on Abies grandis wood degradation

A, Hofrichter M, Wesenberg D, Rogalski J (2001) Fungal laccase: properties and activity on lignin. Journal of Basic Microbiology 41(3-4):185-227

Lundell TK, Mäkelä MR (2010) Lignin-modifying enzymes in filamentous basidiomycetes - ecological, functional and asidiomycet review. Journal of Basic Microbiology 50(1):5-20

Majcherczyk A, Johannes C, Hüttermann A (1999) Oxidation of aromatic alcohols by laccase from Trametes versicolor mediated by the 2,2 "-azino-bis-(3ethylbenzothiazoline-6-sulphonic acid) cation radical and dication. Applied Microbiology and Biotechnology 51(2):267-276

Martínez ÁT, Speranza M, Ruiz-Dueñas FJ, Ferreira P, Camarero S, Guillén F, Martínez MJ, Gutiérrez A, del Río JC (2005) Biodegradation of lignocellulosics: microbial chemical, and enzymatic aspects of the fungal attack of lignin. International Microbiology 8(3):195-204

Mattinen ML, Struijs K, Suortti T, Mattila I, Kruus K, Willför S, Tamminen T, Vincken JP (2009) Modification of lignans by Trametes hirsuta laccase. Bioresources $4(2): 482-496$

Mester T, Field JA (1998) Characterization of a novel manganese peroxidase-lignin peroxidase hybrid isozyme produced by Bjerkandera species strain BOS55 in the absence of manganese. Journal of Biological Chemistry 273:15412-15417

Mohebby B (2004) IR spectroscopy in white rot decayed beech. Proceedings of the Fourth International Iran \& Russia Conference: 903-910 (http://www.tmu.ir/wood/Publications/Mohebby-04-1.pdf)

Moredo N, Lorenzo M, Domínguez A, Moldes D, Camesselle C, Sanroman A (2003) Enhanced ligninolytic enzyme production and degrading capability of Phanerochaete chrysosporium and Trametes versicolor. World Journal of Microbiology \& Biotechnology 19:665-669

Müller G, Schöpper C, Vos H, Kharazipour A, Polle A (2009) FTIR-ATR spectroscopic analyses of changes in wood properties during particle- and fibreboard production of hard- and softwood trees. Bioresources 4(1):49-71

Naumann A, Navarro-González M, Peddireddi S, Kües U, Polle A (2005) Fourier transform infrared microscopy and imaging: Detection of fungi in wood. Fungal Genetics and Biology 42(10):829-835

Navarro-González M, Wei DS, Kües U (2008) Pilze of Holz der Küstentanne (Abies grandis). Holzbiologie 49(6): 23-25 
III. Effects of fungi and fungal enzymes on Abies grandis wood degradation

Orton CR, Parkinson DY, Evans PD, Owen NL (2004) Fourier transform infrared studies of heterogeneity, photodegradation, and lignin/hemicellulose ratios within hardwoods and softwoods. Applied Spectroscopy 58(11):1265-1271

Pandey KK, Pitman AJ (2004) Examination of the lignin content in a softwood and a hardwood decayed by a brown-rot fungus with the acetyl bromide method and Fourier transform infrared spectroscopy. Journal of Polymer Science Part APolymer Chemistry 42(10):2340-2346

Pederson MR, Khanna SN (1999) Electronic and geometrical structure and magnetic ordering in passivated $\mathrm{Mn}_{12} \mathrm{O}_{12}$-acetate nanomagnets. Chemical Physics Letters 307(3-4):253-258

Polle A, Hawighorst P, Spellmann H (2008) Innovative Holzprodukte und Holzwerkstoffe aus nachhaltig produziertem Buchen- und Küstentannenholz. Holztechnologie 49:5-6

Pu YQ, Anderson S, Lucia L, Ragauskas AJ (2004) Investigation of the photo-oxidative chemistry of acetylated softwood lignin. Journal of Photochemistry and Photobiology A-Chemistry 163(1-2):215-221

Rodríguez-López JN, Escribano J, García-Cánovas (1994) A continuous spectrophotometric method for the determination of monophenolase activity of tyrosinase using 3-methyl-2-benzothiazolinone hydrazone. Analytical Chemistry 216:205-212

Röhrig E (1981) Neuere Grundlagen für den Anbau von Abies grandis. Schriften aus der Forstlichen Fakultät der Universität Göttingen und der Niedersächsischen Forstlichen Versuchsanstalt, Sauerländer Verlag, Frankfurt am Main, Germany

Ruel K, Ambert K, Joseleau JP (1994) Influence of the enzyme equipment of whiterot fungi on the patterns of wood degradation. FEMS Microbiology Reviews $13(2-3): 241-254$

Saarinen T, Suurnäkki A, Österberg M, Laine J (2009) Modification of lignin with laccases for the adsorption of anionic ferulic acid studied by quartz cristall microbalance with dissipation and AFM. Holzforschung 63(3):298-306

Shah V, Nerud F (2002) Lignin degrading system of white-rot fungi and its exploitation for dye decolorization. Canadian Journal of Microbiology 48(10):857-870

Spellmann H, Geb M, Nagel J, Nagel R, Schmidt M (2010) Timber product oriented silvicultural strategies for mixed strands of beech (Fagus silvatica) and grand fir (Abies grandis). Forst und Holz 65(1):12-19 
III. Effects of fungi and fungal enzymes on Abies grandis wood degradation

Speranza M, Gutiérrez A, del Río JC, Bettucci L, Martínez ÁT, Martínez MJ (2009)

Sterols and lignin in Eucalyptus globulus Labill. Wood: spatial distribution and fungal removal as revealed by microscopy and chemical analyses.

Holzforschung 63(3):362-370

Speranza M, Ibarra D, Romero J, Martínez ÁT, Martínez MJ, Camarero S (2007)

Microscopy studies reveal delignification and sterol removal from eucalypt kraft pulps by laccase-HBT. Biocatalysis and Biotransformation 25(2-4):251-259

Swamy J, Ramsay JA (1999a) Effect of glucose and $\mathrm{NH}_{4}{ }^{+}$concentrations on sequential dye decoloration by Trametes versicolor. Enzyme and Microbial Technology $25: 278-284$

Swamy J, Ramsay JA (1999b) Effect of $\mathrm{Mn}^{2+}$ and $\mathrm{NH}_{4}{ }^{+}$concentrations on laccase and manganese peroxidase production and amaranth decoloration by Trametes versicolor. Applied Microbiology and Biotechnology 51:391-396

TAPPI T 222 om-02 (2002) Acid-insoluble lignin in wood and pulp. Technical Association of the Pulp and Paper Industry, USA: 5 p

Tomšovský M, Homolka L (2003) Laccase and other ligninolytic enzyme activities of selected strains of Trametes spp. From different localities and substrates. Folia Microbiology 48(3):413-416

Yu HB, Li L, Zhang XY, Huang HY (2008) Effects of wood species and enzyme production on lignocellulose degradation during the biodegradation of three native woods by Trametes versicolor. Forest Products Journal 58(4):62-65

Vos H, Kharazipour A (2010) Properties of light particle boards manufactured using Abies grandis (grand fir). Forst und Holz 65(1):26-30

Wei DS, Houtman CJ, Kapich AN, Hunt CG, Cullen D, Hammel KE (2010) Laccase and its role in production of extracellular reactive oxygen species during wood decay by the brown rot basidiomycete Postia placenta. Applied and Environmental Microbiology 76(7):2091-2097 


\section{III.7 Supplementary material}

\section{III.7.1 Density measurements of $\boldsymbol{A}$. grandis wood blocks with earlywood+latewood} and of $\boldsymbol{A}$. grandis wood blocks with only earlywood

Fig. S1 and Fig. S2 present graphs with the individual values of density as measured for 215 by rough-weight-determination of pre-selected $A$. grandis wood blocks from the earlywood+latewood samples and for 215 by rough-weight-determination of pre-selected A. grandis wood blocks from only earlywood, respectively. These wood blocks with earlywood+latewood (Fig. S1) had a mean density of $0.352 \pm 0.066 \mathrm{~g} \mathrm{~cm}^{-3}$ and a median density of $0.342 \mathrm{~g} \mathrm{~cm}^{-3}$. In comparison, the lot of wood blocks with only earlywood (Fig. S2) had a mean density of $0.367 \pm 0.061 \mathrm{~g} \mathrm{~cm}^{-3}$ and a median density of $0.365 \mathrm{~g} \mathrm{~cm}^{-3}$.

In total, further 36 wood blocks with earlywood+latewood were directly discharged because of an apparent too high density ranging from $0.462 \mathrm{~g} \mathrm{~cm}^{-3}$ to $0.596 \mathrm{~g} \mathrm{~cm}^{-3}$ (mean value $=0.510 \pm 0.035 \mathrm{~g} \mathrm{~cm}^{-3}$ and median value $=0.513 \mathrm{~g} \mathrm{~cm}^{-3}$ ). Likewise, in total further 40 wood blocks were directly discharged because of ranging in a density from $0.393 \mathrm{~g} \mathrm{~cm}^{-3}$ to $0.595 \mathrm{~g} \mathrm{~cm}^{-3}$ (mean value $=0.452 \pm 0.063 \mathrm{~g} \mathrm{~cm}^{-3}$ and median value $=0.425 \mathrm{~g} \mathrm{~cm}^{-3}$ ). The discharged wood blocks with higher relative weight mostly had comparably small year rings $(4-5 \mathrm{~mm})$, explaining possibly by a higher amount of latewood the higher overall density.

All together, the wood blocks with earlywood+latewood including the extra 36 discharged wood blocks had a mean density of $0.376 \pm 0.084 \mathrm{~g} \mathrm{~cm}^{-3}$ and a median density of $0.362 \mathrm{~g} \mathrm{~cm}^{-3}$. In comparison, all wood blocks with only earlywood including the extra discharged 40 wood blocks had a mean density of $0.381 \pm 0.069 \mathrm{~g} \mathrm{~cm}^{-3}$ and a median density of $0.386 \mathrm{~g} \mathrm{~cm}^{-3}$. 


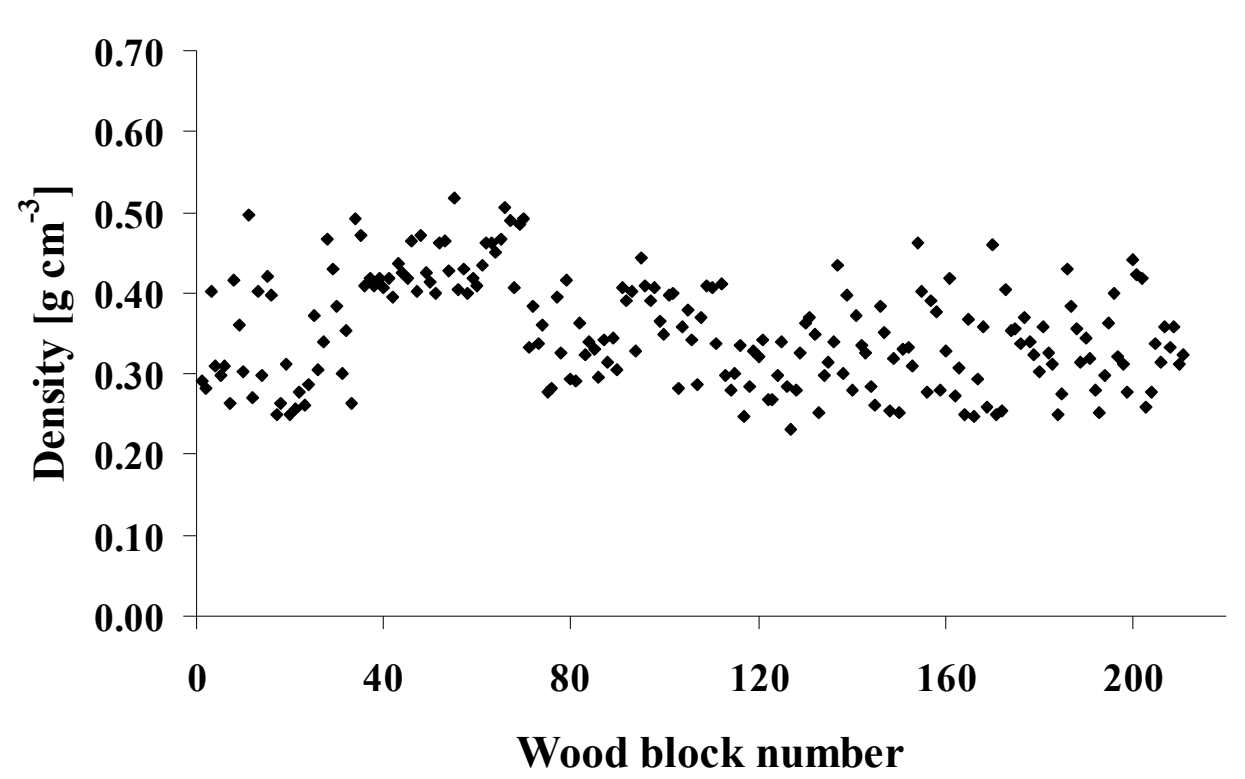

Fig. S1 Wood density of both earlywood+latewood of A. grandis wood blocks of 30 (longitudinal) x 10 (tangential) x 5 (radial) $\mathrm{mm}$ prepared according to European standard EN 113 (1996). The 215 wood blocks were measured in weight and in all dimensions in order to calculate the wood density (mean value $=0.352 \pm 0.066 \mathrm{~g} \mathrm{~cm}^{-3}$ and a median value $=0.342 \mathrm{~g} \mathrm{~cm}^{-3}$ ). Wood blocks for the duration test after EN 113 were selected in the range of $0.25-0.40 \mathrm{~g} \mathrm{~cm}^{-3}$ (mean value $=0.322 \pm 0.045 \mathrm{~g} \mathrm{~cm}^{-3}$ and median value $=0.323 \mathrm{~g} \mathrm{~cm}^{-3}$ in total 156 samples)

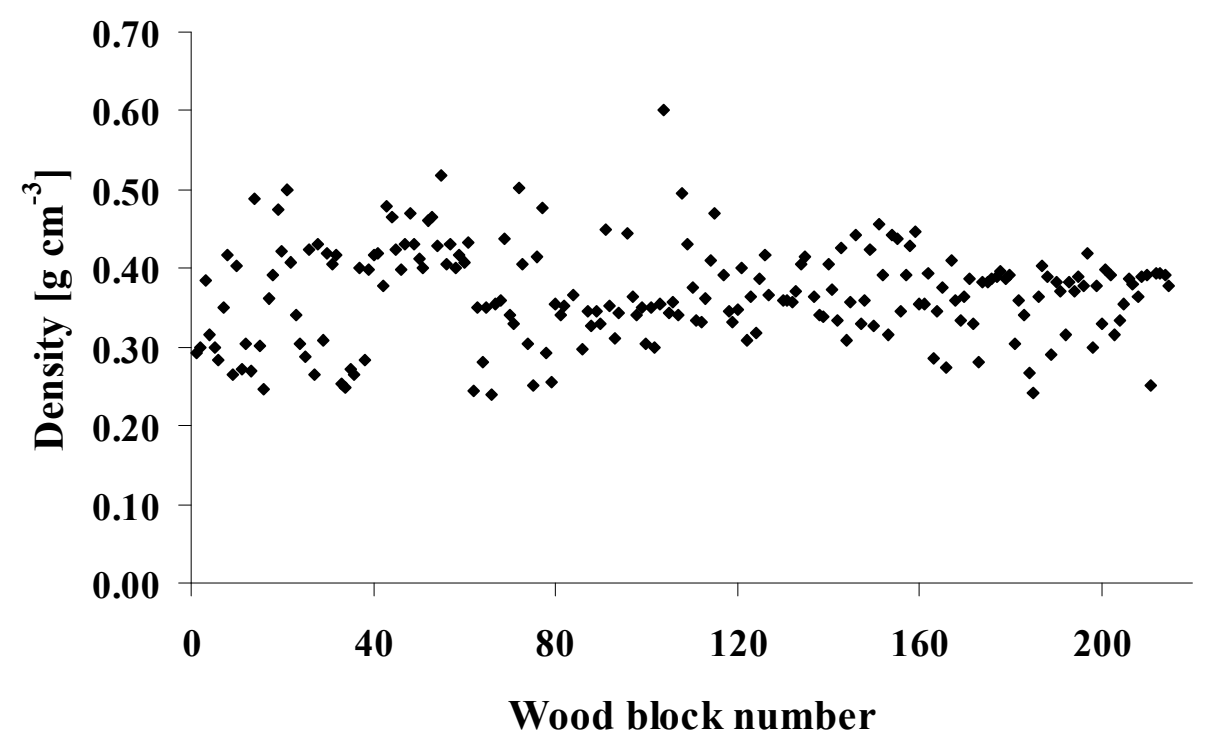

Fig. S2 Wood density of only earlywood of $A$. grandis wood blocks of 30 (longitudinal) x 10 (tangential) x 5 (radial) mm prepared according to European standard EN 113 (1996). The 215 wood blocks were measured in weight and in all dimensions in order to calculate the wood density (mean value $=0.367 \pm 0.061 \mathrm{~g} \mathrm{~cm}^{-3}$ and a median value $=0.365 \mathrm{~g} \mathrm{~cm}^{-3}$ ). Wood blocks for the duration test after EN 113 were selected in the range of $0.25-0.40 \mathrm{~g} \mathrm{~cm}^{-3}$ (mean value $=0.346 \pm 0.041 \mathrm{~g} \mathrm{~cm}^{-3}$ and median value $=0.354 \mathrm{~g} \mathrm{~cm}^{-3}$ in total 162 samples) 
In conclusion, as supported by statistical ANOVA analysis of the samples of 215 wood blocks shown in Fig. S1 and S2 and by statistical ANOVA analysis of only the samples ranging in density between $0.25-0.40 \mathrm{~g} \mathrm{~cm}^{-1}$, wood blocks with only earlywood tended in average to be slightly heavier than the wood blocks with earlywood+latewood, different from what might have been expected. It is possible that during sample preparation, the very "early" earlywood of the about $6 \mathrm{~mm}$ wide year rings has been cut off with the saw (compare materials and methods in this chapter) and that this affects volumes of the calculated average densities. [When considering all samples for both series of wood blocks, due to a higher variation, according to ANOVA analysis differences were statistically not significant]. Since the overall volume of cell walls material in samples with earlywood is however lower than in samples that contain also latewood, one might conclude from the data presented here, that the density of cell walls of fibers in earlywood is generally higher than the density of cell walls of fibers in latewood.

\section{III.7.2 Mass loss of wood by decay fungi}

Mass loss of $A$. grandis wood (density range of $0.25-0.4 \mathrm{~g} \mathrm{~cm}^{-3}$, density mean value $=0.35 \pm 0.07 \mathrm{~g} \mathrm{~cm}^{-3}$ ) by fungi was observed also in an other set of experiments after 6 weeks, 12 weeks and 19 weeks of incubation, respectively. Mass loss values determined after fungal incubation indicates that the $A$. grandis wood was partially decayed by the white-rots $P$. ostreatus $8 \mathrm{~F} 6$ and T. versicolor Strain 6 as indicated from the comparison with the uninfected control (Fig. S3). The mass loss of wood caused was higher with longer incubation time. In 19 weeks of incubation, $13 \pm 3.75 \%$ mass loss was obtained by $P$. ostreatus $8 \mathrm{~F} 6$ and $41 \pm 4.51 \%$ mass loss by $T$. versicolor Strain 6 (Fig. S3). However, compared to the brown-rot species C. puteana Strain 57, A. grandis wood was generally more resistant against white-rot decay fungi. The highest decay of all was obtained with the brown-rot species C. puteana Strain 57 in 19 weeks of incubation with an actual mass loss value of $59 \pm 3.54 \%$. Already after 12 weeks of incubation, the mass loss caused by $C$. puteana was $59 \pm 3.17 \%$. A comparable value for the white-rot fungus $P$. ostreatus $8 \mathrm{~F} 6$ was only achieved after 19 weeks of incubation, and for T. versicolor Strain 6 only after 12 and 19 weeks of incubation, respectively (compare Fig. S3). In conclusion, decay by C. puteana Strain 57 does not only affect stronger the total mass loss but, also, it occurs much earlier than with the white-rots. In 
case of the brown-rot decay test, samples of 12 weeks and 19 weeks incubation showed no difference in mass loss. This could be the result of that cellulose and hemicellulose was completely degraded since the $12^{\text {th }}$ week of incubation. In fact, the brown-rot fungus extensively degrades cellulose and hemicellulose, but acts limited in degradation of lignin (Howell et al. 2009; Lee et al. 2004).

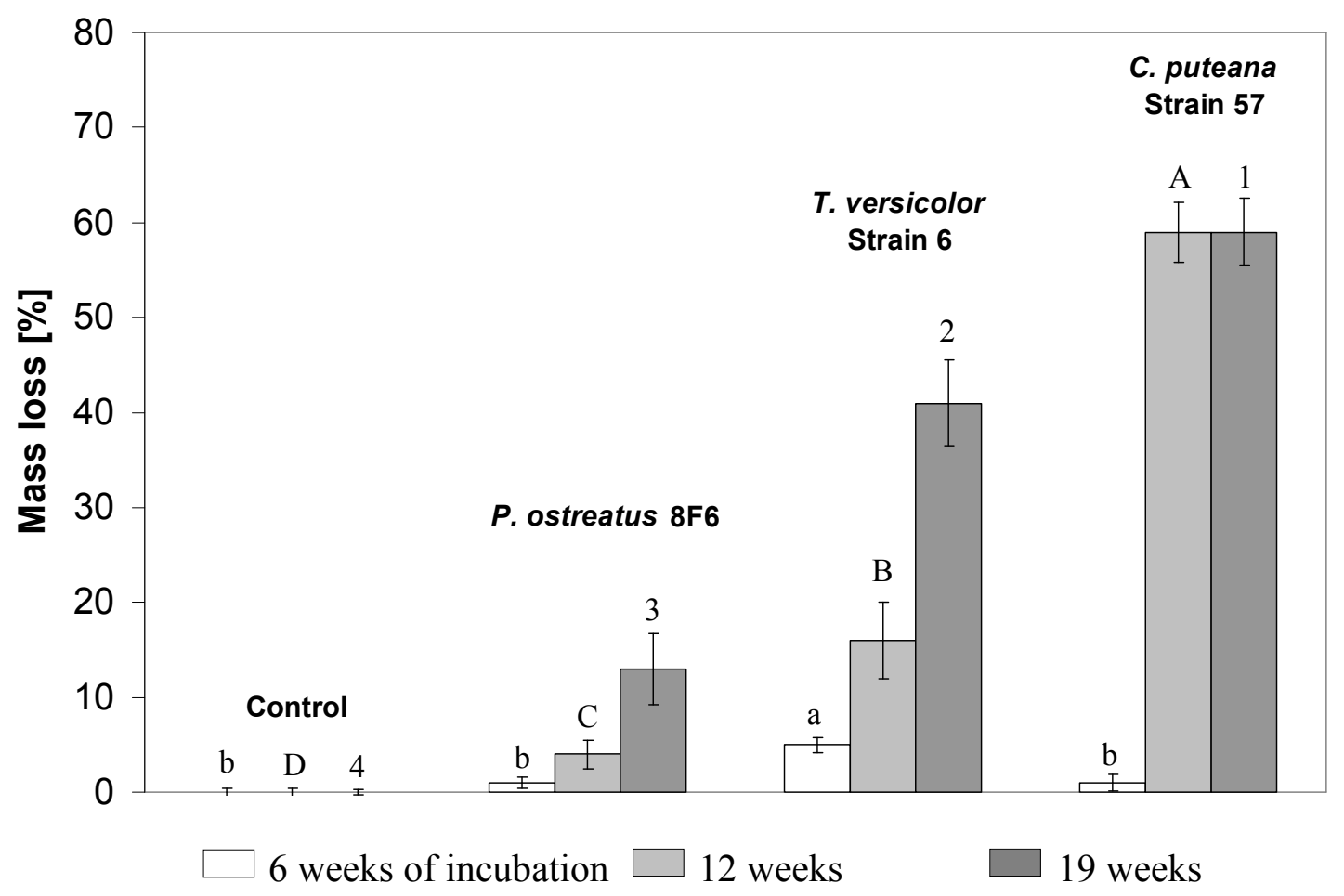

Fig. S3 Mass loss over the time caused by fungal decay of A. grandis wood. Average values of each 5 wood blocks per fungus and per time point were calculated and standard deviations are shown. Statistical analysis of the data was performed with SPSS 8.0 for Windows, the different superscripts on the chart (letters in lower case compare the values after 6 weeks of incubation, capital letters compare the values after 12 weeks of incubation, and numbers compare the values after 19 weeks of incubation) indicate values that differ significantly $(p<0.05)$ between treatments as based on analysis of variance (ANOVA) 
III.7.3 Staining of microtome sections of $A$. grandis wood after incubation with T. versicolor Strain 6 with MBTH and image analysis
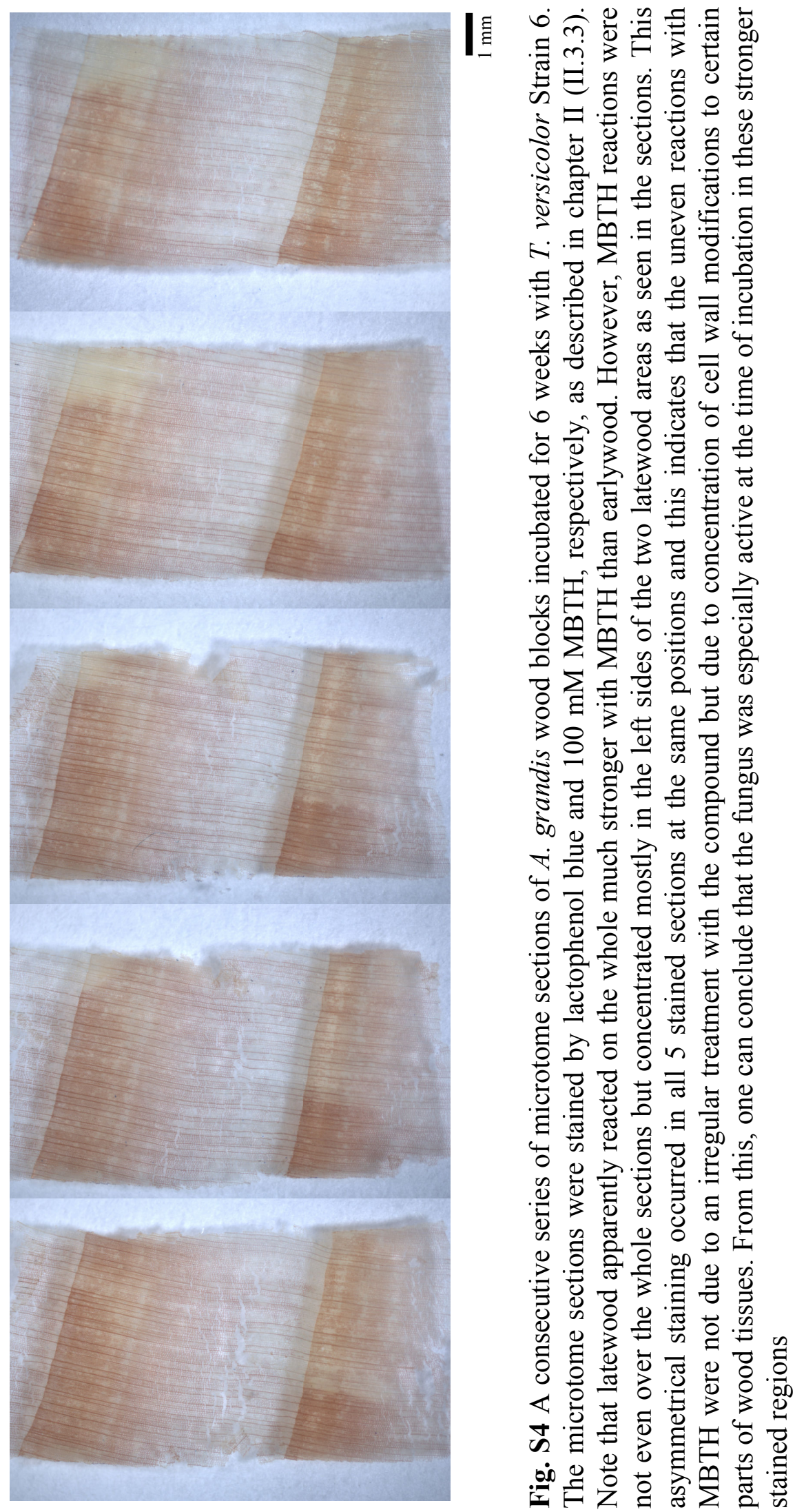
Table S1 Stained cell wall areas of a complete year ring followed up in individual image of a microtome section of an $A$. grandis wood block incubated for 6 weeks at $22^{\circ} \mathrm{C}$ and $65 \% \mathrm{RH}$ on $5 \%$ malt $2 \%$ agar with $T$. versicolor Strain 6

\begin{tabular}{|c|c|c|c|c|}
\hline $\begin{array}{l}* \text { Microtome } \\
\text { section image }\end{array}$ & $\begin{array}{c}\text { Length } \\
(\mu \mathrm{m})\end{array}$ & $\begin{array}{c}\text { Width } \\
(\mu \mathrm{m})\end{array}$ & $\begin{array}{l}\text { Stained cell wall area } \\
(\% \text { of total section area) }\end{array}$ & $\begin{array}{l}\text { Average stained cell } \\
\text { wall area ( } \% \text { of } \\
\text { total section area) }\end{array}$ \\
\hline$\overline{X_{1}}$ & 267 & 550 & 49.29 & \multirow{3}{*}{44.81} \\
\hline$X_{2}$ & 400 & 550 & 46.89 & \\
\hline$X_{3 a}$ & 333 & 550 & 39.80 & \\
\hline $\mathrm{X}_{3 \mathrm{~b}}$ & 67 & 550 & 39.80 & \multirow{4}{*}{40.88} \\
\hline$X_{4}$ & 400 & 550 & 39.84 & \\
\hline $\mathrm{X}_{5}$ & 400 & 550 & 40.59 & \\
\hline$X_{6 a}$ & 133 & 550 & 46.12 & \\
\hline $\mathrm{X}_{6 \mathrm{~b}}$ & 267 & 550 & 46.12 & \multirow{3}{*}{36.52} \\
\hline$X_{7}$ & 400 & 550 & 40.61 & \\
\hline $\mathrm{X}_{8 \mathrm{a}}$ & 333 & 550 & 28.35 & \\
\hline$X_{8 b}$ & 63 & 550 & 28.35 & \multirow{4}{*}{23.02} \\
\hline $\mathrm{X}_{9}$ & 400 & 550 & 27.63 & \\
\hline $\mathrm{X}_{10}$ & 400 & 550 & 20.00 & \\
\hline $\mathrm{X}_{11 \mathrm{a}}$ & 137 & 550 & 20.32 & \\
\hline $\mathrm{X}_{11 \mathrm{~b}}$ & 263 & 550 & 20.32 & \multirow{3}{*}{18.04} \\
\hline $\mathrm{X}_{12}$ & 400 & 550 & 17.19 & \\
\hline$X_{13 a}$ & 337 & 550 & 17.53 & \\
\hline $\mathrm{X}_{13 \mathrm{~b}}$ & 63 & 550 & 17.53 & \multirow{4}{*}{17.39} \\
\hline $\mathrm{X}_{14}$ & 400 & 550 & 18.10 & \\
\hline$X_{15}$ & 400 & 550 & 16.64 & \\
\hline $\mathrm{X}_{16 \mathrm{a}}$ & 137 & 550 & 17.66 & \\
\hline $\mathrm{X}_{16 \mathrm{~b}}$ & 227 & 550 & 17.66 & \\
\hline
\end{tabular}

The microtome sections were stained by lactophenol blue and $100 \mathrm{mM} \mathrm{MBTH}$, respectively, as described in Chapter II.3.3. The Mathematica program was used to calculate the reddish stained cell wall areas as given in Chapter II.3.3 subsequently, the analyzed year ring of $6.227 \mathrm{~mm}$ was divided into sections of $1 \mathrm{~mm}$ in length and average values of stained cell wall areas (\%) of total section area were calculated. * Image that crossed the $1 \mathrm{~mm}$ length border of individual areas of analysis into two parts were divided (each labeled by the letters a and b) so that area of an exact length of $1 \mathrm{~mm}$ were obtained 
Table S2 Stained cell wall areas of a complete year ring followed up in individual images of a microtome section of an A. grandis wood block incubated for 6 weeks at $22^{\circ} \mathrm{C}$ and $65 \% \mathrm{RH}$ on $5 \%$ malt $2 \%$ agar with $T$. versicolor Strain 6

\begin{tabular}{|c|c|c|c|c|}
\hline $\begin{array}{c}\text { Microtome } \\
\text { section }\end{array}$ & $\begin{array}{c}\text { Length } \\
(\mu \mathrm{m})\end{array}$ & $\begin{array}{c}\text { Width } \\
(\mu \mathrm{m})\end{array}$ & $\begin{array}{c}\text { Stained cell wall area } \\
(\% \text { of total section area) }\end{array}$ & $\begin{array}{l}\text { Average stained cell } \\
\text { wall area }(\% \text { of } \\
\text { total section area) }\end{array}$ \\
\hline$\overline{X_{1}}$ & 267 & 550 & 49.37 & \multirow{3}{*}{44.87} \\
\hline$X_{2}$ & 400 & 550 & 46.80 & \\
\hline $\mathrm{X}_{3 \mathrm{a}}$ & 333 & 550 & 39.96 & \\
\hline $\mathrm{X}_{3 \mathrm{~b}}$ & 67 & 550 & 39.96 & \multirow{4}{*}{40.88} \\
\hline $\mathrm{X}_{4}$ & 400 & 550 & 40.01 & \\
\hline $\mathrm{X}_{5}$ & 400 & 550 & 40.31 & \\
\hline $\mathrm{X}_{6 \mathrm{a}}$ & 133 & 550 & 46.45 & \\
\hline $\mathrm{X}_{6 \mathrm{~b}}$ & 267 & 550 & 46.45 & \multirow{3}{*}{36.72} \\
\hline $\mathrm{X}_{7}$ & 400 & 550 & 40.95 & \\
\hline $\mathrm{X}_{8 \mathrm{a}}$ & 333 & 550 & 28.42 & \\
\hline $\mathrm{X}_{8 \mathrm{~b}}$ & 63 & 550 & 28.42 & \multirow{4}{*}{22.75} \\
\hline $\mathrm{X}_{9}$ & 400 & 550 & 27.34 & \\
\hline $\mathrm{X}_{10}$ & 400 & 550 & 19.74 & \\
\hline $\mathrm{X}_{11 \mathrm{a}}$ & 137 & 550 & 20.03 & \\
\hline $\mathrm{X}_{11 \mathrm{~b}}$ & 263 & 550 & 20.03 & \multirow{3}{*}{17.88} \\
\hline$X_{12}$ & 400 & 550 & 16.99 & \\
\hline $\mathrm{X}_{13 \mathrm{a}}$ & 337 & 550 & 17.51 & \\
\hline $\mathrm{X}_{13 \mathrm{~b}}$ & 63 & 550 & 17.51 & \multirow{4}{*}{17.81} \\
\hline$X_{14}$ & 400 & 550 & 18.98 & \\
\hline$X_{15}$ & 400 & 550 & 16.87 & \\
\hline $\mathrm{X}_{16 \mathrm{a}}$ & 137 & 550 & 17.67 & \\
\hline $\mathrm{X}_{16 \mathrm{~b}}$ & 227 & 550 & 17.67 & \\
\hline
\end{tabular}

The microtome sections were stained by lactophenol blue and $100 \mathrm{mM} \mathrm{MBTH}$, respectively, as described in Chapter II.3.3. The images analySIS ${ }^{\circledR}$ program was used to calculate the reddish stained cell wall areas as given in Chapter II.3.3. The analyzed year ring of $6.227 \mathrm{~mm}$ was divided into sections of $1 \mathrm{~mm}$ in length and average values of stained cell wall areas (\% of total section area) were calculated. * See corresponding footnote in Table S1 
Table S3 Total cell wall area of a complete year ring followed up in individual images of a microtome sections of an $A$. grandis wood block incubated for 6 weeks at $22^{\circ} \mathrm{C}$ and $65 \% \mathrm{RH}$ on $5 \%$ malt $2 \%$ agar with $T$. versicolor Strain 6

\begin{tabular}{|c|c|c|c|c|}
\hline$* \begin{array}{l}\text { Microtome } \\
\text { section }\end{array}$ & $\begin{array}{c}\text { Length } \\
(\mu \mathrm{m})\end{array}$ & $\begin{array}{c}\text { Width } \\
(\mu \mathrm{m})\end{array}$ & $\begin{array}{c}\text { Total cell wall area } \\
\text { (\% of total section area) }\end{array}$ & $\begin{array}{c}\text { Average total cell } \\
\text { wall area ( } \% \text { of total } \\
\text { section area) }\end{array}$ \\
\hline$\overline{X_{1}}$ & 267 & 550 & 62.34 & \multirow{3}{*}{64.37} \\
\hline $\mathrm{X}_{2}$ & 400 & 550 & 69.59 & \\
\hline $\mathrm{X}_{3 \mathrm{a}}$ & 333 & 550 & 60.50 & \\
\hline$X_{3 b}$ & 67 & 550 & 60.50 & \multirow{4}{*}{65.12} \\
\hline$X_{4}$ & 400 & 550 & 60.56 & \\
\hline$X_{5}$ & 400 & 550 & 69.95 & \\
\hline $\mathrm{X}_{6 \mathrm{a}}$ & 133 & 550 & 69.40 & \\
\hline $\mathrm{X}_{6 \mathrm{~b}}$ & 267 & 550 & 69.40 & \multirow{3}{*}{61.56} \\
\hline$X_{7}$ & 400 & 550 & 58.39 & \\
\hline $\mathrm{X}_{8 \mathrm{a}}$ & 333 & 550 & 60.04 & \\
\hline $\mathrm{X}_{8 \mathrm{~b}}$ & 63 & 550 & 60.04 & \multirow{4}{*}{46.91} \\
\hline $\mathrm{X}_{9}$ & 400 & 550 & 53.15 & \\
\hline $\mathrm{X}_{10}$ & 400 & 550 & 41.00 & \\
\hline $\mathrm{X}_{11 \mathrm{a}}$ & 137 & 550 & 45.86 & \\
\hline $\mathrm{X}_{11 \mathrm{~b}}$ & 263 & 550 & 45.86 & \multirow{3}{*}{41.34} \\
\hline $\mathrm{X}_{12}$ & 400 & 550 & 39.83 & \\
\hline$X_{13 a}$ & 337 & 550 & 40.07 & \\
\hline$X_{13 b}$ & 63 & 550 & 40.07 & \multirow{4}{*}{43.36} \\
\hline $\mathrm{X}_{14}$ & 400 & 550 & 44.01 & \\
\hline$X_{15}$ & 400 & 550 & 42.18 & \\
\hline $\mathrm{X}_{16 \mathrm{a}}$ & 137 & 550 & 47.00 & \\
\hline $\mathrm{X}_{16 \mathrm{~b}}$ & 227 & 550 & 47.00 & \\
\hline
\end{tabular}

The microtome sections were stained by lactophenol blue and $100 \mathrm{mM} \mathrm{MBTH}$, respectively, as described in Chapter II.3.3. The image analySIS ${ }^{\circledR}$ program was used to calculate the total cell wall areas. The analyzed year ring of $6.227 \mathrm{~mm}$ was divided into sections of $1 \mathrm{~mm}$ in length and average values of cell wall areas $(\%$ total of the section area) were calculated. For an optical view of these areas in comparison with a section from wood not incubated with a fungus, see Fig. S5. * See corresponding footnote in Table S1 


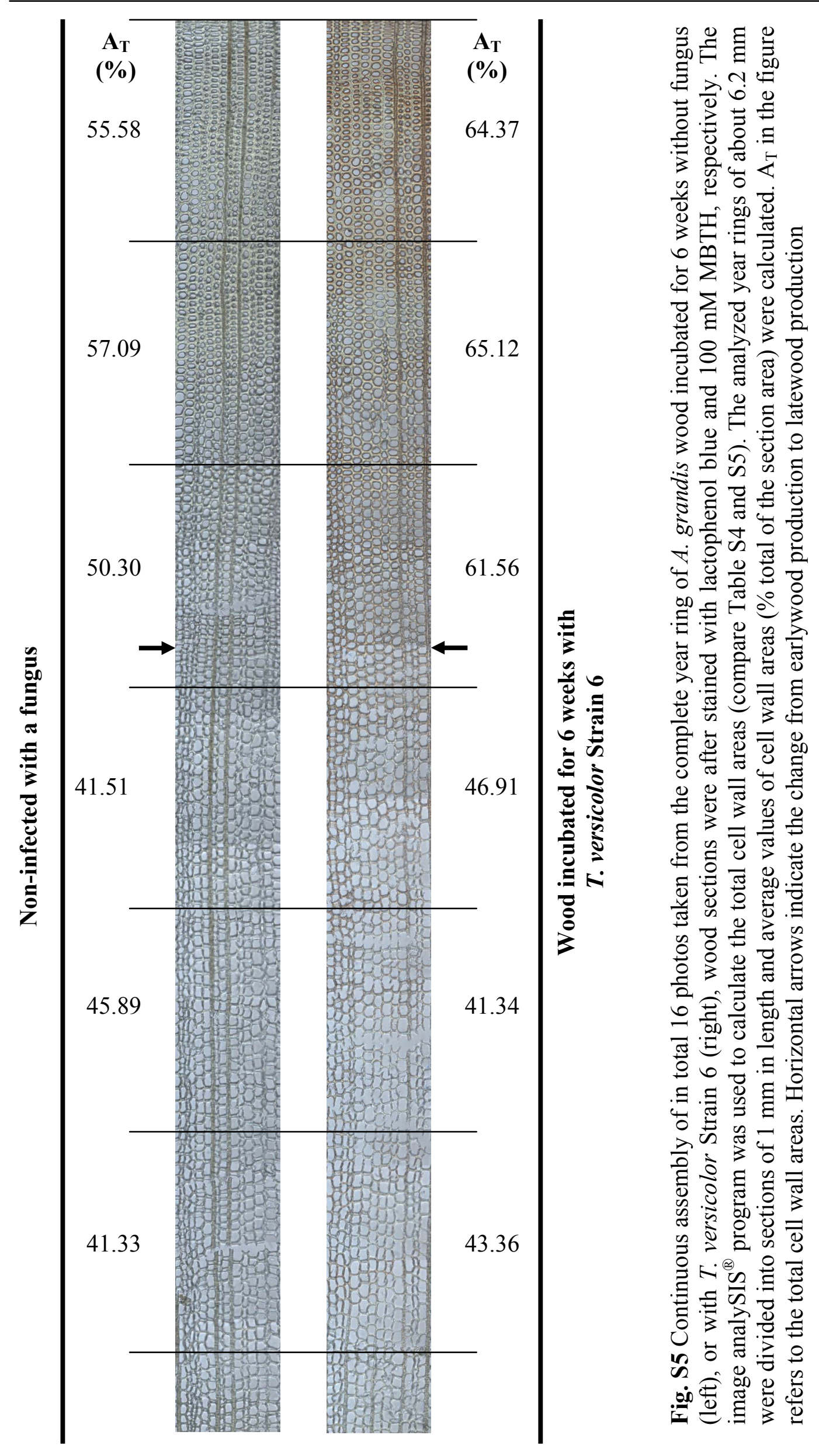


Table S4 Total cell wall area of a complete year ring followed up in individual images of a microtome sections of an $A$. grandis wood block incubated for 6 weeks at $22^{\circ} \mathrm{C}$ and $65 \% \mathrm{RH}$ on $5 \%$ malt $2 \%$ agar without fungus

\begin{tabular}{|c|c|c|c|c|}
\hline $\begin{array}{l}* \text { Microtome } \\
\text { section }\end{array}$ & $\begin{array}{l}\text { Length } \\
(\mu \mathrm{m})\end{array}$ & $\begin{array}{l}\text { Width } \\
(\mu \mathrm{m})\end{array}$ & $\begin{array}{c}\text { Total cell wall area } \\
(\% \text { of total section area) }\end{array}$ & $\begin{array}{c}\text { Average total cell } \\
\text { wall area ( } \% \text { of total } \\
\text { section area) }\end{array}$ \\
\hline$\overline{X_{1}}$ & 267 & 550 & 52.03 & \multirow{3}{*}{55.58} \\
\hline$X_{2}$ & 400 & 550 & 55.32 & \\
\hline $\mathrm{X}_{3 \mathrm{a}}$ & 333 & 550 & 59.14 & \\
\hline$X_{3 b}$ & 67 & 550 & 59.14 & \multirow{4}{*}{57.09} \\
\hline$X_{4}$ & 400 & 550 & 59.03 & \\
\hline $\mathrm{X}_{5}$ & 400 & 550 & 55.53 & \\
\hline $\mathrm{X}_{6 \mathrm{a}}$ & 133 & 550 & 50.98 & \\
\hline $\mathrm{X}_{6 \mathrm{~b}}$ & 267 & 550 & 50.98 & \multirow{3}{*}{50.30} \\
\hline$X_{7}$ & 400 & 550 & 53.63 & \\
\hline $\mathrm{X}_{8 \mathrm{a}}$ & 333 & 550 & 46.36 & \\
\hline $\mathrm{X}_{8 \mathrm{~b}}$ & 63 & 550 & 46.36 & \multirow{4}{*}{41.51} \\
\hline $\mathrm{X}_{9}$ & 400 & 550 & 43.10 & \\
\hline $\mathrm{X}_{10}$ & 400 & 550 & 39.10 & \\
\hline$X_{11 a}$ & 137 & 550 & 42.52 & \\
\hline $\mathrm{X}_{11 \mathrm{~b}}$ & 263 & 550 & 42.52 & \multirow{3}{*}{45.89} \\
\hline $\mathrm{X}_{12}$ & 400 & 550 & 46.23 & \\
\hline$X_{13 a}$ & 337 & 550 & 48.49 & \\
\hline $\mathrm{X}_{13 \mathrm{~b}}$ & 63 & 550 & 48.49 & \multirow{4}{*}{41.33} \\
\hline $\mathrm{X}_{14}$ & 400 & 550 & 41.78 & \\
\hline $\mathrm{X}_{15}$ & 400 & 550 & 39.34 & \\
\hline $\mathrm{X}_{16 \mathrm{a}}$ & 137 & 550 & 43.43 & \\
\hline $\mathrm{X}_{16 \mathrm{~b}}$ & 227 & 550 & 43.43 & \\
\hline
\end{tabular}

The microtome sections were stained by lactophenol blue and $100 \mathrm{mM} \mathrm{MBTH}$, respectively, as described in Chapter II.3.3. The image analySIS ${ }^{\circledR}$ program was used to calculate the total cell wall areas. The analyzed year ring of $6.227 \mathrm{~mm}$ was divided into sections of $1 \mathrm{~mm}$ in length and average values of cell wall areas $(\%$ total of the section area) were calculated with a section from wood treated for 6 weeks with T. versicolor Strain $6 .{ }^{*}$ See corresponding footnote in Table S1 
The total cell wall areas calculated for a wood section for 6 weeks with $T$. versicolor Strain 6 (Table S3) and for a wood section incubated for 6 weeks without a fungus (Table S4) corresponded only roughly to each other. Therefore, a more detailed look on cell wall lumina and rays has been taken on one specific area in the earlywood (section area $\mathrm{X}_{13 \mathrm{~b}}$ to $\mathrm{X}_{16 \mathrm{a}}$ ) and in the latewood (section area $\mathrm{X}_{1}$ to $\mathrm{X}_{3 \mathrm{a}}$ ) in both samples (Table S5) that should present tissue of same age and developmental stage expected to come from the same year ring of a cut larger $A$. grandis wood block.

The values presented in Table S5 indicate that randomly selected wood samples generated from the same year ring area of one larger $A$. grandis wood block (compare Chapter III.3.2 and Fig. 1) can naturally differ in number of cells present in different sections of the same age and developmental stage. When more cells are present in a section of latewood, the total lumina area is likely to decrease as deduced from the latewood data presented in the Table S5. At the same time with more cells per area, the average cell wall width might reduce somewhat (Table S5). In order to get further insight into the variability of wood tissue of the same age and developmental stage, seven images in line of the $X_{1}$ area of wood section incubated without a fungus were analyzed (Table S6).

Table S5 Summary of size and area values as determined from microtome sections from an $A$. grandis wood block incubated for 6 weeks without a fungus and from the wood blocks incubated for 6 weeks with T. versicolor Strain 6

\begin{tabular}{|c|c|c|c|c|c|c|c|}
\hline \multirow{2}{*}{\multicolumn{2}{|c|}{ Wood sample }} & \multicolumn{2}{|c|}{ Cell wall } & \multicolumn{2}{|c|}{ Lumina } & \multicolumn{2}{|c|}{ Rays } \\
\hline & & Average & Total & Number & Total & Number & Total \\
\hline \multirow[b]{2}{*}{ Untreated } & Latewood & 6.93 & 55.58 & 182 & 35.86 & 2 & 8.56 \\
\hline & Earlywood & 2.17 & 41.33 & 131 & 51.56 & 2 & 7.06 \\
\hline \multirow{2}{*}{$\begin{array}{c}\text { Treated } \\
\text { with } \\
\text { fungus }\end{array}$} & Latewood & 6.20 & 64.37 & 236 & 28.86 & 2 & 6.77 \\
\hline & Earlywood & 2.66 & 43.36 & 146 & 53.90 & 1 & 2.74 \\
\hline
\end{tabular}

* Values for cell walls were taken from Table S3, S4 and S5. For latewood, the respective images $X_{1}$ to $X_{3 a}$ and for earlywood the respective images $X_{13 b}$ to $X_{16 a}$ were chosen for comparison of the different wood sections in area of alike age and developmental stage. Values for lumina and rays were determined as described in Chapter III.3.5 
The different area differed in total cell wall in between $54.0 \%$ to $58.3 \%$ indicating the natural variance in cell growth within $A$. grandis wood at the same period of time. Likewise, there was a considerable variation in total lumen area (between $34 \%$ to $42 \%$ ) but this was e.g. influenced by the number and size of rays in an image and partially also by the total number of cells present in the respective sample (Table S6). Accordingly, in order to strengthen the validity of tendencies seen in these data more samples over the complete year rings will have to be analyzed. It is possible that the different effects in the latewood might be larger than in earlywood (compare values in Table S5). When considering the fact that in the analyzed area of the fungal treated sample, there was only one ray in contrast to the corresponding area in the untreated control with about 10 cells more in the total wood fibers' region, the variance of the total lumen area and total cell wall area was less than in the comparison of the latewood values (Table S5).

Table S6 Summary of area values as determined from microtome sections from seven different photos in line to each other of the $\mathrm{X}_{1}$ area (compare Table S5) of latewood from an $A$. grandis wood block incubated for 6 weeks without a fungus

\begin{tabular}{l|ccccccc|c}
\hline $\begin{array}{l}\text { Number of } \\
\text { analyzed } \\
\text { image }\end{array}$ & $\mathbf{1}$ & $\mathbf{2}$ & $\mathbf{3}$ & $\mathbf{4}$ & $\mathbf{5}$ & $\mathbf{6}$ & $\mathbf{7}$ & Average \\
\hline $\begin{array}{l}\text { Number } \\
\text { of cells }\end{array}$ & 177 & 218 & 187 & 173 & 190 & 163 & 161 & $181.29 \pm 19.53$ \\
\hline $\begin{array}{l}\text { Cell wall } \\
\text { area (\%) }\end{array}$ & 57.33 & 58.26 & 57.10 & 58.22 & 58.15 & 54.00 & 53.84 & $56.74 \pm 1.99$ \\
$\begin{array}{l}\text { Lumina } \\
\text { total area } \\
(\%)\end{array}$ & 38.20 & 37.46 & 34.09 & 35.94 & 38.05 & 42.42 & 42.42 & $38.37 \pm 3.11$ \\
$\begin{array}{l}\text { Number of } \\
\text { rays }\end{array}$ & 3 & 3 & 6 & 3 & 3 & 2 & 2 & $3.14 \pm 1.35$ \\
$\begin{array}{l}\text { Ray area } \\
(\%)\end{array}$ & 4.48 & 4.28 & 8.80 & 5.54 & 3.80 & 3.58 & 3.74 & $4.89 \pm 1.85$ \\
\hline
\end{tabular}

Note that photo number 7 corresponds roughly to the $X_{1}$ sample analyzed in Table S5 
III.7.4 Comparison of parameters of $A$. grandis wood blocks after incubation of 6 , 12 and 19 weeks with or without $T$. versicolor

Table S7 Size of complete wood blocks of $A$. grandis incubated for 19 weeks at $22^{\circ} \mathrm{C}$ and $65 \% \mathrm{RH}$ on $5 \%$ malt $2 \%$ agar with $T$. versicolor Strain 6 in comparison with uninfected controls

\begin{tabular}{|c|c|c|c|c|c|c|c|}
\hline \multirow{2}{*}{ Wood } & \multirow{2}{*}{ Block } & \multicolumn{3}{|c|}{ Control } & \multicolumn{3}{|c|}{ Trametes versicolor Strain 6} \\
\hline & & $\begin{array}{c}\begin{array}{c}\text { Length } \\
(\mathrm{mm})\end{array} \\
\end{array}$ & $\begin{array}{l}\text { Width } \\
(\mathrm{mm})\end{array}$ & $\begin{array}{c}\text { Thickness } \\
(\mathrm{mm})\end{array}$ & $\begin{array}{c}\text { Length } \\
(\mathrm{mm})\end{array}$ & $\begin{array}{l}\text { Width } \\
(\mathrm{mm})\end{array}$ & $\begin{array}{c}\text { Thickness } \\
\text { (mm) }\end{array}$ \\
\hline \multirow{5}{*}{ 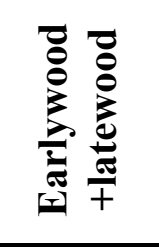 } & 1 & 30.11 & 10.11 & 5.07 & 29.91 & 10.01 & 4.98 \\
\hline & 2 & 30.21 & 10.10 & 5.09 & 30.18 & 10.04 & 5.12 \\
\hline & 3 & 29.83 & 9.96 & 5.09 & 30.18 & 10.11 & 5.10 \\
\hline & 4 & 30.13 & 10.08 & 5.14 & 30.26 & 9.97 & 5.03 \\
\hline & 5 & 30.21 & 10.24 & 5.08 & 30.12 & 10.02 & 5.07 \\
\hline \multirow[t]{2}{*}{ Average } & & 30.10 & 10.10 & 5.09 & 30.13 & 10.03 & 5.06 \\
\hline & & \pm 0.16 & \pm 0.10 & \pm 0.03 & \pm 0.13 & \pm 0.05 & \pm 0.06 \\
\hline \multirow{5}{*}{ 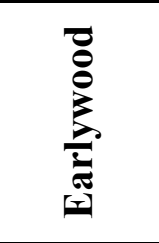 } & 1 & 29.89 & 10.01 & 5.15 & 29.88 & 9.70 & 4.66 \\
\hline & 2 & 29.93 & 10.06 & 5.13 & 30.06 & 9.21 & 4.52 \\
\hline & 3 & 29.94 & 9.92 & 5.01 & 29.80 & 9.58 & 4.36 \\
\hline & 4 & 30.02 & 9.93 & 5.04 & 30.07 & 9.42 & 4.84 \\
\hline & 5 & 30.03 & 10.04 & 5.14 & 29.96 & 9.35 & 4.94 \\
\hline \multirow[t]{2}{*}{ Average } & & 29.96 & 9.99 & 5.09 & 29.95 & 9.45 & 4.66 \\
\hline & & \pm 0.06 & \pm 0.06 & \pm 0.06 & \pm 0.12 & \pm 0.12 & \pm 0.23 \\
\hline
\end{tabular}
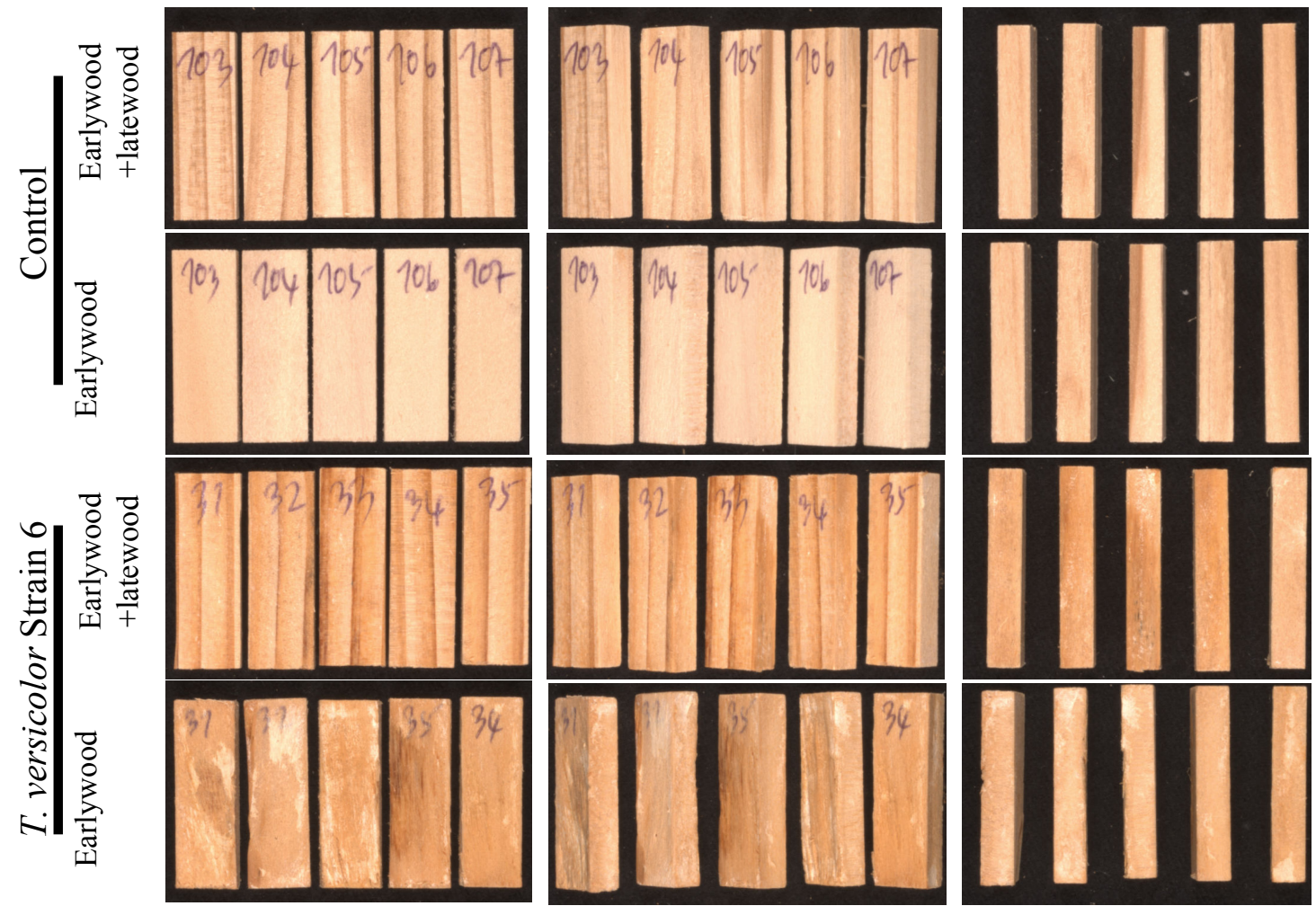

Fig. S6 Dried $A$. grandis wood blocks incubated for 6 weeks at $22^{\circ} \mathrm{C}$ and $65 \% \mathrm{RH}$ on $5 \%$ malt $2 \%$ agar with $T$. versicolor Strain 6 in comparison with uninfected control 


\section{CHAPTER IV}

Contrasting ecological functions of Abies grandis wood extractives on protection against white-rot fungi and induction of laccase activity 

IV. Contrasting ecological functions of Abies grandis wood extractives on protection against white-rot fungi and induction of laccase activity

\section{IV.1 Abstract}

The white-rot fungi Pleurotus ostreatus 8F6 and Trametes versicolor Strain 6 are amongst the best laccase producers on lignocellulosic substrates added to fungal cultures. In this work, the ability of $A$. grandis wood and its wood extractives to influence laccase production of the two fungi was evaluated. Laccase induction of T. versicolor Strain 6 in BSM (basidiomycetes medium) liquid medium with added A. grandis wood particles was found comparable with pine (Pinus sylvestris). A maximum concentration of $600 \mathrm{U}^{-1}$ after 16 days of incubation was reached with T. versicolor Strain 6 in $100 \mathrm{ml} \mathrm{BSM} \mathrm{liquid} \mathrm{medium} \mathrm{with} 0.1 \mathrm{~g}$ added A. grandis wood particles (700 $\mathrm{U}^{-1}$ when using $0.5 \mathrm{~g}$ wood). Laccase activity was also induced in P. ostreatus $8 \mathrm{~F} 6$ with added $0.1 \mathrm{~g} A$. grandis wood particles with a maximum laccase activity of $230 \mathrm{U}^{-1}$ reached after 10 days of incubation $\left(300 \mathrm{U} \mathrm{l}^{-1}\right.$ when using $0.5 \mathrm{~g}$ wood). In contrast to laccase activities, manganese-dependent peroxidase (MnP), lignin peroxidase (LiP) and cellulase activity was not found to be induced by the wood particles.

When $A$. grandis wood extractives have been eliminated through boiling in water or through boiling in water and subsequently in acetone, the washed-out wood had still laccase inducing activity but laccase production was delayed and not as high as with the original wood, suggesting extractives to play an important role in laccase induction. Another general factor in laccase induction was likely the high $\mathrm{C}$ to $\mathrm{N}$ ratio of the wood. Extracted wood lost however also mineral compounds, such as calcium, magnesium, manganese, potassium and zinc but the effects by this were not further followed up. For both the water extractives and the acetone extractives, induction of laccases was documented in both fungi. Laccase activity was obtained at highest amounts of about $186 \mathrm{U}^{-1}$ and $520 \mathrm{U}^{-1}$ by P. ostreatus $8 \mathrm{~F} 6$ and T. versicolor Strain 6 , respectively, when water extractives from $0.5 \mathrm{~g}$ A. grandis wood was added to the BSM cultures. Laccase activities in cultures to which acetone extractives from $0.5 \mathrm{~g}$ A. grandis wood were added, showed best activities of $143 \mathrm{U} \mathrm{l}^{-1}$ and $191 \mathrm{U} \mathrm{l}^{-1}$ with P. ostreatus 8F6 and T. versicolor Strain 6, respectively.

Water extractives were further fractioned on a silica gel column. Extractives eluted with $40 \%$ or $60 \%$ methanol showed highest laccase induction with about $120 \mathrm{U}^{-1}$ in case of $P$. ostreatus 8F6. In case of T. versicolor Strain 6, extractives eluted with $40 \%$ or $60 \%$ methanol gave highest laccase induction of about $800 \mathrm{U} \mathrm{I}^{-1}$ or 
IV. Contrasting ecological functions of Abies grandis wood extractives on protection against white-rot fungi and induction of laccase activity

$600 \mathrm{U}^{-1}$, respectively. Moreover, the first flow through from the column and the $20 \%$ methanol fraction also caused laccase induction in T. versicolor Strain 6 in the order of about $100 \mathrm{U} \mathrm{l}^{-1}$ and they gave also a small increase in laccase production in P. ostreatus $8 \mathrm{~F} 6$.

By gas chromatography, within the water extractives and the acetone extractives, nine distinct phenolic compounds were found: 2-methoxyphenol (guaiacol), 4-hydroxybenzaldehyde, 1,4-dihydroxybenzene (hydroquinone), 3,5-di-tertbut-4hydroxy-toluene (butylhydroxytoluol), 3-methoxy-4-hydroxybenzaldehyde (vanillin), 4-hydroxybenzoic acid, 3-methoxy-4-hydroxybenzoic acid (vanillic acid), 4-hydroxy cinnamic acid ( $p$-coumaric acid), and 4-hydroxy-3-methoxy cinnamic acid (ferulic acid). Of these, at $1 \mathrm{mM}$ concentrations 4-hydroxy cinnamic acid, 3,5-di-tertbut-4hydroxy-toluene and 4- hydroxybenzaldehyde induced laccase production in P. ostreatus 8F6 cultures and 4-hydroxy cinnamic acid and 3,5-di-tertbut-4-hydroxytoluene induced high laccase production in T. versicolor Strain 6 cultures. 4-hydroxy-3methoxy-cinnamic acid, 3-methoxy-4-hydroxybenzaldehyde and 2-methoxyphenol were also effective in P. ostreatus 8F6 and 4-hydroxy-3-methoxy-cinnamic acid and 2-methoxyphenol in T. versicolor Strain 6, but the absolute yields in laccase production were lower. Synergistic effects on laccase induction by T. versicolor Strain 6 were documented for 4-hydroxy cinnamic acid + 3,5-di-tertbut-4-hydroxy-toluene. Laccase activities were two times higher than with 4-hydroxy cinnamic acid alone and about five times higher than with 3,5-di-tertbut-4-hydroxy-toluene alone.

Application of 1,4-dihydroxybenzene and $p$-benzochinone at the concentration of $1 \mathrm{mM}$ applied to liquid cultures inhibited growth of both fungi. Moreover, water extractives and acetone extractives of $A$. grandis were shown to inhibit growth of fungi in agar diffusion tests. Mycelial growth of the dung fungus Coprinopsis cinerea Okayama 7 was highest inhibited by both types of wood extractives, whilst the whiterot species P. ostreatus $8 \mathrm{~F} 6$ and T. versicolor Strain 6 were both less sensitive to the wood extractives. Moreover, wood blocks immersed into water extractives or into acetone extractives inhibited mycelial growth on the surface of $A$. grandis wood blocks in case of $C$. cinerea Okayama 7, of P. ostreatus 8F6 and of T. versicolor Strain 6. Apparently, wood extractives have a protective function to the A. grandis wood. Accordingly, wood extracted with water or with water and with acetone lost resistance against fungal decay. Extracted wood was easily degraded by T. versicolor Strain 6 ( $40 \%$ mass loss versus $15 \%$ of original wood) and somewhat also by $C$. cinerea 
IV. Contrasting ecological functions of Abies grandis wood extractives on protection against white-rot fungi and induction of laccase activity

Okayama 7 ( $2 \%$ mass loss versus $0 \%$ of original wood), although there was no much difference in decay by $P$. ostreatus $8 \mathrm{~F} 6$ (about $10 \%$ mass loss in all cases).

Laccase isoenzymes of culture supernatants were analyzed by gel electrophoresis and mass spectrometry identification. Laccase isoenzymes from T. versicolor Strain 6 detected were laccase I and laccase III and from P. ostreatus 8F6 laccase II. Gel electrophoresis documented that always the same isoenzymes were induced, regardless of whether wood, wood extractives, fractions of wood extractives or specific phenolic compounds were tested for enzyme induction. Using purified laccase III of $T$. versicolor Strain 6 or a commercial recombinant produced laccase (Novozyme, EEC No. 420-150-4 from Aspergillus sp.), chemical actives on A. grandis wood fibers were documented by microscopy, MBTH-staining of the fibers, MBTHstaining of the buffer supernatants of laccase-treated $A$. grandis wood particles and by spectrophotometric and GC-MS-analysis of these supernatants.

Key words: Abies grandis, butylhydroxytoluol, carbon, chromatography fraction, Coprinopsis cinerea, decay fungi, 1,4-dihydroxybenzene, p-benzochinone, 3,5-ditertbut-4-hydroxy-toluene, GC-MS analysis, gel electrophoresis, grand fir, growth inhibition, 4-hydroxy cinnamic acid, laccase induction, lignin peroxidase, manganese peroxidase, nitrogen, phenolic compounds, Pleurotus ostreatus, p-coumaric acid, Trametes versicolor, wood extraction, wood extractives 
IV. Contrasting ecological functions of Abies grandis wood extractives on protection against white-rot fungi and induction of laccase activity

\section{IV.2 Introduction}

Basidiomycetous species are gaining increasing interest because of their capability to cover in degradation a wide variety of natural and synthetic materials, amongst many environmentally persistent organopolutants (Carbajo et al. 2002; Palmieri et al. 2000). Manifold research on this topic has been undertaken because of its great potential in various biotechnological applications, such as bioremediation, animal feed improvement, pulp and paper production, textile dye bleaching, effluent detoxification, wastewater treatment, in the removal of phenolic from wines and transformation of antibiotics and steroid (Mayer and Staples 2002; Terron et al. 2004). On the other hand, wood rotting fungi can be a problem for example for wood constructions. In contrast, whilst white-rot fungi eventually will degrade the cellulose and hemicellulose, they attack either simultaneously or selectively the lignin. Lignin degradation by white-rot fungi depends on the action of extracellular as ligninolytic enzymes, such as lignin peroxidase (LiP), manganese peroxidase $(\mathrm{MnP}), \mathrm{H}_{2} \mathrm{O}_{2}$-generating enzymes, and laccases. White-rot fungi produce various isoforms of extracellular oxidases and peroxidases, which are involved in the degradation of lignin in their natural environments. Low molecular mass compounds can help as mediators the actions of laccase, MnP and LiP. The lignocellulose-degrading enzymatic system is important for the fungi for substrate colonization and carbon acquisition for their nutrition (Elissetche et al. 2006; Sumthong et al. 2008; Valaskova and Baldrian 2006).

Laccase (benzenediol: oxygen oxidoreductase, E.C. 1.10.3.2) being in focus of this study as part of the complex enzymatic system for lignin degradation of white-rot fungi is one of the blue copper oxidases from white-rot fungi. Laccases catalyze the oxidation of a wide variety of organic substrates, specifically aromatic compounds containing hydroxyl and amine groups and thus also participate in the degradation of wood components (Baldrian and Gabriel 2002; Dominguez et al. 2007). In most instances in biotechnological applications, laccases from basidiomycetes are used. Natural amounts of laccase as produced by these fungi are often not sufficient for the applications on industrial scale. Therefore, effects of addition of inducers in laccase production were widely studied. To increase the production of laccase, addition of natural phenolic compounds from wood is one good possibility to obtain a higher yield of laccase production (De Souza et al. 2004; Gregorio et al. 2006; Johansson et al. 1998; Thurston 1994). 
IV. Contrasting ecological functions of Abies grandis wood extractives on protection against white-rot fungi and induction of laccase activity

Laccases may degrade certain wood extractives (Dorado et al. 2001) and laccase production by a white-rot fungus might be induced by specific wood extractives (De Souza et al. 2004; Dorado et al. 2001; Thurston 1994). Wood extractives are a large variety of wood components that are soluble in neutral organic solvents or water and can be collected from the wood as a minor fraction by liquid extractions. The isolation of wood extractives is carried out by extraction with neutral solvent mixtures and/or with single solvents in succession. The main components of the water-soluble part of wood comprise carbohydrates, protein and inorganic salts. Fatty acids, resin acids, waxes, tannins and coloring matter are the most important substances extractable by solvents such as ether, acetone, benzene, ethanol, dichloromethane, or a mixture of them. The volatile fraction containing for example terpenes in the case of softwoods (Fengel and Wegener 1983; Sjostrom 1993) is isolated mainly by steam distillation. If steam distillation is not done, the volatile compounds will be collected either in the water soluble fraction or in the fractions extracted with organic solvent. There are several techniques to determine and identify wood extractive compounds. Gas chromatography followed by mass spectroscopy (GC-MS) is a modern fractional method to determine and identify wood extractives. Several studies on the characterization of wood extractives showed that capillary GC is by far the most convenient and comprehensive technique available for separating individual components in wood extractives for subsequent identification (Fengel and Wegener 1983; Fernandez et al. 2001; Sjostrom 1993).

To maintain the diversified biological functions of wood in the living tree, different types of wood extractives are necessary. For example, phenolic substrates protect the wood against microbiological damage or insect attacks (Sjostrom 1993). Extractives give wood its color, its odor and to some extent, there is a positive correlation of content of extractives to its physical properties such as wood density and strength. Wood extractives have various effects on other physical properties with consequences in applications. For example, quinones extremely affect the quality of wood finishing and performance of wood adhesives. Moreover, nonpolar extractives (e.g. terpenoids, oils, fats and wax) can influence hydroscopicity and permeability, and on other hand increase the flammability of the wood by their high caloric value (Imamura 1989). Further, the role of wood extractives in protection of wood from biological is of considerable significance not only for the living tree and but also for wood products made from a tree (Dorado et al. 2001). 
IV. Contrasting ecological functions of Abies grandis wood extractives on protection against white-rot fungi and induction of laccase activity

The neophyte $A$. grandis has been shown to have a high potential both for sustainable wood production and for applications in the wood products industry. Information on this tree species and its wood has been collected since it had been introduced from North America to Germany. The tree species shows good and fast growth in German locations (Röhrig 1981; Spellmann et al. 2010) and the wood properties make it an interesting species for all kinds of wood products (Vos and Kharazipour 2010). Therefore, the wood industries in the country might be supported by an extension of silviculture with the fast growing conifer and by long-term supply from plantations with the fast growing tree in short rotation cycles (Polle et al. 2008). The behavior of wood degrading-fungi with living and with dead $A$. grandis wood has been observed either in natural forests and plantations or in laboratory tests with solid wood blocks or wood-derived products. Mainly, A. grandis wood is prone to attack by brown-rot species but it possesses a certain degree of resistance against white-rot species (Hawighorst et al. 2010; Kües et al. 2009; Navarro-González et al. 2008, Chapter II and III of this thesis). Wood extractives may play a role in this (Chapter III of this thesis).

To better understand the functions of $A$. grandis wood extractives and their influences on wood decay fungi, the work presented in this chapter focused on the production of laccase induced by $A$. grandis wood extractives and specific phenolic compounds in it as natural inducers. In contrast, wood extractives as a whole function to protect $A$. grandis wood against enzymatic degradation by white-rot fungi. Mycelial growth of different fungi was shown to be inhibited by $A$. grandis wood extractives.

\section{IV.3 Materials and methods}

\section{IV.3.1 Wood}

A. grandis and Pinus sylvestris wood was obtained from the Stadtwald Meschede, Sauerland, Germany. Mini-wood blocks for the duration test EN 113 (1996) of a size of 30 (longitudinal) x 10 (tangential) x 5 (radial) $\mathrm{mm}$ with earlywood+latewood were obtained as described in Chapter II.3.2.

Wood particles of $A$. grandis and P. sylvestris were prepared in cooperation with the work group of Prof. Kharazipour from the Division of Molecular Wood Biology and Technology and Technical Mycology, Büsgen-Institute, Göttingen, Germany, by 
IV. Contrasting ecological functions of Abies grandis wood extractives on protection against white-rot fungi and induction of laccase activity

chipping wood log into wood shaving (length about $1 \mathrm{~cm}$, width about $1 \mathrm{~cm}$ and height about $2 \mathrm{~cm}$ ) in a drum chipper (Klöckner Trommelhacker KTH 120 x 400 H2WT, Klöckner Wood Technology GmbH, Hirtscheid, Germany), flaking these with a knife ring flaker (Condux HS 350, Condux Maschinenbau GmbH. and. Co. KG, Hanau, Germany) into smaller particles and then sieving the wood particles (60-40 mesh), following the methods presented in Müller et al. (2009).

In order to preserve the entire volatile compounds and not to loose them by heating up during wood grinding, wood powder of $A$. grandis was prepared from chipped wood blocks [50 (longitudinal) x 20 (tangential) x 15 (radial) mm] by using a ball mill (Retsch MM 2000, F. Kurt Retsch GmbH \& Co. KG, Düsseldorf, Germany) with liquid nitrogen as cooling system during processing. Wood chips were grinded with the ball mill at $80 \mathrm{rpm}$ for periods of $3 \mathrm{~min}$ until the wood was fully powdered. After grinding, the wood powder was stored at $-20^{\circ} \mathrm{C}$ until Soxhlet extraction (Fig. 1).



Fig. 1 A. grandis (left to right) wood block [50 (longitudinal) x 20 (tangential) x 15 (radial) $\mathrm{mm}$ ], wood shaving after chipping, wood powder after grinding and wood powder after Soxhlet extraction

\section{IV.3.2 Wood extractives}

Wood powder (10 g) was extracted with a Soxhlet apparatus in $450 \mathrm{ml}$ boiling water for 6 hrs, following TAPPI Test Method T 204 om-88 (1988). After extraction with water, wood powders were kept at $4^{0} \mathrm{C}$ until acetone extraction. Acetone extraction of wood powders was performed from a half of the wood amount used in the water extraction ( $5 \mathrm{~g}$ ) with $450 \mathrm{ml}$ boiling acetone for $6 \mathrm{hrs}$. After the acetone extraction, wood particles 
IV. Contrasting ecological functions of Abies grandis wood extractives on protection against white-rot fungi and induction of laccase activity

were kept at $4^{0} \mathrm{C}$ until hexane extraction. Hexane extraction of wood particles was performed from a half of the wood amount used in acetone extraction $(2.5 \mathrm{~g})$ with $450 \mathrm{ml}$ boiling hexane for $6 \mathrm{hrs}$. After the hexane extraction, wood particles were kept at $4^{\circ} \mathrm{C}$ until vacuum drying in a vacuum chamber (Heraeus Holding $\mathrm{GmbH}$, Hamburg, Germany) at $50^{\circ} \mathrm{C}$ for $24 \mathrm{hrs}$.

The water, acetone and hexane extractives were concentrated and evaporated to dryness using a rotatory evaporator (Heidolph, W105/38, V 220, and $\mathrm{Hz} 50$ ) at $60^{\circ} \mathrm{C}$ (water extractives) and $40^{\circ} \mathrm{C}$ (acetone and hexane extractives). Wood extractives were dissolved in dimethyl sulfoxide (DMSO) in a concentration of $1 \mathrm{ml}$ DMSO per $1 \mathrm{~g}$ extracted wood. One half $(5 \mathrm{ml}$ for the water extractives, $2.5 \mathrm{ml}$ for the acetone extractives, and $1.25 \mathrm{ml}$ for the hexane extractives) of the DMSO solution with dissolved extractives was used in $1.5 \mathrm{ml}$ portions for extraction in chloroform with a separating funnel that contained $50 \mathrm{ml}$ water, $50 \mathrm{ml}$ chloroform and, as an catalyst, $0.5 \mathrm{ml}$ phosphoric acid per $1.5 \mathrm{ml}$ of extractives solution applied. After shortly mixing, the $\mathrm{pH}$ value of the water phase was measured to adjust the $\mathrm{pH}$ to about 2 . Then, the mixture was shaken for 10-15 min prior to finally separate the chloroform fraction from the water phase. For all types of wood extractives, chloroform extraction was once repeated with $20 \mathrm{ml}$ chloroform each. The chloroform fraction was concentrated and evaporated to dryness by rotatory evaporation at $40^{\circ} \mathrm{C}$. Wood extractives from chloroform fractions were dissolved in DMSO at a concentration of $1 \mathrm{ml}$ DMSO per $1 \mathrm{~g}$ extracted wood.

Water soluble and acetone-soluble extractives were further resolved by Soxhlet extraction from $10 \mathrm{~g}$ dry wood particles (60-40 mesh) with boiling distilled water and acetone as described above, respectively for $6 \mathrm{hrs}$ each, following TAPPI Test Method T 204 om-88 (1998), then extracted in chloroform and dried and the dry extractives were quantified gravimetrically. Extractives were dissolved in $1 \mathrm{ml}$ DMSO per $1 \mathrm{~g}$ wood particles.

Wood extractives were analyzed by GC-MS (Gas Chromatography Mass Spectrometry, Agilent technologies, 6890N, network GC system, USA) to define individual extractive constituents in the solution. The extractives in $0.5 \mathrm{ml}$ DMSO were concentrated to dryness by speed-vacuum-centrifugation (Concentrator 5301, Eppendorf, Wesseling-Berzdorf, Germany) at $45^{\circ} \mathrm{C}$. Dry samples were dissolved in $50 \mu 1$ pyridine (PIERCE Biotechnology, Rockford, USA) and $50 \mu \mathrm{l}$ bis-N,Otrimethylsilyl trifluoroacetamide (BSTFA: PIERCE Biotechnology, Rockford, USA) 
IV. Contrasting ecological functions of Abies grandis wood extractives on protection against white-rot fungi and induction of laccase activity

for derivatization in order to increase the volatile phase of compounds and give more thermal stability to the samples. The extractives were directly dried and resuspended in $300 \mu 1$ toluene to be injected into the GC-MS (capillary column, Agilent 122-5532). Compounds were identified by comparing resulting data with standard references in the data program NIST (National Institute of Standards and Technology, Maryland, USA). Concentration of specific compounds were determined by injecting mixtures of specific compounds (2-methoxyphenol, 4-hydroxybenzaldehyde, 1,4-dihydroxybenzene, 3,5-ditertbut-4-hydroxy-toluene, 3-methoxy-4-hydroxybenzaldehyde, 4-hydroxybenzoic acid, 3-methoxy-4-hydroxybenzoic acid, 4-hydroxy cinnamic acid, and 4-hydroxy-3methoxy cinnamic acid) in defined amounts $(0.025 \mu \mathrm{g}$ each, $0.05 \mu \mathrm{g}$ each, $0.1 \mu \mathrm{g}$ each, $0.25 \mu \mathrm{g}$ each, $0.5 \mu \mathrm{g}$ each, $2.5 \mu \mathrm{g}$ each, and $5.0 \mu \mathrm{g}$ each) into the GC-MS and creating for comparison a standard curve of abundances for each tested compounds.

In another set of experiments, a chromatography fraction technique was used to fraction freeze-dried water extractives from $50 \mathrm{~g}$ wood. Extractives were dissolved with $20 \mathrm{ml}$ DMSO (20\% in water), centrifuged at $4000 \mathrm{rpm}$ for $20 \mathrm{~min}$ to separate the precipitate from the supernatant which was stored in a $25 \mathrm{ml}$ Falcon tube (SARSTEDT, Nümbrecht, Germany). The precipitate was then dissolved in $5 \mathrm{ml} 25 \%$ DMSO in water, sonicated and centrifuged at $4000 \mathrm{rpm}$ for $20 \mathrm{~min}$ in order to harvest the supernatant. Solving the precipitate in $5 \mathrm{ml} 25 \%$ DMSO was twice more repeated and all supernatants were collected in the $25 \mathrm{ml}$ Falcon tube. The extractives dissolved in DMSO were loaded onto an activated $5 \mathrm{ml}$ silica gel column [Adsorbex RP-18 (400 mg), E. Merck, Darmstadt, Germany] as supplied by Chromabond for selective sample preparation, Macherey-Nagel. The column was activated by running $12 \mathrm{ml}$ $100 \%$ methanol (LC-MS grade) through the column and, afterwards, twice $24 \mathrm{ml}$ water (HPLC grade). The effluent was kept as first elution fraction subsequently extractives bond to the column. Extractives loaded onto the column were successively released by each $5 \mathrm{ml}$ of $20 \%, 40 \%, 60 \%$, and $100 \%$ methanol and by finally $2 \mathrm{ml} \mathrm{DMSO}$. The chromatography fractions were concentrated to dryness by freeze drying. The dry fractions were dissolved with $10 \mathrm{ml}$ DMSO in order to use $200 \mu \mathrm{l}$ (corresponding to $1 \mathrm{~g}$ wood) aliquots of these for adding to fungal cultures. 
IV. Contrasting ecological functions of Abies grandis wood extractives on protection against white-rot fungi and induction of laccase activity

\section{IV.3.3 Fungal cultivations}

\section{IV.3.3.1 Microorganisms and general culture conditions}

All fungal strains used in this study were from the strain collection of the Division of Molecular Wood Biotechnology and Technical Mycology of the Büsgen-Institute, Göttingen. The white-rot strains Trametes versicolor Strain 6, Pleurotus ostreatus 8F6, and Coprinopsis cinerea Okayama 7 were maintained on BSM medium (see Chapter III.3.1 for recipe). Agar plugs (6 $\mathrm{mm}$ diameter) from the outer edge of a mycelial colony grown on YMG/T solid medium plates (per liter: $4 \mathrm{~g}$ yeast extract, $10 \mathrm{~g}$ malt extract, $4 \mathrm{~g}$ glucose, $100 \mathrm{~g}$ tryptophan, $10 \mathrm{~g}$ agar) were used as inocula for $100 \mathrm{ml}$ liquid BSM pre-cultures in $250 \mathrm{ml}$ Erlenmeyer flasks. Fungal liquid stock cultures were incubated stationary at $25^{\circ} \mathrm{C}$, but in case of $C$. cinerea Okayama 7 at $37^{\circ} \mathrm{C}$. Pre-cultures were 7 days old when using them for further cultivations.

\section{IV.3.3.2 Fungal cultures with wood, wood extractives or specific phenolic compounds}

Experiments were conducted in $100 \mathrm{ml}$ sterilized liquid BSM medium in $250 \mathrm{ml}$ Erlenmeyer flasks with added $0.5 \mathrm{~g}$ air-dried wood particles (original or after water or acetone or hexane extraction) or with added wood extractives corresponding to $0.5 \mathrm{~g}$ of wood. Flasks were inoculated with $4 \mathrm{ml}$ of mycelium of a fungal pre-culture, macerated for 1 min with a homogenizer (Ultra-turrax T25, Janke \& Kunkel GmbH. and Co. KG, Staufen, Germany) and cultures ( 3 per treatment) were stationary incubated for a total period of 3 weeks at $25^{\circ} \mathrm{C}$. Laccase, MnP, LiP and cellulase activity were determined every 2 days of cultivation.

In another set of experiments, each $4 \mathrm{ml}$ of macerated mycelium from a fungal pre-culture were added to $100 \mathrm{ml}$ of sterilized liquid BSM medium containing either $0.25,0.5,1.0$ or $2.0 \mathrm{ml}$ of wood extractives (at a concentration of wood extractives from $1 \mathrm{~g}$ of wood dissolved in $1 \mathrm{ml}$ DMSO) and 1.75 to $0 \mathrm{ml}$ DMSO (summing up to a total of $2 \mathrm{ml}$ DMSO per culture), or $0.25,0.5,1.0$ or $2.0 \mathrm{~g}$ of original, water extracted or acetone extracted or hexane extracted wood particles (60-40 mesh). Controls (with $2 \mathrm{ml}$ DMSO added) and controls with just BSM medium were run in parallel. Per treatment, 
IV. Contrasting ecological functions of Abies grandis wood extractives on protection against white-rot fungi and induction of laccase activity

three cultures were performed. Laccase activities were determined every two days of cultivation.

In another set of experiments, each $1 \mathrm{ml}$ of purified extractives from chromatography fractionation were added to $100 \mathrm{ml}$ of sterilized liquid BSM medium inoculated with $4 \mathrm{ml}$ of macerated mycelium from a fungal pre-culture. Controls (with $1 \mathrm{ml} \mathrm{DMSO}$ added) and controls with just BSM medium were run in parallel. Per treatment, three cultures were performed. Laccase activities were determined every two days of cultivation.

In another set of experiments, phenolic compounds (from Fluka: 2-methoxyphenol, 4-hydroxybenzoic acid, 4-hydroxy cinnamic acid, and 4-hydroxy-3methoxy cinnamic acid; from Sigma-Aldrich: 4-hydroxybenzaldehyde, 3,5-di-tertbut-4hydroxy-toluene, and 3-methoxy-4-hydroxybenzoic acid; from Merck: 1,4-dihydroxybenzene, 3-methoxy-4-hydroxybenzaldehyde, and $p$-benzochinone) were individually added into different fungal cultures (100 $\mathrm{ml}$ sterilized liquid BSM medium in $250 \mathrm{ml}$ Erlenmeyer flasks inoculated with $4 \mathrm{ml}$ of macerated mycelium from a fungal pre-culture) at final concentrations of $1 \mathrm{mM}$ of the respective phenolic compound. Cultures were stationary incubated for a total period of three weeks at $25^{\circ} \mathrm{C}$. All treatments including controls without added phenolic compound were performed in triplicate. Laccase activities were determined every two days of cultivation.

\section{IV.3.3.3 Influence of nutrients on laccase production}

Upon inoculation with $4 \mathrm{ml}$ of macerated fungal mycelium, T. versicolor Strain 6 was grown for 9 days at $25^{\circ} \mathrm{C}$ in stationary $100 \mathrm{ml}$ BSM cultures and laccase activity was determined at days 5, 7 and 9 of cultivation. After 9 days of cultivation, the culture supernatants were removed from the mycelia by sucking off the liquid with sterilized $25 \mathrm{ml}$ glass pipettes mounted to an electronic pipetting aid (Gilson, Pipetting Aid, Mettmenstetten, Switzerland). The fungal mycelia in the flask were washed for 3 times with each $50 \mathrm{ml} 0.9 \% \mathrm{NaCl}$ in order to remove all resident nutrient sources. For each washing step, the cultures were for $15 \mathrm{~min}$ shaken on a shaker at $60 \mathrm{rpm}$. Then, either $100 \mathrm{ml}$ of liquid autoclaved BSM or of liquid autoclaved modified BSM (BSM without glucose, asparagine, $\mathrm{Mn}\left(\mathrm{CH}_{3} \mathrm{COO}\right)_{2} \mathrm{x} 4 \mathrm{H}_{2} \mathrm{O}, \mathrm{Zn}\left(\mathrm{NO}_{3}\right) \times 4 \mathrm{H}_{2} \mathrm{O}$, and $\mathrm{Ca}\left(\mathrm{NO}_{3}\right)_{2} \mathrm{x} 4 \mathrm{H}_{2} \mathrm{O}$; BSM plus $0.5 \mathrm{~g}$ A. grandis wood particles) was added into flasks onto the mycelia and cultures were further stationary incubated at $25^{\circ} \mathrm{C}$ for 13 days. Laccase activity was 
IV. Contrasting ecological functions of Abies grandis wood extractives on protection against white-rot fungi and induction of laccase activity

measured every two days. After 13 days, total biomasses of harvested fungal mycelium were calculated from the weight of fresh mass and of dry mass. Fungal mycelium was harvested from culture supernatants by vacuum filtration with a Büchner funnel and filter papers (Macherey-Nagel GmbH and Co. KG, Düren, Germany). Mycelium was dried at $103 \pm 2^{\circ} \mathrm{C}$ for $24 \mathrm{hrs}$.

\section{IV.3.4 Wood decay tests following EN 113 and fungal growth test of wood blocks immersed in wood extractives}

For negative testing of wood extractives on fungal decay, A. grandis wood blocks of 120 (longitudinal) x 10 (tangential) x 5 (radial) $\mathrm{mm}$ with earlywood+latewood were oven dried at $103 \pm 2^{\circ} \mathrm{C}$ for $24 \mathrm{hrs}$, then mounted onto the ends of about $2 \mathrm{~m}$-long rubber tubes $(9 \mathrm{~mm}$ in diameter) with about $5 \mathrm{~mm}$ length in the longitudinal direction within the tubes. In order to prevent solvent to leak out from the other surfaces of the wood blocks, the rest of the wood blocks were sealed with Parafilm (Laboratory Film, Chicago, USA) leaving only the surface of the cross-section at the open ends of wood blocks free. The rubber tubes were fitted onto outlets affixed close to their bottoms to $1,000 \mathrm{ml}$ Erlenmeyer bottles. The Erlenmeyer flasks were positioned on a shelf at a height of about $2.2 \mathrm{~m}$ and the wood sticks on the rubber tubes were inserted with their free ends hanging into the opening of $250 \mathrm{ml}$ Erlenmeyer flasks standing on the floor. Water or acetone were filled into the $1,000 \mathrm{ml}$ flasks and with a natural hydrostatic pressure of about $200 \mathrm{hPa}$ [calculated from $\mathrm{P}=\rho \mathrm{gh} ; \mathrm{P}=$ hydrostatic pressure $(\mathrm{Pa})$, $\rho=$ fluid density $\left(\mathrm{kg} \mathrm{m}^{-3}\right), \mathrm{g}=$ gravitational acceleration $\left(\mathrm{m} \mathrm{s}^{-2}\right), \mathrm{h}=$ the height $\left.(\mathrm{m})\right]$, $200 \mathrm{ml}$ per wood block liquid ran in a time of about $3 \mathrm{hrs}$ through the plastic tubes and through the wood blocks into the $250 \mathrm{ml}$ flasks in order to thereby elute all extractives from the wood. Then, wood blocks were cut into three wood blocks of smaller sizes of 30 (longitudinal) x 10 (tangential) x 5 (radial) mm, thereby, discarding the $1.5 \mathrm{~cm}$ from both ends. Wood blocks were sterilized and used in wood decay tests following EN 113 (1996). Sterile wood blocks with the original extractives [size 30 (longitudinal) x 10 (tangential) x 5 (radial) $\mathrm{mm}$ ] served as control.

In another set of experiments, oven dried A. grandis wood blocks [30 (longitudinal) x 10 (tangential) x 5 (radial) mm; dried at $103 \pm 2^{\circ} \mathrm{C}$ for $24 \mathrm{hrs}$ ] were sterilized, immersed for $5 \mathrm{sec}$ into wood extractives (from an extraction of $4 \mathrm{~g}$ wood particles dissolved in $1 \mathrm{ml}$ DMSO), and air dried for 6 hrs. For each type of wood 
IV. Contrasting ecological functions of Abies grandis wood extractives on protection against white-rot fungi and induction of laccase activity

treatment (treated wood with extractives, or control wood) and each fungus tested (T. versicolor Strain 6, P. ostreatus 8F6 and C. cinerea Okayama 7), six wood blocks were laid on metal holders (1.5 mm in height) on fungal mycelium grown for 1 week (at $25^{\circ} \mathrm{C}$ for T. versicolor Strain 6 and P. ostreatus $8 \mathrm{~F} 6$ and $37^{\circ} \mathrm{C}$ for C. cinerea) on $5 \%$ malt extract, $2 \%$ agar for further treatment as described in Chapter II.3.2, following EN 113 (1996) with the modifications of Bravery (1978). Petri dishes were incubated for 10 weeks at $22^{\circ} \mathrm{C}$ and $65 \% \mathrm{RH}$. The extents of surface growth were recorded and photographed by a digital camera.

\section{IV.3.5 Effect of wood extractives on growth of fungi}

Antibiotic activities of wood extractives (150 $\mu$ l water or acetone extractives in a concentration of $1 \mathrm{ml}$ DMSO per $1 \mathrm{~g}$ dried wood) were tested in Petri plates (90 $\mathrm{mm}$ in diameter) containing $20 \mathrm{ml}$ of solid $2 \%$ malt extract agar medium (1\% agar) against spore suspensions of $C$. cinerea Okayama 7 and against suspensions of aerial hyphae of T. versicolor Strain 6 and P. ostreatus 8F6, respectively. In some experiments with C. cinerea Okayama 7, alternatively plates with $20 \mathrm{ml} \mathrm{YMG/T} \mathrm{(Yeast} \mathrm{and} \mathrm{Malt} \mathrm{Extract}$ with Glucose Media, Granado et al. 1997) were used to which 5 mM ABTS [2,2'-azinobis (3-ethylbenzthiazoline-6-sulfonate)] was added. Spores and mycelial debris were collected from fungal cultures grown for 7 days on $20 \mathrm{ml} 2 \%$ solid malt extract medium (T. versicolor Strain 6 and $P$. ostreatus $8 \mathrm{~F} 6$ at $25^{\circ} \mathrm{C}$, C. cinerea Okayama 7 at $37^{\circ} \mathrm{C}$ ) by scraping them off from the aerial mycelium with a spatula from the agar surface into $10 \mathrm{ml}$ of sterile $\mathrm{H}_{2} \mathrm{O}$. Spores (oidia) of $C$. cinerea and small mycelial fragments from the two other species were separated from larger mycelial fragments by filtration through sterile glass wool placed in a bell-shaped funnel. The number of oidia in C. cinerea Okayama 7 solutions and the number of small hyphal fragments left from T. versicolor Strain 6 and P. ostreatus $8 \mathrm{~F} 6$ after filtration in solution was then determined with a hematocytometer (type Thoma-chamber: $0.05 \mathrm{~mm}$ in deepness and $0.0025 \mathrm{~mm}^{2}$ per 1 small square of the hematocytometer counting area). Spore and hyphal suspensions were adjusted to a concentration of $10^{4}$ spores or mycelial pieces $/ \mathrm{ml}$ solution. Each $0.5 \mathrm{ml}$ of a given fungal cell suspension were evenly spread per Petri dish over the entire surface of the respective agar medium, and a hole $(5 \mathrm{~mm}$ in diameter) was made with a cork borer in the agar in the center of the respective plate. An amount of $75 \mu 1$ wood extractives in DMSO was added into such a hole, and when 
IV. Contrasting ecological functions of Abies grandis wood extractives on protection against white-rot fungi and induction of laccase activity

the medium had completely absorbed the extractives, another $75 \mu 1$ was added. Agar plates with plated fungal cells with a total $150 \mu \mathrm{DMSO}$ added into their holes served as controls. In all cases, for controls and for the tests of extractives on the different fungi, three plates (replicates) were used. The plates were placed at $25^{\circ} \mathrm{C}$ (T. versicolor Strain 6 and P. ostreatus $8 \mathrm{~F} 6)$ or at $37^{\circ} \mathrm{C}$ (C. cinerea Okayama 7 ) for culturing. Inhibition zones of growth around punched holes were determined by measuring the diameter of the zones without any fungal growth after 5 days of incubation.

\section{IV.3.6 Enzymes}

\section{IV.3.6.1 Enzyme tests}

Laccase, $\mathrm{MnP}$ and Lip activities were tested in volume of $20 \mu 1$ in microtiter plates using a microplate spectrophotometer (Spectra max340PC, Molecular Devices, California, USA) for detection. Laccase activity was determined with $5 \mathrm{mM}$ ABTS in $120 \mathrm{mM}, \mathrm{pH} 5.0$ sodium acetate buffer. Oxidation of ABTS was followed up by an absorbance increase at $420 \mathrm{~nm}$. The enzyme activity was expressed in units defined as $1 \mathrm{U}=1 \mu \mathrm{mol}$ ABTS oxidized/min (Childs and Bardsley 1975; Majcherczyk et al. 1999). Activity of MnP was measured at $270 \mathrm{~nm}$ by following the description of Kuwahara et al. (1984) and Michel et al. (1991) using $5 \mathrm{mM}$ phenol red in $66 \mathrm{mM}$ sodium-succinate buffer, $\mathrm{pH}$ 5.0. The enzyme activity was expressed in units defined as $1 \mathrm{U}=1 \mu \mathrm{mol}$ phenol red oxidized/min. LiP activity was determined at $310 \mathrm{~nm}$ using $10 \mathrm{mM}$ veratryl alcohol in $250 \mathrm{mM}$, sodium-tartrate buffer, $\mathrm{pH} 3.0$ by following the description of Tien and Kirk (1984). The enzyme activity was expressed in units defined as $1 \mathrm{U}=1 \mu \mathrm{mol}$ veratryl alcohol oxidized $/ \mathrm{min}$.

Cellulase activity was assayed at $530 \mathrm{~nm}$ in $100 \mathrm{mM}$ sodium acetate buffer, $\mathrm{pH} 5.0$ at $50^{\circ} \mathrm{C}$ by release of reducing sugars as measured with 3,5-dinitrosalicylic acid (DNS) reagent, using the appropriate substrate 4\% (w/v) carboxymethylcellulose (CMC) ultra low viscosity (Fluka) in $100 \mathrm{mM}$ sodium acetate buffer, $\mathrm{pH} 5.0$ (König et al. 2002) in $1 \mathrm{ml}$ plastic cuvettes in a spectrophotometer (UV-DU800, Beckman coulter, Inc., California, USA). The enzyme activity was expressed in units defined as $1 \mathrm{U}=1 \mu \mathrm{mol}$ reducing of sugar (glucose) $/ \mathrm{min}$. 
IV. Contrasting ecological functions of Abies grandis wood extractives on protection against white-rot fungi and induction of laccase activity

\section{IV.3.6.2 Laccase treatment of $\boldsymbol{A}$. grandis wood particles}

A. grandis wood particles ( $0.1 \mathrm{~g}$ in $10 \mathrm{ml}$ of $50 \mathrm{mM}$ sodium acetate buffer, $\mathrm{pH} 5.0)$ were incubated in $2 \mathrm{U} \mathrm{ml}^{-1}$ T. versicolor Strain 6 laccase and in $2 \mathrm{U} \mathrm{ml}^{-1}$ of 15 min cooked T. versicolor Strain 6 laccase [laccase III as purified by Dwivedi (2006)] at $10 \mathrm{rpm}$ in $20 \mathrm{ml}$ Schott glass tubes (Schott, Mainz, Germany) fixed onto a moving rotary drum (Synchronous Motor, Belgium). The $100 \mu 1$ aliquot samples were periodically 1, 2, 3, 4 and $12 \mathrm{hrs}$ removed and centrifuged for $16,000 \mathrm{~g}$ for $5 \mathrm{~min}$. Changes in absorbance in the sample's liquid reflecting chemical alterations on the wood particles were measured by using spectrophotometer Beckman coulter, UV-DU800.

Recombinant Novo laccase $\left(2 \mathrm{U} \mathrm{ml}^{-1}\right)$ from Aspergillus sp. (Novozyme, EEC No. 420-150-4) was added to $0.1 \mathrm{~g}$ of A. grandis wood particles in $10 \mathrm{ml}$ of $50 \mathrm{mM}$ sodium acetate buffer, pH 5.0 and incubated for $1 \mathrm{hr}$ at $10 \mathrm{rpm}$ in $20 \mathrm{ml}$ Schott glass tubes fixed onto a moving rotary drum. In parallel, the same amounts of wood particles were incubated in $10 \mathrm{ml}$ sodium acetate buffer without laccase. Subsequently, wood particles were separated from the supernatant containing the enzyme. Some wood particles were taken up with forceps and transferred into $0.5 \mathrm{ml}$ of $100 \mathrm{mM} \mathrm{MBTH}$ (3-methyl-2-benzothiazolinon-hydrazone hydrochloride; from Fluka, Netherlands) added in a well of a porcelain spot dish. Staining was done at RT for $1 \mathrm{~min}$, and the MBTH solution was replaced $3 \mathrm{x}$ by $0.5 \mathrm{ml}$ of water prior to microscopy of the wood particles. Photos were taken at enlargements of $40 \mathrm{x}$ and $100 \mathrm{x}$ with a digital CCD camera (Color View II, Soft Imaging System GmbH, Münster, Germany) that was mounted onto the microscope and linked to a computer with an installed copy of the image software analySIS ${ }^{\circledR}$ (Soft Imaging System GmbH, Münster, Germany).

The supernatant containing laccase harvested after incubation of the wood particles was stained by $100 \mathrm{mM}$ MBTH in $1: 1$ relation $(0.5 \mathrm{ml}$ each). Supernatant harvested from incubation without laccase with and without MBTH, and supernatant with laccase without adding MBTH served as controls. For photographing, per treatment $160 \mu \mathrm{l}$ of sample solution were filled into individual wells of a 96-wellmicrotiter plate. Photographs were taken from the microtiter plate on a light box with illuminating from below. The absorbance of solutions was measured in a spectrophotometer at 320-700 $\mathrm{nm}$. The same supernatant containing laccase or harvested from incubation without laccase with and without MBTH or supernatant with laccase without adding MBTH was analyzed by GC-MS as described in Chapter IV.3.2. 
IV. Contrasting ecological functions of Abies grandis wood extractives on protection against white-rot fungi and induction of laccase activity

\section{IV.3.6.3 Gel electrophoresis and staining}

For separation of proteins and native in-gel staining, polyacrylamide gel-electrophoresis (PAGE) was performed with $4 \%$ stacking and $12 \%$ resolving gels in a double gel Multigel-long Biometra gel chamber (Biometra GmbH, Göttingen, Germany). 4\% stacking gels were prepared as follows: $2.5 \mathrm{ml}$ of $0.5 \mathrm{M}$ Tris $\mathrm{pH} 6.8,1.3 \mathrm{ml}$ of $37 \%$ acrylamide (from Fluka), $100 \mu 1$ 10\% (w/v) SDS (sodium dodecyl sulfate), $50 \mu 1$ 10\% (w/v) APS (ammonium peroxodisulfate, Sigma-Aldrich, Steinheim, Germany), $10 \mu 1$ TEMED (N,N,N',N'-tetramethylethylenediamine, Amersham Bioscience AB, Uppsala, Sweden), filled up to $10 \mathrm{ml}$ with $\mathrm{dH}_{2} \mathrm{O}_{2} .12 \%$ resolving gels were prepared as: $2.5 \mathrm{ml}$ of 1.5 M Tris pH 8.8, $4.0 \mathrm{ml}$ acrylamide (37\%), $100 \mu 1$ 10\% (w/v) SDS, $50 \mu 1$ 10\% (w/v) APS $5 \mu 1$ TEMED, filled up to $10 \mathrm{ml}$ with $\mathrm{dH}_{2} \mathrm{O}_{2}$.

Protein samples of $P$. ostreatus 8F6 and T. versicolor Strain 6 cultures $(20 \mu 1$ per slot; $10 \mu 1$ sample aliquots $+10 \mu 1$ loading buffer [contained Tris-Cl ( $\mathrm{pH} 6.8$ ), 2\% SDS, $10 \%$ glycerol, $0.025 \%(\mathrm{w} / \mathrm{v})$ bromphenol blue] or of Novo laccase from Aspergillus sp. (Novozyme, EEC No. 420-150-4; $893.4 \mathrm{U} \mathrm{ml}^{-1}$ in $10 \mu 1$ loading buffer for one gel slot) were reduced by $10 \mathrm{mM}$ DTT (DL-dithiothreitol) and denatured by boiling for $5 \mathrm{~min}$. From cultures with added $A$. grandis wood or with added water extractives or with added acetone extractives and from parallel cultures with added DMSO solvent, each $10 \mu 1$ of original supernatant was loaded per slot. In case of P. ostreatus 8F6 and T. versicolor Strain 6 cultures supplemented with specific phenolic compounds, each $2 \mathrm{ml}$ of the respective supernatants were 10-fold concentrated with a Vivaspin column (10.000 MWCO) from Sartorius (Sartorius GmbH, Göttingen, Germany) prior to loading $10 \mu \mathrm{l}$ concentrated sample aliquots onto the gels. In parallel native electrophoresis, for each of the analyzed cultures, the proteins in same amounts and volumes were neither reduced nor denatured. To determine the size of the denatured proteins, a molecular weight marker (\#SM0431, Fermentas GmbH, St. Leon-Rot, Germany) was used. The loading buffer for denatured and native probes contained $0.6 \mathrm{M}$ Tris-HCL (pH 6.8), 2\% SDS, 10\% glycerol, and 0.025\% bromphenol blue (w/v). Separation was performed at a constant current of $15 \mathrm{~mA}$ until the samples reached the resolving gel and gel electrophoresis was continued at $25 \mathrm{~mA}$ for separation in the resolving gel.

For Coomassie staining, gels with denatured proteins were fixed in $12 \%(\mathrm{w} / \mathrm{v})$ TCA (trichloroacetic acid) for at least $1 \mathrm{hr}$ and stained in a colloidal Coomassie staining 
IV. Contrasting ecological functions of Abies grandis wood extractives on protection against white-rot fungi and induction of laccase activity

solution $[10 \%$ phosphoric acid $(\mathrm{v} / \mathrm{v}), 10 \%$ ammonium sulphate $(\mathrm{w} / \mathrm{v})$ and $0.12 \%(\mathrm{w} / \mathrm{v})$ Coomassie Brilliant Blue G250 (Serva Electrophoresis GmbH, Heidelberg, Germany) in water/methanol $(4: 1, \mathrm{v} / \mathrm{v})$ solution] for over night. The gels were washed with water until the background level was reduced for an adequate band to background ratio. In case of native laccase staining, gels were washed in $100 \mathrm{ml}$ of $10 \mathrm{mM}$ sodium acetate buffer (pH 5.0) for at least $10 \mathrm{~min}$ and then incubated with $5 \mathrm{mM} \mathrm{MBTH}$ and $5 \mathrm{mM}$ DHPPA (3,4-dihydroxyhydrocinnamic acid; from Sigma-Aldrich) in the same buffer.

\section{IV.3.6.4 Protein identification}

Protein bands of Coomassie-stained gels were cut with a razor blade and gel pieces were digested as described by Havlis and Shevchenko (2004) with some modification. Briefly, gel pieces were washed twice with water for $15 \mathrm{~min}$ and subsequently once with $50 \%$ ethanol for destaining. Gel pieces were dehydrated with $100 \%$ acetonitril (from Fisher Scientific) for $20 \mathrm{~min}$ and dried in a vacuum centrifuge (Concentrator 5301, Eppendorf, Wesseling-Berzdorf, Germany) for $10 \mathrm{~min}$. Subsequently, the gel pieces were soaked in $2 \mu \mathrm{M}$ trypsin (from Promega) in $50 \mathrm{mM}$ ammonium bicarbonate buffer $(\mathrm{pH} 8.0)$ at $4^{\circ} \mathrm{C}$ for $1 \mathrm{hr}$ and digested for $30 \mathrm{~min}$ at $58^{\circ} \mathrm{C}$. Peptides were extracted from the gel pieces on a shaker first with $150 \mu 15 \%(\mathrm{v} / \mathrm{v})$ formic acid for $1 \mathrm{hr}$, followed by extraction with $150 \mu 150 \%$ acetonitril in 5\% formic acid. Extracts were combined, dried in the vacuum centrifuge and stored at $-20^{\circ} \mathrm{C}$ until further proceeding. For further processing, peptides were dissolved in $15 \mu \mathrm{l}$ of 5\% formic acid and 3 times $4 \mu 1$ were injected to an $12 \mathrm{~cm}$ capillary column packed with $3 \mu \mathrm{m}$ particles of Reprosil-Pur C18AQ (Dr. Manisch GmbH, Ammerbuch, Germany) for peptide separation. Analysis of the peptides was performed by mass spectrometry (ESI-LC-MS, HP 1100 Agilent; Esquire 3000, Bruker Daltonik). Each sample was analyzed in a mass range of the ion trap from 200 to $3,000 \mathrm{~m} / \mathrm{z}$. Analysis of the raw data was done with program Daltonic DataAnalysis (version 3.0 Bruker Daltonic $\mathrm{GmbH}$ ). Proteins were identified using mascot (version 2.2, Matrix Science, London, UK) by searching the obtained data against a protein database established by Dr. A. Majcherczyk from the whole NCBI GenBank entries (http://www.ncbi.nlm.nih.gov/) including all up to now known laccases of $P$. ostreatus and of $T$. versicolor. The following settings were used for the database search: fixed modification: C-carbamidomethylation; variable modification: 
IV. Contrasting ecological functions of Abies grandis wood extractives on protection against white-rot fungi and induction of laccase activity

M-oxidation; peptide mass tolerance: $1.4 \mathrm{Da}$; peptide charge: $1+, 2+$ and 3+; MS/MS tolerance: $0.4 \mathrm{Da}$; missed cleavages allowed: 1 .

\section{IV.3.7 Statistical analysis}

Group values for all parameters in the tests were compared by analysis of variance (ANOVA) tests using the Fisher's least significant difference (LSD) and Duncan's test procedure for multiple comparison (SPSS 8.0 for Windows; USA). Relations among the value of samples were compared for each factor to the controls kept under the same conditions than the samples of interest.

\section{IV.4 Results}

\section{IV.4.1 Enzyme production in fungal cultures in presence of $A$. grandis wood particles}

Previous experiments showed for the strains P. ostreatus 8F6 and T. versicolor Strain 6 that they can to certain extend decay A. grandis wood (see Chapter II and III). It was therefore of interest to define the ligninolytic enzymatic activities of the two strains when grown in presence of $A$. grandis wood. Therefore, the fungi were inoculated into $100 \mathrm{ml} \mathrm{BSM} \mathrm{liquid} \mathrm{medium} \mathrm{with} 0.5 \mathrm{~g} \mathrm{~A}$. grandis wood particles added and laccase, MnP, LiP and cellulase activities were measured over the time. Laccase activities were detected from both fungi (Fig. 2A and 2B). Laccase activities reached maximum values on day 14 and day 16 of cultivation for T. versicolor Strain 6 and P. ostreatus 8F6, respectively. The highest laccase activity obtained in cultures of $P$. ostreatus 8F6 was $227 \pm 4.8 \mathrm{U}^{-1}$ (Fig. 2A). The highest laccase activity was obtained by T. versicolor Strain 6 with $423 \pm 23.9 \mathrm{U} \mathrm{l}^{-1}$ (Fig. 2B). MnP, LiP and cellulase production were not detected for both fungi (Fig. 2A and 2B).

Laccase production by $P$. ostreatus and $T$. versicolor has previously been reported to be induced by some unspecified wood shaving and saw dust (Arora and Sandhu 1987; Moredo et al. 2003; Lorenzo et al. 2002) but knowledge on wood effects on laccase production of the fungal species is still scarce. Here, the quality of laccase production during growth with $A$. grandis wood compared against $P$. sylvestris wood was therefore tested. Addition of both wood types to BSM medium had a similar 
IV. Contrasting ecological functions of Abies grandis wood extractives on protection against white-rot fungi and induction of laccase activity

positive effect on laccase production of both fungi (Fig. 3). Laccase activities obtained by $T$. versicolor Strain 6 with $A$. grandis or $P$. sylvestris wood particles reached highest
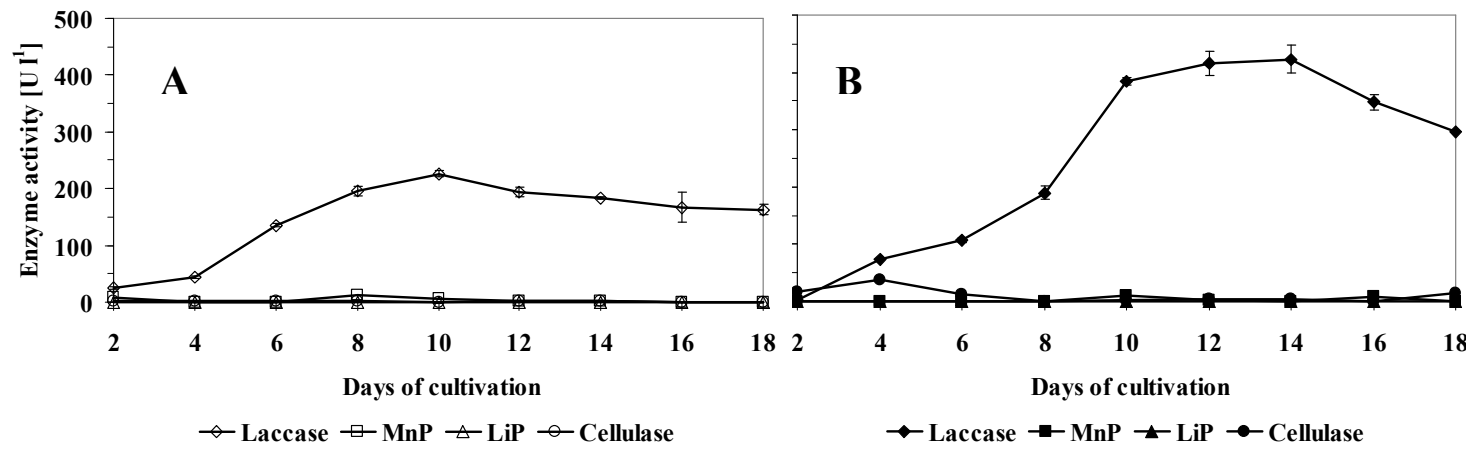

Fig. 2 Extracellular laccase, MnP, LiP and cellulase activities of A) P. ostreatus 8F6 and B) $T$. versicolor Strain 6 in stationary $100 \mathrm{ml} \mathrm{BSM}$ cultures with $0.5 \mathrm{~g}$ added A. grandis wood particles incubated at $25^{\circ} \mathrm{C}$ over the time. Average values and standard deviations were calculated from three parallel cultures each
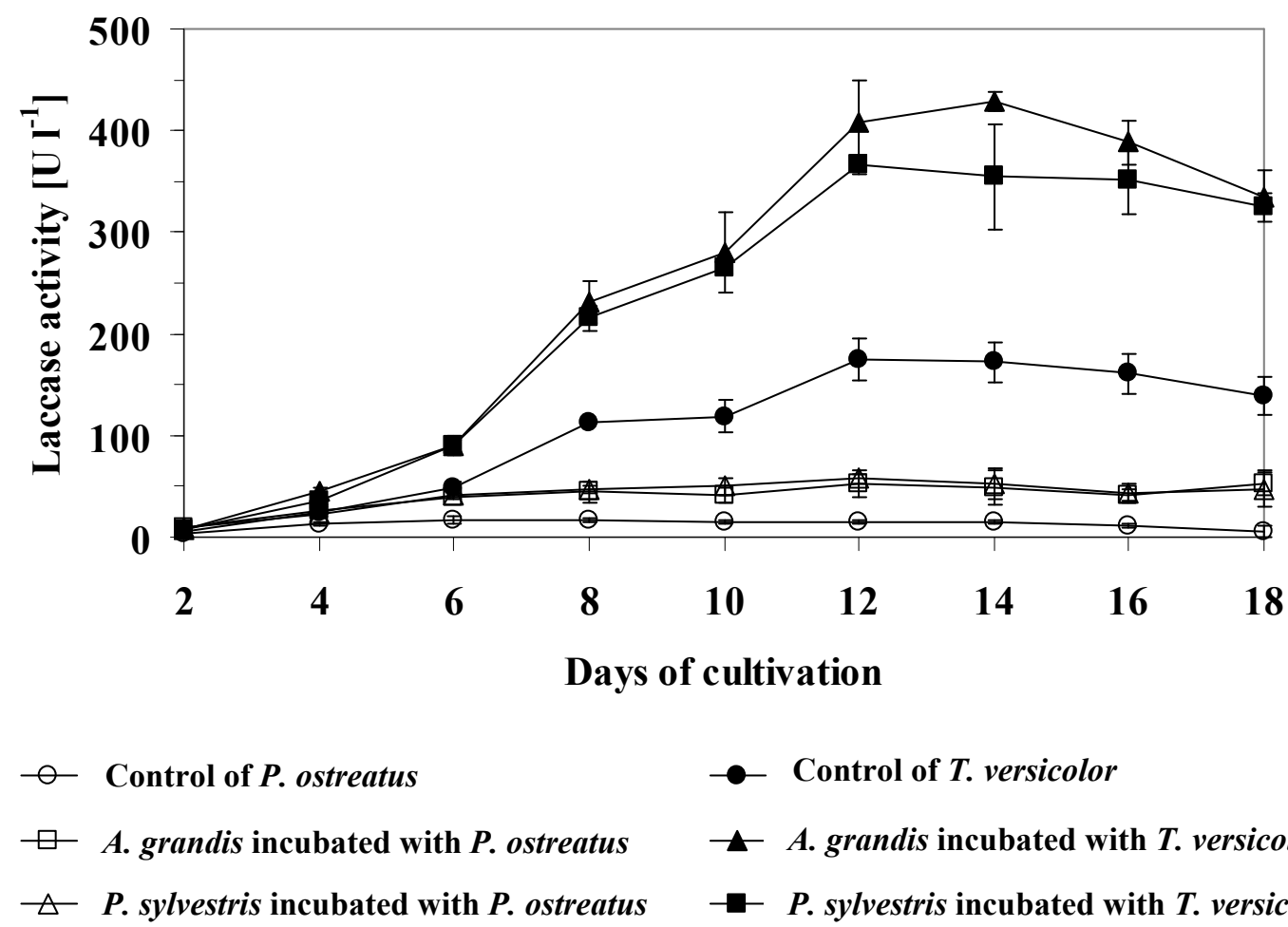

- Control of $T$. versicolor

- A. grandis incubated with $T$. versicolor

$\rightarrow-P$. sylvestris incubated with $T$. versicolor

Fig. 3 Laccase production of P. ostreatus 8F6 and T. versicolor Strain 6 incubated at $25^{\circ} \mathrm{C}$ in standing cultures with $100 \mathrm{ml}$ BSM liquid medium with $0.1 \mathrm{~g}$ of added $A$. grandis or $P$. sylvestris wood particles. Average values and standard deviations were calculated from three parallel cultures each 
IV. Contrasting ecological functions of Abies grandis wood extractives on protection against white-rot fungi and induction of laccase activity

levels of $370-400 \mathrm{U} \mathrm{l}^{-1}$ at day 12 to 16 of incubation compared to the best yield of $175 \pm 21.03 \mathrm{U} \mathrm{l}^{-1}$ of controls without any wood added obtained at day 12 of incubation. Highest laccase activities in cultures of $P$. ostreatus $8 \mathrm{~F} 6$ were about 53-58 $\mathrm{U}^{-1}$ at day 12 of incubation with $A$. grandis or with $P$. sylvestris wood as compared to $15 \pm 1.13 \mathrm{U}^{-1}$ of control cultures without addition of any wood. Thus, laccase production was generally higher by $T$. versicolor Strain 6 than by P. ostreatus 8F6, regardless of whether the fungi were cultured with and without added wood.

\section{IV.4.2 Effects of wood extractives on laccase production}

Since conifer wood was shown to have an inductive effect on laccase production of the two fungi (Fig. 3), wood extractives corresponding to $0.5 \mathrm{~g}$ of original $A$. grandis wood particles (obtained from a preparation of wood extractives from $10 \mathrm{~g}$ of $\mathrm{A}$. grandis wood particles as described in Chapter IV.3.3.2) and dissolved in DMSO were tested for inductive activities on enzyme production. Water extractives and acetone extractives were positive in both fungi in laccase induction whereas hexane extractives had no inducing effect (Fig. 4). In all cultures, laccase production began on the second day of incubation and increased continuously to reach a maximum at about day 14 of incubation. Water extractives showed the strongest inducer effect on P. ostreatus 8F6 and T. versicolor Strain 6 laccase production compared to acetone extractives and to the background laccase production in cultures with hexane extractives, in controls with DMSO but without extractives, and in controls with medium only, respectively (Fig. 4).

Cultures to which hexane extractives were added behaved in $T$. versicolor Strain 6 alike BSM control cultures to which nothing extra was given. Best activities for both types of cultures added with hexane extractives were about $50 \mathrm{U}^{-1}$ for P. ostreatus $8 \mathrm{~F} 6$ at day 8 of cultivation and about $120 \mathrm{U}^{-1}$ for T. versicolor Strain 6 at day 14 of cultivation (Fig. 4). In P. ostreatus 8F6 this level fell subsequently down to about $40 \mathrm{U}^{-1}$ during the end of incubation whereas the activities in $T$. versicolor Strain 6 remained at the level of $120 \mathrm{U} \mathrm{l}^{-1}$ till the end of incubation. Cultures of P. ostreatus $8 \mathrm{~F} 6$ to which only DMSO was added showed at longer time of incubation constant slightly higher laccase activities of about $60 \mathrm{U}^{-1}$ compared to the values of

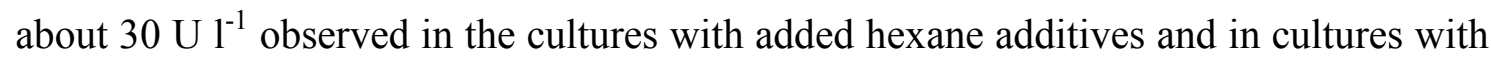
medium only (Fig. 4). These data suggests that there might be a low level inductive 
IV. Contrasting ecological functions of Abies grandis wood extractives on protection against white-rot fungi and induction of laccase activity

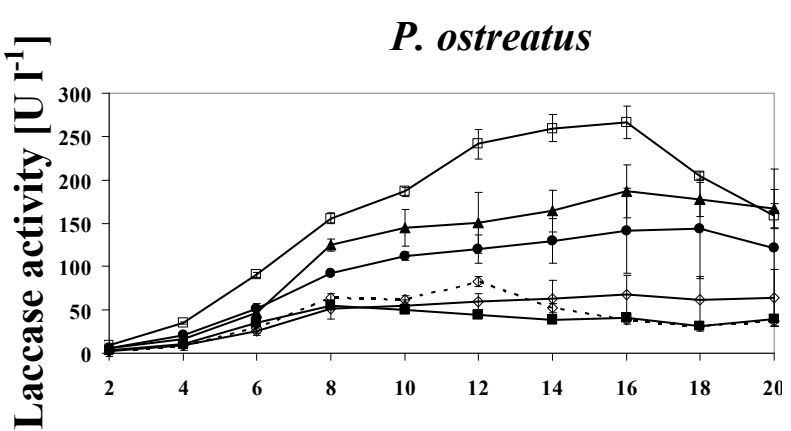

Days of cultivation
T. versicolor

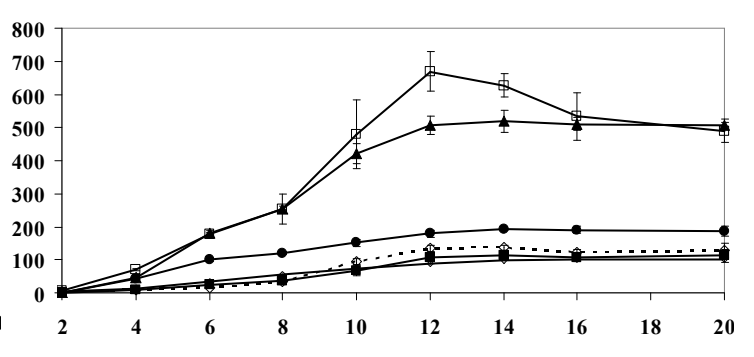

Days of cultivation
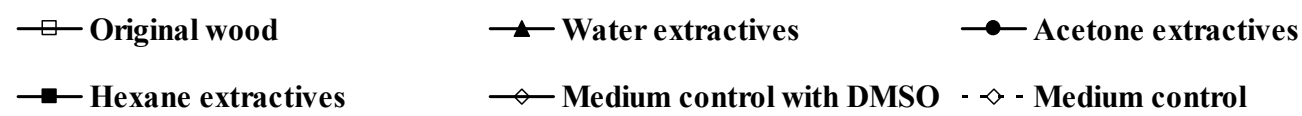

Fig. 4 Laccase production of $P$. ostreatus 8F6 and T. versicolor Strain 6 incubated at $25^{\circ} \mathrm{C}$ in standing cultures with $100 \mathrm{ml} \mathrm{BSM}$ medium to which different types of wood extractives (each corresponding to $0.5 \mathrm{~g}$ wood) dissolved in DMSO were added. In total $0.5 \mathrm{ml}$ DMSO were added to cultures with extractives. Cultures with $0.5 \mathrm{~g}$ added original wood particles, cultures with added $0.5 \mathrm{ml}$ DMSO and cultures with only medium were included as controls. No difference was seen in growth between the various cultures of the same fungus. Average values and standard deviations were calculated from three parallel cultures each

effect of DMSO on laccase production in P. ostreatus 8F6. It is to be noted that in none of the cultures any negative effect of DMSO on mycelial growth was observed.

For both fungi, water extractives were highly effective, nearly as much as the original A. grandis wood added in form of wood particles (Fig. 4). The highest laccase activities in all cultures were obtained from $0.5 \mathrm{~g}$ original $A$. grandis wood particles, for P. ostreatus $8 \mathrm{~F} 6$ activities of $266 \pm 18.7 \mathrm{U}^{-1}$ at day 16 of cultivation as compared to $670 \pm 59 \mathrm{U}^{-1}$ at day 12 of cultivation with T. versicolor Strain 6. Subsequently in cultures with original wood particles of both fungi, laccase activities decreased significantly with time of incubation (Fig. 4). With water extractives, for P. ostreatus $8 \mathrm{~F} 6$ highest activities of $186 \pm 30.3 \mathrm{U} \mathrm{l}^{-1}$ were obtained at day 16 of cultivation as compared to highest $T$. versicolor Strain 6 activities of $520 \pm 33 \mathrm{U} \mathrm{l}^{-1}$ obtained at day 14 of cultivation with parallel cultures with original wood particles giving (Fig. 4). Upon reaching maximum activity, laccase activities remained at similar high levels in cultures with added water extractives of P. ostreatus 8F6 and T. versicolor Strain 6 till the end of the experiment (Fig. 4). 
IV. Contrasting ecological functions of Abies grandis wood extractives on protection against white-rot fungi and induction of laccase activity

Effects of acetone extractives were generally lower, with highest activities of $143 \pm 57.3 \mathrm{U} \mathrm{l}^{-1}$ and $191 \pm 1.9 \mathrm{U}^{-1}$ in cultures of P. ostreatus $8 \mathrm{~F} 6$ and $T$. versicolor Strain 6 at day 18 and 14 of incubation, respectively (Fig. 4). For both fungi, it is further interesting to note that laccase production in cultures with added acetone extractives had also not a defined peak in best production around day 12 to 16 of incubation after which laccase production significantly decreased in levels, as repeatedly was observed with original wood particles (Fig. 2 to 4). Instead, laccase production continued on the range of best reached levels till the end of cultivation at day 20 (Fig. 4).

Parallel in the same set of experiments elucidating the effects of wood extractives as presented in Fig. 4, also effects of extracted wood particles on laccase production of $P$. ostreatus 8F6 and T. versicolor Strain 6 were tested (Fig. 5). After all types of extraction, A. grandis wood particles were still effective in laccase induction in both P. ostreatus 8F6 and T. versicolor Strain 6 but always at reduced levels (Fig. 5).
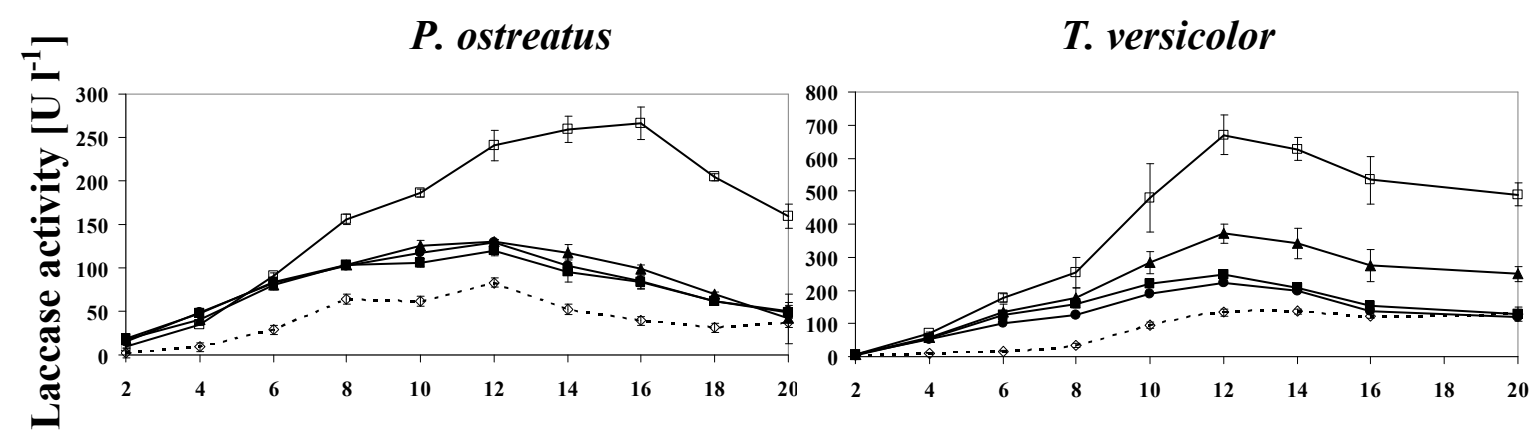

Days of cultivation
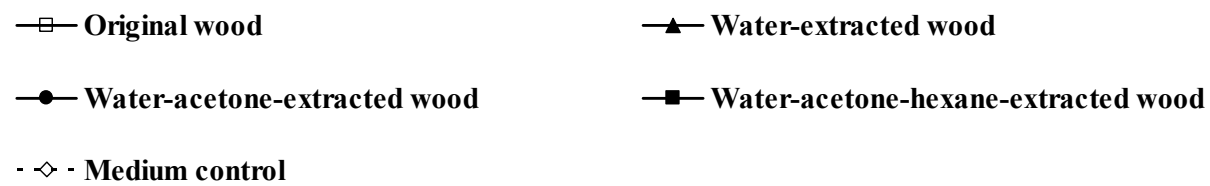

$-\diamond$ - Medium control

Fig. 5 Laccase production of $P$. ostreatus $8 \mathrm{~F} 6$ and T. versicolor Strain 6 incubated at $25^{\circ} \mathrm{C}$ in standing cultures with $100 \mathrm{ml} \mathrm{BSM}$ medium to which $0.5 \mathrm{~g} \mathrm{~A}$. grandis wood particles after extraction with water, with water-acetone or water-acetone-hexane, respectively, were alternatively added. Cultures with $0.5 \mathrm{~g}$ added original wood particles and cultures with only medium were included as controls. Note that the two types of controls are the same as in Fig. 4 since all cultivation shown in Fig. 4 and Fig. 5 were done in parallel in the same set of experiment. No difference was seen in growth between the various cultures of the same fungus. Average values and standard deviations were calculated from three parallel cultures each 
IV. Contrasting ecological functions of Abies grandis wood extractives on protection against white-rot fungi and induction of laccase activity

In P. ostreatus 8F6, water extracted wood on day 12 of cultivation lead to highest activities of $130 \pm 2.8 \mathrm{U} \mathrm{l}^{-1}$, water-acetone-extracted wood to $129 \pm 3.7 \mathrm{U} \mathrm{l}^{-1}$ and water-acetone-hexane-extracted wood to $120 \pm 6 \mathrm{U} \mathrm{l}^{-1}$, respectively and laccase activities were thus higher than in the controls without wood $\left(83 \pm 5.4 \mathrm{U}^{-1}\right)$ but lower than with the original wood $\left(241 \pm 17.4 \mathrm{U}^{-1}\right)$. Generally, between the three different types of extracted wood, there were only marginal differences in laccase activity in P. ostreatus 8F6 cultures over the time (Fig. 5).

In T. versicolor Strain 6, water-extracted wood on day 12 of cultivation lead to highest activities of $371 \pm 28.4 \mathrm{U} \mathrm{l}^{-1}$, water-acetone-extracted wood to $224 \pm 39.6 \mathrm{U} \mathrm{l}^{-1}$ and water-acetone-hexane-extracted wood to $248 \pm 29.3 \mathrm{U}^{-1}$ as compared to samples without wood with activities of $133 \pm 11.9 \mathrm{U}^{-1}$ and samples with original wood particles with activities of $670 \pm 59 \mathrm{U} \mathrm{l}^{-1}$. Thus, water extracted wood was still somewhat more effective in enhancing laccase production in cultures of T. versicolor Strain 6 as compared to the wood samples that in addition were extracted with organic solvents. However, water-extracted wood was not as effective as the original wood. Furthermore, over the whole time of incubation, there were no differences seen in samples treated with water-acetone-extracted wood compared to samples treated with water-acetonehexane-extracted wood (Fig. 5).

For all different types of wood samples, levels of laccase activities in the cultures of both fungi rapidly decreased after a best value was obtained, leading to bellshaped activities curves over the time (Fig. 5) unlike to what was seen in the same experiment with the active wood extractives (Fig. 4).

Since no differences were seen for both fungi in between cultures with wateracetone-extracted wood particles and cultures with water-acetone-hexane-extracted wood particles, in the following only water-extracted wood particles and water-acetoneextracted wood particles were further considered. As shown by adding different amounts of original wood particles or of wood particles after water extraction or of wood particles after water and acetone extraction to fungal cultures (Fig. 6), increasing wood particles concentrations can positively influence the outcome of laccase production of both fungi, regardless of the type of wood particles applied in the fungal cultures. In all instances, in cultures to which any type of wood particles were added, higher laccase activities were detected as compared to BSM medium control cultures of P. ostreatus $8 \mathrm{~F} 6$ (highest activities of $54 \pm 5.4 \mathrm{U}^{-1}$ at day 6 of cultivation of the 
IV. Contrasting ecological functions of Abies grandis wood extractives on protection against white-rot fungi and induction of laccase activity

controls) and of T. versicolor Strain 6 (highest activities of $105 \pm 29.3 \mathrm{U} \mathrm{l}^{-1}$ at 13 day of cultivation of the controls).

P. ostreatus

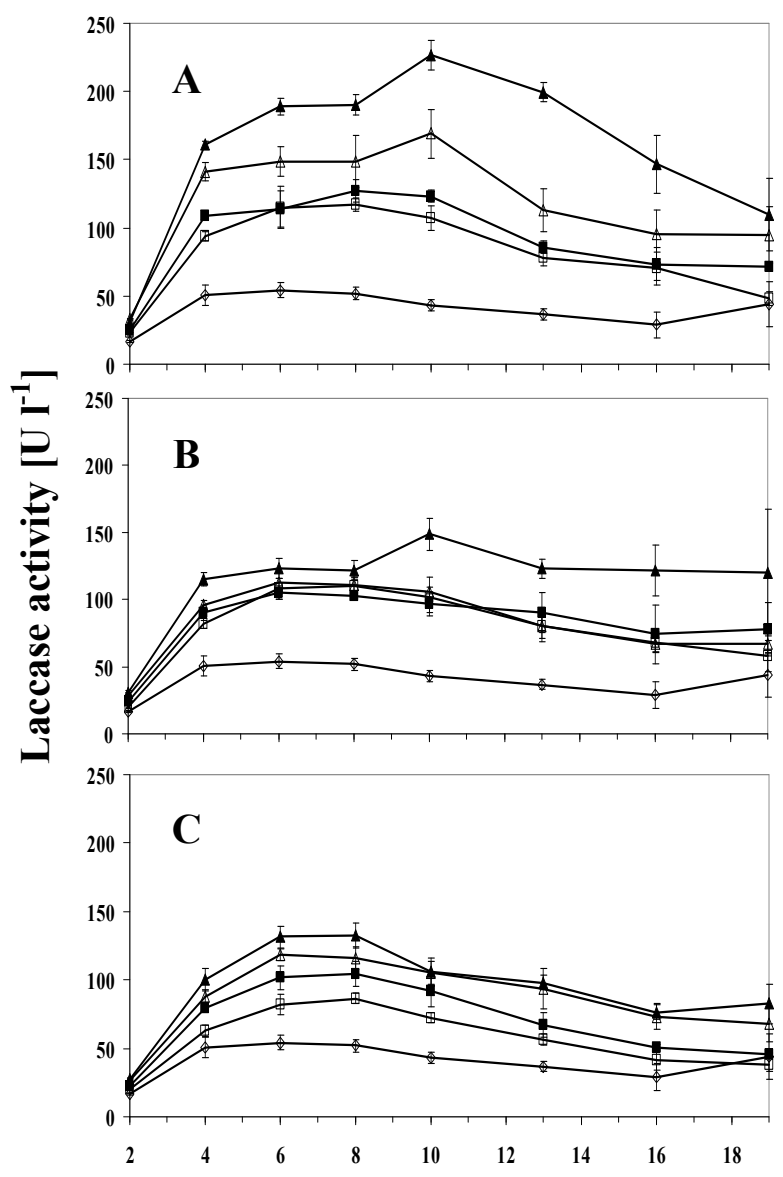

Days of cultivation
T. versicolor
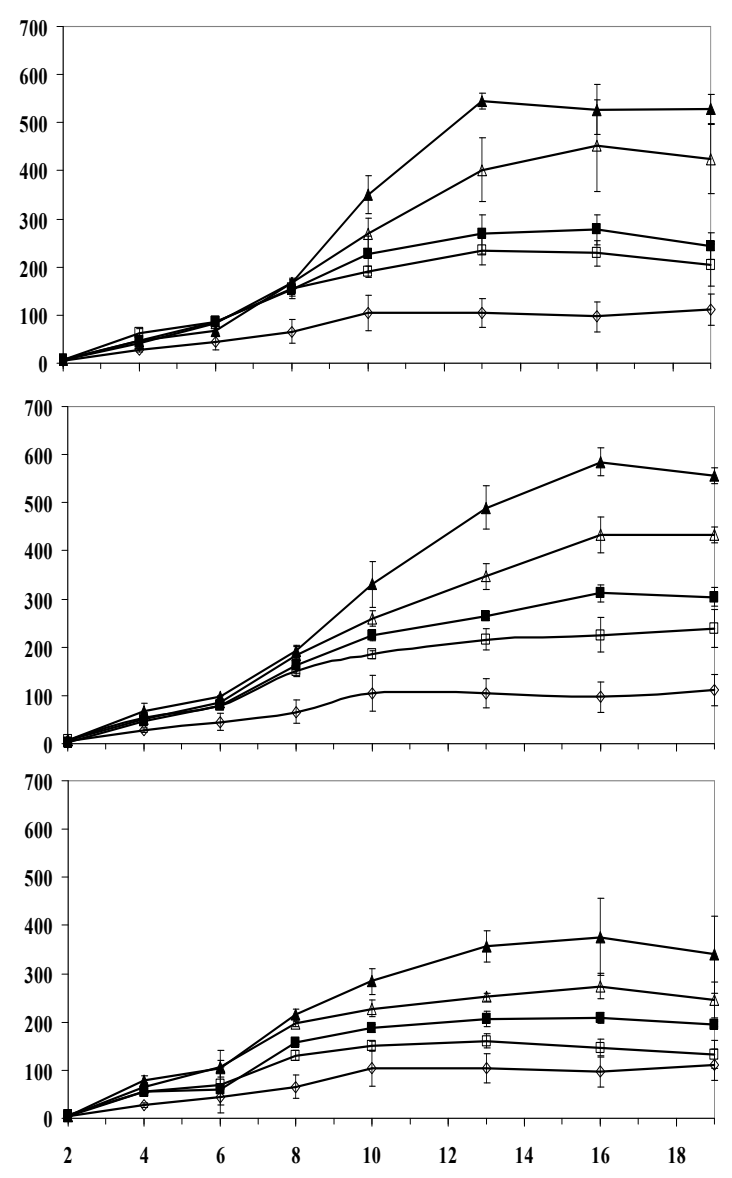

Days of cultivation

$\sqsubset 0.25 \mathrm{~g}$ wood $\rightarrow-0.5 \mathrm{~g}$ wood $\triangle 1 \mathrm{~g}$ wood $\leftarrow 2 \mathrm{~g}$ wood $\rightarrow$ Medium control

Fig. 6 Laccase production of $P$. ostreatus 8F6 and T. versicolor Strain 6 cultivated at $25^{\circ} \mathrm{C}$ in $100 \mathrm{ml} \mathrm{BSM} \mathrm{liquid} \mathrm{medium} \mathrm{to} \mathrm{which} \mathrm{different} \mathrm{amounts} \mathrm{of} \mathrm{A)} \mathrm{original}$ A. grandis wood particles, B) wood particles after water extraction, and C) wood particles after water and acetone extraction were added. For comparison of effects of different amounts of wood extractives (chloroform-purified water extractives and chloroform-purified acetone extractives) on laccase production, see Fig. 7 in which enzyme activities are shown of cultures run in parallel to the cultures presented here. No difference was seen in growth between the various cultures of the same fungus. Average values and standard deviations were calculated from three parallel cultures each 
IV. Contrasting ecological functions of Abies grandis wood extractives on protection against white-rot fungi and induction of laccase activity

Fig. 6 shows that laccase production in cultures with added original $A$. grandis wood particles was always higher than in cultures with added wood particles that had undergone steps of extraction, especially when compared to water-acetone-extracted wood particles. The highest activities were obtained from $2 \mathrm{~g}$ original wood particles (227 $\pm 10.9 \mathrm{U} \mathrm{l}^{-1}$ for $P$. ostreatus $8 \mathrm{~F} 6$ after 10 days of cultivation, $544 \pm 16.4 \mathrm{U} \mathrm{l}^{-1}$ for T. versicolor Strain 6 at 13 day of cultivation, respectively). Highest activities with $1 \mathrm{~g}$ of added original wood particles were $170 \pm 17.8 \mathrm{U}^{-1}$ for $P$. ostreatus $8 \mathrm{~F} 6$ at day 10 of cultivation and $452 \pm 95.5 \mathrm{U} \mathrm{l}^{-1}$ for $T$. versicolor Strain 6 at day 16 of cultivation. For both fungi, enzymatic activities over the time were comparable in cultures with added $0.5 \mathrm{~g}$ and with added $0.25 \mathrm{~g}$ original wood, respectively, with best values of about $130 \mathrm{U}^{-1}$ in P. ostreatus $8 \mathrm{~F} 6$ cultures at day 8 of incubation and of about $270 \mathrm{U} \mathrm{l}^{-1}$ in T. versicolor Strain 6 cultures at day 16 of incubation (Fig. 6).

Concentration-dependent effects on laccase production were also seen for both species in cultures to which extracted wood particles were added. Water-extracted wood particles in cultures with $P$. ostreatus $8 \mathrm{~F} 6$ gave at a concentration of $2 \mathrm{~g} / 100 \mathrm{ml} \mathrm{BSM}$ culture on day 12 of cultivation highest activities of $149 \pm 7.3 \mathrm{U} \mathrm{l}^{-1}$, and water-acetoneextracted wood particles at a concentration of $2 \mathrm{~g} / 100 \mathrm{ml} \mathrm{BSM}$ culture on day 8 of cultivation highest activities of $133 \pm 9.1 \mathrm{U} \mathrm{l}^{-1}$. Lower concentrations of water-extracted wood particles lead to similar laccase activities in the respective P. ostreatus 8F6 cultures (highest activities of about $110 \mathrm{U}^{-1}$ on day 6 of incubation), whilst in cultures with water-acetone-extracted wood particles laccase activities were slightly more variable; highest activities in cultures with $1,0.5$ and $0.25 \mathrm{~g}$ water-acetone-extracted wood particles were $118 \pm 4.3 \mathrm{U} \mathrm{l}^{-1}, 102 \pm 8.5 \mathrm{U} \mathrm{l}^{-1}$ and $82 \pm 7.3 \mathrm{U} \mathrm{l}^{-1}$ at day 6 of incubation, respectively.

In case of $T$. versicolor Strain 6, addition of $2 \mathrm{~g}$ of water-extracted wood particles lead to highest activities of $585 \pm 29.7 \mathrm{U}^{-1}$ on day 16 of cultivation, and addition of $2 \mathrm{~g}$ of water-acetone-extracted wood particles to highest activities of $376 \pm 79.4 \mathrm{U}^{-1}$ (Fig. 6). Thus, the highest activity achieved with water-extracted wood particles was comparable to the highest activity obtained with $2 \mathrm{~g}$ of original wood particles but the day of highest activity in comparison was however delayed. Similarly, with lower amounts of added water-extracted wood particles, it is interesting to note for T. versicolor Strain 6 that for water extracted wood the best obtained activities were comparable to these obtained with the original wood but they were delayed always by several days (Fig. 6). 
IV. Contrasting ecological functions of Abies grandis wood extractives on protection against white-rot fungi and induction of laccase activity

In contrast in cultures of $P$. ostreatus $8 \mathrm{~F} 6$, peaks in highest laccase production correlated in time more or less with each other and with ongoing incubation, always significant decreases in laccase activities within cultures were observed (Fig. 6).

Like increasing wood concentrations (Fig. 6), also increasing the amounts of added wood extractives correlated positively with laccase induction (Fig. 7). The cultures with different amounts of added wood additives were run in parallel to the cultures with different amounts of wood particles added; so, the results shown in Fig. 6 and in Fig. 7 are directly comparable. Water extractives were chosen for this next experiment on concentration effects because of them having a nearly as good effect on laccase induction than the original wood (Fig. 4), and acetone extractives were chosen for this experiment since for P. ostreatus 8F6 and for T. versicolor Strain 6 acetone extractives had also an inductive effect on laccase production (Fig. 4).

Different from the previous experiments documented in Fig. 5, water extractives and acetone extractives were chloroform-purified prior to application to fungal cultures. Chloroform-purification is expected to extract un-polar organic compounds from the solutions. From analysis of other tree species, it is known that various compounds with such nature (lipids, terpenoids and steroids, fat and wax, and phenolic compounds) are present in the mixtures of wood extractives (Fengel and Wegener 1983; Sjostrom 1993).

In case of chloroform-purified water extractives and in case of chloroformpurified acetone extractives added in different concentrations to BSM cultures of P. ostreatus $8 \mathrm{~F} 6$, all the cultures showed laccase production rising up over the whole time of cultivation (over 20 days at $25^{\circ} \mathrm{C}$ ) even in the cultures of medium control with added DMSO, but not for cultures that served as medium control (Fig. 7). Accordingly, DMSO itself has laccase inducing activity, confirming an earlier observation by Shah et al. (2006). With $1 \%$ DMSO in cultures of $P$. ostreatus, these authors had laccase activities in cultures of $75 \mathrm{U}^{-1}$ with and $50 \mathrm{U}^{-1}$ without adding DMSO whilst fungal growth was not affected by DMSO. Here in this study, 2\% of DMSO in cultures were used and this that a profound effect on fungal growth. The mycelium grew only as a thin film and did not cover all the culture surface and there was only little aerial mycelium, different from cultures without added DMSO that formed compact mycelial mats on the surfaces of cultures with much of fluffy aerial mycelium that at the edges of the cultures grew onto the glass of the Erlenmeyer flasks up to $2 \mathrm{~cm}$ into the height (not shown). Accordingly, DMSO is inhibiting fungal growth and as one reaction of the stress caused to the fungus, laccase production might be induced. Since growth was also 
IV. Contrasting ecological functions of Abies grandis wood extractives on protection against white-rot fungi and induction of laccase activity

affected, a relatively high amount of induced laccase in cultures with wood extractives added as a $2 \mathrm{ml}$ DMSO solution is therefore likely due to the effects of DMSO on growth and laccase production. Nevertheless, with all types of wood extractives tested there were extra portions of further laccase activities, indicating that also the extractives have an inducing effect on laccase in P. ostreatus 8F6 (Fig. 7).

\section{P. ostreatus}

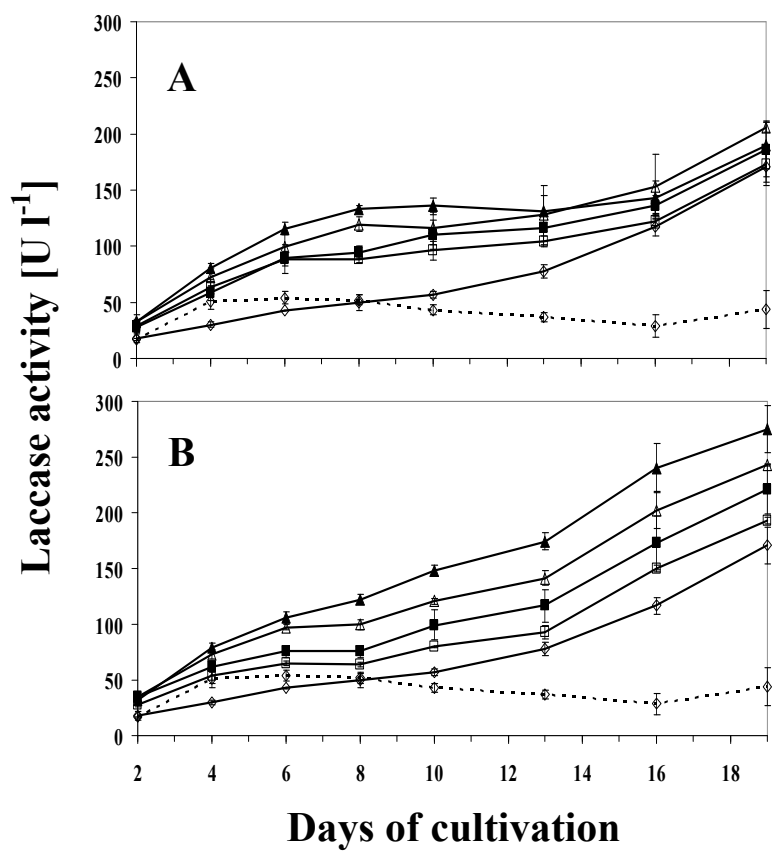

T. versicolor
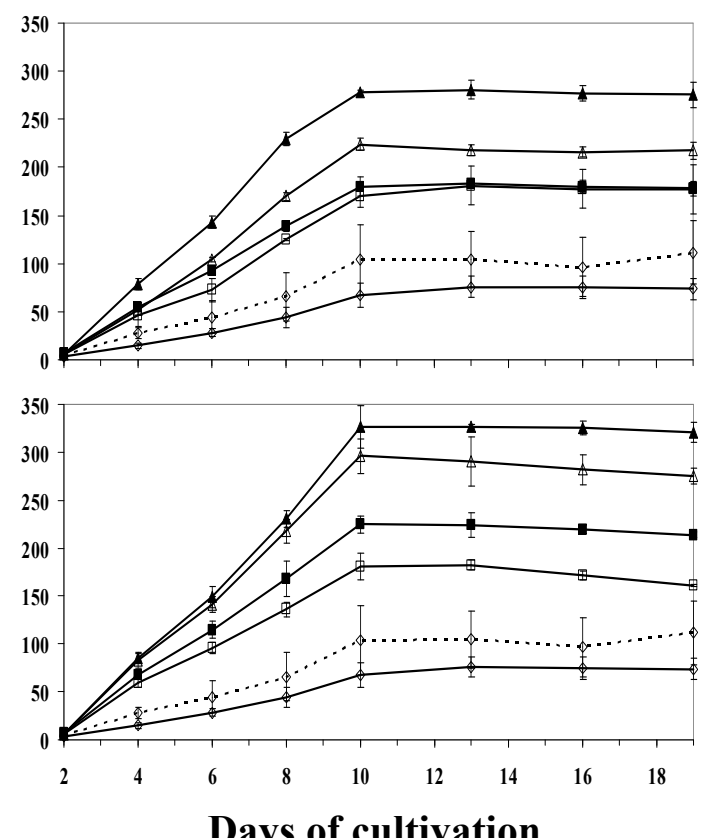

Days of cultivation

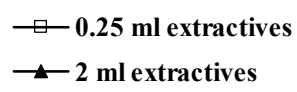

$\square-0.25 \mathrm{ml}$ extractive
$\square-2 \mathrm{ml}$ extractives $\triangle 1 \mathrm{ml}$ extractives

$-\diamond$ - Medium control

Fig. 7 Laccase production of $P$. ostreatus $8 \mathrm{~F} 6$ and $T$. versicolor Strain 6 cultivated at $25^{\circ} \mathrm{C}$ in $100 \mathrm{ml} \mathrm{BSM}$ liquid medium to which different amounts of $\mathbf{A}$ ) the chloroform fraction of water extractives and $\mathbf{B}$ ) the chloroform fraction of acetone extractives of $A$. grandis wood were added. In order to avoid unwanted falsifying effects from different volumes added, in all instances extra DMSO was added, giving a total volume of $2 \mathrm{ml} \mathrm{DMSO}$ added per culture. For comparison of effects of different amounts of added wood particles (original or extracted) on laccase production see Fig. 6 in which enzyme activities are shown of cultures run in parallel to the cultures presented here. No difference was seen in growth between the cultures of T. versicolor Strain 6 , but in case of the cultures of $P$. ostreatus $8 \mathrm{~F} 6$, fungal mycelia did not grow as well in the medium with the added DMSO in comparison to medium without DMSO. Average values and standard deviations were calculated from three parallel cultures each 
IV. Contrasting ecological functions of Abies grandis wood extractives on protection against white-rot fungi and induction of laccase activity

The chloroform fraction from water extractives applied at different concentration resulted in highest laccase activities of about 170-200 $\mathrm{U} \mathrm{1}^{-1}$ at day 20 of cultivation in cultures of $P$. ostreatus 8F6 (Fig. 7A). Over the whole incubation time, cultures with different amounts of added water-extractives behaved very similar in laccase production although there was always a slightly higher amount of laccase activities in cultures correlating with from one amount of water extractives added to the next amount of water extractives added. However, also cultures with added DMSO alone reached finally nearly similar high laccase activities levels whilst earlier in cultivation laccase activities in cultures with only DMSO were lower compared to cultures with added extractives (at day 10 of cultivation e.g. only $50 \mathrm{U} \mathrm{l}^{-1}$ compared to $100 \mathrm{U}^{-1}$ to $150 \mathrm{U}^{-1}$ in cultures with added extractives, respectively; Fig. 7A).

Similarly with different amounts of chloroform-purified acetone extractives in P. ostreatus $8 \mathrm{~F} 6$ cultures, over the time of 20 days of cultivation at $25^{\circ} \mathrm{C}$, laccase activities constantly increased in all cultures in a manner correlating with the amount of added acetone extractives (Fig. 7B). At the end of the experiment, highest laccase activity between $193 \pm 3.0 \mathrm{U} \mathrm{l}^{-1}$ (when $0.25 \mathrm{ml}$ acetone extractives were added) and $275 \pm 7.7 \mathrm{U} \mathrm{l}^{-1}$ (when $2 \mathrm{ml}$ of acetone extractives were added) by P. ostreatus 8F6 cultures were obtained. For comparison, highest amounts of laccase in cultures with added DMSO alone were 170 $\pm 16.5 \mathrm{U}^{-1}$ (Fig. 7B).

Overall, the highest amount of laccase obtained upon addition of chloroformpurified wood extractives to $P$. ostreatus $8 \mathrm{~F} 6$ cultures corresponded in value to the best activities observed when adding original wood to the cultures (compare Fig. 6A and Fig. 7). The timings of production was however different with a delay of about 10 days in case of added wood particles (Fig. 6A, Fig. 7). From the observations on fungal mycelia of $P$. ostreatus $8 \mathrm{~F} 6$ cultures, one reason of the delay in laccase production was likely the delay of fungal growth. Moreover, DMSO is a very hard to degrade compound by microorganism and by chemical mineralization (Murakami-Nitta et al. 2002; Lee et al. 2002). Thus, DMSO might be kept high in concentration in the fungal cultures over the time, explaining in the $P$. ostreatus $8 \mathrm{~F} 6$ cultures the ever increasing laccase activities over the time and also in the $T$. versicolor Strain 6 cultures the constant laccase activities reached after day 10 of cultivation in all cultures with added DMSO with or without wood extractives (Fig. 7).

In cultures of $T$. versicolor Strain 6, both with chloroform-purified water extractives and with chloroform-purified acetone extractives, effects of different 
IV. Contrasting ecological functions of Abies grandis wood extractives on protection against white-rot fungi and induction of laccase activity

concentrations added to cultures where more obvious. With increasing amounts of wood extractives, the yield in laccase activities raised visibly from concentration to concentration (Fig. 7A and 7B). Highest activities with $2 \mathrm{ml}$ of added water extractives were achieved on day 13 of cultivation with a value of $280 \pm 9.8 \mathrm{U} \mathrm{l}^{-1}$ (Fig. 7A) and highest activities with $2 \mathrm{ml}$ of added acetone extractives with a value of $327 \pm 2.2 \mathrm{U}^{-1}$ (Fig. 7B). In comparison, highest activities with only added DMSO were $60 \mathrm{U} \mathrm{l}^{-1}$ and this was even slightly lower than with the control cultures of only medium that reached $50 \mathrm{U}^{-1}$. Subsequently for the next 10 days till the end of cultivation, the laccase activities in all these cultures remained at similar levels.

Comparable curves of laccase activities over the time were obtained with T. versicolor Strain 6 for lower amounts of added chloroform-purified water extractives and for lower amounts of added chloroform-purified acetone extractives (Fig. 7A and 7B). The highest values of laccase activities reached with addition of wood extractives (in $2 \mathrm{ml} \mathrm{DMSO}$ ) corresponded to the yields obtained in parallel cultures with addition of $0.25 \mathrm{~g}$ and $0.5 \mathrm{~g}$ original wood particles (compare Fig. 6A and Fig. 7A, 7B), although in timings, laccase production in cultures with added wood particles was delayed by seven days (Fig 6A, Fig. 7A and 7B). DMSO in T. versicolor Strain 6 apparently had no inductive effect and the growth behavior of cultures with and without DMSO were comparable well (not shown). DMSO is shown not to inhibit laccase activity of Trametes sp. (Xiao et al. 2004) but to have at higher concentrations (10\%) a stabilizing effect on laccases (Tavares et al. 2008). Whether such effect is responsible for the observed constance in laccase activity at later times of incubation (Fig. 7) was not further addressed in this study.

\section{IV.4.3 Laccase induction by different fractions of $\boldsymbol{A}$. grandis wood extractives}

Water extractives from A. grandis wood were fractionated on a silica gel column as described in Chapter IV.3.2. A volume of $10 \mathrm{ml}$ of water extractives were applied to the column corresponding to a dry weight of $2.0 \mathrm{~g}$ obtained from water extraction of $50 \mathrm{~g}$ A. grandis wood particles. The pass-through was collected as first fraction. With increasing concentration of methanol used afterwards for elution of extractives from the column, the weight of dry mass of eluted extractives was decreasing from fraction to fraction from about $0.6 \mathrm{~g}$ (first pass-through fraction obtained prior to the elution with methanol) to about no dry mass when using $80 \%$ and more methanol for extraction, or 
IV. Contrasting ecological functions of Abies grandis wood extractives on protection against white-rot fungi and induction of laccase activity

when finally using DMSO in the last elution (Fig. 8). In total, a dry mass of $1.6 \mathrm{~g}$ water extractives was recovered from the column.

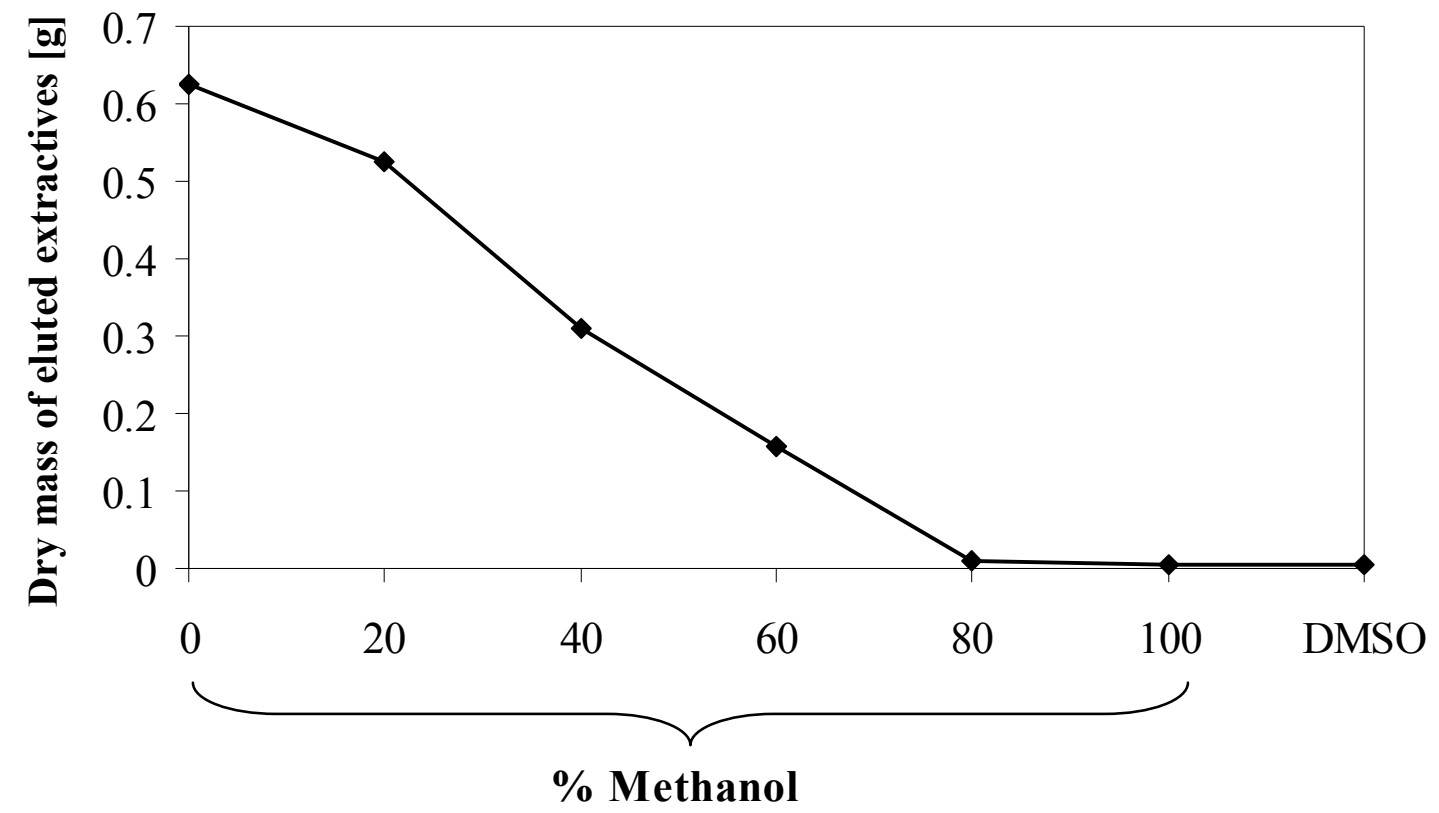

Solvent used for elution

Fig. 8 Weights of dry masses of fractions of water extractives from $A$. grandis wood particles serially eluted from a silica gel column as first pass-through ( $0 \%$ methanol) and with $20 \%$ to $100 \%$ methanol and DMSO, respectively

In total, seven different fractions of $A$. grandis extractives were obtained by the chromatography elution. $200 \mu \mathrm{l}$ of dissolved extractives (corresponding to $1 \mathrm{~g}$ wood) in DMSO per each fraction (from a total of each $10 \mathrm{ml}$ solution obtained from solving the individual fractions of eluted extractives in DMSO) was added into BSM cultures of the white-rot fungi $P$. ostreatus $8 \mathrm{~F} 6$ and $T$. versicolor Strain 6, respectively, in order to investigate effects on laccase production. In both cases, the fractions eluted with $40 \%$ and $60 \%$ methanol showed the highest effect on laccase production with a maximum of laccase activity of $111 \pm 6 \mathrm{U}^{-1}$ and $112 \pm 3.8 \mathrm{U} \mathrm{l}^{-1}$ on day 10 of cultivation, respectively in cultures of $P$. ostreatus 8F6 (Fig. 9A) and a maximum of laccase activities of $582 \pm 43.2 \mathrm{U} \mathrm{l}^{-1}$ and $797 \pm 13.7 \mathrm{U} \mathrm{l}^{-1}$ on day 20 of cultivation in respective cultures of T. versicolor Strain 6 (Fig. 9B). In addition, the pass-through fraction obtained at the beginning of the chromatography and the $20 \%$ methanol fraction lead to significant 
IV. Contrasting ecological functions of Abies grandis wood extractives on protection against white-rot fungi and induction of laccase activity
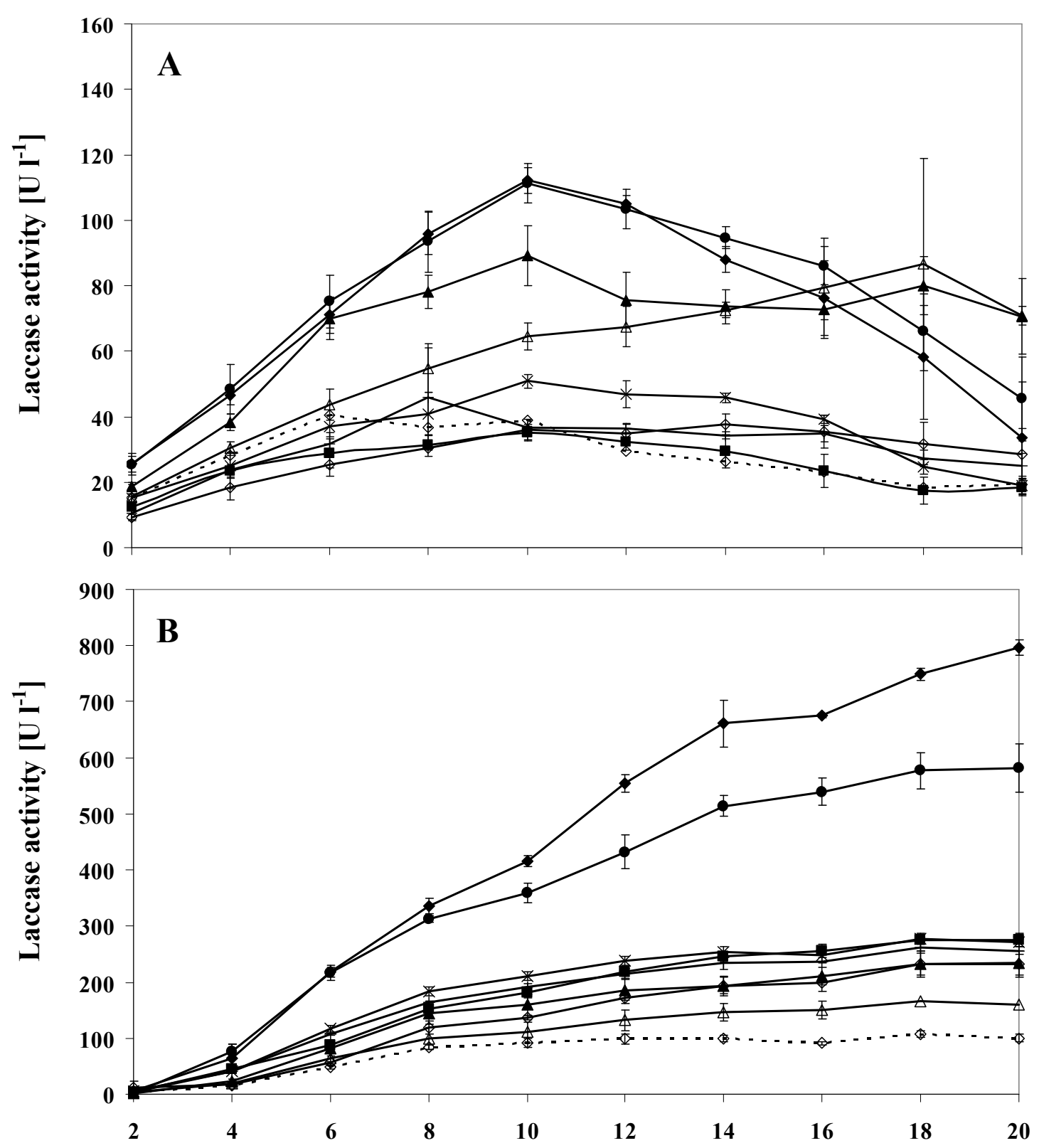

\section{Days of cultivation}

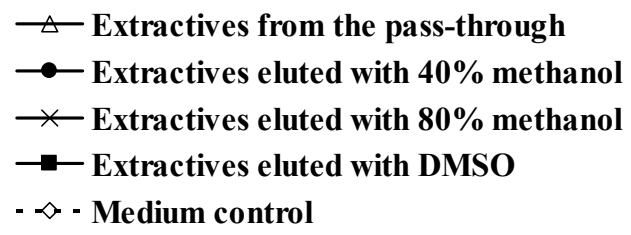

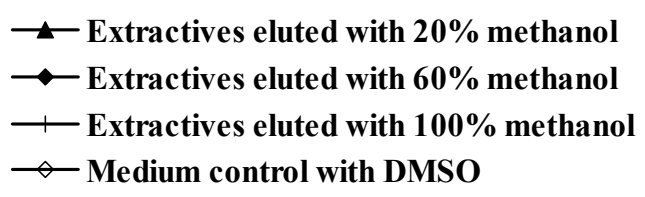

- Extractives eluted with $20 \%$ methanol

$\neg$ Medium control with DMSO

Fig. 9 Laccase production at $25^{\circ} \mathrm{C}$ by $P$. ostreatus $8 \mathrm{~F} 6 \mathrm{~A}$ ) and $T$. versicolor Strain 6 B) in stationary cultures of $100 \mathrm{ml}$ BSM liquid medium without and with added chromatography fractions of water extractives in $200 \mu \mathrm{l}$ of DMSO. Added amounts of extractives within the different chromatography fractions corresponded to $1 \mathrm{~g}$ of A. grandis wood. $200 \mu \mathrm{l}$ only DMSO was added into control cultures to observe the effect of the solvent on laccase production. No difference was seen in growth between the various cultures of the same fungus. Average values and standard deviations were calculated from three parallel cultures each 
IV. Contrasting ecological functions of Abies grandis wood extractives on protection against white-rot fungi and induction of laccase activity

higher laccase production when added to the BSM medium of cultures of P. ostreatus $8 \mathrm{~F} 6$ as compared to control cultures with or without added $200 \mu 1 \mathrm{DMSO}$ or as compared to cultures with other added fractions obtained from the chromatography of the water extractives of $A$. grandis wood (Fig. 9A). Control cultures with only added $200 \mu \mathrm{DMSO}$ had slightly more laccase activity (about $30 \mathrm{U} \mathrm{l}^{-1}$ ) at longer times of incubation as compared to cultures with pure medium (about $20 \mathrm{U} \mathrm{l}^{-1}$ ) but this differences are likely due to statistical variations and not to specific effects of adding DMSO (Table S1 in the supplementary material). Cultures with the added fraction from DMSO elution behaved alike cultures of pure medium, cultures with the added fraction from the $100 \%$ methanol elution like the control cultures with added $200 \mu 1$ DMSO. Only cultures with the added fraction from $80 \%$ methanol elution were slightly better in laccase production at day 10 to 14 of incubation with about $50 \mathrm{U}^{-1}$ laccase activities (Fig. 9A).

In comparison, cultures of T. versicolor Strain 6 with added eluates from the chromatography obtained by elution with $40 \%$ and $60 \%$ methanol showed highest laccase production of about $600 \mathrm{U}^{-1}$ and about $800 \mathrm{U} \mathrm{l}^{-1}$ at day 18 to 20 of incubation, respectively, whilst all other fractions had a comparably little effect on increasing laccase production as compared to the medium controls with an without added $200 \mu 1$ DMSO (Fig. 9B). Much of the comparably low effects on laccase induction however might be due to the addition of DMSO (Fig. 9B) or more likely just due to statistical variations (compare values for laccase activities with and without DMSO in other experiments in Table S1 in the supplementary material). Fractions eluted with $80 \%$ or $100 \%$ methanol or with DMSO from the chromatography columns had at the end of incubation about $270 \mathrm{U}^{-1}$ laccase activities, cultures with pure DMSO and with the fraction eluted with $20 \%$ methanol had about $230 \mathrm{U} \mathrm{l}^{-1}$ laccase activity (Fig. 9B). Moreover, the pass-through fractions even lead to slightly lower amounts laccase in the cultures (about $160 \mathrm{U}^{-1}$ at the end of incubation) as compared to cultures with only added DMSO (Fig. 9B).

The results shown in Fig. 9 indicate that fractions of extractives eluted with $40 \%$ and $60 \%$ methanol of $A$. grandis wood extractives have a potential in both fungi to induce strongly laccase production. These two fractions were further purified with chloroform as described in Chapter IV.3.2. Aliquots of $200 \mu 1$ of chloroform-purified $40 \%$ and $60 \%$ methanol fractions (corresponding to $1 \mathrm{~g}$ of original $A$. grandis wood) were added into cultures of P. ostreatus 8F6 and T. versicolor Strain 6 in order to 
IV. Contrasting ecological functions of Abies grandis wood extractives on protection against white-rot fungi and induction of laccase activity

compare the effects on laccase production in comparison to the original fraction of extractives eluted with $40 \%$ or $60 \%$ methanol from the silica-gel column. Original wood and the original solution of water extractives were used as controls for further comparison (Fig. 10).

In P. ostreatus 8F6 cultures, chloroform-purified fractions of extractives eluted with $40 \%$ or $60 \%$ methanol from the silica-gel column resulted in alike laccase activities over the time than the respective unpurified fractions. P. ostreatus 8F6 cultures showed highest laccase activity on day 8 of cultivation of about 90-100 U $1^{-1}$ with both purified and unpurified fractions of extractives eluted with $40 \%$ and $60 \%$ methanol from the silica-gel column, respectively (Fig. 10A and Fig. S1 in the supplementary material). Accordingly in $P$. ostreatus $8 \mathrm{~F} 6$, the laccase inducing compounds seem to be un-polar and extractable by chloroform. However, activities of the different unpurified and purified fractions did not reach peaks of laccase activities of cultures with added original extractives $\left(150 \pm 5.1 \mathrm{U} \mathrm{l}^{-1}\right.$ at day 8 of incubation) nor that of added original wood (226 $\pm 37.5 \mathrm{U} \mathrm{l}^{-1}$ at day 8 of incubation). Further to be noted from this experiment are the controls with and without DMSO. In this experiment, the controls without adding $200 \mu \mathrm{D}$ DSO gave slightly higher laccase activities at longer time of incubation (about $40 \mathrm{U}^{-1}$ at day 16 to 20 of incubation) than the cultures with $200 \mu \mathrm{l}$ DMSO of about $30 \mathrm{U}^{-1}$ at day 16 to 20 of incubation (Fig. 10A). Accordingly, differences noted in this (Fig. 10A) and in former experiments (Fig. 9A) with and without addition of $200 \mu 1 \mathrm{DMSO}$ to the cultures are subject to experimental variation.

In cultures of $T$. versicolor Strain 6 , the highest laccase activities occurred on day 14 of cultivation with about $300 \mathrm{U}^{-1}$ and $500 \mathrm{U} \mathrm{l}^{-1}$ in cultures to which the original $40 \%$ or $60 \%$ methanol fractions of extractives were added. In comparison, somewhat lower values were obtained with the chloroform-purified fractions: $260 \mathrm{U}^{-1}$ in the case of the purified $40 \%$ methanol fraction and $450 \mathrm{U}^{-1}$ in the case of the purified $60 \%$ methanol fraction, respectively (Fig. 10B). According to these results, the main inducer of laccase production in T. versicolor Strain 6 in the $40 \%$ and $60 \%$ methanol fractions of chromatography-separated water extractives are also expected to be un-polar and extractable by chloroform. Also in this experiment, laccase activities of unpurified and chloroform purified $40 \%$ and $60 \%$ methanol fractions of the water extractives remained below the values obtained with the original water extractives $\left(611 \pm 46.5 \mathrm{U} \mathrm{l}^{-1}\right.$ at day 14 of incubation) or with the original wood $\left(691 \pm 126.4 \mathrm{U}^{-1}\right.$ at day 14 of incubation). Controls with $200 \mu \mathrm{l}$ added DMSO showed in this experiment (Fig. 10B) as in the 
IV. Contrasting ecological functions of Abies grandis wood extractives on protection against white-rot fungi and induction of laccase activity
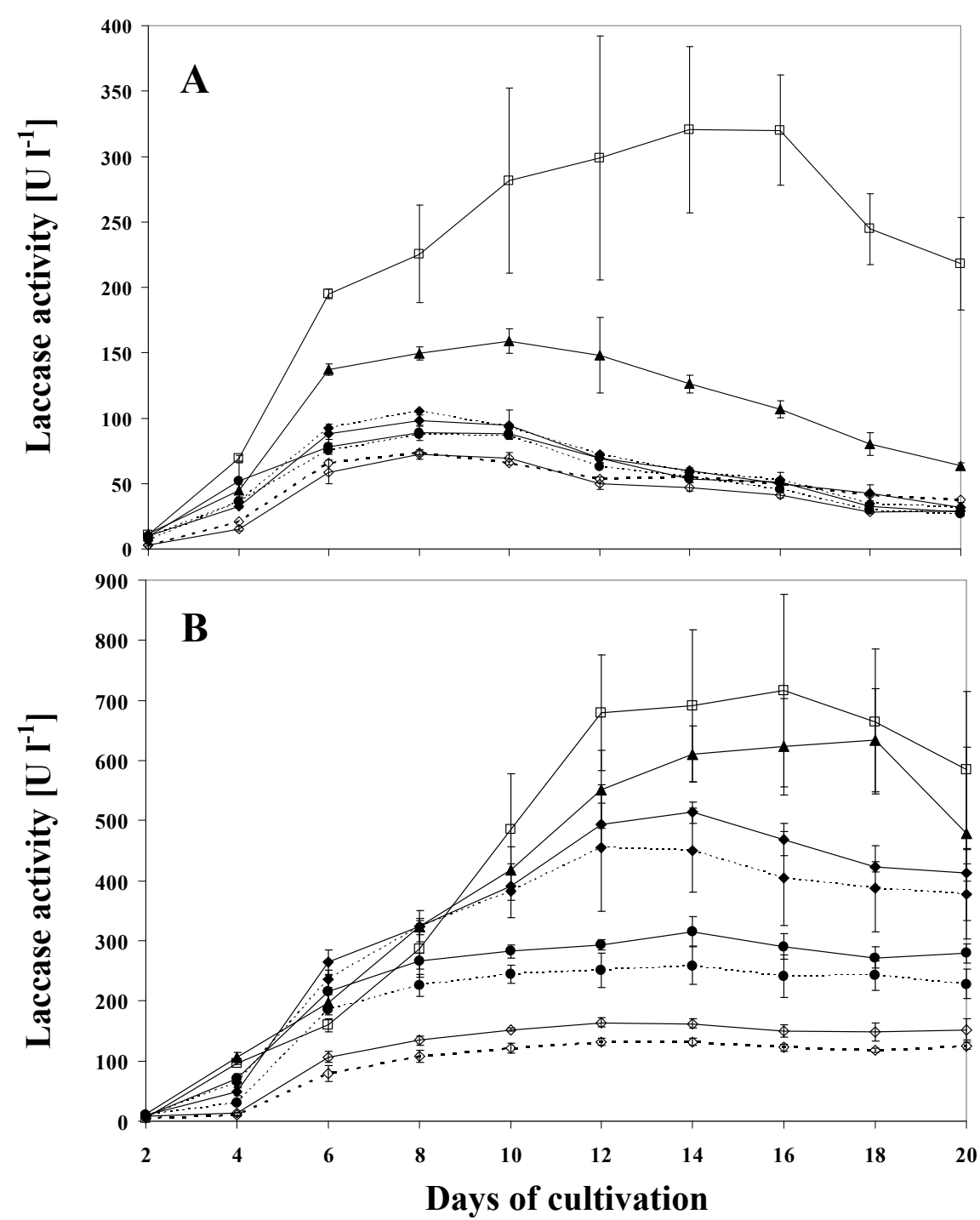

\footnotetext{
$\square$ Original wood

- Extractives eluted with $40 \%$ methanol

•.. Chloroform fraction of extractives eluted with $40 \%$ methanol

$\multimap$ Medium control with DMSO
}

—-Water extractives

- Extractives eluted with $60 \%$ methanol

$\diamond$ - Medium control

Fig. 10 Laccase production at $25^{\circ} \mathrm{C}$ by $P$. ostreatus $8 \mathrm{~F} 6 \mathrm{~A}$ ) and T. versicolor Strain 6 B) in stationary cultures of $100 \mathrm{ml}$ BSM liquid medium without and with added original wood ( $1 \mathrm{~g}$ per culture), or with added extractives corresponding to $1 \mathrm{~g}$ of $\mathrm{A}$. grandis wood, dissolved in $200 \mu \mathrm{l}$ DMSO. For testing effects of water extractives, either aliquots of the original water extractives, or of chromatography fractions of water extractives eluted by $40 \%$ or $60 \%$ methanol from the silica gel column or of chloroform-purified fractions of the extractives eluted by $40 \%$ or $60 \%$ methanol from the column were added. $200 \mu 1$ only DMSO was added into control cultures to observe the effect of the solvent on laccase production. No difference was seen in growth between the various cultures of the same fungus. For better view on the cultures of $P$. ostreatus $8 \mathrm{~F} 6$ with added fractions of extractives see Fig. S1 in the supplementary material. Average values and standard deviations were calculated from three parallel cultures each 
IV. Contrasting ecological functions of Abies grandis wood extractives on protection against white-rot fungi and induction of laccase activity

previous experiment (Fig. 9B) somewhat higher values of laccase activities (152 $\pm 17.4 \mathrm{U} \mathrm{l}^{-1}$ at day 20 of incubation) as compared with the controls of pure medium (125 $\mathrm{U} \mathrm{l}^{-1}$ at day 20 of incubation).

\section{IV.4.4 Identification of $A$. grandis wood extractive compounds by GC-MS}

Water extractives and acetone extractives in original and in chloroform-purified form were subjected to a GC-MS analysis in order to identify their individual compounds (Fig. 11 and Fig. 12). Particularly unpurified water extractives and to less extend also unpurified acetone extractives showed a quite nervous peak pattern in the chromatograms and many of the peaks could not unambiguously assigned to compounds, because peaks were unsharp likely by presenting more the one compounds.

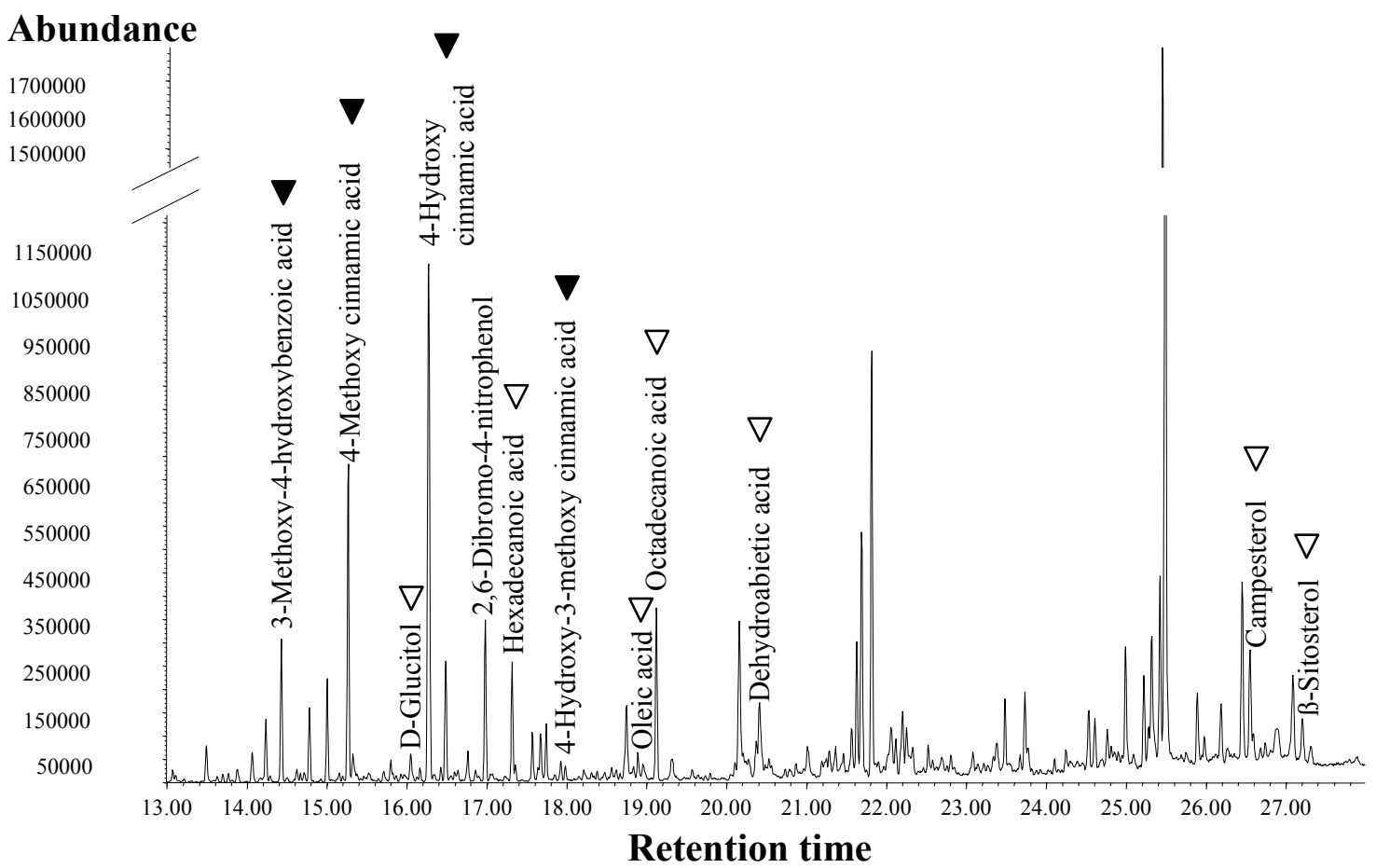

Fig. 11 GC-MS analysis of compounds in the chloroform fraction of water extractives from A. grandis wood particles. Filled arrows mark compounds shared with the chloroform fraction of the acetone extractives of $A$. grandis wood and expected to induce laccase. Open arrows mark other compounds shared with the chloroform fraction of the acetone extractives of $A$. grandis wood (see Table 1, Fig. 11 and 12). Only a part of the complete chromatogram is shown with interesting readily visible peaks (for the complete chromatogram of chloroform-purified extractives and of extractives prior to chloroform-purified see Fig. S2 in the supplementary material) 


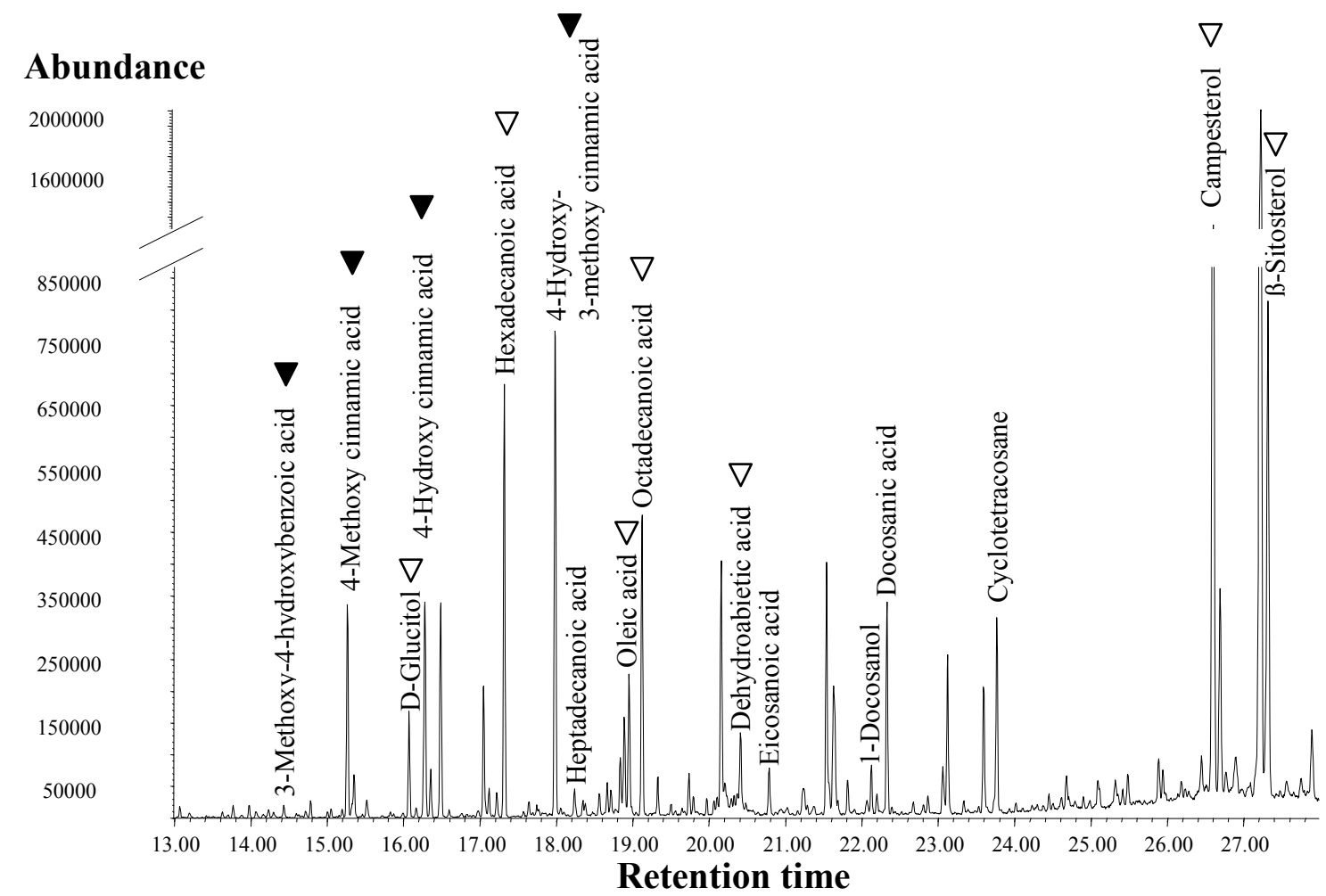

Fig. 12 GC-MS analysis of compounds in the chloroform fraction of acetone extractives from A. grandis wood particles. Filled arrows mark compounds shared with the chloroform fraction of the water extractives of $A$. grandis wood and expected to induce laccase. Open arrows mark other compounds shared with the chloroform fraction of the water extractives of $A$. grandis wood (see Table 1, Fig. 11 and 12). 3-Methoxy-4hydroxybenzoic acid was present but with only an identification quality at $90 \%$. Only a part of the complete chromatogram is shown with readily visible peaks (for the complete chromatogram of chloroform-purified extractives and of extractives prior to chloroform-purified see Fig. S3 in the supplementary material)

Nevertheless, a range of different compounds were identified by GC-MS the two types of wood extractives (Fig. S2 and S3). Many of the unidentified peaks and peaks identified to e.g. represent hydrophilic sugars (Fig. 11, 12 and 22) were eliminated upon chromatography-purification (compare Fig. 11, 12, S2, S3 and S4) and chromatograms became clearer for identification.

Accordingly, in total, 18 different compounds were present in the chromatograms of chloroform-purified water extractives with an identification quality higher than $95 \%$ and 21 different compounds in the chloroform-purified acetone extractives. Between the two types of extractives, 17 compounds were shared: 2-methoxyphenol, 4-hydroxybenzaldehyde, 1,4-dihydroxybenzene, 3,5-di-tertbut- 
IV. Contrasting ecological functions of Abies grandis wood extractives on protection against white-rot fungi and induction of laccase activity

4-hydroxy-toluene, 3-methoxy-4-hydroxybenzaldehyde, 4-hydroxybenzoic acid, 3-methoxy-4-hydroxybenzoic acid, 4-methoxy cinnamic acid, D-glucitol, 4-hydroxy cinnamic acid, hexadecanoic acid, 4-hydroxy-3-methoxy cinnamic acid, oleic acid, octadecanoic acid, dehydroabietic acid, campesterol, and $\beta$-sitosterol (see Fig. 11 and 12, Table 1 and Fig. S2 and S3 in the supplementary material). Amongst were ten compounds of phenolic nature (shown above in bold) that could be expected to have an inductive effect on laccase production.

Table 1 The contents of specific wood extractives in $1 \mathrm{~g}$ dry wood

\begin{tabular}{|c|c|c|c|c|c|c|}
\hline \multirow{3}{*}{ No. } & \multirow{3}{*}{ Compound* } & \multirow{3}{*}{$\mathbf{R T}^{* *}$} & \multicolumn{4}{|c|}{ Concentration $(\mu \mathrm{g})$ in $1 \mathrm{~g}$ dry wood } \\
\hline & & & \multicolumn{2}{|c|}{$\begin{array}{l}\text { Original solution of } \\
\text { extractives }\end{array}$} & \multicolumn{2}{|c|}{$\begin{array}{c}\text { Extractives after } \\
\text { chloroform } \\
\text { purification }\end{array}$} \\
\hline & & & Water & Acetone & Water & Acetone \\
\hline 1 & 2-Methoxyphenol (guaiacol) & 7.51 & 0.38 & 0.44 & 0.44 & 0.35 \\
\hline 2 & 4- Hydroxybenzaldehyde & 9.76 & 0.29 & 0.43 & 0.41 & 0.47 \\
\hline 3 & $\begin{array}{l}\text { 1,4-Dihydroxybenzene } \\
\text { (hydroquinone) }\end{array}$ & 10.50 & 0.10 & 0.10 & 0.09 & 0.09 \\
\hline 4 & $\begin{array}{l}\text { 3,5-Di-tertbut-4-hydroxy- } \\
\text { toluene (butylhydroxytoluol) }\end{array}$ & 11.49 & 0.21 & 0.18 & 0.20 & 0.22 \\
\hline 5 & $\begin{array}{l}\text { 3-Methoxy-4- } \\
\text { hydroxybenzaldehyde } \\
\text { (vanillin) }\end{array}$ & 11.96 & 1.38 & 0.90 & 1.84 & 1.24 \\
\hline 6 & 4-Hydroxybenzoic acid & 12.91 & 3.00 & 1.14 & 2.65 & 0.81 \\
\hline 7 & $\begin{array}{l}\text { 3-Methoxy-4- } \\
\text { hydroxybenzoic acid } \\
\text { (vanillic acid) }\end{array}$ & 14.43 & 11.52 & 2.45 & 12.05 & 2.41 \\
\hline 8 & $\begin{array}{l}\text { 4-Hydroxy cinnamic acid } \\
\text { ( } p \text {-coumaric acid) }\end{array}$ & 16.28 & 10.10 & 9.27 & 10.59 & 7.46 \\
\hline 9 & $\begin{array}{l}\text { 4-Hydroxy-3-methoxy } \\
\text { cinnamic acid (ferulic acid) }\end{array}$ & 17.82 & 1.31 & 1.13 & 1.55 & 2.21 \\
\hline
\end{tabular}

*The identity of these compounds was confirmed and the amount of compound present was determined by analyzing in the same set of experiments standards of compounds in different known concentrations

** RT: retention time 
IV. Contrasting ecological functions of Abies grandis wood extractives on protection against white-rot fungi and induction of laccase activity

Table 1 shows the content of the phenolic compounds of interest for laccase induction in the two types of wood extractives. Slight differences were in a number of cases observed in amounts of compounds prior and after chloroform purification but, on the whole, values corresponded to each other (Table 1). Between the analyzed types of extractives, some shared compounds differed in concentration. When comparing water extractives with acetone extractives, striking differences were detected for 3-methoxy4-hydroxybenzoic acid (ca 5x higher in amount in water extractives than in acetone extractives), 4-hydroxybenzoic acid (ca 3x higher in amount in water extractives than in acetone extractives) and smaller differences for 3-methoxy-4-hydroxybenzaldehyde and 4-hydroxy cinnamic acid (both ca 1.1x higher in amount in water extractives than in acetone extractives). When adding values for individual compounds together (values after chloroform-purification were used), the results showed that 4-hydroxy cinnamic acid was the compound of the highest amount with about $18 \mu \mathrm{g}$ in $1 \mathrm{~g}$ dry wood, followed by 3-methoxy-4-hydroxybenzoic acid with about $14.5 \mu \mathrm{g}$ in $1 \mathrm{~g}$ dry wood and by 4-hydroxybenzoic acid and 4-hydroxy-3-methoxy cinnamic acid with each about $3.5 \mu \mathrm{g}$ in $1 \mathrm{~g}$ dry wood, respectively, and by 3-methoxy-4-hydroxybenzyldehyde with about $3 \mu \mathrm{g}$ in $1 \mathrm{~g}$ dry wood. Other compounds ranged in amounts of 0.4 to $0.9 \mu \mathrm{g}$ in $1 \mathrm{~g}$ dry wood whilst 1,4-dihydroxybenzene showed the lowest amount with about $0.2 \mu \mathrm{g}$ in $1 \mathrm{~g}$ dry wood (compare Table 1 and see Table S2 in the supplementary material).

The compounds in the different chromatography fractions of water extractives were also analyzed by GC-MS (Table 2, Fig. S4 in the supplementary material). The saturated fatty acid hexadecanoic acid and octadecanoic acid were found in all fractions eluted with different concentrations of methanol (20-100\% methanol) and octadecanoic acid in addition also in the DMSO-eluted fraction. The phenolic compounds 4-hydroxy cinnamic acid ( $p$-coumaric acid) and 4-hydroxy-3-methoxy cinnamic acid (ferulic acid) were only detected in the chromatography fraction eluted with $20 \%$ methanol, whereas 3-methoxy-4-hydroxybenzoic acid (vanillic acid) was detected in the chromatography fractions eluted with $40 \%$ and $60 \%$ methanol, respectively (Table 2). 
IV. Contrasting ecological functions of Abies grandis wood extractives on protection against white-rot fungi and induction of laccase activity

Table 2 Qualitative profile for compounds found in the different chromatography fraction of $A$. grandis wood extractives as detected by GC-MS analysis

\begin{tabular}{|c|c|c|c|c|c|c|c|c|c|}
\hline \multirow{2}{*}{ No. } & \multirow{2}{*}{ Compounds* } & \multirow{2}{*}{$\mathbf{R T} * *$} & \multicolumn{7}{|c|}{ Chromatography fraction eluted by } \\
\hline & & & $\begin{array}{c}0 \% \\
\text { methanol }\end{array}$ & $\begin{array}{c}20 \% \\
\text { methanol }\end{array}$ & $\begin{array}{c}40 \% \\
\text { methanol }\end{array}$ & $\begin{array}{c}60 \% \\
\text { methanol }\end{array}$ & $\begin{array}{c}80 \% \\
\text { methanol }\end{array}$ & $\begin{array}{c}100 \% \\
\text { methanol }\end{array}$ & DMSO \\
\hline 1 & $\begin{array}{l}\text { 3-Methoxy-4- } \\
\text { hydroxybenzoic } \\
\text { acid (vanillic } \\
\text { acid) }\end{array}$ & 14.43 & - & - & + & + & - & - & - \\
\hline 2 & $\begin{array}{l}\text { 4-Hydroxy } \\
\text { cinnamic acid } \\
\text { ( } p \text {-coumaric } \\
\text { acid) }\end{array}$ & 16.28 & - & + & - & - & - & - & - \\
\hline 3 & $\begin{array}{l}\text { 4-Hydroxy-3- } \\
\text { methoxy } \\
\text { cinnamic acid } \\
\text { (ferulic acid) }\end{array}$ & 17.82 & - & + & - & - & - & - & - \\
\hline 4 & $\begin{array}{l}\text { Hexadecanoic } \\
\text { acid }\end{array}$ & 17.32 & - & + & + & + & + & + & - \\
\hline 5 & $\begin{array}{l}\text { Octadecanoic } \\
\text { acid }\end{array}$ & 19.12 & - & + & + & + & + & + & + \\
\hline
\end{tabular}

* Amounts of compounds were not defines in this experiment in which standards of known concentrations were not applied

$* * \mathrm{RT}$ : retention time

\section{IV.4.5 Laccase induction and inhibition by phenolic compounds}

The phenolic compounds shown in Table 1 were added in finial concentrations of $1 \mathrm{mM}$ (between 1.1-2.2 $\mu \mathrm{g}$, depending on the specific compound, see Fig. 13) into $100 \mathrm{ml}$ BSM liquid medium inoculated with either P. ostreatus 8F6 or T. versicolor Strain 6. One of the compounds [1,4-dihydroxybenzene (hydroquinone)] negatively affected growth of both fungi, with $P$. ostreatus $8 \mathrm{~F} 6$ being more strongly effected than T. versicolor Strain 6 (Fig. 14). The compound was applied at 10-fold higher concentration than found in the water extractives or acetone extractives eluted of $1 \mathrm{~g}$ of A. grandis wood. According to the poor growth of the fungi, no laccase activity was detected in the cultures with $1 \mathrm{mM}$ added 1,4-dihydroxybenzene. In cultures with other 
IV. Contrasting ecological functions of Abies grandis wood extractives on protection against white-rot fungi and induction of laccase activity
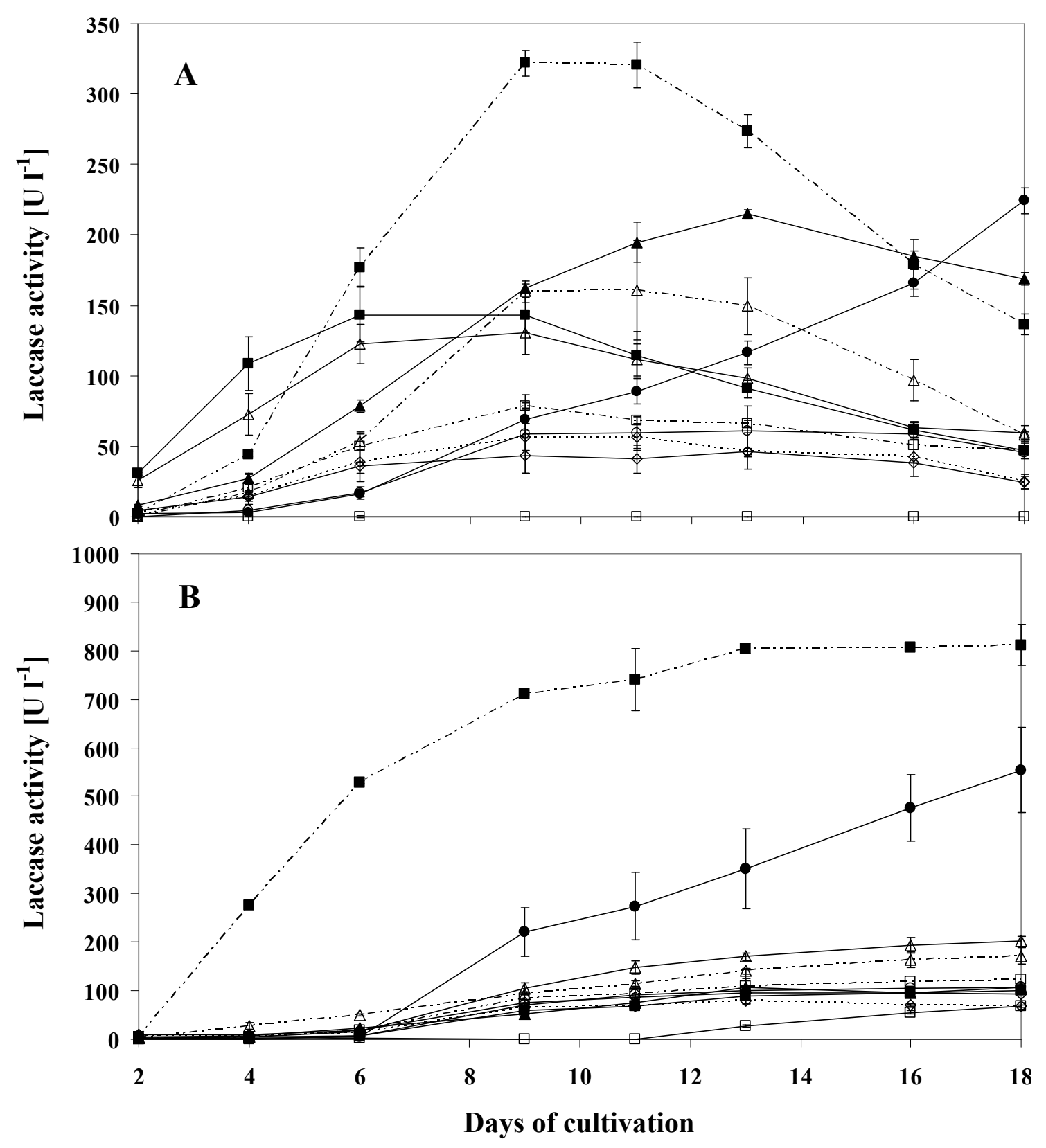

\footnotetext{
$\triangle$ 2-Methoxyphenol (1.2 $\mu \mathrm{g})$

$\square-1,4$ Dihydroxybenzene $(1.1 \mu \mathrm{g})$

$\rightarrow$ 3-Methoxy-4-hydroxybenzaldehyde (1.5 $\mu \mathrm{g})$

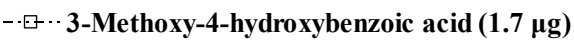

$-\diamond \cdots 4-H y d r o x y-3-m e t h o x y$ cinnamic acid $(1.9 \mu \mathrm{g})$

$\diamond \cdot$ Medium control
}

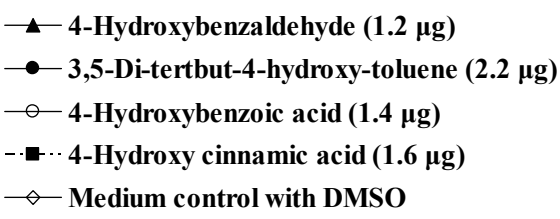

$\neg$ Medium control with DMSO

Fig. 13 Laccase production at $25^{\circ} \mathrm{C}$ over the time by $P$. ostreatus $8 \mathrm{~F} 6$ A) and T. versicolor Strain 6 B) in stationary cultures of $100 \mathrm{ml} \mathrm{BSM}$ liquid medium without and with added $1 \mathrm{mM}$ (in $100 \mu \mathrm{l}$ of DMSO) final concentration of phenolic compounds (for absolute amounts see above the symbol the respective explanations). $100 \mu 1$ of DMSO was added into controls cultures to observe the effect of the solvent on laccase production. No difference was seen in growth between the various cultures of the same fungus but with 1,4-dihydroxybenzene that suppressed growth (see Fig. 14) and 3,5-ditertbut-4-hydroxy-toluene showed the delay growth of both fungi in the first week of incubation. Average values and standard deviations were calculated from three parallel cultures each 
IV. Contrasting ecological functions of Abies grandis wood extractives on protection against white-rot fungi and induction of laccase activity

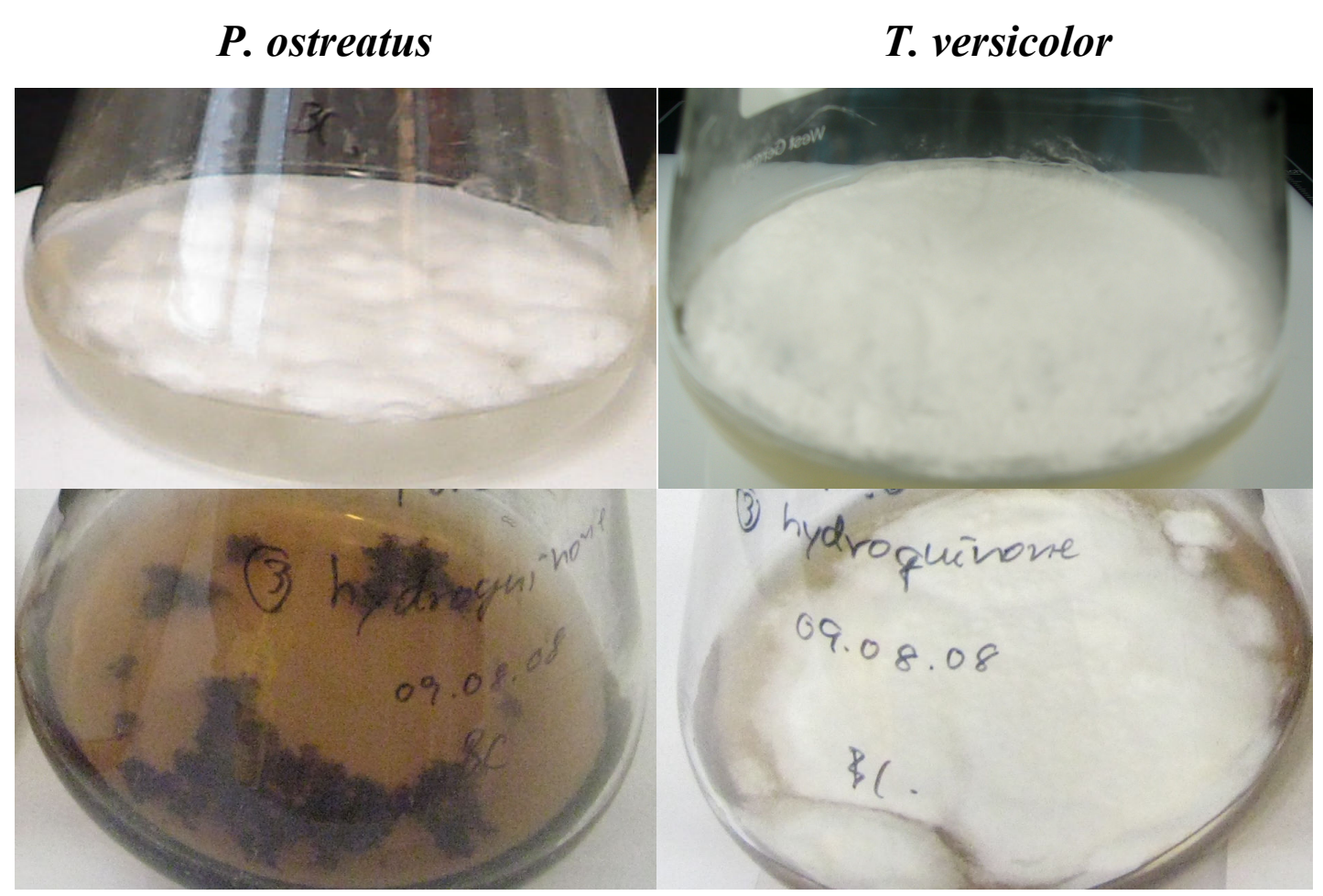

Fig. 14 One week old standing cultures of P. ostreatus 8 F6 and T. versicolor Strain 6 incubated at $25^{\circ} \mathrm{C}$ in $100 \mathrm{ml}$ BSM liquid medium added with $1 \mathrm{mM}$ 1,4dihydroxybenzene (lower row) and medium controls (upper row). For growth effects on a similar compound ( $p$-benzochinone), see Fig. S5 in the supplementary material

compounds with the exception of compound (see below), no differences were seen in fungal growth as compared to the medium controls (Fig. 14).

In both fungi, 4-hydroxy cinnamic acid was most successful in induction of laccase activity. Although with $1 \mathrm{mM}$ final concentration the amount of the added compound was several-fold lower than in the water extractives or in the acetone extractives obtained from $1 \mathrm{~g}$ of $A$. grandis wood (see Table 1), 4-hydroxy cinnamic acid induced highest laccase production in $P$. ostreatus $8 \mathrm{~F} 6$ of $322 \pm 9.1 \mathrm{U}^{-1}$ (at day 9 of incubation) and in T. versicolor Strain 6 of $811 \pm 41.7 \mathrm{U} \mathrm{l}^{-1}$ (at day 18 of incubation), respectively (Fig. 14). 3,5-di-tertbut-4-hydroxy-toluene (applied in about 10-fold higher concentration as found in the water extractives and acetone extractives obtained from $1 \mathrm{~g}$ A. grandis wood, see Table 1) showed second highest laccase induction in the fungal cultures (Fig. 14). Levels of laccase activity with $1 \mathrm{mM}$ 3,5-di-tertbut-4hydroxy-toluene added into the BSM liquid medium reached of $224 \pm 8.9 \mathrm{U}^{-1}$ in P. ostreatus $8 \mathrm{~F} 6$ cultures at day 18 of incubation and $554 \pm 88.4 \mathrm{U} \mathrm{l}^{-1}$ in $T$. versicolor 
IV. Contrasting ecological functions of Abies grandis wood extractives on protection against white-rot fungi and induction of laccase activity

Strain 6 cultures at day 18 of incubation, respectively. However, 3,5-di-tertbut-4hydroxy-toluene also caused a delay of growth of both fungi in the first week of incubation (not shown), but afterward fungal growth increased and the compound functioned well in laccase induction (Fig. 13).

Four further compounds positively affected laccase production in P. ostreatus 8F6: 4-hydroxybenzaldehyde with laccase yields of $215 \pm 3.2 \mathrm{U} \mathrm{l}^{-1}$ at day 13 of incubation, 4-hydroxy-3-methoxy cinnamic acid with laccase yields of $161 \pm 35.2 \mathrm{U}^{-1}$ at day 11 of incubation, 3-methoxy-4 hydroxybenzaldehyde with laccase yields of $143 \pm 12.2 \mathrm{U} \mathrm{l}^{-1}$ at day 9 of incubation, and 2-methoxyphenol with laccase yields of $130 \pm 15.4 \mathrm{U}^{-1}$ at day 9 of incubation, respectively (Fig. 13A). The amounts of three of these compounds in this experiment were comparable to their amounts in water extractives and acetone extractives obtained of $1 \mathrm{~g}$ of $A$. grandis (compare Table 1) whilst the amount of 2-methoxyphenol added to cultures in this experiment by a factor of about 10 lower. However, still there was an inductive effect in laccase production. Other tested compounds (4-hydroxybenzoic acid and 3-methoxy-4-hydroxybenzoic acid) were without effects (Fig. 13A). However, the applied amounts were at least from 3-methoxy-4-hydroxybenzoic acid slightly lower than the concentration obtained from A. grandis wood with acetone extractives and in both cases the applied concentration was (much) lower compared to the amounts found in water extractives isolated from $1 \mathrm{~g}$ of $A$. grandis wood.

When examining the effects in T. versicolor Strain 6 cultures, 2-methoxyphenol and 4-hydroxy-3-methoxy cinnamic acid gave laccase activity of $202 \pm 11.2 \mathrm{U}^{-1}$ and of $172 \pm 17.4 \mathrm{U} \mathrm{l}^{-1}$ at day 18 of incubation, respectively; as compared to $70 \pm 6.5 \mathrm{U} \mathrm{l}^{-1}$ of controls with or without added $100 \mu 1$ DMSO (Fig. 13B). All other compounds gave no or only neglectable effects on laccase induction (Fig. 13B).

Since 4-hydroxy cinnamic acid and 3,5-di-tertbut-4-hydroxy-toluene were found to induced in both fungi very well the laccase production (Fig. 13) synergistic effects were tested between them by adding either $1 \mathrm{mM}$ of the individual compound into culture or each $1 \mathrm{mM}$ of both compounds. Again, 4-hydroxy cinnamic acid induced very well laccase production in similar amounts as in the previous experiments (Fig. 13) with best activity of $332 \pm 37.0 \mathrm{U}^{-1}$ at day 12 of incubation in the case of $P$. ostreatus 8 F6 (Fig. 15A) and best activity of $826 \pm 140.3 \mathrm{U} \mathrm{l}^{-1}$ at day 12 of incubation in the case of T. versicolor Strain 6 (Fig. 15B). When applying $1 \mathrm{mM}$ of 3,5-di-tertbut-4-hydroxytoluene fungal growth was again delayed by about 8-10 days as compared to the 
IV. Contrasting ecological functions of Abies grandis wood extractives on protection against white-rot fungi and induction of laccase activity

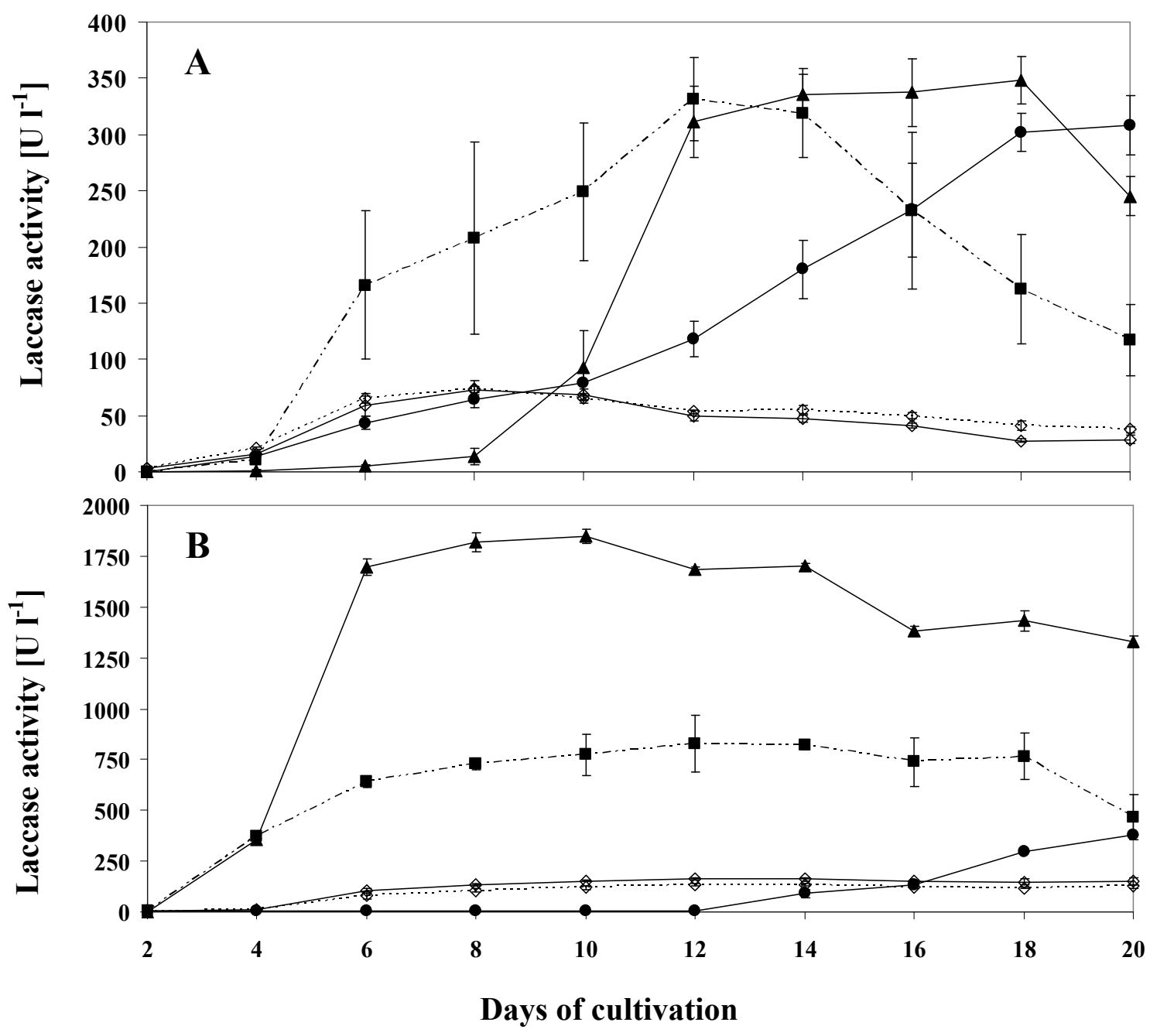

- 4-Hydroxy cinnamic acid (1.6 $\mu \mathrm{g})$

$\longrightarrow 3,5$-Di-tertbut-4-hydroxy-toluene $(2.2 \mu \mathrm{g})$

$\triangle 4$-Hydroxy cinnamic acid $(1.6 \mu \mathrm{g})+3,5$-di-tertbut-4-hydroxy-toluene $(2.2 \mu \mathrm{g})$

$\diamond$ Medium control with DMSO

$\cdots \diamond \cdot$ Medium control

Fig. 15 Laccase production at $25^{\circ} \mathrm{C}$ by $P$. ostreatus $8 \mathrm{~F} 6$ A) and T. versicolor Strain 6 B) in stationary cultures of $100 \mathrm{ml} \mathrm{BSM}$ liquid medium without and with $1 \mathrm{mM}$ (in $200 \mu 1$ of DMSO) final concentration of phenolic compounds. $200 \mu 1$ of DMSO was added into control cultures to observe the effect of the solvent on laccase production. Differences were seen in growth for both fungi compared to the cultures added with 3,5-di-tertbut-4-hydroxy-toluene and control, and with 4-hydroxy cinnamic acid + 3,5di-tertbut-4-hydroxy-toluene for $P$. ostreatus 8F6. Average values and standard deviations were calculated from three parallel cultures each 
IV. Contrasting ecological functions of Abies grandis wood extractives on protection against white-rot fungi and induction of laccase activity

medium control cultures and consequently also laccase production. Highest levels laccase of $308 \pm 26.2 \mathrm{U} \mathrm{l}^{-1}$ were reached at day 20 of incubation in the P. ostreatus $8 \mathrm{~F} 6$ cultures (Fig. 15A) and $382 \pm 0.01 \mathrm{U} \mathrm{l}^{-1}$ at day 20 of incubation in the T. versicolor Strain 6 cultures (Fig. 15B).

Growth delay by $8-10$ days was also observed with $P$. ostreatus $8 \mathrm{~F} 6$ when applying both compounds. Once growth started, laccase production however was induced to best level of $349 \pm 21.3 \mathrm{U} \mathrm{l}^{-1}$ at day 18 of incubation, which corresponds in amounts of enzymes to the best yields when the compounds were individually applied (Fig. 15A).

Different was the behavior of $T$. versicolor Strain 6 with both compounds added together there was no growth delay and the onset of high laccase production was early (day 6 of incubation) to amazing amount of $1,850 \pm 36.1 \mathrm{U}^{-1}$ and this high level of enzymatic yields was hold up to day 14 of incubation upon which enzymatic activities in the cultures decreased for the rest of the incubation time to about 1,500 $\mathrm{U}^{-1}$ (Fig. 15B). Laccase yields with the combination of both compounds were thus about two times higher than with 4-hydroxy cinnamic acid alone and about five times higher than 3,5-di-tertbut-4-hydroxy-toluene alone. In conclusion, there was a clear-cut synergistic effect between the two compounds in T. versicolor Strain 6 but not in P. ostreatus $8 \mathrm{~F} 6$.

\section{IV.4.6 Enzyme identification}

Supernatants from $P$. ostreatus 8F6 and T. versicolor Strain 6 cultures with or without added wood or with added wood extractives or with added fractions of wood extractives or with added specific compounds were loaded onto native PAGE gels and proteins were separated by gel electrophoresis (as described in Chapter IV.3.6). Laccase activities in the supernatants ranged in between $20 \mathrm{U} \mathrm{l}^{-1}$ to $2500 \mathrm{U} \mathrm{l}^{-1}$ (Table 3). However, laccase activities detected upon activity staining in the native PAGE gels did not always correspond well with the measured activities in the culture supernatants (compare Fig. 16 and Table 3). No laccase activity was seen on the gel with the original supernatants of $P$. ostreatus $8 \mathrm{~F} 6$ culture with added water extractives, with added acetone extractives and with added solvent DMSO (Fig. 16A). Since laccase activities might have been too low for detection in the samples, supernatant samples from cultures with added specific phenols were 10-fold concentrated prior to loading. 
IV. Contrasting ecological functions of Abies grandis wood extractives on protection against white-rot fungi and induction of laccase activity

Native in-gel staining revealed one main laccase isoenzyme in culture supernatant of $P$. ostreatus $8 \mathrm{~F} 6$ and two different isoenzymes in cultures of T. versicolor Strain 6. Mass spec analysis of the in gel trypsin-digested proteins from the bands of the supernatants of wood supplemented cultures from parallel Coomassie stained gels (see Chapter IV.3.6 for methodical details) identified by database comparison laccase II (NCBI: gi|116499197) from P. ostreatus 8F6 and laccase I (NCBI: gi|23200086) and laccase III (NCBI: gi|19848920) from T. versicolor Strain 6, respectively (see Table 4).
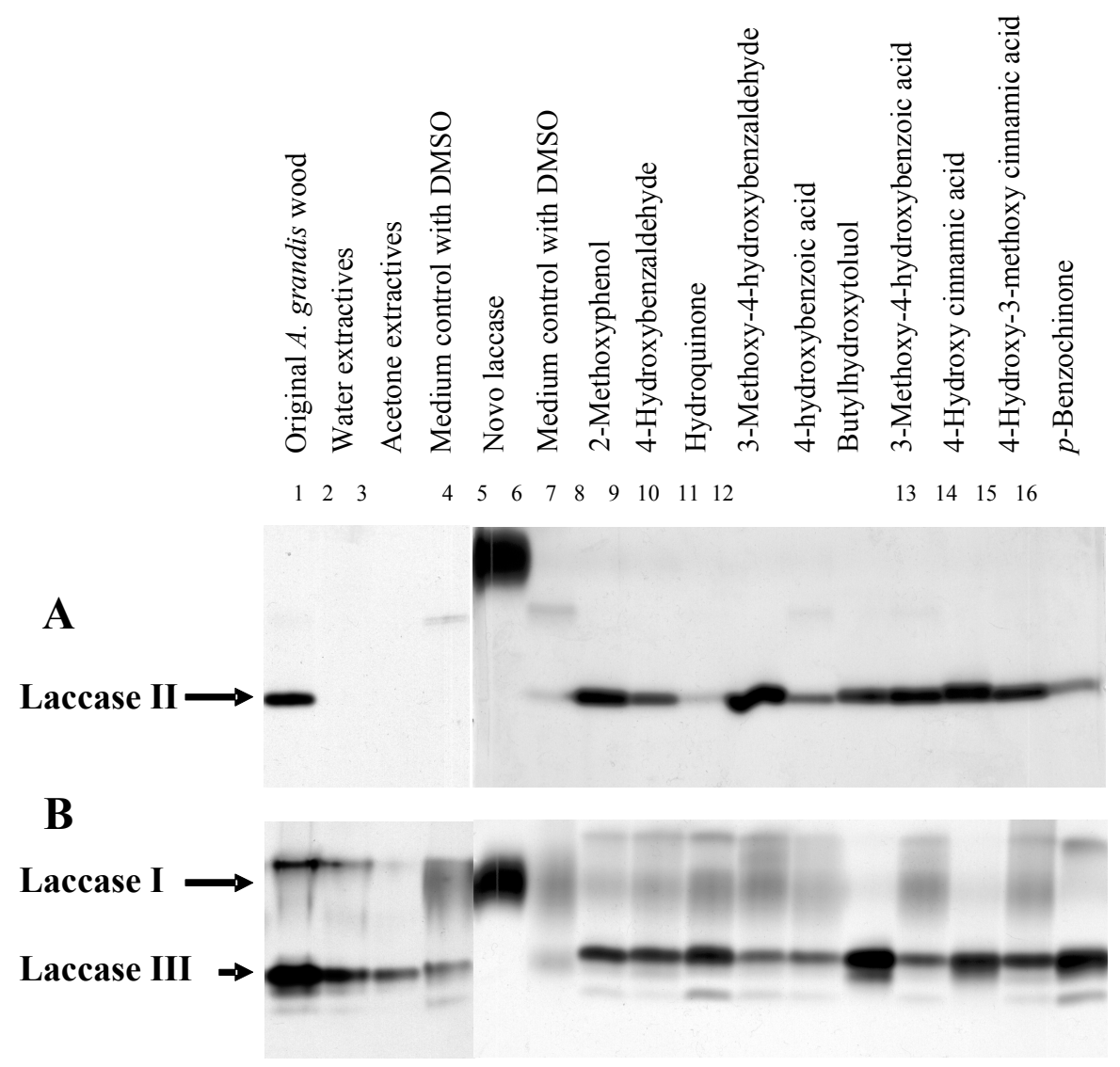

Fig. 16 P. ostreatus 8F6 A) and T. versicolor Strain 6 B) laccase isoenzyme analysis by native PAGE of culture supernatants with added original wood or with added wood extractives (lane 2 and 3) or with added specific phenolic compounds (lane 6 to 16) or with added solvent DMSO (lane 4 and 6), (compare Table 3 for the individual samples). In most cases, $10 \mu \mathrm{l}$ of culture supernatant was loaded into a gel slot. Samples numbered 6 to 16 for both fungi were 10x concentrated with a Vivaspin column before loading samples of a volume of $10 \mu \mathrm{l}$. Recombinant $893 \mathrm{U} \mathrm{ml}^{-1}$ Novo laccase (Novozyme, EEC No. 420-150-4) from Aspergillus sp. was added in lane 5 for comparison before loading samples of a volume of $10 \mu \mathrm{l}$. Laccase activities within the gels were detected by MBTH staining as described in Chapter IV.3.6. The samples in slots 1 to 4 and in slots 5 to 16 came from different gels 
IV. Contrasting ecological functions of Abies grandis wood extractives on protection against white-rot fungi and induction of laccase activity

Table 3 Laccase activities in supernatants of $P$. ostreatus $8 \mathrm{~F} 6$ and $T$. versicolor Strain 6 cultures use in a native PAGE (Fig. 16) for isoenzyme analysis

\begin{tabular}{|c|c|c|c|c|c|}
\hline \multirow[t]{2}{*}{ No.* } & \multirow[t]{2}{*}{ Sample } & \multicolumn{2}{|c|}{$\begin{array}{c}\text { Laccase activity } \\
\qquad\left[\mathrm{U} \mathrm{l}^{-1}\right]\end{array}$} & \multicolumn{2}{|c|}{$\begin{array}{c}\text { Day of harvest (highest } \\
\text { laccase activity)*** }\end{array}$} \\
\hline & & P. ostreatus & T. versicolor & P. ostreatus & T. versicolor \\
\hline 1 & Original $A$. grandis wood & 260 & 670 & 14 & 12 \\
\hline 2 & Water extractives & 186 & 520 & 16 & 14 \\
\hline 3 & Acetone extractives & 143 & 191 & 18 & 14 \\
\hline 4 & $\begin{array}{l}\text { Medium control with } \\
\text { DMSO }\end{array}$ & 67 & 102 & 16 & 20 \\
\hline 6 & $\begin{array}{l}\text { Medium control with } \\
\text { DMSO }\end{array}$ & 424 & 1252 & 13 & 16 \\
\hline 7 & 2-Methoxyphenol & 692 & 1650 & 9 & 18 \\
\hline 8 & 4- Hydroxybenzaldehyde & 223 & 1556 & 13 & 13 \\
\hline 9 & Hydroquinone & 21 & 1761 & 4 & 18 \\
\hline 10 & $\begin{array}{l}\text { 3-Methoxy-4- } \\
\text { hydroxybenzaldehyde }\end{array}$ & 1258 & 1586 & 9 & 18 \\
\hline 11 & 4-Hydroxybenzoic acid & 218 & 2532 & 13 & 18 \\
\hline 12 & Butylhydroxytoluol & 1043 & 1726 & 18 & 18 \\
\hline 13 & $\begin{array}{l}\text { 3-Methoxy-4- } \\
\text { hydroxybenzoic acid }\end{array}$ & 870 & 1675 & 9 & 18 \\
\hline 14 & 4-Hydroxy cinnamic acid & 702 & 1618 & 11 & 18 \\
\hline 15 & $\begin{array}{l}\text { 4-Hydroxy-3-methoxy } \\
\text { cinnamic acid }\end{array}$ & 839 & 1720 & 11 & 18 \\
\hline 16 & $p$-Benzochinone & 118 & 1478 & 18 & 18 \\
\hline
\end{tabular}

* Note: Sample numbers refer to the gels shown in Fig. 16. Samples numbered 6 to 16 were 10x concentrated with Viva spin columns before use; samples 1 to 4 originated from the experiment documented in Fig. 4, samples 6 to 16 from the experiment documented in Fig. 15.

** Day of harvest was always the day of highest laccase activity when culturing the fungi over the time 
IV. Contrasting ecological functions of Abies grandis wood extractives on protection against white-rot fungi and induction of laccase activity

Table 4 Identification of purified laccases in supernatants of cultures of $P$. ostreatus 8F6 and T. versicolor Strain 6

\begin{tabular}{|c|c|c|c|c|c|c|c|}
\hline $\begin{array}{l}\text { Peptides identified by } \\
\text { ESI-LC-MS }\end{array}$ & $\begin{array}{l}\text { Peptide } \\
\text { mass } \\
\text { M+H }\end{array}$ & $\begin{array}{l}\text { Peptide } \\
\text { score }\end{array}$ & Protein & $\begin{array}{l}\text { Total } \\
\text { score }\end{array}$ & Coverage & pI & MW \\
\hline R.YDVDNESTITLTDWYHTAAR.L & 2469.14 & 55 & Laccase I & 153 & $10 \%$ & 5.9 & 53885 \\
\hline R.FPLGADATLINGLGR.S & 1513.83 & 44 & & & & & \\
\hline R.SAGSTTYNDPIFR.D & 1704.77 & 52 & & & & & \\
\hline R.ANPNLGSTGFVGGINSAILR.Y & 1957.04 & 32 & Laccase II & 202 & $3 \%$ & 4.7 & 56027 \\
\hline K.LGPAFPLGADATLINGK.G & 1653.91 & 55 & Laccase III & 207 & $9 \%$ & 4.8 & 55724 \\
\hline R.SPSTTTADLTVISVTPGKR.Y & 1930.04 & 26 & & & & & \\
\hline R.SAGSTVYNYDNPIFER.D & 1702.80 & 41 & & & & & \\
\hline
\end{tabular}

\section{IV.4.7 Spectrophotometric determination of laccase activity on wood particles}

A high performance purified laccase [laccase III (accession no. AAL93622.1) from T. versicolor Strain 6 (Dwivedi 2006)] or Novo laccase from Aspergillus sp. (Novozyme, EEC No. 420-150-4) was used to treat $A$. grandis wood particles in order to detect specific effects by the enzyme on the wood. Always in all experiments, three replicates of samples per tested condition were performed.

The laccase III modifies $A$. grandis wood particles as observed by color changes of both, of wood particles and of aliquot solutions (Fig. 17). Compared to the light color of untreated wood particles, wood particles treated with laccase were more dark yellow and also the color of the liquid of sample with laccase was more intense yellow (Fig. 17). Such color changes were observed with different amounts of the laccase $\left(0.67 \mathrm{U} \mathrm{ml}^{-1}\right.$ and $\left.2 \mathrm{U} \mathrm{ml}^{-1}\right)$ applied for $15 \mathrm{~min}$ to $0.1 \mathrm{~g}$ wood particles present in $10 \mathrm{ml}$ of BSM medium (Fig. 17). The lower amount of laccase activities corresponds to the laccase activities observed in BSM cultures with added $A$. grandis wood particles wood particles and T. versicolor Strain 6 (Fig. 5). Technically, $20 \mathrm{ml}$ Schott glass tubes fixed on a moving rotary drum gave best results when using $0.1 \mathrm{~g}$ of wood particles in $10 \mathrm{ml}$ solutions. With smaller tubes (e.g. $10 \mathrm{ml}$ ), wood particles clumped together hindering the enzyme the access to the fiber surfaces (not shown). 
IV. Contrasting ecological functions of Abies grandis wood extractives on protection against white-rot fungi and induction of laccase activity

Control

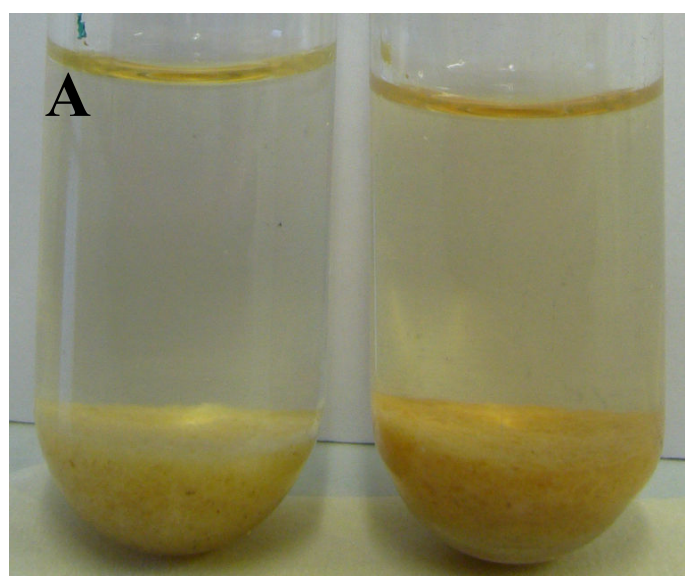

Control

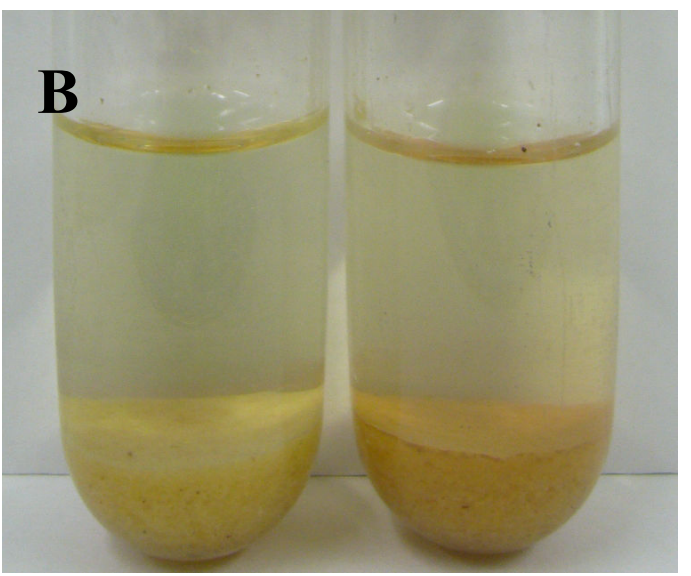

Fig. 17 Color changes of $0.1 \mathrm{~g}$ A. grandis wood particles after incubation for $15 \mathrm{~min}$ at room temperature with laccase III (accession no. AAL93622.1) from $T$. versicolor Strain 6 at concentration of A) $0.67 \mathrm{U} \mathrm{ml}^{-1}$ and B) $2 \mathrm{U} \mathrm{ml}^{-1}$ in $10 \mathrm{ml}$ of BSM medium in $20 \mathrm{ml}$ Schott glass tubes fixed onto a moving rotary drum. Note that the yellowishorange change in color in case of wood particles incubated with laccase is due to modification of wood cell walls (compare Fig. 18)

The enhanced yellow coloring in supernatants of wood particles treated with laccase (Fig. 17) likely reflected the release of compounds into the sample liquids. Therefore, after incubation of $0.1 \mathrm{~g}$ wood particles in $50 \mathrm{mM}$ sodium acetate buffer together with $2 \mathrm{U} \mathrm{ml}^{-1}$ laccase III for different times, chemical changes were measured by detecting release of compounds into the buffer through changes in absorbance in the sample's liquid. Spectrograms over the whole spectrums of wavelengths from $620 \mathrm{~nm}$ to $220 \mathrm{~nm}$ were scanned for both samples with laccase-treated and samples with untreated wood particles (Fig. 18). Higher absorbance was measured in the range of wavelength $220 \mathrm{~nm}$ to $420 \mathrm{~nm}$ with highest absorbance in the range of lower wavelengths and a clear peak at a wavelength of $280 \mathrm{~nm}$. In samples of wood particles incubated only in buffer, basically no change in absorbance was detected over the time of $12 \mathrm{hrs}$. In contrast, in samples with laccase III visible changes occurred with time with increasing levels of absorbance over the whole range of wavelengths from $220 \mathrm{~nm}$ to $420 \mathrm{~nm}$. In contrast to the buffer of non-treated wood particles, the peak at $280 \mathrm{~nm}$ was generally more flat, also due to a generally higher absorbance in the wavelength region from $245 \mathrm{~nm}$ to $270 \mathrm{~nm}$ (Fig. 18, Fig. 19).

Changes in absorbance in the samples' liquid reflecting chemical alterations on A. grandis wood particles or on compounds released into the buffer are apparent at some specific wavelengths as shown in Fig. 20. Whereas in the control samples the 
IV. Contrasting ecological functions of Abies grandis wood extractives on protection against white-rot fungi and induction of laccase activity

absorbance levels at the three especially selected wavelengths at $245 \mathrm{~nm}, 280 \mathrm{~nm}$ and $295 \mathrm{~nm}$ remained constant over the time, in samples with laccase III at all three wavelengths there were obvious increases in absorbance. Particularly at $245 \mathrm{~nm}$, a highest change in absorbance was observed (Fig. 20).

Differences in the absorbance pattern between wood particles treated with laccase III and wood particles not treated with the laccase could be due to decay of released compounds by laccase III, or due to release of further compounds from the wood due to laccase actions on the cells, or due to combinations thereof. That there was a decrease in absorbance in between wavelengths $270 \mathrm{~nm}$ and $290 \mathrm{~nm}$ and otherwise at other wavelengths an increase in absorbance (Fig. 19) argues for a combination of both types of activities.

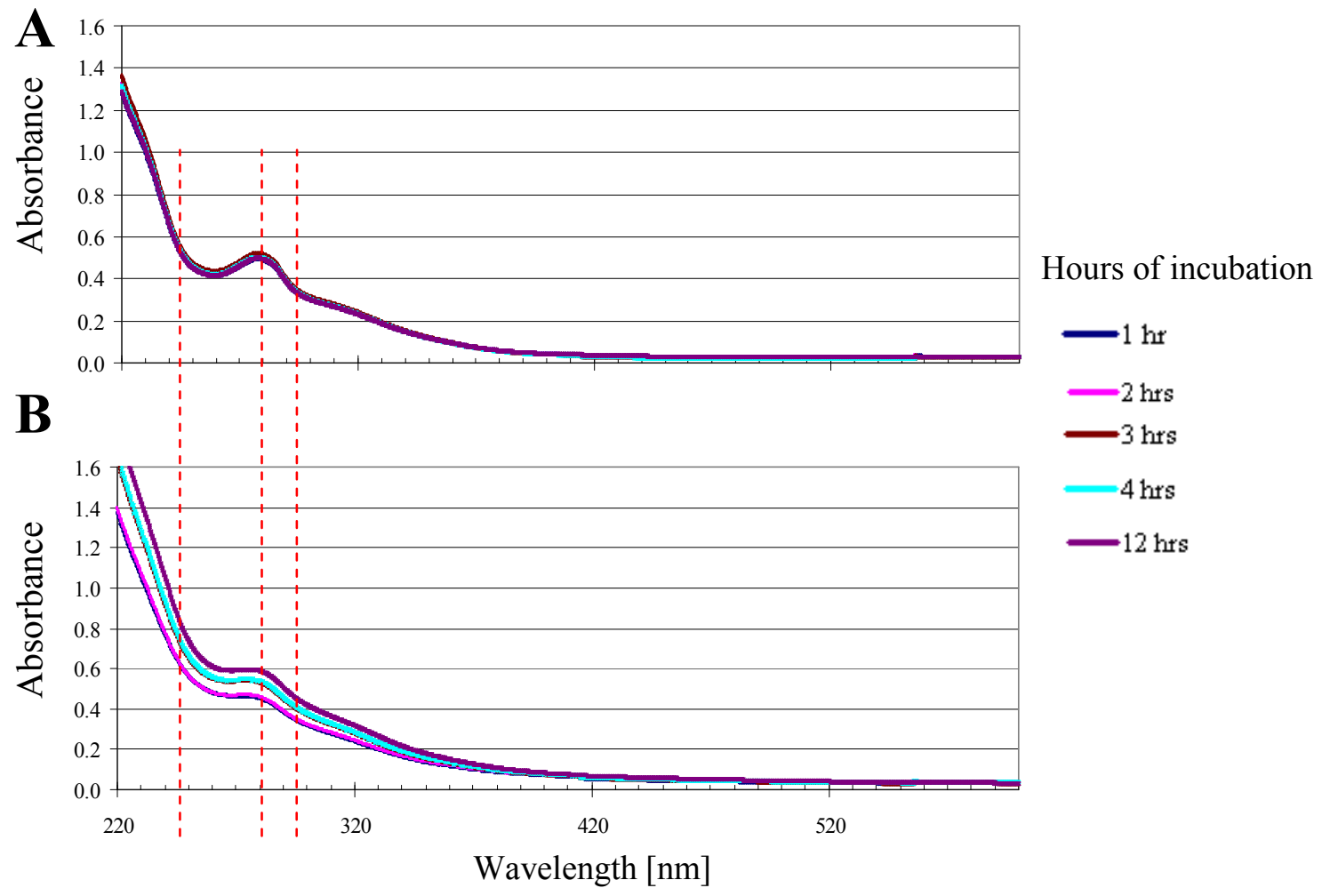

Fig. 18 Changes in absorbance in $50 \mathrm{mM}$ sodium acetate buffer, $\mathrm{pH} 5.0$ of samples of A. grandis wood particles, reflecting release of chemicals over the time from untreated (control) wood particles A) and from wood particles treated with $2 \mathrm{U} \mathrm{ml}^{-1}$ laccase III (accession no. AAL93622.1) from T. versicolor Strain 6 B). Dashed lines indicate specific wavelengths of 245, 280 and $295 \mathrm{~nm}$ (from left to right), respectively, that corresponded to obvious changes in absorbance due to laccase treatment. For an enlarged view of this range of wavelength see Fig. 19. Note that the buffer alone does not give any absorbance (not shown). Absorbances were measured in a spectrophotometer (UV-DU800, Beckman coulter, Inc., California, USA) with a white light source 
IV. Contrasting ecological functions of Abies grandis wood extractives on protection against white-rot fungi and induction of laccase activity

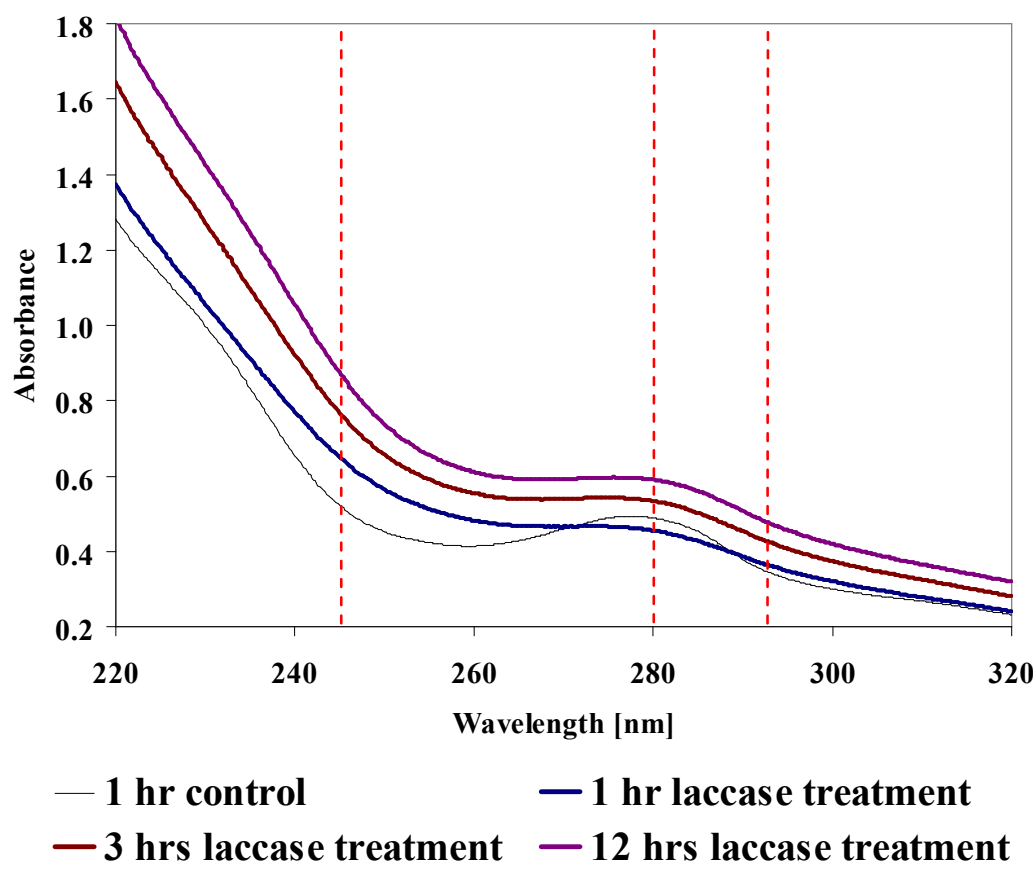

Fig. 19 Enlargement view of the wavelength range from $220 \mathrm{~nm}$ to $320 \mathrm{~nm}$ of the spectrogram shown in Fig. 18, showing changes in absorbance in $50 \mathrm{mM}$ sodium acetate buffer, $\mathrm{pH} 5.0$ of samples of wood particles, reflecting release of chemicals over the time on the $A$. grandis wood particles of untreated (control) wood particles and wood particles treated with $2 \mathrm{U} \mathrm{ml}^{-1}$ laccase III (accession no. AAL93622.1) from T. versicolor Strain 6, respectively. Dashed lines indicate specific wavelengths of 245, 280 and $295 \mathrm{~nm}$ (from left to right), respectively, that corresponded to obvious changes in absorbance due to laccase treatment

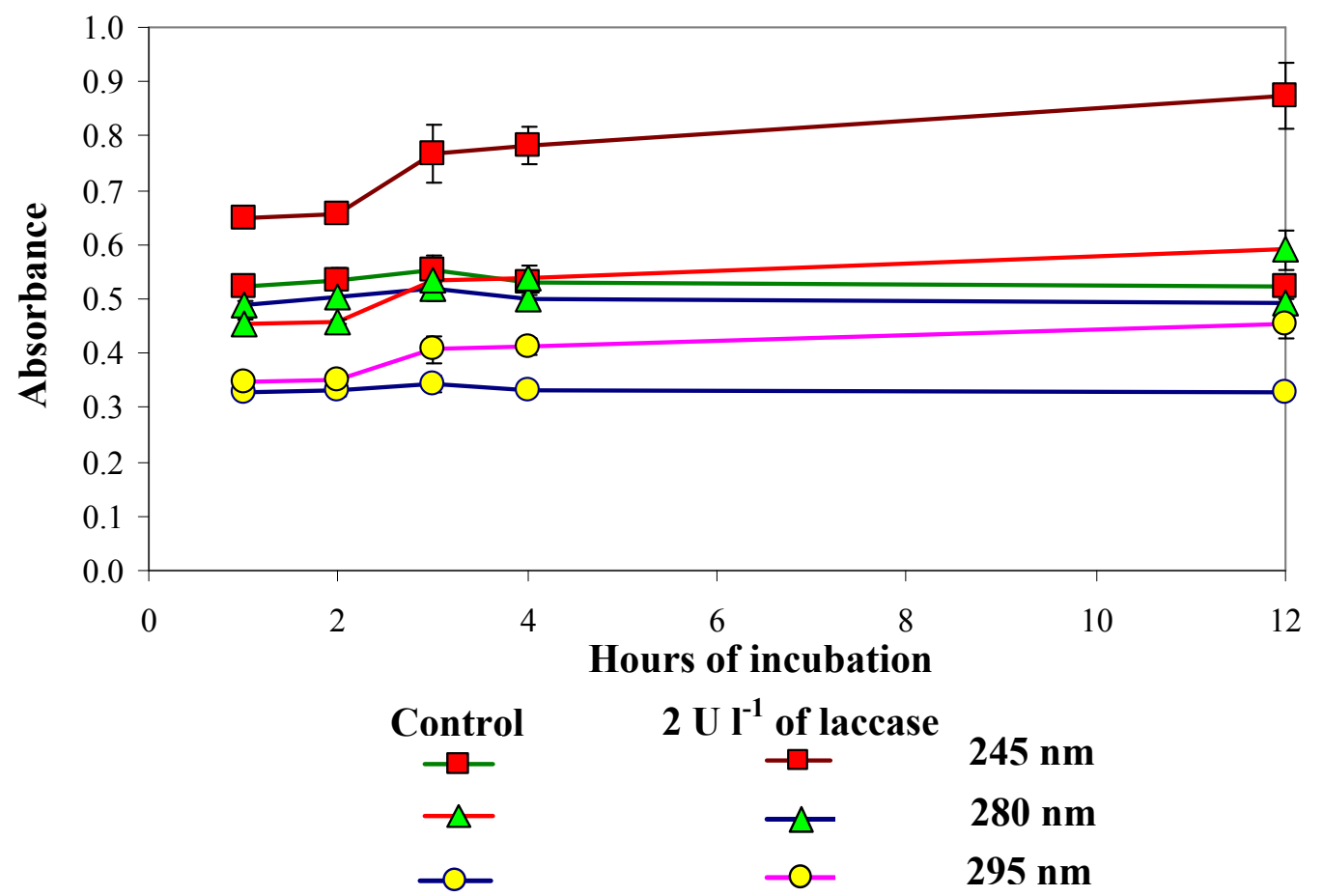

Fig. 20 Measured absorbances at specific wavelengths over the time in $10 \mathrm{ml}$ of $50 \mathrm{mM}$ sodium acetate buffer with added $0.1 \mathrm{~g} \mathrm{~A}$. grandis wood particles and with or without added $2 \mathrm{U} \mathrm{ml}^{-1}$ of laccase (see Fig. 19 for the whole diagrams) 
IV. Contrasting ecological functions of Abies grandis wood extractives on protection against white-rot fungi and induction of laccase activity

Further, after the purified laccase III of Dwivedi (2006) was used up, effects of Novo laccase of Aspergillus sp. on A. grandis wood particles was observed in $50 \mathrm{mM}$ sodium acetate buffer. In all experiments, $0.1 \mathrm{~g}$ of wood particles were incubated in $10 \mathrm{ml}$ of buffer with $2 \mathrm{U} \mathrm{ml}^{-1}$ Novo laccase. A color change of wood particles and a color change of the buffer was also observed when incubating wood particles with Novo laccase in the buffer (not shown) as previously with laccase III in the BSM medium (Fig. 17). The darker yellow color of the wood particles suggested that changes at the wood cell walls might have happened subsequent to incubation, wood particles were therefore observed under the microscope (see Fig. 21).

In Novo-laccase-treated wood particles, areas of light cell-wall-staining were found already after $15 \mathrm{~min}$ of incubation unlike in comparison in control wood particles not treated with the enzyme. Light yellowish-brown staining was seen in cell walls in between ray cells and at the opening of pits (Fig. 21), indicating laccase activities at these structures. Treatment with MBTH did not change the color of the wood in the control cultures but had an effect on wood particles that were treated with laccase (Fig. 21). Cell walls in between ray cells and at the opening of pits were much stronger brownish colored after staining with MBTH (Fig. 21), supporting also the earlier observation (Fig. 11 in Chapter III) that laccase acts most easily at the ray cell walls and at the pits.

In the following, GC-MS analysis was then used to identify the compounds released from $0.1 \mathrm{~g} \mathrm{~A}$. grandis wood particles when incubated for $1 \mathrm{hr}$ at room temperature alone in $10 \mathrm{ml}$ of $50 \mathrm{mM}$ sodium acetate buffer, $\mathrm{pH} 5.0$ or with $2 \mathrm{U} \mathrm{ml}^{-1}$ Novo laccase in $50 \mathrm{mM}$ sodium acetate buffer, $\mathrm{pH} 5.0$ in Schott glass tubes fixed onto a moving rotary drum (Fig. 22).

In total, 12 compounds were detected in the buffer solutions with an identification quality higher than 95\%: 3-methoxy-4-hydroxybenzaldehyde, glucofuranose, D-glucose, octadecane, cinnamic acid, $\alpha$-D-galactopyranose, hexadecanoic acid, oleic acid, octadecanoic acid, 9-octadecanamide, docosanoic acid and lignan. Compounds shown in italic were present significantly by higher in abundance in samples treated with laccase. Compounds shown in bold were present in significant higher amounts in samples without Novo laccase. Further note that of the phenolic compounds found in wood extractives from Soxhlet extraction at boiling temperature (Chapter IV.4.4), only 3-methoxy-4-hydroxybenzaldehyde, cinnamic acid, 
IV. Contrasting ecological functions of Abies grandis wood extractives on protection against white-rot fungi and induction of laccase activity

hexadecanoic acid, oleic acid, octadecanoic acid, and docosanoic acid were present in the supernatants in the current experiment performed at RT.

Without staining

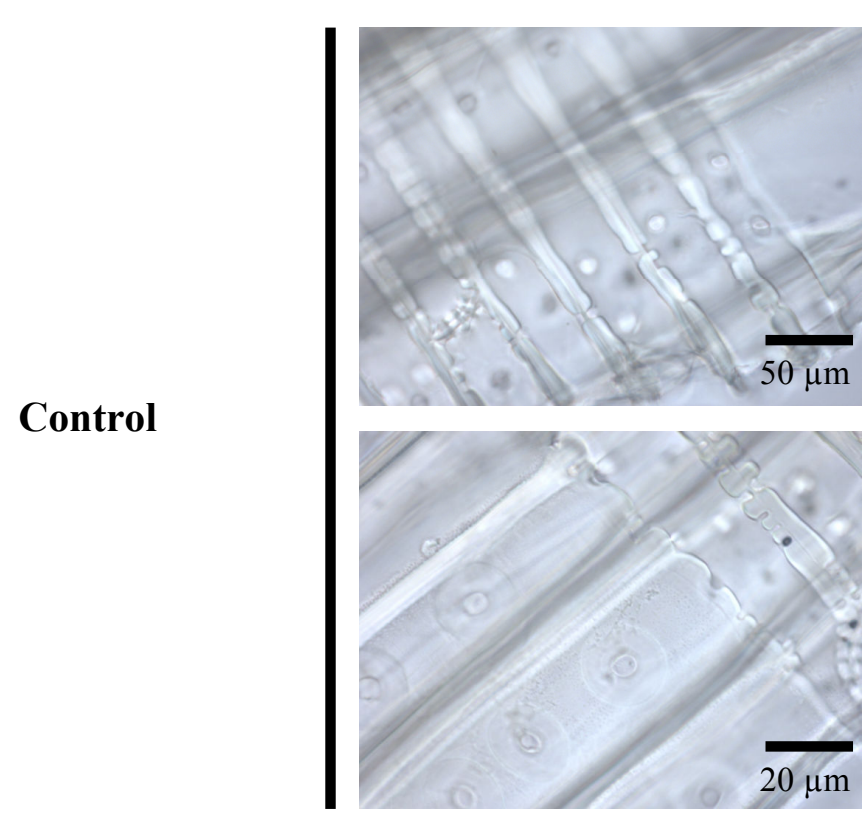

Laccase treatment

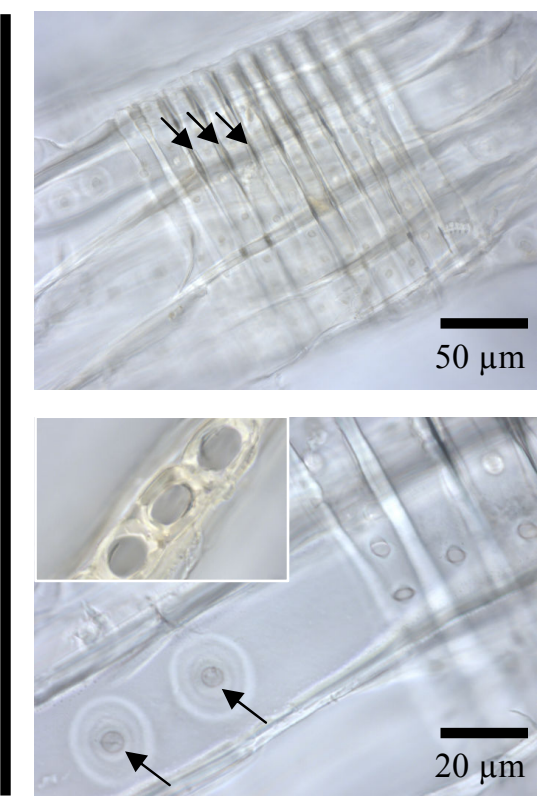

MBTH-staining
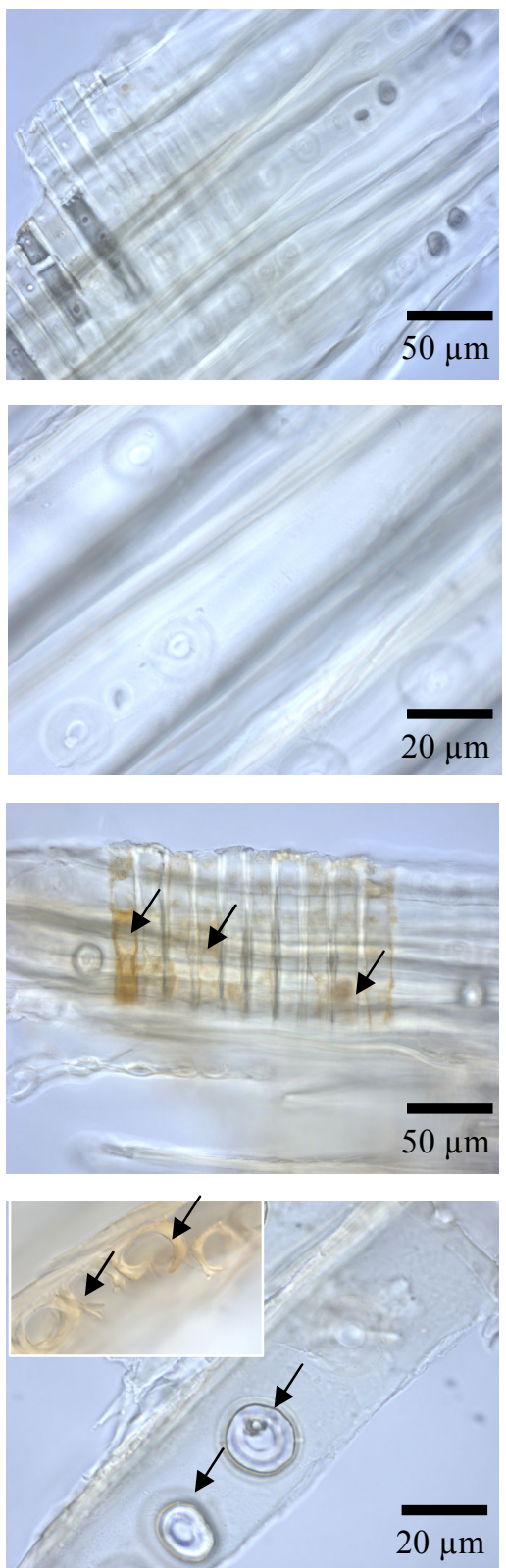

Fig. 21 Enlarged views of $A$. grandis wood fiber cells after $1 \mathrm{hr}$ incubation at RT in $50 \mathrm{mM}$ sodium acetate buffer, $\mathrm{pH} 5.0$ without laccase (control) or with $2 \mathrm{U} \mathrm{ml}^{-1}$ Novo laccase from Aspergillus sp. (laccase treatment) without MBTH-staining (left column) or with MBTH-staining (right column). Arrows indicate cell walls around pits and cell walls between ray cells that were stained by laccase treatment or, in addition, by MBTH treatment 


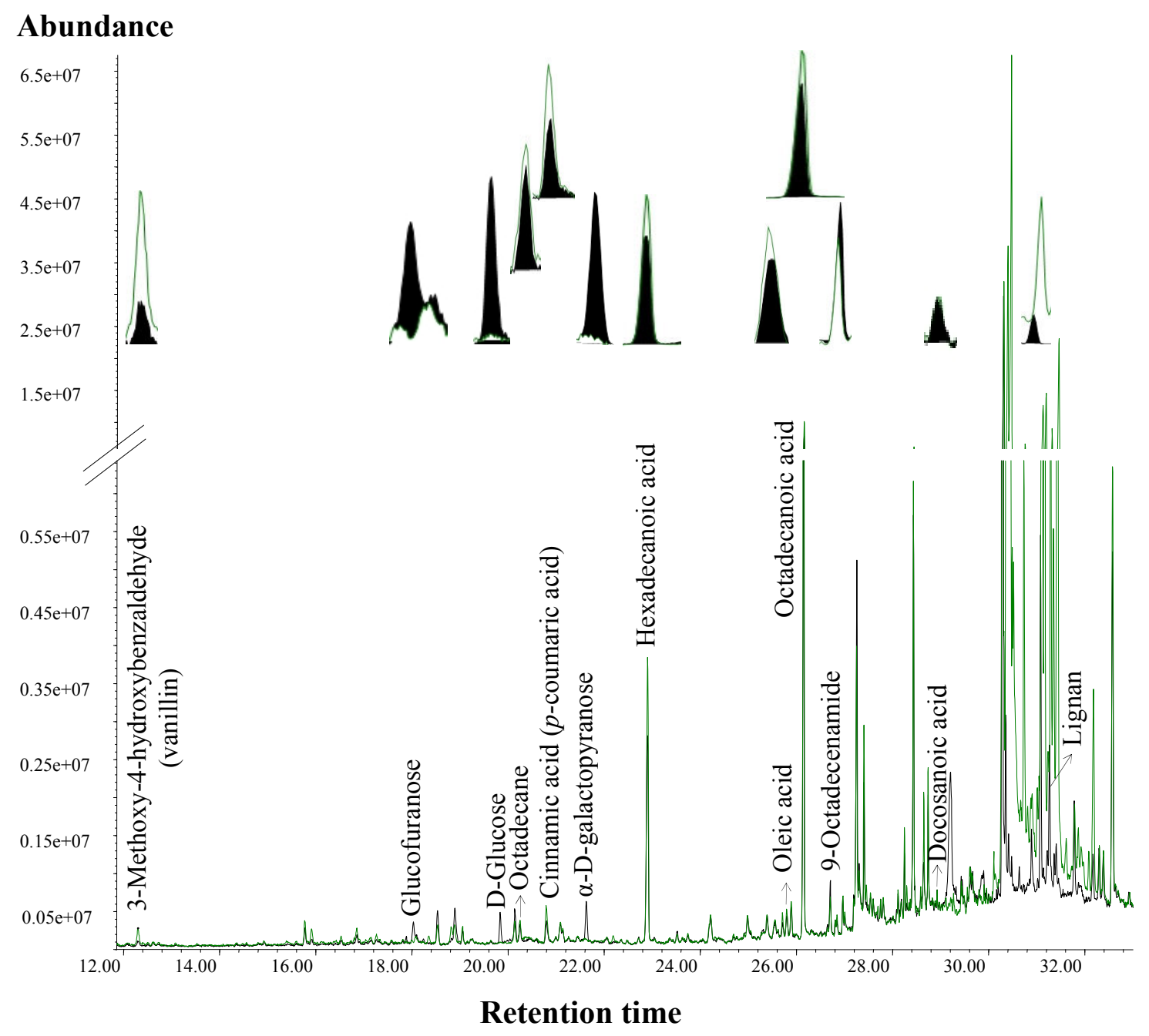

Fig. 22 GC-MS analysis of compounds in the liquid supernatant of $0.1 \mathrm{~g} \mathrm{~A}$. grandis wood particles in $10 \mathrm{ml} 50 \mathrm{mM}$ sodium acetate buffer, $\mathrm{pH} 5.0$ treated for $1 \mathrm{hr}$ at room temperature in a $20 \mathrm{ml}$ Schott glass tubes fixed onto a moving rotary drum with $2 \mathrm{U} \mathrm{ml}^{-1}$ Novo laccase from Aspergillus sp. (Novozyme, EEC No. 420-150-4) and of compounds in a parallel control of $0.1 \mathrm{~g}$ wood particles incubated in $10 \mathrm{ml} 50 \mathrm{mM}$ of sodium acetate buffer, $\mathrm{pH} 5.0$ without addition of enzyme. Note that in the main chromatograms and in the compared individual peaks above the main chromatograms, the green line and the white areas indicate the control without laccase and the black line and the black areas indicate the sample treated with laccase

Obvious substrates for laccases such as 3-methoxy-4-hydroxybenzaldehyde and cinnamic acid as well as lignan occur in the laccase samples at lower abundances than in the controls, arguing for a degradation of these phenolic compounds by the enzyme. Interesting to note is also the massive increase of the sugars glucofuranose, D-glucose and $\alpha$-D-galactopyranose in the sample with Novo laccase compared to the control 
IV. Contrasting ecological functions of Abies grandis wood extractives on protection against white-rot fungi and induction of laccase activity

samples. D-glucose occurs in cellulose and glucofuranose as a mutarotation of D-glucose and $\alpha$-D-galactopyranose as a ring form of galactose being present in arabinogalactan in softwood (Hoegger et al. 2007). Recombinant Novo laccase from Aspergillus sp. used in this experiment was not a highly purified product in buffer as the highlz purified laccase III from Dwivedi (2006) applied in the first experiments of incubating $A$. grandis wood fibers with a laccase. Since not present in purified form, contaminations by cellulases and hemicellulases from the fungus Aspergillus sp. might be responsible for the release of sugars from the $A$. grandis wood particles, by decaying celluloses and hemicelluloses of the wood, respectively. Aspergillus strains are found to usually secrete mixtures of multiple of such enzymes into culture media (Stricker et al. 2008; Wang et al. 2006), but a test for contaminating cellulase, xylanase and $\beta$-glucosidase activity of the Novo laccase preparation failed to detected such activities (see Chapter V.3.4). However, the bought Novo laccase (2 $\mathrm{U} \mathrm{ml}^{-1}$ in $50 \mathrm{mM}$ sodium acetate buffer) was also independently analyzed by GC-MS. As a sugar, only $\beta$-D-glucopyranose was detected in the enzyme solution, indicating that the three sugars D-glucose, glucofuranose and $\alpha$-D-galactopyranose found in the laccase-treated wood samples were not a sugar contamination due to the unpurified Novo laccase sample. Therefore, the source of the three sugars remains currently unknown.

Aliquots of $50 \mathrm{mM}$ sodium acetate buffer solutions from $0.1 \mathrm{~g}$ A. grandis wood particles incubated for $1 \mathrm{hr}$ in $10 \mathrm{ml}$ sodium acetate buffer in $20 \mathrm{ml}$ Schott glass tubes fixed onto a rotary drum with or without $2 \mathrm{U} \mathrm{ml}^{-1}$ Novo laccase were stained for $15 \mathrm{~min}$ with $100 \mathrm{mM} \mathrm{MBTH}$. Clearly, there was a color change to red-pinkish in the buffer solution from wood particles treated with laccase unlike in the control without laccase (Fig. 23A). The buffer from the wood sample without laccase, the MBTH-stained buffer from the wood sample without laccase, the buffer from the wood sample treated with Novo laccase and the MBTH-stained buffer from the wood sample treated with Novo laccase (Fig. 23A) were analyzed by a spectrophotometer (UV-DU800, Beckman coulter, Inc., California, USA) using an UV light source, as suggested by Blanchette (1997) and Rodríguez-López et al. (1994) for measuring pinkish adducts formed between MBTH and quinone. Staining with MBTH in the buffer from laccase-treated wood particles caused the formation of a broad peak in the wavelengths range of $370 \mathrm{~nm}$ to $450 \mathrm{~nm}$ with the highest absorbance at $400 \mathrm{~nm}$, as it is typical for MBTHquinone adducts (Medda et al. 1995; Rodríguez-López et al. 1994). 
A
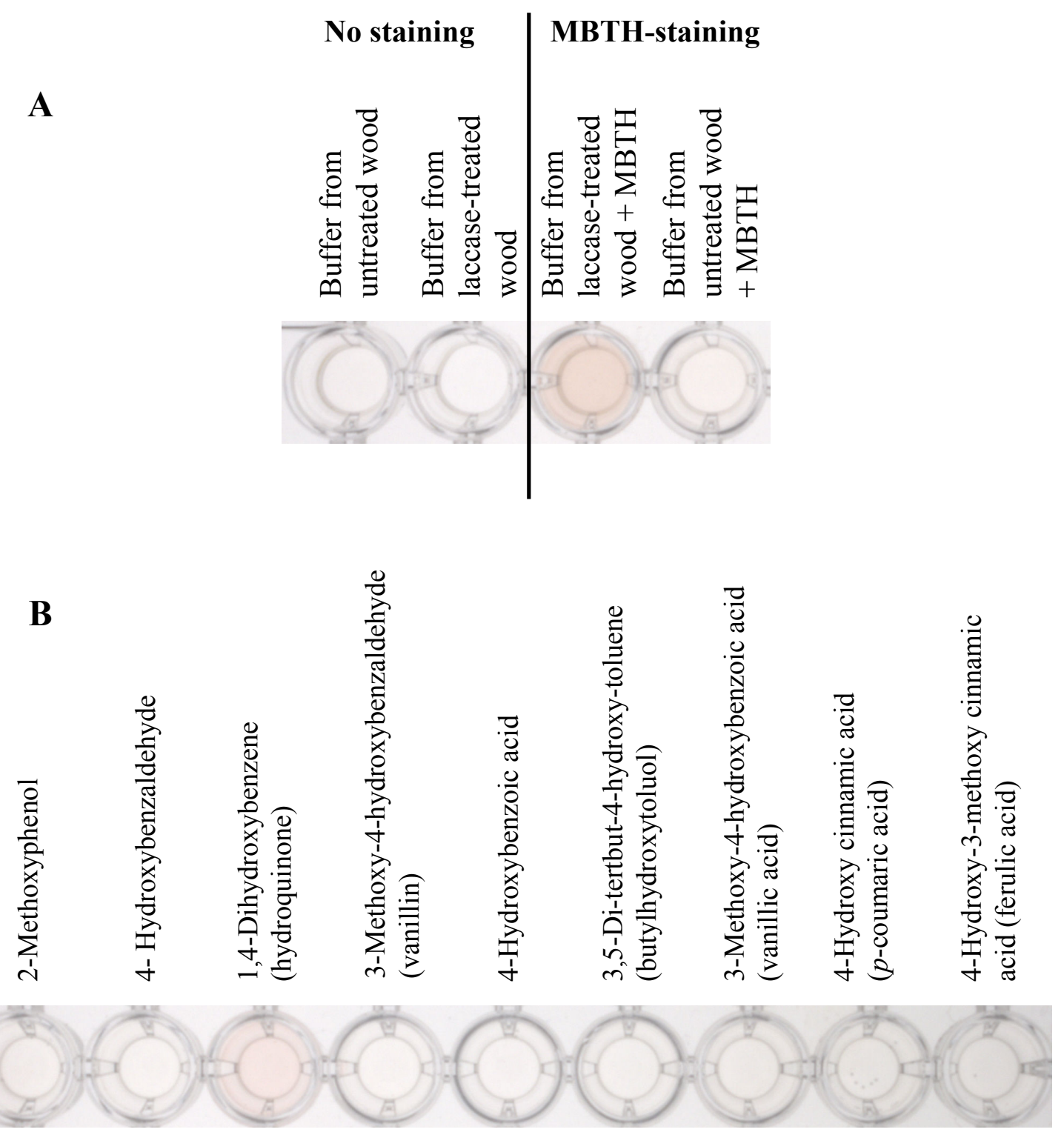

Fig. 23 Buffer from untreated and from laccase-treated wood (of $1 \mathrm{hr}$ incubation) unstained and stained with MBTH A) and specific phenolic compounds in $1 \mathrm{mM}$ concentration in $50 \mathrm{mM}$ sodium acetate buffer, $\mathrm{pH} 5.0$ stained with MBTH B) for 15 min of incubation at RT. For staining $80 \mu \mathrm{l}$ of buffer of wood samples or $80 \mu \mathrm{l}$ buffer with a specific phenolic compound were mixed in individual wells a 96well-microtiter plate with each $80 \mu \mathrm{l}$ of $100 \mathrm{mM}$ MBTH. Photographs were taken from the microtiter plate on a light box with illuminating from below

The MBTH-stained buffer from non-treated wood did however not show a similar peak in the range of wavelengths from $370 \mathrm{~nm}$ to $450 \mathrm{~nm}$, indicating clearly that reactions by the laccase were responsible for the red-pinkish adduct formation with MBTH. When incubating at a concentration each of $1 \mathrm{mM}$ of individual pure phenolic 
IV. Contrasting ecological functions of Abies grandis wood extractives on protection against white-rot fungi and induction of laccase activity

compounds identified previously in chromatograms of samples of laccase-treated wood particles (Table 1 and Fig. 22) with MBTH, only 1,4 dihydroxybenzene (hydroquinone) gave a positive reaction with MBTH (Fig. 23B). This compound however was not detected in the chromatogram shown in Fig. 22. It is thus possible that other unknown laccase-released quinones are present in the buffer solution ready to act with MBTH. However, it should be noted that the chromatogram shown in Fig. 22 and the buffers shown in Fig. 23B came from two independent sets of experiments and it is thus possible that hydroquinone by chance was not present in the sample analyzed in Fig. 22 but instead in the sample shown in Fig. 23.

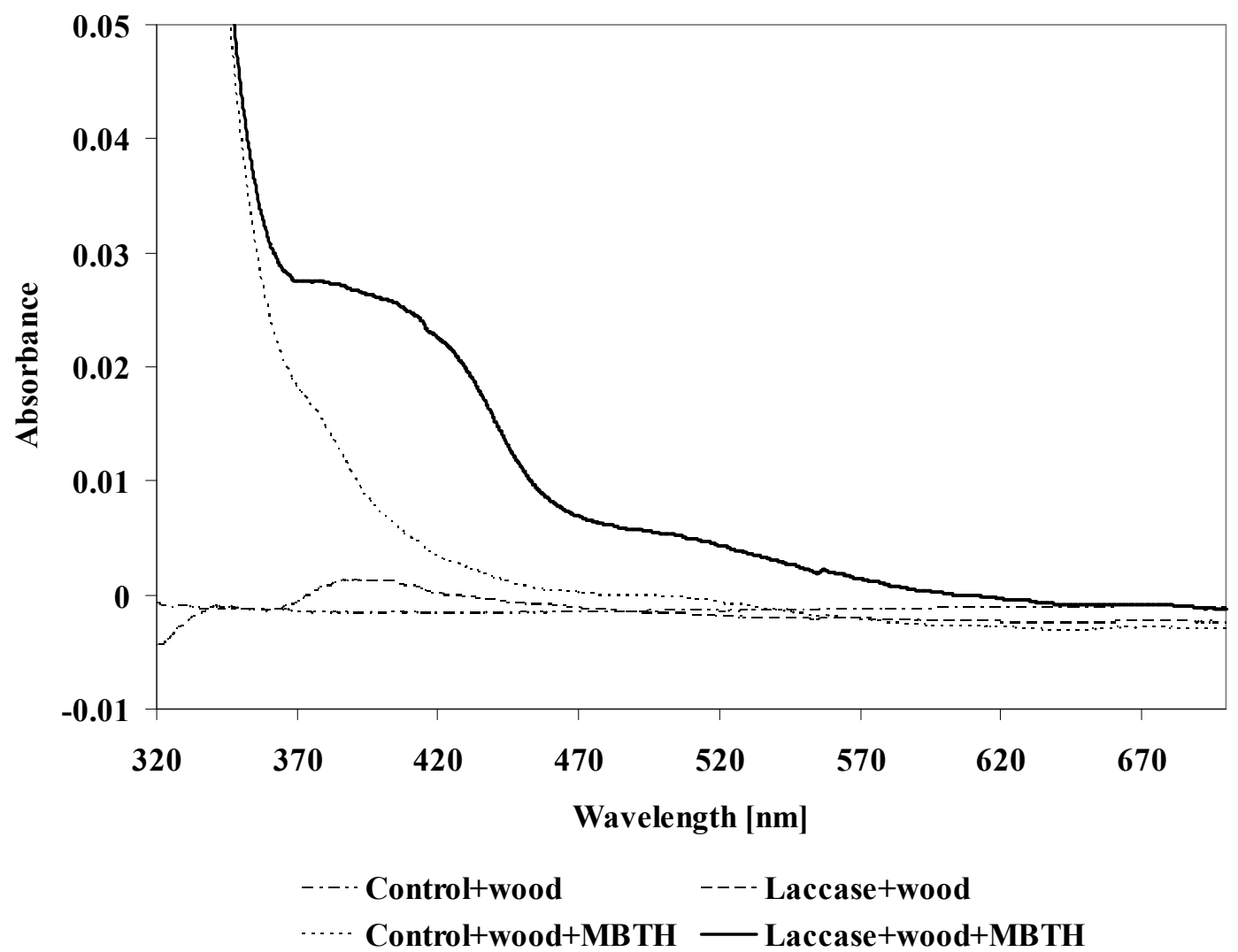

Fig. 24 Absorbance spectra of the liquid supernatant of $0.1 \mathrm{~g} \mathrm{~A}$. grandis wood particles in $10 \mathrm{ml} 50 \mathrm{mM}$ sodium acetate buffer, $\mathrm{pH} 5.0$ treated for $1 \mathrm{hr}$ at RT with $2 \mathrm{U} \mathrm{ml}^{-1}$ Novo laccase from Aspergillus sp. (Novozyme, EEC No. 420-150-4) and in parallel controls of $0.1 \mathrm{~g}$ wood particles incubated for $1 \mathrm{hr}$ at RT in $10 \mathrm{ml} 50 \mathrm{mM}$ of sodium acetate buffer, pH 5.0 without addition of enzyme. Incubation was done in $20 \mathrm{ml}$ Schott glass tubes fixed onto a moving rotary drum. Staining with MBTH of respective $0.5 \mathrm{ml}$ aliquots of the samples were done by mixing $0.5 \mathrm{ml}$ of $100 \mathrm{mM} \mathrm{MBTH}$ in $50 \mathrm{mM}$ sodium acetate buffer, $\mathrm{pH} 5.0$ and incubating for $15 \mathrm{~min}$ at RT. The absorbance of solutions was measured in a spectrophotometer (UV-DU800, Beckman coulter, Inc., California, USA) at 320-700 nm with an UV light source 
IV. Contrasting ecological functions of Abies grandis wood extractives on protection against white-rot fungi and induction of laccase activity

\section{IV.4.8 Wood extractives in protection of $A$. grandis wood against fungal decay}

A. grandis mini wood blocks [30 (longitudinal) x 10 (tangential) x 5 (radial) mm] after water extraction and after water-acetone extraction was used to determine in EN 113 tests the degree of wood mass loss caused by different basidiomycete fungi $(C$. cinerea Okayama 7, P. ostreatus 8F6, T. versicolor Strain 6). Wood extractives were eliminated from the wood blocks as described in Chapter IV.3.4. Mass loss of A. grandis mini wood blocks decayed by fungi was observed in a period of 6 weeks and 18 weeks of incubation with the fungi at $22^{\circ} \mathrm{C}$ and $65 \% \mathrm{RH}$ (relative humidity) in the dark. Effects of loss of wood extractives were seen on C. cinerea Okayama 7 and $T$. versicolor Strain 6 but not on P. ostreatus 8F6 (Fig. 25).

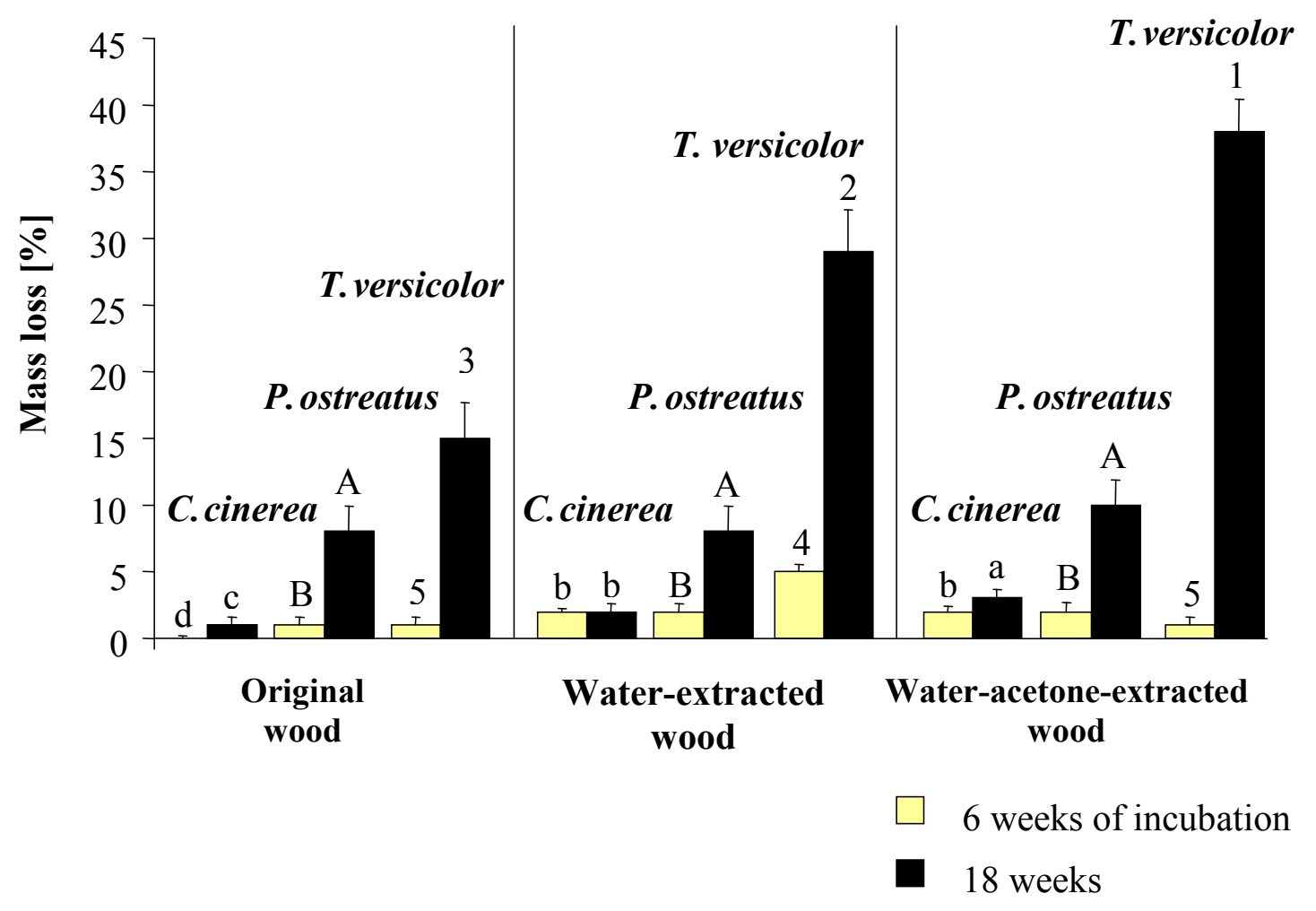

Fig. 25 Mass loss over the time of original, water-extracted and water-acetone-extracted A. grandis mini wood blocks [30 (longitudinal) x 10 (tangential) x 5 (radial) $\mathrm{mm}$ ] incubated with $C$. cinerea Okayama 7, P. ostreatus 8F6 and T. versicolor Strain 6 at $22^{\circ} \mathrm{C}$ and $65 \% \mathrm{RH}$ in fungal decay tests of the EN 113. Average values and standard deviations were calculated from three parallel samples each. Statistical analysis of the data was performed with SPSS 8.0 for Windows, the different superscripts on the chart (letters in lower case compare the values obtained at different times incubation with C. cinerea Okayama 7, capital letters compare the respective values of $P$. ostreatus 8F6, and numbers compare the respective values of $T$. versicolor Strain 6) indicate values that differ significantly $(p<0.05)$ between treatments as based on analysis of variance (ANOVA) 
IV. Contrasting ecological functions of Abies grandis wood extractives on protection against white-rot fungi and induction of laccase activity

Of all three species, T. versicolor Strain 6 obtained the highest decay of A. grandis wood in 18 weeks of incubation. Untreated $A$. grandis wood was more resistant for white-rot decay than the extracted wood. Water-extracted wood and wateracetone-extracted wood showed in 18 weeks of incubation with $T$. versicolor $29 \pm 3.12 \%$ and $38 \pm 2.43 \%$ of mass loss, respectively, compared to only $15 \pm 2.67 \%$ of mass loss in original wood samples incubated with the fungus. This effect of enhanced decay by T. versicolor Strain 6 upon loss of wood extractives was not yet as obvious at the earlier time of incubation of 6 weeks (Fig. 25).

Loss of extractives affected also the behavior of $C$. cinerea Okayama 7 with A. grandis wood. No mass loss were detected in incubation of this dung fungus with the original wood whilst a rate of about $1 \%$ of mass loss was achieved upon water extraction and about $2 \%$ of mass loss upon water-acetone extraction of the $\mathrm{A}$. grandis wood (Fig. 25).

P. ostreatus $8 \mathrm{~F} 6$ showed no significant different rates of mass loss between the three types of tested samples. In all cases, mass loss of $A$. grandis wood after 18 weeks of incubation ranged at about $10 \%$ (Fig. 25).

Since decay the wood decay tests indicated that wood extractives have a certain protection role in hindering fungal decay of $A$. grandis wood, water extractives and acetone extractives were tested against the fungi C. cinerea Okayama 7, P. ostreatus 8F6 and T. versicolor Strain 6 growing on 5\% malt extract, 2\% agar plates (Fig. 26 and 27). Of all three species, the mycelial growth of the most sensitive $C$. cinerea Okayama 7 was highest inhibited by wood extractives. Growth inhibition zones with water extractives and with acetone extractives were $40 \mathrm{~mm}$ and $30 \mathrm{~mm}$ in diameter on day 5 of incubation, respectively. T. versicolor Strain 6 was the least sensitive to the wood extractives, with a growth inhibition zone of only $15 \mathrm{~mm}$ and $10 \mathrm{~mm}$ in diameter with water extractives and with acetone extractives at the same day of incubation, respectively. The growth inhibition zone of $P$. ostreatus $8 \mathrm{~F} 6$ on day 5 of incubation were $25 \mathrm{~mm}$ and $15 \mathrm{~mm}$ in diameter with water extractives and with acetone extractives, respectively (Table 4). P. ostreatus 8F6 was thus more sensitive to the wood extractives than T. versicolor Strain 6 but less sensitive as compared to C. cinerea Okayama 7. Accordingly, the effects of wood extractives on fungal growth correlated with their ability to decay A. grandis wood (compare Fig. 25). 
IV. Contrasting ecological functions of Abies grandis wood extractives on protection against white-rot fungi and induction of laccase activity

Table 4 Growth inhibition by diffusion of wood extractives into $5 \%$ malt extract, $2 \%$ agar medium of fungi grown for 5 days at $25^{\circ} \mathrm{C}(P$. ostreatus $8 \mathrm{~F} 6$ and $T$. versicolor Strain 6) and $37^{\circ} \mathrm{C}$ (C. cinerea Okayama 7)

\begin{tabular}{lcccc}
\hline \multirow{2}{*}{ Fungus } & \multicolumn{3}{c}{ Growth inhibition zone (mm in diameter) } \\
& Water & Acetone & DMSO & Untreated \\
& extractives & extractives & control & control \\
\hline C. cinerea Okayama 7 & $40 \pm 0.50$ & $30 \pm 0.29$ & 0 & 0 \\
P. ostreatus 8F6 & $25 \pm 0.58$ & $15 \pm 0$ & 0 & 0 \\
T. versicolor Strain 6 & $15 \pm 0.29$ & $10 \pm 0$ & 0 & 0 \\
\hline
\end{tabular}

${ }^{a}$ Data are means of three replicates, and standard deviations are presented

${ }^{\mathrm{b}}$ Wood extractives were added into punched holes within agar plates in a solution of in total $150 \mu \mathrm{l}$ DMSO, why control controls with $150 \mu \mathrm{l}$ DMSO added into holes within agar plates were also included

C. cinerea

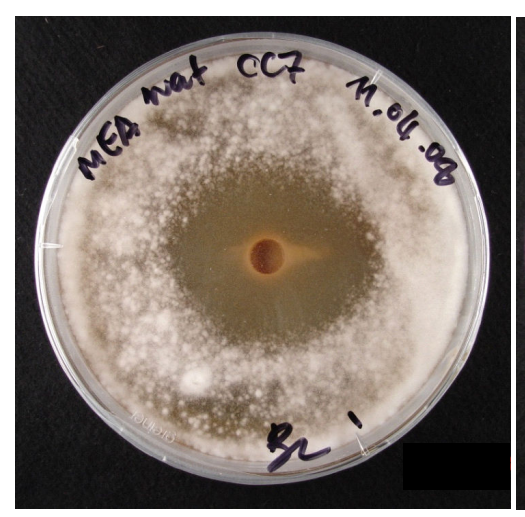

P. ostreatus

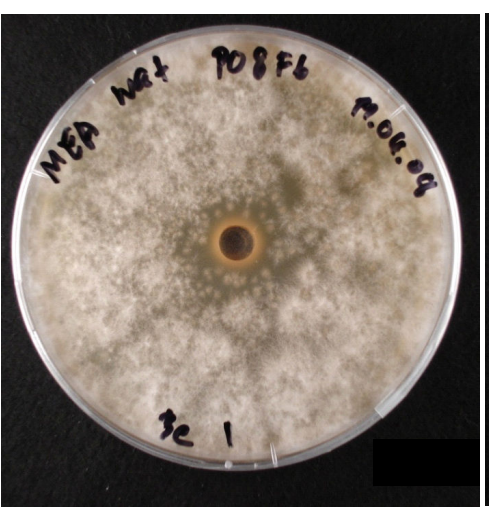

T. versicolor

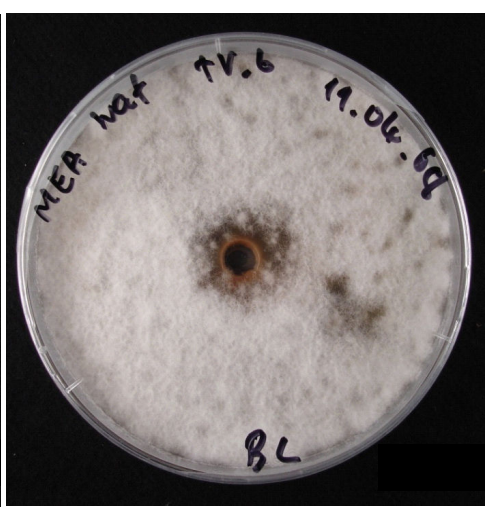

Fig. 26 Growth inhibition of fungi by $A$. grandis water extractives. Fungi were incubated for 5 days at $25^{\circ} \mathrm{C}$ for P. ostreatus $8 \mathrm{~F} 6$ and T. versicolor Strain 6 and $37^{\circ} \mathrm{C}$ for $C$. cinerea Okayama 7 on plates with $5 \%$ malt extract, $2 \%$ agar medium to which $150 \mu \mathrm{l}$ water extractives in DMSO were added into a hole made in the middle of the plates. The amount of water extractives added corresponded to $0.15 \mathrm{~g}$ of $A$. grandis wood particles

Fig. 27 shows in a second experiment that $A$. grandis wood extractives inhibited mycelial growth of $C$. cinerea Okayama 7. In this experiment, acetone was used as a solvent for the extractives, added into holes made within the YMG/T agar medium supplemented with $5 \mathrm{mM}$ ABTS. Again, growth of fungal mycelium was inhibited by 


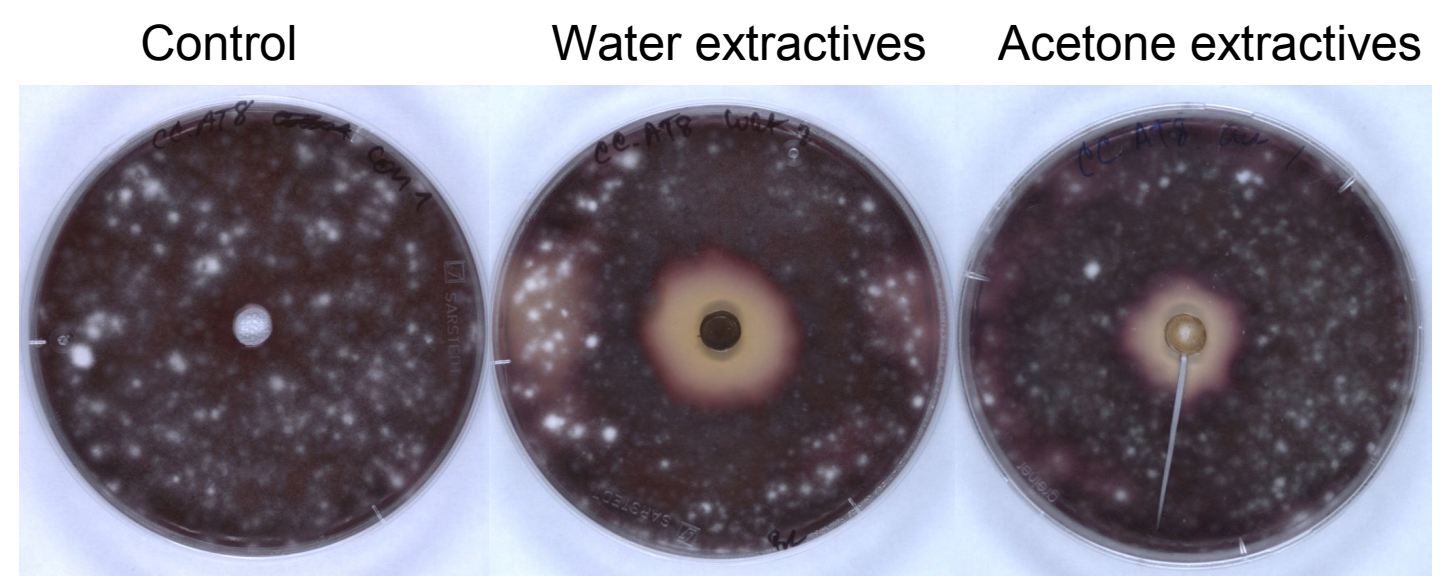

Fig. 27 Mycelial growth and laccase secretion of C. cinerea Okayama 7 at $37^{\circ} \mathrm{C}$ incubated for 5 days on YMG/T medium supplemented with $5 \mathrm{mM}$ ABTS to which $150 \mu 1$ water extractives or acetone extractives of $A$. grandis wood particles dissolved in acetone were added into a hole made in the middle of the plates or alternatively in case of the controls $150 \mu \mathrm{l}$ of acetone. The amount of water extractives and acetone extractives added corresponded to $0.15 \mathrm{~g}$ of $A$. grandis wood particles. After growth, plates were turned upside down and placed onto a light box. A digital camera was used to take photos of the plates making use of the luminary light source from the light box from below

both, water extractives and acetone extractives. The inhibition zone was clearly seen by lack of oxidation of ABTS. Lilac coloration in the plates indicated fungal growth and conversion of the colorless ABTS into a lilac compound likely due to a laccase reaction. Accordingly, in controls no growth inhibition was observed, whilst the halo of growth inhibition was larger in plates with wood extractives $(\varnothing 30 \mathrm{~mm})$.

The effect of wood extractives on protecting $A$. grandis wood from fungal decay was further tested by wood immersion tests. A. grandis mini wood blocks [30 (longitudinal) x 10 (tangential) x 5 (radial) $\mathrm{mm}$ ] were immersed into solutions of wood extractives and air-dried as described in Chapter IV.3.4 and subsequently incubated for 10 weeks on plates with $5 \%$ malt extract, $2 \%$ agar medium on which the different fungi C. cinerea Okayama 7, P. ostreatus 8F6 and T. versicolor Strain 6 were grown. Whilst all three species showed good up to excellent surface growth on the original A. grandis wood (Fig. 28 top), especially water-extractives and, for P. ostreatus 8 F6 and for T. versicolor Strain 6 in less amount, the acetone extractives dramatically reduced the surface growth on treated mini wood block (Fig. 28 middle and bottom). 
IV. Contrasting ecological functions of Abies grandis wood extractives on protection against white-rot fungi and induction of laccase activity

Accordingly, the mycelial growth of all fungi tested was inhibited by the wood extractives.

The time course documented in Fig. 29 indicates that there are differences in timings in which treated $A$. grandis wood was protected. C. cinerea Okayama 7 cultures were well protected by extractives over most of the time of incubation. Only in the $10^{\text {th }}$ week, thin surface growth became obvious for wood previously immersed in water extractives and for wood previously immersed in acetone extractives (Fig. 29). In case of $P$. ostreatus $8 \mathrm{~F} 6$, traces of surface growth were visible for mini wood blocks immersed in water extractives at week 6 of incubation and for mini wood blocks
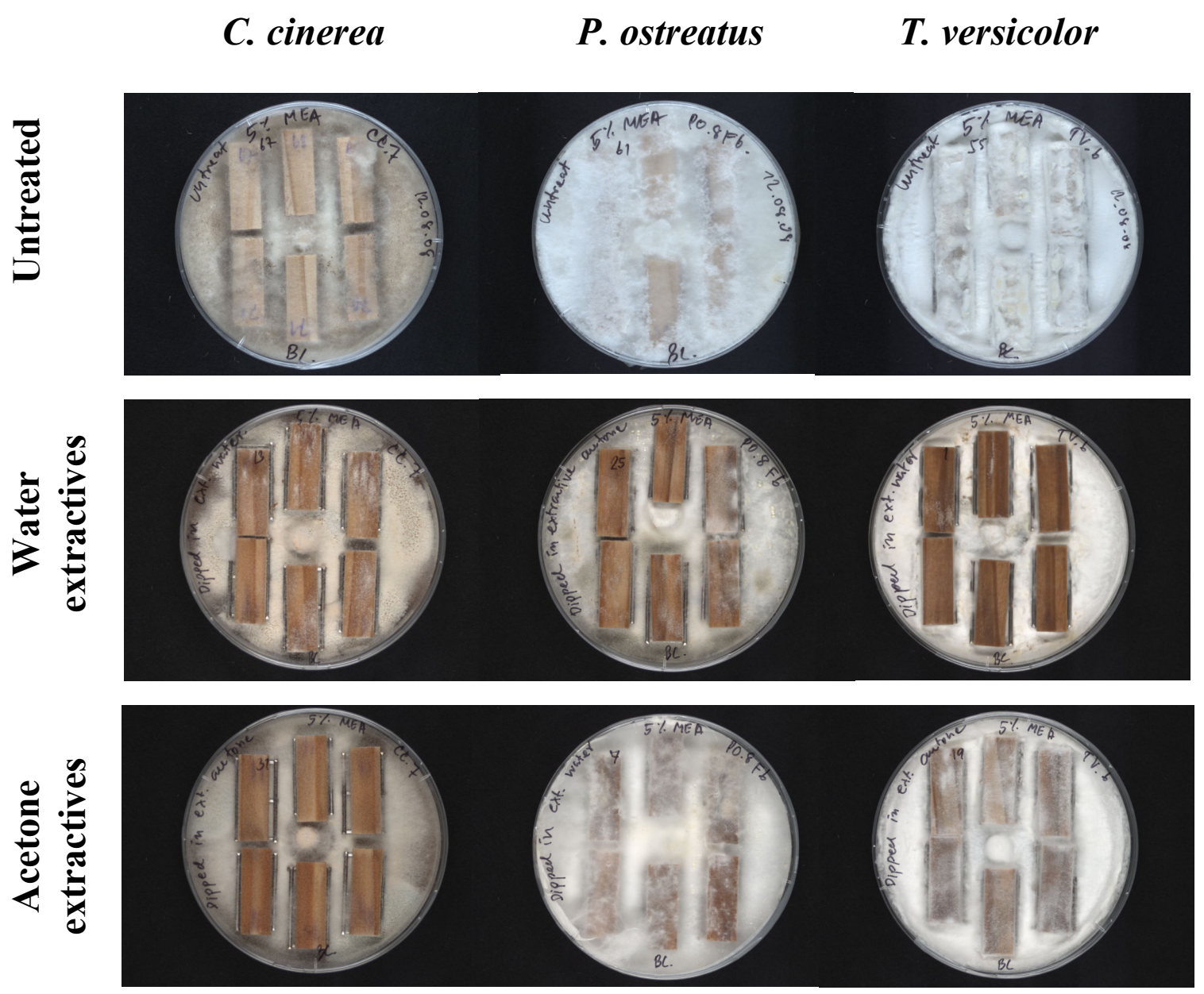

Fig. 28 Original $A$. grandis mini wood blocks and $A$. grandis mini wood blocks were immersed for $5 \mathrm{sec}$ at RT in $10 \mathrm{ml}$ of water extractives or acetone extractives (corresponding to $4 \mathrm{~g}$ wood) in DMSO, air-dried and then incubated for 10 weeks with different basidiomycetes grown on $5 \%$ malt extract, $2 \%$ agar plates (at $37^{\circ} \mathrm{C}$ for C. cinerea Okayama 7 and at $25^{\circ} \mathrm{C}$ for $P$. ostreatus $8 \mathrm{~F} 6$ and T. versicolor Strain 6 ) 


\section{$1^{\text {st }}$ week $\quad 2^{\text {nd }}$ week $\quad 4^{\text {th }}$ week $\quad 6^{\text {th }}$ week $\quad 1^{\text {th }}$ week}

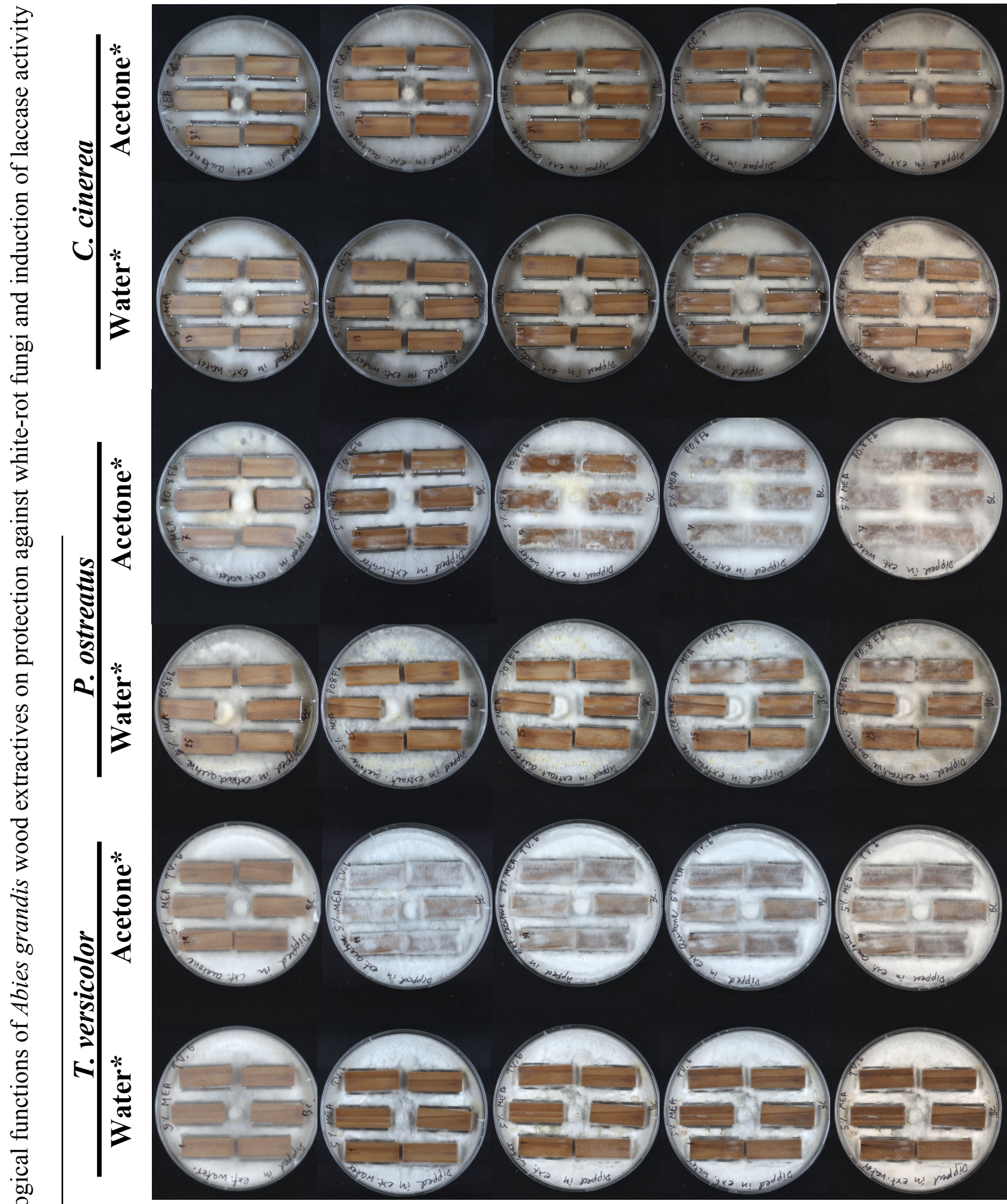

Fig. 29 Original $A$. grandis mini wood blocks and $A$. grandis wood blocks immersed in water extractives or in acetone extractives (corresponding to $4 \mathrm{~g}$ wood) and incubated after air-drying the wood at RT on 5\% malt extract, $2 \%$ agar medium with different basidiomycetes (at $37^{\circ} \mathrm{C}$ for $C$. cinerea Okayama 7 and at $25^{\circ} \mathrm{C}$ for $P$. ostreatus $8 \mathrm{~F} 6$ and for $T$. versicolor Strain 6, respectively) for 10 weeks. * Water and acetone refer to water extractives or acetone extractives, respectively, in which wood blocks were immersed 
IV. Contrasting ecological functions of Abies grandis wood extractives on protection against white-rot fungi and induction of laccase activity

immersed in acetone extractives at week 4 of incubation. Surface growth on the later samples increased further over the time (Fig. 29). In case of T. versicolor Strain 6, it was interesting to note that water extractives protected the A. grandis wood over the whole period of 10 weeks of incubation, whereas effects of acetone extractives lasted well only in the first week of incubation (Fig. 29). Already in week 10 of incubation, mini wood blocks showed some degree of surface growth of fungal mycelium (Fig. 29) that however never reached the density of T. versicolor Strain 6 surface growth on control wood blocks that were not extra treated with wood extractives (Fig. 28).

\section{IV.5 Discussion}

\section{IV.5.1 Phenolic compounds in wood extractives play a role in laccase production}

Experiments presented in this Chapter IV address effects of fungi and of produced laccase on A. grandis wood degradation. A. grandis wood particles and wood extractives have been shown to possess a potential to induce laccase production in the white-rot fungi P. ostreatus 8F6 and T. versicolor Strain 6 (Fig. 4). In contrast, activity of MnP, LiP and cellulase were not detected when applying $A$. grandis wood particles to cultures of P. ostreatus 8F6 and T. versicolor Strain 6, respectively (Fig. 2).

Laccase production by a fungus might be induced in order to attack wood for accessing the potential nutrients in the material (Eriksson et al. 1990). Wood usually has a low nitrogen-content (Fengel and Wegener 1983 and Fig. S9 in supplementary material) and under low nitrogen conditions, fungal production of laccase might be induced (Eugenio et al. 2009; Lee et al. 2006; Kachlishvili et al. 2006; Patel et al. 2009). BSM used in this work contains per $100 \mathrm{ml}$ about $0.206 \mathrm{~g} \mathrm{C}$ and about $0.034 \mathrm{~g}$ $\mathrm{N}$. Thus, the $\mathrm{C}$ to $\mathrm{N}$ ration in original BSM was about $6: 1$. In this medium without addition of any other compounds, laccase production of $P$. ostreatus 8F6 and of T. versicolor Strain 6 was always low (e.g. see Fig. 5 and Fig. S9 in the supplementary material). Altering the $\mathrm{C}$ to $\mathrm{N}$ ratio to $0.04: 1$ in low $\mathrm{C}, \mathrm{N}$ BSM medium by eliminating all $\mathrm{C}$ and $\mathrm{N}$ sources from the medium but the yeast extract $(0.8 \% \mathrm{C}$ and $18 \% \mathrm{~N}$, see information given by the company Oxoid to their product LP0021; www.oxoid.com) did not induce laccase production in T. versicolor Strain 6 (Fig. S9 in the supplementary material). Only upon addition of wood particles to original BSM medium or to low C,N BSM medium, laccase production was much enhanced (Fig. 5 and Fig. S9 in the 
IV. Contrasting ecological functions of Abies grandis wood extractives on protection against white-rot fungi and induction of laccase activity

supplementary material). Indeed, laccase production started much earlier in low C,N BSM medium than in normal BSM medium (indeed directly after addition of the wood) but it reached not as high total levels (Fig. S9 in the supplementary material). Laccase activity of about $600 \mathrm{U}^{-1}$ was obtained with $T$. versicolor Strain 6 from low C,N BSM culture medium when $1 \mathrm{~g}$ A. grandis wood particles were added, 7 days after the addition of wood particles. Apparently, these experiments indicate that wood particles are the main factors to induce laccase production. However, $\mathrm{C}$ and $\mathrm{N}$ relationships might be most effective for laccase induction and also fungal biomass production. The highest laccase induction reached up about $840 \mathrm{U}^{-1}$ in original BSM medium with added of $A$. grandis wood particles (Fig. S9), 11 days after the addition of the wood to the fungal mycelium.

The fresh original BSM medium with new easy accessible $\mathrm{C}$ and $\mathrm{N}$ sources (see above) added together with the wood to the T. versicolor Strain 6 allowed the fungus to grow first on the components of the liquid medium prior to switching to laccase production and growth on the A. grandis wood. That the fungus enters under the environmental conditions the wood and grows within the wood can be seen in Fig. S10. With the added wood in the original BSM medium, the fungal biomass reached up about $0.425 \pm 0.009 \mathrm{~g}$ (Fig. S8) compared to $0.209 \pm 0.004 \mathrm{~g}$ without added wood. It was thus only little more in the original BSM medium plus the added wood than the fungal biomass of $0.397 \pm 0.006 \mathrm{~g}$ in the low C,N BSM medium with added wood (compared to $0.095 \pm 0.006 \mathrm{~g}$ in the low $\mathrm{C}, \mathrm{N}$ BSM medium without added wood). The $\mathrm{C}$ to $\mathrm{N}$ ratios upon addition of wood were about 400:1 in original BSM medium and about 500:1 in low C,N BSM medium (for data on $\mathrm{C}$ and $\mathrm{N}$ contents of the A. grandis wood see Chapter IV.7.5). Thus, the relative $\mathrm{C}$ to $\mathrm{N}$ ratio increased in favor to carbon, in agreement with reports that low nitrogen favors laccase production in T. versicolor (Lee et al. 2006). Leaving aside the differences in total nutrient contents in these experiments that certainly had effects on total biomass values, the data do suggest that the nutrient status in a medium has an influence on laccase production by T. versicolor Strain 6. However, it might not be the only reason for induction or alternatively possibly also repression of laccase production.

Laccase production upon addition of inducers was widely studied, for example in order to close a gap of non-sufficient naturally laccase production of a fungus for applications in biotechnology. Natural and synthetic inducers were used to increase the production of laccase for applications of industrial scales. Woody, plant or agricultural 
IV. Contrasting ecological functions of Abies grandis wood extractives on protection against white-rot fungi and induction of laccase activity

residues of high $\mathrm{C}$ to $\mathrm{N}$ ratios are amongst the natural substrates available in plentiful amounts to act well in fungal cultivations as putative laccase inducers (Elissetche et al. 2006; Rühl et al. 2007). For example, by adding of wood chips of Drimys winteri into cultures of Ganoderma australe, the fungus was found to produce laccase (Elissetche et al. 2006). Alike results were obtained by adding lignocellulosic substrates such as wheat straw, barley straw, wood shavings and barley bran into cultures of $T$. versicolor: laccase production of $T$. versicolor was induced, with e.g. highest laccase production of about 1,200 U $1^{-1}$ obtained with barley bran (Couto et al. 2002; Rodríguez-López et al. 1994).

If not (solely) due to the low relative nitrogen content in the lignocellulosic substrates, specific compounds in the biological materials might be expected to have an inductive effect. Results on wood extractives in this work (Fig. 4) support this assumption. In case of the extractives, water extractives of $A$. grandis wood particles as applied were stronger inducers of laccase production than acetone extractives and hexane extractives, respectively, isolated from the same amount of wood. Induction of laccase production in cultures of $P$. ostreatus $8 \mathrm{~F} 6$ and of T. versicolor Strain 6 was however not as good as with the original wood (Fig. 4).

Chromatography fractions of water extractives eluted with $20 \%, 40 \%$ and $60 \%$ methanol from a silica gel column all had the potential to induce laccase production in cultures of $P$. ostreatus 8F6 and of T. versicolor Strain 6, respectively (Fig. 9). Thereby, entire fractions were further purified with chloroform to identify the specific soluble organic compounds. Chloroform extracts of the chromatography fraction were shown to behave not different from the original chromatography fractions. The highest laccase induction achieved by the chloroform-purified fractions of $40 \%$ and $60 \%$ methanol in T. versicolor Strain 6 cultures yielded about $500 \mathrm{U} \mathrm{l}^{-1}$ and $300 \mathrm{U} \mathrm{l}^{-1}$, respectively (Fig. 10). Highest laccase production of about $100 \mathrm{U}^{-1}$ was obtained in cultures of P. ostreatus 8F6 with both fractions (Fig. 10). Further, the results indicated that there are some inducing compounds that seem to be un-polar in overall nature and extractable to chloroform.

Various research groups studied induction of laccase by adding phenols as natural substrates of laccases into fungal cultures, in view of their redox potential, which is conveniently low and in the 0.5-1.0 V vs. NHE (normal hydrogen electrode) range allowing easy electron abstraction from the $\mathrm{T} 1 \mathrm{CuII}$ binding site in an activated i.e. oxidized laccase. Resulting aryloxyl radical intermediates may catalyze the 
IV. Contrasting ecological functions of Abies grandis wood extractives on protection against white-rot fungi and induction of laccase activity

oxidation of non-phenolics. Plentiful amounts of phenolic monomers may thus drive the laccase-induced oxidative degradation of lignocellulosic material (Calcaterra et al. 2008; Tadesse et al. 2008) and the degradation of the material may release compounds (phenolic) acting in turn as inducers on fungal laccase production (compare Fig. 13).

The effects of phenolic compounds on laccase production were widely studied (De Souza et al. 2004; Kollmann et al. 2005; Terron et al. 2004). The group of De Souza et al. (2004) reported for example that various phenolic compounds, such as p-coumaric acid, ferulic acid, guaiacol, syringol, $p$-methoxyphenol, pyrocatechol, phloroglucinol, 3,5-dihydroxybenzoic acid, and syringaldazine induced laccase production in Trametes sp. I-62, and highest enzymatic activity obtained by adding of p-coumaric acid and guaiacol was about 273-fold and 73-fold, respectively, higher than in control cultures. Terron et al. (2004) using $0.2 \mathrm{mM}$ ferulic acid and $0.2 \mathrm{mM}$ vanillin in cultures of Trametes sp. I-62 achieved an increase in laccase activity up to 10-fold. Moreover, Kollmann et al. (2005) showed that small amounts of $12.5 \mu \mathrm{M}$ of 2,5-xylidine could induce laccase production by $T$. versicolor about 9-fold.

The chemical composition of lipophilic extracts obtained in this study by chloroform purification of water extractives and of acetone extractives of A. grandis wood was analyzed by GC-MS in a same manner as previously done for other wood types such as by Silverio et al. (2007) who analyzed components of eucalyptus extractives and as by Fernandez et al. (2001); Gutierrez et al. (1998); Gutierrez et al. (1999) and Lavoie and Stevanovic (2006) who studied the chemical composition of eucalyptus, pine, birch and aspen wood extractives, respectively. Common types of compounds identified in extractives of the different woods wood include: tetradecanoic acid, hexadecanoic acid, oleic acid, dehydroabietic acid, eicosanoic acid, docosanic acid, 1-docosanol, eicosane, campesterol and $\beta$-sitosterol. In this study here, all the mentioned compounds but tetradecanoic acid and eicosane were found also in A. grandis wood extractives (Fig. 11 and 12), but in this study in addition various specific phenolic compounds were detected in the A. grandis wood extractives. In chloroform-purified water extractives, and in chloroform-purified acetone extractives in total nine phenolic compounds were found (Table 1) that could be suspected to be potential inducers of laccase production of the white-rot fungi P. ostreatus 8F6 and T. versicolor Strain 6. All were tested with the two fungi and for P. ostreatus 8F6, 4-hydroxy cinnamic acid, 3,5-di-tertbut-4-hydroxy-toluene, and 4-hydroxybenzaldehyde were found to be well effective and for T. versicolor Strain 6, it 
IV. Contrasting ecological functions of Abies grandis wood extractives on protection against white-rot fungi and induction of laccase activity

was 4-hydroxy cinnamic acid and 3,5-di-tertbut-4-hydroxy-toluene. 4-hydroxy-3methoxy cinnamic acid, 3-methoxy-4-hydroxybenzaldehyde and 2-methoxyphenol were also effective with $P$. ostreatus $8 \mathrm{~F} 6$ but achieved laccase activities were lower. Some minor level induction was also seen with 2-methoxyphenol and 4-hydroxy-3methoxy cinnamic acid in $T$. versicolor Strain 6 . The results show that fungi differ in the reaction on different compounds but that the great variety of different compounds in the wood extractives might offer one or more inducers to laccase production in different species.

In the experiment shown in Fig. 13, conveniently always $1 \mathrm{mM}$ final concentration per compound (between 1.1 to $2.2 \mu \mathrm{g}$ per individual compound per $100 \mathrm{ml}$ culture) were chosen for testing with the fungi. In a number of instances, this was in the range of amounts of the specific compounds found in the water extractives or in the acetone extractives of $1 \mathrm{~g}$ A. grandis wood particles (Table 1 and Table 5) which was the taken amount of wood particles usually applied in control cultures performed for reasons of comparison (Fig. 13). Two main exceptions regarding concentration of compounds and enzyme activities need however to be mentioned: 4-hydroxy cinnamic acid was applied in an amount about 5-fold lower than as found in $1 \mathrm{~g}$ of A. grandis wood (Table 5). At this lower concentration, there were good inductive effects in laccase production for both fungi. The other interesting cases are 2-methoxyphenol, 4-hydroxy-3-methoxy cinnamic acid and 3,5-di-tertbut-4-hydroxytoluene that were found effective in laccase induction in T. versicolor Strain 6 at a concentration about 5 to 10 -fold higher than it occurred in the A. grandis wood (see Table 5). It remains to be established whether lower concentrations will also show effects on laccase inhibition in the two fungi.

When comparing the own data with data from the literature, in this study $1 \mathrm{mM}$ 3-methoxy-4-hydroxybenzalhyde was not enough to induce laccase in $T$. versicolor Strain 6 , different from a case in the literature where $0.2 \mathrm{mM}$ was sufficient to induce laccase in Trametes sp. I-62 (Terron et al. 2004). $0.2 \mathrm{mM}$ was also sufficient in inducing laccase with 4-hydroxy-3-methoxy cinnamic acid in Trametes sp. I-62 (Terron et al. 2004) and concentration of $0.2 \mathrm{mM}$ of 4-hydroxybenzaldehyde and 3,5- di-tertbut4-hydroxy-toluene were enough to enhance laccase production in $P$. ostreatus pulmonarius and in Pleurotus sajor-caju (De Souza et al. 2004; Mumaran et al. 1997). Again, this raises the question whether lower concentrations of these compounds would also be effective with $T$. versicolor Strain 6 (4-hydroxy-3methoxy cinnamic acid) and 
IV. Contrasting ecological functions of Abies grandis wood extractives on protection against white-rot fungi and induction of laccase activity

with P. ostreatus 8F6 (3-methoxy-4-hydroxybenzalhyde and 4-hydroxy-3methoxy cinnamic acid).

Table 5 Highest laccase activities observed in $100 \mathrm{ml} \mathrm{BSM}$ cultures of $P$. ostreatus $8 \mathrm{~F} 6$ and T. versicolor Strain 6 upon addition of phenolic compounds at a final concentration of $1 \mathrm{mM}$ each and comparison with concentrations of the respective compounds upon addition of water extractives or acetone extractives obtained from $1 \mathrm{~g}$ of $A$. grandis wood particles to $100 \mathrm{ml} \mathrm{BSM}$ medium

\begin{tabular}{|c|c|c|c|c|c|}
\hline \multirow[t]{2}{*}{ No. } & \multirow[t]{2}{*}{ Compound } & \multicolumn{2}{|c|}{$\begin{array}{l}\text { Highest laccase activity } \\
\text { obtained upon addition of } \\
1 \mathrm{mM} \text { compound * }\left[\mathrm{U} \mathrm{l}^{-1}\right]\end{array}$} & \multicolumn{2}{|c|}{$\begin{array}{l}\mathrm{mM} \text { in } 100 \mathrm{ml} \text { of } \mathrm{BSM} \\
\text { upon addition of }\end{array}$} \\
\hline & & P. ostreatus & T. versicolor & $\begin{array}{l}\text { Water } * * \\
\text { extractives }\end{array}$ & $\begin{array}{l}\text { Acetone } * * \\
\text { extractives }\end{array}$ \\
\hline 1 & 2-Methoxyphenol (guaiacol) & $130 \pm 15.4$ & $202 \pm 11.2$ & 0.31 & 0.23 \\
\hline 2 & 4- Hydroxybenzaldehyde & $215 \pm 3.2$ & $108 \pm 35.9$ & 0.24 & 0.22 \\
\hline 3 & $\begin{array}{l}\text { 1,4-Dihydroxybenzene } \\
\text { (hydroquinone) } * * *\end{array}$ & - & $69 \pm 2.2$ & 0.09 & 0.05 \\
\hline 4 & $\begin{array}{l}\text { 3,5-Di-tertbut-4-hydroxy- } \\
\text { toluene (butylhydroxytoluol) }\end{array}$ & $224 \pm 8.9$ & $554 \pm 88.4$ & 0.10 & 0.09 \\
\hline 5 & $\begin{array}{l}\text { 3-Methoxy-4- } \\
\text { hydroxybenzaldehyde } \\
\text { (vanillin) }\end{array}$ & $143 \pm 19.4$ & $100 \pm 5.2$ & 0.91 & 0.46 \\
\hline 6 & 4-Hydroxybenzoic acid & $61 \pm 17.9$ & $106 \pm 10.9$ & 2.17 & 0.59 \\
\hline 7 & $\begin{array}{l}\text { 3-Methoxy-4-hydroxybenzoic } \\
\text { acid (vanillic acid) }\end{array}$ & $78 \pm 2.2$ & $124 \pm 19.2$ & 6.85 & 1.26 \\
\hline 8 & $\begin{array}{l}\text { 4-Hydroxy cinnamic acid } \\
\text { (p-coumaric acid) }\end{array}$ & $322 \pm 9.1$ & $811 \pm 41.7$ & 6.16 & 4.77 \\
\hline 9 & $\begin{array}{l}\text { 4-Hydroxy-3-methoxy } \\
\text { cinnamic acid (ferulic acid) }\end{array}$ & $161 \pm 35.2$ & $172 \pm 17.4$ & 0.67 & 0.58 \\
\hline & Medium control with DMSO & $46 \pm 13.0$ & $96 \pm 14.9$ & - & - \\
\hline$* *$ & $\begin{array}{l}\text { are Fig. 13; values given ir } \\
\text { induction, respectively } \\
\text { ues refer to amounts of co } \\
\text { extractives obtained fror } \\
\text { re Table } 1 \text { ) } \\
\text { mpound hindered fungal gr }\end{array}$ & $\begin{array}{l}\text { ld and in ita } \\
\text { ounds deter } \\
\mathrm{g} \text { of } A . \varepsilon \\
\text { (Fig. 14) }\end{array}$ & $\begin{array}{l}\text { refer to } \mathrm{h} \\
\text { ned for } \mathrm{pt} \\
\text { andis woo }\end{array}$ & $\begin{array}{l}\text { and mec } \\
\text { water ex }\end{array}$ & $\begin{array}{l}\text { Im levels of } \\
\text { actives and } \\
\text { espectively }\end{array}$ \\
\hline
\end{tabular}


IV. Contrasting ecological functions of Abies grandis wood extractives on protection against white-rot fungi and induction of laccase activity

Fig. 14 showed that the combination of two phenolic laccase inducers can have synergistic effects. The combination of 4-hydroxy cinnamic acid and 3,5- di-tertbut-4hydroxy-toluene at each $1 \mathrm{mM}$ final concentration resulted in a 2-fold and 5-fold increase in laccase activity in T. versicolor Strain 6 cultures than the laccase activity obtained with the individual compounds 4-hydroxy cinnamic acid and 3,5-di-tertbut-4hydroxy-toluene, respectively. In contrast, with $P$. ostreatus $8 \mathrm{~F} 6$ there was interestingly no such synergistic effect, indicating again that different fungi may react differentially on given chemical compounds.

Synergism might not only exist between different phenolic compounds present in the wood, but dissolvable inorganic compounds might also play a role. For example, heavy metals as putative inducers, naturally present in most of the environments, are also an important group of enzyme activity modulators. Thus, positive regulation of laccase production by copper has repeatedly been reported for various fungal species. Copper concentrations of 0.25 to $3 \mathrm{mM}$ added into cultures of different white-rot fungi (T. versicolor, Trametes sp. 420, P. ostreatus, Lentinula edodes, Panus tigrinus, Rhizoctonia solani, Marasmius quercophilus) resulted in better laccase yields (Baldrian and Gabriel 2002; Cavallazzi et al. 2005; Chernykh et al. 2005; Crowe and Olsson 2001; Dominguez et al. 2007; Farnet et al. 1999; Palmieri et al. 2000; Patel et al. 2009; Tong et al. 2007). Of these cases, highest induced laccase production of about $7000 \mathrm{U}^{-1}$ was achieved with $0.5 \mathrm{mM} \mathrm{Cu}^{2+}$ with Trametes sp. 420 (Tong et al. 2007). Cavallazzi et al. (2005) used $0.25 \mathrm{mM}$ copper in an artificial medium added together with ammonium tartrate in cultures of Trametes sp. 420. The authors found that laccase induction was higher than when copper was added alone. Effects of other inducers has been reported by Crowe and Olsson (2001) who tested substances and compounds such as copper (II), manganese (II), lithium chloride, calcium chloride, in addition to the calcium ionophore A23187 and to organic compounds such as cyclic AMP (cAMP), caffeine, amphotericin $\mathrm{B}$, paraquat, ethanol, isopropanol and $p$-anisidine and all were found to induce laccase production of Rhizoctonia solani. However, there are also cases of inhibition of laccase production compounds. $\mathrm{NaN}_{3}$ for example was found strongly to inhibit laccase production of Trametes sp. 420 (Tong et al. 2007). Gallic acid and catechol in concentrations of $1 \mathrm{mM}$ were seen as phenolic inhibitors of laccase activity of L. edodes (Cavallazzi et al. (2005). The examples discussed document the expected complexity of how enzyme production and activities might be influenced in a multicompound environment. 
IV. Contrasting ecological functions of Abies grandis wood extractives on protection against white-rot fungi and induction of laccase activity

In frame of this work, the mineral contents in A. grandis wood of $\mathrm{Ca}, \mathrm{Mg}, \mathrm{Mn}$, $\mathrm{K}, \mathrm{Zn}, \mathrm{Na}, \mathrm{S}, \mathrm{P}$ and Al were determined (Chapter IV.7.4 in the supplementary material). Considerable amounts were detected for $\mathrm{Ca}, \mathrm{Mg}, \mathrm{Mn}, \mathrm{K}$ and $\mathrm{Na}$ as is typical for conifer wood (Fengel and Wegener 1983). Of the compounds analyzed, Mn and Ca could be of relevance in laccase induction (compare the work by Crowe and Olsson 2001). However, in frame of this study, $\mathrm{Ca}, \mathrm{Mg}, \mathrm{Mn}, \mathrm{K}$ and $\mathrm{Zn}$ were (mostly) removed from wood blocks by extraction with water (Fig. S7 in supplementary material) and extracted wood still had the ability to induce laccase, albeit at lower level (Fig. 5). This observation however does not exclude that $\mathrm{Mn}$ and $\mathrm{Ca}$ do not have a kind of influence on laccase production in P. ostreatus 8F6 and T. versicolor Strain 6.

\section{IV.5.2 Wood extractives in protection of wood against fungi}

In the previous chapters (Chapter II and Chapter III) and in a few available publications (Hawighorst et al. 2010; Kües et al. 2009; Navarro-González et al. 2008), A. grandis was shown to be sensitive against brown-rot fungi such as Coniophora puteana but to have a certain resistance against white-rot fungi. Further experiments presented in this study revealed that wood extractives were capable of inhibiting to different degrees actions of wood decay fungi in $A$. grandis wood. Compared to the original wood, mass loss caused by incubation with $T$. versicolor Strain 6 was significantly increased when the wood extractives were eliminated from tested wood (Fig. 25). Upon extraction with water or with water and acetone, even the dung fungus $C$. cinerea Okayama 7 was able to grow on the wood resulting in low amounts of wood mass loss (Fig. 25). Only P. ostreatus $8 \mathrm{~F} 6$ showed no difference in causing mass loss with $A$. grandis wood with extractives still present or with extractives being eliminated by water or by water and acetone treatment (Fig. 25). Moreover, when immersing A. grandis wood blocks in solutions of wood extractives prior to incubation with a fungus, this had profound effects on the surface growth of the species (Fig. 28 and 29). All three tested species reacted but at different degrees. P. ostreatus 8F6 was least affected, followed by T. versicolor Strain 6 and finally by $C$. cinerea Okayama 7. In case of T. versicolor Strain 6 , even more there was a difference regarding the type of extractives. When treating the wood blocks with water extractives, the inhibitory effect on surface growth was stronger than with the wood blocks treated with acetone extractives (Fig 29). Thus, there appears to be a correlation between the protective behaviors of water extractives 
IV. Contrasting ecological functions of Abies grandis wood extractives on protection against white-rot fungi and induction of laccase activity

against $T$. versicolor Strain 6 and the much improved amount of mass loss observed after acetone extractives were eliminated from the wood blocks as compared to when only water extractives were eliminated from the wood (Fig. 25).

The inhibitory effect of wood extractives against fungi was further demonstrated in agar-plate-diffusion-tests with suppression of growth of fungal mycelium (Fig. 26 and 27). C. cinerea Okayama 7 was most sensitive, P. ostreatus 8F6 and T. versicolor Strain 6 less against the wood extractives.

Various phenolic compounds have been reported to be inhibitory against fungi (Leonowicz et al. 2001; Martínez-Iñigo et al. 1999), amongst 1-aminobenzotriazole against the white-rot P. ostreatus and T. versicolor (Hundt et al. 1999). Here, clear negative effects of hydroquinone on fungal growth was demonstrated (Fig. 14). Moreover, $p$-benzochinone, a compound closely related to hydroquinone, had similar inhibiting effects on growth of the two fungi (Fig. S5 in the supplementary material). In conclusion, within the mixtures of wood extractives there are certainly compounds of negative effects on growth of fungi. Whether there are more of such negative effects by other compounds than the two identified in this study needs to be clarified in the future.

\section{IV.5.3 General conclusions on functions of wood extractives on fungi}

The experiments presented in this chapter indicate two opposing functions of $A$. grandis wood extractives on fungi. In favor of the tree species, wood extractives inhibit the growth of different fungi and thereby protect the wood from decay. This will be of particular importance whilst the tree is still alive. In contrast, wood extractives have also an inductive effect on laccase production of at least the two white-rots $P$. ostreatus 8F6 and T. versicolor Strain 6. This is of importance in ecology for the whole forest ecosystem. For $\mathrm{C}$ and $\mathrm{N}$ cycling within the forest ecosystem, it is important that the dead wood in reasonable time gets degraded, thereby releasing the nutrients for new tree biomass production (Knops et al. 2002; Knops and Tilman 2000).

As has been shown in this study, fungal laccase affects the $A$. grandis wood (Fig. 6). Since other phenoloxidase activities such as manganese peroxidase and lignin peroxidase were apparently not induced by $A$. grandis wood (Fig. 2), laccase probably has an important role in the decay of the difficult to degrade lignin (Eriksson et al. 1990, Leonowicz et al. 2001; Martínez-Iñigo et al. 1999). Induction of fungal enzymes 
IV. Contrasting ecological functions of Abies grandis wood extractives on protection against white-rot fungi and induction of laccase activity

for decay of dead wood by extractives not longer required for a protective function is thus reasonable in view of the whole ecological system.

That effects on inhibition of growth and on induction of enzyme production are individually different between fungal species, even different between two white-rot species, likely reflects just variations in the evolutionary adaptations to environmental situations such as different habitats (for the fungi treated here wood against dung) and such as different woods that may differ e.g. individual mixtures of compounds present in wood extractives and/or in the overall lignin contents.

\section{IV.6 References}

Abdallah SM, Mohamed G, Zayed MA, bou El-Ela MS (2009) Spectroscopic study of molecular structures of novel Schiff base derived from o-phthaldehyde and 2aminophenol and its coordination compounds together with their biological activity. Spectrochimica Acta Part A: Molecular and Biomolecular Spectroscopy 73(5)

Agren G, Bosatta E, Magill AH (2001) Combining theory and experiment to understand effects of inorganic nitrogen on litter decomposition. Oecologia 128(3):464-464

Arora DS, Sandhu DK (1987) Decomposition of angiospermic wood sawdust and laccase production by 2 Pleurotus Species. Journal of Basic Microbiology 27(4):179-184

Baldrian P (2004) Increase of laccase activity during interspecific interactions of whiterot fungi. FEMS Microbiology Ecology 50(3):245-253

Baldrian P, Gabriel J (2002) Copper and cadmium increase laccase activity in Pleurotus ostreatus. FEMS Microbiology Letters 206(1):69-74

Bergstrom B (2003) Chemical and structural changes during heartwood formation in Pinus sylvestris. Forestry 76(1):45-53

Binbuga N, Ruhs C, Hasty JK, Henry WP, Schultz TP (2008) Developing environmentally benign and effective organic wood preservatives by understanding the biocidal and non-biocidal properties of extractives in naturally durable heartwood. Holzforschung 62(3):264-269 
IV. Contrasting ecological functions of Abies grandis wood extractives on protection against white-rot fungi and induction of laccase activity

Blagodatskiy SA, Larionova AA, Yevdokimov IV (1993) Effect of mineral nitrogen on the respiration rate and growth efficiency of soil-microorganisms. Eurasian Soil Science 25(4):85-95

Blanchette RA, Krueger EW, Haight JE, Akhtar M, Akin DE (1997) Cell wall alterations in loblolly pine wood decayed by the white-rot fungus, Ceriporiopsis subvermispora. Journal of Biotechnology 53(2-3):203-213

Boddy L, Watkinson SC (1995) Wood decomposition, higher fungi, and their role in nutrient redistribution. Canadian Journal of Botany-Revue Canadienne de Botanique 73:1377-1383

Bravery AF (1978) A minimized wood-block test for rapid evaluation of wood preservative fungicides. International Research Group on Wood Preservation. Document No. IRG/WP 2113, Stockholm

Calcaterra A, Galli C, Gentili P (2008) Phenolic compounds as likely natural mediators of laccase: A mechanistic assessment. Journal of Molecular Catalysis BEnzymatic 51(3-4):118-120

Carbajo JM, Junca H, Terron MC, Gonzalez T, Yague S, Zapico E, Gonzalez AE (2002) Tannic acid induces transcription of laccase gene cglcc1 in the white-rot fungus Coriolopsis gallica. Canadian Journal of Microbiology 48(12):10411047

Carey JK, Savory JG, Mendes F, Bravery AF (1984) Loss of resistance to decay of Scots pine heartwood in a biologically-active environment. Applied Biochemistry and Biotechnology 9(4):343-344

Cavallazzi JRP, Kasuya CM, Soares MA (2005) Screening of inducers for laccase production by Lentinula edodes in liquid medium. Brazilian Journal of Microbiology 36(4):383-387

Chernykh AM, Leont'evskii AA, Golovleva LA (2005) New approaches to increasing the yield of laccase from Panus tigrinus. Applied Biochemistry and Microbiology 41(5):508-511

Childs RE, Bardsley WG (1975) Steady-state kinetics of peroxidase with 2,2'-azinodi-(3-ethylbenzthiazoline-6-sulphonic acid) as chromogen. Biochemical Journal 145(1):93-103

Chirkova J, Irbe I, Andersone I, Andersons B (2009) Study of the sorption-desorption properties of pine wood at the initial stage of decay by wood-rot fungi 10(th) EWLP, Stockholm, Sweden, August 25-28, 2008. Holzforschung 63(6):761-766 
IV. Contrasting ecological functions of Abies grandis wood extractives on protection against white-rot fungi and induction of laccase activity

Couto SR, Gundin M, Lorenzo M, Sanroman MN (2002) Screening of supports and inducers for laccase production by Trametes versicolor in semi-solid-state conditions. Process Biochemistry 38(2):249-255

Crowe JD, Olsson S (2001) Induction of laccase activity in Rhizoctonia solani by antagonistic Pseudomonas fluorescens strains and a range of chemical treatments. Applied and Environmental Microbiology 67(5):2088-2094

del Rio JC, Speranza M, Gutierrez A, Martinez MJ, Martinez AT (2002) Lignin attack during eucalypt wood decay by selected basidiomycetes: a Py-GC/MS study. Journal of Analytical and Applied Pyrolysis 64(2):421-431

De Souza CGM, Tychanowicz GK, De Souza DF, Peralta RM (2004) Production of laccase isoforms by Pleurotus pulmonarius in response to presence of phenolic and aromatic compounds. Journal of Basic Microbiology 44(2):129-136

Dominguez A, Gomez J, Lorenzo M, Sanroman A (2007) Enhanced production of laccase activity by Trametes versicolor immobilized into alginate beads by the addition of different inducers. World Journal of Microbiology \& Biotechnology 23(3):367-373

Dorado J, van Beek TA, Claassen FW, Sierra-Alvarez R (2001) Degradation of lipophilic wood extractive constituents in Pinus sylvestris by the white-rot fungi Bjerkandera sp. and Trametes versicolor. Wood Science and Technology 35(12):117-125

Ejechi BO (1996) Growth inhibition of Pseudomonas aeruginosa and Enterococcus faecalis by crude extractives of Mansonia altisima timber sawdust. Current Microbiology 32(5):297-298

Elissetche JP, Ferraz A, Freer J, Mendonca R, Rodriguez J (2006) Thiobarbituric acid reactive substances, $\mathrm{Fe}^{3+}$ reduction and enzymatic activities in cultures of Ganoderma australe growing on Drimys winteri wood. FEMS Microbiology Letters 260(1):112-118

EN 113 (1996) Wood preservatives - test method for determining the protective effectiveness against wood destroying basidiomycetes - Determination of the toxic values. European Committee for Standardization, Brüssel, Belgium: $35 \mathrm{p}$

Eriksson KEL, Blanchette RA, Ander P (1990) Microbial and enzymatic degradation of wood and wood components. Springer-Verlag, Berlin, Germany 
IV. Contrasting ecological functions of Abies grandis wood extractives on protection against white-rot fungi and induction of laccase activity

Eugenio ME, Carbajo JM, Martin JA, Gonzalez AE, Villar JC (2009) Laccase production by Pycnoporus sanguineus under different culture conditions. Journal of Basic Microbiology 49(5):433-440

Evans PD, Banks WB (1990) Degradation of wood surfaces by water - weight losses and changes in ultrastructural and chemical-composition. Holz als Roh-und Werkstoff 48(4):159-163

Farnet AM, Tagger S, Le Petit J (1999) Effects of copper and aromatic inducers on the laccases of the white-rot fungus Marasmius quercophilus. Comptes Rendus de 1 Academie des Sciences Serie Iii-Sciences de la Vie-Life Sciences 322(6):499503

Fengel D, Wegener G (1983) Wood. Chemistry, ultrastructure, reactions. Walter de Gruyter, Berlin

Fernandez MP, Watson PA, Breuil C (2001) Gas chromatography-mass spectrometry method for the simultaneous determination of wood extractive compounds in quaking aspen. Journal of Chromatography A 922(1-2):225-233

Ferraz A, Cordova AM, Machuca A (2003) Wood biodegradation and enzyme production by Ceriporiopsis subvermispora during solid-state fermentation of Eucalyptus grandis. Enzyme and Microbial Technology 32(1):59-65

Ghosh SC, Militz H, Mai C (2008) Decay resistance of treated wood with functionalised commercial Silicones. Bioresources 3(4):1303-1314

Gierlinger N, Jacques D, Schwanninger M, Wimmer R, Paques LE (2004) Heartwood extractives and lignin content of different larch species (Larix sp.) and relationships to brown-rot decay-resistance. Trees-Structure and Function $18(2): 230-236$

Gregorio APF, Da Silva IR, Sedarati MR, Hedger JN (2006) Changes in production of lignin degrading enzymes during interactions between mycelia of the tropical decomposer basidiomycetes Marasmiellus troyanus and Marasmius pallescens. Mycological Research 110:161-168

Gutierrez A, del Rio JC, Gonzalez-Vila FJ, Martin F (1998) Analysis of lipophilic extractives from wood and pitch deposits by solid-phase extraction and gas chromatography. Journal of Chromatography A 823(1-2):449-455

Gutierrez A, del Rio JC, Martinez MJ, Martinez AT (1999) Fungal degradation of lipophilic extractives in Eucalyptus globulus wood. Applied and Environmental Microbiology 65(4):1367-1371 
IV. Contrasting ecological functions of Abies grandis wood extractives on protection against white-rot fungi and induction of laccase activity

Gutierrez A, Rodriguez IM, del Rio JC (2006) Chemical characterization of lignin and lipid fractions in industrial hemp bast fibers used for manufacturing high-quality paper pulps 54(6):2138-2144

Havlis J, Shevchenko A (2004) Absolute quantification of proteins in solutions and in polyacrylamide gels by mass spectrometry. Analytical Chemistry 76:3029-3036

Hawighorst P, Müller GS, Navarro-González M, Malik I, Kües U, Polle A (2010) Knowledge-based production and durability of innovation beech and grand fir wood-base panels. Forst und Holz 65(1):20-25

Hoegger P, Majcherczyk A, Dwivedi RC, Svobodová K, Kilaru S, Kües U (2007) Enzyme in wood degradation. In: Kües U (Ed.) Wood Production, Wood Technology, and Biotechnological Impacts. Universitätsverlag Göttingen, Göttingen, 383-432

Johansson M, Lundgren L, Asiegbu FO (1998) Differential phenol-induced laccase activity and total oxidative capacity of the $\mathrm{S}$ and $\mathrm{P}$ intersterility groups of the conifer root pathogen Heterobasidion annosum. Microbiological Research 153(1):71-80

Kachlishvili E, Penninckx MJ, Tsiklauri N, Elisashvili V (2006) Effect of nitrogen source on lignocellulolytic enzyme production by white-rot basidiomycetes under solid-state cultivation. World Journal of Microbiology \& Biotechnology 22(4):391-397

Klaassen RKWM (2008) Water flow through wooden foundation piles: A preliminary study. International Biodeterioration \& Biodegradation 61(1):61-68

Klepzig KD, Smalley EB, Raffa KF (1996) Combined chemical defenses against an insect-fungal complex. Journal of Chemical Ecology 22(8):1367-1388

Knops JMH, Tilman D (2000) Dynamics of soil nitrogen and carbon accumulation for 61 years after agricultural abandonment. Ecology 81(1):88-98

Knops JMH, Bradley KL, Wedin DA (2002) Mechanisms of plant species impacts on ecosystem nitrogen cycling. Ecology Letters 5(3):454-466

Kollmann A, Boyer FD, Ducrot PH, Kerhoas L, Jolivalt C, Touton I, Einhorn J, Mougin C (2005) Oligomeric compounds formed from 2,5-xylidine (2,5dimethylaniline) are potent enhancers of laccase production in Trametes versicolor ATCC 32745. Applied Microbiology and Biotechnology 68(2):251258 
IV. Contrasting ecological functions of Abies grandis wood extractives on protection against white-rot fungi and induction of laccase activity

König J, Grasser R, Pikor H, Vogel K (2002) Determination of xylanase, $\beta$ glucanase, and cellulase activity. Analytical and Bioanalytical Chemistry 371(1):80-87

Kuwahara M, Glenn JK, Morgan MA, Gold MH (1984) Separation and characterization of 2 extracellular $\mathrm{H}_{2} \mathrm{O}_{2}$-dependent oxidases from ligninolytic cultures of Phanerochaete chrysosporium. FEMS Letters 169(2):247-250

Kües U, Navarro-González M, Cherdchim B, Majcherczyk A (2009) Anfälligkeit von lebendem und totem Holz der Küstentanne gegenüber Pilzen. Mykologisches Kolloquium, Molekularbiologische Pilzdiagnostik, 7./8.Mai 2009 in Dresden, Institut für Holztechnologie, Dresden :1-14

Lavoie JM, Stevanovic T (2006) Yield and composition of lipophylic extracts of yellow birch (Betula alleghaniensis Britton) as a function of wood age and aging under industrial conditions. Holzforschung 60(2):184-189

Lee C, Lee Y, Yoon J (2006) Oxidative degradation of dimethylsulfoxide by locally concentrated hydroxyl radicals in streamer corona discharge process. Chemosphere 65(7):1163-1170

Lee Y, Park C, Lee B, Han EJ, Kim TH, Lee J, Kim S (2006) Effect of nutrients on the production of extracellular enzymes for decolorization of reactive blue 19 and reactive black 5. Journal of Microbiology and Biotechnology 16(2):226-231

Lekounougou S, Mounguengui S, Dumarpy,S, Rose C, Courty PE, Garbaye J, Gerardin P, Jacquot JP, Gelhaye E (2008) Initial stages of Fagus sylvatica wood colonization by the white-rot basidiomycete Trametes versicolor: Enzymatic characterization. International Biodeterioration \& Biodegradation 61(4):287-293

Leonowicz A, Cho NS, Luterek J, Wilkolazka A, Wojtas-Wasilewska M, Matuszewska A, Hofrichter M, Wesenberg D, Rogalski J (2001) Fungal laccase: properties and activity on lignin. Journal of Basic Microbiology 41(3-4):185-227

Lorenzo M, Moldes D, Couto SR, Sanroman A (2002) Improving laccase production by employing different lignocellulosic wastes in submerged cultures of Trametes versicolor. Bioresource Technology 82(2):109-113

Mai C, Kües U, Militz H (2004) Biotechnology in the wood industry. Applied Microbiology and Biotechnology 63(5):477-494

Majcherczyk A, Johannes C, Hüttermann A (1999) Oxidation of aromatic alcohols by 
IV. Contrasting ecological functions of Abies grandis wood extractives on protection against white-rot fungi and induction of laccase activity

laccase from Trametes versicolor mediated by the 2,2 '-azino-bis-(3-

ethylbenzothiazoline-6-sulphonic acid) cation radical and dication. Applied

Microbiology and Biotechnology 51(2):267-276

Martínez-Iñigo MJ, Immerzeel P, Gutierrez A, del Río JC, Sierra-Alvarez R (1999)

Biodegradability of extractives in sapwood and heartwood from Scots pine by sapstain and white rot fungi. Holzforschung 53(3):247-252

Mayer AM, Staples RC (2002) Laccase: new functions for an old enzyme.

Phytochemistry 60(6):551-565

Medda R, Padiglia A, Pedersen JZ, Rotilio G, Agro AF, Floris G (1995) The reaction mechanism of copper amine oxidase: Detection of intermediates by the use of substrates and inhibitors. Biochemistry 34(50):16375-16381

Michel FC, Dass SB, Grulke EA, Reddy CA (1991) Role of manganese peroxidases and lignin peroxidases of Phanerochaete chrysosporium in the decolorization of kraft beach pant effluent. Applied and Environmental Microbiology 57(8):23682375

Moredo N, Lorenzo M, Dominguez A, Moldes D, Cameselle C, Sanroman A (2003) Enhanced ligninolytic enzyme production and degrading capability of Phanerochaete chrysosporium and Trametes versicolor. World Journal of Microbiology \& Biotechnology 19(7):665-669

Müller G, Schöpper C, Vos H, Kharazipour A, Polle A (2009) FTIR-ATR spectroscopic analyses of changes in wood properties during particle- and fibreboard production of hard- and softwood trees. Bioresources 4(1):49-71

Murakami-Nitta T, Kurimura H, Kirimura K, Kino K, Usami S (2002) Continuous degradation of dimethyl sulfoxide to sulfate ion by Hyphomicrobium denitrificans WU-K217. Journal of Bioscience and Bioengineering 94(1):52-56

Navarro-González M, Wei DS, Kües U (2008) Pilze auf Holz der Küstentanne (Abies grandis). Holzbiologie 49(6): 23-25

Palmieri G, Giardina P, Bianco C, Fontanella B, Sannia G (2000) Copper induction of laccase isoenzymes in the ligninolytic fungus Pleurotus ostreatus. Applied and Environmental Microbiology 66(3):920-924

Patel H, Gupte A, Gupte S (2009) Effect of different culture conditions and inducers on production of laccase by a basidiomycete fungal isolate Pleurotus ostreatus Hp-1 under solid state fermentation. Bioresources 4(1):268-284 
IV. Contrasting ecological functions of Abies grandis wood extractives on protection against white-rot fungi and induction of laccase activity

Philip RW, Bruce A, Munro AG (1995) The effect of water soluble Scots pine (Pinus sylvestris L) and Sitka spruce [Picea sitchensis (bong) carr] heartwood and sapwood extracts on the growth of selected Trichoderma species. International Biodeterioration \& Biodegradation 35(4):355-367

Pino V, Ayala JH, Gonzalez V, Afonso AM (2007) Determination of the alkyl- and methoxy-phenolic content in wood extractives by micellar solid-phase microextraction and gas chromatography-mass spectrometry. Talanta 73(3):505513

Polle A, Hawighorst P, Spellmann H (2008) Innovative Holzprodukte und Holzwerkstoffe aus nachhaltig produziertem Buchen- und Küstentannenholz. Holztechnologie 49:5-6

Popescu CM, Popescu MC, Singurel G, Vasile C, Argyropoulos DS, Willfor S (2007) Spectral characterization of eucalyptus wood. Applied Spectroscopy 61(1):1168-1177

Rodríguez-López JN, Escribano J, García-Cánovas (1994) A continuous spectrophotometric method for the determination of monophenolase activity of tyrosinase using 3-methyl-2-benzothiazolinone hydrazone. Analytical Chemistry 216:205-212

Röhrig E (1981) Neuere Grundlagen für den Anbau von A. grandis. Schriften aus der Forstlichen Fakultät der Universität Göttingen und der Niedersächsischen Forstlichen Versuchsanstalt, Sauerländer Verlag, Frankfurt am Main

Rühl M, Kilaru S, Navarro-González M, Hoegger P, Kharazipour A, Kües U (2007) Production of laccase and other enzymes for the wood industry. In: Kües U (Ed.) Wood Production, Wood Technology, and Biotechnological Impacts. Universitätsverlag Göttingen, Göttingen, 469-507

Schultz TP, Nicholas DD (2000) Naturally durable heartwood: evidence for a proposed dual defensive function of the extractives. Phytochemistry 54(1):47-52

Schultz TP, Nicholas DD (2002) Development of environmentally-benign wood preservatives based on the combination of organic biocides with antioxidants and metal chelators. Phytochemistry 61(5):555-560

Shah V, Baldrian P, Eichlerova I, Dave R, Madamwar D, Nerud F, Gross R (2006) Influence of dimethyl sulfoxide on extracellular enzyme production by Pleurotus ostreatus. Biotechnology Letters 28(9):651-655 
IV. Contrasting ecological functions of Abies grandis wood extractives on protection against white-rot fungi and induction of laccase activity

Shah V, Nerud F (2002) Lignin degrading system of white-rot fungi and its exploitation for dye decolorization. Canadian Journal of Microbiology 48(10):857-870

Silverio FO, Barbosa LCA, Silvestre AJD, Pilo-Veloso D, Gomide JL (2007) Comparative study on the chemical composition of lipophilic fractions from three wood tissues of Eucalyptus species by gas chromatography-mass spectrometry analysis. Journal of Wood Science 53(6):533-540

Sjostrom E (1993) Wood chemistry fundamentals and applications. Academic Press, San Diego

Spellmann H, Geb M, Nagel J, Nagel R, Schmidt M (2010) Timber product oriented silvicultural strategies for mixed strands of beech ( Fagus silvatica) and grand fir (Abies grandis). Forst und Holz 65(1):12-19

Stricker AR, Mach R, de Graaff LH (2008) Regulation of transcription of cellulasesand hemicellulases-encoding genes in Aspergillus niger and Hypocrea jecorina (Trichoderma reesei). Applied Microbiology and Biotechnology 78(2):211-220

Sumthong P, Romero-Gonzalez RR, Verpoorte R (2008) Identification of anti-wood rot compounds in teak (Tectona grandis L.f.) sawdust extract. Journal of Wood Chemistry and Technology 28(4):247-260

Tadesse MA, D'Annibale A, Galli C, Gentili P, Sergi F (2008) An assessment of the relative contributions of redox and steric issues to laccase specificity towards putative substrates. Organic \& Biomolecular Chemistry 6(5):868-878

TAPPI T 204 om-88 (1988) Soxhlet systems for determining extractives content. Technical Association of the Pulp and Paper Industry, USA: $2 \mathrm{p}$

Tateno M, Chapin FS (1997) The logic of carbon and nitrogen interactions in terrestrial ecosystems. American Naturalist 149(4):723-744

Taylor AM, Gartner BL, Morrell JJ, Tsunoda K (2006) Effects of heartwood extractive fractions of Thuja plicata and Chamaecyparis nootkatensis on wood degradation by termites or fungi. Journal of Wood Science 52(2):147-153

Tavares APM, Rodriguez O, Macedo EA (2008) Ionic liquids as alternative co-solvents for laccase: Study of enzyme activity and stability. Biotechnology and Bioengineering 101(1):201-207

Terron MC, Gonzalez T, Carbajo JM, Yague S, rana-Cuenca A, Tellez A, Dobson ADW, Gonzalez AE (2004) Structural close-related aromatic compounds have different effects on laccase activity and on $l c c$ gene expression in the 
IV. Contrasting ecological functions of Abies grandis wood extractives on protection against white-rot fungi and induction of laccase activity

ligninolytic fungus Trametes sp I-62. Fungal Genetics and Biology 41(10):954962

Thiet RK, Frey SD, Six J (2006) Do growth yield efficiencies differ between soil microbial communities differing in fungal: bacterial ratios? Reality check and methodological issues. Soil Biology \& Biochemistry 38(4):837-844

Thurston CF (1994) The structure and function of fungal laccases. MicrobiologySGM 140:19-26

Tien M, Kirk TK (1984) Lignin-degrading enzyme from Phanerochaete chrysosporium -purification, characterization, and catalytic properties of a unique $\mathrm{H}_{2} \mathrm{O}_{2}$ requiring oxygenase. Proceedings of the National Academy of Sciences of the United States of America-Biological Sciences 81(8):2280-2284

Tong PG, Hong YZ, Xiao YZ, Zhang M, Tu XM, Cui TJ (2007) High production of laccase by a new basidiomycete, Trametes sp. Biotechnology Letters 29(2):295301

van der Wal A, de Boer W, Smant W, van Veen JA (2007) Initial decay of woody fragments in soil is influenced by size, vertical position, nitrogen availability and soil origin. Plant and Soil 301(1-2):189-201

Venalainen M, Harju AM, Terziev N, Laakso T, Saranpaa P (2006) Decay resistance, extractive content, and water sorption capacity of Siberian larch (Larix sibirica Lebed.) heartwood timber. Holzforschung 60(1):99-103

Verma P, Junga U, Militz H, Mai C (2009) Protection mechanisms of DMDHEU treated wood against white and brown rot fungi. Holzforschung 63(3):371-378

Vos H, Kharazipour A (2010) Properties of light particle boards manufactured using Abies grandis (grand fir). Forst und Holz 65(1):26-30

Wang XJ, Bai JG, Liang YX (2006) Optimization of multienzyme production by two mixed strains in solid-state fermentation. Applied Microbiology and Biotechnology 73(3):533-540

Willfor S, Hemming J, Reunanen M, Holmbom B (2003) Phenolic and lipophilic extractives in Scots pine knots and stemwood. Holzforschung 57(4):359-372

Yang DQ, Wang XM, Wan H (2007) Biological treatment of aspen strands to improve mold resistance and reduce resin consumption of strand panels. Forest Products Journal 57(7-8):58-62 
IV. Contrasting ecological functions of Abies grandis wood extractives on protection against white-rot fungi and induction of laccase activity

Yang DQ, Wan H, Wang XM, Liu ZM (2007) Use of fungal metabolites to protect wood-based panels against mould infection. Biocontrol 52(3):427-436

Xiao YZ, Chen Q, Hang J, Shi YY, Xiao YZ, Wu J, Hong YZ, Wang YP (2004)

Selective induction, purification and characterization of a laccase isozyme from the basidiomycete Trametes sp AH28-2. Mycologia 96(1):26-35

Zaidon A, Nizam AMN, Faizah A, Paridah MT, Jalaluddin H, Nor MYM, Yuziah MYN (2008) Efficacy of pyrethroid and boron preservatives in protecting particleboards against fungus and termite. Journal of Tropical Forest Science 20(1):57-65 
IV. Contrasting ecological functions of Abies grandis wood extractives on protection against white-rot fungi and induction of laccase activity

\section{IV.7 Supplementary material}

\section{IV.7.1 Laccase induction by different fractions of $\boldsymbol{A}$. grandis wood extractives and by DMSO}

Results in Fig. 10A showed that original A. grandis wood particles and water extractives had a profound effect on laccase induction in cultures of $P$. ostreatus $8 \mathrm{~F} 6$. Chromatography fractions of water extractives have also an inductive effect but at much lower efficiency. To show this effect in better way, Fig. S1 presents the curves with a more stretched $y$-axis than in Fig. 10A of laccase production obtained with P. ostreatus $8 \mathrm{~F} 6$ over the time in BSM cultures with added chromatography fractions in comparison to media controls.

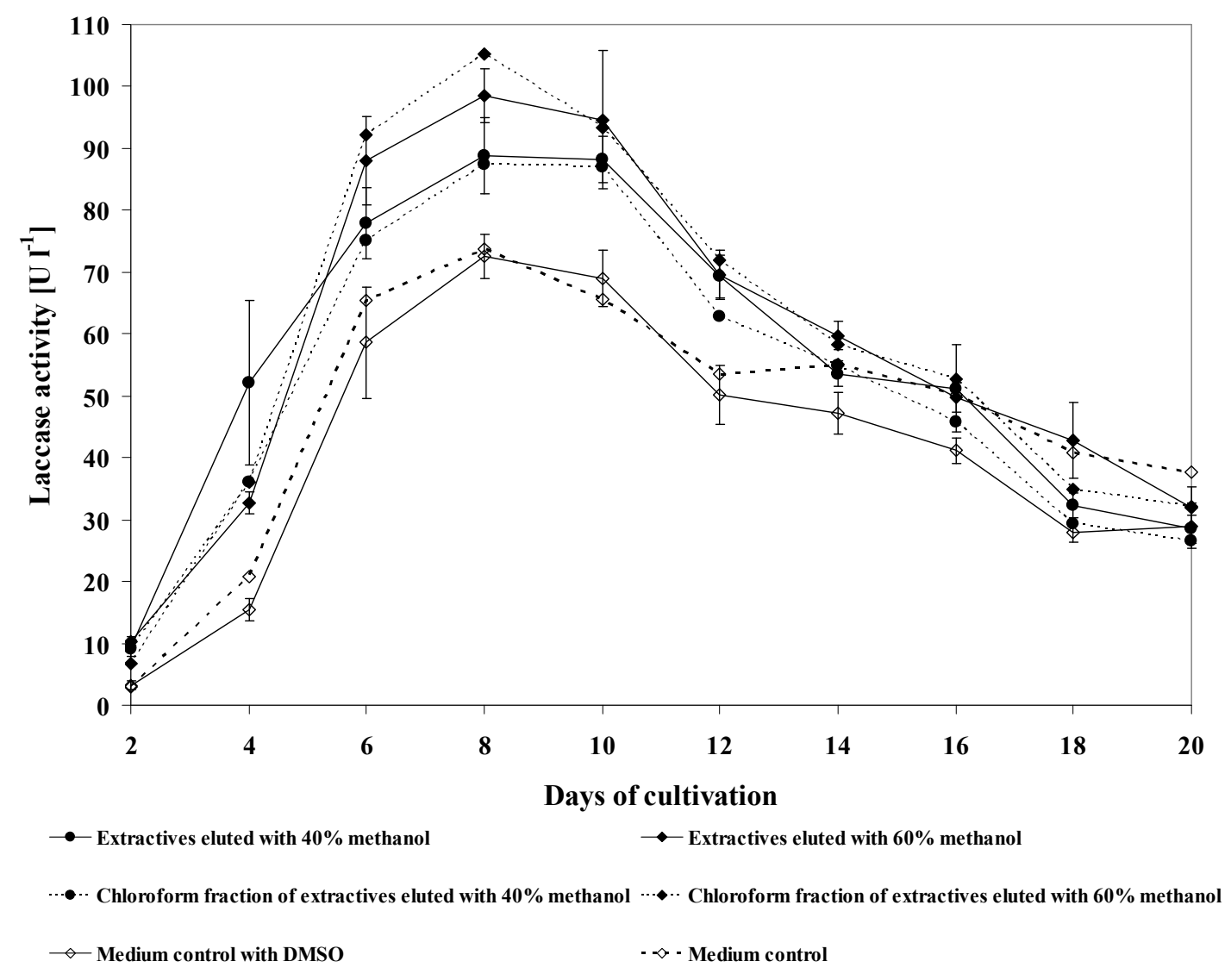

Fig. S1 Laccase production at $25^{\circ} \mathrm{C}$ by $P$. ostreatus $8 \mathrm{~F} 6$ in stationary cultures of $100 \mathrm{ml}$ BSM liquid medium with added extractives corresponding to $1 \mathrm{~g}$ of $A$. grandis wood, dissolved in $200 \mu \mathrm{l}$ DMSO (detailed presentation of the results shown in Fig. 10A). For testing effects of water extractives, either aliquots of the original water extractives, or of chromatography fractions of water extractives eluted by $40 \%$ or $60 \%$ methanol from the silica gel column, or of chloroform-purified fractions of the extractives eluted by $40 \%$ or $60 \%$ methanol from the column were added. $200 \mu 1$ of only DMSO was added into control cultures to observe the effect of the solvent on laccase production. No difference was seen in growth between the various cultures. Average values and standard deviations were calculated from three parallel cultures each 
IV. Contrasting ecological functions of Abies grandis wood extractives on protection against white-rot fungi and induction of laccase activity

Table S1 Laccase activities in fungal cultures with added DMSO at the end of incubation periods (day 19 to 20 of incubation in $100 \mathrm{ml} \mathrm{BSM}$ liquid medium at $25^{\circ} \mathrm{C}$ )

\begin{tabular}{|c|c|c|c|c|}
\hline \multirow{3}{*}{$\begin{array}{l}\text { Experiment } \\
\text { (ml DMSO) }\end{array}$} & \multicolumn{4}{|c|}{ Laccase activity $\left[\mathrm{U} \mathrm{I}^{-1}\right]$} \\
\hline & \multicolumn{2}{|c|}{ P. ostreatus 8F6 } & \multicolumn{2}{|c|}{ T. versicolor Strain 6} \\
\hline & With DMSO & Without DMSO & With DMSO & Without DMSO \\
\hline \multicolumn{5}{|l|}{$0.1 \mathrm{ml}$} \\
\hline Fig. 13 & $24 \pm 4.2$ & $25 \pm 5.0$ & $93 \pm 9.9$ & $68 \pm 6.5$ \\
\hline \multicolumn{5}{|l|}{$0.2 \mathrm{ml}$} \\
\hline Fig. 9 & $28 \pm 7.9$ & $19 \pm 7.5$ & $232 \pm 24$ & $100 \pm 6.3$ \\
\hline Fig. 10/15 & $29 \pm 3.7$ & $38 \pm 2.5$ & $152 \pm 17.4$ & $125 \pm 5.9$ \\
\hline \multicolumn{5}{|l|}{$0.4 \mathrm{ml}$} \\
\hline Fig. 11 & $78 \pm 13.4$ & $31 \pm 5.7$ & $78 \pm 1.3$ & $83 \pm 7.0$ \\
\hline \multicolumn{5}{|l|}{$0.5 \mathrm{ml}$} \\
\hline Fig. 4 & $64 \pm 3.3$ & $37 \pm 5.4$ & $102 \pm 9.6$ & $128 \pm 21.1$ \\
\hline \multicolumn{5}{|l|}{$2 \mathrm{ml}$} \\
\hline Fig. 7 & $171 \pm 16.5$ & $44 \pm 16.8$ & $74 \pm 11.0$ & $112 \pm 33$ \\
\hline
\end{tabular}


IV. Contrasting ecological functions of Abies grandis wood extractives on protection against white-rot fungi and induction of laccase activity

IV.7.2 Identification of specific compound in $A$. grandis wood extractive by GCMS

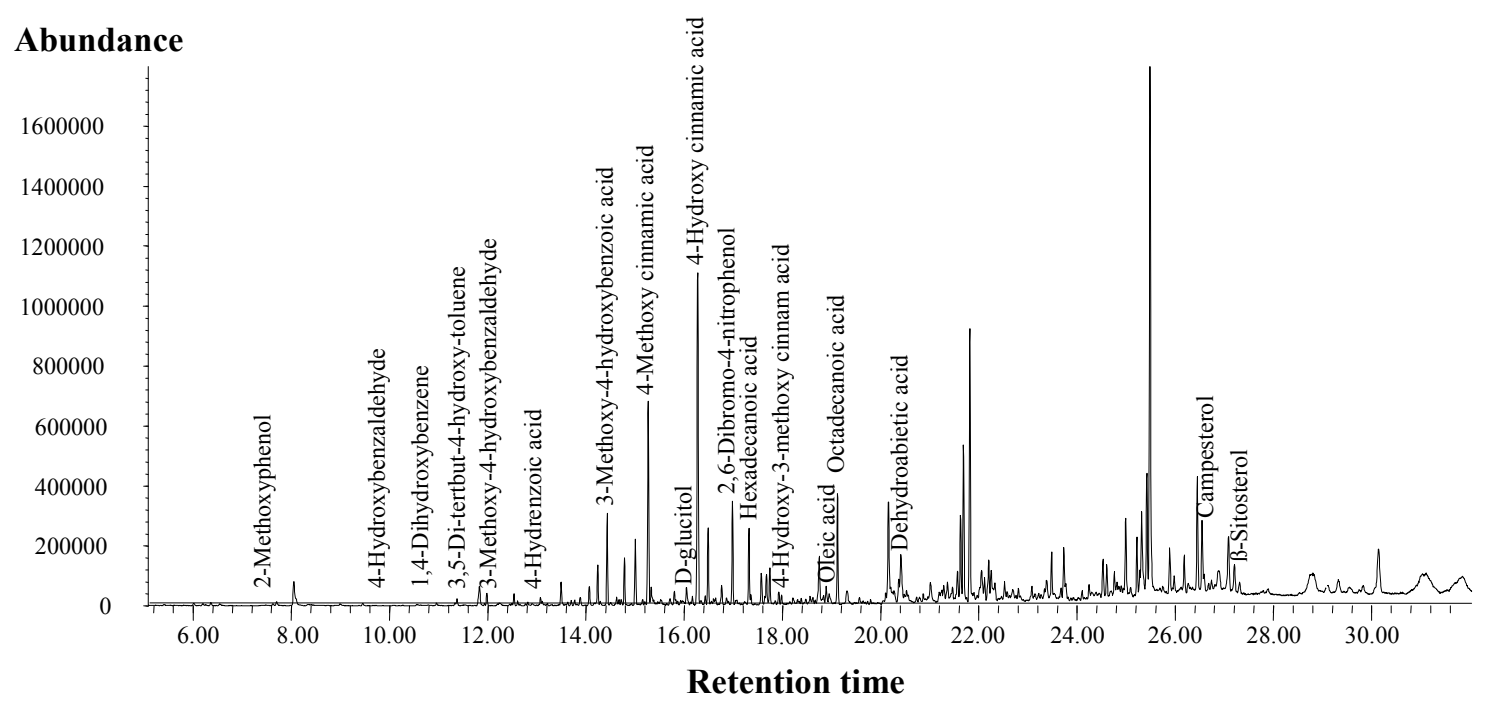

Fig. S2 Complete chromatogram of the GC-MS analysis of compounds in the chloroform-purified fraction of water extractives from $A$. grandis wood particles. Compounds identified with an identification quality higher than $95 \%$ are indicated (for further details compare Fig. 11)

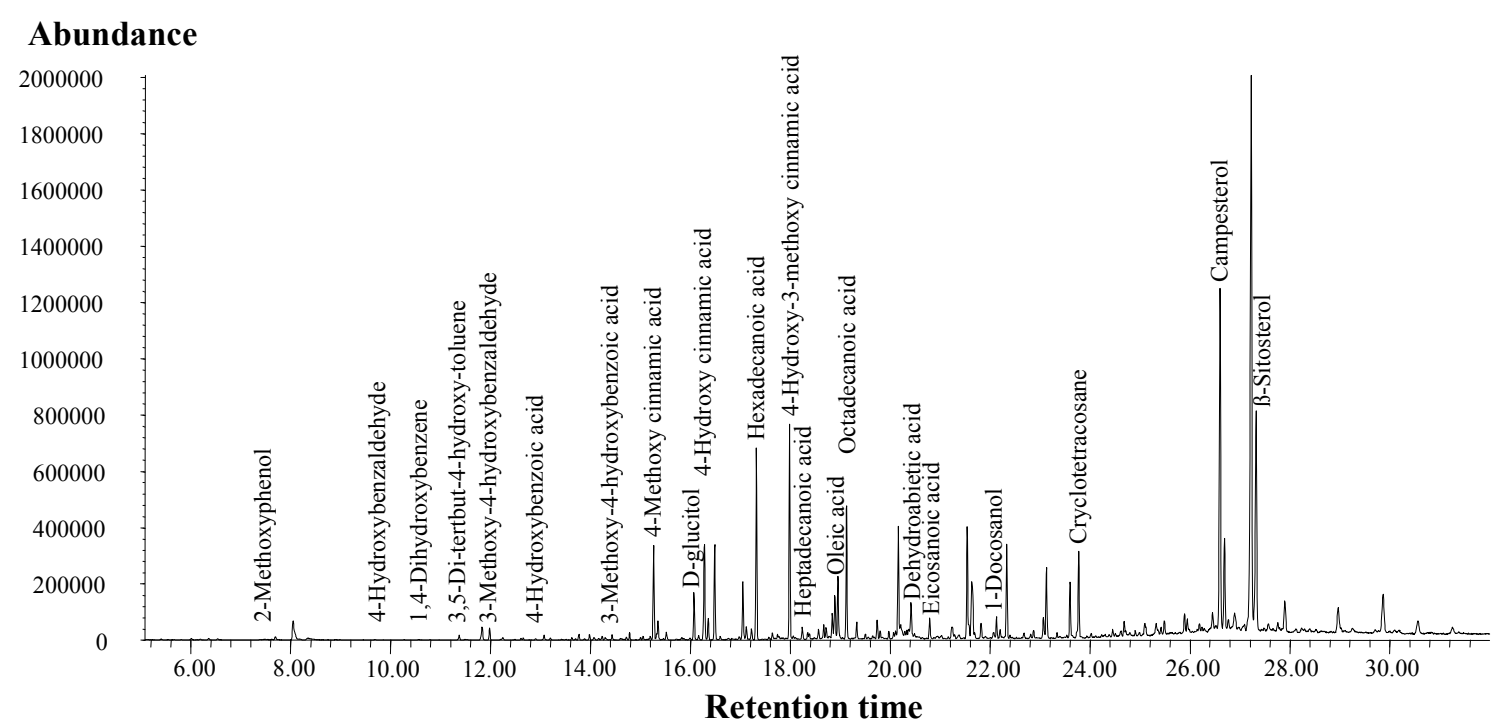

Fig. S3 Complete chromatogram of the GC-MS analysis of compounds in the chloroform-purified fraction of acetone extractives from A. grandis wood particles. Compounds identified with an identification quality higher than $95 \%$ are indicated (for further details compare Fig. 12) 
IV. Contrasting ecological functions of Abies grandis wood extractives on protection against white-rot fungi and induction of laccase activity

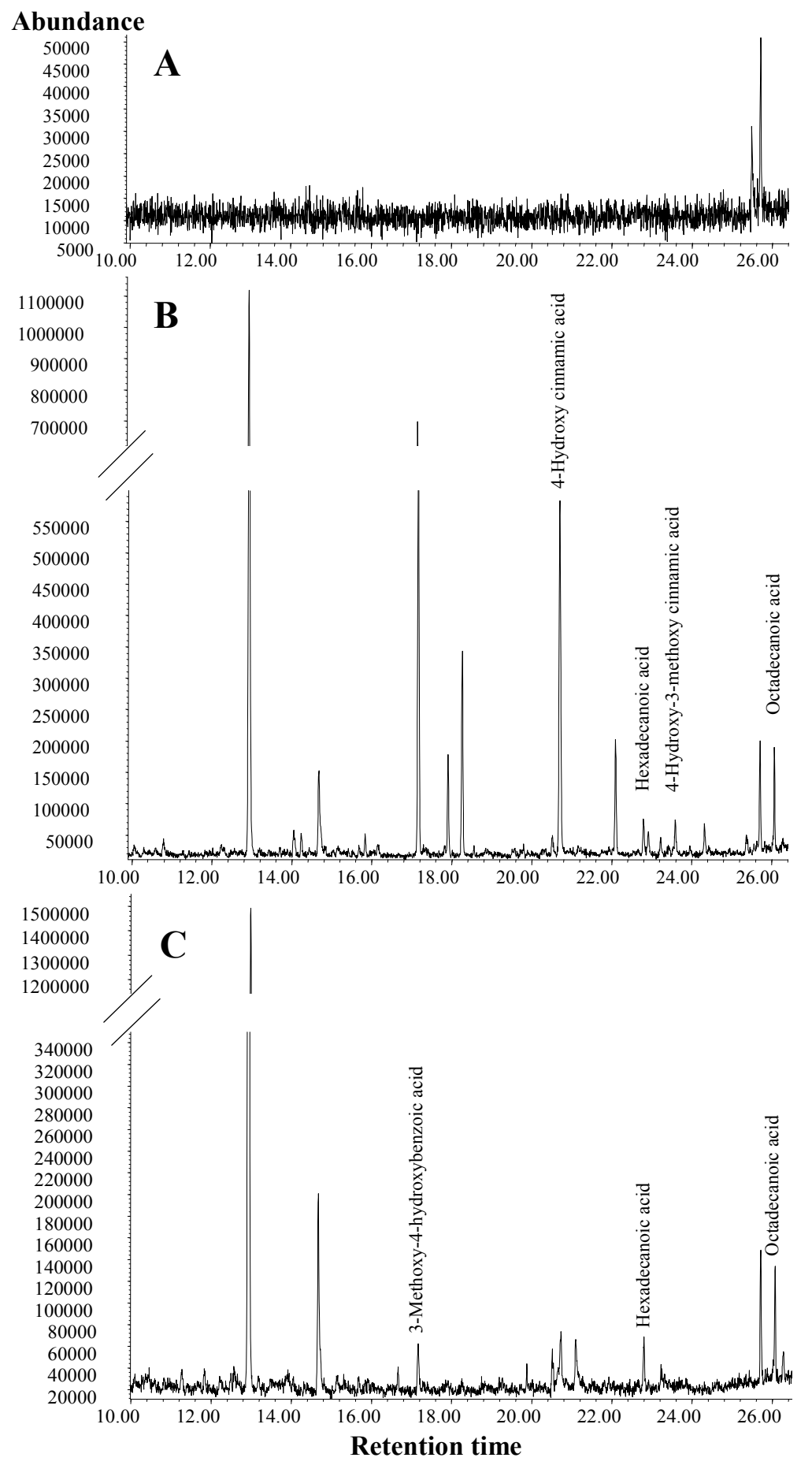

Fig. S4 Complete chromatogram of the GC-MS analysis of compounds in the different chromatography fractions of water extractives of $A$. grandis wood: the pass-through $\mathbf{A}$ ), extractives eluted with $20 \%$ methanol B), extractives eluted with $40 \%$ methanol C), extractives eluted with $60 \%$ methanol D), extractives eluted with $80 \%$ methanol E), extractives eluted with $100 \%$ methanol F), and extractives eluted with DMSO G) from the silica gel column. Compounds identified with an identification quality higher than $95 \%$ are indicated 
IV. Contrasting ecological functions of Abies grandis wood extractives on protection against white-rot fungi and induction of laccase activity
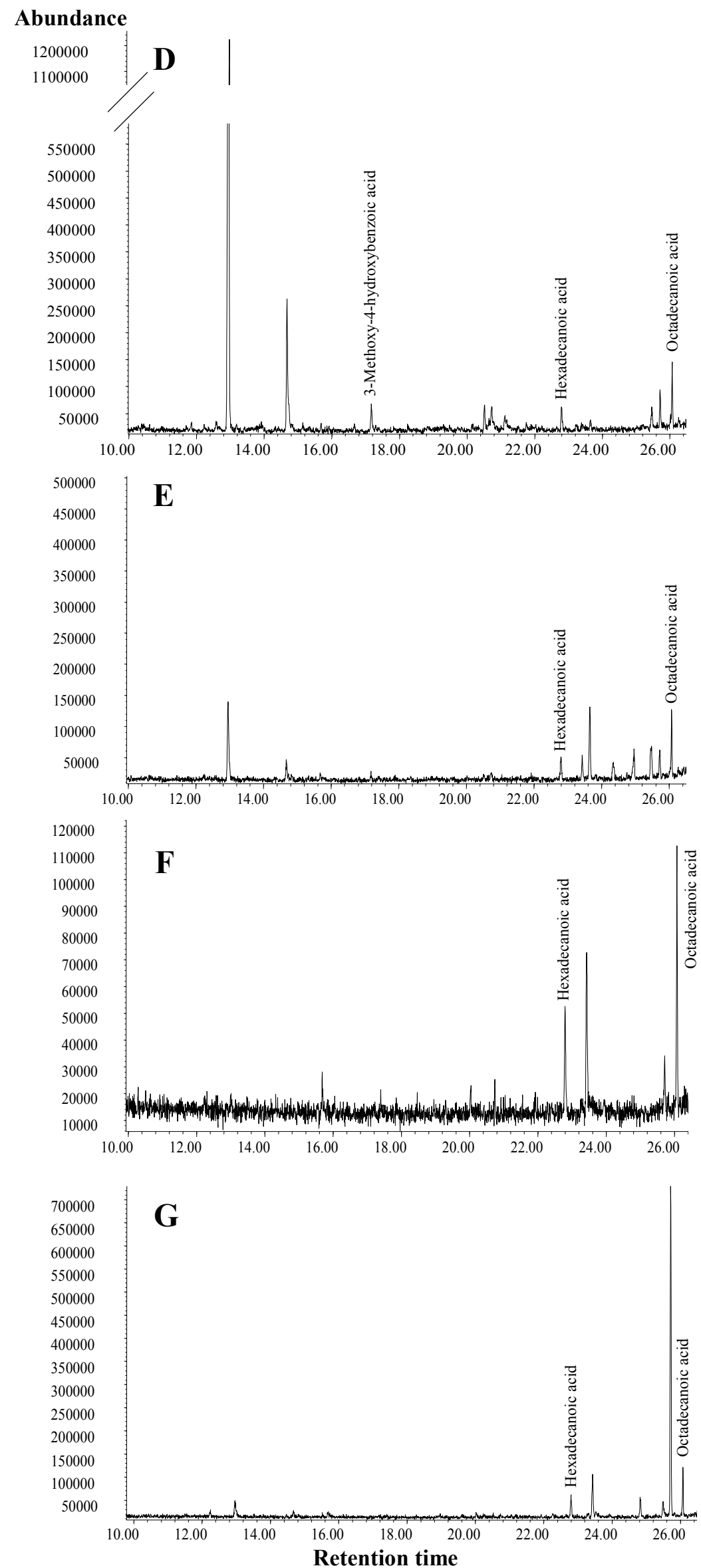

Fig. S4 (continued) 
IV. Contrasting ecological functions of Abies grandis wood extractives on protection against white-rot fungi and induction of laccase activity

Table S2 Chemical structures of phenolic compounds identified in wood extractives of A. grandis

\begin{tabular}{ccc}
\hline Compounds & Chemical structure \\
\hline 2-Methoxyphenol (guaiacol) &
\end{tabular}

4- Hydroxybenzaldehyde

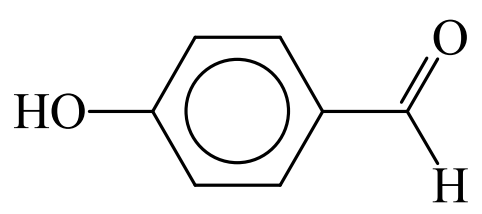

1,4-Dihydroxybenzene (hydroquinone)<smiles>Oc1ccc(O)cc1</smiles>

3,5-Di-tertbut-4-hydroxy-toluene (butylhydroxytoluol)

3-Methoxy-4-hydroxybenzaldehyde (vanillin)<smiles>Cc1cc(C(C)(C)C)c(O)c(C(C)(C)C)c1</smiles><smiles>COc1cc(C=O)ccc1O</smiles>

4-Hydroxybenzoic acid<smiles>O=C(O)c1ccc(O)cc1</smiles> 
IV. Contrasting ecological functions of Abies grandis wood extractives on protection against white-rot fungi and induction of laccase activity

3-Methoxy-4-hydroxybenzoic acid (vanillic acid)

4-Methoxy cinnamic acid<smiles>COc1cc(C(=O)O)ccc1O</smiles><smiles>COc1ccc(/C=C/C(=O)O)cc1</smiles>

4-Hydroxy cinnamic acid ( $p$-coumaric acid)<smiles>O=C(O)/C=C/c1ccc(O)cc1</smiles>

3-Hydroxy-4-methoxy cinnamic acid<smiles>COc1ccc(/C=C/C(=O)O)cc1O</smiles>

4-Hydroxy-3-methoxy cinnamic acid (ferulic acid)<smiles>COc1cc(/C=C/C(=O)O)ccc1O</smiles> 
IV. Contrasting ecological functions of Abies grandis wood extractives on protection against white-rot fungi and induction of laccase activity

\section{IV.7.3 Inhibition of fungal growth by phenolic compounds}

Results presented in Fig. 14 indicated that 1,4-dihydroxybenzene (hydroquinone) had a negative effect on the growth of the white-rot fungi $P$. ostreatus $8 \mathrm{~F} 6$ and $T$. versicolor Strain 6. In Fig. S5 it is shown that the related compound $p$-benzochinone has similar negative effects on the growth of the fungi. Likewise, there was no laccase production in P. ostreatus 8F6 cultures because of lack of fungal growth (not shown) and only marginal laccase production by $T$. versicolor Strain 6 ( $\mathrm{U}^{-1}$ at day 20 of incubation).

\section{$\begin{array}{ll}\text { P. ostreatus Tersicolor } & \text { T. ver }\end{array}$}

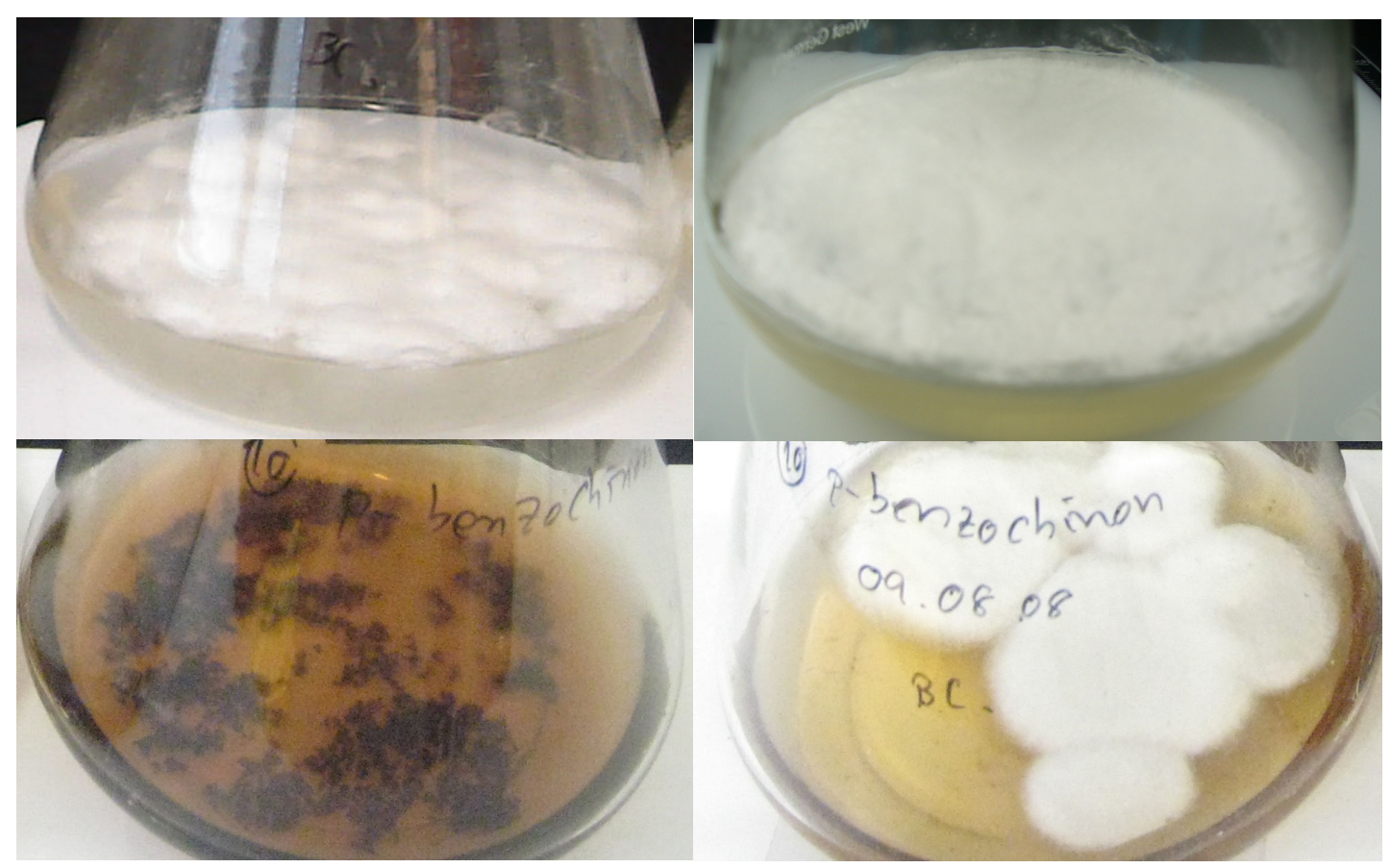

Fig. S5 One week old cultures of $P$. ostreatus $8 \mathrm{~F} 6$ and T. versicolor Strain 6 incubated at $25^{\circ} \mathrm{C}$ in $100 \mathrm{ml}$ liquid $\mathrm{BSM}$ medium (upper row) and in $100 \mathrm{ml}$ liquid BSM medium supplemented with $1 \mathrm{mM} p$-benzochinone (lower row)

\section{IV.7.4 Component composition of $A$. grandis wood}

Fine A. grandis wood powder was prepared from wood chips (obtained in hand-cuts with a razor blade from blocks of original wood, from blocks of water-extracted wood, and of water-acetone-extracted wood) by ball mill grinding in order to measure the content of specific components in the respective wood samples. Analysis of the $\mathrm{C}$ and 
IV. Contrasting ecological functions of Abies grandis wood extractives on protection against white-rot fungi and induction of laccase activity

$\mathrm{N}$ content of the samples was carried out with a CHNS-O element analyzer (Carlo Erba Instruments, EA 1108-Element Analyzer, Milan, Italy) according to the instructions of the supplier. Mineral components in original wood and in water-extracted wood were measured at the Department of Soil Science of Temperature and Boreal Ecosystem (Faculty of Forest Sciences and Forest Ecology, Göttingen, Germany) using an Inductively Coupled Plasma-Atomic Emission Spectrometer (ICP-AES; Spectro Analytical Instruments, Kleve, Germany) according to the instructions of the supplier.

Fig. S6 shows the results of the $\mathrm{C}$ and the $\mathrm{N}$ content of the original wood, of the water-extracted wood and of the water-acetone-extracted wood, respectively. The $\mathrm{C}$ ratio in the different wood samples ranged between 47 to $48 \%$ in total dry weight. Slight differences in the $\mathrm{C}$ content after water extraction and after water-acetone extraction was insignificant due to the high overall $\mathrm{C}$ content in the wood (Fig. S6). In contrast, the slightly higher $\mathrm{N}$ content in extracted wood $(0.112 \%$ in case of water extraction and $0.107 \%$ in case of water-acetone extraction, respectively) compared to the original wood with $0.085 \%$ appears by statistical analysis to possibly significant, obviously due to the fact that the $\mathrm{N}$ content of the wood is generally very low. From the obtained values, the $\mathrm{C}$ to $\mathrm{N}$ ratio within the three wood samples were calculated 562:1, 430:1, and 450:1 for original wood, water-extracted wood and water-acetone-extracted wood, respectively.

The content of selected mineral compounds in the original wood is shown in Fig. S7 in comparison to water-extracted wood. The main minerals were Ca (ca $650 \mu \mathrm{g}$ per g original wood), K (ca $550 \mu \mathrm{g}$ per g original wood) and Zn (ca $470 \mu \mathrm{g}$ per g original wood), whilst $\mathrm{Mg}$ (ca $70 \mu \mathrm{g}$ per g original wood) and $\mathrm{Mn}$ (ca $70 \mu \mathrm{g}$ per g original wood) were somewhat lower. According to the data, parts of the $\mathrm{Ca}, \mathrm{Mg}, \mathrm{Mn}$, $\mathrm{K}$ and $\mathrm{Zn}$ were removed from the original wood by water extraction, whereas $\mathrm{Na}, \mathrm{S}, \mathrm{P}$ and $\mathrm{Al}$ showed no differences in the content in water-extracted wood in comparison to the original wood. The results are in trend with reports by Fengel and Wegener (1983) that the mineral components of woods from the temperate zone are predominantly the elements $\mathrm{K}, \mathrm{Ca}$ and also $\mathrm{Mg}$. 
IV. Contrasting ecological functions of Abies grandis wood extractives on protection against white-rot fungi and induction of laccase activity
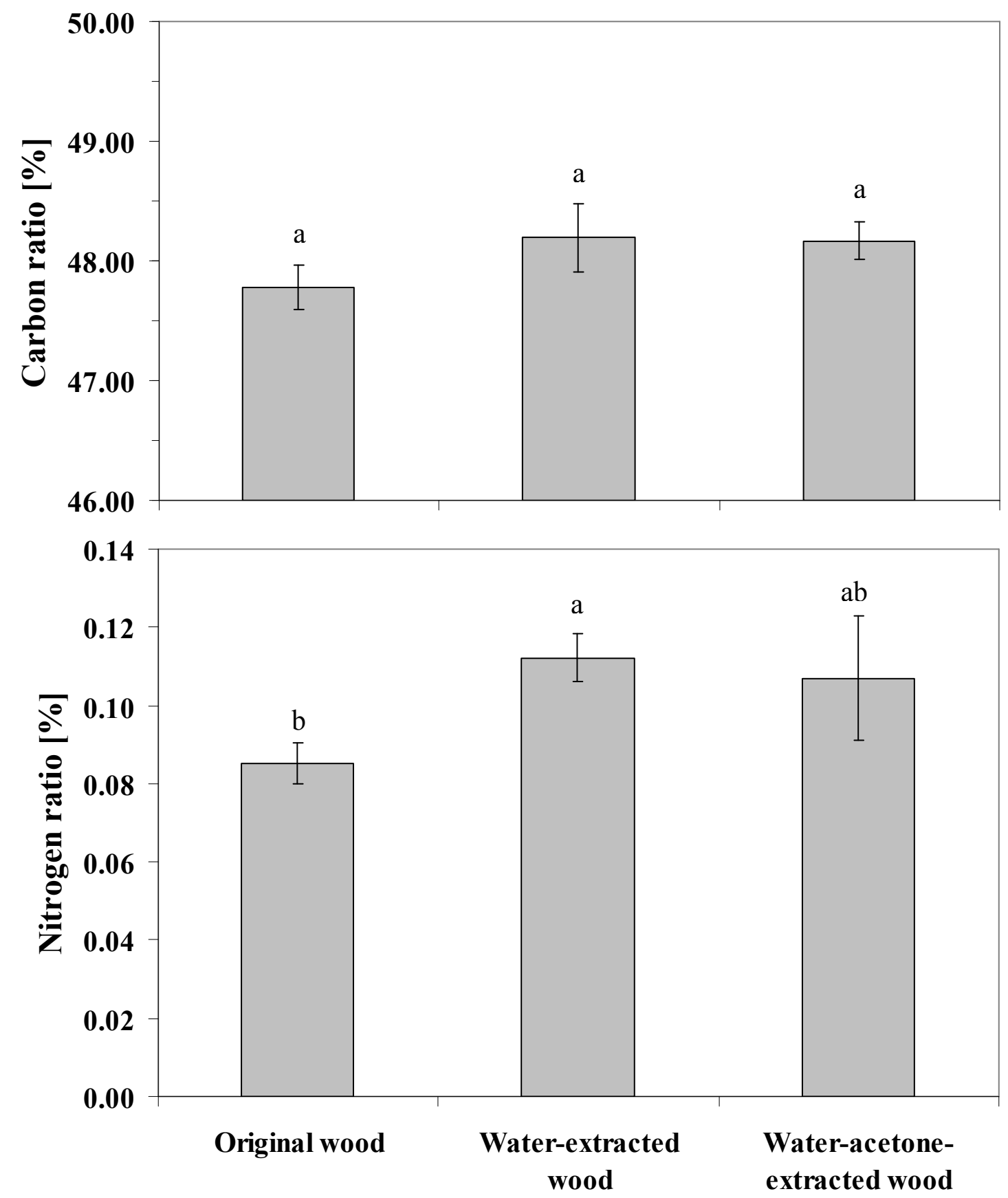

Fig. S6 Carbon and nitrogen ratios of original $A$. grandis wood and of $A$. grandis wood after water extraction and after water-acetone extraction. The different superscripts on the chart indicate values that differ significantly $(p<0.05)$ to each other according to the tests of the different $A$. grandis wood samples. Values are averages from three technical repeats of measurements plus standard deviations 
IV. Contrasting ecological functions of Abies grandis wood extractives on protection against white-rot fungi and induction of laccase activity

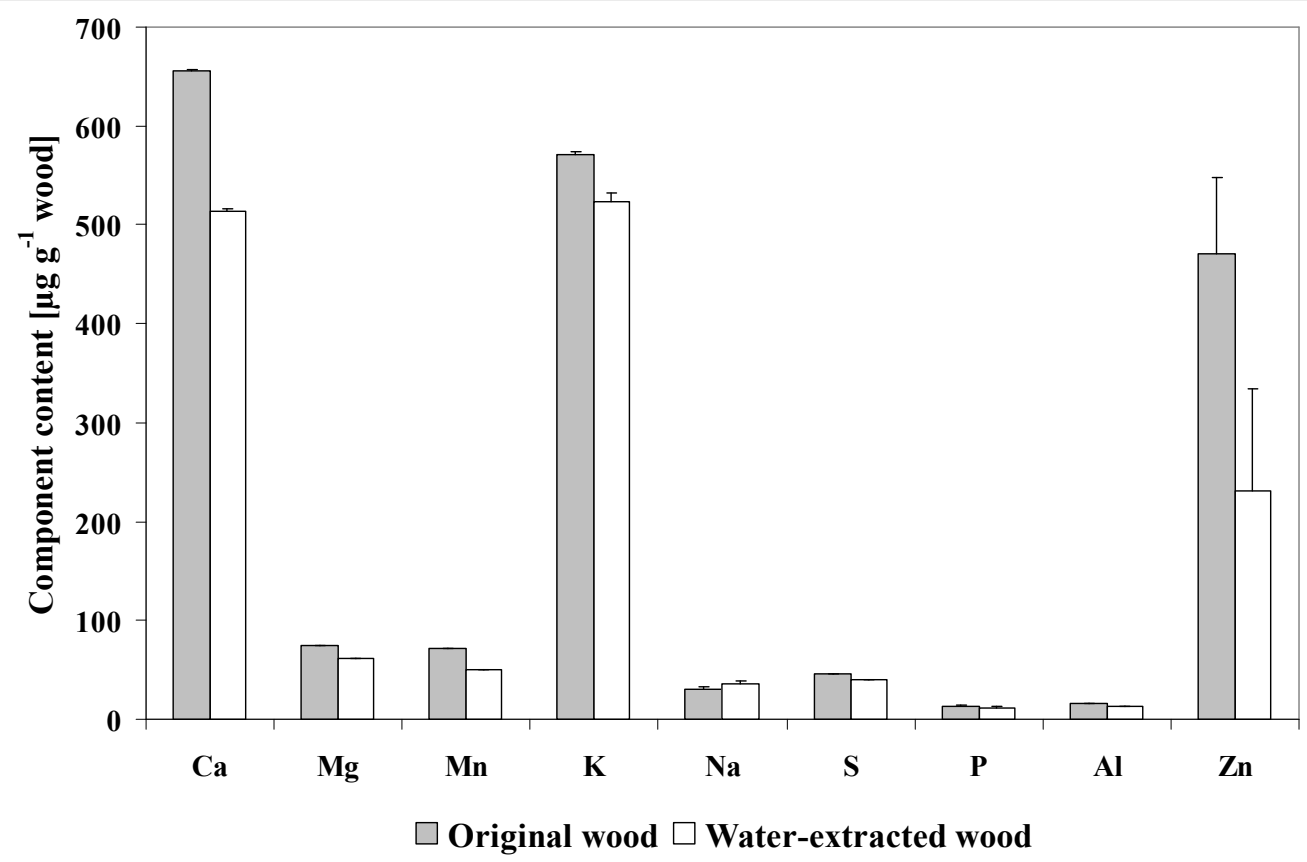

Fig. S7 Mineral component composition in A. grandis wood prior and after water extraction. Values are averages from three technical repeats of measurements plus standard deviations

\section{IV.7.5 Influence of nutrients on laccase production}

Fungi as wood colonizers are an integral part of ecosystems $\mathrm{C}$ and $\mathrm{N}$ cycling (Knops et al. 2002). In wood, $\mathrm{C}$ and $\mathrm{N}$ nutrients consist in parenchyma cells, resin ducts and other woody tissue of which wood decay fungi may make use off. White-rot basidiomycetes in special are also very effective in degradation of wood extractives (Taylor et al. 2006; Venalainen et al. 2006). In Chapter IV.7.4, it is shown that A. grandis wood has a $\mathrm{C}$ to $\mathrm{N}$ ratio of about 560:1 (about 450:1 in wood after water extraction and after water-acetone extraction) and that the nitrogen-poor wood has generally limited available nutrients. In particular, nitrogen may influence the yield of laccase production by white-rot fungi such as T. versicolor (Lee et al. 2006). The aims of this experiment targeted at elucidating the role of the nutrients $(\mathrm{C}$ and $\mathrm{N})$ for fungi to grow in BSM culture media with and without added $A$. grandis wood particles.

Upon inoculation with $4 \mathrm{ml}$ of macerated fungal mycelium, $T$. versicolor Strain 6 was grown for 9 days at $25^{\circ} \mathrm{C}$ in stationary cultures with $100 \mathrm{ml} \mathrm{BSM} \mathrm{medium}$ [basidiomycetes medium; per liter: $5 \mathrm{~g}$ glucose, $0.65 \mathrm{~g}$ L-asparagine, $1 \mathrm{~g} \mathrm{KH}_{2} \mathrm{PO}_{4}, 0.5 \mathrm{~g}$ $\mathrm{MgSO}_{4} \times \mathrm{H}_{2} \mathrm{O}, 0.5 \mathrm{~g} \mathrm{KCL}, 0.5 \mathrm{~g}$ yeast extract, $50 \mathrm{ml}$ stock solution I (0.2 g/1 FeSO 4 x $\left.7 \mathrm{H}_{2} \mathrm{O}\right), 50 \mathrm{ml}$ stock solution II $\left(0.16 \mathrm{~g} / \mathrm{l} \mathrm{Mn}\left(\mathrm{CH}_{3} \mathrm{COO}\right)_{2} \times \mathrm{H}_{2} \mathrm{O}, 0.04 \mathrm{~g} / 1 \mathrm{Zn}\left(\mathrm{NO}_{3}\right)_{2} \mathrm{x}\right.$ $\left.\left.4 \mathrm{H}_{2} \mathrm{O}, 1 \mathrm{~g} / \mathrm{l} \mathrm{Ca}\left(\mathrm{NO}_{3}\right)_{2} \times 4 \mathrm{H}_{2} \mathrm{O}, 0.06 \mathrm{~g} / 1 \mathrm{CuSO}_{4} \times 5 \mathrm{H}_{2} \mathrm{O}\right)\right]$ and laccase activity was determined at days 5, 7 and 9 of cultivation. After 9 days of cultivation, the culture 
IV. Contrasting ecological functions of Abies grandis wood extractives on protection against white-rot fungi and induction of laccase activity

supernatants were removed from the mycelia by sucking off the liquid with sterilized $25 \mathrm{ml}$ glass pipettes mounted to an electronic pipetting aid (Gilson, Pipetting Aid, Mettmenstetten, Switzerland). The fungal mycelia in the flasks were washed for 3 times with each $50 \mathrm{ml} 0.9 \% \mathrm{NaCl}$, in order to remove all resident nutrient sources. For each washing step, the cultures were for $15 \mathrm{~min}$ shaken on a shaker at $60 \mathrm{rpm}$. Then, either $100 \mathrm{ml}$ of liquid autoclaved BSM or $100 \mathrm{ml}$ of liquid autoclaved modified BSM (low $\mathrm{C}, \mathrm{N}$ BSM, i.e. without the compounds glucose, asparagine, $\mathrm{Mn}\left(\mathrm{CH}_{3} \mathrm{COO}\right)_{2} \mathrm{X} 4 \mathrm{H}_{2} \mathrm{O}$, $\mathrm{Zn}\left(\mathrm{NO}_{3}\right) \times 4 \mathrm{H}_{2} \mathrm{O}$, and $\mathrm{Ca}\left(\mathrm{NO}_{3}\right)_{2} \mathrm{x} 4 \mathrm{H}_{2} \mathrm{O}$, leaving just yeast extract as $\mathrm{C}$ and $\mathrm{N}$ source) either plus $0.5 \mathrm{~g}$ A. grandis wood particles or without $A$. grandis wood particles were added into flasks onto the mycelia and cultures were further stationary incubated at $25^{\circ} \mathrm{C}$ for 13 days. Laccase activity was measured every two days. After 13 days, total biomasses of harvested fungal mycelium were calculated from the weight of fresh mass and of dry mass. Fungal mycelium was harvested from culture supernatants by vacuum filtration with a Büchner funnel and filter paper (glass fiber papers, Macherey-Nagel GmbH and Co. KG, Düren, Germany). Note that by surface growth, the mycelium of cultures with added wood particles could easily be separated from the latter that accumulated on the bottom of the flasks. Mycelium was dried at $103 \pm 2^{\circ} \mathrm{C}$ for $24 \mathrm{hrs}$ and weighed (Fig. S8).

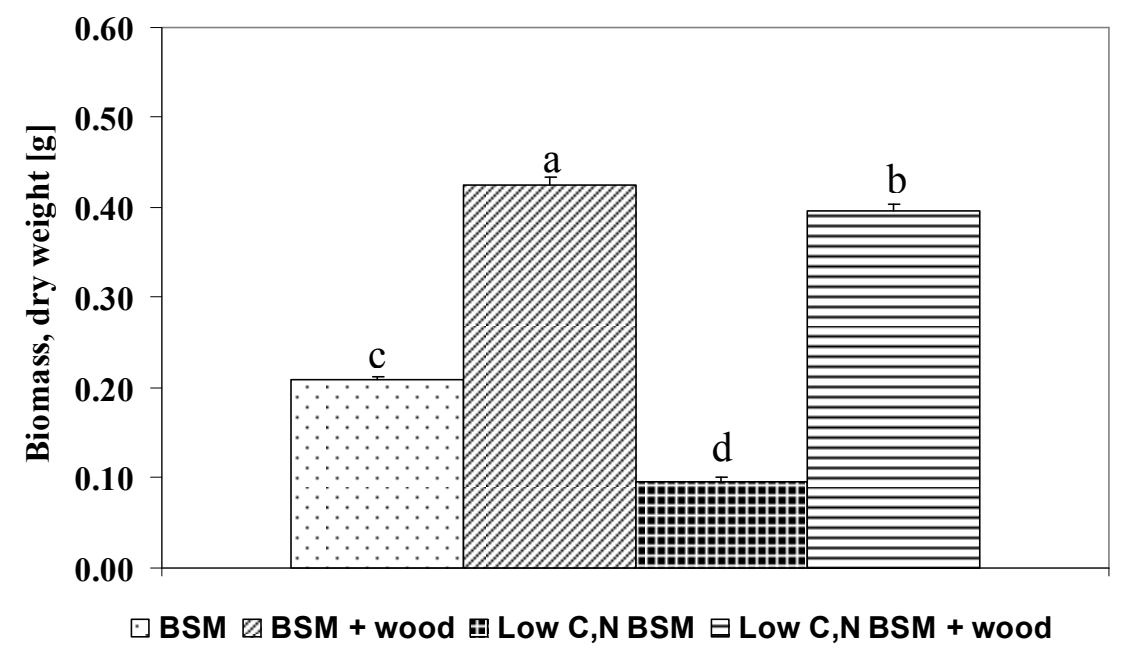

Fig. S8 Biomass of $T$. versicolor accumulating upon first growth for 9 days at $25^{\circ} \mathrm{C}$ in standing $100 \mathrm{ml}$ cultures with BSM liquid medium and subsequent growth for 13 days at $25^{\circ} \mathrm{C}$ in standing $100 \mathrm{ml}$ cultures with BSM liquid medium or in standing $100 \mathrm{ml}$ cultures with low $\mathrm{C}, \mathrm{N}$ BSM liquid medium, with or without added $0.5 \mathrm{~g} \mathrm{~A}$. grandis wood particles. The different superscripts in the chart indicate values that differ significantly $(p<0.05)$ from each other according to analysis of variance (ANOVA). $\mathrm{C}=$ carbon, $\mathrm{N}=$ nitrogen 
IV. Contrasting ecological functions of Abies grandis wood extractives on protection against white-rot fungi and induction of laccase activity

Reducing the $\mathrm{C}$ and $\mathrm{N}$ contents and with it the $\mathrm{Mn}, \mathrm{Zn}$ and $\mathrm{Ca}$ contents in the culture medium at day 9 of cultivation negatively affected the total biomass production by $T$. versicolor Strain 6 by a more than two fold reduction (Fig. S9) but only marginally the laccase production over the time (Fig. S10). Highest laccase yields in the standard BSM medium were $140 \pm 11.4 \mathrm{U}^{-1}$ at day 20 of cultivation and in low C,N BSM $109 \pm 10.2 \mathrm{U}^{-1}$ at day 20 of cultivation (Fig. S10), respectively. In contrast, upon replacement of the medium under addition of $A$. grandis wood particles, biomass production was much stimulated, regardless of the composition of the liquid medium. At day 22 of cultivation, the highest biomass of $0.425 \mathrm{~g}$ was measured in BSM liquid medium with added wood particles, compared to $0.209 \mathrm{~g}$ biomass from simple BSM cultures. In case of low C,N BSM liquid medium with added wood particles, the maximum of $0.397 \mathrm{~g}$ biomass was reached at day 22 of cultivation, compared to $0.095 \mathrm{~g}$ biomass from pure low C,N BSM (Fig. S10). Thus, not only the reduction of C and N but also of the minerals $\mathrm{Mn}, \mathrm{Zn}$ and $\mathrm{Ca}$ in the modified low $\mathrm{C}, \mathrm{N}$ BSM medium might have been compensated for by adding of the A. grandis wood (for contents of C, N, Mn, Zn and $\mathrm{Ca}$ in $A$. grandis wood, see Chapter IV.7.4).

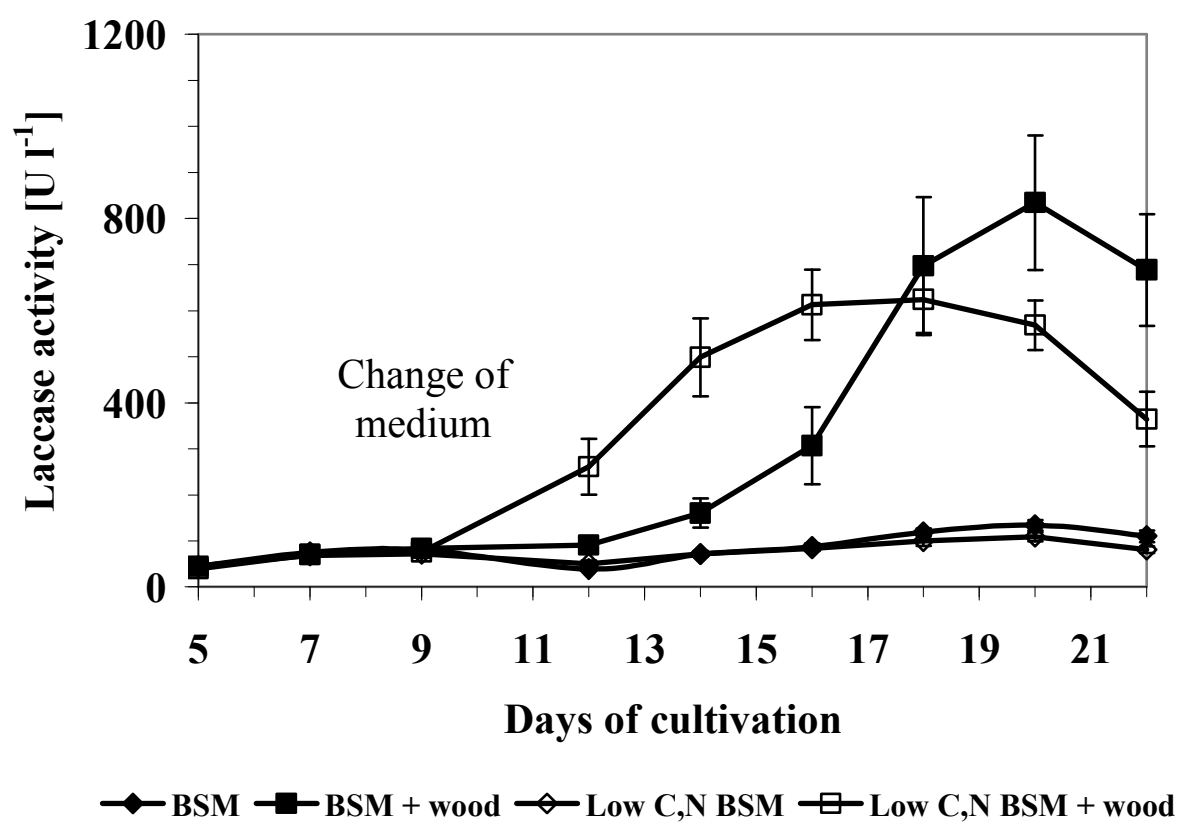

Fig. S9 Laccase production of T. versicolor Strain 6 at $25^{\circ} \mathrm{C}$ incubated first for 9 days in $100 \mathrm{ml} \mathrm{BSM}$ liquid medium and then after change of the medium, either in $100 \mathrm{ml}$ normal BSM liquid medium or in low C,N BSM medium, with or without added $0.5 \mathrm{~g}$ of $A$. grandis wood. Average values and standard deviations were calculated from three parallel cultures each 


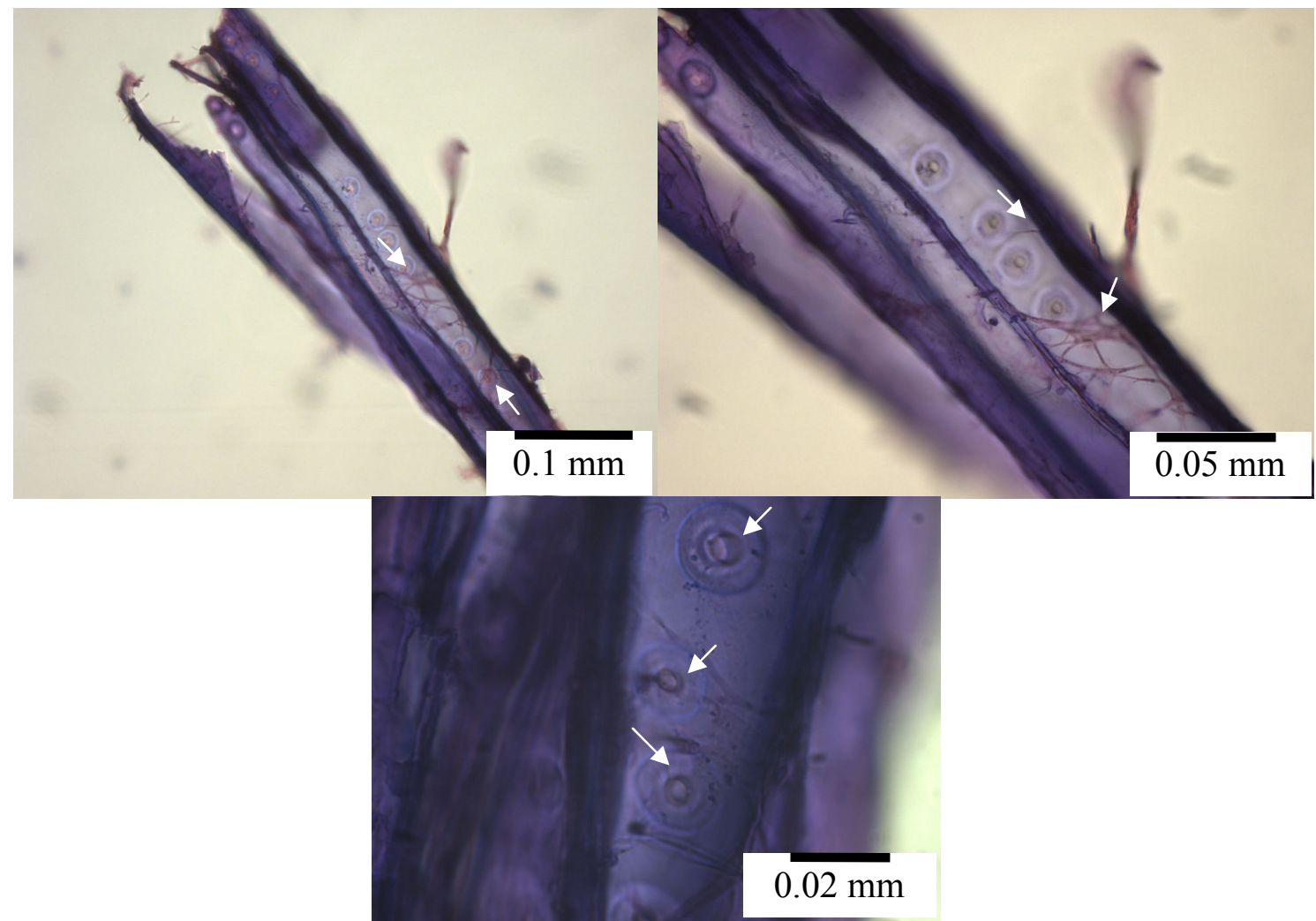

Fig. S10 A. grandis wood fibers from standing $100 \mathrm{ml}$ liquid BSM cultures at day 22 of total cultivation (day 13 of cultivation after replacing the original BSM medium with $100 \mathrm{ml}$ of fresh BSM medium with added $0.5 \mathrm{~g}$ A. grandis wood) with $T$. versicolor Strain 6 at $25^{\circ} \mathrm{C}$. The fibers were stained with $1 \%$ toluidin blue in order to observe the mycelium colonizing on the fibers surfaces or penetrating into the fiber cells by passing through the pits (arrows)

However, there were differences in cultures to which $A$. grandis wood particles were added. Significant amounts of laccase activities were detected in cultures with low C,N BSM two days earlier than in cultures of normal BSM medium. A peak in laccase production with $624 \pm 72.8 \mathrm{U} \mathrm{l}^{-1}$ was reached 4 days earlier in the cultures with low $\mathrm{C}, \mathrm{N}$ BSM and added $A$. grandis wood particles, whilst the best laccase activities in normal BSM cultures with added A. grandis wood particles were much higher with $835 \pm 146 \mathrm{U} \mathrm{l}^{-1}$. Likely, in the normal BSM medium the fungus could still make use of the easily to access $\mathrm{C}$ and $\mathrm{N}$ sources in the liquid, differently, from the cultures with low C,N BSM. Under the latter conditions, the fungus probably needed to quickly access the nutrients offered by the $A$. grandis wood. That $A$. grandis wood has been converted into fungal biomass can be concluded from the doubling of fungal biomass in the normal BSM with added $A$. grandis wood compared to just BSM medium and from the four-fold increase in biomass in the low C,N BSM cultures with added wood as 
IV. Contrasting ecological functions of Abies grandis wood extractives on protection against white-rot fungi and induction of laccase activity

compared to cultures with just low C,N BSM medium (Fig. S8). That the fungus penetrates into the wood, possibly for accessing the residing nutrients resources, can be seen in Fig. S10, where A. grandis wood fibers from the BSM cultures with added A. grandis wood particles are shown.

\section{IV.7.6 References}

Fengel D, Wegener G (1983) Wood. Chemistry, ultrastructure, reactions. Walter de Gruyter, Berlin

Knops JMH, Tilman D (2000) Dynamics of soil nitrogen and carbon accumulation for 61 years after agricultural abandonment. Ecology 81(1):88-98

Lee Y, Park C, Lee B, Han EJ, Kim TH, Lee J, Kim S (2006) Effect of nutrients on the production of extracellular enzymes for decolorization of reactive blue 19 and reactive black 5. Journal of Microbiology and Biotechnology 16(2):226-231

Taylor AM, Gartner BL, Morrell JJ, Tsunoda K (2006) Effects of heartwood extractive fractions of Thuja plicata and Chamaecyparis nootkatensis on wood degradation by termites or fungi. Journal of Wood Science 52(2):147-153

Venalainen M, Harju AM, Terziev N, Laakso T, Saranpaa P (2006) Decay resistance, extractive content, and water sorption capacity of Siberian larch (Larix sibirica Lebed.) heartwood timber. Holzforschung 60(1):99-103 

CHAPTER V

Application of Abies grandis wood for technical
use in biofuel production 



\section{V.1 Abstract}

This study focused on effects chemical and physico-chemical pretreatments as well as biological pretreatments of Abies grandis wood on enzymatic hydrolysis of cellulose into glucose in view of potential applications in biofuel production. Wood particles were treated with various liquid acids [phosphoric acid, formic acid, acetic acid or a combination of phosphoric acid and ionic liquid (Aliquat)], incubated in a hot water bath at $50^{\circ} \mathrm{C}$ or they were submitted to microwave irradiation. In biological pretreatment, $A$. grandis wood was incubated for 19 weeks with either the brown-rot basidiomycete Coniophora puteana Strain 57 or with one of the white-rot species Pleurotus ostreatus 8F6 and Trametes versicolor Strain 6, respectively. Upon a pretreatment, left materials (cellulosic pellets) were hydrolyzed with different enzymes (cellulase, xylanase, $\beta$-glucosidase and/or laccase) in order to achieve a conversion of cellulose to glucose. The highest glucose yields of up to $100 \%$ of converted cellulose were reached by using $1 \mathrm{~g}$ of wood particles in $8 \mathrm{ml}$ of $80 \%$ phosphoric acid irradiated in a microwave for 20 second and applying a combination of $4 \mathrm{U} \mathrm{ml}^{-1}$ cellulase, $4 \mathrm{U} \mathrm{ml}^{-1}$ xylanase and $4 \mathrm{U} \mathrm{ml}^{-1}$ laccase in $5 \mathrm{ml}$ of $50 \mathrm{mM}$ sodium acetate buffer, $\mathrm{pH} 5.0$ for cellulose hydrolysis for $24 \mathrm{hrs}$. With only chemical pretreatment, application of $8 \mathrm{ml}$ $85 \%$ phosphoric acid for $1 \mathrm{hr}$ at $50^{\circ} \mathrm{C}$ resulted in best conversion of glucose from cellulosic pellets left after the pretreatment. Longer times of incubation and higher concentrations and amounts of phosphoric acid lead to mass loss of up to $50 \%$ and to relative increase of the cellulose content up to $100 \%$. However, such enrichment in cellulose gave not necessarily higher glucose yields. Changes in structure of the cellulose during a pretreatment produce of the A. grandis wood and/or production of compounds with inhibitory effects on the hydrolyzing enzymes can be reasons for a failure of glucose production. Evidence for the latter was given in studies where glucose yields from cellulosic pellets obtained from physico-chemical pretreatments were dramatically increased when laccase was added to the samples prepared for cellulose hydrolysis: generally, the combination of $4 \mathrm{U} \mathrm{ml}^{-1}$ cellulase, $4 \mathrm{U} \mathrm{ml}^{-1}$ xylanase and $4 \mathrm{U} \mathrm{ml}^{-1}$ laccase was more effective in increasing glucose yields than $4 \mathrm{U} \mathrm{ml}^{-1}$ cellulase and $4 \mathrm{U} \mathrm{ml}^{-1}$ xylanase alone. Likely, laccase has a potential to decrease inhibitory effects of phenolic compounds present wood extractives on enzymatic hydrolysis. Evidence for this was further given by experiments where wood extractives or specific phenolic compounds as found in wood extractives were added to enzymatic reactions. Water extractives, and $p$-coumaric acid, vanillic acid and ferulic acid in concentration- 
dependent manner all inhibited the actions of cellulase on carboxymethylcellulose (CMC). Addition of laccases in most instances could partially or fully reverse the inhibitory effects but in the case of ferulic acid. This compound in presence of laccase or its products obtained by laccase-mediated oxidation partially blocks cellulase activity, possibly by chemical interactions (dimerization of cysteines, cross-linking of tyrosines) on the cysteine and tyrosines-rich cellulose-binding-motifs (CBMs) present in distinct cellulases at the outmost $\mathrm{C}$-terminal ends of the enzymes.

In biological pretreatments, the effects of fungal actions were determined as mass losses, changes in content of extractives and in released sugar in enzymatic hydrolysis of material left after 19 weeks of incubation with a fungus according to EN 113 (1996). The highest glucose yields from material from a biological pretreatment were obtained from wood decayed by $T$. versicolor Strain $6, P$. ostreatus $8 \mathrm{~F} 6$ and C. puteana Strain 57 , by reaching about $50 \%, 47 \%$ and $40 \%$ glucose yields, from $100 \mathrm{mg}$ of left material, respectively. Concerning the yield as compared to the original amount of wood prior to fungal decay, application of $8 \mathrm{ml} 85 \%$ phosphoric acid was most successful with conversion of $42.8 \%$ of the total original wood mass into glucose. This corresponds to $92.4 \%$ of the cellulose that was originally present in the $A$. grandis wood. Similar high conversion rates or even better rates were obtained upon chemical pretreatment of $A$. grandis wood particles with $8 \mathrm{ml} 85 \%$ phosphoric acid at $50^{\circ} \mathrm{C}$ in a hot water bath for $1 \mathrm{hr}$, whereas in physico-chemical pretreatment such best yields were obtained with $8 \mathrm{ml} 80 \%$ phosphoric acid in $20 \mathrm{sec}$ microwave irradiation. Whilst the biological pretreatment with $P$. ostreatus 8F6 gives excellent cellulose yields and is highly environmentally friendly, by the long incubation periods it is economically better to turn to a chemical or to a physico-chemical pretreatment. From the study presented here, most advisable appears to be in economical and ecological terms the use of physico-chemical pretreatment with optimal chemical (low concentration of phosphoric acid) and low energy supply (short time microwave irradiation) and a combination of high performance enzymes (laccase and cellulase) in hydrolysis. Future studies should direct on further optimization of the procedure and scaling it up to amounts and volumes interesting for technical applications and industries.

Key words: biofuels, chemical pretreatment, physico-chemical pretreatment, biological pretreatment, microwave irradiation, liquid acid, Trametes versicolor, Pleurotus ostreatus, Coniophora puteana, rate of production of glucose, kinetic curve of production of glucose, influence of wood extractive, influence of phenolic compound 


\section{V.2 Introduction}

Bioethanol has been promoted to replace fossil fuel and becomes more and more interesting for this target because of the large growing demand for the world energy consumption, limited resources on petroleum and gas, and global warming also linked to consumption of fossil fuels. Crude-oil resources are rapidly decreasing by using fossil-based energy carriers, thereby increasing also the speed of climate change. Political attention and commitment therefore turned currently towards improved utilization of renewable energy resources. Presently, ethanol is the most important renewable fuel in terms of volume and market value. However, the first generation of biofuels was produced from food and fodder, which competes with human and animal consumption. To overcome this point of disadvantage for the first generation biofuels, the second generation of biofuels was generated from non-food and non-fodder materials. Plant lignocellulosic biomass is the most attractive choice in biofuel production. Biomass represents the most abundant of all bio-materials for renewable energy resources, including for biofuel production and value added chemicals. Wood biomass from forest land and fast growing tree plantations could be supplier of large amounts of raw materials, supporting biofuel production for many regions of the world. Woody biomass is most attractive to use as a feedstock in production of bio-based materials because of its many advantages in terms of production, harvesting, storage, and transportation as well as overall energy content in comparison to herbaceous materials (Li et al. 2008; Majcherczyk and Hüttermann 2007; Stöcker et al. 2008; Taherzadeh and Karimi 2008; Zhu and Pan 2010; Zhu et al. 2010a; Zhu et al. 2010b).

The biofuel production from lignocellulosic materials concerns to convert cellulose to glucose. Cellulose in the plant materials generally is covered by lignin and hemicellulose as protectors for enzymatic hydrolysis (Mtui 2009; Olofsson et al. 2008). To remove lignin and hemicellulose, and to make cellulose easily accessible for enzymatic degradation, specific pretreatments are used in biofuel production. The optimum wood pretreatment considers effectiveness and economical and environmental aspects such as: cost reducing of raw material and equipment (such as pretreatment reactor), cost reducing of feedstock size reduction, optimizing of energy demand through all steps of the procedure till the end product is there, best end-production of cellulose for enzymatic hydrolysis, avoiding of destructing of cellulose and hemicellulose, avoiding inhibitory factors of enzymatic hydrolysis or in fermentation 
with microorganisms fermentation, avoidance of use of chemicals in general, and producing waste residues as little as possible or, if necessary, use of cheapest and best environmentally friendly chemicals. The optimum, most effective pretreatments should lead to high ethanol production efficiency and to lowest costs over the whole production chain. However, currently processes to produce only ethanol from plant biomass are of very poor economics. Approaching of value added bio-based chemicals, for example butanol, are one type of possible measures to achieve better values of the economics scale (Mtui 2009; Briens et al. 2008; Olofsson et al. 2008; Sainz 2009; Sun and Cheng 2002; Taherzadeh and Karimi 2008). Others may address the way of biomass pretreatments.

Generally, wood pretreatments might be chemically, biologically and combinations of chemical/physical measures referred to as physico-chemical (Table 1). Any of the pretreatment processes requires at first significant wood size reduction, e.g. by first mechanical chopping and then by milling the wood chips or wood particles. Costs of such physical treatments can significantly be reduced with applying a chemical treatment e.g. with hot water, acids or SPORL (sulfite pretreatment to overcome recalcitrance of lignocellulose) prior to milling. Milling wood under such conditions is referred to as post-chemical pretreatment and such post-chemical pretreatment may reduce the energy cost of milling up to $78 \%$, in dependence for example of the $\mathrm{pH}$ at which chemical pretreatment (hot water, acid or SPORL) occurred. Savings in energy consumption as $>100-800 \mathrm{Wh} \mathrm{kg}^{-1}$ can be achieved (Zhu et al. 2010a).

Enzymatic hydrolysis of cellulose to glucose is carried out by cellulase enzymes that are specialized in breaking up the $\beta-1-4$ glycosidic bonds of glucans. Cellulase cleaving the $\beta-1-4$ glycosidic bonds and $\beta$-glucosidase as one enzyme type in a subcategory of cellulases that cut glucose chains into its monomers can work in combination for cellulose digestion, thereby reducing the degree of polymerization of the substrates, shorten the glucan molecules and split the disaccharide cellobiose into two units of glucose (Dadi et al. 2007; Jørgensen et al. 2008; Mtui 2009; Olofsson et al. 2008). Enzymes attacking hemicelluloses can also support cellulose digestion. Xylanases for example have a positive effect on cellulose digestion which is generally attributed to breaking the existing links of xylans between cellulose and lignin (Valls and Roncero 2009). Palonen and Viikari (2004) have first reported that use of laccase on steam-pretreated softwood could improve the subsequent hydrolysis process with cellulases. Moreover, laccase-mediator treatments in combination with commercial 
cellulase further increased the sugar yields in the enzymatic hydrolysis. Combinations of enzymatic hydrolysis were generally tested in order to approach higher sugar yields from the hydrolysis process (Chapter I).

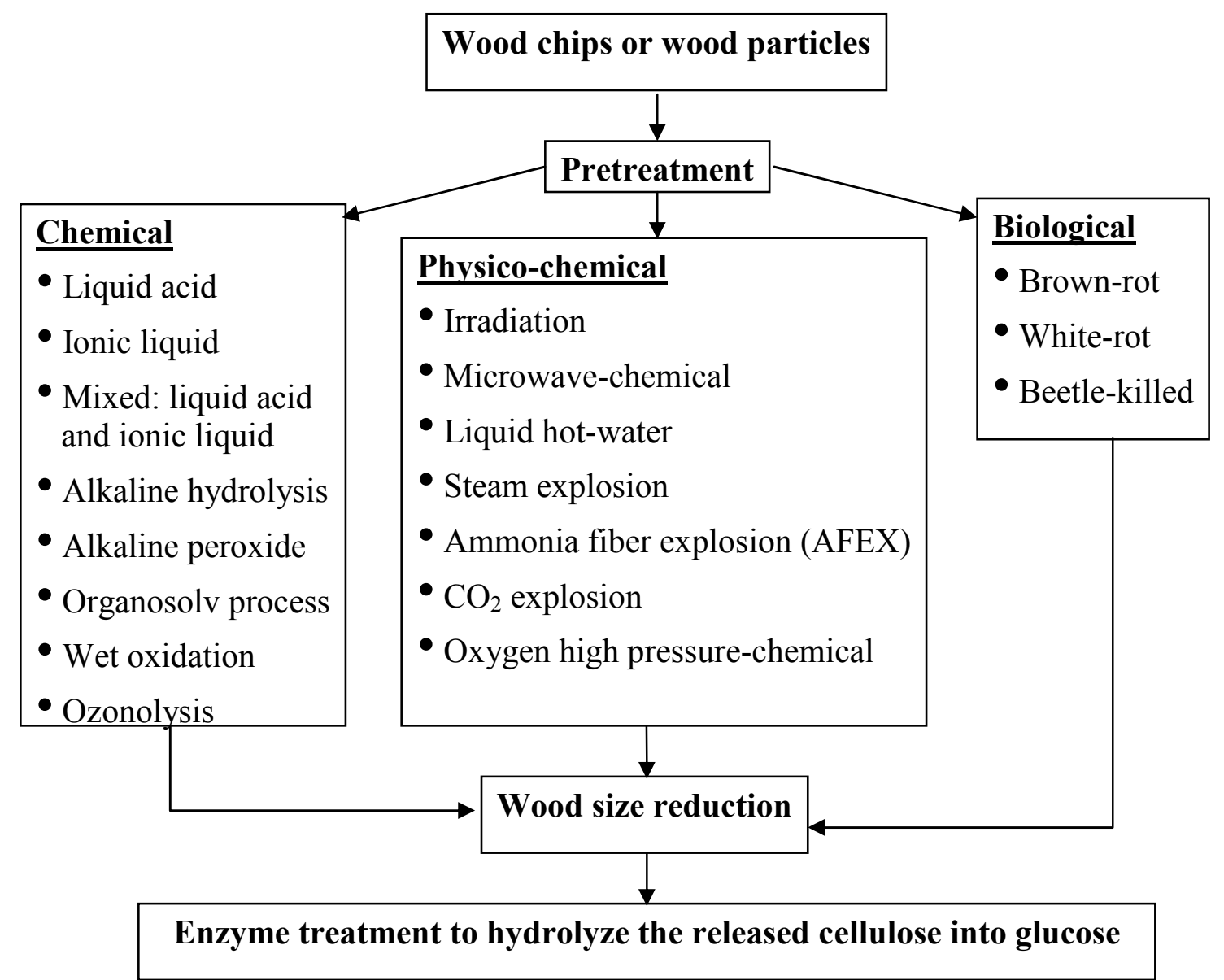

Fig. 1 Overview of possible types of wood pretreatments after chopping the materials for preparing the biomass for enzymatic hydrolysis for producing fermentable sugars. The variety of pretreatment methods will be followed by post-pretreatment sizereduction usually by milling for the next step of enzymatic hydrolysis of released cellulose into glucose (adapted from Zhu et al. 2010a)

However, as indicated already above the cumulative high cost of biofuel production is based on three main sector: commercial pretreatment, enzymatic hydrolysis and the final fermentation process. Generally, the costs of the large amount of enzymes required to access cellulose and hemicellulose in order to obtain sugars to be subsequently transformed to fermentable ethanol are evidently a major point in the 
commercial success of such biofuel production. It is of eminent economic interest to identify not only new, more active enzymes, but also to optimize of the matching between enzymes and material pretreatments, and to increase the knowledge on inhibitory effects in the material of enzymatic hydrolysis in order in best possible way rising up the full performance of the enzymatic cellulose hydrolysis (Mtui 2009).

The research presented here addresses the problem of making the so far little studied softwood better accessible to efficient cellulose hydrolysis. Softwoods have generally not yet been as thoroughly investigated in their potential for biofuel production. Abies grandis is a fast growing coniferous tree which can easily be cultivated in plantations and thus could offer a great source of wood biomass (Polle et al. 2008; Spellmann et al. 2010). In the previous Chapter IV, it was shown that laccase can attack $A$. grandis wood particles. Here, these observations are transferred to potential applications for future biofuel production with $A$. grandis wood. In this work, not only the release of sugars from $A$. grandis wood upon treatment with laccase in combination with other plant polymer-degrading enzymes is followed up but different types of pretreatments are also considered. Amongst are chemical pretreatments with phosphoric acid and physico-chemical pretreatments with phosphoric acid and microwave incubation as well as biological pretreatments using the white-rot and brown-rot fungi for loosening the wood structure by partial biopolymer degradation. Furthermore, potential inhibitory factors in the wood on final cellulose hydrolysis in presence of laccase are considered.

\section{V.3 Materials and methods}

\section{V.3.1 Wood and straw}

Wood particles (size 60-40 mesh) of A. grandis and Picea abies and wheat straw (size 60-40 mesh) were prepared in cooperation with the work group of Prof. Kharazipour from the Division of Molecular Wood Biology and Technology and Technical Mycology, Büsgen-Institute, Göttingen, Germany, as described in Chapter IV.3.1. In case of wood powder preparation, A. grandis was prepared from chipped wood blocks [30 (longitudinal) x 10 (tangential) x 5 (radial) mm] by using a ball mill (Retsch MM 2000, F. Kurt Retsch GmbH \& Co. KG, Düsseldorf, Germany). Wood chips were grinded with the ball mill at $80 \mathrm{rpm}$ for periods of $3 \mathrm{~min}$ until the wood was fully powdered. After grinding, the wood powder was stored at $-20^{\circ} \mathrm{C}$ until use. In case of 
extractives free wood preparation, wood particles $(10 \mathrm{~g})$ was extracted with a Soxhlet apparatus in $450 \mathrm{ml}$ boiling water for $6 \mathrm{hrs}$, following TAPPI Test Method T $204 \mathrm{om}-88$ (1988) as described in Chapter IV.3.2.

\section{V.3.2 Chemical and physico-chemical pretreatment}

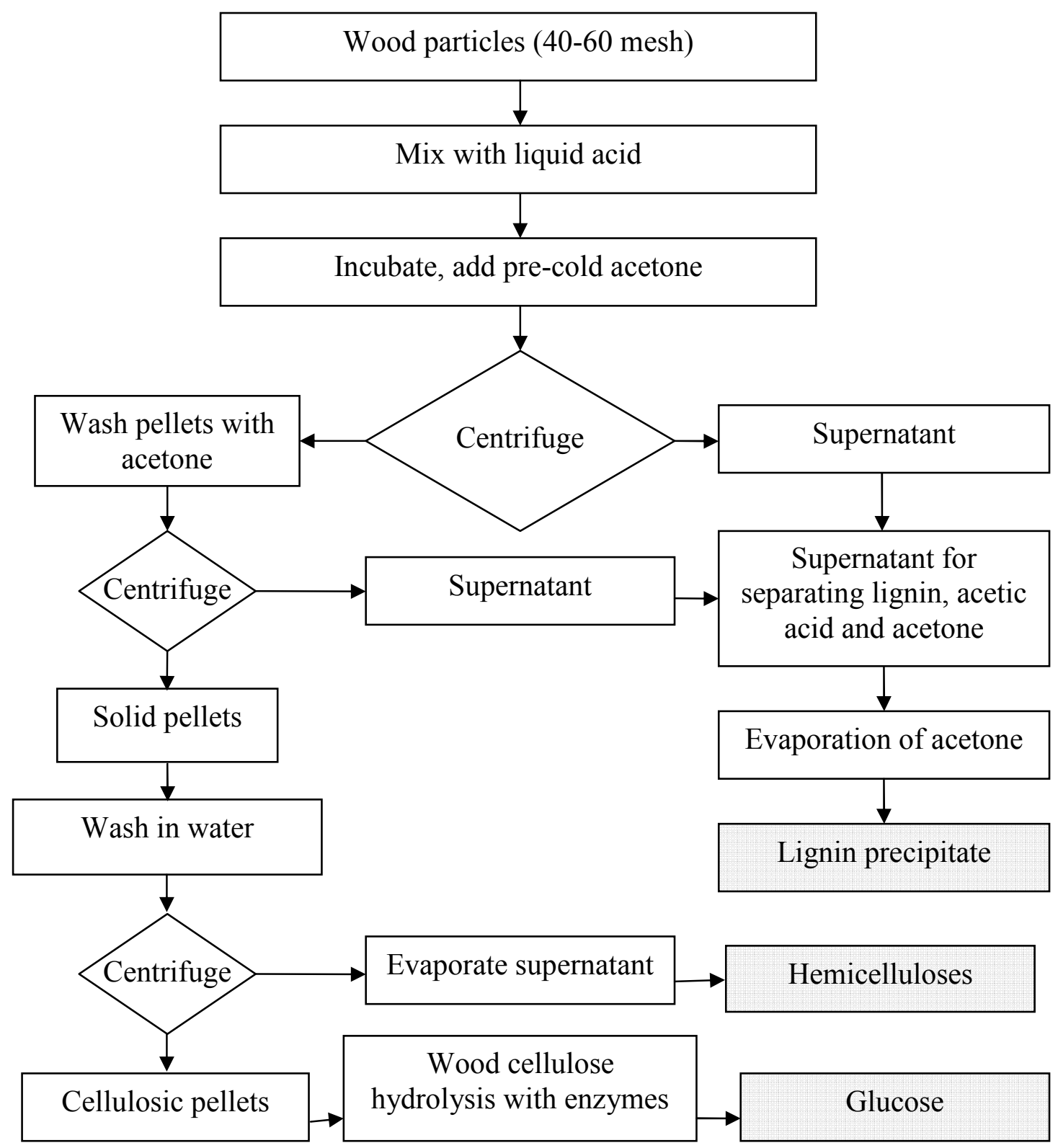

Fig. 2 General flow chart for phosphoric acid pretreatment wood and subsequent steps of enzymatic wood hydrolysis, final products are marked by grey boxes

Chemical and physico-chemical pretreatments followed with modifications (see Fig. 2) the procedure presented by Zhang et al. (2007). In different treatments, 
wood particles or wheat straw particles were treated with individual liquid acids $(8 \mathrm{ml}$ per $1 \mathrm{~g}$ wood in $25 \mathrm{ml}$ glass beaker) for each treatment, such as phosphoric acid, formic acid, acetic acid or in combination of phosphoric acid and ionic liquid (aliquat), then incubated in a hot water bath at $50^{\circ} \mathrm{C}$ or incubated with microwave irradiation (Intellowave microwave, LG Co., Ltd, Germany). For irradiation of $1 \mathrm{~g}$ wood particles in $8 \mathrm{ml}$ phosphoric acid in a $25 \mathrm{ml}$ glass beaker, a rating power $1000 \mathrm{~W}$, an out power $700 \mathrm{~W}$, and working voltage $230 \mathrm{~V}$ and $50 \mathrm{~Hz}$ was used.

\section{V.3.3 Microorganism and culture conditions for biological pretreatment}

All fungal strains used in this study were from the strain collection of the Division of Molecular Wood Biotechnology and Technical Mycology of the Büsgen-Institute, Göttingen. Solid culture media [malt extract-agar (5\% malt $/ 2 \%$ agar)] were inoculated in the middle of the Petri dishes (90 $\mathrm{mm}$ in diameter) with one mycelium-overgrown agar plug per plate of either one of the white-rot fungi Pleurotus ostreatus 8F6 and Trametes versicolor Strain 6 or of the brown-rot strain Coniophora puteana Strain 57. Inoculated plates were sealed with Parafilm (Laboratory Film, Chicago, USA) and grown for seven days at $25^{\circ} \mathrm{C}$ until plates were filled with mycelium for use in the EN 113 (1996) wood duration test as described in Chapter III.3.2. Wood samples incubated for 19 weeks with or without a fungus were used to determine for each treatment the mass loss of the wood blocks and the contents of extractives and they were used for milling for the enzymatic hydrolysis experiments.

\section{V.3.4 Chemicals and enzymes}

All chemicals were reagent grade and purchased from Sigma-Aldrich (St. Louis, MO), unless otherwise noted. A commercial cellulase enzyme (Cellulase "Onozuka R-10" from Trichoderma viride, SERVA Electrophoresis GmbH, Heidelberg, Germany), xylanase from Trichoderma viride (Fluka), $\beta$-glucosidase from almonds and recombinant laccase from Aspergillus sp. (Novozyme, EEC No. 420-150-4) and pure laccase V [as purified by Rühl (2009)] from C. cinerea Okayama 7 were used for enzymatic hydrolysis. Spectrophotometric measurements of cellulase, xylanase and $\beta$-glucosidase activities, cellulase and xylanase activities were measured as described in Chapter IV.3.6.1. $\beta$-glucosidase activity was assayed at $400 \mathrm{~nm}$ in $100 \mathrm{mM}$ sodium 
acetate buffer, $\mathrm{pH} 5.0$ at $45^{\circ} \mathrm{C}$ by release of reducing $p$-nitro-phenol (pNP-Glc) as measured with sodium carbonate $\left(\mathrm{Na}_{2} \mathrm{CO}_{3}\right)$, using the appropriate substrate $1 \mathrm{mM}$ p-nitro-phenol in $100 \mathrm{mM}$ sodium acetate buffer, pH 5.0 (Yoshida et al. 2008) in $1 \mathrm{ml}$ plastic cuvettes in a spectrophotometer (UV-DU800, Beckman coulter, Inc., California, USA). The enzyme activity was expressed in units defined as $1 \mathrm{U}=1 \mu \mathrm{mol}$ reducing of p-nitro-phenol/min.

\section{V.3.5 Enzymatic cellulose hydrolysis}

All enzymatic hydrolysis experiments were carried out at $37^{\circ} \mathrm{C}$ in $5 \mathrm{ml}$ of $50 \mathrm{mM}$ sodium acetate buffer, pH 5.0 for $100 \mathrm{mg}$ substrate in $50 \mathrm{ml}$ Falcon tubes (SARSTEDT, Nümbrecht, Germany) on a standing shaker (Uniform, Infors AG, Bottmingen, Switzerland) applying $200 \mathrm{rpm}$ for shaking. $500 \mu 1$ samples were periodically removed and centrifuged with $16,000 \mathrm{~g}$ for $5 \mathrm{~min}$. The production of sugar was determined by using a glucose detection GAGO-20 assay, Sigma-Aldrich, Steinheim, Germany (Fig. 3) through measurements in a spectrophotometer (Spectra max340PC, Molecular Devices, California, USA).

$100 \mathrm{mg}$ substrate with added enzymes for hydrolysis in $5 \mathrm{ml}$ of $50 \mathrm{mM}$ sodium acetate buffer, $\mathrm{pH} 5.0$ incubated under shaking at $200 \mathrm{rpm}$ at $37{ }^{\circ} \mathrm{C}$

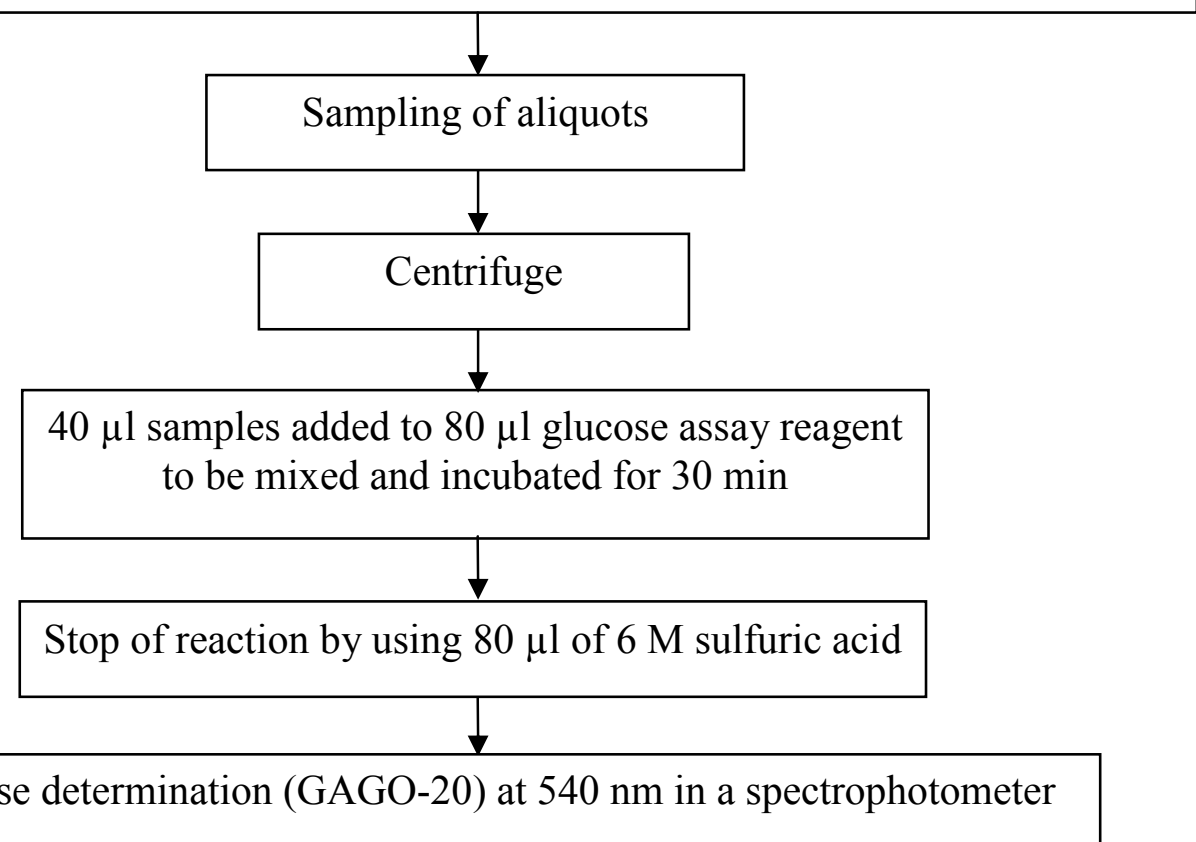

Fig. 3 General flow chart for enzymatic hydrolysis and glucose measurement 
In another set of experiments, the influences of wood extractives and specific phenolic compounds on cellulose hydrolysis were determined by analyzing a kinetic curve between production of glucose and incubation time. The experiment was carried out by using $5 \mathrm{mg}$ carboxymethylcellulose (CMC) as a substrate in a $50 \mathrm{ml}$ Falcon tube on a standing shaker $200 \mathrm{rpm}, 37^{\circ} \mathrm{C}$ in $5 \mathrm{ml}$ of $50 \mathrm{mM}$ sodium acetate buffer, $\mathrm{pH} 5.0$. $500 \mu \mathrm{l}$ samples were periodically collected after 60,90 and $120 \mathrm{~min}$ of incubation, and boiled for $5 \mathrm{~min}$ to stop the enzymatic reaction, and centrifuged for $16,000 \mathrm{~g}$ for $5 \mathrm{~min}$. The production of sugar was measured by using the glucose oxidase/peroxidase method of the GAGO-20 assay, Sigma-Aldrich.

\section{V.3.6 Sugar assay}

Production of sugar was quantified by using a glucose (GO) assay kit, GAGO-20 (Sigma) following the suppliers directions. An adapted methodology was carried out using a 96-microplate format. Per individual test, $40 \mu \mathrm{l}$ of centrifuged, diluted sample was mixed with $80 \mu \mathrm{l}$ glucose reagent and incubated at $37^{\circ} \mathrm{C}$ on a standing shaker $200 \mathrm{rpm}$ for $30 \mathrm{~min}$. Reactions were stopped at 30-60 seconds by adding $80 \mu \mathrm{l}$ of $12 \mathrm{~N}$ sulphuric acid $\left(\mathrm{H}_{2} \mathrm{SO}_{4}\right)$. Absorbance at $540 \mathrm{~nm}$ was measured in a microplate spectrophotometer. In order to convert absorbance values to glucose concentration, measured values were interpolated using a glucose calibration curve which kept a linear relationship between 37.04 and $1.37 \mu \mathrm{g} \mathrm{ml}^{-1}$.

\section{V.3.7 Wood component characterization}

Lignin contents in wood samples were measured by using Klason lignin following the small scale instructions of TAPPI T22 om-02 (2002). Cellulose content was measured by using the anthrone assay (Morris, 1948) and hemicellulose content by using the orcinon assay (Mejbaum, 1939). Total hexose and total pentose were spectrophotometrically determined at $620 \mathrm{~nm}$ and $665 \mathrm{~nm}$, respectively. The total wood extractive content in degraded wood by fungi was determined by using $2 \mathrm{~g}$ of completely dry wood powder (see Chapter V.3.7). The samples of wood powder were poured with $100 \mathrm{ml}$ distilled water into $200 \mathrm{ml}$ round flask, and boiled for $2 \mathrm{hrs}$. Filters were marked and put in the oven at $103 \pm 2^{\circ} \mathrm{C}$ to dry for $2 \mathrm{hrs}$. Afterward, filters were kept in a desiccator for $30 \mathrm{~min}$ and then they were weighed. The boiled $100 \mathrm{ml}$ 
solutions with the wood particles were filtered through the marked filters. The filters with the solid material of the samples were dried in an oven at $103 \pm 2^{\circ} \mathrm{C}$ until a constant weight was achieved (about 2 hrs), upon which dry masses were measured and the extractive contents calculated.

\section{V.3.8 Statistical analysis}

Group values for all parameters in the tests were compared by analysis of variance (ANOVA) tests using the Fisher's least significant difference (LSD) and Duncan's test procedure for multiple comparison (SPSS 8.0 for Windows; USA). Relations among the values of samples were compared for each factor to the controls kept under the same conditions than the samples of interest.

\section{V.4 Results}

\section{V.4.1 Chemical composition of $\boldsymbol{A}$. grandis wood in comparison to Norway spruce wood and wheat straw}

There are several effective parameters on lignocellulose hydrolysis, such as characters of the wood, contents of lignin, hemicellulose, wood extractives, applied pretreatment methods and the general enzymatic hydrolysis (Olofsson et al. 2008; Sainz 2009), that will affect the yields of sugars for biofuel production. Therefore, first the principal chemical composition of $A$. grandis wood (original and extractives free), lignin content, hemicellulose content and cellulose content has been determined (Table 1) in comparison to Norway spruce wood and wheat straw, respectively.

A. grandis has a comparable cellulose content to wheat straw $(46.3 \pm 0.15 \%$ versus $47.6 \pm 1.60 \%)$, a lower hemicellulose content $(8.2 \pm 0.16 \%$ versus $28.6 \pm 1.15 \%)$ and a higher lignin content $(31.6 \pm 0.66 \%$ versus $19.4 \pm 0.17 \%)$. A grandis and wheat straw distinguish from Norway spruce in cellulose, in hemicellulose and in lignin content $(52.2 \pm 0.32,13.8 \pm 0.09 \%$ and $26.5 \pm 0.14 \%$ in Norway spruce, respectively). Hemicellulose and lignin might both be seen as a problem in accessing the cellulose in enzymatic treatments (see Chapter V.2). A lower hemicellulose content in A. grandis wood might therefore be of benefit for enzymatic cellulose degradation. However, the high lignin content could instead be of hindrance. 
Table 1 Chemical composition of different lignocellulosic materials

\begin{tabular}{lcccl}
\hline \multicolumn{1}{c}{ Wood } & $\begin{array}{c}\text { Cellulose } \\
\text { content (\%) }\end{array}$ & $\begin{array}{c}\text { Hemicellulose } \\
\text { content (\%) }\end{array}$ & $\begin{array}{c}\text { Lignin } \\
\text { content (\%) }\end{array}$ & $\begin{array}{c}\text { Rest } \\
(\%)\end{array}$ \\
\hline Grand fir & $46.3 \pm 0.15$ & $8.2 \pm 0.16$ & $31.6 \pm 0.66$ & 13.9 \\
$\begin{array}{l}\text { Grand fir (after } \\
\text { water extraction) }\end{array}$ & $51.7 \pm 0.24$ & $7.5 \pm 0.13$ & $29.8 \pm 0.25$ & 11.0 \\
$\begin{array}{l}\text { Norway spruce } \\
\text { Wheat straw }\end{array}$ & $52.2 \pm 0.32$ & $13.8 \pm 0.09$ & $26.5 \pm 0.14$ & 7.5 \\
\hline
\end{tabular}

The average values and standard deviations came from technical repeats

Original A. grandis wood and water-extracted wood were also compared (Table 1). Water-extracted wood of $A$. grandis gave similar hemicellulose and lignin values but the total cellulose content $(51.7 \pm 0.24 \%)$ increased in value by elimination of wood extractives. Hot water treatment prior to enzymatic hydrolysis might therefore be of advantage.

\section{V.4.2 Effect of enzymatic hydrolysis on glucose production of lignocellulosic materials}

Conversion of cellulose to glucose from $100 \mathrm{mg}$ original wood particles was first attempted by individually applying $4 \mathrm{U} \mathrm{ml}^{-1}$ cellulase or $4 \mathrm{U} \mathrm{ml}^{-1}$ xylanase or by applying a combination of $4 \mathrm{U} \mathrm{ml}^{-1}$ cellulase and $4 \mathrm{U} \mathrm{ml}^{-1}$ xylanase in $5 \mathrm{ml} 50 \mathrm{mM}$ sodium acetate buffer, $\mathrm{pH}$ 5.0, under shaking at $200 \mathrm{rpm}$ and at an incubation temperature of $37^{\circ} \mathrm{C}$ (Fig. 4). Glucose content in the supernatants of the samples was checked after $24 \mathrm{hrs}$ and $48 \mathrm{hrs}$ of incubation, respectively.

The glucose yields of $A$. grandis, Norway spruce and wheat straw for the single enzymes and for the enzyme combination were after 24 hrs about $4-5 \%, 8-10 \%$ and $14-17 \%$, respectively of total cellulose content (Fig. 4A). Conversion of cellulose to glucose from wheat straw with enzymes gave generally the highest glucose yield, whereas $A$. grandis had the lowest. The cellulose to glucose conversion relating to the original total cellulose content in the material after $48 \mathrm{hrs}$ of enzyme treatment were about 4-6\%, 12-13\% and 16-17\% for A. grandis wood, Norway spruce and wheat straw, respectively (Fig. 4B). 


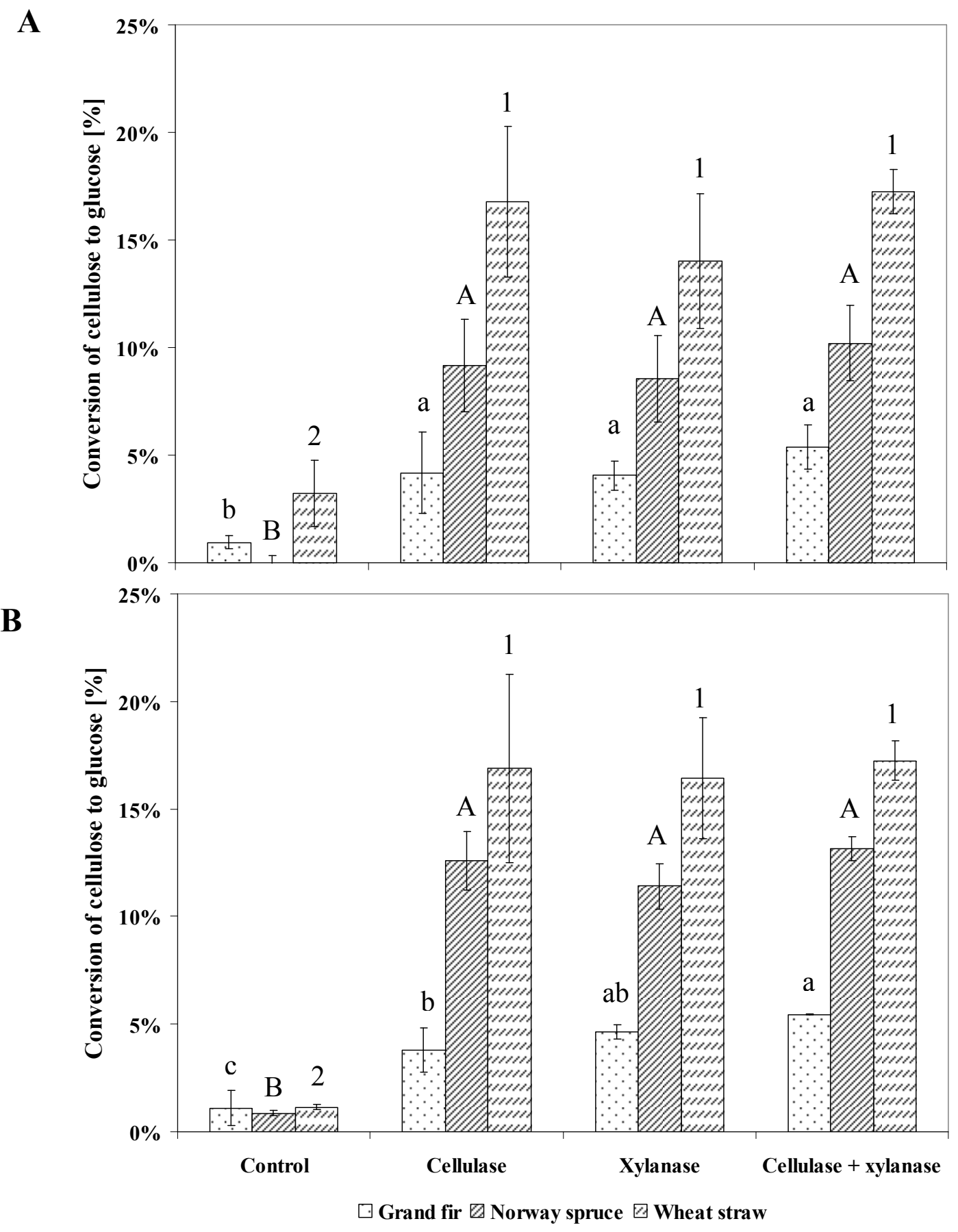

Fig. 4 Conversion of total cellulose to glucose from $100 \mathrm{mg}$ raw wood particles from A. grandis or Norway spruce or from $100 \mathrm{mg}$ wheat straw particles, hydrolyzed by $4 \mathrm{U} \mathrm{ml}^{-1}$ of either cellulose or xylanase or of both enzymes each in $50 \mathrm{mM}$ sodium acetate buffer, pH 5.0 in $24 \mathrm{hrs} \mathrm{A}$ ) and $48 \mathrm{hrs}$ B) incubation at $37^{\circ} \mathrm{C}$ under constant shaking of $200 \mathrm{rpm}$. Controls for all three different materials without addition of enzyme were run in parallel. Per wood type and treatment, three different samples were analyzed and average yields with standard deviations were calculated. The different superscripts on the individual chart (letters in lower case compare the values obtained at different treatments of grand fir wood particles, capital letters compare the respective values of Norway spruce wood particles, and numbers compare the respective values of 
wheat straw) indicate values of glucose yields that differ significantly $(p<0.05)$ between treatments as based on analysis of variance (ANOVA)

Accordingly, also after two days of incubation, values in most cases did not differ very much at the longer incubation time from the shorter one. In case of A. grandis wood, yields of glucose from conversion of the total cellulose amounts on A. grandis wood were still around 5\%. However, the combination of cellulase and xylanase in A. grandis hydrolysis showed a significantly higher glucose yield, but only in an order of very low amounts ( $6 \%$ glucose yield from conversion of the total cellulose content compared to $4 \%$ obtained with cellulase alone and $5 \%$ compared to $4 \%$ obtained with xylanase alone). When one compares the 2-day incubation values and the 1-day incubation values with each other, one might however conclude that this slight increase might just be due to methodic-based variations (Fig. 4A, 4B).

In contrast, an increase in yield over the time was obvious in all three different enzyme treatments for Norway spruce wood with glucose yields of around $12 \%$ of total cellulose in the $A$. grandis wood after 2 days of incubation with enzymes (Fig. 4A, 4B). In case of wheat straw, when using only xylanase there was an increase from $14 \%$ to about 16\% from $24 \mathrm{hrs}$ to $48 \mathrm{hrs}$ incubation whereas samples with added cellulase did not visibly increase in yields after $48 \mathrm{hrs}$ incubation compared to those from the day before (Fig. 4A, 4B).

Both, cellulase and xylanase samples originate from Trichoderma viride. The cellulase ("Onozuka R-10" from SERVA, catalog no. 16419) indeed contains hemicellulase active on beech wood xylan to form xylose in alike amounts as the cellulase converting cellulose into glucose (information by supplier SERVA, Heidelberg). In contrast, the xylanase EC No. 2534397 (Fluka, Netherlands) has possibly less foreign enzyme activity since glucose production has been found delayed with this enzyme production when applied alone on wood or wheat straw as compared to samples treated with cellulase (Fig. 4A and 4B). Further information on enzymatic impurities of xylanase EC No. 2534397 is not available (information given by the supplier).

In conclusion from this experiment, the original $A$. grandis wood did not behave best in the test of enzymatic glucose production as compared to Norway spruce wood and wheat straw. Moreover, a further experiment, a comparison of original A. grandis wood with water-extracted wood, revealed that water extractives were not the reason of the limited glucose yields (Table 2). Because of the possibly slightly better yield of 
glucose with cellulase and xylanase (Fig. 4B), the combination of the two enzymes was applied in this comparison of the two A. grandis wood types (Table 2), as well in experiments presented in the following.

Table 2 Glucose production from $100 \mathrm{mg}$ original and from $100 \mathrm{mg}$ water-extracted A. grandis wood particles, respectively, during $24 \mathrm{hrs}$ incubation at $37^{\circ} \mathrm{C}$ under constant shaking in $5 \mathrm{ml}$ of $50 \mathrm{mM}$ sodium acetate buffer, pH 5.0 with added $4 \mathrm{U} \mathrm{ml}^{-1}$ cellulase and $4 \mathrm{U} \mathrm{ml}^{-1}$ xylanase

Conversion of cellulose to glucose

\begin{tabular}{lcccc}
\cline { 2 - 5 } Experiment & \multicolumn{2}{c}{ Original wood } & \multicolumn{2}{c}{ Water-extracted A. grandis wood } \\
\cline { 2 - 5 } & $\begin{array}{c}\text { mg Glucose/g } \\
\text { wood }\end{array}$ & $\begin{array}{c}\text { \% Conversion of } \\
\text { total cellulose* }\end{array}$ & $\begin{array}{c}\text { mg Glucose/g } \\
\text { wood }\end{array}$ & $\begin{array}{c}\text { \% Conversion of } \\
\text { total cellulose* }\end{array}$ \\
\hline I (Fig. 4) & $53.7 \pm 8.42$ & $5.5 \pm 1.01$ & n.d. & n.d. \\
II (Fig. 5) & $46.6 \pm 5.11$ & $3.7 \pm 0.051$ & $49.1 \pm 1.41$ & $3.6 \pm 0.01$
\end{tabular}

n.d. $=$ not done

* Note that the equal relative glucose yield per total cellulose is the same in both types of wood because of the generally higher relative cellulose content in the extracted A. grandis wood (compare Table 1)

\section{V.4.3 Effects of $\boldsymbol{A}$. grandis wood pretreatments on production of glucose}

In order to increase the susceptibility of $A$. grandis wood for enzymatic hydrolysis, chemical, physico-chemical and biological pretreatments were carried out with the A. grandis wood in order to identify advantages of using such techniques for producing a better glucose yield. In the following, the different sub-categories of pretreatment are presented.

\section{V.4.3.1 Chemical pretreatment of $A$. grandis wood particles}

Chemical pretreatment was done by using liquid acids (85\% phosphoric acid, or $85 \%$ phosphoric acid mixed with ionic liquid, Aliquat in 1:1 w/w relation, or Aliquat alone) to incubate $1 \mathrm{~g}$ of $A$. grandis wood particles (original or in one case also waterextracted) in $8 \mathrm{ml}$ liquid acid at $50^{\circ} \mathrm{C}$ in a hot water bath for up to $1 \mathrm{hr}$ in order to 
destroy both lignin and hemicellulose (Dadi et al. 2006; Zhang et al. 2007). Under these conditions, furthermore, concentrated phosphoric acid can break up orderly hydrogen bonds among glucan chains in crystalline cellulose, resulting in shorter molecule chains of an amorphous form of cellulose suitable for hydrolysis (Zhang et al. 2007).

After washing 3 times the acid-treated material with water and centrifuging, the left solid material (cellulosic pellets; Zhang et al. 2007) were weighed (see data in Table S1 in the supplementary material). It is to be noted that left cellulosic pellets differed in amounts between the different samples, in values of $0.642 \mathrm{~g}$ to $0.697 \mathrm{~g}$ left after pretreatment produces with phosphoric acid with the theoretical maximum cellulose content of $66.4 \%$ to $79.7 \%$. In contrast after treatment with pure Aliquat, $0.865 \mathrm{~g}$ with a theoretical maximum cellulose content of $53.5 \%$ was still left (Exp I, Table S1 in the supplementary material). Cellulose contents in these samples were also determined experimentally (Exp I, Table S1 in the supplementary material). The measured values corresponded very well with theoretical values of cellulose achieved under the assumption that no cellulose would have been lost from a sample by the pretreatment but (parts of the) lignin and hemicellulose (Exp I, Table S1 in the supplementary material). Clearly with all of the phosphoric acid pretreatments, there was a good enhancement of cellulose content in the material for enzymatic hydrolysis.

$100 \mathrm{mg}$ of the cellulosic pellets of the different samples were treated for $24 \mathrm{hrs}$ with both $4 \mathrm{U} \mathrm{ml}^{-1}$ cellulase and $4 \mathrm{U} \mathrm{ml}^{-1}$ xylanase in $50 \mathrm{mM}$ sodium acetate buffer, $\mathrm{pH}$ 5.0, and, subsequently, glucose yields were determined. Glucose levels in the samples were calculated against the measured cellulose content being present in treated $100 \mathrm{mg}$ samples of cellulosic pellets. The treatment with phosphoric acid drastically increased subsequent yields of glucose from cellulose conversion to values of over $55 \%$ up to $70 \%$ (\% of conversion of the cellulose to glucose that was left in the cellulosic pellets) as compared to the about $5 \%$ yields of cellulose to glucose conversion from wood control samples handled without chemical pretreatment or as compared to the $10 \%$ of wood samples treated just with Aliquat (Fig. 5). 56\% cellulose conversion into glucose was already reached of cellulosic pellets from A. grandis wood particles obtained from 15 min incubation with $85 \%$ phosphoric acid as pretreatment whilst prolongation of incubation to $1 \mathrm{hr}$ further increased the glucose yield to $70 \%$ conversion of the cellulose present in the left cellulosic pellet to glucose. Incubation for $1 \mathrm{hr}$ with $85 \%$ phosphoric acid plus Aliquat in $1: 1 \mathrm{w} / \mathrm{w}$ relation (final concentration of $42.5 \%$ of phosphoric acid) was less efficient with $60 \%$ glucose yield. Also, only a comparable 
slight difference in glucose yield was obtained (60\%), when water extractives were eliminated from the $A$. grandis wood particles prior to 15 min pretreatment with $85 \%$ phosphoric acid (Fig. 5).

In further experiments, the effects of different amounts of $85 \%$ phosphoric acid were tested by incubation of $1 \mathrm{~g}$ A. grandis wood particles for one hour in different

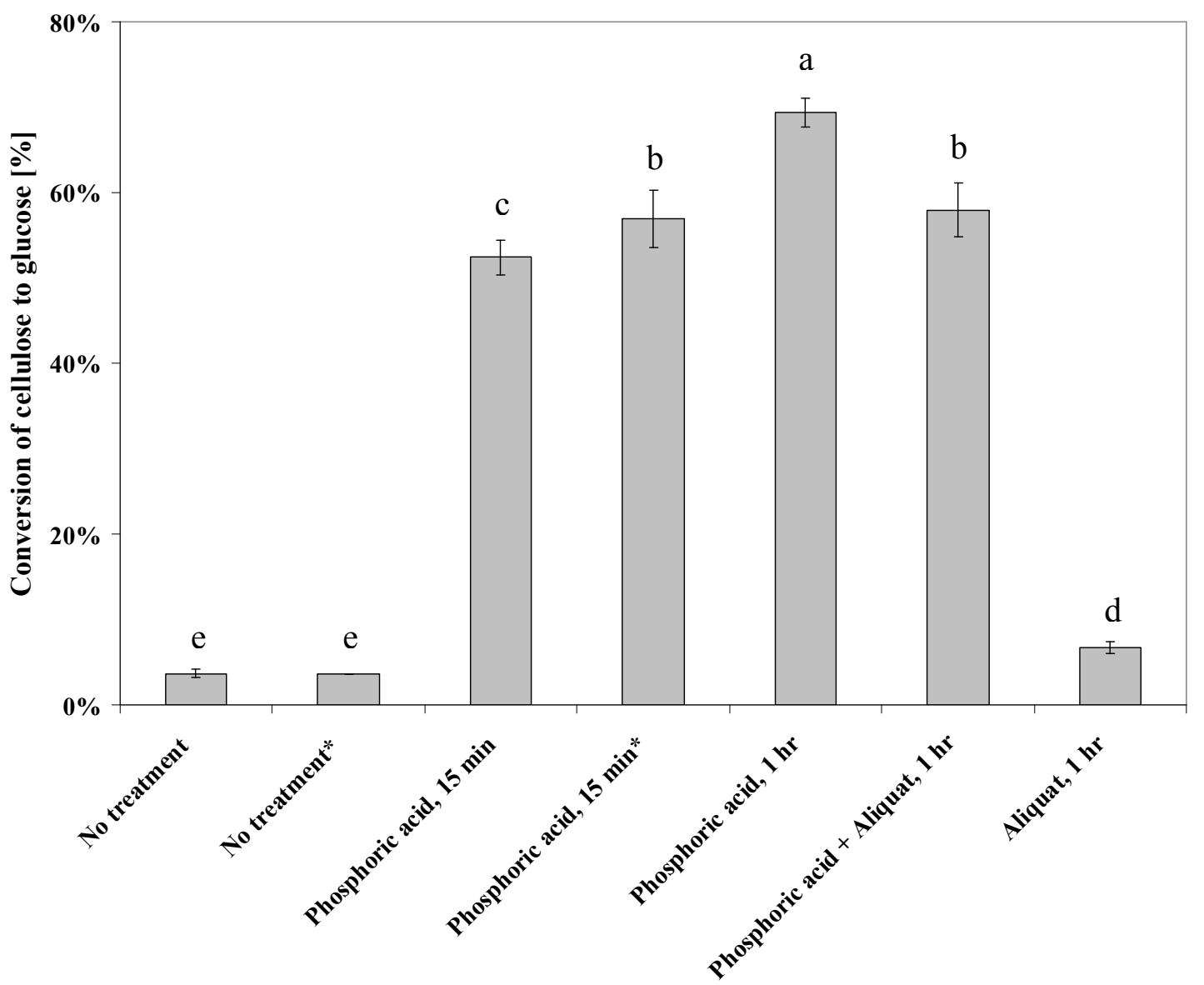

Fig. 5 Enzymatic conversion of cellulose to glucose of $100 \mathrm{mg}$ of cellulosic pellets left from $A$. grandis wood particles pretreated prior to enzymatic hydrolysis with $8 \mathrm{ml}$ liquid acid [of either $85 \%$ phosphoric acid or of $85 \%$ phosphoric acid mixed with ionic liquid, Aliquat in 1:1 w/w relation resulting in a total phosphoric acid content of $42.5 \%$, or of Aliquat alone] during $15 \mathrm{~min}$ or $1 \mathrm{hr}$ incubation at $50^{\circ} \mathrm{C}$ in a hot water bath and, subsequently, the left material was washed with water. For enzymatic hydrolysis, in each three parallel samples the respective materials were incubated with $4 \mathrm{U} \mathrm{ml}^{-1}$ of cellulase and $4 \mathrm{U} \mathrm{ml}^{-1}$ xylanase in $50 \mathrm{mM}$ sodium acetate buffer, $\mathrm{pH} 5.0$ for $24 \mathrm{hrs}$ at $37^{\circ} \mathrm{C}$ under constant shaking. In all instances, average yields of glucose and standard deviations were calculated relative to the cellulose content experimentally determined in $100 \mathrm{mg}$ of the wood particles or in $100 \mathrm{mg}$ of the cellulosic pellets (see Exp I, Table S1 in the supplementary material). The different superscripts on the chart indicate values of glucose yields that differ significantly $(p<0.05)$ between treatments as based on analysis of variance (ANOVA). * Wood after water extraction 
volumes of the acid. Subsequently, each time $100 \mathrm{mg}$ of the respective left cellulosic pellets were hydrolyzed for one day by $4 \mathrm{U} \mathrm{ml}^{-1}$ of cellulase and $4 \mathrm{U} \mathrm{ml}^{-1}$ of xylanase in $5 \mathrm{ml}$ of $50 \mathrm{mM}$ sodium acetate buffer, $\mathrm{pH} 5.0$ at $37^{\circ} \mathrm{C}$ (Fig. 6). Glucose yields were found to decrease in a manner in dependence of the increasing amounts of phosphoric acid.

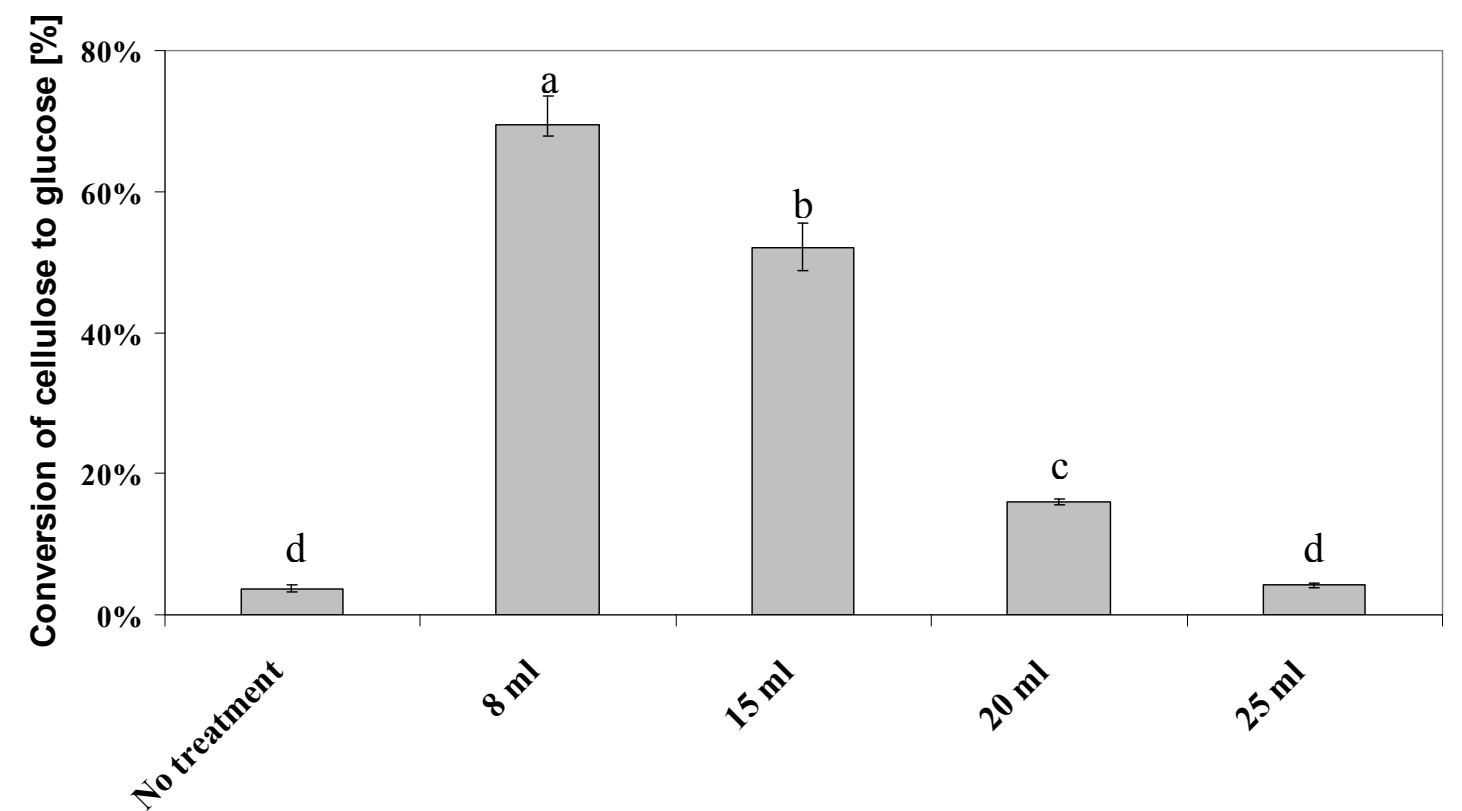

Fig. 6 Enzymatic conversion of cellulose to glucose of $A$. grandis wood particles pretreated prior to enzymatic hydrolysis for one hour at $50^{\circ} \mathrm{C}$ in a hot water bath with 8 , 15,20 , and $25 \mathrm{ml}$ of $85 \%$ phosphoric acid, respectively. Upon washing the left material with water, each $100 \mathrm{mg}$ of left cellulosic pellets were incubated with $4 \mathrm{U} \mathrm{ml}^{-1}$ of cellulase and $4 \mathrm{U} \mathrm{ml}^{-1}$ xylanase in $50 \mathrm{mM}$ sodium acetate buffer, $\mathrm{pH} 5.0$ for $24 \mathrm{hrs}$ at $37^{\circ} \mathrm{C}$ under constant shaking. Three different samples per pretreatment and three controls with $100 \mathrm{mg}$ original $A$. grandis wood particles were treated with enzymes in parallel and average values of glucose yields and standard deviations were calculated against the cellulose content (respectively the hexose content after treatment with sulfuric acid) experimentally determined in $100 \mathrm{mg}$ of cellulosic pellets and in $100 \mathrm{mg}$ of $A$. grandis wood particles, respectively (see Exp II, Table $\mathrm{S} 1$ in the supplementary material). The different superscripts on the chart indicate values of glucose yields that differ significantly $(p<0.05)$ between treatments as based on analysis of variance (ANOVA)

The glucose yield obtained by using $8 \mathrm{ml}, 15 \mathrm{ml}, 20 \mathrm{ml}$ or $25 \mathrm{ml}$ of $85 \%$ phosphoric acid in the pretreatment procedure of $A$. grandis wood particles corresponded to yields of $70 \%, 50 \%, 15 \%$ and $5 \%$ of conversion of cellulose in the left cellulosic pellets to glucose, respectively (Fig. 6). This result does however not relate 
with a high loss of cellulose by the different treatments. Indeed, cellulose was enriched in all samples with about $66 \%$ of total mass at treatments with $8 \mathrm{ml}$ and $15 \mathrm{ml}$ of $85 \%$ phosphoric acid, respectively, and about $90 \%$ in the treatments of higher values of phosphoric acid presenting the maximum theoretical yields of cellulose in the cellulosic pellets (Exp II, Table S1 in the supplementary material). Enzymatic degradation depends on the general structure of cellulose, i.e. whether accessible or not by enzymes (Zhang et al. 2007) and the results of poor cellulose conversion upon application of higher phosphoric acid concentrations may point to an un-favored chemical structural stage generated in applications of high phosphoric acid volumes.

\section{V.4.3.2 Microwave-chemical pretreatment of $A$. grandis wood particles}

This category of pretreatments includes physical pretreatment and combines measures of chemical to measures of physical pretreatment. Physical pretreatment can increase pores size and accessible surface area in the wood cell walls, and it can decrease the degree of polymerization and crystallinity of cellulose (Galbe and Zacchi 2007; Taherzadeh and Karimi 2007). For a physico-chemical pretreatment, this work focused on a cheap energy source and the easy use of microwave (Guo et al. 2009; Zhang and Zhao 2009). For the first time, microwave pretreatment in this study was generally combined with a phosphoric acid treatment.

Microwave pretreatment (rating power $1000 \mathrm{~W}$, out power $700 \mathrm{~W}$, working voltage $230 \mathrm{~V}, 50 \mathrm{~Hz}$ ) of $1 \mathrm{~g}$ of $A$. grandis wood particles in 8 or $25 \mathrm{ml} 50 \mathrm{mM}$ sodium acetate buffer, $\mathrm{pH} 5.0$ for $20-60 \mathrm{sec}$ did not lead to a significant mass loss of material and the relative cellulose content did also not differ much as compared to the nontreated original A. grandis wood particles (Exp III, Table S1 in the supplementary material). According to low changes in the material, cellulose hydrolysis of $100 \mathrm{mg}$ of micro-waved materials with $4 \mathrm{U} \mathrm{ml}^{-1}$ cellulase and $4 \mathrm{U} \mathrm{ml}^{-1}$ xylanase in $50 \mathrm{mM}$ sodium acetate buffer, $\mathrm{pH} 5.0$ for 24 hrs did yield only low amounts of glucose (about 1\% of cellulose conversion referred to the wood mass), comparable to non-treated wood particles $(0.5 \%$ of cellulose conversion referred to the wood mass), (Fig. 7$)$.

The situation was different, when for the microwave procedure the buffer was replaced by $85 \%$ phosphoric acid (Fig. 7). Cellulosic pellets left from $1 \mathrm{~g}$ of $\mathrm{A}$. grandis wood after the microwave-phosphoric acid pretreatment in either 8 or $25 \mathrm{ml}$ volume of $85 \%$ phosphoric acid ranged in weight in between 0.5 and $0.679 \mathrm{~g}$ (Exp III, Table S1 in 
the supplementary material). With cellulosic pellets of about $0.5 \mathrm{~g}$ and below, apparently only or nearly only cellulose was left (ca $90 \%$ to $98 \%$ of cellulose were present) in contrast to the $20 \mathrm{sec}$ microwave treatment in $25 \mathrm{ml} 85 \%$ phosphoric acid that resulted in values of $68 \%$ cellulose content in the $0.679 \mathrm{~g}$ cellulosic pellets (Exp III, Table S1 in the supplementary material). Interestingly, subsequently enzymatic hydrolysis with $4 \mathrm{U} \mathrm{ml}^{-1}$ cellulase and $4 \mathrm{U} \mathrm{ml}^{-1}$ xylanase in $50 \mathrm{mM}$ sodium acetate

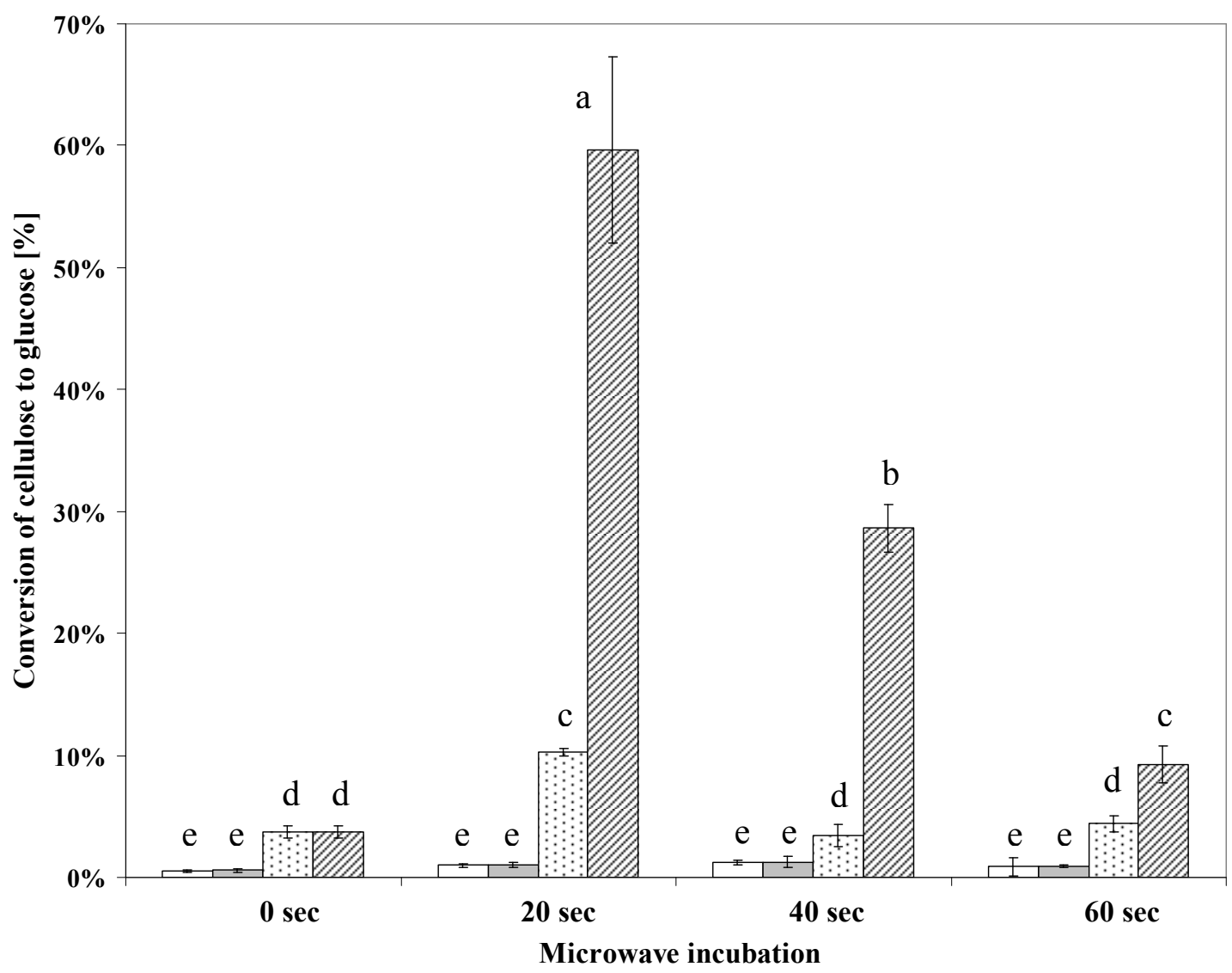

$\square 8 \mathrm{ml}$ Buffer $\square 25 \mathrm{ml}$ Buffer $\square 8 \mathrm{ml}$ Phosphoric acid $\square 25 \mathrm{ml}$ Phosphoric acid

Fig. 7 Enzymatic conversion of cellulose to glucose of $A$. grandis wood particles pretreated prior to enzymatic hydrolysis for 20,40 and $60 \mathrm{sec}$ in a microwave (Intellowave microwave, LG Co., Ltd, Germany) at a rating power of $1000 \mathrm{~W}$, an out power of $700 \mathrm{~W}$, and a working voltage of $230 \mathrm{~V}$ and $50 \mathrm{~Hz}$, with 8 and $25 \mathrm{ml}$ of $85 \%$ phosphoric acid. Each $100 \mathrm{mg}$ of left cellulosic pellets were incubated with $4 \mathrm{U} \mathrm{ml}^{-1}$ of cellulase and $4 \mathrm{U} \mathrm{ml}^{-1}$ xylanase in $50 \mathrm{mM}$ sodium acetate buffer, $\mathrm{pH} 5.0$ for $24 \mathrm{hrs}$ at $37^{\circ} \mathrm{C}$ under constant shaking. Three different samples per pretreatment and three controls with $100 \mathrm{mg}$ original $A$. grandis wood particles were treated in parallel and average values of conversion of cellulose to glucose (referred to the experimentally determined amount of cellulose in the respective samples; compare Exp III, Table S1 in the supplementary material) and standard deviations were calculated. The different superscripts on the chart indicate values of glucose yields that differ significantly $(p<0.05)$ between treatments as based on analysis of variance (ANOVA) 
buffer $\mathrm{pH} 5.0$ for $24 \mathrm{hrs}$ obtained the highest glucose yield (60\%) of cellulose conversion into glucose when microwave incubation for $20 \mathrm{sec}$ with $25 \mathrm{ml}$ of $85 \%$ phosphoric acid was applied (Fig. 7). Microwaving the material for $20 \mathrm{sec}$ in only $8 \mathrm{ml}$ $85 \%$ phosphoric acid resulted subsequently in only $10 \%$ of cellulose conversion into glucose (Fig. 7). Generally, the glucose yield from microwaving for different time of $1 \mathrm{~g}$ of $A$. grandis wood particles in $25 \mathrm{ml} \mathrm{85 \%}$ phosphoric acid obtained always a significant higher glucose yield than what was achieved with a parallel pretreatment in $8 \mathrm{ml} \mathrm{85 \%}$ phosphoric acid without microwaving. However, with both volumes of $85 \%$ phosphoric acid applied, the glucose yields decreased with longer incubation time in the microwave (Fig. 7). Again, also in this series of experiments, it appears that application of more phosphoric acid for times above optimum results in making cellulose nonaccessible for enzymatic conversion.

Upon microwave treatment in presence of $85 \%$ phosphoric acid, the $A$. grandis materials changed visibly in consistence by aggregation and in color by staining dark as if burned in an overview of the material (not further shown). Therefore, materials were observed under the microscope (Fig. 8). Before any pretreatment, cell structures and even entire wood fibers were clearly identified in the A. grandis wood particles (Fig. 8A). If only a pretreatment of $8 \mathrm{ml} 85 \%$ phosphoric acid was done for $1 \mathrm{hr}$ at $50^{\circ} \mathrm{C}$ by incubating in a hot water bath, the fibril structures loosened, although cellular structures were still recognizable (Fig. 8B) or the structures totally dissolved in case of the $25 \mathrm{ml}$ pretreatment (Fig. 8C).

In contrast, upon the incubation with the microwave in presence of 8 and $25 \mathrm{ml}$ phosphoric acid, no fiber structures were anymore observed (Fig. 8D, 8E and 8F). With prolongation of incubation time in the microwave, the brown material formed clumps of increasing size and increasing crystalline structure and increasing dark staining (Fig. 8C to $8 \mathrm{D}$ ). Looking at the phosphoric acid-microwave pretreatment, how stronger the morphological changes (aggregation) and the color changes were, how less the yields in glucose were in subsequent enzymatic hydrolysis (compare Fig. 7 and $8 \mathrm{D}$ to $8 \mathrm{~F}$ ). However, just from the appearance of the material after pretreatment it is not possible to do any reliable prediction on possible yields in enzymatic glucose production. Nevertheless, a too dark staining of the material and a too compact aggregation appears to be negative (compare Fig. 8 with Fig. 6, 7 and 9).

Since microwaving of $1 \mathrm{~g}$ of $A$. grandis wood particles for $20 \mathrm{sec}$ in $25 \mathrm{ml}$ of $85 \%$ phosphoric acid was found to be best for subsequent enzymatic glucose 


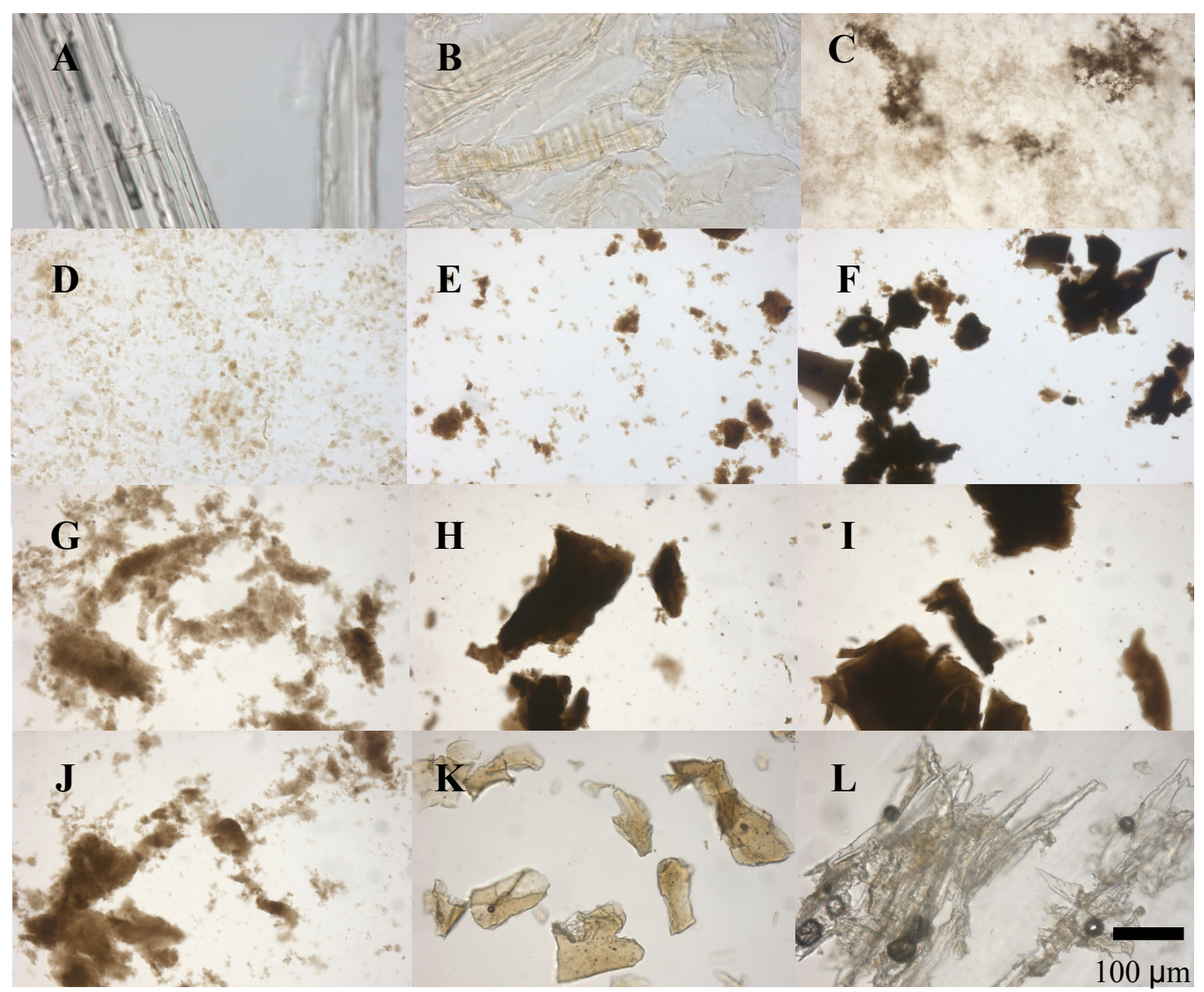

Fig. 8 A. grandis wood particles before any pretreatment $\mathbf{A}$ ), after pretreatment with $8 \mathrm{ml}$ and with $25 \mathrm{ml}$ of $85 \%$ phosphoric acid for $1 \mathrm{hr}$ at $50^{\circ} \mathrm{C}$ in a hot water bath B) and C), respectively, after pretreatment with $8 \mathrm{ml}$ of $85 \%$ phosphoric acid and microwave incubation (at a rating power of $1000 \mathrm{~W}$, an out power of $700 \mathrm{~W}$, and a working voltage of $230 \mathrm{~V}$ and $50 \mathrm{~Hz}$ ) for 20,40 and $60 \mathrm{sec}$, respectively, D), E) and F) after pretreatment with $25 \mathrm{ml} \mathrm{85 \%}$ phosphoric acid and microwave incubation (at a rating power of $1000 \mathrm{~W}$, an out power of $700 \mathrm{~W}$, and a working voltage of $230 \mathrm{~V}$ and $50 \mathrm{~Hz}$ ) for 20, 40 and $60 \mathrm{sec}$, respectively, G), H) and I) and after pretreatment with $25 \mathrm{ml} \mathrm{85 \%}$ phosphoric acid, $25 \mathrm{ml}$ formic acid and $25 \mathrm{ml}$ acetic acid and microwave incubation (at a rating power of $1000 \mathrm{~W}$, an out power of $700 \mathrm{~W}$, and a working voltage of $230 \mathrm{~V}$ and $50 \mathrm{~Hz}$ ) for $20 \mathrm{sec}$, respectively, J), K) and $\mathbf{L}$ ). The samples shown in $\mathbf{B}$ to $\mathbf{L}$ correspond to the experiments on glucose production presented in Fig. 6 (Fig. 8B and 8C), Fig. 7 (Fig. 8D to 8I) and Fig. 9 (Fig. 8J to 8L)

production, alike volumes of different acids (100\% formic acid and $100 \%$ acetic acid) were tested in parallel to $85 \%$ phosphoric acid for their efficiency in making by microwaving $(20 \mathrm{sec}$ ) cellulose in $A$. grandis wood particles accessible for enzymatic degradation. The microwave treatment with $85 \%$ phosphoric acid lead to highest mass loss ( $0.68 \mathrm{~g}$ left upon irradiation with a cellulose content of $60 \%)$, whereas the mass loss with $100 \%$ formic acid and $100 \%$ acetic acid was lower, resulting in about $0.875 \mathrm{~g}$ 
of left materials with about 53\% cellulose content (Exp IV, Table S1 in the supplementary material). Here, the material treated with $85 \%$ phosphoric acid was again much loosened in structure as in the previous alike pretreatment, although an aggregation relative started already when adding $25 \mathrm{ml}$ instead of $8 \mathrm{ml}$ (compare Fig. $8 \mathrm{G}$ and $8 \mathrm{D})$. Aggregation increased over the time of microwave incubation and the material stained dark (Fig. 8H and 8I). In contrast, clear crystalline aggregates were formed after treatment with formic acid and with acetic acid, respectively (Fig. 8K and $8 \mathrm{~L})$.

The materials in the treatments where wood particles were pretreated with the $85 \%$ phosphoric acid obtained again highest glucose yield with $70 \%$ conversion of cellulose to glucose (Fig. 9, compare with Fig. 7). Wood particles after treatment with

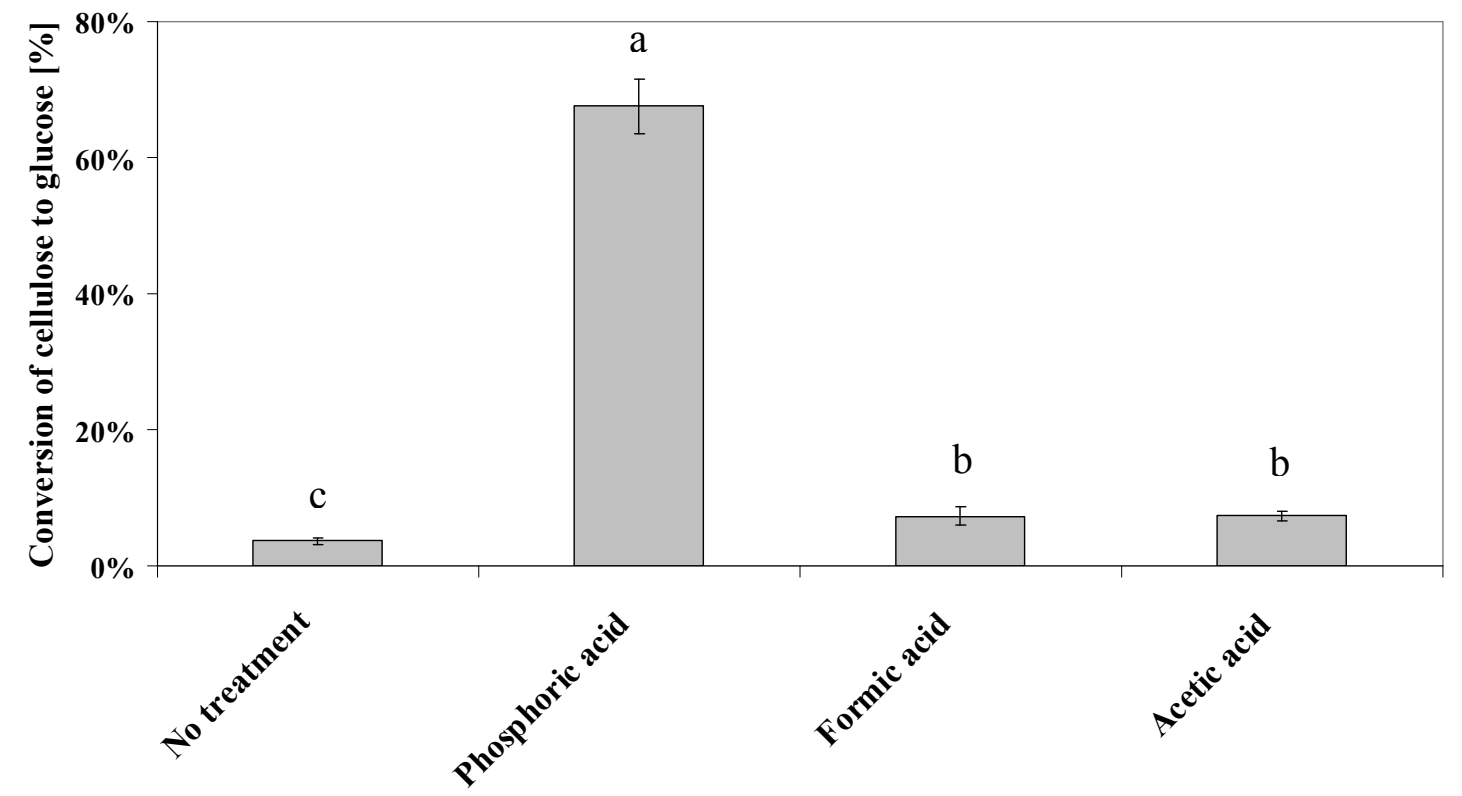

Fig. 9 Enzymatic conversion of cellulose to glucose of $A$. grandis wood particles pretreated prior to enzymatic hydrolysis for $20 \mathrm{sec}$ in a microwave (Intellowave microwave, LG Co., Ltd, Germany) at a rating power of $1000 \mathrm{~W}$, an out power of $700 \mathrm{~W}$, and a working voltage of $230 \mathrm{~V}$, and $50 \mathrm{~Hz}$, with $25 \mathrm{ml}$ of $85 \%$ phosphoric acid, of $100 \%$ formic acid or of $100 \%$ acetic acid, respectively. Each $100 \mathrm{mg}$ of left cellulosic pellets were incubated with $4 \mathrm{U} \mathrm{ml}^{-1}$ of cellulase and $4 \mathrm{U} \mathrm{ml}^{-1}$ xylanase in $50 \mathrm{mM}$ sodium acetate buffer, $\mathrm{pH} 5.0$ for $24 \mathrm{hrs}$ at $37^{\circ} \mathrm{C}$ under constant shaking. Three different samples per pretreatment and three controls with $100 \mathrm{mg}$ original A. grandis wood particles were treated in parallel and average values of conversion of cellulose to glucose referred to the experimentally determined amounts of cellulose in the respective samples (compare Exp IV, Table S1 in the supplementary material) and standard deviations were calculated. The different superscripts on the chart indicate values of glucose yields that differ significantly $(p<0.05)$ between treatments as based on analysis of variance (ANOVA) 
formic acid or acetic acid resulted in less glucose yields of both only about 10\% (Fig. 9) and these acids are therefore less suitable for wood pretreatment than $85 \%$ phosphoric acid. However, the amounts of cellulose conversion to glucose were still significantly higher than those obtained from non-treated wood (cellulose conversion to glucose of $4 \%$ of the original mass; see Fig. 9).

The overall results in Fig. 7 suggested that in microwaving of $A$. grandis wood particles a combination of timing of irradiation and of volume play a role in optimum pretreatment of the wood for later enzymatic cellulose to glucose conversion. A higher volume of $85 \%$ phosphoric acid at a same energy supply from microwaves might be less aggressive as a smaller, easier to heat volume of $85 \%$ phosphoric acid. To possibly compensate this, shorter times might be applied in microwaving with lower amounts in volume of the acid and /or lower concentrations of the acid. Accordingly, reducing the time to $5 \mathrm{sec}$ of incubation in presence of $8 \mathrm{ml}$ of $85 \%$ phosphoric acid gave much better yields of glucose (50\% of cellulose conversion; Fig. 10), whilst the overall mass loss by the pretreatment was comparably low $(0.892 \mathrm{~g}$ material left after $5 \mathrm{sec}$ of microwaving, see Exp V, Table $\mathrm{S} 1$ in the supplementary material). Supplying microwave energy in four $5 \mathrm{sec}$ steps interrupted by each $5 \mathrm{sec}$ of non-irradiation (incubation for $5 \mathrm{sec}$, stop for $5 \mathrm{sec}$; repeated for 4 cycles; total $20 \mathrm{sec}$ ) did not significantly change the outcome on glucose yields ( $30 \%$ of cellulose conversion) compared to a constant irradiation of $20 \mathrm{sec}$ (Fig. 10). In both cases, the mass loss was significant $(0.531$ and $0.481 \mathrm{~g}$ cellulosic pellets left with cellulose contents of 87.1 and 96.1\%, respectively; see Exp V, Table S1 in the supplementary material).

Reducing the acid concentration to $80 \%$ phosphoric acid with $5 \mathrm{sec}$ of microwave irradiation obtained a cellulosic pellet of $0.739 \mathrm{~g}$ with $59 \%$ cellulose content (Exp V, Table S1 in the supplementary material) and about $40 \%$ glucose yield in subsequent enzymatic hydrolysis (Fig. 10), whilst with microwave irradiation at this phosphoric acid concentration for $20 \mathrm{sec}$ or $20 \mathrm{sec}^{*}$ (incubation for $5 \mathrm{sec}$, stop for $5 \mathrm{sec}$, repeated for 4 cycles; total $20 \mathrm{sec})$ the mass loss was higher $(0.492$ and $0.522 \mathrm{~g}$ of cellulosic pellets left with a cellulose content of 94.1 and $88.7 \%$, respectively; see Exp V, Table S1 in the supplementary material) and the glucose yields in enzymatic conversion of cellulose were reduced to about $30 \%$ and comparable to the treatment with $85 \%$ phosphoric acid as applied for the same timings (Fig. 10).

Finally for comparison, wood particles pretreated for some minutes (during mixing the material with the acid) at RT with $85 \%$ or $80 \%$ phosphoric acid without any 


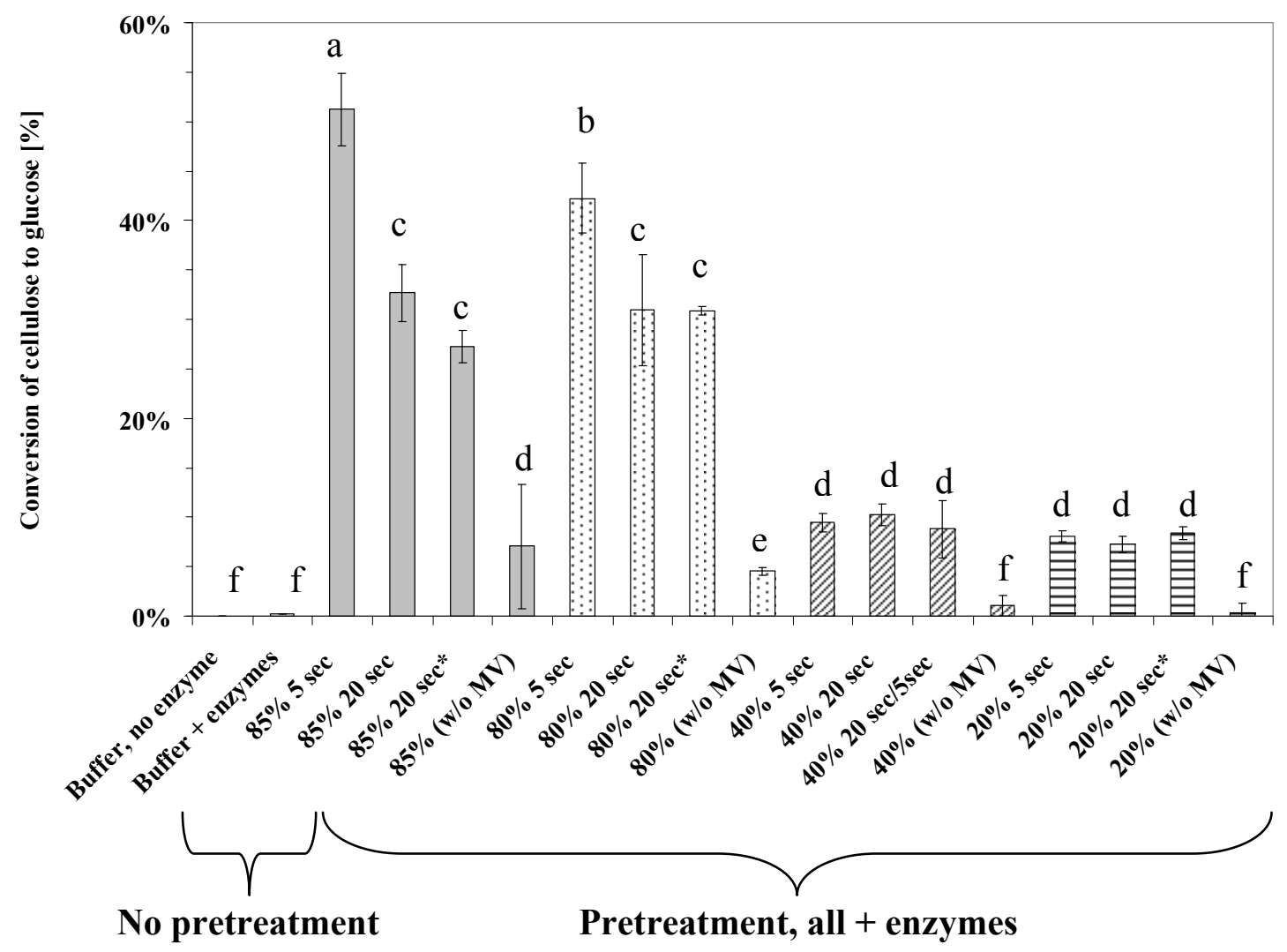

$85 \%$ 团 $80 \%$ 目 $20 \%$ phosphoric acid

Fig. 10 Enzymatic conversion of cellulose to glucose of $A$. grandis wood particles pretreated prior to enzymatic hydrolysis for 5 or $20 \mathrm{sec}$ in a microwave (Intellowave microwave, LG Co., Ltd, Germany) at a rating power of $1000 \mathrm{~W}$, an out power of $700 \mathrm{~W}$, and a working voltage of $230 \mathrm{~V}$ and $50 \mathrm{~Hz}$, with $8 \mathrm{ml}$ of various concentrations of phosphoric acid. Each $100 \mathrm{mg}$ of left cellulosic pellets were incubated with $4 \mathrm{U} \mathrm{ml}^{-1}$ of cellulase and $4 \mathrm{U} \mathrm{ml}^{-1}$ xylanase in $50 \mathrm{mM}$ sodium acetate buffer, $\mathrm{pH} 5.0$ for $24 \mathrm{hrs}$ at $37^{\circ} \mathrm{C}$ under constant shaking. Three different samples per pretreatment and three controls with $100 \mathrm{mg}$ original $A$. grandis wood particles were treated in parallel and average values of conversion of cellulose to glucose referred to the experimentally determined cellulose amount in the respective samples (compare Exp V, Table S1 in the supplementary material) and standard deviations were calculated. The different superscripts on the chart indicate values of glucose yields that differ significantly $(p<0.05)$ between treatments as based on analysis of variance (ANOVA). * Incubation for $5 \mathrm{sec}$, stop for $5 \mathrm{sec}$, repeated for 4 cycles; total $20 \mathrm{sec}$

incubated microwave incubation obtained very low glucose yield of less than $10 \%$ in subsequent enzymatic conversion of cellulose to glucose. This was however still higher than the yields of $1 \%$ obtained in enzymatic conversion in the control of A. grandis wood particles having obtained no acid pretreatment at all and also no microwaving (Fig. 10). No glucose $(0 \%$ conversion of cellulose) was obtained from 
A. grandis wood particles without addition of enzyme (Fig. 10), indicating that the $1 \%$ of glucose obtained from the original wood in presence of the enzyme result from their enzymatic activities. Unlike the untreated $A$. grandis wood, wood treated at RT with $85 \%$ or $80 \%$ phosphoric acid had a slightly reduced weight $(0.915 \mathrm{~g}$ and $0.852 \mathrm{~g}$, respectively) with cellulose contents of $50.6 \%$ and $54.3 \%$, respectively (see Exp V, Table S1 in the supplementary material).

When reducing in the pretreatments with microwaving the phosphoric concentration further to $40 \%$ and $20 \%$, respectively, cellulosic pellets obtained from wood particles incubated with $40 \%$ and $20 \%$ phosphoric acid for different microwaving times resulted in enzymatic incubation in glucose yields of about $10 \%$, irrespectively of the timing for microwaving used (Fig. 10). Moreover, at these low phosphoric concentrations, incubation for some minutes at RT (during handling of the samples) did not result in better glucose yields in subsequent enzymatic cellulose conversion (about $1-2 \%$ yield of glucose) as in the controls non-treated with the acid and with microwaving (Fig. 10). Also in these instances of samples treated without microwaving, there was a low mass loss upon addition of 40 and 20\% phosphoric acid, respectively, resulting in cellulosic pellets of 0.852 and $0.897 \mathrm{~g}$ with cellulose contents of $54.3 \%$ and $57.6 \%$ (see Exp V, Table S1 in the supplementary material).

Also in these series of experiments presented in Fig. 10, loss of material and changes in morphology in structure and coloration by the pretreatments and increases in the relative cellulose content should carefully be considered. Samples with high yields of glucose (about 40 to $60 \%$; Fig. 10) had a mass left after the pretreatment of usually about 70 to $90 \%$ of the original weight with a cellulose content of about 50 to $60 \%$ (see Exp V, Table S1 in the supplementary material). Medium yields of glucose (about $30 \%$; Fig. 10) were achieved in samples characterized by high mass losses (about 50\%) and by high cellulose contents (about 100\%; see Exp V, Table S1 in the supplementary material). However, low yields of glucose (about 10\%; Fig. 10) were achieved with samples that had a mass left after the pretreatment of about 74 to $90 \%$ with a cellulose content of about 50 to $60 \%$ (see Exp V, Table S1 in the supplementary material). Most importantly, glucose yields were generally low (Fig. 10) when mass losses after phosphoric acid treatments reached about 50\% (compare Exp V, Table S1 in the supplementary material). Accordingly, mass loss values and cellulose contents can not be the only criteria for predictions of achievable glucose yields from enzymatic treatments of samples. This conclusion reinforces also the results presented in Fig. 6 
and Fig. 7, where glucose yields were usually only low (Fig. 10) when mass loss after phosphoric acid treatments reached about 50\% although cellulose levels were up to $100 \%$

The $A$. grandis wood particles irradiated for different times in the microwave with different concentrations of phosphoric acid are shown in Fig. 11. Wood particles with longer pretreatments by microwave irradiation had a much darker color than those of short time incubation. In addition, higher concentrations of phosphoric acid also favored darkening of the material as compared to the lower concentrations. Thus, especially $1 \mathrm{~g}$ of $A$. grandis wood particles pretreated for $20 \mathrm{sec}$ in microwave irradiation in $8 \mathrm{ml}$ of $80 \%$ phosphoric acid had an enhanced black color, whereas with $20 \mathrm{sec}$ of microwave irradiation at the lower concentration ( $20 \%$ phosphoric acid) the material had a dark green color (Fig. 11). Wood particles after incubation in cycles of $5 \mathrm{sec}$ for total $20 \mathrm{sec}\left(20 \mathrm{sec}^{*}\right)$ appeared generally lighter in color than wood particles

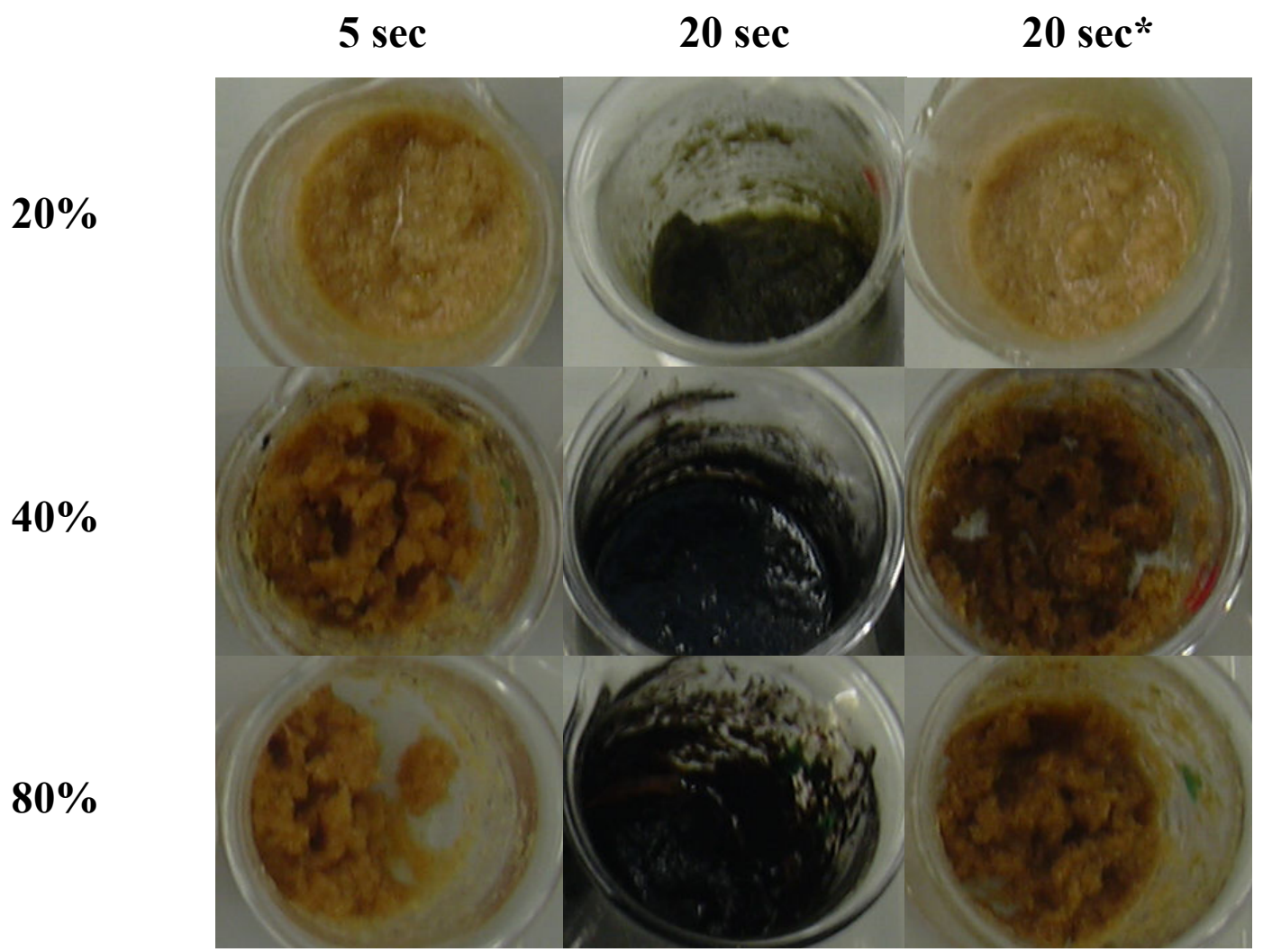

Fig. 11 A. grandis wood particles (1 g) after pretreatment by using microwave irradiation for different times in $8 \mathrm{ml}$ of $20 \%, 40 \%$ and $80 \%$ of phosphoric acid, respectively (compare Fig. 10 for glucose yields). ${ }^{*}$ Incubation for $5 \mathrm{sec}$ and stop for 5 sec, repeated for 4 cycles 
treated constantly for $20 \mathrm{sec}$. However, when comparing enzymatic release of glucose from cellulose (Fig. 10), there was also no clear-cut correlation with glucose yields.

The observations from Fig. 11 generally support the results presented in Fig. 8 that dark clumps of wood cell material appear after the wood has been incubated by longer time in microwave irradiation in the presence of phosphoric acid. Microwave incubation in shorter cycles, however, can improve or avoid the problematic of dark clump formation from the wood cell structures. Nevertheless, the results in Fig. 11 as well as results presented earlier in Fig. 8 in combination with the trials of enzymatic hydrolysis of the obtained materials (Fig. 10) show that formation of clumps and darkening is less a problem than likely the good chemical conversion of recalcitrant compounds such as lignin that generally hinder the enzymatic access to the celluloses.

In conclusion from all the data of the series of experiments shown in Fig. 10, wood pretreatment with phosphoric acid gave optimized glucose yields at a high concentration of phosphoric acid (at least 80\%), and a short time of microwave incubation of e.g. $5 \mathrm{sec}$ in $8 \mathrm{ml}$ phosphoric acid, required in order to obtain highest glucose yields (40 to $60 \%$ glucose yields from conversion of cellulose left in the cellulosic pellet; Fig. 10) in subsequent enzymatic treatment. In contrast, the results shown in Fig. 7 and 9 indicate that the optimal time for microwaving was $20 \mathrm{sec}$ when $25 \mathrm{ml}$ of phosphoric acid was applied in microwave treatment, representing the best condition at which the highest glucose yields (about 65\% glucose yields from conversion of cellulose left in the cellulosic pellet; Fig. 7 and 9) were produced in this work. For economic and ecological reasons, by application of less amounts of harmful acid and by application of less energy, the first pretreatment might however be favored.

It also should be stated from all these experiments that whenever good glucose yields were obtained the total mass of material left after pretreatment was quite often about $65-70 \%$, and that the corresponding cellulose content in the cellulosic pellets left after pretreatment were about $70 \%$ (see Table S1 in supplementary material). It might thus be safest for good glucose yields to concentrate on pretreatment that loosen somewhat the lignocellulosic materials without fully degrading lignin or also hemicellulose in order to avoid too much stress on the cellulose resulting in possible unfavorable structural changes of the cellulose. 


\section{V.4.4 Effects of laccase in combination with other enzymes on hydrolysis of cellulose to glucose}

\section{V.4.4.1 Chemical pretreatment and effects of laccase and other enzymes on glucose production}

Results presented so far in this Chapter $\mathrm{V}$ indicate that a reduction of lignin in the wooden A. grandis material helps in enzymatic access of cellulose for glucose production. In previous work (Chapter IV, Fig. 2), the activity of fungal laccase was demonstrated. Thus, an obvious further development in wood treatment for best release of sugar molecules from cellulose is the appointment of laccase for attacking lignin in the material. Moreover, to some samples also $\beta$-glucosidase, an enzyme that acts on $\beta-1-4$ glycosidic bonds between two glucose or glucose-substituted molecules and that therefore can lead to chain reductions of cellulose molecules (Dadi et al. 2007; Jørgensen et al. 2008; Mtui 2009; Olofsson et al. 2008), was added for possible better cellulose degradation. Further experiments (Fig. 12) tested therefore the effects of different combinations of enzymes in cellulose hydrolysis by using each time $100 \mathrm{mg}$ of cellulosic pellets obtained in an up-scaled pretreatment process in which in $1 \mathrm{hr}$ incubation $5 \mathrm{~g}$ of $A$. grandis wood were incubated in $40 \mathrm{ml}$ of $85 \%$ phosphoric acid in a $100 \mathrm{ml}$ beaker kept at $50^{\circ} \mathrm{C}$ in a hot water bath. Of the $5 \mathrm{~g}$ of $\mathrm{A}$. grandis wood, after the $85 \%$ phosphoric acid treatment, $3.615 \mathrm{~g}$ cellulosic pellets were left with a total cellulose content of $71.09 \%$ (see Table S1 in supplementary material).

Each $100 \mathrm{mg}$ of the cellulosic pellet were hydrolyzed either by a single enzyme or by combinations of enzymes in $50 \mathrm{mM}$ sodium acetate buffer, $\mathrm{pH} 5.0$ at $37^{\circ} \mathrm{C}$ for one day. In a comparative analysis, single enzyme hydrolysis was followed up, thereby using $4 \mathrm{U} \mathrm{ml}^{-1}$ of either cellulase, xylanase, laccase, laccase + mediator (mediator: HBT; 1-hydroxybenzotriazole; $1 \mathrm{mM}$ ) or $\beta$-glucosidase, respectively (Fig. 12). Of these, only cellulase and xylanase hydrolysis obtained about $60 \%$ glucose yields, whereas laccase, laccase + HBT and $\beta$-glucosidase obtained glucose yields lower than $10 \%$ (Fig. 12) and no glucose yield was obtained by un-hydrolyzed controls. Combinations of enzymes (laccase and/or $\beta$-glucosidase together with cellulase and xylanase) in contrast were in all instances more successful in yielding glucose from cellulose degradation (up to $100 \%$ glucose yield) but the combination of only cellulase and xylanase (Fig. 12). This 


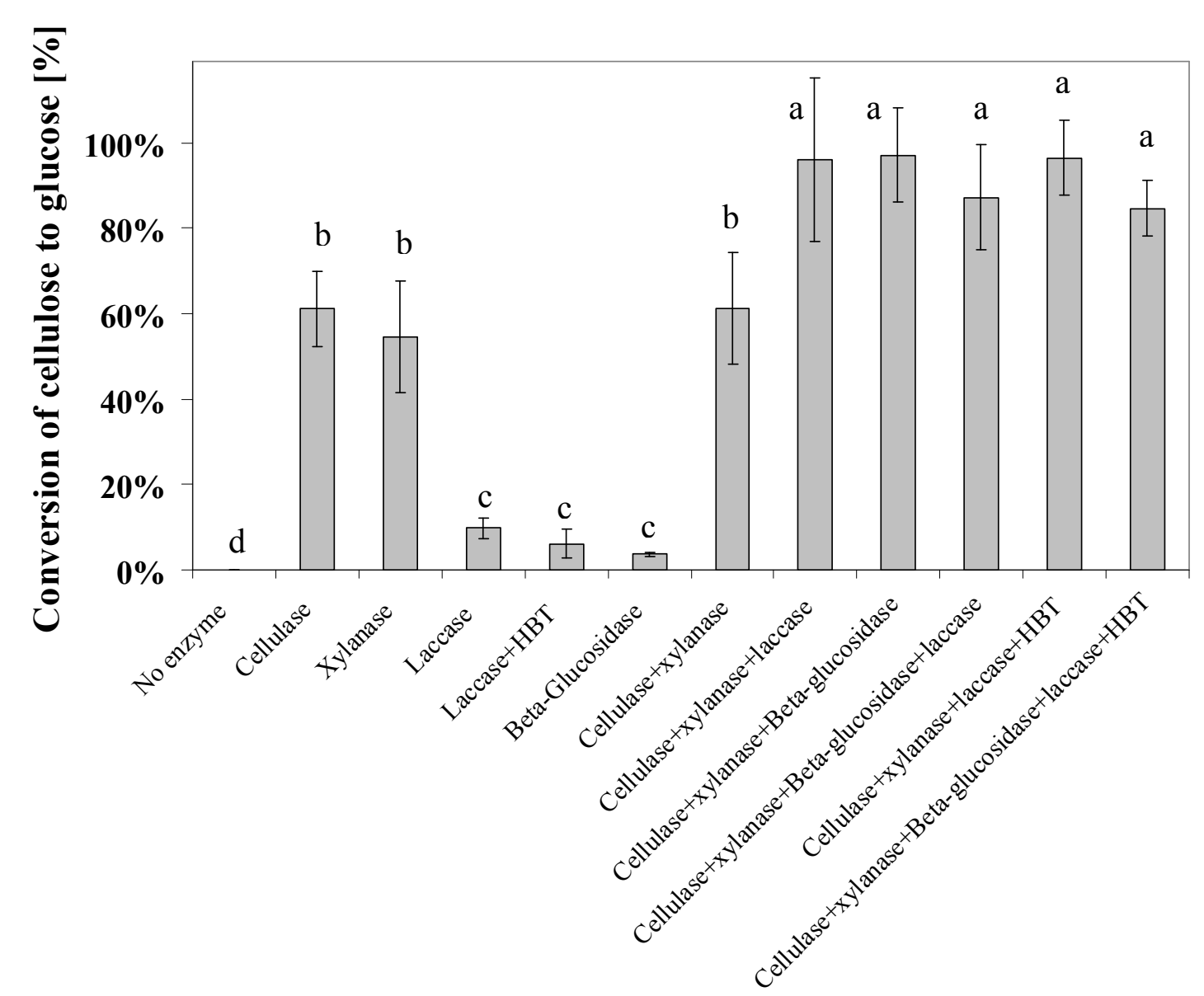

Fig. 12 Conversion of cellulose to glucose in cellulosic pellets of $A$. grandis wood particles pretreated prior to enzymatic hydrolysis with $8 \mathrm{ml}$ of $85 \%$ phosphoric acid at $50^{\circ} \mathrm{C}$ for one hour in a hot water bath, and washed with water. Each time $100 \mathrm{mg}$ of left cellulosic pellet were incubated with enzymes in $50 \mathrm{mM}$ sodium acetate buffer, $\mathrm{pH} 5.0$ for $24 \mathrm{hrs}$ at $37^{\circ} \mathrm{C}$ under constant shaking. Per different treatment, three samples were incubated in parallel and average values and standard deviations were calculated. The different superscripts on the chart indicate values of glucose yields that differ significantly $(p<0.05)$ between treatments as based on analysis of variance (ANOVA)

combination of only two enzymes reached just about $60 \%$ glucose yield, similarly to that what was achieved with the two enzymes in individual digestion (Fig. 12).

Interesting to note is that regardless of whether laccase, laccase with a mediator (HBT), $\beta$-glucosidase or combinations of these were added, the yield of glucose was always optimal (Fig. 12). Previously in Chapter IV.4.7, some results on determining sugars in samples' supernatants indicated that in the laccase preparation there might be some type of contaminating enzymatic activity. The laccase applied were supplied from Novo, Denmark (Novozyme, laccase EEC No. 420-150-4). To elucidate possible contaminations, $0.12 \mathrm{U}$ of the laccase solved in $30 \mu 1$ of $50 \mathrm{mM}$ sodium acetate buffer, 
pH 5.0 were used in final total volumes of $1 \mathrm{ml}$ of $100 \mathrm{mM}$ sodium lactate buffer, $\mathrm{pH} 5.0$ to determine cellulase activity with $4.0 \%(\mathrm{w} / \mathrm{v}) \mathrm{CMC}$ and xylanase activity with $1.5 \%(\mathrm{w} / \mathrm{v})$ with xylan form oat spelts as described in Chapter V.3.4. To determine $\beta$-glucosidase activity with $1 \mathrm{mM}$-nitro phenol (pNP-Glc), $2 \mathrm{U}$ of laccase solved in $500 \mu \mathrm{l}$ of $100 \mathrm{mM}$ sodium acetate buffer, $\mathrm{pH} 5.0$ were tested for foreign enzymatic activities. In no case, such enzymatic activities were detected as contaminations in the preparations. Accordingly, if these types of enzymes are at all present, they should occur in very minor amounts in the solutions of $4 \mathrm{U} \mathrm{ml}^{-1}$ of laccase applied in the A. grandis wood pretreatments.

\section{V.4.4.2 Microwave-chemical pretreatment and effects of laccase on glucose production}

Effects of laccase were further tested on A. grandis wood that had undergone phosphoric acid-microwave pretreatments by five or $20 \mathrm{sec}$ irradiation in a volume of $8 \mathrm{ml}$ phosphoric acid at the different concentrations of $80 \%, 40 \%$ and $20 \%$ acid, respectively (for mass loss and total cellulose contents after the pretreatments see Exp. VI, Table S1 in the supplementary material). The same materials were used as previously in the experiments shown in Fig. 10. Samples pretreated with $85 \%$ phosphoric acid were not included since the application of laccase targets at avoiding as best as possible the use of phosphoric acid and conversion rates of cellulose to glucose of cellulosic pellets from $80 \%$ and $85 \%$ phosphoric acid pretreatments were not as much different (Fig. 10).

Each time for enzymatic hydrolysis, $100 \mathrm{mg}$ of resulting cellulosic pellets were hydrolyzed by a combination of $4 \mathrm{U} \mathrm{ml}^{-1}$ cellulase, $4 \mathrm{U} \mathrm{ml}^{-1}$ xylanase and $4 \mathrm{U} \mathrm{ml}^{-1}$ laccase in $50 \mathrm{mM}$ sodium acetate buffer, $\mathrm{pH} 5.0$ for $24 \mathrm{hrs}$. The glucose yields after enzymatic hydrolysis obtained were highest with about $100 \%$ when wood particles were pretreated with $80 \%$ phosphoric acid in $20 \mathrm{sec}$ microwave irradiation, whereas a pretreatment with $80 \%$ phosphoric acid in $5 \mathrm{sec}$ microwave irradiation resulted in a glucose yield of about $60 \%$ (Fig. 13). Application of lower phosphoric acid concentrations in the pretreatment procedures resulted in lower glucose yields (around $40 \%$ to $50 \%$ ) in subsequent enzymatic cellulose conversion (Fig. 13). When applying a scheme of microwave irradiation of 4 rounds of $5 \mathrm{sec}$ irradiation and $5 \mathrm{sec}$ of no irradiation, when higher concentrations of phosphoric acid were applied $(80 \%$ and 
$40 \%$ ), the resulting glucose yields in the subsequent enzymatic hydrolysis were significantly lower as in microwave experiments in which $20 \mathrm{sec}$ in a constant incubation was applied (Fig. 13). In contrast, for the lowest applied phosphoric acid concentration (20\%), no differences in the yields were seen regardless of the time of microwaving applied (Fig. 13).

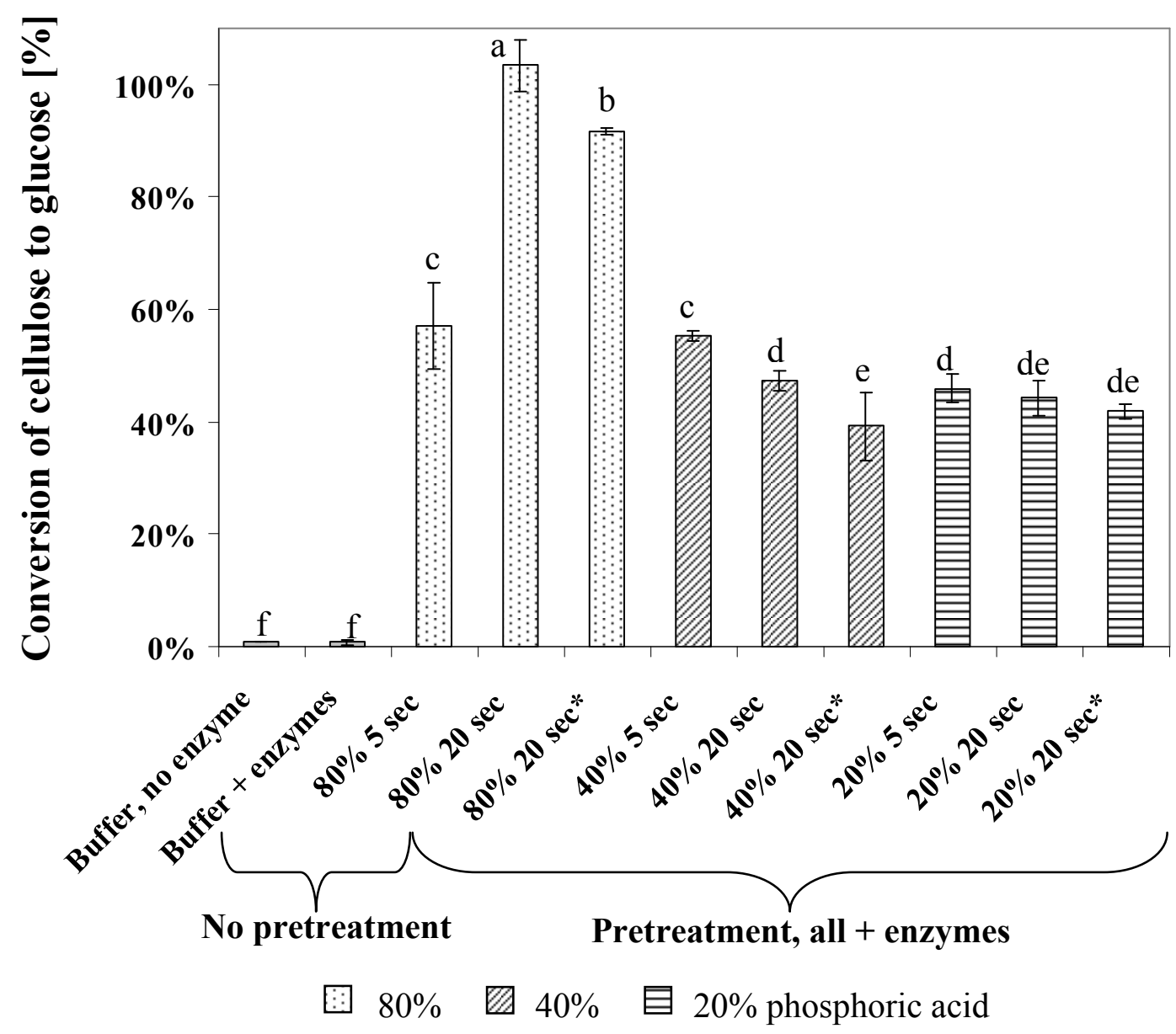

Fig. 13 Enzymatic conversion of cellulose to glucose of $A$. grandis wood particles pretreated prior to enzymatic hydrolysis for $5 \mathrm{or} 20 \mathrm{sec}$ in a microwave (Intellowave microwave, LG Co., Ltd, Germany) at a rating power of $1000 \mathrm{~W}$, an out power of $700 \mathrm{~W}$, and a working voltage of $230 \mathrm{~V}$ and $50 \mathrm{~Hz}$, with $8 \mathrm{ml}$ of various concentrations of phosphoric acid. Each $100 \mathrm{mg}$ of left cellulosic pellets were incubated with $4 \mathrm{U} \mathrm{ml}^{-1}$ of cellulase and $4 \mathrm{U} \mathrm{ml}^{-1}$ xylanase and $4 \mathrm{U} \mathrm{ml}^{-1}$ laccase in $50 \mathrm{mM}$ sodium acetate buffer, pH 5.0 for $24 \mathrm{hrs}$ at $37^{\circ} \mathrm{C}$ under constant shaking. Three different samples per pretreatment and three controls with $100 \mathrm{mg}$ original $\mathrm{A}$. grandis wood particles were treated in parallel and average values of conversion of cellulose to glucose the experimentally determined amount in the respective samples (compare Table S1 in the supplementary material) and standard deviations were calculated. The different superscripts on the chart indicate values of glucose yields that differ significantly $(p<0.05)$ between treatments as based on analysis of variance (ANOVA). * Incubation for $5 \mathrm{sec}$, stop for $5 \mathrm{sec}$, repeated for 4 cycles; total $20 \mathrm{sec}$ 
When comparing the overall results shown in Fig. 13 with those presented in Fig. 10, there is a general tendency seen that due to addition of laccase to samples, higher yields of glucose by enzymatic conversion of cellulose were achieved. The positive input in glucose yields were between 10 to $70 \%$. The lowest increase in glucose yields were obtained with the cellulosic pellets left after $5 \mathrm{sec}$ microwave pretreatment in presence of $85 \%$ phosphoric acid, i.e. material that already gave prior to laccase addition comparably good glucose yields (Fig. 10). The highest upraise in yields were seen with the samples treated for $20 \mathrm{sec}$ with $80 \%$ phosphoric acid $(70 \%$ and $60 \%$ increase in glucose yields as compared to the enzymatic treatment without laccase; Fig. 10 and Fig. 13). Previously, it was suggested that longer incubation with phosphoric acid in the microwave might cause in unknown manner a negative effect on the cellulose structure, making the cellulose less accessible for enzymes to hydrolyze it (Chapter IV.4.3.2). The results in Fig. 13 in contrast might indicate that during the pretreatments chemical compounds of possibly phenolic nature are produced or that linkages of cellulase with compounds of phenolic character are formed that may block the actions of cellulase on the cellulose and that might be overcome due to degradation processes mediated by the added laccase.

Another interesting result from the experiments presented in Fig. 13 is, that reasonable yields of glucose were obtained both with application of $40 \%$ and of $20 \%$ phosphoric acid in the microwave pretreatments but that overall there were no or only marginal differences in the glucose yields. Thus, a combination of very low amounts of phosphoric acid with laccase gives the ecological favorable situation.

\section{V.4.4.3 Biological pretreatment of $\boldsymbol{A}$. grandis wood for efficient glucose production}

Biological pretreatment of $A$. grandis wood was selected in order to also determine its efficiency on gaining glucose yields in subsequent enzymatic hydrolysis of cellulose. White-rot and brown-rot fungi degraded wood were used as a natural pretreatment. Material of a mini block test run for 19 weeks with the brown-rot $C$. puteana Strain 57 and the white-rots T. versicolor Strain 6 and P. ostreatus 8F6 according to the European standard EN 113 (1996), (see Fig. S3 in Chapter III), was used in this experiment. C. puteana Strain 57 caused the highest amount of decay of $A$. grandis wood resulting in about $60 \%$ of mass loss. Wood blocks incubated for 19 weeks of incubation by T. versicolor Strain 6 and P. ostreatus $8 \mathrm{~F} 6$ obtained a lower mass loss of about $40 \%$ and 
$15 \%$, respectively. There was no mass loss or decay detected in uninfected controls (Fig. S3 in Chapter III).

After incubation for 19 weeks, per fungal treatment and per control five mini block wood blocks were grinded with a ball mill apparatus as described in Chapter IV.3.1 for subsequent enzymatic hydrolysis. After grinding, $5.48 \mathrm{~g}$ of wood powder was available from wood decayed by $P$. ostreatus, $2.68 \mathrm{~g}$ of wood powder was available from wood decayed by $T$. versicolor Strain, $2.32 \mathrm{~g}$ of wood powder was available from wood decayed by C. puteana, and $5.6 \mathrm{~g}$ of wood powder was available from the control untreated with a fungus. Of these, two times $1 \mathrm{~g}$ of wood powder was taken in order to determine the content of extractives. Fig. 14 shows that the wood extractive contents after wood degradation by fungi was doubled in samples treated with C. puteana Strain 57 and T. versicolor Strain 6. In contrast, with the comparably low degradation caused by $P$. ostreatus 8F6, no differences in amounts of wood extractives were obtained in comparison to untreated controls (Fig. 14). Enzymatic hydrolysis experiments were carried out with $100 \mathrm{mg}$ milled wood particles per wooden material from the EN $113(1996)$ test at $37^{\circ} \mathrm{C}$ in $50 \mathrm{ml}$ Falcon tubes in $5 \mathrm{ml}$ of $50 \mathrm{mM}$ sodium

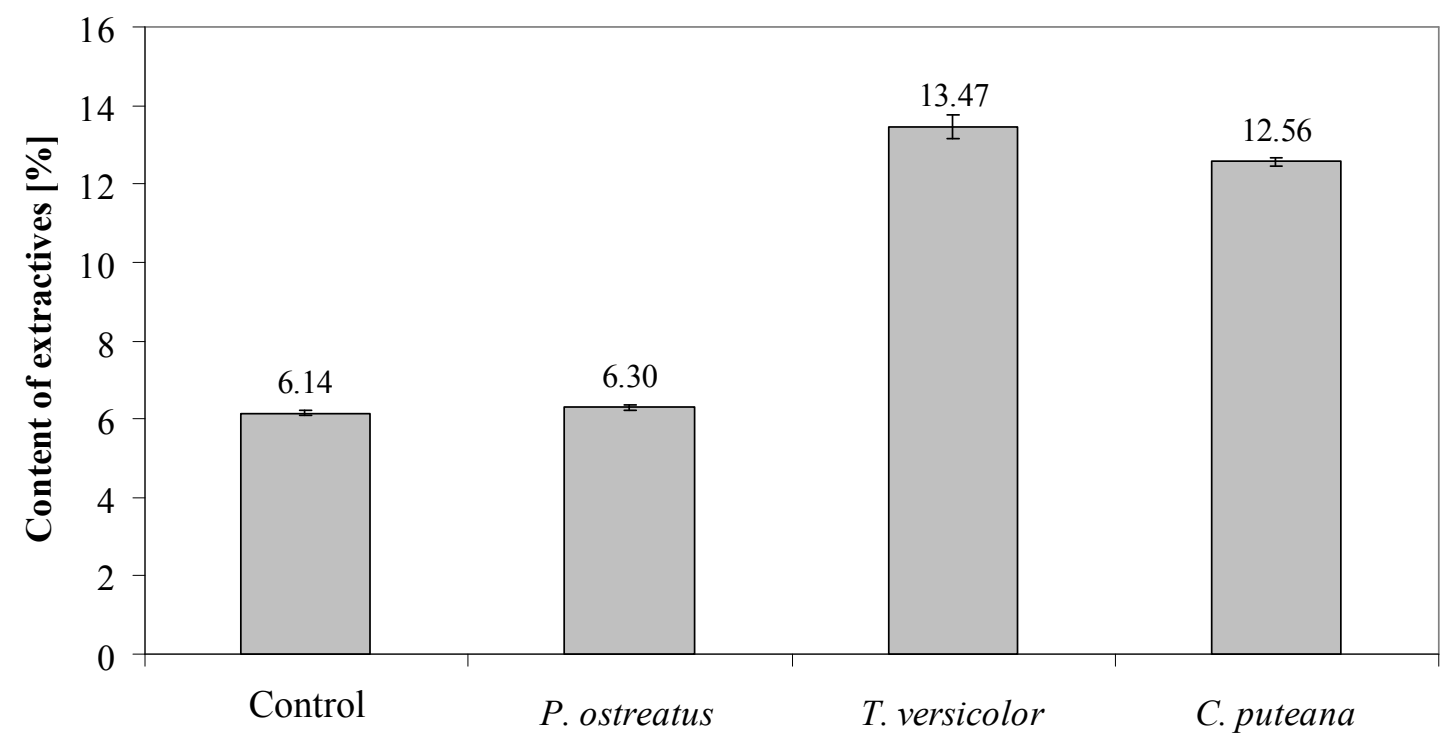

Fig. 14 Contents of extractives in A. grandis mini wood blocks after 19 weeks of incubation with different fungi (compare Fig. S3 in Chapter III) in comparison of uninfected control wood blocks. Mini wood blocks were grinded and wood extractives were eliminated two times $1 \mathrm{~g}$ wood powder by boiling then in water as described in Chapter V.3.7 and the solid material obtained after $2 \mathrm{hrs}$ of boiling was weighed and put in relation of the original wood weight (compare Fig. S3 in Chapter III). Values shown in the figure were calculated from the two parallel treatments done for each case 
acetate buffer, $\mathrm{pH} 5.0$ under shaking at $200 \mathrm{rpm}$. Grinded wood with no fungal treatment was used as control and wood particles pretreated with $85 \%$ phosphoric acid for $20 \mathrm{sec}$ in the microwave (see Fig. 9 and Exp. IV, Table S1 in the supplementary material) were also used for comparison in this test. To all samples, each $4 \mathrm{U} \mathrm{ml}^{-1}$ of cellulase, of xylanase and of laccase were added for enzymatic hydrolysis. After $24 \mathrm{hrs,}$ $500 \mu \mathrm{l}$ samples were removed from the tubes and centrifuged for $16,000 \mathrm{~g}$ for $5 \mathrm{~min}$ and the glucose content in the samples was measured using the GAGO kit.

Fig. 15 presents the results of a conversion of cellulose to glucose from enzymatic hydrolysis of $A$. grandis wood incubated for 19 weeks with fungi or without a fungus according to EN 113 (1996) and, in comparison, of original wood pretreated with $85 \%$ phosphoric acid for $20 \mathrm{sec}$ in a microwave, by using $4 \mathrm{U} \mathrm{ml}^{-1}$ cellulase, $4 \mathrm{U} \mathrm{ml}^{-1}$ xylanase and $4 \mathrm{U} \mathrm{ml}^{-1}$ laccase. The glucose yield was highest (70\%) from wood particles of the original wood (i.e. non-degraded wood) pretreated with $25 \mathrm{ml}$ of $85 \%$ phosphoric acid in the microwave for $20 \mathrm{~min}$. No glucose was yielded in samples with or without added enzymes from wood particles that experienced no pretreatment. On the other hand, wood material obtained from a biological pretreatment (fungal degraded wood) reached up to $47 \%$ and $50 \%$ glucose yields from left material when the white-rots $P$. ostreatus 8F6 and T. versicolor Strain 6 (mass loss of 13\% and 41\%, respectively; see Fig. S3 in Chapter III) were used for wood decay, respectively. The brown-rot fungus $C$. puteana Strain 57 (highest mass loss of 59\%; see Fig. S3 in Chapter III) obtained lowest glucose yields of about 40\% from the material left, which was significantly lower than the values reached with the two white-rot fungi.

White-rot basidiomycetes are known to extensively degrade lignin and they can also somewhat degrade hemicellulose and cellulose (Erikkson et al. 1989; Fengel and Wegener 1983). In contrast, when wood is degraded by brown-rot fungi it generally losses much more cellulose biomass, whereas the lignin and maybe some hemicelluloses remain in the wood cell wall structures (Erikkson et al. 1990; Fengel and Wegener 1983; Kremer 1999). Therefore not surprisingly, wood particles from biological brown-rot pretreatment obtained in this experiment the lowest glucose yields. When considering furthermore the high loss of material through incubation with the brown-rot fungus, the obtained yield of glucose in relation to the amount of original material is with $17.3 \%$ comparably low (Table 3 ). Although the glucose yield from left material after incubation with $T$. versicolor Strain 6 was much better, by the overall mass loss caused by the fungus, the glucose yields relative to the original wood were 


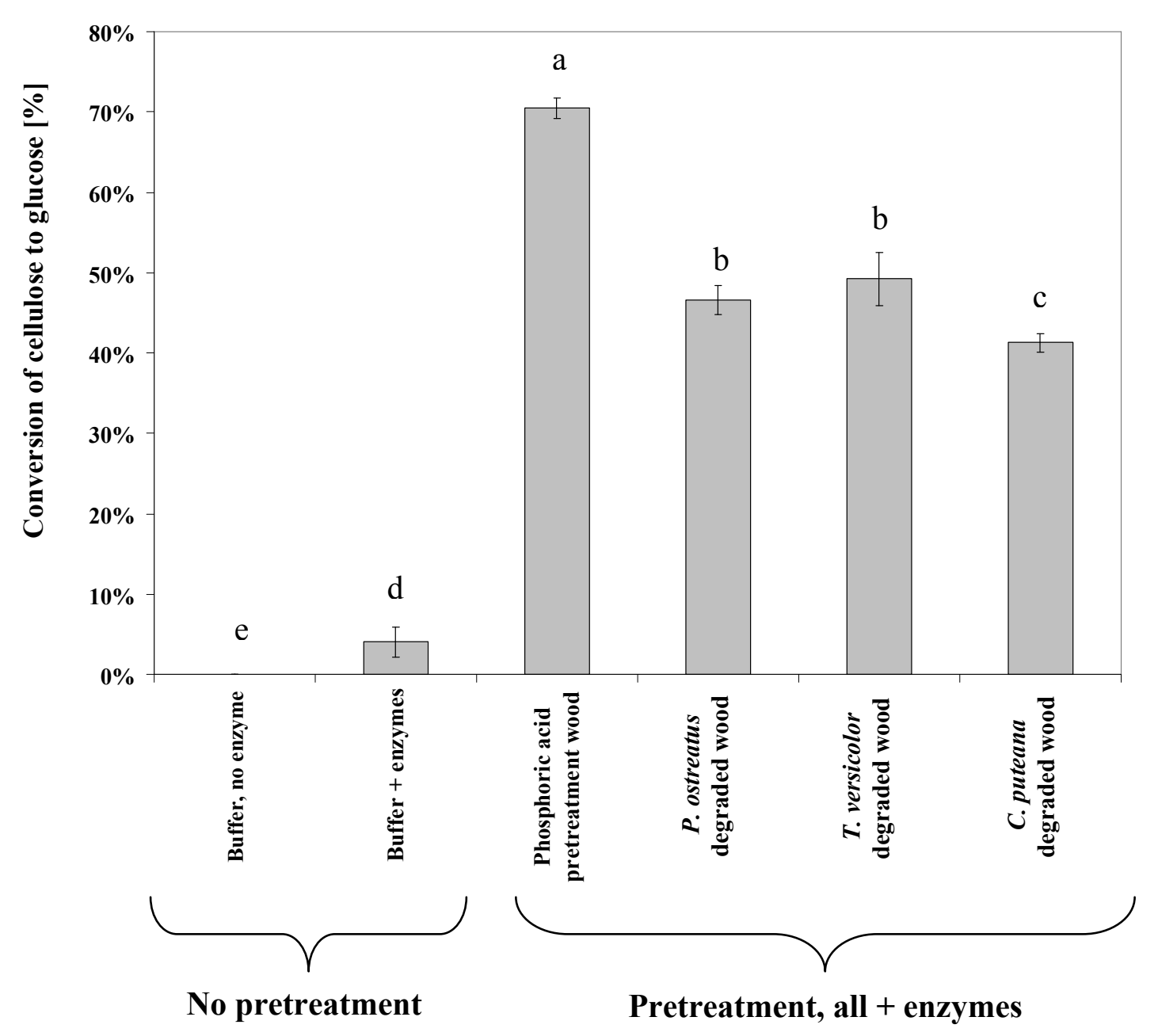

Fig. 15 Enzymatic conversion of cellulose to glucose of $A$. grandis wood particles pretreated prior to enzymatic hydrolysis for $20 \mathrm{sec}$ in a microwave (Intellowave microwave, LG Co., Ltd, Germany) at rating power $1000 \mathrm{~W}$, out power $700 \mathrm{~W}$, working voltage $230 \mathrm{~V}, 50 \mathrm{~Hz}$, with $25 \mathrm{ml}$ of $85 \%$ phosphoric acid or with wood degraded by fungi. Each $100 \mathrm{mg}$ of left cellulosic pellets or wood degraded by fungi were incubated with $4 \mathrm{U} \mathrm{ml}^{-1}$ of cellulase, $4 \mathrm{U} \mathrm{ml}^{-1}$ xylanase and $4 \mathrm{U} \mathrm{ml}^{-1}$ laccase in $50 \mathrm{mM}$ sodium acetate buffer, $\mathrm{pH} 5.0$ for $24 \mathrm{hrs}$ at $37^{\circ} \mathrm{C}$ under constant shaking. Three different samples per pretreatment and three controls with $100 \mathrm{mg}$ original A. grandis wood particles were treated in parallel and average values and standard deviations were calculated. The different superscripts on the chart indicate values of glucose yields that differ significantly $(p<0.05)$ between treatments as based on analysis of variance (ANOVA)

with $26 \%$ only medium high (Table 3 ). In contrast, with $P$. ostreatus $8 \mathrm{~F} 6$ incubation, the overall mass loss was low, and the relative glucose yield of $32.2 \%$ from the original mass therefore comparably high. Knowing that the original A. grandis wood has a cellulose content of $46.3 \%$ (see Table 1), $78 \%$ of the original cellulose has been 
converted to glucose mediated through the biological pretreatment with $P$. ostreatus 8 F6. This compares well with the conversion rates of original cellulose in the $A$. grandis wood following physico-chemical pretreatment of wood with $25 \mathrm{ml}$ phosphoric acid at $20 \mathrm{sec}$ microwaving ( $42.8 \%$ yield of conversion of cellulose to glucose with regards to the original wood applied; compare Fig. 14 and Table 3).

Table 3 Calculation of glucose yields from wooden material of $A$. grandis left after 19 weeks of incubation with fungi according to EN 113 (1996) and in relation to the absolute amounts of original wood applied

\begin{tabular}{lccccc}
\hline Treatment & $\begin{array}{c}\text { Original } \\
\text { weight of } \\
\text { mini wood } \\
\text { blocks } \\
\mathbf{( g ) *}\end{array}$ & $\begin{array}{c}\text { Weight after } \\
\mathbf{1 9} \text { weeks of } \\
\text { incubation after } \\
\text { EN 113 (1996) } \\
(\mathbf{( g )}\end{array}$ & $\begin{array}{c}\text { Glucose } \\
\text { yield from } \\
\mathbf{1 0 0} \mathbf{~ m g ~ l e f t ~} \\
\text { material } \\
\mathbf{( \% )}\end{array}$ & $\begin{array}{c}\text { Calculated } \\
\text { glucose yield } \\
\text { from total } \\
\text { left material } \\
\mathbf{( g ) * *}\end{array}$ & $\begin{array}{c}\text { Yield of glucose } \\
\text { in relation to the } \\
\text { total original } \\
\text { wood mass } \\
\mathbf{( \% ) * *}\end{array}$ \\
\hline Control & 5.60 & 5.60 & 4 & 0.20 & 3.6 \\
P. ostreatus & 6.30 & 5.48 & 41 & 2.03 & 32.2 \\
T. versicolor & 4.54 & 2.68 & 49 & 1.18 & 26.0 \\
C. puteana & 5.67 & 2.32 & 47 & 0.98 & 17.3 \\
\hline
\end{tabular}

* In the calculation was taken into account that $180 \mathrm{~g}$ of hexose $(1 \mathrm{Mol})$ will give $162 \mathrm{~g}$ cellulose when linked together in cellulose chains

** In total, 5 mini blocks wood block of a size of 30 (longitudinal) x 10 (tangential) x 5 (radial) $\mathrm{mm}$ were applied

\section{V.4.5 Influence of wood extractives and specific phenolic compounds on cellulase hydrolysis}

As indicated already from the conclusion from the results presented in Fig. 13 and Chapter V.4.4.2, wood extractives are one of the possible drawbacks of enzymatic hydrolysis processes since the functions of the enzymes might be inhibited by the wood extractives. This section therefore investigates the influences of wood extractives in cellulase hydrolysis carried out in $50 \mathrm{mM}$ sodium acetate buffer, pH 5.0 (Fig. 16). A commercial carboxymethylcellulose (CMC) preparation (product No. 21901, Fluka) was used as a substrate in cellulase hydrolysis at a concentration of $5 \mathrm{mg}$ in $5 \mathrm{~m}$ 

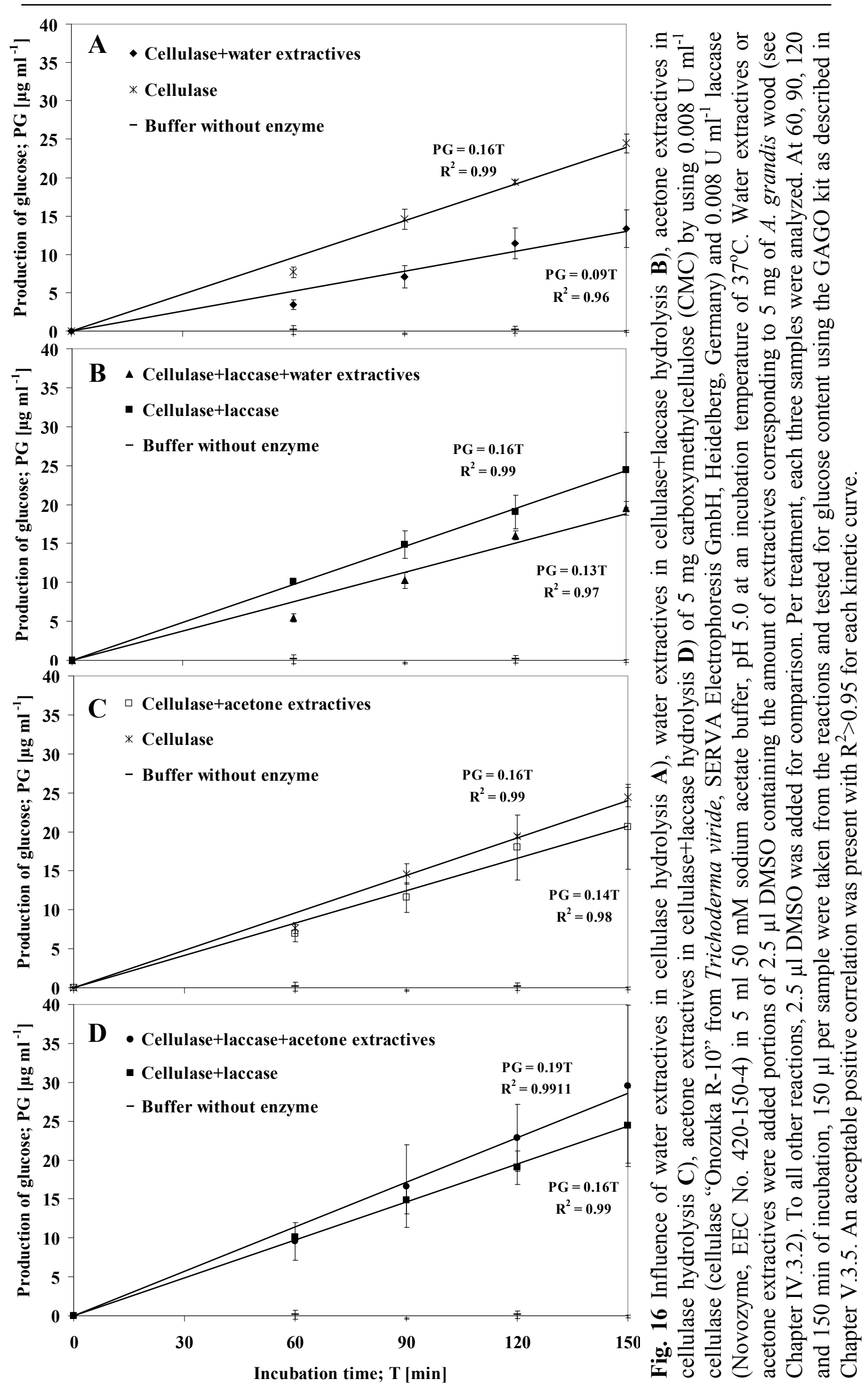
$50 \mathrm{mM}$ sodium acetate buffer $\mathrm{pH} 5.0$ with either $0.008 \mathrm{U} \mathrm{ml}^{-1}$ cellulase (Cellulase “Onozuka R-10" from Trichoderma viride, SERVA Electrophoresis GmbH, Heidelberg, Germany) or $0.008 \mathrm{U} \mathrm{ml}^{-1}$ cellulase and $0.008 \mathrm{U} \mathrm{ml}^{-1}$ laccase (Novozyme, EEC No. 420-150-4) added to the samples for a 2 and a half hour incubation at $37^{\circ} \mathrm{C}$. Water and acetone extractives (obtained from A. grandis wood as described in Chapter V.3.7) were used to simulate the influence of extractives on enzymatic cellulose hydrolysis. Moreover, addition of laccase was also used to possibly improve cellulase hydrolysis under such circumstances.

Fig. 16A shows that water extractives negatively influenced hydrolysis of $5 \mathrm{mg}$ CMC in $50 \mathrm{mM}$ sodium acetate buffer, $\mathrm{pH} 5.0$ by cellulase over the whole time of incubation observed, although the kinetic curve of production of glucose (PG) by CMC hydrolysis through cellulase increased linearly according to the time of incubation $(T)$, (Fig. 16A). At the end of the experiment, the glucose yield was $13 \mu \mathrm{g} \mathrm{ml}^{-1}$ and thus only half as much as achieved with the enzyme without addition of wood extractives $(24 \mu \mathrm{g}$ $\mathrm{ml}^{-1}$; Fig. 16A). However, when adding in addition laccase to the samples, the negative effect by the wood extractives was counteracted and a nearly identical kinetic curve for $\mathrm{CMC}$ hydrolysis was obtained than for $\mathrm{CMC}+$ cellulase alone (compare Fig. 16A and 16B).

Acetone extractives in contrast affected hydrolysis by cellulase and by cellulase+laccase comparably little (Fig. 16C). There was no apparent difference in production of glucose in samples with cellulose with and without acetone extractives and also not in comparison to hydrolysis of CMC with cellulase+laccase in presence of acetone extractives (Fig. 16C and 16D). Very likely, the majority of hydrolysis inhibitors of celluloses were present in water extractives. Specific compounds within the wood extractives of the A. grandis wood were therefore tested in reaction with enzymatic hydrolysis of CMC.

According to results presented in Chapter IV.4.5, p-coumaric acid (4-hydroxy cinnamic acid), vanillic acid (3-methoxy-4-hydroxybenzoic acid) and ferulic acid (4-hydroxy-3-methoxy cinnamic acid) are active in inducing a high laccase production. These phenolic compounds ( $p$-coumaric acid, vanillic acid and ferulic acid) were also found in the highest amounts within water extractives (Table 1 in Chapter IV). These phenolic compounds in pure commercial form were therefore added in different concentrations ( $1 \mathrm{mM}$ and $1 \mu \mathrm{M})$ to reactions of enzymatic CMC hydrolysis carried out in $50 \mathrm{mM}$ sodium acetate buffer, $\mathrm{pH} 5.0$ (Fig. 17). 


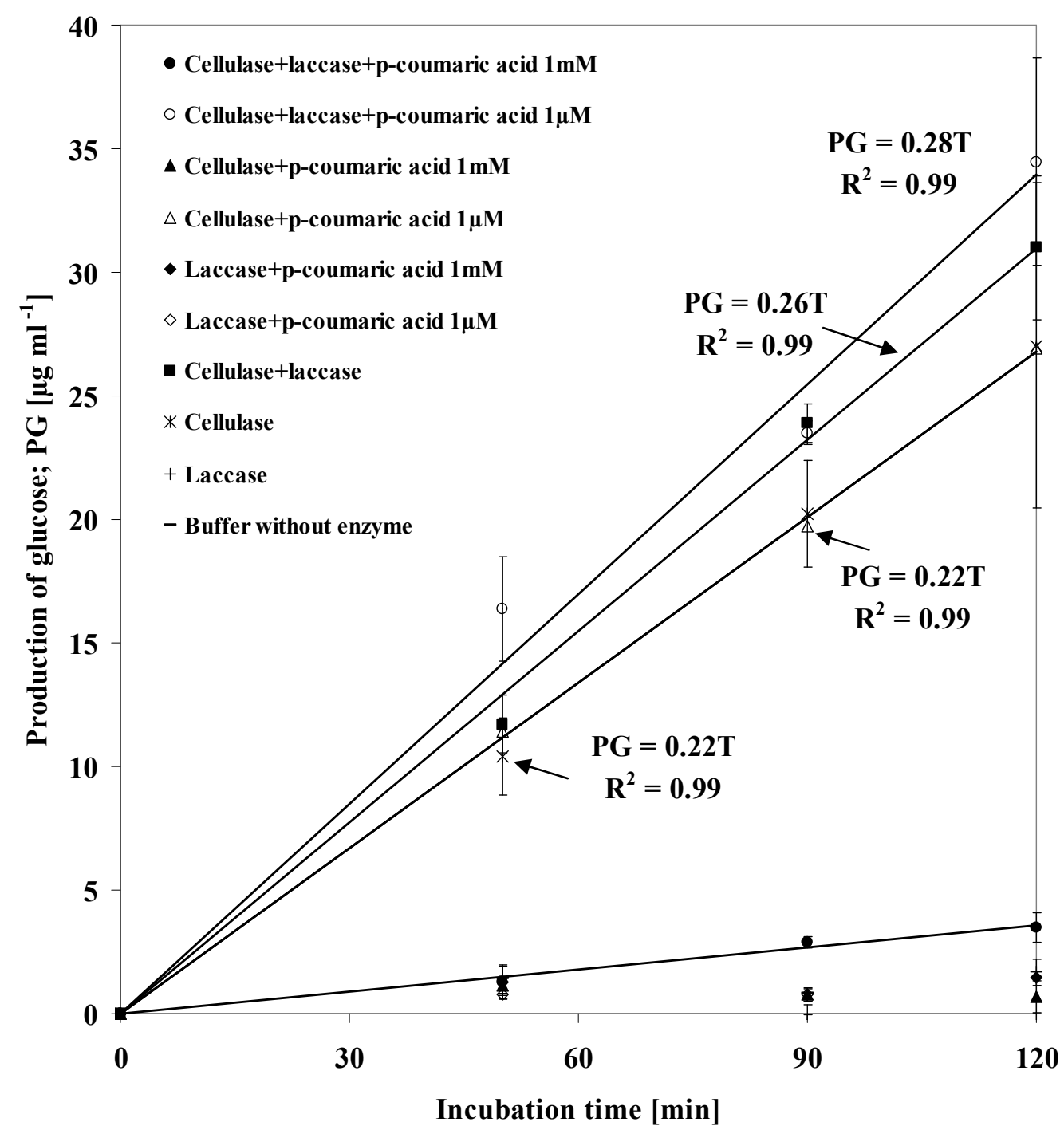

Fig. 17 Influence of addition of $p$-coumaric acid on hydrolysis of $5 \mathrm{mg}$ CMC by cellulase in presence and absence of laccase. $0.008 \mathrm{U} \mathrm{ml}^{-1}$ cellulase (Cellulase "Onozuka R-10" from Trichoderma viride, SERVA Electrophoresis GmbH, Heidelberg, Germany) and $0.5 \mathrm{U} \mathrm{ml}^{-1}$ of purified laccase $\mathrm{V}$ of $C$. cinerea Okayama 7 in $5 \mathrm{ml}$ of $50 \mathrm{mM}$ sodium acetate buffer, $\mathrm{pH} 5.0$ were used. $p$-Coumaric acid was dissolved in $100 \mu \mathrm{l}$ DMSO. To all other samples, for reasons of comparison, $100 \mu 1$ of pure DMSO was added. Per treatment, three different samples were followed up in glucose formation over the time. At 60, 90, and $120 \mathrm{~min}$ of incubation, per sample aliquots of $150 \mu \mathrm{l}$ were taken and analyzed for glucose content with the GAGO kit as described in Chapter V.3.5

In this series of tests with $5 \mathrm{mg}$ of commercial CMC preparation, again $0.008 \mathrm{U} \mathrm{ml}^{-1}$ cellulase was used and $0.5 \mathrm{U} \mathrm{ml}^{-1}$ of purified laccase $\mathrm{V}$ from Coprinopsis cinerea Okayama 7. As expected, the purified laccase added into samples with just 
CMC did not lead to recognizable conversion of cellulose into glucose like in the samples with no added enzyme and unlike in the samples where just cellulase was added to the CMC (Fig. 16 till Fig. 19). Additions of the three different phenolic compounds always lead to a reduction cellulase activity.

The results in Fig. 16 show that $p$-coumaric acid at a high concentration (1mM) nearly fully blocked the enzymatic hydrolysis of CMC whereas production of glucose was to a certain level reduced at the lower concentration of $1 \mu \mathrm{M}$ of $p$-coumaric acid (Fig. 17). At the high concentration of $p$-coumaric acid (1 mM), as in controls without added enzymes, a neglectable rate of production of glucose much below than $0.03 \mu \mathrm{g} \mathrm{ml}^{-1} \mathrm{~min}^{-1}$ were obtained in comparison to a rate of production of glucose of $0.22 \mu \mathrm{g} \mathrm{ml}^{-1} \mathrm{~min}^{-1}$ at the low concentration of $p$-coumaric acid. When laccase was added to samples with cellulase and $1 \mu \mathrm{M} p$-coumaric acid, the hydrolysis activity was fully restored with a highest rate of glucose production of about $0.28 \mu \mathrm{g} \mathrm{ml}^{-1} \mathrm{~min}^{-1}$, respectively even increased when compared to rates of production of glucose in samples with cellulase alone $\left(0.22 \mu \mathrm{g} \mathrm{ml}^{-1} \mathrm{~min}^{-1}\right)$. Also, some activity was restored upon addition laccase to samples with cellulase and $1 \mathrm{mM}$-coumaric acid, although the production rate of glucose was very low with a rate of $0.03 \mu \mathrm{g} \mathrm{ml}^{-1} \mathrm{~min}^{-1}$ (Fig. 17).

Notable is further that the combination of the cellulase + laccase without addition of $p$-coumaric acid had also a better rate of glucose production $\left(0.26 \mu \mathrm{g} \mathrm{ml}^{-1}\right.$ $\left.\min ^{-1}\right)$ than cellulase alone $\left(0.22 \mu \mathrm{g} \mathrm{ml}^{-1} \mathrm{~min}^{-1}\right)$, (Fig. 17).

Addition of vanillic acid (Fig. 18) followed the same trend in the production of glucose than previously observed with samples with added $p$-coumaric acid (Fig. 17). A high concentration of vanillic acid $(1 \mathrm{mM})$ resulted in a rate of production of glucose of less than $0.12 \mu \mathrm{g} \mathrm{ml}^{-1} \mathrm{~min}^{-1}$, whereas in comparison a low concentration of vanillic acid $(1 \mu \mathrm{M})$ resulted in a rate of production of glucose of $0.20 \mu \mathrm{g} \mathrm{ml}^{-1} \mathrm{~min}^{-1}$ which was somewhat reduced as compared to the rate of production of $0.22 \mu \mathrm{g} \mathrm{ml}^{-1} \mathrm{~min}^{-1}$ of glucose in samples with only cellulase and CMC as substrate. Upon addition of laccase to samples with cellulase and vanillic acid at a concentration of $1 \mu \mathrm{M}$ vanillic acid, highest rate of production of glucose with about $0.34 \mu \mathrm{g} \mathrm{ml}^{-1} \mathrm{~min}^{-1}$ a same trend was measured and at a concentration of $1 \mathrm{mM}$ vanillic acid, a reduced rate of about $0.12 \mu \mathrm{g} \mathrm{ml}^{-1} \mathrm{~min}^{-1}$. Thus, rates of production of glucose in presence of laccase in combination with cellulase and a low amount of a phenolic compound $(1 \mu \mathrm{M})$ and in presence of laccase in combination with cellulase without any added phenolic 


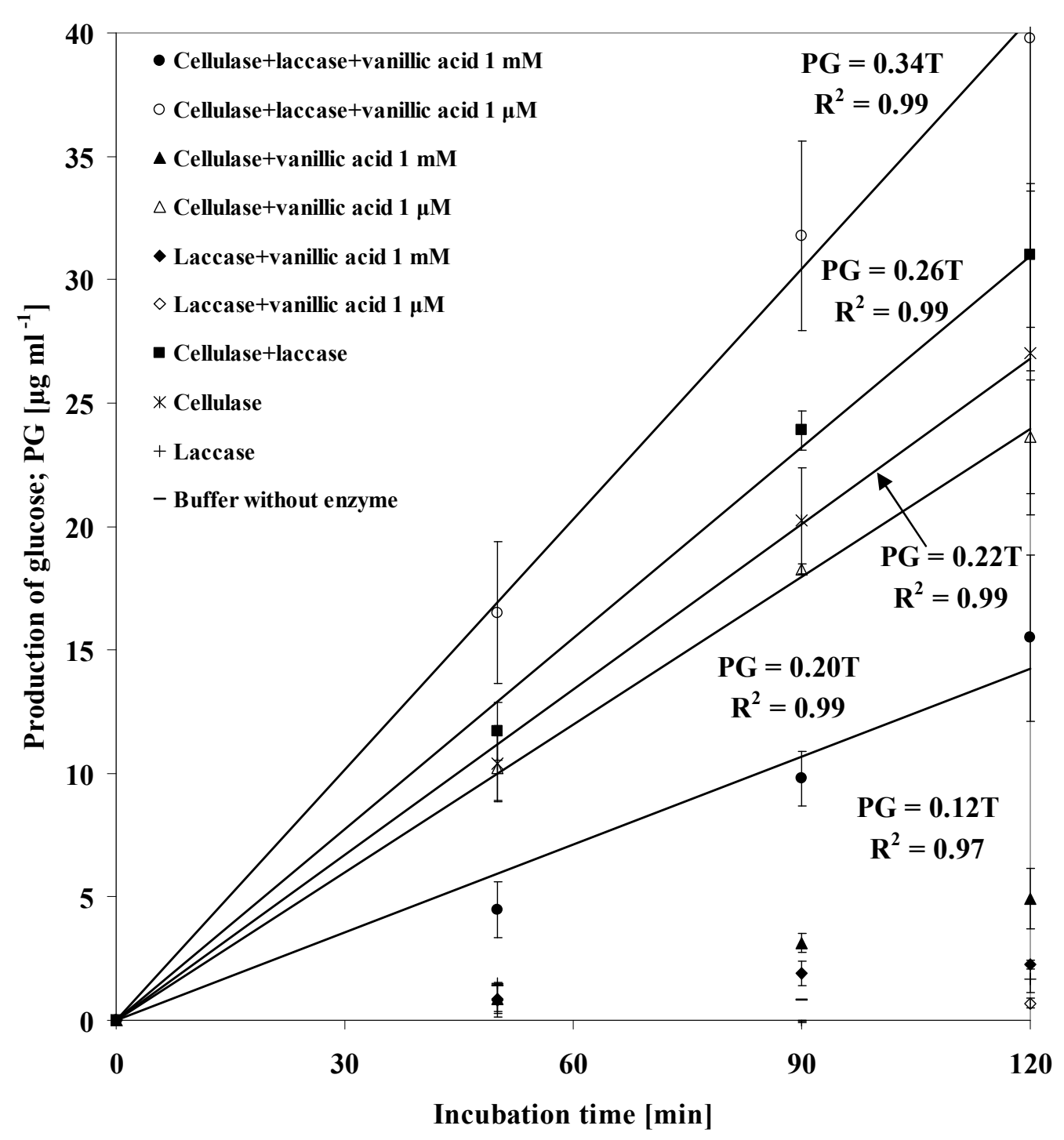

Fig. 18 Influence of addition of vanillic acid on hydrolysis of $5 \mathrm{mg}$ CMC by cellulase in presence and absence of laccase. $0.008 \mathrm{U} \mathrm{ml}^{-1}$ cellulase (Cellulase "Onozuka R-10" from Trichoderma viride, SERVA Electrophoresis GmbH, Heidelberg, Germany) and $0.5 \mathrm{U} \mathrm{ml}^{-1}$ of purified laccase $\mathrm{V}$ of $C$. cinerea Okayama 7 in $5 \mathrm{ml}$ of $50 \mathrm{mM}$ sodium acetate buffer, $\mathrm{pH} 5.0$ were used. Vanillic acid was dissolved in $100 \mu 1$ DMSO. To all other samples, for reasons of comparison, $100 \mu 1$ of pure DMSO was added. Per treatment, three different samples were followed up in glucose formation over the time. At 60, 90, and 120 min of incubation, per sample aliquots of $150 \mu 1$ were taken and analyzed for glucose content with the GAGO kit as described in Chapter V.3.5

compound $\left(0.26 \mu \mathrm{g} \mathrm{ml}^{-1} \mathrm{~min}^{-1}\right)$ were obtained that were higher than the rate of production of glucose in samples with just cellulase alone.

When testing effects of ferulic acid (Fig. 19), at the high concentration of $1 \mathrm{mM}$ no CMC hydrolysis by cellulase was achieved but a nearly normal rate of production of 
glucose of less than $0.21 \mu \mathrm{g} \mathrm{ml}^{-1} \mathrm{~min}^{-1}$ was observed when the low concentration of $1 \mathrm{mM}$ of ferulic acid was added to samples. In comparison, upon addition of laccase to samples with the low concentration of ferulic acid $(1 \mu \mathrm{M})$ a rate of production of glucose over $0.15 \mu \mathrm{g} \mathrm{ml}^{-1} \mathrm{~min}^{-1}$ was obtained. These results suggest that by the actions of laccase in combination with ferulic acid, negative actions on the hydrolysis of cellulose to glucose via the applied cellulase occur. Laccases transfer ferulic acids into different radicals and these may form several different ferulic acid dimers and trimers (Carunchio et al. 2001; Steffensen et al. 2009; Ward et al. 2001). Moreover, in presence of ferulic acid, laccase may oligomerize peptides containing cysteine and crosslink tyrosine containing peptides (Carunchio et al. 2001; Steffensen et al. 2009). On longer peptides, cross-linking may particularly often occur when tyrosines are present close to the C-terminal end (Steffensen et al. 2009). The Onozuka cellulose R-10 applied in this study is indeed a mixture of various cellulases for T. viride: six endoglucanases (Endo I to Endo VI), three exoglucanases (Exo I to Exo III) and a $\beta$-glucosidase are present in the enzyme mixture (Beldman et al. 1985). Of these, Exo I and Endo I for example have an outmost C-terminal 30 amino-acid long CBM domain (cellulose binding motif) with four cysteine and four tyrosines (Cheng et al. 1990; GenBank accession number ACC59774), offering a good target for ferulic-acid-mediated cross-linking through laccase action, presenting an explanation for then here observed reduction in cellulase activity on CMC by the presence of laccase plus $1 \mu \mathrm{M}$ ferulic acid (Fig. 19). In contrast, other cellulases of $T$. viride such as Endo II (GenBank BAA 36216) and Endo III (GenBank AAQ 21383) and two other endoglucanases with not yet assigned number (GenBank ADJ10627 and ABQ95572) have an N-terminal CBM domain, offering both protection from ferulic-acid mediated cross-linking by laccase. Accordingly, the low hydrolysis activity $\left(0.15 \mu \mathrm{g} \mathrm{ml}^{-1} \mathrm{~min}^{-1}\right)$ on cellulose in samples with added laccase and $1 \mu \mathrm{M}$ ferulic acid may be due to such cellulase being less accessible from inactivation. 


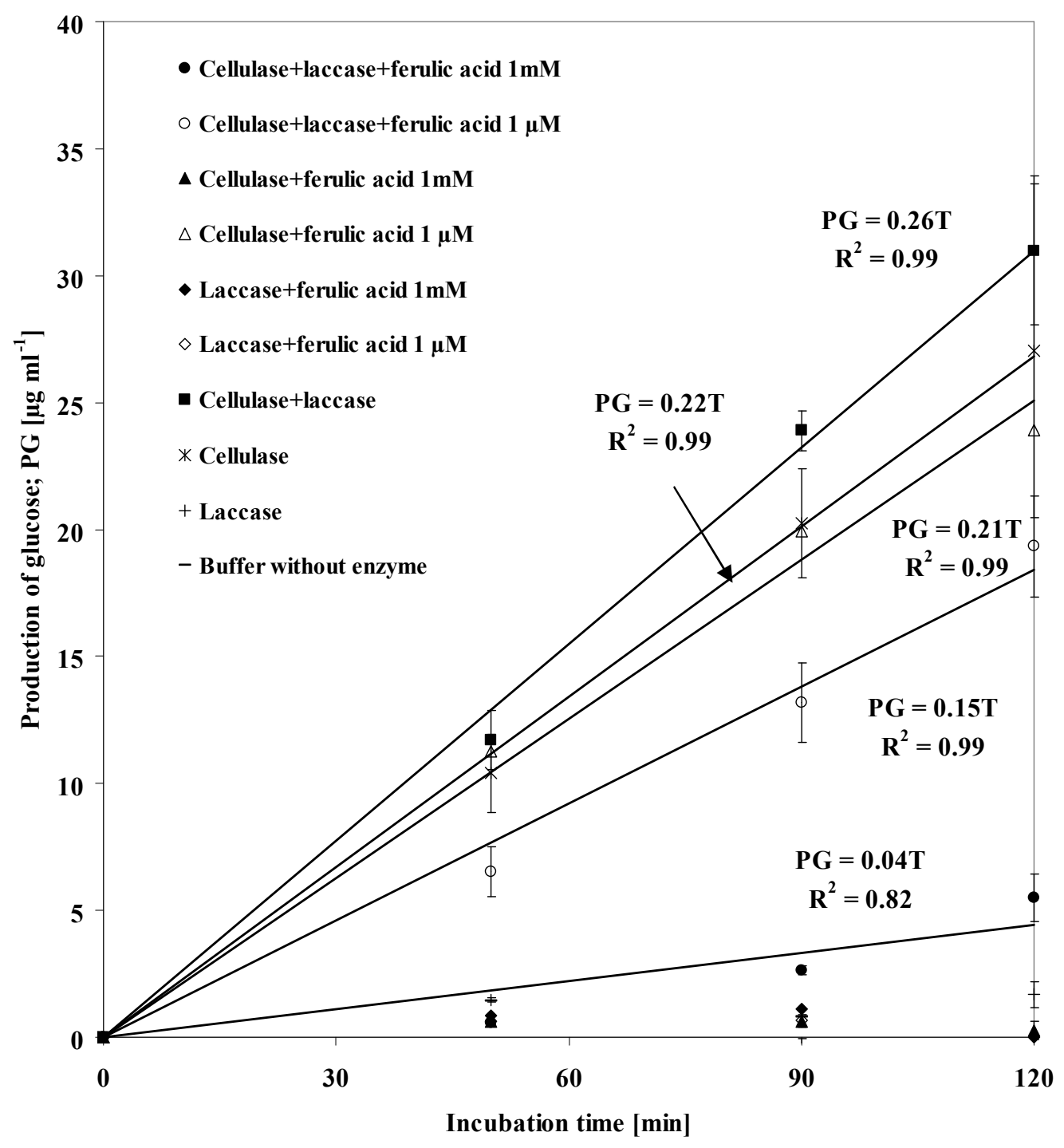

Fig. 19 Influence of addition of ferulic acid on hydrolysis of $5 \mathrm{mg}$ CMC by cellulase in presence and absence of laccase. $0.008 \mathrm{U} \mathrm{ml}^{-1}$ cellulase (Cellulase "Onozuka R-10" from Trichoderma viride, SERVA Electrophoresis GmbH, Heidelberg, Germany) and $0.5 \mathrm{U} \mathrm{ml}^{-1}$ of purified laccase $\mathrm{V}$ of $C$. cinerea Okayama 7 in $5 \mathrm{ml}$ of $50 \mathrm{mM}$ sodium acetate buffer, $\mathrm{pH} 5.0$ were used. Ferulic acid was dissolved in $100 \mu \mathrm{l}$ DMSO. To all other samples, for reasons of comparison, $100 \mu 1$ of pure DMSO was added. Per treatment, three different samples were followed up in glucose formation over the time. At 60, 90, and 120 min of incubation, per sample aliquots of $150 \mu 1$ were taken and analyzed for glucose content with the GAGO kit as described in Chapter V.3.5. Note that for the curve calculated for samples with added cellulase, laccase and $1 \mathrm{mM}$ ferulic acid, the $\mathrm{R}^{2}$ was only low with 0.82 , indicating a low reliability of the calculated value for the rate of production of glucose via the applied cellulose occur 


\section{V.5 Discussion}

\section{V.5.1 $\mathrm{A}$. grandis softwood as a resource in environmentally friendly production of glucose for biofuels}

Nowadays, much research has been performed and more and more research concentrates on transforming lignocellulose based on enzymatic hydrolysis to produce bioethanol. Most efforts on the first generation of biofuels have been focused on using biological resources that are also used as food and fodder (such as sugarcane, cereals, soybeans, seed from oil plants, animal fat) and this development was induced by the various kinds of the world's demand and supply of energy consumption. However, the first generation biofuel competes directly with nourishing humans and animals. Therefore, these resources are limited in available amounts. According to this problematic (Pimentel and Patzek 2005; Stöcker et al. 2008), the new (second) generation of biofuels is targeted to use non-food and non-fodder materials as substrates in enzymatic hydrolysis (Li et al. 2008; Taherzadeh and Karimi 2008; Zhu and Pan 2010). Straw and other agricultural residues as well as certain fast growing grasses (e.g. Miscanthus) are established biomaterials for second generation biofuels' production (Majcherczyk and Hüttermann 2007). Much work has now also been carried out on using e.g. hardwood trees from forests and plantations, such as on wood of poplars and olive trees, respectively (Cara et al. 2007; Kumar 2009; Zhang et al. 2007). Less work has been invested into using softwood in biofuel production (Galbe and Zacchi 2007).

A. grandis wood is especially attractive because $A$. grandis is a fast growing coniferous tree with a high potential for sustainable wood production and also interesting applications in the wood products industries (Röhrig 1981; Spellmann et al. 2010; Vos and Kharazipour 2008, 2010). Moreover, as shown by the research in this $\mathrm{PhD}$ thesis, the renewable wood biomass from $A$. grandis is also a potential source for enzymatic treatment technologies to unlock the energy stored in its lignocellulose for transferring it into biofuels (this chapter). In this work, the A. grandis softwood has been explored using different pretreatment methods and different combinations of hydrolytic and oxidizing enzymes (cellulase, xylanase, $\beta$-glucosidase, laccase) in wood hydrolysis. The different pretreatments resulted in the production of fermentable sugars from the wood cellulose to possibly be used as a bio-base for fermentation processes of bioethanol with e.g. suitable yeast (Galbe and Zacchi 2007; Kumar et al. 2009). In the 
near future, process integration and optimization to transform the whole wood of trees such as from $A$. grandis into value-added and biochemical products, such as ethanol, but also butanol and other bio-based chemicals such as lactic acid, succinic acid etc. could become promising attempts to fulfill the world's energy consumption (Mtui 2009; Sun and Cheng 2002).

The research presented here addresses new enzymatic technologies as required if biofuels by environmentally friendly production are to significantly contribute to planetary energy needs and, with it, to the reduction of greenhouse gas emission (Pimentel and Patzek 2005). Next to other developing technologies such as creating better yeast strains for sugar conversion (Hu et al. 2008; Kumar et al. 2009), efficient hydrolysis protocols for wood have to be established in finally enabling the commercial viability of lignocellulosic ethanol (Mtui 2009; Li et al. 2008; Taherzadeh and Karimi 2008). The purpose for this is on the development of approaches and technologies with a significant promise in reducing production cost and in caring for environmentally friendly procedures in order to make lignocellulosic ethanol economically competitive with the first generation biofuel production. This knowledge is thus urgently needed and also knowledge from timely research in how to integrate enzymatic hydrolysis of material such as wood into already existing basic fermentation processes in order to adjust the available biotechnology for biofuel production to the specific challenges meet when transforming cellulose from wood e.g. into ethanol ( $\mathrm{Li}$ et al. 2008; Stöcker et al. 2008; Taherzadeh and Karimi 2008).

\section{V.5.2 Chemical and physico-chemical pretreatment of $A$. grandis wood for glucose production}

Well established is by the high resistancy of the material, that hydrolyzing enzymes added to raw wood material are inefficient in transferring the lignocellulose into fermentable sugars (Galbe and Zacchi 2007; Kumar et al. 2009). Not surprisingly, in this study this was confirmed to be also true for A. grandis wood (see Fig 5 to Fig. 10). Different types of physical pretreatment, such as milling, irradiation by gamma rays, ultrasound, application of electron beams, microwaving, hydrothermal incubation, high pressure steaming, extrusion, expansion and pyrolysis were studied on different wood types such as from poplar, Douglas fir, eucalyptus, maple, olive tree, red oak, beech, rubber wood, pine, and spruce (Galbe and Zacchi 2007; Hu et al. 2008; Taherzadeh and 
Karimi 2007; Taherzadeh and Karimi 2008). The results generally show that physical treatment alone will not be good enough for good subsequent release of sugars from the wooden material.

Combinations of chemical and physical pretreatment are more efficient (Galbe and Zacchi 2002; Hu et al. 2008; Taherzadeh and Karimi 2008). A combination such as phosphoric acid pretreatment and energy-efficient incubation with microwave irradiation could therefore turn out to be optimal in terms of economical and environmental aspects. So far, there are however no intensive studies in the literature on the effectiveness of such combination of measures on lignocellulosic material. Only Zhang et al. (2009) studied the effect of the combined measures on pure cellulose. Cellulose consists in different forms: soluble, non-crystalline, amorphous cellulose and crystalline cellulose (Fengel and Wegener 1983). Fungal enzymes such as found in the Onozuka R-10 preparation consisting of a mixture of different endo- and exocellulases have different affinity to this different forms of cellulose (Beldman et al. 1985). Particularly the crystalline form presents a challenge to enzymatic degradation due to the presence of extensive intra- and inter-molecular hydrogen bonds and van der Waals interactions (Fengel and Wegener 1983). Depolymerization of the cellulose by strong acids such as sulfuric acid is well assisted when performed simultaneously with ionic liquids in a microwave. Total reducing sugars up to $37 \%$ yields of the cellulose were obtained within times of $8 \mathrm{~min}$ irradiation (Zhang and Zhao 2009). Concentrated sulfuric acid or phosphoric acids can fully dissolve the crystalline structure of cellulose (Xiang et al. 2003; Zhang et al 2006). However, enzymatic treatment requires mild acid concentrations in order not to destroy the enzymes' functions (Zhang et al. 2007). Reducing the required amounts of applied acids as well as energy in the pretreatments can be economical and environmental friendly advantages of using an acid-microwave pretreatment prior to an enzymatic reaction, in particular when more complex material than pure cellulose is concerned, such as here in this study the wood from A. grandis. Phosphoric acid even in concentrated form is a modest acid as compared to sulfuric acid and it acts rather as a solvent of cellulose than in an aggressive way in cellulose hydrolysis. Application of phosphoric acid is thus preferred over application of sulfuric acid when wanting to separate polysaccharides of different length (different degree of polymerization) since the degree of hydrolysis can easily be controlled by temperature and incubation times (Zhang et al. 2007). 
Microwave-chemical pretreatment with phosphoric acid per se and in comparison with just chemical pretreatment using phosphoric acid and with biological pretreatments was therefore an interesting approach to follow up in this study with A. grandis wood. The results revealed that several parameters involved in glucose yields, such as amount, type and concentration of the liquid acid applied or the incubation time of microwave irradiation play important roles for the success of releasing high amounts of glucose from the A. grandis wood (Fig. 5 to Fig. 10).

In this study, upon the optimization of $A$. grandis wood pretreatment, highest glucose yields were obtained with $1 \mathrm{~g}$ A. grandis wood particles in $8 \mathrm{ml}$ of $85 \%$ phosphoric acid incubated for one hour at $50^{\circ} \mathrm{C}$ in a hot water bath with $70 \%$ conversion of cellulose into glucose regarding the mass loss of the left cellulosic pellet (Fig. 5). Importantly, this relates to a cellulose conversion of $92.4 \%$ of the cellulose present in the original wood. With microwave incubation of $1 \mathrm{~g}$ A. grandis wood particles for $5 \mathrm{sec}$ in $8 \mathrm{ml}$ of $85 \%$ phosphoric acid, and with microwave incubation for $20 \mathrm{sec}$ of $1 \mathrm{~g}$ A. grandis wood particles in $25 \mathrm{ml}$ of $85 \%$ phosphoric acid, similar high values of absolute cellulose to glucose conversion were achieved (Fig. 9).

Zhang et al. (2007), (Table 4) and Zhang et al. (2009), (Table 5) by their studies have pointed out the different advantages of chemical and physico-chemical pretreatments for release of sugars from complex biological materials. The purposes of these type of studies aim to reduce the cost for size reduction of feedstocks, and to reduce the cost of materials for construction of pretreatment reactors which when aggressive chemicals (acids or alkalines) are to be an applied must be of non-corrosive material (Rabelo et al. 2009).

Table 4 Digestibility of cellulose (containing) substrates after pretreatment of the materials by phosphoric acid in subsequent enzymatic hydrolysis exerted by a combination of cellulase and $\beta$-glucosidase (Zhang et al. 2007)

\begin{tabular}{clc}
\hline Entry & Cellulose type & Cellulose digestibility (\%) \\
\hline 1 & Avicel & 100 \\
2 & a-cellulose & 100 \\
3 & Corn stove & 97 \\
4 & Switchgrass & 96 \\
5 & Hybrid poplar & 97 \\
6 & Douglas fir & 75 \\
\hline
\end{tabular}


Table 5 Glucose yields of different cellulose (containing) substrates after chemical hydrolysis with ionic + solid acid catalysts ( $\mathrm{Si}$ and $\mathrm{Al}$ ) using microwave incubation (Zhang et al. 2009)

\begin{tabular}{clc}
\hline Entry & Cellulose type & Glucose yield (\%) \\
\hline 1 & Avicel & 36.9 \\
2 & a-cellulose & 34.9 \\
3 & Spruce & 34.5 \\
4 & Sigmacell & 32.5 \\
\hline
\end{tabular}

Zhang et al. (2009) analyzed just pure cellulose in microwave irradiation in presence of sulfuric acid (Table 5). However, lignocellulosic materials including wood present further problems: not only the crystallinity of cellulose and the degree of polymerization has to be addressed, but also the available surface area that is reduced due to covering the cellulose by lignin and hemicellulose, and the general degree of lignin content in the material, and also the reduced moisture content in dependence of lignin present further new challenges (Chang and Holtzapple 2000). Treatments by acid-assisted microwave irradiation of more "easy" substrates such as plant-materialcontaining swine manure and biodegradable municipal solid waste fractions have been reported ( $\mathrm{Li}$ et al. 2007; Lie et al. 2009) as well as treatment by alkali-assisted microwave irradiation of wheat and rice straw (Saha et al. 2008; Zhu et al. 2005, 2006a, b, c) and of Switchgrass (Hu and Wen 2008; Keshwani and Cheng 2010). Since wood is much more recalcitrant than all these materials, high steam pressure during extensive microwave treatment might be applied as a type of steam explosion treatment. However, this high energy consuming step does not necessarily lead to the high release of cellulose for subsequently enzymatic hydrolysis since it may only be the cellular tissue structure that will be destroyed by such treatment ( $\mathrm{Li}$ et al. 2010). Cooperation of the combined chemical and physical pretreatments it is instead expected that the physical pretreatment would increase pores sizes and accessible surface area and that the chemical partner in the treatment would decrease the degree of polymerization and crystallinity of cellulose (Zhang et al. 2009).

Several kinds of substrates, such as Avicel cellulose, $\alpha$-cellulose, corn stove, Switchgrass, hybrid poplar and Douglas fir wood were pretreated in a study by 
Zhang et al. (2007) in an 1:8 reaction of 83-85.9\% phosphoric acid:dry wood biomass and incubated in a rotary water bath with $90 \mathrm{rpm}$ at $50 \pm 2^{\circ} \mathrm{C}$ for $(45-60 \mathrm{~min})$ and the resulting material (10 $\mathrm{g} \mathrm{l}^{-1}$ glucan) was hydrolyzed for $24 \mathrm{hrs}$ at $50 \pm 2^{\circ} \mathrm{C}$ with $15 \mathrm{FPU}$ Genencor Spezyme CP cellulase and 60 IU novozyme $188 \beta$-glucosidase. The results of the treatments in terms of cellulose digestibility were calculated based on the definition of the conversion of cellulose, i.e. the ratio of the amount of soluble sugars (glucose equivalent) in the liquid phase after hydrolysis to the initial amount of glucose equivalent in the solid cellulose sample before hydrolysis. In the study by Zhang et al. (2007), the lowest value of $8 \mathrm{ml}$ of $85 \%$ phosphoric acid was found e.g. for $1 \mathrm{~g}$ of poplar and $1 \mathrm{~g}$ of Douglas fir wood to be the optimal condition for highest glucose yield. Of these two wood types, hardwood poplar reached a higher cellulose digestibility (97\%) than softwood Douglas fir (75\%). However, all the other substrates tested by the authors obtained cellulose digestibility values in orders as high as 95-100\% (Table 4).

In a later study, the same authors applied individual substrates such as Avicel, $\alpha$-cellulose, spruce, and Sigmacell to study the effects of microwave incubation on chemical release of sugars (Table 5). Microwaving was carried out with different types of celluloses in order to hydrolyze these with ionic liquid and solid acid catalysts ( $\mathrm{Si}$ and $\mathrm{Al}$ ) through incubation in the microwave with subsequent measurements of determining the reached glucose yields. Microwave incubation showed thus a potential to produce glucose yields from the different materials in the range of $32-37 \%$ (Zhang et al. 2009; Table 5). Whether a subsequent enzyme treatment would result in even higher yields of glucose was not addressed by these authors.

As stated already above, in this $\mathrm{PhD}$ study comparable good glucose yields with A. grandis wood were obtained with the chemical pretreatment of $1 \mathrm{~g}$ of material in $8 \mathrm{ml} 85 \%$ phosphoric acid at $50^{\circ} \mathrm{C}$ in a hot bath for $1 \mathrm{hr}(70 \%$ of conversion of mass of the cellulosic pellet left into glucose, Fig. 5) and with the physico-chemical pretreatments of $1 \mathrm{~g}$ of material in $8 \mathrm{ml} 85 \%$ phosphoric acid in $5 \mathrm{sec}$ microwave irradiation or with physico-chemical pretreatments of $1 \mathrm{~g}$ of material in $25 \mathrm{ml} \mathrm{85 \%}$ phosphoric acid in $20 \mathrm{sec}$ microwave irradiation (giving similar values of $70 \%$ conversion of mass of the cellulosic pellet into glucose, Fig. 9). In order to consume as less as possible energy, the microwaving pretreatment is rather preferred and of the microwaving pretreatments the one with application of only $8 \mathrm{ml} 85 \%$ phosphoric acid with short time irradiation in order to save as much as possible on the 
costs for acids and use as little as possible of an acidic compound of a potential environmental hazard.

In this study, various experiments have been performed to analyze effects of different parameters on chemical and physico-chemical pretreatments: different types of acids, different volumes of incubation, different concentrations of acid, different supplementary liquids, different types of heat, and different times of microwaving and heat supply (Fig. 5 to Fig. 10). The study is certainly not complete in what type of effects can be studied. However, from the results so far available, the most advisable treatment would be to use short time of microwaving and low amounts of $80-85 \%$ phosphoric acid. In the future, not only timings of microwaving might be further addressed but most importantly also the amount of microwave energy applied in these timings in order to see whether still more energy can be saved in the treatments without a reduction in later enzymatic glucose yields. As discussed in the following Chapter V.5.3, application of suitable enzymes such as laccase or also $\beta$-glucosidase can dramatically reduce the microwave energy and the amount of acid required for later good release of glucose from cellulose by enzymatic hydrolysis. However, as long as enzymes are matters of high cost, concentration needs further to be given on physicochemical pretreatments of wood that allows best yields of glucose from cellulose under the appointment of amounts of enzymes as little as possible.

Usually, enzyme mixtures containing various cellulases are appointed in degradation of (pretreated) lignocellulosic materials (Beldman et al. 1985). Here we used cellulase "Onozuka R-10" alone or in combination with a xylanase from $T$. viride (Fluka). Whilst the cellulase mixture was efficient in glucose release, the xylanase alone was not as much. In combination, the final yields of glucose by both enzymes together were not much better than when using the cellulase Onozuka R-10 alone (Fig. 12). It is thus possible that addition of xylanase is just a waste of enzyme. However, in this study, enzyme amounts in relation of applied acid-treated, microwave-irradiated material have not been optimized and this will be another task for future studies to address. Most importantly, all experiments done in this study were on small laboratory scale and for technical application, it remains to be elucidated whether the pretreatments as well as the subsequent enzymatic hydrolysis reactions can be scaled up by factors of thousands or even millions, i.e. from $1 \mathrm{~g}$ to $1 \mathrm{~kg}$ or from $1 \mathrm{~g}$ to $1000 \mathrm{~kg}$ or more, in order to become finally interesting for industries. 


\section{V.5.3 Effects of laccase and $\beta$-glucosidase in enzymatic hydrolysis by cellulase and xylanase of $\boldsymbol{A}$. grandis cellulosic pellets for glucose production}

Laccase in combination of enzymatic hydrolysis of cellulose by application of Onozuka R-10 cellulase in combination or in a mixture with also xylanase improved the glucose yields by factors of 60 to $100 \%$ as compared to enzymatic hydrolysis reactions performed without laccase. The conversion of cellulose to glucose reached up to $100 \%$ when using for $1 \mathrm{~g}$ of $A$. grandis wood particles $8 \mathrm{ml}$ of $85 \%$ phosphoric acid for one hour incubation at $50^{\circ} \mathrm{C}$ in a hot water bath and in subsequent enzymatic hydrolysis of $100 \mathrm{mg}$ of left cellulosic pellets each $4 \mathrm{U} \mathrm{ml}^{-1}$ of cellulase, of xylanase and of laccase, or each $4 \mathrm{U} \mathrm{ml}^{-1}$ of cellulase, of xylanase and of $\beta$-glucosidase, or each $4 \mathrm{U} \mathrm{ml}^{-1}$ of cellulase, of xylanase, of $\beta$-glucosidase and of laccase, or each $4 \mathrm{U} \mathrm{ml}^{-1}$ of cellulase, of xylanase and of laccase plus $1 \mathrm{mM} \mathrm{HBT}$, or each $4 \mathrm{U} \mathrm{ml}^{-1}$ of cellulase, of xylanase, of $\beta$-glucosidase and of laccase plus $1 \mathrm{mM} \mathrm{HBT}$, respectively, in $50 \mathrm{mM}$ sodium acetate buffer, $\mathrm{pH} 5.0$ for one day incubation (Fig. 12), or alternatively, when using $1 \mathrm{~g}$ of A. grandis wood particles in $8 \mathrm{ml}$ of $80 \%$ phosphoric acid pretreatment for incubation with microwave irradiation for $20 \mathrm{sec}$ and in subsequent enzymatic hydrolysis $100 \mathrm{mg}$ of left cellulosic pellets each $4 \mathrm{U} \mathrm{ml}^{-1}$ of cellulose, of xylanase and of laccase in $50 \mathrm{mM}$ sodium acetate buffer, pH 5.0 for one day incubation (Fig. 12).

As discussed above in Chapter V.5.2 for enzymatic hydrolysis of cellulosic pellets obtained from just chemical pretreatment, various conditions have been tested here for enzymatic hydrolysis but for a systematic evaluation of pretreatments and effects of different enzymes, a much more detailed study has to be performed. From the results available so far, it appears that a combination of just Onozuka R-10 cellulase and laccase or also a combination of Onozuka R-10 cellulase and $\beta$-glucosidase are sufficient to reach optimal glucose yields, but reactions have to be optimized e.g. in timings and in amounts of enzymes applied and reactions have to be scaled up to larger volumes with higher amounts of wood.

In this study, different laccases have been used as available in the laboratory by the work of others [purified T. versicolor laccase from Dwivedi (2006), purified C. cinerea laccase V from Rühl (2009); commercial raw laccase from Novozyme, EEC No. 420-150-4 used in the work of Euring (2009)]. It is currently not known whether these enzymes differ in quality or whether expensive enzymes purification versus 
simple enzyme harvests from fungal cultures will make a difference to the reactions and also this remains to be elucidated in the future.

In principle, as shown in this study (Fig. 12), the combination of enzymatic hydrolysis by cellulase and xylanase with oxidative reactions of enzymes such as performed by laccase acting on lignin and wood extractives can improve the production of glucose yields in hydrolysis processes (Fig. 12). Similar positive outcomes on glucose yields were reached in combination of enzymatic hydrolysis of cellulosic pellets by cellulase plus xylanase in the presence of $\beta$-glucosidase (Fig.12).

Cellulase and $\beta$-glucosidase generally act on the digestibility of cellulose. Cellulases distinguish in endo- and exocellulases which synergistically act in crystalline cellulose hydrolysis (Wood and McCrae 1978), with endocellulases cleaving internal $\beta-1-4$ linkages between two neighboring glucose residues and exocellulases cleaving $\beta-1-4$ bonding at the end of cellulose chains (Dadi 2007). $\beta$-Glucosidases will remove cellobiose (two glucose molecules linked in a $\beta-1-4$ bond) which is a strong inhibitor of both endo- and exocellulases (Woodward and Wiseman 1982). Therefore, an improvement in glucose yields from cellulase action on cellulosic pellets of $A$. grandis wood is easily explainable.

In most of the experiments presented in this work, xylanase was added to the enzymatic reactions since xylanase was reported to have a positive effect on cellulose hydrolysis which is generally attributed to helping to break off the existing links between the cellulose, hemicellulose and the lignin (Valls and Roncero 2009). This might be very important for material that is not well broken up by suitable pretreatments. However, in this study, a slightly positive effect of addition of xylanase to wood particles without any pretreatment was observed (Fig. 4) but no one when phosphoric acid pretreated wood particles were used (Fig. 12) and a reason for this might be that by the phosphoric acid or also by the combined phosphoric acid and microwave treatment much of the lignin has successfully been destroyed. By the pretreatment, mass losses of up to $50 \%$ of the original weights of $A$. grandis wood were observed with enrichments of cellulose of up to $98 \%$ (Table S2 in the supplementary material). It is very likely that, due to this, not many hindering linkages between cellulose, hemicellulose and lignin are left that might be degraded by the xylanase. Thus, in future experiments, addition of this enzyme might not anymore be done.

Laccase-mediator treatments with commercial cellulases increased sugar yields in enzymatic hydrolysis from cellulosic pellets of $A$. grandis wood dramatically, as did 
also laccase alone (Fig. 12). Laccase was also shown in another study to improve enzymatic cellulose hydrolysis of steam-pretreated (unspecified) softwood by partial removal of the lignin. Laccase alone or laccase with mediator were reported to give an improvement on enzymatic cellulose hydrolysis (Palonen and Viikari 2004). In the study here with $A$. grandis softwood, laccase in combination with cellulase and xylanase was more effective in increasing the glucose yield than the enzyme combination of cellulase and xylanase alone (Fig. 12). However, no significant differences were detected when laccase plus the mediator HBT was applied compared to using the laccase without a mediator in the enzymatic hydrolysis of the cellulosic pellets (Fig. 12). In all instances with addition of laccase, yields of glucose from cellulose reached the level of $100 \%$. This indicated that the system was likely positively oversaturated with laccase. Accordingly in the future, lower amounts of laccase with or without mediator should be tested, possibly also for shorter times of incubation.

What are the reasons for optimizing the glucose yields from cellulosic pellets in the presence of laccase? Cellulases may bind to lignin and this can hinder their enzymatic actions (Palonen et al. 2003). In the study here, by addition of wood extractives and by addition of specific phenolic compounds shown to be present in A. grandis wood extractives (see Chapter IV.4.4) to CMC and cellulase it was shown that such compounds also hinder the cellulolytic enzymatic activities (Fig. 16).

Water extractives, $p$-coumaric acid, vanillic acid and ferulic acid all influenced the production of glucose from cellulose by cellulase hydrolysis, whereas acetone extractives had no such effects on glucose production (Fig. 16). Water extractives and acetone extractives of $A$. grandis wood both contain $p$-coumaric acid, vanillic acid and ferulic acid (Fig. 11 and Fig. 12 in Chapter IV). The concentration in water extractives of $A$. grandis wood applied in the enzymatic test with CMC (water extractives from $1 \mathrm{mg}$ A. grandis wood transferred into $1 \mathrm{ml}$ buffer; Fig. 16) were $0.123 \mathrm{mM}$ of p-coumaric acid, $0.137 \mathrm{mM}$ vanillic acid and $0.013 \mathrm{mM}$ ferulic acid (compare Table 5 in Chapter IV) and thus in between the two concentrations of $1 \mathrm{mM}$ and $1 \mu \mathrm{M}$ used in the test for the purified compounds (Fig. 17 to Fig. 19). From the results shown in Fig. 17 to 19 , it is to be expected that at the natural concentrations the three compounds will also provide negative effects to cellulase activity, in particular when considering in addition that in the wood extractives the negative actions of the different compounds will likely add up to a stronger effect. Therefore, since addition of laccase to the cellulase overcomes in concentration-dependent manner the negative effects of the 
phenolic compounds, it can be concluded that laccase has a potential to decrease inhibitors in form of phenolic compounds in wood extractives blocking cellulases in cellulose hydrolysis (Fig. 16). Whilst the data show that laccase can very much improve the enzymatic generation of glucose from cellulosic pellets, the example of ferulic acid shows that in certain situations their might be also some negative effects by addition of laccase. As discussed in Chapter V.4.5, ferulic acid potentially blocks upon laccaseactivation a subrange of cellulase in their activities.

In summary, however, laccase has a good potential to improve the drawbacks of lignin and lignin-related compounds on enzymatic cellulose degradation because laccase is efficient in degrading of lignin and of phenolic compound as present in wood extractives or even somewhat of hemicellulose (Palonen and Viikari 2004). Thus, use of laccase in combination of enzymatic hydrolysis can improve the hydrolysis process and gain higher glucose yields, as found in the study by Palonen and Viikari (2004) and also in this study.

\section{V.5.4 Biological pretreatment of $A$. grandis wood for enzymatic hydrolysis for glucose production from cellulose}

Lignin and hemicellulose are some of the drawbacks of using lignocellulosic materials in enzymatic hydrolysis as it makes lignocellulose resistant to chemical and biological degradation, especially for the enzymatic accessibility of the surface of celluloses (Olofsson et al. 2008). Moreover, lignin and degradation products of lignin are also inhibitors of cellulases, xylanases and glucosidases in the hydrolysis process of the cellulose and the hemicellulose being present in the biological materials (Galbe and Zacchi 2007; Mtui 2009). Biological treatment is an alternative measure to address

these problems (see Table 1 in Chapter I). Biological pretreatment can be performed by applying lignin and hemicellulose degrading microorganisms, such as white-rot and brown-rot fungi, respectively. On the other hand, insect-infesting of wood has a potential to remove lignin, carbohydrate and extractives (Chandra et al. 2009; Ewanick et al. 2007; Pan et al. 2007). Such natural pretreatments of wood are considered to be environmentally friendly and energy-saving as there is no need for use of chemicals or for energy for heating, steam-explosion or microwaving. However, through such biological methods, some biomass will be going lost and such pretreatments need their time and are thus far too long ones to be applied on industrial scale in developed 
countries. However, as an optimization with other types of wood pretreatment such biological methods could be used as an initial step in a combination of pretreatment measures (Carmona et al. 2009; Pan et al. 2007; Schilling et al. 2009). Yu et al. (2010) presented recently a study of 15 days bio-treatment of cornstalks with Irpex lacteus, leading to $14 \%$ increase in digestibility of the material. Likewise, studies can be found where a biological pretreatment has been applied to types of straw. For example, rice straw was incubated with various fungi including $P$. ostreatus, with the result of subsequent better glucose yields in enzymatic degradation (Taniguchi et al. 2005). Pretreatment of wood chips with white-rot fungi (bio-pulping) has been shown to reduce the costs in mechanical pulping for the paper industry (e.g. see Hakala et al. 2004). However, in more recent time, selective white-rot fungi have also been applied with success in pretreatments of woods for enzymatic release of sugars, for example for hardwood and softwood species growing in China with the fungus Echinodontium taxodii (Yu et al. 2009). Moreover, results from this $\mathrm{PhD}$ study support the use of natural pretreatment of wood with fungi (Chapter II). Wood after partial degradation by the white-rot fungi $P$. ostreatus 8F6 and T. versicolor Strain 6 was more effective in increasing glucose yields in subsequent enzymatic hydrolysis of left cellulose, differently from the pretreatment with the brown-rot fungus C. puteana Strain 57 (Fig. 15). In the work here, best hydrolysis of cellulose was achieved upon incubation of A. grandis wood with the weak white-rot fungus P. ostreatus 8F6 (Fig. 15). If wanting to optimize the process of bio-treatment in the future, it might be advisable to use such weak degrader in order to prevent unwanted decay of valuable material. On the other hand, it could also be that short time incubation with a more aggressive fungus such as T. versicolor (Fig. 15) or even more aggressive species will give perfect results in subsequent enzymatic cellulose hydrolysis that might make a bio-treatment finally more interesting also from the economical view.

Although the process of biological pretreatment is time-consuming, so that in most instances it might not be applied in the future for industries, it might still be of interest in places where outside storage of material does not as much matter. As advantages, short-time biological pretreatment could fulfill some part of the industrial biofuel process, particularly in the use of felled forest trees left for a certain while in the nature for either natural fungal infestation or, also thinkable if not contradictory to ecological aspects, by artificial inoculation with suitable fungal species. Otherwise, there might be also large natural resources of partially degraded wood available in the 
forests for an easy enzymatic hydrolysis, material that may be fed in addition into established biofuel processing procedures, such as microorganism-infected wood taken from the nature, not forgetting about wood from forests mass-infested by beetles (Table 1 in Chapter I). Partially decayed wood as can be present in high amounts in the forests such as when conifer trees are infected by Heterobasidion (Kharazipour et al. 2007, Kües et al. 2007). Such partially degraded wood may for example offer a welcome source of easily degradable material for biofuel production.

\section{V.6 References}

Beldman G, Searle-van Leeuwen MF, Rombouts FM, Voragen FGJ (1985) The cellulase of Trichoderma viride purification, characterization and comparison of all detectable endoglucanases, exoglucanases and $\beta$-glucosidases. European Journal of Biochemistry 146:301-308

Briens C, Piskorz J, Berruti F (2008) Biomass valorization for fuel and chemicals Production - a review. International Journal of Chemical Reactor Engineering $6: 1-49$

Cara C, Romero I, Oliva JM, Sa'ez F, Castro E (2007) Liquid hot water pretreatment of olive tree pruning residues. Applied Biochemistry Biotechnology 136-140:379394

Carmona R, Lienqueo ME, Salazar O, García A (2009) Bioenergy II: biological pretreatment with fungi as a tool for improvement of the enzymatic saccharification of Eucalyptus globulus Labill to obtain bioethanol. International Journal of Chemical Reactor Engineering 7:1-13

Carunchio F, Crescenzi C, Girelli AM, Messina A, Tarola AM (2001) Oxidation of ferulic acid by laccase: identification of the products and inhibitory effects of some dipeptides. Talanta 55:189-200

Chandra RP, Ewanick SM, Chung PA, Au-Yeung K, Rio LD, Mabee W, Saddler JN (2009) Comparison of methods to assess the enzyme accessibility and hydrolysis of pretreated lignocellulosic substrates. Biotechnology Letters $31: 1217-1222$

Chang VS, Holtzapple MT (2000) Fundamental factors affecting biomass enzymatic reactivity. Applied Biochemistry and Biotechnology 84-86:1-37 
Cheng C, Tsukagoshi N, Udaka S (1990) Nucleotide sequence of the cellobiohydrolases gene from Trichoderma viride. Nucleic Acids Research 18(18):5559

Dadi AP, Schall CA, Varanasis S (2007) Mitigation of cellulose recalcitrance to enzymatic hydrolysis by ionic liquid pretreatment. Applied Biochemistry and Biotechnology 136-140/407-422

Dwivedi RC (2006) Extracellular proteins from lignocellulose degrading Basidiomycetes: Redox enzymes from Trametes versicolor and Coprinopsis cinerea. Dissertation, Georg-August University, Göttingen

EN 113 (1996) Wood preservatives - test method for determining the protective effectiveness against wood destroying basidiomycetes - Determination of the toxic values. European Committee for Standardization, Brüssel, Belgium: $35 \mathrm{p}$

Eriksson KEL, Blanchette RA, Ander P (1990) Microbial and enzymatic degradation of wood and wood components. Springer-Verlag, Berlin

Euring M (2009) Einsatz von Mediatoren bei der enzymatischen Aktivierung der fasereigenen Bindekräfte zur Herstellung von enzymgebundenen, bindemittelfreien Holzwerkstoffen. Dissertation, Georg-August-University, Göttingen

Ewanick SM, Bura R, Saddler JN (2007) Acid-catalyzed steam pretreatment of lodgepole pine and subsequent enzymatic hydrolysis and fermentation to ethanol. Biotechnology and Bioengineering 98(4):737-746

Fengel D, Wegener G (1983) Wood chemistry ultrastructure reactions. Walter de Gruyter, Berlin

Galbe M, Zacchi G (2002) A review of the production of ethanol from softwood. Applied Microbiology and Biotechnology 59:618-628

Galbe M, Zacchi G (2007) Pretreatment of lignocellulosic materials for efficient bioethanol production. Advances in Biochemical Engineering/Biotechnology 108:41-65

Guo Y, Zhou J, Song Y, Zhang L (2009) An efficient and environmentally friendly method for the synthesis of cellulose carbamate by microwave heating. Macromolecular Rapid Communications 30:1504-1508

Hakala TK, Maijala P, Konn J, Hatakka A (2004) Evaluation of novel wood-rotting polypores and corticioid fungi for the decay and biopulping of Norway spruce (Picea abies) wood. Enzyme and Microbial Technology 34(3-4):255-263

Hu G, Heitmann JA, Rojas OJ (2008) Feedstock pretreatment strategies for producing 
ethanol from wood, bark and forest residues. BioResources 3(1):270-294

$\mathrm{Hu}$ Z, Wen Z (2008) Enhancing enzymatic digestibility of Switchgrass by microwaveassisted alkali pretreatment. Biochemical Engineering Journal 38(3):369-378

Jørgensen H, Kristensen JB, Felby C (2007) Enzymatic conversion of lignocellulose into fermentable sugars: challenges and opportunities. Biofuels, Bioproducts and Biorefining 1:119-134

Keshwani DR, Cheng JJ (2010) Microwave-based alkali pretreatment of Switchgrass and coastal Bermudagrass for bioethanol production. 26(3):644-652

Kharazipour A, Ludwig K, Chaisaena W, Polle A, Kües U (2007) Wood and other plant fibers in the production of peat substitutes and pot plant container. In: Kües $U$ (Ed.) Wood Production, Wood Technology, and Biotechnological Impacts. Universitätsverlag Göttingen, Göttingen, 609-635

Kremer ML (1999) Mechanism of the Fenton reaction. Evidence for a new intermediate. Physical Chemistry Chemical Physics 1(15):3595-3605

Kües U, Bohn C, Euring M, Müller C, Polle A, Kharazipour A (2007) Enzymatic modified wood in panel board production. In: Kües U (Ed.) Wood Production, Wood Technology, and Biotechnological Impacts. Universitätsverlag Göttingen, Göttingen, 433-467

Kumar P, Barrett DM, Delwiche MJ, Stroeve P (2009) Methods for pretreatment of lignocellulosic biomass for efficient hydrolysis and biofuel production. Industrial Engineering Chemistry Resources 48:3713-3729

Kumar R (2009) Effects of cellulase and xylanase enzymes on the deconstruction of solids from pretreatment of poplar by leading technologies. Biotechnology Progress 25(2):302-314

Li A, Antizar-Ladislao B, Khraisheh M (2007) Bioconversion of municipal solid waste to glucose for bio-ethanol production. Bioprocess and Biosystems Engineering 30(3):189-196

Li C, Wang Q, Zhao YK (2008) Acid in ionic liquid: An efficient system for hydrolysis of lignocellulose. Green Chemistry 10:177-182

Li J, Yang Y, Chen H, Jiang F, Ling J, Liu MC, Yan FL, Xu JJ (2009) Comparison of saccharification process by acid and microwave-assisted acid pretreated swine manure. Bioprocess and Biosystems Engineering 32(5):649-654

Li XJ, Zhou YD, Yan YL Cai ZY, Feng F (2010) A single cell model for pretreatment of wood by microwave explosion. Holzforschung 64(5):633-637 
Mejbaum W (1939) Über die Bestimmung kleiner Pentosemengen insbesondere in Derivaten der Adenylsäure. Hoppe-Seyler's Zeitschrift für physiologische Chemie 258(2-3):117-120

Morris DL (1948) Quantitative determination of carbohydrates with Dreywood's Anthrone reagent. Science 107:254-255

Mtui GYS (2009) Recent advances in pretreatment of lignocellulosic wastes and production of value added products. African Journal of Biotechnology 8(8):1398-1415

Olofsson K, Bertilsson M, Lidén G (2008) A short review on SSF - an interesting process option for ethanol production from lignocellulosic feedstocks. Biotechnology for Biofuels 1(7):1-14

Palonen H, Tjerneld F, Zacchi G, Tenkanen M (2003) Adsorption of Trichoderma reesei $\mathrm{CBH}$ I and EG II and their catalytic domains to steam pretreated softwood and lignin. Journal Biotechnology 107:65-72

Palonen H, Viikari L (2004) Role of oxidative enzymatic treatments on enzymatic hydrolysis of softwood. Biotechnology and Bioengineering 86(5):550-557

Pan X, Xie D, Yu RW, Lam D, Saddler JN (2007) Pretreatment of lodgepole pine killed by mountain pine beetle using the ethanol organosolv process: fractionation and process optimization. Industrial and Engineering Chemistry Research 46:26092617

Pimentel D, Patzek TW (2005) Ethanol production using corn, switchgrass, and wood; biodiesel production using soybean and sunflower. Natural Resources Research 14(1):65-76

Polle A, Hawighorst P, Spellmann H (2008) Innovative Holzprodukte und Holzwerkstoffe aus nachhaltig produziertem Buchen- und Küstentannenholz. Holztechnologie 49:5-6

Rabelo SC, Maciel R, Costa AC (2009) Lime pretreatment of sugarcane bagasse for bioethanol production. Applied Biochemistry and Biotechnology 153(1-2):139150

Röhrig E (1981) Neuere Grundlagen für den Anbau von A. grandis. Schriften aus der Forstlichen Fakultät der Universität Göttingen und der Niedersächsischen Forstlichen Versuchsanstalt, Sauerländer Verlag, Frankfurt am Main Rühl M (2009) Laccase and other ligninolytic enzymes of the basidiomycetes 
Cropinopsis cinerea and Pleurotus ostreatus. Dissertation, Georg-August University, Germany

Sainz MB (2009) Commercial cellulosic ethanol: the role of plant-expressed enzymes. In Vitro Cellular and Developmental Biology-Plant 45:314-329

Saha BC, Biswas A, Cotta MA (2008) Microwave pretreatment, enzymatic saccharification and fermentation of wheat straw to ethanol. Journal of Biobased Materials and Bioenergy 2(3):210-217

Schilling JS, Tewalt JP, Duncan SM (2009) Synergy between pretreatment lignocellulose modifications and saccharification efficiency in two brown rot fungal systems. Applied Microbiology and Biotechnology 84:465-475

Spellmann H, Geb M, Nagel J, Nagel R, Schmidt M (2010) Timber product oriented silvicultural strategies for mixed strands of beech (Fagus silvatica) and grand fir (Abies grandis). Forst und Holz 65(1):12-19

Steffensen CL, Stensballe A, Kidmose U, Deng PD, Andersen ML, Nielsen JH (2009) Modifications of amino acids during ferulic acid-mediated, laccase-catalysed cross-linking of peptides. Free Radical Reseach 43(12):1167-1178

Stöcker M (2008) Biofuels and biomass-to-liquid fuels in the biorefinery: catalytic conversion of lignocellulosic biomass using porous materials. Angewandte Chemie International Edition 47:9200-9211

Sun Y, Cheng J (2002) Hydrolysis of lignocellulosic materials for ethanol production: a review. Bioresource Technology 83:1-11

Taherzadeh MJ, Karimi K (2007) Enzyme-based hydrolysis processes for ethanol from lignocellulosic materials: a review. BioResources 2(4):707-738

Taherzadeh MJ, Karimi K (2008) Pretreatment of lignocellulosic wastes to improve ethanol and biogas production: a review. International Journal of Molecular Sciences 9:1621-1651

Taniguchi M, Takahashi D, Watanabe D, Sakai K, Hoshino K, Kouya T, Tanaka T (2010) Evaluation of fungal pretreatments for enzymatic saccharification of rice straw. Journal of Chemical Engineering of Japan 43(4):401-405

TAPPI T 222 om-02 (2002) Acid-insoluble lignin in wood and pulp. Technical Association of the Pulp and Paper Industry, USA: $5 \mathrm{p}$

Tu M, Chandra RP, Saddler JN (2007) Recycling cellulases during the hydrolysis of steam exploded and ethanol pretreated lodgepole pine. Biotechnology Progress 23:1130-1137 
Valls C, Roncero MB (2009) Using both xylanase and laccase enzymes for pulp bleaching. Bioresource Technology 6:2032-2039

Verma P, Mai C (2010) Hydrolysis of cellulose and wood powder treated with DMDHEU by a hydrolase enzyme complex, Fenton's reagent, and in a liquid culture of Trametes versicolor. Holzforschung 64(1):69-75

Vos H, Kharazipour A (2008) Utilization of grand fir timber and beech timber for the production of novel sandwich boards. Holztechnologie 6:20-22

Vos H, Kharazipour A (2010) Properties of light particle boards manufactured using Abies grandis (grand fir). Forst und Holz 65(1):26-30

Ward G, Hadar Y, Billkis I, Konstantinovsky L, Dosoretz CG (2001) Initial steps of ferulic acid polymerization by lignin peroxidase. The Journal of Biological Chemistry 276:18734-18741

Wood TM, Mccrae SI (1978) Cellulase of Trichoderma koningii - Purification and properties of some endoglucanase components with special reference to their action on cellulose when acting alone and in synergism with cellobiohydrolases. Biochemical Journal 171(1):61-72

Woodward J, Wiseman A (1982) Fungal and other $\beta$-D-glucosidases - their properties and applications. Enzyme and Microbial Technology 4(2):73-79

Xiang Q, Lee YY, Pettersson PO, Torget RW (2003) Heterogeneous aspects of acid hydrolysis of $\alpha$-cellulose. Applied Biochemistry and Biotechnology 105108:505-514

Yoshida M, Liu Y, Uchida S, Kawarada K, Ukagami Y, Ichinose H, Kaneko S, Fukuda K (2008) Effects of cellulose crystallinity, hemicellulose, and lignin on the enzymatic hydrolysis of Miscanthus sinensis to monosaccharides. Bioscience, Biotechnology, and Biochemistry 72(3):805-810

Yu HB, Guo GB, Zhang XY, Yan KL, Xu CY (2009) The effect of biological pretreatment with the selective white-rot fungus Echinodontium taxodii on enzymatic hydrolysis of softwoods and hardwoods. Bioresource Technology 100(21):5170-5175

Yu HB, Du WQ, Zhang J, Ma FY, Zhang XY, Zhong WX (2010) Fungal treatment of cornstalks enhances the delignification and xylan loss during mild alkaline pretreatment and enzymatic digestibility of glucan. Bioresource Technology 101(17):6728-6734

Zhang YHP, Ding SY, Mielenz JR, Cui JB, Elander RT, Laser M, Himmel ME, 
McMillan JR, Lynd LR (2007) Fractionating recalcitrant lignocellulose at modest reaction conditions. Biotechnology and Bioengineering 97(2):214-223

Zhang YHP, Lynd LR (2006) A functionally based model for hydrolysis of cellulose by fungal cellulase. Biotechnology and Bioengineering 94(5):888-898

Zhang Z, Zhao ZK (2009) Solid acid and microwave-assisted hydrolysis of cellulose in ionic liquid. Carbohydrate Research 344:2069-2072

Zhu JY, Pan XJ (2010) Woody biomass pretreatment for cellulosic ethanol production: technology and energy consumption evaluation. Bioresource Technology 100(13):4992-5002

Zhu JY, Pan X, Zalesny Jr RS (2010a) Pretreatment of woody biomass for biofuel production: energy efficiency, technologies, and recalcitrance. Applied Microbiology and Biotechnology. doi: 10.1007/s00253-010-2654-8

Zhu JY, Zhu W, OBryan P, Dien BS, Tian S, Gleisner R, Pan XJ (2010b) Ethanol production from SPORL-pretreated lodgepole pine: preliminary evaluation of mass balance and process energy efficiency. Applied Microbiology Biotechnology. doi: 10.1007/s00253-009-2408-7

Zhu SD, Wu YX, YU ZN, Chen QM, Wu GY, Yu FQ, Wang CW, Jin SW (2006) Microwave-assisted alkali pre-treatment of wheat straw and its enzymatic hydrolysis. Biosystems Engineering 94(3):437-442

Zhu SD Wu YX, Yu ZN, Liao JT, Zhang Y (2005) Pretreatment by microwave/alkali of rice straw and its enzymic hydrolysis. Process Biochemistry 40(9):3082-3086

Zhu SD, Wu YX, Yu ZN, Wang CW, Yu FQ, Jin SW, Ding YQ, Chi RA, Liao JT, Zhang Y (2006) Comparison of three microwave/chemical pretreatment processes for enzymatic hydrolysis of rice straw. Biosystems Engineering 93(3):279-283

Zhu SD, Wu YX, Yu ZN, Zhang X, Wang CW, Yu FQ, Jin SW (2006) Production of ethanol from microwave-assisted alkali pretreated wheat straw. Process Biochemistry 41(4):869-873 


\section{V.7 Supplementary material}

\section{V.7.1 Chemical pretreatment of $A$. grandis wood}

Chemical treatment with phosphoric acid with phosphoric acid and Aliquat and with phosphoric acid in microwaving reduced to different degrees the overall amount of A. grandis wood components left after the treatment (Table S1). The Table S1 also gives the absolute amounts of cellulose determined by experimental analysis (in \% of the weight of the left cellulosic pellets), the expected theoretical maximum cellulose amounts (in \%) calculated for the case that during the pretreatment no cellulose has gone lost, and the actual yield in \% of the theoretical maximum value. Cellulose contents were calculated from three parallel samples by measuring the hexose content as described in Chapter V.3.7 by treating $0.5 \mathrm{~g}$ original $A$. grandis wood particles with $7.5 \mathrm{ml}$ of $72 \%$ sulfuric acid, respectively $0.1 \mathrm{~g}$ cellulosic pellets with $1.5 \mathrm{ml}$ of $72 \%$ sulfuric acid prior to treatment with anthrone and spectrophotometric determination of the sugar contents in $\mathrm{mg} \mathrm{ml}^{-1}$. Cellulose contents were calculated under the assumption that $180 \mathrm{~g}$ of hexose $(1 \mathrm{Mol})$ will give $162 \mathrm{~g}$ cellulose when linked together in cellulose chains.

Table S1 Weight of cellulosic pellets left after different chemical pretreatments of $1 \mathrm{~g}$ A. grandis wood particles or $1 \mathrm{~g}$ of water-extracted $A$. grandis wood particles (sample with $\vee$ ) and calculations of actual and theoretical cellulose contents in the left materials

\begin{tabular}{|c|c|c|c|c|c|c|}
\hline Experiment & Pretreatment & $\begin{array}{c}\text { Material } \\
\text { left } \\
\text { [cellulosic } \\
\text { pellets] } \\
\text { (g) }\end{array}$ & $\begin{array}{c}\text { Measured } \\
\text { hexose content } \\
\text { after sulfuric } \\
\text { acid treatment } \\
\text { (\%) }\end{array}$ & $\begin{array}{c}\text { Calculated } \\
\text { cellulose } \\
\text { content after } \\
\text { pretreatment } \\
(\%)\end{array}$ & $\begin{array}{c}\text { Theoretical } \\
\text { maximum } \\
\text { cellulose content } \\
\text { after treatment } \\
(\%)\end{array}$ & $\begin{array}{l}\text { Actual yield of } \\
\text { theoretical } \\
\text { maximum } \\
\text { value of } \\
\text { cellulose }(\%)\end{array}$ \\
\hline \multirow[t]{9}{*}{ I (Fig. 5) } & $8 \mathrm{ml} \mathrm{85 \%}$ & 0.642 & 79.4 & 71.5 & 72.1 & 99.2 \\
\hline & $\begin{array}{l}\text { Phosphoric acid, } \\
15 \mathrm{~min}\end{array}$ & & & & & \\
\hline & $8 \mathrm{ml} \mathrm{85 \%}$ & 0.648 & 88.6 & 79.7 & 79.7 & 100 \\
\hline & $\begin{array}{l}\text { Phosphoric acid, } 15 \\
\text { min (water-extracted } \\
\text { wood) }\end{array}$ & & & & & \\
\hline & $8 \mathrm{ml} \mathrm{85 \%}$ & 0.697 ـ & 73.2 & 65.9 & 66.4 & 99.3 \\
\hline & Phosphoric acid, $1 \mathrm{hr}$ & & & & & \\
\hline & $8 \mathrm{ml} \mathrm{85 \%}$ & 0.693 & 73.6 & 66.2 & 66.7 & 99.2 \\
\hline & $\begin{array}{l}\text { Phosphoric acid }+ \\
\text { Aliquat, } 1 \mathrm{hr}\end{array}$ & & & & & \\
\hline & $8 \mathrm{ml}$ Aliquat, $1 \mathrm{hr}$ & 0.865 & 58.9 & 53.0 & 53.5 & 99.2 \\
\hline
\end{tabular}


V. Application of Abies grandis wood for technical use in biofuel production

\begin{tabular}{|c|c|c|c|c|c|c|}
\hline \multirow[t]{8}{*}{ II (Fig. 6) } & $8 \mathrm{ml} \mathrm{85 \%}$ & 0.697 & 73.2 & 65.9 & 66.4 & 99.3 \\
\hline & \multicolumn{6}{|l|}{ Phosphoric acid, $1 \mathrm{hr}$} \\
\hline & $15 \mathrm{ml} \mathrm{85 \%}$ & 0.704 & 73.0 & 65.7 & 65.7 & 100 \\
\hline & \multicolumn{6}{|l|}{ Phosphoric acid, $1 \mathrm{hr}$} \\
\hline & $20 \mathrm{ml} \mathrm{85 \%}$ & 0.508 & 101.1 & 91.0 & 91.1 & 99.9 \\
\hline & \multicolumn{6}{|l|}{ Phosphoric acid, $1 \mathrm{hr}$} \\
\hline & $25 \mathrm{ml} 85 \%$ & 0.495 & 103.8 & 93.4 & 93.4 & 100 \\
\hline & \multicolumn{6}{|l|}{ Phosphoric acid, $1 \mathrm{hr}$} \\
\hline \multirow[t]{23}{*}{ III (Fig. 7) } & $\begin{array}{l}8 \mathrm{ml} \mathrm{Buffer}, 0 \mathrm{sec} \\
\text { microwave }\end{array}$ & 1.000 & 51.4 & 46.3 & 46.3 & 100 \\
\hline & $8 \mathrm{ml}$ Buffer, $20 \mathrm{sec}$ “ & 1.030 & 50.0 & 45.0 & 45.0 & 100 \\
\hline & $8 \mathrm{ml}$ Buffer, $40 \mathrm{sec}$ “ & 1.020 & 50.4 & 45.4 & 45.4 & 100 \\
\hline & $8 \mathrm{ml}$ Buffer, $60 \mathrm{sec}$ “ & 1.010 & 50.9 & 45.8 & 45.8 & 100 \\
\hline & $25 \mathrm{ml}$ Buffer, $0 \mathrm{sec}$ “" & 1.020 & 50.4 & 45.4 & 45.4 & 100 \\
\hline & $25 \mathrm{ml}$ Buffer, $20 \mathrm{sec}$ & 1.000 & 51.4 & 46.3 & 46.3 & 100 \\
\hline & $\begin{array}{l}25 \mathrm{ml} \text { Buffer, } 40 \mathrm{sec} \\
\text { ، }\end{array}$ & 1.001 & 51.3 & 46.2 & 46.2 & 100 \\
\hline & $\begin{array}{l}25 \mathrm{ml} \text { Buffer, } 60 \mathrm{sec} \\
\text { ، }\end{array}$ & 1.000 & 51.4 & 46.3 & 46.3 & 100 \\
\hline & $8 \mathrm{ml} \mathrm{85 \%}$ & 0.502 & 102.3 & 92.1 & 92.2 & 99.9 \\
\hline & $\begin{array}{l}\text { Phosphoric acid, } \\
20 \mathrm{sec} \text { microwave }\end{array}$ & & & & & \\
\hline & $8 \mathrm{ml} \mathrm{85 \%}$ & 0.501 & 102.6 & 92.3 & 92.3 & 100 \\
\hline & Phosphoric acid, & & & & & \\
\hline & $40 \mathrm{sec}$ microwave & & & & & \\
\hline & $8 \mathrm{ml} \mathrm{85 \%}$ & 0.512 & 100.4 & 90.4 & 90.4 & 100 \\
\hline & $\begin{array}{l}\text { Phosphoric acid, } \\
60 \text { sec microwave }\end{array}$ & & & & & \\
\hline & $25 \mathrm{ml} \mathrm{85 \%}$ & 0.679 & 75.7 & 68.1 & 68.1 & 100 \\
\hline & Phosphoric acid, & & & & & \\
\hline & $20 \mathrm{sec}$ microwave & & & & & \\
\hline & $25 \mathrm{ml} \mathrm{85 \%}$ & 0.484 & 106.2 & 95.6 & 95.6 & 96.5 \\
\hline & Phosphoric acid, & & & & & \\
\hline & $40 \mathrm{sec}$ microwave & & & & & \\
\hline & $25 \mathrm{ml} \mathrm{85 \%}$ & 0.473 & 108.6 & 97.7 & 97.8 & 99.9 \\
\hline & $\begin{array}{l}\text { Phosphoric acid, } \\
60 \mathrm{sec} \text { microwave }\end{array}$ & & & & & \\
\hline \multirow[t]{3}{*}{ IV (Fig. 9) } & $25 \mathrm{ml} \mathrm{85 \%}$ & 0.680 & 75.6 & 68.0 & 68.0 & 100 \\
\hline & $\begin{array}{l}\text { Phosphoric acid, } \\
20 \text { sec microwave }\end{array}$ & & & & & \\
\hline & $\begin{array}{l}25 \mathrm{ml} \text { Formic acid, } \\
20 \mathrm{sec} \text { microwave }\end{array}$ & 0.862 & 59.6 & 53.6 & 53.6 & 100 \\
\hline
\end{tabular}




\begin{tabular}{|c|c|c|c|c|c|c|}
\hline & $\begin{array}{l}25 \mathrm{ml} \text { Acetic acid, } \\
20 \mathrm{sec} \text { microwave }\end{array}$ & 0.869 & 59.1 & 53.1 & 53.2 & 99.9 \\
\hline \multirow[t]{42}{*}{ V (Fig. 10) } & $8 \mathrm{ml} \mathrm{85 \%}$ & 0.892 & 57.6 & 51.8 & 51.8 & 100 \\
\hline & Phosphoric acid, & & & & & \\
\hline & $5 \mathrm{sec}$ microwave & & & & & \\
\hline & $8 \mathrm{ml} \mathrm{85 \%}$ & 0.481 & 106.8 & 96.1 & 96.2 & 99.9 \\
\hline & Phosphoric acid, & & & & & \\
\hline & $20 \mathrm{sec}$ microwave & & & & & \\
\hline & $8 \mathrm{ml} \mathrm{85 \%}$ & 0.531 & 96.8 & 87.1 & 87.1 & 100 \\
\hline & Phosphoric acid, & & & & & \\
\hline & $20 \mathrm{sec} *$ microwave & & & & & \\
\hline & $8 \mathrm{ml} \mathrm{85 \%}$ & 0.915 & 56.2 & 50.6 & 50.6 & 100 \\
\hline & Phosphoric acid, RT & & & & & \\
\hline & without microwave & & & & & \\
\hline & $8 \mathrm{ml} \mathrm{80 \%}$ & 0.739 s & 65.6 & 59.0 & 62.6 & 94.3 \\
\hline & Phosphoric acid, & & & & & \\
\hline & $5 \mathrm{sec}$ microwave & & & & & \\
\hline & $8 \mathrm{ml} \mathrm{80 \%}$ & 0.492 s & 104.5 & 94.1 & 94.1 & 100 \\
\hline & Phosphoric acid, & & & & & \\
\hline & $20 \mathrm{sec}$ microwave & & & & & \\
\hline & $8 \mathrm{ml} \mathrm{80 \%}$ & 0.522 & 98.5 & 88.7 & 88.7 & 100 \\
\hline & Phosphoric acid, & & & & & \\
\hline & $20 \mathrm{sec}^{*}$ microwave & & & & & \\
\hline & $8 \mathrm{ml} \mathrm{80 \%}$ & 0.852 & 60.3 & 54.3 & 54.3 & 100 \\
\hline & Phosphoric acid, RT & & & & & \\
\hline & without microwave & & & & & \\
\hline & $8 \mathrm{ml} \mathrm{40 \%}$ & 0.744 & 69.1 & 62.2 & 62.2 & 100 \\
\hline & Phosphoric acid, & & & & & \\
\hline & $5 \mathrm{sec}$ microwave & & & & & \\
\hline & $8 \mathrm{ml} \mathrm{40 \%}$ & 0.739 s & 69.6 & 62.6 & 62.6 & 100 \\
\hline & Phosphoric acid, & & & & & \\
\hline & $20 \mathrm{sec}$ microwave & & & & & \\
\hline & $8 \mathrm{ml} \mathrm{40 \%}$ & 0.884 s & 58.1 & 52.3 & 52.3 & 100 \\
\hline & Phosphoric acid, & & & & & \\
\hline & $20 \mathrm{sec}^{*}$ microwave & & & & & \\
\hline & $8 \mathrm{ml} \mathrm{40 \%}$ & 0.897 & 57.3 & 57.6 & 57.6 & 100 \\
\hline & Phosphoric acid, RT & & & & & \\
\hline & without microwave & & & & & \\
\hline & $8 \mathrm{ml} \mathrm{20 \%}$ & 0.847 s & 60.7 & 54.6 & 54.6 & 100 \\
\hline & Phosphoric acid, & & & & & \\
\hline & $5 \mathrm{sec}$ microwave & & & & & \\
\hline & $8 \mathrm{ml} \mathrm{20 \%}$ & 0.898 s & 57.2 & 51.5 & 51.5 & 100 \\
\hline & Phosphoric acid, & & & & & \\
\hline & $20 \mathrm{sec}$ microwave & & & & & \\
\hline
\end{tabular}


V. Application of Abies grandis wood for technical use in biofuel production

\begin{tabular}{|c|c|c|c|c|c|c|}
\hline & $8 \mathrm{ml} \mathrm{20 \%}$ & 0.876 ـ & 58.7 & 52.8 & 52.8 & 100 \\
\hline & \multicolumn{6}{|l|}{ Phosphoric acid, } \\
\hline & \multicolumn{6}{|l|}{$20 \mathrm{sec}^{*}$ microwave } \\
\hline & $8 \mathrm{ml} \mathrm{20 \%}$ & 0.911 & 56.4 & 50.8 & 50.8 & 100 \\
\hline & \multicolumn{6}{|l|}{$\begin{array}{l}\text { Phosphoric acid, RT } \\
\text { without microwave }\end{array}$} \\
\hline \multirow[t]{27}{*}{ VI (Fig. 13) } & $8 \mathrm{ml} \mathrm{80 \%}$ & 0.739 s & 65.6 & 59.0 & 62.6 & 94.3 \\
\hline & \multirow{2}{*}{\multicolumn{6}{|c|}{$\begin{array}{l}\text { Phosphoric acid, } \\
5 \mathrm{sec} \text { microwave }\end{array}$}} \\
\hline & & & & & & \\
\hline & $8 \mathrm{ml} \mathrm{80 \%}$ & 0.492 ـ & 104.5 & 94.1 & 94.1 & 100 \\
\hline & \multirow{2}{*}{\multicolumn{6}{|c|}{$\begin{array}{l}\text { Phosphoric acid, } \\
20 \mathrm{sec} \text { microwave }\end{array}$}} \\
\hline & & & & & & \\
\hline & $8 \mathrm{ml} \mathrm{80 \%}$ & 0.522 \& & 98.5 & 88.7 & 88.7 & 100 \\
\hline & \multicolumn{6}{|l|}{ Phosphoric acid, } \\
\hline & \multicolumn{6}{|l|}{$20 \mathrm{sec}^{*}$ microwave } \\
\hline & $8 \mathrm{ml} \mathrm{40 \%}$ & $0.744 \&$ & 69.1 & 62.2 & 62.2 & 100 \\
\hline & \multirow{2}{*}{\multicolumn{6}{|c|}{$\begin{array}{l}\text { Phosphoric acid, } \\
5 \text { sec microwave }\end{array}$}} \\
\hline & & & & & & \\
\hline & $8 \mathrm{ml} \mathrm{40 \%}$ & 0.739 مـ & 69.6 & 62.6 & 62.6 & 100 \\
\hline & \multirow{2}{*}{\multicolumn{6}{|c|}{$\begin{array}{l}\text { Phosphoric acid, } \\
20 \mathrm{sec} \text { microwave }\end{array}$}} \\
\hline & & & & & & \\
\hline & $8 \mathrm{ml} \mathrm{40 \%}$ & 0.884 م & 58.1 & 52.3 & 52.4 & 99.9 \\
\hline & \multirow{2}{*}{\multicolumn{6}{|c|}{$\begin{array}{l}\text { Phosphoric acid, } \\
20 \mathrm{sec}^{*} \text { microwave }\end{array}$}} \\
\hline & & & & & & \\
\hline & $8 \mathrm{ml} \mathrm{20 \%}$ & 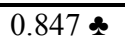 & 60.7 & 54.6 & 54.6 & 100 \\
\hline & \multirow{2}{*}{\multicolumn{6}{|c|}{$\begin{array}{l}\text { Phosphoric acid, } \\
5 \mathrm{sec} \text { microwave }\end{array}$}} \\
\hline & & & & & & \\
\hline & $8 \mathrm{ml} \mathrm{20 \%}$ & $0.898 \div$ & 57.2 & 51.5 & 51.6 & 99.9 \\
\hline & \multicolumn{6}{|l|}{ Phosphoric acid, } \\
\hline & \multicolumn{6}{|l|}{$20 \mathrm{sec}$ microwave } \\
\hline & $8 \mathrm{ml} \mathrm{20 \%}$ & 0.876 م & 58.7 & 52.8 & 52.8 & 100 \\
\hline & \multicolumn{6}{|l|}{ Phosphoric acid, } \\
\hline & $20 \mathrm{sec}^{*}$ microwave & & & & & \\
\hline
\end{tabular}

- This calculation is done under the expectation that no cellulose has been gone lost by the pretreatment procedure applied

$\checkmark$ Water-extracted wood had a cellulose content of $51.7 \%$ in contrast to the original wood that had a cellulose content of $46.3 \%$ (Table 1)

- The $8 \mathrm{ml} \mathrm{85 \%}$ phosphoric acid samples in Experiment I and Experiment II were identical

The $8 \mathrm{ml}$ of $80 \%, 40 \%$ and $20 \%$ phosphoric acid samples in Experiment V and VI were identical

* Incubation with the acid for $5 \mathrm{sec}$, stop for $5 \mathrm{sec}$, repeated for 4 cycles; total $20 \mathrm{sec}$ 



\section{CHAPTER VI}

Final discussion and prospects 



\section{VI.1 Final discussion}

\section{VI.1.1 General overview on the results of this work and their implications for technical applications}

Laccase is an oxidative enzyme that occurs in bacteria, fungi, higher plants and arthropods (Hoegger et al. 2006). Fungal laccases are often secreted, especially laccases from the white-rot fungi (Tadesse et al. 2008). The actions of fungal laccases on Abies grandis wood with the final target for applications in the wood products industry and in biofuel production were studied in this thesis. In this work, it was shown that in cultures with $A$. grandis wood laccase was secreted by the white-rot fungi $P$. ostreatus and T. versicolor, likely in order to degrade the A. grandis wood by modifying and then degrading lignin within the wood cell walls (Chapter II and III). A. grandis wood and, within wood extractives, were found to induce laccase production, but on the other hand, A. grandis wood extractives apparently mediate also protection against wood decay by fungi by inhibiting fungal growth (Chapter IV).

A. grandis wood has a high potential for sustainable wood production (Polle et al. 2008) and, as recently has been shown, for applications in the wood product industries (Müller et al. 2009; Vos and Kharazipour 2010). Next to making usage of the solid A. grandis wood e.g. in constructions (Hof et al. 2008), possible further applications of $A$. grandis wood are found in the wood panel industries (Kloeser et al. 2008). The estimated European demand for raw material for panel board production will increase from 26 million tons in the year 2005 to 37 million tons in the year 2015 in absolutely amounts of dry wood (Alteheld 2007). These wood product industries have therefore an urgent need far long-term supply of feedstock and an enlargement of plantations of the fast growing North-American A. grandis in short rotation cycles, its excellent drought tolerance and ecological compatibility with domestic tree species in Europe is one of most promising options to answer this challenge (Polle et al. 2008; Röhrig 1981; Spellmann et al. 2010). Next to the established potential for applications of $A$. grandis wood as a solid material, this $\mathrm{PhD}$ thesis addresses for the first time the potential use of $A$. grandis wood in biofuel production (Chapter V). Biofuel production generally meets also the challenge of the availability of sufficient wood supply which needs to be fulfilled from lignocellulosic feedstocks of forests or also from wood residues from industrial products (Galbe and Zacchi 2007). Also by these reasons, 
plantations of $A$. grandis promise to well support the supply of sustainable wood material sources.

Fig. 1 presents a conception of this thesis and projects the handled fields of study into four main sectors which include: i. wood particles, ii. wood extractives, iii. decay fungi, and iv. application in biofuels. This cycle summarizes the different effects and functions of wood particles and wood extractives in natural fungal degradation of the wood and their participation to a biofuel production process on the one hand and of the fungi and their enzymes on the other hand.

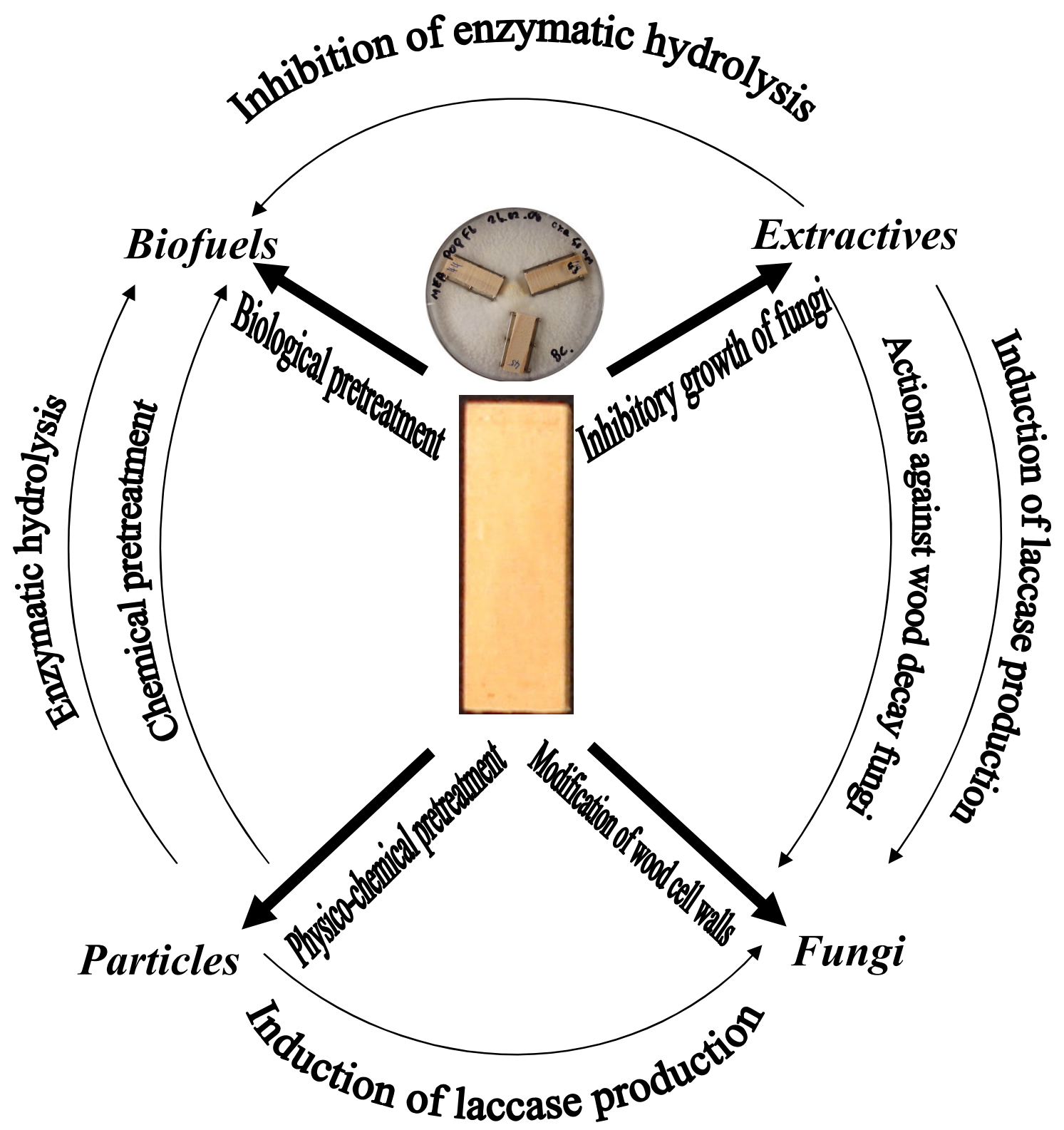

Fig. 1 Interlinkages of different targets of this study (wood, extractives, fungi and biofuel) in production, function and application of enzymes including laccase 
In detail, fungi may attack the $A$. grandis wood for degradation in order to win nutrients for their own growth (Chapter II and III). Both brown-rot and at least some white-rots are active on A. grandis wood, whilst the brown-rot fungus C. puteana was however much more severe than the white-rots $P$. ostreatus and $T$. versicolor. Generally, A. grandis wood has a certain protection against white-rots, a result of importance when wanting to use $A$. grandis wood in indoor or outdoor applications. Even more important for applications might be the findings that wood composites, possibly by the addition of nitrogen-containing chemicals, have an even better resistance than the pure wood (Chapter II). Further to in Chapter II, the mechanisms of fungal decay were also addressed in Chapter III of this thesis. A wood-staining method with MBTH (3-methyl-2-benzothiazolinone-hydrazone hydrochloride monohydrate) was used to follow up chemical modifications in the wood cell walls by the formation of quinones that interact with MBTH. Evidence is given that fungal laccases can contribute to wood decay by the fungi (Chapter II and III). Chapter IV addresses functions and effects of wood particles and of wood extractives on enzymatic hydrolysis of the wood via such contrasting inputs as being inducers of production of fungal enzymes such as laccases and being inhibitors of fungal growth when wood and fungi are kept together under adequate conditions. In applications for release of sugars from the $A$. grandis wood for biofuel production, wood extractives were found to inhibit enzymatic hydrolysis processes which, in contrast, could be overcome by the application of laccase (Chapter V). Wood pretreatments (chemical, physico-chemical pretreatments in terms of wood particles, and fungal incubation in terms of biological pretreatments of solid wood blocks) are required for efficient biotechnological conversion of cellulose into fermentable sugars (Chapter V). Pretreatment methods are required to resolve the lignin and the hemicellulose cover of the cellulose fibrils in the wood cell walls and measures to increase the overall cellulose content might either be done chemically (e.g. in form of phosphoric acid) assisted by heating or by energy supply through microwaving or it might be done by partial decay through fungi, in particular through white-rot species (Chapter V). The latter is possible since fungi secrete enzymes to modify wood cell walls for their degradation. Selective lignin and hemicellulose degradation allows the cellulose surface area to increase in order to be better accessible for later enzymatic hydrolysis of the cellulose by cellulases (Chapter III). Laccase plays a role to improve enzymatic hydrolysis of celluloses by decreasing the content of inhibitory phenolic compounds from wood extractives or from the decay 
of lignin by chemical, thermal or biological measures. In consequence, laccase indirectly improves the hydrolysis of cellulose by stabilization of cellulase reactions, which in the experiments performed resulted in high glucose yields of up to $100 \%$ cellulose conversion (Chapter V). For example, free hydroxyl groups from lignin benzene rings play an important role in inhibiting enzymes such as cellulases (Pan 2008). Laccase in combination with cellulases and xylanase also improved the hydrolysis process of $A$. grandis wood (Chapter V) and this confirmed earlier results from Palonen et al. (2004) on an other unknown type of softwood. It is finally to be noted from this $\mathrm{PhD}$ work that cellulase functions can also be stabilized for efficient conversion of cellulose from cellulosic pellets when adding $\beta$-glucosidase (Chapter V) as an enzyme that degrades cellulase-inhibiting cellobiose molecules (Dadi et al. 2007; Jørgensen et al. 2008). Since actions addressing two different types of cellulose inhibitory compounds inactivated by two different enzymatic mechanisms (degradation of phenolic compounds by laccases, degradation of cellobiose by $\beta$-glucosidase) both lead to glucose yields of up to $100 \%$ cellulose conversion (Chapter V), it is very likely that in the future after optimization of the enzyme step procedures with reduced amounts of cellulase might be applied in evenly good reactions.

Whilst in this subchapter the interconnections between different tasks, experiments and results of this thesis are documented and more globally be discussed, selected issues linked to biological principles and to potential applications are presented in further details in the following subchapters. Moreover, the reader is referred also to the specific discussions in the Chapters II to $\mathrm{V}$ for more details on the more specific aspects handled in these individual chapters, e.g. such as on chemical details of MBTHstaining.

\section{VI.1.2 Laccase and wood extractives, colleagues or enemies?}

The typical role of laccase in nature as secreted by white-rot fungi is assumed in possible association with other extracellular oxidases (e.g. manganese peroxidase, lignin peroxidase) to degrade complex natural polymers, such as lignin and possibly also some of the hemicellulose or cellulose in the cell walls of wood fibers (Eriksson et al. 1990; Fengel and Wegener 1983). Phenols which principally are present in wood extractives play a central role as natural substrates of laccases. Suitable phenolic 
compounds such as hydroquinone, 2,6-dimethoxyphenol, 4-methoxy phenol and 2,4,6tri-methoxy phenol were suggested to act as natural mediators of laccase in the depolimerization process of wood (Calcaterra et al. 2008; Tadesse et al. 2008). Mediators are low molecular weight compounds with a high redox potential that function to increase the substrate ranges and reaction (i.e. oxidation) rates of the enzymatic laccase system also to compounds with which laccase can not directly react (Call and Mücke 1997; Johannes and Majcherczyk 2000).

This study gave evidences for a dual role of wood extractives: laccase induction was obtained in liquid cultures of white-rot fungi when wood or wood extractives were added (Chapter IV). Extracted wood with eliminated wood extractives were also active, but not as efficient in degree of induction and the induction was delayed in time as compared to wood still containing the extractives. These observations support also the result that wood extractives have an inductive effect on laccase production, in addition to that they revealed that degradation products from the wood will also serve this function (Chapter IV). On the other hand, in growth tests of Coprinopsis cinerea with added wood extractives strong inhibition of growth was observed, and somewhat weaker growth inhibition when wood extractives were applied to P. ostreatus and T. versicolor, respectively. Thus, wood extractives showed bi-functions in terms of fungi. In a cooperative action, behaving like good colleagues, laccase production is induced which results ultimately in modifying the lignin within the wood cell walls and in degradation of the wood cell (Chapter III). In an antagonistic action, "as an enemy", wood extractives act to inhibit growth of fungi and, in consequence, this inhibits the possibility to secrete laccase as an enzyme to degrade wood and other lignocellulosic material (Chapter IV). Moreover, as shown in Chapter V, wood extractives also have the ability to inhibit enzymatic actions, such as those on cellulases, which is another way as how to protect wood from decay by fungal enzymes. Thus, evolution has created an interplay between wood extractives and laccase that in some situations are synergistic and in other situations are antagonistic. Very likely, wood extractives as possible metabolites left from reactions performed for lignification of plant cell walls may have adopted a protective role against microbial growth prior to move to a function that induces production of enzymes by fungi that then target at plant cell degradation and at the degradation of the wood extractives themselves.

In any case, the "ping-pong" interplay between wood extractives and induction of laccase production and the decay of wood extractives and compounds of the wood 
lignin by the enzyme is likely to play a role in processes of biodegradation. Biodegradation of wood by white-rot fungi is a promising natural wood treatment process in terms of its low energy requirement and environmental friendly action (see Chapter I and V). Undegraded wood has generally a high resistance to degradation of its cellulose but after the biodegradation by for example $T$. versicolor eliminating the protective lignin-hemicellulose-cover, the resistance of cellulose to degradation decreases (Yu et al. 2008 and Chapter V).

Work in this thesis addressed the process of biodegradation of wood with the target to get ideal material freed from hindering compounds for the conversion of cellulose into fermentable sugars. The results from Chapter III showed that laccases secreted by $T$. versicolor could modify lignin within the wood cell walls over the time with the effect that the thicknesses of the secondary cell walls were reduced. Thereby, the actions of $T$. versicolor and its enzymes act in form of a biological pretreatment of wood by attacking lignin and hemicellulose and by decreasing the resistance of wood cellulose against degradation by cellulases in order to then allow enzymatic hydrolysis to digest the cellulose. Experiments presented in Chapter V showed that wood after such biodegradation by the white-rot fungi $P$. ostreatus and $T$. versicolor gave much better relative conversion rates of cellulose than wood that had not undergone a biodegradation process. However, biodegradation of wood by brown-rot fungi also lead to relative higher glucose yields as compared to untreated wood (Chapter V). The results showed that it is very important not only to calculate relative conversion rates of cellulose into glucose but also the absolute amounts of glucose with respect of the actual cellulose amount originally present in the wood. Wei et al. (2010) recently reported that the brown-rot Postia placenta initiates wood decay by producing extracellular reactive oxygen species that depolymerize the structural polysaccharides of lignocellulose. Whilst it has still to be tested whether only a short time incubation with such a brown-rot fungus will lead therefore on the one hand to very good cellulose-glucose conversion rates with only a neglectable loss of total wood material, longer incubation times result in loss of large amounts of the cellulose as was confirmed in this work for A. grandis wood incubated with the brown-rot C. puteana and such high loss of material will be to uneconomical for the conversion of wood into biofuel. The situation with (selective) white-rot fungi decaying preferentially the lignin in the wood is thus much more attractive for such a purpose. However, as shown in Chapter II and in Chapter $\mathrm{V}$ and discussed there in more detail, a weak white-rot fungus such as 
P. ostreatus causing only low level of mass loss with a simultaneous high absolute glucose yield from the cellulose being laid open from its lignin-hemicellulose-cover might be a better organism for biodegradation of wood for biofuel production as compared to a more aggressive white-rot fungus such as $T$. versicolor that consumes much of wood biomass including parts of the cellulose which in total results then only in moderate glucose yields (Chapter V).

\section{VI.1.3 The possibility to use modification of wood cell walls to calculate stages of decay by fungi}

According to the results regarding modification of wood cell wall by fungi (Chapter III), extracellular oxidative enzymes secreted by fungi apparently could attack and modify lignin within the wood cell walls. The chemical proof to document the entire modifications of wood cell walls was done by staining with MBTH reagent (Chapter II and III). Since MBTH-staining locally correlated with likely the production of quinones from enzymatic decaying of lignin by laccase, the wood cell wall areas attacked by the enzyme could be evaluated by mathematical analysis based on results on the area fraction of stained cell walls $\left(\mathrm{A}_{\mathrm{S}}\right)$. Stages of decay by fungi can apparently be followed up by the MBTH staining method and the degree of decay can be evaluated by differences of $A_{S}$ value, which within year rings of $A$. grandis wood were found to decrease non-linearly in a slightly sigmoid form from the border of earlywood to the latewood region. Indeed, the turn in the slightly sigmoid curve correlated well with the switch from earlywood production to latewood production. Moreover, MBTH-staining allows identification of the stage of wood decay by fungi since the degree and distribution of MBTH-staining in the wood cell walls illustrate stages of decaying. However, during initial stages of wood decay by fungi, MBTH-staining was generally more enhanced by having higher $A_{S}$ values than final stages of decay being only faintly stained with MBTH, likely because of the progressed degradation of lignin and its degradation products and thereby the general decrement of the cell walls. In conclusion, integrating of $A_{S}$ values together with observations on reductions of cell wall widths makes it possible to identify specific stages of decay by fungi, which are selective on degradation of lignin. 


\section{VI.1.4 Enzymatic hydrolysis of wood and pretreated wood material for biofuel production and its technical improvement}

Wood extractives comprise a large number of individual compounds of both lipophilic and hydrophilic types. Different types of extractives refer to the diversity of biological functions of wood, such as phenolic substances can protect wood against microorganism or insect attack or give the wood its color and its odor and also can influence physical properties (Dorado et al. 2001; Fernandez et al. 2001; Sjostrom 1993). Specific phenolic compounds from wood extractive were found to induce laccase production, and wood extractives were found to inhibit growth of fungi (Chapter IV). On other hand, some specific compounds, such as $p$-coumaric acid, vanillic acid and ferulic acid inhibited the enzymatic hydrolysis process of cellulose by cellulases. Addition of laccase in cellulase hydrolysis could improve enzymatic hydrolysis (Chapter V). Enzymatic hydrolysis with addition of laccase improved in efficiency by increasing glucose yields, likely because laccase functions to decrease the amount of phenolic compounds in the wood or in partially decayed lignocellulosic material. As discussed above (Chapter VI.1.3), these interactions between wood extractives and/or also phenolic degradation products of the lignin and laccase and cellulases are important issues in future attempts for bioethanol biofuel production from wood, such as from $A$. grandis. Improvement of the individual steps in the process chain leading from the raw wood to the biofuel in order to gain the highest glucose yields for subsequent fermentative conversion e.g. into ethanol might emphasize first on the important step of wood pretreatment for decreasing the bio-organic negative-acting molecules such as lignin and wood extractives and their related phenolic compounds that act as inhibitors on enzymatic hydrolysis. From the work presented in this thesis, most advisable is to improve this step in the process.

In this study, several methods were tested to improve the yield of glucose production by cellulose, amongst were testing the effectiveness of different pretreatment technologies and optimizing strategies for enhancing enzymatic saccharification of cellulose. The conversion of cellulose to glucose reached up to $100 \%$ by using optimized conditions of two different types of wood pretreatment with an optimized enzymatic hydrolysis process (Chapter V). In a first approach, pretreatment of $1 \mathrm{~g}$ of $A$. grandis wood particles in $8 \mathrm{ml}$ of $85 \%$ phosphoric acid was performed by incubation for one hour at $50^{\circ} \mathrm{C}$ in a hot water bath. The material left in the resulting 
cellulosic pellet was then hydrolyzed by combinations of enzymes, each at a $4 \mathrm{U} \mathrm{ml}^{-1}$ in concentration. Enzyme mixtures included: cellulase + xylanase + laccase, cellulase + xylanase $+\beta$-glucosidase, cellulase + xylanase $+\beta$-glucosidase + laccase, cellulase + xylanase + laccase plus $1 \mathrm{mM}$ mediator HBT (1-hydroxybenzotriazole), and cellulase + xylanase $+\beta$-glucosidase + laccase plus $1 \mathrm{mM}$ HBT, all kept in $50 \mathrm{mM}$ sodium acetate buffer, pH 5.0 for one day of incubation at $37^{\circ} \mathrm{C}$. In a second approach, pretreatment of $1 \mathrm{~g}$ of $A$. grandis wood particles was done in $8 \mathrm{ml}$ of $85 \%$ phosphoric acid by using microwave irradiation for $20 \mathrm{sec}$. Then, the hydrolysis of the resulting cellulosic pellet was done at a concentration of $4 \mathrm{U} \mathrm{ml}^{-1}$ per enzyme, i.e. cellulase + xylanase + laccase in $50 \mathrm{mM}$ sodium acetate buffer, $\mathrm{pH} 5.0$ for one day of incubation at $37^{\circ} \mathrm{C}$ (Chapter $\mathrm{V}$ ). In this second approach, microwave irradiation as a wood pretreatment was improved on saving on energy and improving the energy efficiency in the pretreatment technologies. By applying the microwave irradiation as a cheapest form of energy source, by the easy use of microwave and by reducing the amount of hazardous acids during the irradiation, the microwave-chemical pretreatment was manifested by both economical and environmental aspects. The short time use of the microwave can generally save much higher energy amounts than the one hour use of hot bath incubation. In the case of microwave incubation, only $20 \mathrm{sec}$ irradiation was required in order to obtain from pretreated material the same glucose yields than from one hour incubation in a hot water bath at $50^{\circ} \mathrm{C}$ (Chapter V). The total energy consumption for wood pretreatment in a hot water bath and in a microwave included thermal energy for both chemical and physical-chemical pretreatments of rising temperatures. Generally, usage of thermal energy consumption is influenced by three factors: the liquid to wood ratio $(\mathrm{L} / \mathrm{W})$ and the pretreatment temperature and the time of pretreatment (Zhu et al. 2010). Another energy-consuming step is prior to the pretreatment when crushing the wooden material for pretreatment: the smaller the material is made the more energy will be needed. Zhu et al. (2010) in their recent review suggested that post-chemical pretreatment size reduction could be approached for further reducing energy costs and the authors explain the benefits for energy saving by avoiding to much mechanical breakup of wood as follows: i. eliminating the energy required for operations of chipping and milling steps to produce small size material, ii. lesser energy resources are needed to separate supernatants and the loosened cellulosic pellets that remain after wood pretreatment then chipping and milling of wood prior to the pretreatment; and iii. reducing the $\mathrm{L} / \mathrm{W}$ of a pretreatment due to reduced thermal energy for heating up 
water because larger wood particles (e.g. wood chips) have much less water intake than small wood particles (e.g. wood powder or mixed wood fibers). However, in this thesis work after energy consuming ball-milling of wood chips, only wood particles were used as a raw material for wood pretreatment for the release of cellulose as best as possible. Future experiments of process optimizing will include the use of bigger sizes of wood in order to improve microwave-chemical pretreatments through reducing the energy intense under final gain of highest glucose yields.

Within the last few years, the use of microwave irradiation as a type wood pretreatment has been reported from a few research groups (Li et al. 2010; Palm and Zacchi 2003). Palm and Zacchi (2003) for example reported on spruce wood chips sized 2-10 $\mathrm{mm}$ and incubated in a microwave under the condition of $0.09 \mathrm{~g}$ of dry wood $/ \mathrm{g}$ of water at a temperature at $180-230^{\circ} \mathrm{C}$ and a time of incubation for $2-10 \mathrm{~min}$. By this microwave treatment, the authors obtained $13 \%$ of total oligomeric mannose, glucose, and xylose from their material without any enzymatic hydrolysis. An important other advantage of microwave irradiation as compared with conventional steam treatment in the experiments performed by Palm and Zacchi (2003) was the easy possibility of maintaining always constant conditions. The amounts of chemical and water were fixed, and there was no loss of material in the whole pretreatment process. In the future, such advantages in microwaving larger wood chips might be combined with a subsequent enzymatic hydrolysis of the loosened cellulosic material left after such a microwave treatment.

Enzymatic hydrolysis is a part of the process from wood to biofuel that so far has least been approached for optimization of glucose yields from cellulose, also in terms of ecology and economy. Especially the costs for the enzymatic hydrolysis play an important role of barrier to commercialization of bio-based products. One major target to reduce the cost of biofuel production by biotechnological techniques is to find the optimal combinations of hydrolytic enzymes with synergistic enzymes for eliminating all the possible inhibitory compounds in enzymatic hydrolysis of cellulose. In this study, cellulase in combination with laccase or with $\beta$-glucosidase was shown to be best in order to gain the high glucose yields from cellulose hydrolysis (Chapter VI and V). A systematic future study should target at optimizing these enzyme combinations in terms of concentrations of enzymes, of volume per wood weight, time of incubation etc. With such studies it is expected to save on the overall amounts of enzymes required for biofuel production. However, parallel developments need to be 
done by other researchers for identifying new enzymes with better properties for application and for establishing better production processes of enzymes (Kilaru 2006; Rühl 2009), both of which will contribute to reduction of the overall costs.

\section{VI.2 General conclusions and prospects for future own studies}

Work in this thesis concentrated on $A$. grandis as a fast growing tree from North America that has been imported into Europe for possible fast wood production in plantations with promising excellent yields of wood (Polle et al. 2008). It was therefore very reasonable to test in this thesis the features of this wood in combination with wood decaying fungi and to study the effects of their lignolytic and cellulolytic enzymes on the wood, singular and in combination. For application of $A$. grandis in solid form as well as in processed form in wood composites, the knowledge gained in this thesis on fungal decay will be very valuable. Even more seminal are the studies on $A$. grandis wood for application in biofuel production.

Future own studies for upcoming applications will concentrate on the predominant wood industries in Thailand, especially in Southern Thailand, that rely economically mainly on rubberwood and palm tree plantations. Knowledge from this $\mathrm{PhD}$ thesis gained by studying $A$. grandis wood will be transferred and adapted to such wood species playing on important role in Thailand as the home country of the author of this thesis. As a result of the Thai wood industries, huge amounts of wood product residues and wood sawdust from the local tree species and fruit bunches of palm trees accumulate. Petpradab et al. (2009) reported that countries in Southeast Asia including Thailand are the biggest rubberwood producers in the world, making use of the wood for furniture and particleboard production. Surprisingly, only $10 \%$ of the total wood end up as products, while about $90 \%$ of the material represent wood residues. From this, a ratio of $54 \%$ comes from small branches, and a ratio of $32 \%$ wastes from wood sawmills and the $4 \%$ rest wood comes from furniture factories. In total, there is an annual availability of rubberwood residues of 1,100,000 tons from rubberwood plantations and sawmills for which optimal economical and ecological uses have to be found. Moreover, a palm oil research unit was established in 2008 in the province of Surat Thani, where the Prince of Songkla University (author work's office) is located, by implementing the province policy to develop in the next few years the Surat Thani province to be a "Palm city" (http://opru.surat.psu.ac.th). Also this development will 
lead to huge amounts of further waste materials that should economically und ecologically be made use of. According to the official Thai policy for generating sustainable biomass products, producing bioethanol from wastes sources of both tree species are highly interesting challenges, for Thailand in the near future's research, both for industry and for society.

The Asia Biomass Energy Cooperation Promotion Office (http://www.asiabiomass.jp/english/topics/0907_07.html) and other sources report that Thailand promotes the production of bioethanol from plant biomass. Due to national since the resolutions, since $1^{\text {st }}$ January of 2009 ethanol-gasoline-mixtures (gasohol) are for example promoted by the state. In the year 2008, daily 1.57 million liter of ethanol were produced, usually from sugar or starch. During the chemical conversion of the plant substrates, per liter ethanol 2,500 1 water are used, which represents a large current environmental problem for the Asian rivers. New techniques are looked for, in order to make the processes cheaper, more efficient and less weakening for the environment with replacing biomass competing with food and fodder by non-food and non-fodder material (agrarian and forest wastes) to finally result in environmental friendly techniques producing efficiently bioethanol in economical and social best way. At the "Prince of Songkla University" is a strong group of researchers supported by the Princess Sirinthorn that focus on research to newly develop such techniques to bioethanol production. As the university owns very good technical plants, testing new methods for the transformation of biomass e.g. from rubber wood and palm trees into bioethanol permits a possible up-scaling of laboratory processes to an industrial-scale. There is therefore a very good chance to integrate knowledge from this study and new perspectives into ongoing research at "the Prince of Songkla University" and to bring along novel techniques and ideas for the research present here.

\section{VI.3 References}

Altehel R (2007) Veränderte Stoffströme bei Sägeresthölzern: Auswirkungen auf den Holzmarkt. Kongress Rohholzmanagement in Deutshchland, Hannover (http:www.kompetenznetzholz.de/aktuelles/rohholzmanagement/vortraege/11_ Referat_Alteheld.pdf)

Call HP, Mücke I (1997) History, overview and applications of mediated lignolytic 
systems, especially laccase-mediator-systems (Lignozym ${ }^{\circledR}$-process). Journal of Biotechnology 53(2-3):163-202

Dadi AP, Schall CA, Varanasis S (2007) Mitigation of cellulose recalcitrance to enzymatic hydrolysis by ionic liquid pretreatment. Applied Biochemistry and Biotechnology 136-140/407-422

Dorado J, van Beek TA, Claassen FW, Sierra-Alvarez R (2001) Degradation of lipophilic wood extractive constituents in Pinus sylvestris by the white-rot fungi Bjerkandera sp. and Trametes versicolor. Wood Science and Technology 35(12):117-125

Eriksson KEL, Blanchette RA, Ander P (1990) Microbial and enzymatic degradation of wood and wood components. Springer-Verlag, Berlin

Fengel D, Wegener G (1983) Wood. Chemistry, ultrastructure, reactions. Walter de Gruyter, Berlin

Fernandez MP, Watson PA, Breuil C (2001) Gas chromatography-mass spectrometry method for the simultaneous determination of wood extractive compounds in quaking aspen. Journal of Chromatography A 922(1-2):225-233

Galbe M, Zacchi G (2007) Pretreatment of lignocellulosic materials for efficient bioethanol production. Advances in Biochemical Engineering Biotechnology $108: 41-65$

Hakala TK, Lundell T, Galkin S, Maijala P, Kalkkinen N, Hatakka A (2005)

Manganese peroxidases, laccases and oxalic acid from the selective white-rot fungus Physisporinus rivulosus grown on spruce wood chips. Enzyme and Microbial Technology 36(4):461-468

Hoegger PJ, Kilaru S, James TY, Thacker JR, Kües U (2006) Phylogenetic comparison and classification of laccase and related multicopper oxidase protein sequences. FEBS Journal 273:2308-2326

Hof C, Kielmann BC, Hapla F (2008) Verwendungsorientierte Untersuchungen am Schnittholz der Abies grandis. Holztechnologie 49:7-10

Johannes C, Majcherczyk A (2000) Natural mediators in the oxidation of polycyclic aromatic hydrocarbons by laccase mediator systems. Applied and Environmental Microbiology 66(2):524-528

Jørgensen H, Kristensen JB, Felby C (2007) Enzymatic conversion of lignocellulose into fermentable sugars: challenges and opportunities. Biofuels, Bioproducts and Biorefining 1:119-134 
Kilaru S (2006) Identification of fungal multi-copper oxidase gene families:

Overexpression and characterization of Coprinopsis cinerea laccases for applications in biotechnology. Dissertation, Cuvillier Verlag, Göttingen

Kloser L, Kües U, Schöpper C, Hosseinkhani H, Schütze S, Dantzl S, Malik I, Vos H, Bartholme M, Müller C, Polle A, Kharazipour A (2008) Panel board and conventional adhesive. In: Kües U. (Ed.) Wood Production, Wood Technology and Biological Impacts. Universitätsverlag Göttingen, Göttingen, 297-346

Li XJ, Zhou YD, Yan YL Cai ZY, Feng F (2010) A single cell model for pretreatment of wood by microwave explosion. Holzforschung 64(5):633-637

Müller G, Schöpper C, Vos H, Kharazipour A, Polle A (2009) FTIR-ATR spectroscopic analyses of changes in wood properties during particle- and fibreboard production of hard- and softwood trees. Bioresources 4(1):49-71

Palm M, Zacchi G (2003) Extraction of hemicellulosic oligosaccharides from spruce using microwave oven or steam treatment. Biomacromolecules 4:617-623

Polle A, Hawighorst P, Spellmann H (2008) Innovative Holzprodukte und Holzwerkstoffe aus nachhaltig produziertem Buchen- und Küstentannenholz. Holztechnologie 49:5-6

Palonen H, Viikari L (2004) Role of oxidative enzymatic treatments on enzymatic hydrolysis of softwood. Biotechnology and Bioengineering 86(5):550-557

Pan XJ, Xie D, Yu RW, Saddler JN (2008) The bioconversion of mountain pine beetlekilled lodgepole pine to fuel ethanol using the organosolv process.

Biotechnology and Bioengineering 101(1):39-48

Petchpradab P, Yoshida T, Charinpanitkul T, Matsumura Y (2009) Hydrothermal pretreatment of rubber wood for the saccharification process. Industrial \& Engineering Chemistry Research 48: 4587-4591

Röhrig E (1981) Neuere Grundlagen für den Anbau von Abies grandis. Schriften aus der Forstlichen Fakultät der Universität Göttingen und der Niedersächsischen Forstlichen Versuchsanstalt, Sauerländer Verlag, Frankfurt am Main, Germany Rühl M (2009) Laccase and other ligninolytic enzymes of the basidiomycetes Coprinopsis cinerea and Pleurotus ostreatus. Dissertation, Georg-August University, Germany

Sjostrom E (1993) Wood chemistry fundamentals and applications. Academic Press, San Diego

Spellmann H, Geb M, Nagel J, Nagel R, Schmidt M (2010) Timber product oriented 
silvicultural strategies for mixed strands of beech (Fagus silvatica) and grand fir (Abies grandis). Forst und Holz 65(1):12-19

Tadesse MA, D'Annibale A, Galli C, Gentili P, Sergi F (2008) An assessment of the relative contributions of redox and steric issues to laccase specificity towards putative substrates. Organic \& Biomolecular Chemistry 6(5):868-878

Vos H, Kharazipour A (2010) Properties of light particle boards manufactured using Abies grandis (grand fir). Forst und Holz 65(1):26-30

Wei DS, Houtman CJ, Kapich AN, Hunt CG, Cullen D, Hammel KE (2010) Laccase and its role in production of extracellular reactive oxygen species during wood decay by the brown rot basidiomycete Postia placenta. Applied and Environmental Microbiology 76(7):2091-2097 





\section{Curriculum Vitae}

$\begin{array}{ll}\text { First name: } & \text { Banyat } \\ \text { Surname: } & \text { Cherdchim } \\ \text { Date of birth: } & \text { 28 June } 1974\end{array}$

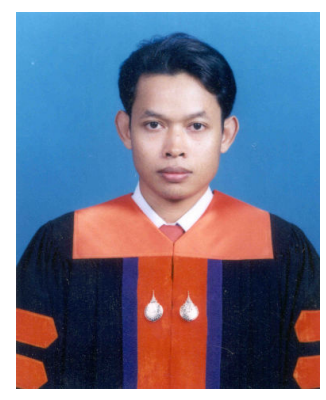

Nationality: Thai

\section{Contact address:}

Büsgen-Institut der Georg-August Universität Göttingen Abteilung für Molekulare Holzbiotechnologie und technische Mycologie, 37077 Göttingen, Büsgenweg 2

Tel.: 0551-3914085, Fax: 0551-392705

E-mail: bcherdc@gwdg.de

Faculty of Science and Industrial Technology

Prince of Songkla University, Surat Thani campus

Surat Thani, 84000, Thailand.

Tel.: +66-77355040, Fax: +66-77355041

Email: banyat.c@psu.ac.th

\section{Educational qualifications:}

B.Sc. in Physics

Prince of Songkla University, Thailand

M.Sc. in Physics (Material Science)

Walailak University, Thailand 
Work experience:

1997 - 2005: $\quad$ Scientist and Assistant Lecturer in Physics and Remote Sensing, Geographic Information System (GIS)

File Server and Network Administrator

2005 - Present Lecturer in Faculty of Science and Industrial Technology, Prince of Songkla University, Surat Thani Campus, Thailand

2006 - Present: $\quad$ Ph.D. student in Wood Biology and Wood Technology, GeorgAugust-University, Göttingen, Germany

\section{Research publications:}

1. W. Kurdthongmee and B. Cherdchim. 1998. Development of an Instrument to Measure the Velocity of Moving Objects for Physics Laboratory, pp. 992-993. In The $24^{\text {th }}$ Congress on Science and Technology of Thailand. 19-21 October 1998, The Science Society of Thailand under the Patronage of his Majesty the King and Mahidol University.

2. P. Kurdthongmee, B. Cherdchim, J. Kalakan and S. Eadklub. 1999. The Application of Electromagnetic Field in Searching for Natural Frequency of Wires, pp. 1240-1241. In The $\mathbf{2 5}^{\text {th }}$ Congress on Science and Technology of Thailand. 20-22 October 1999, The Science Society of Thailand under the Patronage of his Majesty the King and Naresuan University.

3. B. Cherdchim, N. Matan and B. Kyokong. 2001. Diffusion of Water in Black Sweet-Bamboo Culms (Dendrocalamus asper Backer), p. 761. In The $27^{\text {th }}$ Congress on Science and Technology of Thailand. 16-18 October 2001, The Science Society of Thailand under the Patronage of his Majesty the King and Prince of Songkla University.

4. B. Cherdchim, N. Matan and B. Kyokong. 2002. Investigation and Simulation of Water and Linseed Oil Movements in Black Sweet-Bamboo (Dendrocalamus asper Backer), pp. 124-126. In The Second Thailand Materials Science and Technology Conference: Materials Science and Technology for a Sustainable Development of Thailand. 6 - 7 August 2002, National Metal and Materials Technology Center MTEC. (Best award for presentation of a postgraduate research paper) 
5. B. Cherdchim, N. Matan and B. Kyokong. 2003. Thermal Softening of Black Sweet-Bamboo Culms (Dendrocalamus asper Backer) in Linseed Oil, p. 200. In The $29^{\text {th }}$ Congress on Science and Technology of Thailand. 20-22 October 2003, The Science Society of Thailand under the Patronage of his Majesty the King and Khon Kean University.

6. B. Cherdchim, N. Matan and B. Kyokong. 2004. Effect of Temperature on Thermal Softening of Black Sweet-Bamboo Culms (Dendrocalamus asper Backer) in Linseed Oil. Songklanakarin J. Sci. Technol 26(6):855-866

7. Kües U, Navarro-González M, Cherdchim B, Majcherczyk A (2009) Anfälligkeit von lebendem und totem Holz der Küstentanne gegenüber Pilzen. Mykologisches Kolloquium, Molekularbiologische Pilzdiagnostik, 7./8.Mai 2009 in Dresden, Institut für Holztechnologie, Dresden:1-14

\section{Poster:}

1. B. Cherdchim, M. Navarro-Gonzalez, I. Malik, A. Majcherczyk, H. Vos, A. Kharazipour, M. Rühl and U. Kües. Abbau von Holzwerkstoffen auf Küstentannenbasis durch Pilze. Poster presented on a symposium on sustainable use of natural raw material including wood. March 27-28 ${ }^{\text {th }}$, 2007. Oldenburg, Germany.

2. P. J. Hoegger, I. Malik, B. Cherdchim, A. Majcherczyk, M. Navarro-Gonzalez and U. Kües. Degradation of wood composites by fungi. VAAM 2007 Sonderausgabe, Osnabrück. April 1-4 ${ }^{\text {th }}, 2007$. Osnabrück, Germany. Abstract: Biospektrum Sonderausgabe, 2007, ISSN 0947-0867. Page 102

3. B. Cherdchim, M. Navarro- González, M. Zommorodi, A. Majcherczyk, and U. Kües. Fungal degradation of grand fir (Abies grandis) and induction of oxidative enzymes by wood extractives. VAAM 2008 Frankfurt, March 9-11 $1^{\text {th }}, 2008$. Frankfurt, Germany. Abstract: Biospektrum Sonderausgabe, 2008, ISSN 09470867. Page 138

4. B. Cherdchim, A. Majcherczyk, and U. Kües. Effects of wood extractives against fungal decay and induction of fungal laccase decaying Abies grandis wood. Poster presented on a symposium on biotransformation by fungal cells or fungal enzymes. September $30^{\text {th }}$-October $2^{\text {nd }}, 2010$. Senftenberg, Germany. 
Talks:

1. B. Cherdchim. Modification of wood fibers by phenoloxydases. Presented in the seminar of the $\mathrm{PhD}$ program Wood biology and wood technology. January $23^{\text {rd }}, 2007$. Göttingen, Germany.

2. B. Cherdchim. Effect of enzymatic treatment of wood and wood products. Presented in the seminar of the PhD program Wood biology and wood technology. June $13^{\text {th }}, 2007$. Göttingen, Germany.

3. B. Cherdchim. Induction of oxidative enzymes by wood extractives and actions of laccase on wood fibers. Presented in the Forest botany seminar of the Büsgen-Institute, Georg-August-University. May $5^{\text {th }}$, 2008. Göttingen, Germany.

4. B. Cherdchim. Conventional versus natural binder in wood. Presented in the seminar of the PhD program Wood biology and wood technology. June $24^{\text {th }}$, 2008. Göttingen, Germany.

5. B. Cherdchim. Wood extractives as a whole protect grand fir (Abies grandis) wood against enzymatic degradation by white-rot fungi but specific compounds induce laccases for attack on lignin. VAAM 2009, Ruhr-University Bochum, Bochum, Germany. March 8-11 ${ }^{\text {th }}, 2009$. Abstract: Biospektrum Sonderausgabe, 2009, ISSN 0947-0867. Page 48

6. B. Cherdchim. Influence of wood extractives on wood degradation. Presented in the Forest botany seminar of the Büsgen-Institute, Georg-August University. June $29^{\text {th }}, 2009$. Göttingen, Germany.

7. B. Cherdchim. Effects of fungi and of enzymes on Abies grandis wood degradation. Presented in the seminar of the PhD program Wood biology and wood technology. November $25^{\text {th }} 2009$. Göttingen, Germany.

8. B. Cherdchim. Biofuels from lignocellulose of wood. Presented in the Forest botanz seminar of the Büsgen-Institute, Georg-August University. January $18^{\text {th }}$, 2010. Göttingen, Germany.

\section{Other research projects:}

2000 -2005: $\quad$ Effects of Fluids and Heat Transport on Plasticization of Sweet-Bamboo Culms (Dendrocalamus asper Backer.) 
Award:

Best award for presentation of a postgraduate research paper at the Second Thailand Materials Science and Technology Conference: Materials Science and Technology for a Sustainable Development of Thailand, August 6 - 7 2002, Kasetsart University

\section{Overseas training experience:}

1. Data Studio Teaching with Technology course at PASCO Scientific Company Ltd., Roseville, CA, USA (2000)

2. National Science Centre, Malaysia (2001)

\section{Scholarships:}

- Prince of Songkla University, Thailand: August 2006-July 2009

- International student service, University of Göttingen: November 2009December 2009

- Scholarship with care achievement STIBET I (DAAD), University of Göttingen: December 2009-February 2010

- Katholischer Akademischer Ausländer-Dienst (KAAD): March 2010-April 2010

\section{Membership:}

- Katholischer Akademischer Ausländer-Dienst: KAAD ideelle Förderung

- Vereinigung für Allgemeine und Angewandte Mikrobiologie (VAAM)

\section{Fields of Interest:}

- Materials Science

- Wood Science

- Wood Physics

- Wood decay fungi

- Wood biofuels 
